Rudolf de Cillia | Jutta Ransmayr

\title{
Österreichisches \\ Deutsch macht \\ Schule
}

Bildung und Deutschunterricht im Spannungsfeld von sprachlicher Variation und Norm

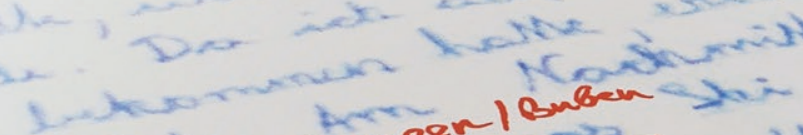

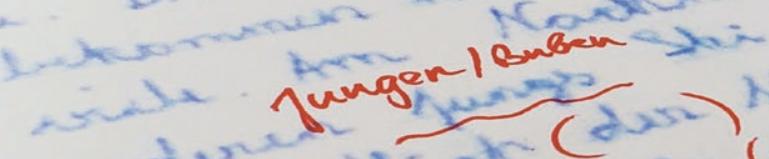

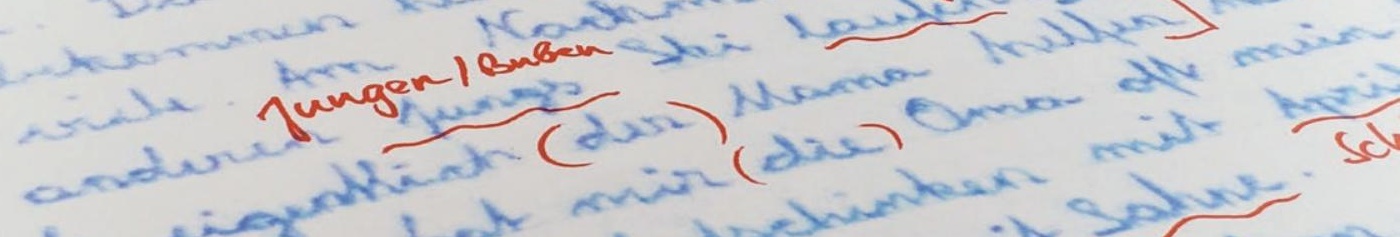

in sian

and

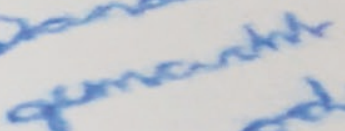
Ron bin

ivo 
콩 
Rudolf de Cillia/Jutta Ransmayr

\section{Österreichisches Deutsch macht Schule}

Bildung und Deutschunterricht im Spannungsfeld von sprachlicher Variation und Norm

unter Mitarbeit von Ilona Elisabeth Fink 
Der Wissenschaftsfonds.

\section{Veröffentlicht mit Unterstützung des Austrian Science Fund (FWF): PUB 579-G30}

Open Access:Wo nicht anders festgehalten, ist diese Publikation lizenziert unter der CreativeCommons-Lizenz Namensnennung 4.0; siehe http://creativecommons.org/licenses/by/4.0/

Diese Publikation wurde einem anonymen, internationalen Peer-Review-Verfahren unterzogen.

Bibliografische Information der Deutschen Nationalbibliothek: Die Deutsche Nationalbibliothek verzeichnet diese Publikation in der Deutschen Nationalbibliografie; detaillierte bibliografische Daten sind im Internet über http://dnb.de abrufbar.

(C) 2019 by Böhlau Verlag GmbH \& Co.KG, Kölblgasse 8-10, 1030 Wien

Bildnachweis: Foto Jutta Ransmayr

Satz und Layout: büro mn, Bielefeld

Druck und Bindung: Hubert \& Co. BuchPartner, Göttingen

Printed in the EU

Vandenhoeck \& Ruprecht Verlage I www.vandenhoeck-ruprecht-verlage.com 


\section{Inhalt}

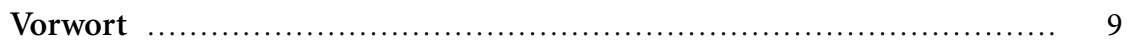

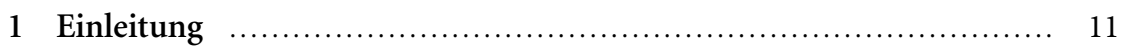

2 Theoretische Einordnung des Forschungsgegenstandes

Innere Mehrsprachigkeit - sprachliche Variation -

Sprach/en/unterricht

2.1 (Innersprachliche) Mehrsprachigkeit und sprachliche Variation .. 15

2.2 Status und Rolle/Funktion der deutschen Sprache in den deutschsprachigen Ländern/Regionen $\ldots \ldots \ldots \ldots \ldots \ldots \ldots \ldots \ldots . \ldots \ldots$

2.2.1 Die deutsche Sprache in Österreich $\ldots \ldots \ldots \ldots \ldots \ldots \ldots \ldots \ldots 20$

2.3 Sprachliche Variation und deutsche Sprache $\ldots \ldots \ldots \ldots \ldots \ldots \ldots . \ldots 22$

2.4 Konzeptualisierungen der Variation im Standarddeutschen ...... 25

2.4.1 Plurizentrik ....................................... 25

2.4.2 Pluriarealität .............................................. 32

2.4.3 Plurizentrisch - Pluriareal? .............................. 40

2.5 Sprachliche Variation der deutschen Sprache in Österreich ...... 47

2.6 Sprachnorm und Sprachenunterricht ........................ 53

2.7 Forschungslage zum österreichischen Deutsch als Unterrichtssprache und ExpertInnenbefragung $\ldots \ldots \ldots \ldots \ldots \ldots \ldots \ldots \ldots \ldots . \ldots \ldots$

2.7.1 Forschungslücken/Forschungsfragen $\ldots \ldots \ldots \ldots \ldots \ldots \ldots \ldots 60$

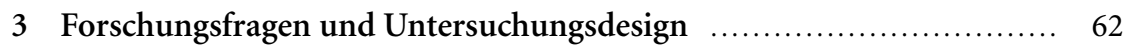

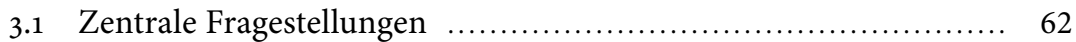

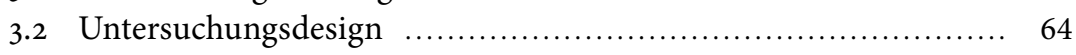

4 Analyse von unterrichtsrelevanten Dokumenten

(Lehrpläne, Studienpläne, Lehrbücher) ............................ 69

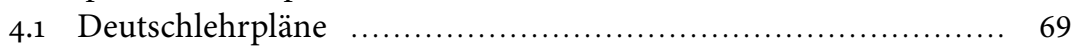

4.2 Studienpläne für die Ausbildung von DeutschlehrerInnen ....... 73

4.3 Deutsch-Lehrwerke ..................................... 76

4.4 Zusammenfassung der Lehrwerksanalysen $\ldots \ldots \ldots \ldots \ldots \ldots \ldots \ldots . \ldots 6$

4.5 Zusammenfassung der Dokumentenanalyse $\ldots \ldots \ldots \ldots \ldots \ldots \ldots . \quad 88$ 
5 Empirische Erhebung bei LehrerInnen und SchülerInnen an österreichischen Schulen

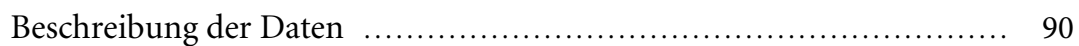

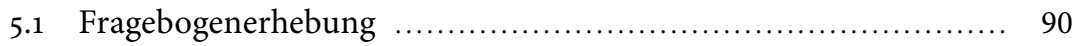

5.2 Fragebogenerhebung: Stichprobe der LehrerInnen .............. 94

5.3 Fragebogenerhebung: Stichprobe der SchülerInnen .............. 104

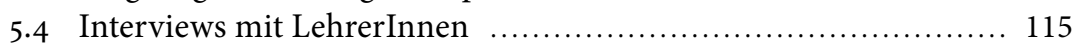

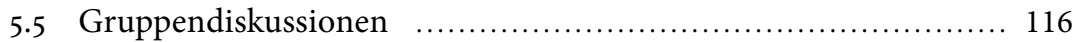

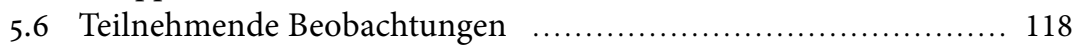

6 Ergebnisse der empirischen Erhebung an Schulen .................... 121

6.1 Konzeptualisierung der Variation des Deutschen in Österreich ... 121

6.1.1 Wie wird die Mehrheitssprache in Österreich benannt? .... 121

6.1.2 Mit welchen Varietäten wird österreichisches

Deutsch assoziiert? ....................................... 125

6.1.3 Unterschiede im Deutschen aus der Perspektive von LehrerInnen und SchülerInnen ..................... 132

6.1.4 Deutsch als plurizentrische Sprache? .................... 136

6.2 Spracheinstellungen gegenüber den Varietäten des Deutschen .... 145

6.2.1 Korrektheit des österreichischen Deutsch .................. 145

6.2.2 Einstellungen gegenüber dem österreichischen, deutschen und Schweizer Standarddeutsch: Polaritätsprofile .......... 153

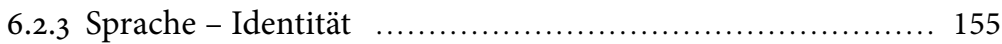

6.2.4 Zusammenfassung der Ergebnisse zu den Einstellungen gegenüber den Varietäten des Deutschen unter

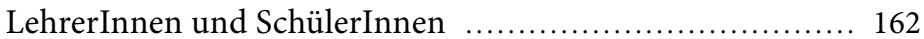

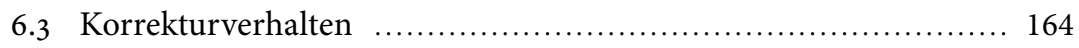

6.3.1 Normen und Korrektur bei schriftlicher Kommunikation

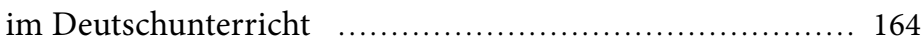

6.3.2 Normen und Korrektur bei mündlicher Kommunikation

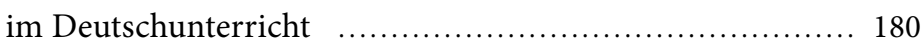

6.4 Sprachverwendung: Präferenz von Deutschlandismen/ Austriazismen

6.5 Dialekt - Umgangssprache - Standard? Angaben zum Varietätengebrauch innerhalb und außerhalb der Schule

6.5.1 Thematisierung des österreichischen Deutsch im Unterricht

6.6 Zusammenfassung der wichtigsten Ergebnisse der empirischen Erhebung an den Schulen 
7 Schlussbetrachtung und Ausblick f.................................. 222

7.1 Zusammenfassung der Ergebnisse ….......................... 222

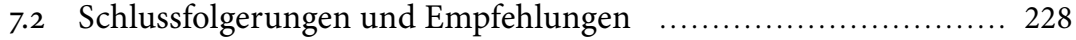

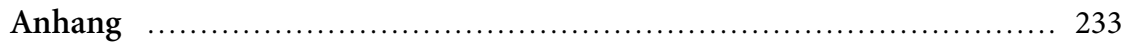

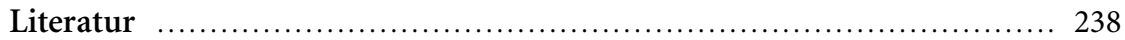

Verzeichnis der Tabellen und Abbildungen ................................. 253

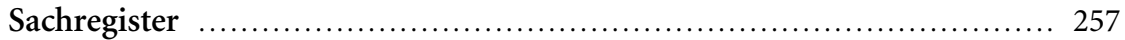




\section{Vorwort}

Ausgangspunkt für dieses Buch war ein vom FWF gefördertes Forschungsprojekt (FWF-Projekt Nr. P23913-G18) zur Rolle des österreichischen Deutsch als Unterrichts- und Bildungssprache. Zum Stellenwert des österreichischen Deutsch im Deutsch-als Fremdsprache-Unterricht im Ausland sind in den letzten Jahren eine Reihe von Studien durchgeführt worden, mit dem Ergebnis, dass sich das plurizentrische Konzept in der Fachdidaktik für Deutsch als Fremdsprache mittlerweile etabliert hat, die österreichische Varietät international aber trotzdem mit Dialektgleichsetzungen und einem Prestigedefizit kämpft. Wie aber geht es dem österreichischen Deutsch im Inland? Im Zuge unserer Erhebung hat ein Tiroler Lehrer Folgendes gesagt:

Also i denk, man kann durchaus a gewisses Selbstbewusstsein für des österreichische Deutsch entwickeln, und ... net dieses latente Minderwertigkeitsgefühl gegenüber dem bundesdeutschen Standard mit sich herumtragen, sondern es eben als eine durchaus auch legitime Variante sehen. ${ }^{1}$

Stellt so eine Aussage eine Einzelmeinung dar oder ist sie charakteristisch für die Einstellungen österreichischer LehrerInnen? Es war aus unserer Sicht an der Zeit, den Stellenwert des österreichischen Deutsch im heimischen Deutschunterricht näher zu untersuchen - nicht zuletzt deshalb, weil in der wissenschaftlichen Literatur seit geraumer Zeit ein sprachlicher Minderwertigkeitskomplex der ÖsterreicherInnen gegenüber ihren bundesdeutschen NachbarInnen geortet wird, der sich auch darin zeige, dass die ÖsterreicherInnen zwischen dem eigenen Sprachempfinden und einer bundesdeutschen Kontrastnorm hin- und hergerissen wären. Der Ort, an dem angesichts dessen jungen Menschen ein selbstbewusster Umgang mit dem österreichischen Deutsch vermittelt werden könnte, wäre sinnvollerweise der Deutschunterricht. Womit auch die Frage auf der Hand liegt, inwiefern das Plurizentrik-Konzept im „Muttersprachen“-Unterricht für Deutsch - oder präziser im Unterrichts- und Bildungssprachenunterricht - Beachtung findet und umgesetzt wird.

Über die Spracheinstellungen der LehrerInnen, die gemeinsam mit den im Unterricht verwendeten Unterrichtsmaterialien die Spracheinstellungen der SchülerInnen prägen, ist ebenfalls wenig bekannt. Nochmals allgemeiner formuliert: Wie wird innersprachliche Variation im Unterricht an österreichischen Schulen berücksichtigt und thematisiert? Ein Anliegen des Projekts war es, zu

1 Das Zitat stammt aus einem Interview, das im Rahmen des genannten Projekts durchgeführt wurde. 
untersuchen, inwiefern österreichisches Deutsch „Schule macht“, das heißt, welche Rolle es im Kontext von Unterricht und sprachlicher Bildung an Österreichs Schulen spielt.

Um diese Punkte zu klären, haben wir im Rahmen unseres Forschungsprojekts eine Reihe von empirischen Untersuchungen durchgeführt. Ein Kernstück der Untersuchung war die Analyse von Dokumenten wie Lehrplänen und Studienplänen sowie Deutsch-Lehrbüchern. Dafür, dass sie uns die Lehrbücher zur Verfügung gestellt haben, danken wir den betreffenden Schulbuchverlagen. Die Erhebungen für das Projekt wurden im Wesentlichen in den Schuljahren 2012/13 und 2013/14 durchgeführt. Die analysierten Lehrpläne, Studienpläne und Schulbücher (Kapitel 4) spiegeln den Stand in diesen beiden Schuljahren wider. ${ }^{2}$

Ein weiteres Kernstück des Datenmaterials war eine österreichweite Befragung von LehrerInnen und Oberstufen-SchülerInnen. Wir möchten uns an dieser Stelle bei den vielen LehrerInnen und SchülerInnen bedanken, die an der Fragebogenerhebung teilgenommen haben und die sich für Interviews und Gruppendiskussionen zur Verfügung gestellt haben. Unser Dank gilt auch jenen LehrerInnen und SchülerInnen, die damit einverstanden waren, dass wir sie im Unterricht beobachten.

Um all das organisieren zu können, war die Unterstützung der Schulbehörden unerlässlich. Wir danken den beteiligten DirektorInnen für ihr freundliches Entgegenkommen. Gleichzeitig möchten wir uns bei allen Landesschulräten in den Bundesländern und beim Wiener Stadtschulrat für die Erlaubnis bedanken, unsere Erhebungen an den Schulen durchführen zu können.

Unser Dank gilt auch Dagmar Gilly in Graz und Martin Kasy in Wien für die Organisation der Gruppendiskussionen. Für die Transkriptionsarbeiten und Dateneingaben danken wir Birgit Plöchl und Katharina Kranewetter.

Ganz besonderer Dank geht an Martina Maria Koller, die uns in statistischen Fragen mit Geduld und großer Umsicht betreut und beraten hat.

Wir danken zudem Ursula Huber vom Böhlau Verlag für die Betreuung des Buchprojekts.

Rudolf de Cillia

Jutta Ransmayr

Wien, im Herbst 2018

2 Bis zur Drucklegung des Buches wurden sowohl Lehrpläne als auch Schulbücher zum Teil überarbeitet bzw. werden derzeit überarbeitet. Auch neue Schulbücher sind zwischenzeitlich erschienen. Manche haben die Thematik „Variation in der deutschen Standardsprache“ und „Österreichisches Deutsch“ mittlerweile bereits aufgegriffen. So finden sich z. B. in den Lehrwerken Sprachräume 3 (öbv) und Kompetenz: Deutsch 7 (htp) Passagen, die die Themen „Österreichisches Deutsch“ und „Plurizentrik“ explizit und fachgerecht behandeln. 


\section{Einleitung}

Das vorliegende Buch befasst sich mit der Rolle und der Bedeutung des österreichischen Deutsch an österreichischen Schulen, vor allem mit der deutschen Sprache als Unterrichts- und Bildungssprache. Im Vordergrund steht daher die Standardsprache, „Hochdeutsch“, und zwar in ihrer Funktion als Medium des Unterrichts bzw. als Gegenstand des Deutschunterrichts, der einerseits „Muttersprachen "-Unterricht ${ }^{1}$, aber andererseits auch Zweit- oder Drittsprachenunterricht sein kann. Darüber hinaus werden auch andere Varietäten - dem tatsächlichen Sprachgebrauch in Österreich entsprechend - zur Sprache kommen.

Grundlage für dieses Buch war das im Vorwort erwähnte FWF-Projekt „Österreichisches Deutsch als Unterrichts- und Bildungssprache“. In einer ersten großen Säule des Projekts ging es zunächst um die Thematisierung von sprachlichen Normen und Normkonzepten sowie um den Themenkomplex der Konzeptualisierungsmodelle des Deutschen (wie z. B. der Plurizentrik und Pluriarealität), und um soziolinguistische Variation in Lehrplänen, in der Aus- und Weiterbildung für PädagogInnen sowie in der Unterrichtspraxis bzw. in Lehrwerken für Deutsch als Muttersprache/Bildungssprache. Was die LehrerInnenausbildung betrifft, lag bisher keine Analyse des Aspekts der sprachlichen Variation (staatsund regionsspezifische bzw. plurizentrische Standardvariation/österreichisches Deutsch/Varietätenbewusstsein/soziolinguistische Variation) in den Studienplänen vor. ${ }^{2}$ Das trifft auch auf die Lehrpläne für österreichische Schulen zu: In den Projektmodulen "Lehrplananalyse“ und „Studienplananalyse“ sollte daher die Frage beantwortet werden, ob und - wenn ja - wie sprachliche Variation (plurizentrische/staatsbezogene und andere soziolinguistische Varietäten) in den schulischen Lehrplänen und Studienplänen der DeutschlehrerInnenausbildung berücksichtigt wird. Das Projektmodul "Studienplananalyse“ wurde darüber hinaus mit einer exemplarischen Analyse des Lehrangebots an österreichischen Universitäten der Studienrichtung Germanistik (Lehramt) verknüpft (siehe Kapitel 4.1 und 4.2).

1 Für die Sprache, die die Kinder als erste erwerben, werden in der Literatur unterschiedliche Bezeichnungen verwendet: „Muttersprache“, „Erstsprache“, „L11, „Primärsprache“, manchmal auch „Familiensprache“ oder „Herkunftssprache“, wobei auch Bedeutungsunterschiede mit den Termini verbunden sind. Zweitsprache (L2) wird für eine Umgebungssprache verwendet, die erst später, hauptsächlich in der Form des ungesteuerten Spracherwerbs, erworben wird und die sich durch die Erwerbsform von einer eventuellen Fremdsprache unterscheidet, wobei es sich im konkreten Fall im Migrationskontext bei Deutsch auch um die Dritt- oder Viertsprache handeln kann (vgl. de Cillia 2013, auch www.schule-mehrsprachig.at [18.12. 2018]).

2 Zur Ausbildung von UnterrichtspraktikantInnen siehe jedoch Redl 2014. 
Was das Unterrichtsmaterial angeht, legte eine vor Projektbeginn durchgeführte Vorstudie den Schluss nahe, dass die gängigen Lehrwerke für den DaF-/ DaZ (Deutsch-als-Fremdsprache- bzw. Zweitsprache)-Unterricht weitgehend monozentrisch konzipiert sind. Dies war auch für den Deutsch-als-Muttersprache(DaM)-Bereich zu vermuten. Im Modul „Lehrbuchanalyse“ sollte durch eine Untersuchung der zu Projektbeginn (Schuljahr 2012/13) gängigsten Lehrbuchserien (je drei Deutsch-Lehrwerke aus Volksschule, Sekundarstufe I und Sekundarstufe II) diese Vermutung überprüft werden (siehe Kapitel 4.3).

Verschiedene LehrerInnen- und SchülerInnen-Befragungsmodule bildeten die zweite große Projektsäule. Es wurden unterschiedliche Methoden der Befragung eingesetzt: eine große Fragebogenerhebung mit 164 LehrerInnen bzw. 1.253 SchülerInnen, 21 Interviews mit LehrerInnen, je eine Gruppendiskussion mit LehrerInnen und SchülerInnen sowie das Instrument der teilnehmenden Beobachtung in sieben Schulklassen. ${ }^{3}$ Dabei wurden zum einen Angaben von Lehrkräften zur Unterrichtspraxis im Hinblick auf sprachliche Variation gesammelt (d.h. zu ihrer Ausbildung in Bezug auf Variation in der Sprache, zur Verwendung unterschiedlicher Varietäten im Unterricht, zur Thematisierung von sprachlicher Variation und zu Unterrichtskonzepten zur sprachlichen Variation, zu Materialien und Lehrbüchern, zum Umgang mit dem Kodex im Unterricht, zu Nachschlagwerken sowie zum Korrekturverhalten der LehrerInnen). Ein anderer Schwerpunkt der Befragungen befasste sich mit Spracheinstellungen der LehrerInnen und SchülerInnen gegenüber dem österreichischen Deutsch und dem deutsch(ländisch)en Deutsch und ihren Konzeptualisierungen der Variation der deutschen Sprache. Letzteres betraf sowohl die Konzeptualisierung der sprachlichen Variation im gesamt-deutschsprachigen Raum als auch innerhalb Österreichs. Es hat sich hier etwa die Frage gestellt, wie die ProbandInnen die in Österreich verwendete(n) Varietät(en) des Deutschen benennen (Deutsch/ Österreichisches Deutsch/Österreichisch/...). Von Interesse war auch, wie es um die Konzeptualisierung der deutschen Standardsprache bei LehrerInnen und SchülerInnen (monozentrisch, plurizentrisch/pluriareal) bestellt ist, d. h., ob sie die deutsche Standardsprache als einheitliche Sprache wahrnehmen oder ob sie eher drei gleichwertige Varietäten des deutsch(ländisch)en Standarddeutsch, des österreichischen Deutsch und des Schweizer Hochdeutsch sehen. Weiters galt es herauszufinden, welche Sprachform konkret mit dem österreichischen Deutsch assoziiert wird (standardnahe, umgangssprachliche, dialektale Varietäten). Eine häufig geäußerte Annahme, die ebenfalls in diesem Kontext Gegenstand der Untersuchung war, betrifft die Unterschiede zwischen Ost- und Westösterreich, die es nicht erlauben würden, von einem einheitlichen österreichischen Deutsch zu sprechen.

3 Eine detaillierte Darstellung und Begründung des Projektdesigns findet sich in Kap. 3.2. 
Einige weitere Fragen haben sich erst im Laufe der Forschungsarbeit während der ersten Projektsäule bei den Modulen Lehrbuchanalyse, Lehrplananalyse und Studienplananalyse herauskristallisiert und wurden daher zusätzlich ins Forschungsprogramm der Befragungsmodule aufgenommen. So hat etwa die Lehrbuchanalyse aufgezeigt, dass der Kodex des österreichischen Deutsch Schwächen und Lücken hat, die auch von anderen konstatiert worden sind. (vgl. Ender/Kaiser 2009, 268) ${ }^{4}$. Es erschien uns sinnvoll, die Gelegenheit zu nutzen und entsprechende Fragestellungen in die Erhebung mit aufzunehmen. Wir haben beispielhaft danach gefragt, wie sich das im Kodex beschriebene österreichische Standarddeutsch im Sprachgebrauch der LehrerInnen und SchülerInnen nach deren Selbstauskunft abbildet, wie LehrerInnen und SchülerInnen einige ausgewiesene bzw. präsumptive Austriazismen zu verwenden angeben.

Untersucht wurde auch, ob es wesentliche Unterschiede in der angegebenen Sprachverwendung, in den Spracheinstellungen und in den Konzeptualisierungen gibt - und ob zum Beispiel zwischen LehrerInnen und SchülerInnen sowie altersmäßig oder regional (und anhand von weiteren sprachlichen und außersprachlichen Variablen) Unterschiede feststellbar sind.

Eine wichtige, immer wieder in der Literatur formulierte Hypothese ist die, dass ÖsterreicherInnen eine geringe Sprachloyalität gegenüber der eigenen Varietät aufweisen (z. B. Clyne 1995, Muhr 2005b, Schmidlin 2011, Ammon/Bickel/ Lenz 2016), die eigene Varietät nicht als gleichwertig betrachten bzw. nicht als gleich korrekt wie deutsches Standarddeutsch, ja dass österreichische LehrerInnen exonorm-orientiert korrigieren würden, d.h., sich nach der deutschen Norm orientieren. Eine Reihe von Items in den Befragungsmodulen Fragebogen, Interviews und Gruppendiskussionen widmeten sich diesen Fragestellungen. Im LehrerInnen-Fragebogen ging es bei einer Frage explizit um das lehrerseitige Korrekturverhalten, in das wir anhand eines exemplarischen Schüleraufsatzes, der von LehrerInnen in Hinblick auf normative Sprachrichtigkeit sowie stilistische Angemessenheit korrigiert und kommentiert wurde, Einblick nehmen konnten. Auch das Spannungsfeld zwischen österreichischem Deutsch und nationaler Identitätskonstruktion wurde in diesem Kontext in den Blick genommen. Ein weiterer Teil der Erhebung befasste sich mit der Verwendung von unterschiedlichen Varietäten bei LehrerInnen und SchülerInnen, und zwar nicht nur in der Schule, sondern auch im Kontext Familie/Freundschaft. Ergänzt wurden diese Sprachverwendungsdaten schließlich mit der teilnehmenden Beobachtung in sieben Klassen in vier Bundesländern, die die Daten der Befragung komplettierten.

Die Befragung wurde, wie schon erwähnt, unter LehrerInnen und SchülerInnen durchgeführt. LehrerInnen und SchülerInnen stehen dabei zum einen natürlich für die jeweilige Gruppe und gleichzeitig auch für unterschiedliche

4 „Der österreichische Standard war und ist dabei relativ wenig kodifiziert.“ (Ender/Kaiser 2009, 268). 
Altersgruppen und Generationen. Auf der anderen Seite repräsentieren sie wiederum die Gruppe der linguistischen LaiInnen (SchülerInnen), denen die Berufsgruppe der professionell mit Sprache befassten Sprachnormautoritäten (LehrerInnen) gegenübersteht. Die Daten der beiden Gruppen können somit auch als Daten von LaiInnen/Professionellen und von unterschiedlichen Altersgruppen interpretiert werden. Insofern können die Ergebnisse - obwohl nur eine Querschnittstudie vorliegt - auch Auskunft über eventuelle generationsspezifische Sprachwandel- und Spracheinstellungsphänomene geben, zumal die Gruppe der LehrerInnen im Alter von 22-63 Jahren streut.

Das folgende Buch gibt - der Logik des Forschungsprozesses folgend - zunächst einen Überblick über die wissenschaftliche Literatur zu Fragen von Variation und Varietäten der deutschen Sprache. Auf ein Kapitel zu innersprachlicher Mehrsprachigkeit und sprachlicher Variation folgt eine Darstellung der Funktion und Rolle der deutschen Sprache in den deutschsprachigen Ländern. Die Variation der deutschen Sprache und die unterschiedlichen gängigen Formen der Konzeptualisierung (plurizentrisch - plurinational - pluriareal) werden anschließend dargestellt und ausführlich diskutiert. Nach einer Darstellung der Modellierung (Standard-Umgangssprache-Dialekt-Modell; Diglossie) und empirischen Beschreibung der sprachlichen Variation in Österreich werden Fragen rund um Variation, Standard und Norm im Unterricht besprochen. Schließlich kommen Befunde zum Deutschunterricht in Österreich aus der ExpertInnenbefragung im Rahmen der eingangs erwähnten Vorstudie zur Sprache.

Dem folgt eine explizite Formulierung der im Projekt verfolgten Forschungsfragen und überprüften Annahmen, des Forschungsdesigns, eine Darstellung der im Projekt verwendeten triangulierend eingesetzten Forschungsmethoden sowie die Darstellung der im Projekt erhobenen Datensätze. Anschließend werden die Ergebnisse der einzelnen Projektmodule präsentiert. Dem schließt sich eine Zusammenfassung an, die auf die anfangs formulierten Fragestellungen Bezug nimmt. Das Buch führt abschließend zu einem Ausblick, in dem Forschungsdesiderata benannt werden und vor allem Schlussfolgerungen für die Unterrichtspraxis gezogen werden. 


\section{Theoretische Einordnung des Forschungsgegenstandes}

Innere Mehrsprachigkeit - sprachliche Variation Sprach/en/unterricht

Das dem vorliegenden Buch zugrundeliegende Forschungsprojekt verortet sich einerseits in einer einem weiten Begriff von Mehrsprachigkeit verpflichteten Mehrsprachigkeitsforschung, und andererseits in der Variationslinguistik. Darüber hinaus befasst es sich mit der Vermittlung von Sprachnormen im Sprachunterricht. Deshalb sollen im Folgenden zentrale Konzepte und Begrifflichkeiten dargestellt werden, um die vorliegende Arbeit in Forschungstraditionen und Forschungsrichtungen einzuordnen. Dabei werden zunächst Mehrsprachigkeit, sprachliche Variation und innersprachliche Mehrsprachigkeit behandelt, bevor kurz die sprachliche Situation im deutschsprachigen Raum und genauer in Österreich skizziert wird. Anschließend werden zwei unterschiedliche Konzeptualisierungen bzw. Modellierungen der Variation der deutschen Sprache diskutiert - das plurizentrische und das pluriareale Modell -, bevor die Variation der deutschen Sprache in Österreich konkret thematisiert wird. Und schließlich kommt die Frage von Sprachnorm und Sprachenunterricht zur Sprache.

\section{1 (Innersprachliche) Mehrsprachigkeit und sprachliche Variation}

Gesellschaften sind so wie Staaten und Individuen prinzipiell mehrsprachig man denke nur daran, dass den ca. 2.50o bis 7.00o Sprachen der Erde (Störig 1987, Lewis/Simons/Fennig 2016) ca. 200 Staaten gegenüberstehen, oder an die neue Mehrsprachigkeit durch die weltweiten Migrationsbewegungen. Auch Schulen sind durch sprachliche Vielfalt gekennzeichnet (vgl. de Cillia 2013, 2010): Sie sind geprägt von fremdsprachlicher Mehrsprachigkeit, lebensweltlicher Mehrsprachigkeit von autochthonen Sprachminderheiten, von Gebärdensprach-Minderheiten und zugewanderten Minderheiten, die andere Sprachen als die Mehrheitssprache Deutsch als Erstsprache/Familiensprache verwenden. Während in Forschung, Didaktik und Medien vor allem diese „äußere“ Mehrsprachigkeit, besonders die lebensweltliche Mehrsprachigkeit der Migrationssprachen, thematisiert wird, ist das mit einer anderen Form der Mehrsprachigkeit - der inneren oder innersprachlichen oder „muttersprachlichen“ Mehrsprachigkeit, wie sie Mario Wandruzska genannt hat (Wandruszka 1979) - weniger der Fall. In den 1970er-Jahren war sie unter dem Vorzeichen „Dialekt und Sprachbarrieren“ noch intensiv diskutiert 
worden - heute ist sie in der Sprachdidaktik unseres Erachtens nach zu Unrecht kaum ein Thema (vgl. auch Ammon/Kellermeier 1997).

Mit innersprachlicher Mehrsprachigkeit ist die Tatsache gemeint, dass kompetente SprecherInnen einer Sprache über unterschiedliche Ausprägungen, sprich „Varietäten“ dieser Sprache verfügen - und gerade im deutschsprachigen Raum spielt diese innersprachliche Mehrsprachigkeit im schulischen Kontext eine wichtige Rolle: einerseits durch die so genannte Diglossie ,Dialekt - Standardsprache bzw. die Triglossie ,Dialekt - Umgangssprache - Standardsprache ${ }^{{ }_{11}}$ (siehe Kap. 2.3 und 2.5), andererseits durch die sprachliche Variation innerhalb der deutschen Hochsprache bzw. Standardsprache (der letzte Begriff wird im Folgenden vorwiegend verwendet), die einerseits mit dem Konzept der Plurizentrik mit den drei Varietäten des Schweizer Standarddeutsch, des österreichischen Standarddeutsch und des deutschen Standarddeutsch modelliert wird, andererseits mit dem Konzept der Pluriarealität (s. u.).

Dass die Variation des Deutschen in österreichischen Schulen etwa in Lehrplänen, Lehrmaterialien und auch im Unterricht kaum bis gar nicht berücksichtigt wird, ließen vor Projektbeginn die ExpertInnen-Einschätzungen aus der eingangs erwähnten Vorstudie sowie die Ergebnisse zweier Diplomarbeiten (Legenstein 2008, Heinrich 2010) vermuten. Wie Bildungsinstitutionen wie Schulen (und auch Kindergärten) mit mehr oder minder weit vom bildungssprachlichen Standarddeutschen entfernten Erstsprachen-Varietäten der Kinder, also mit deren innersprachlicher Mehrsprachigkeit, umgehen, beeinflusst die Bildungschancen der SchülerInnen. Denn die deutsche Sprache ist nicht nur die Erstsprache der Mehrheit der SchülerInnen - ihre zentrale Funktion im Bildungssystem ist die der Bildungssprache. Mit Bildungssprache ist ein formelles sprachliches Register mit spezifischen syntaktischen, lexikalischen und diskursiven Merkmalen gemeint, in dem man abstrakte und kognitiv anspruchsvolle Informationen unabhängig vom jeweiligen Kontext speichern und übermitteln kann; dasjenige sprachliche Register also, in dem man sich mit den Mitteln der Schulbildung ein grundlegendes Orientierungswissen verschaffen kann (Habermas 1977, Gogolin/Lange 2011).

Da sich die vorliegende Arbeit in erster Linie mit staatsbezogenen, nationalen Varietäten der deutschen Sprache befasst, seien im Folgenden zunächst Informationen zur Rolle und zum Status der deutschen Sprache in den entsprechenden Ländern gegeben.

1 Diglossie bezeichnet die Zweisprachigkeit innerhalb einer Gesellschaft/Sprachgemeinschaft, bei der es eine klare funktionale Differenzierung zwischen zwei eng verwandten Sprachvarietäten gibt, die unterschiedliches Prestige haben (High bzw. Low Language, vgl. Ferguson 1982/1959). Von Triglossie kann man in Parallelität dazu sprechen, wenn es sich um drei Varietäten handelt. 


\subsection{Status und Rolle/Funktion der deutschen Sprache in den deutschsprachigen Ländern/Regionen}

Das häufig so genannte "geschlossene deutsche Sprachgebiet in Mitteleuropa“ (Ammon 2006, 1765) der deutschen Sprache erstreckt sich auf die so genannten „Vollzentren“ der deutschen Sprache Deutschland, Österreich, die deutschsprachige Schweiz und die „Halbzentren“ Liechtenstein, die autonome Provinz BozenSüdtirol, Luxemburg und Ostbelgien (vgl. Variantenwörterbuch des Deutschen $\mathrm{VWB}=$ Ammon et al. 2004). In diesen Staaten bzw. Landesteilen ist die deutsche Sprache die Muttersprache der Mehrheit der Bevölkerung (mit Ausnahme von Luxemburg, wo dies Letzeburgisch ist) und die staatliche Amtssprache. Die relative Geschlossenheit des deutschen Sprachgebiets einerseits und die Verteilung auf mehrere angrenzende Staaten sowie der Varietätenreichtum andererseits erklären sich aus der historischen Entwicklung (zur Geschichte der deutschsprachigen Länder siehe Ammon 2006, 1765f). Die früher benutzte Bezeichnung „Binnendeutsch“ für die Varietät in Deutschland, der die „Peripherie“ in Österreich und der Schweiz gegenübergestellt wurde, ist heute linguistisch überholt. Die deutsche Sprache wird nicht als monozentrisch, sondern meist als plurizentrisch/plurinational oder pluriareal (s. u.) beschrieben.

Die sprachliche Situation im deutschsprachigen Raum ist durch eine reiche dialektale Gliederung gekennzeichnet, die von einer gemeinsamen Standardsprache überdacht wird. Dabei wird häufig eine schwer zu operationalisierende Zwischenebene „Umgangssprache“ (s. u.) angenommen. Die Dialekte werden einerseits nach den germanischen Stämmen bezeichnet (Alemannisch, Bairisch, Fränkisch, Sächsisch etc.), andererseits wird nach einem stark vereinfachten Schema das deutsche Sprachgebiet meist in nord-südlicher Richtung dreigeteilt (in Niederdeutsch für den Norden, Oberdeutsch für den Süden und Mitteldeutsch für das Gebiet dazwischen) und in west-östlicher Richtung zweigeteilt (also z. B. Westniederdeutsch für Nordniedersächsisch und West-/Ostfälisch) oder Ostmitteldeutsch (für Obersächsisch und Thüringisch) bzw. Ostoberdeutsch für das bairische Sprachgebiet (vgl. die Karte bei Ammon 2008, 160). Die Nord-SüdEinteilung erfolgt im Wesentlichen nach den Ergebnissen der Hochdeutschen Lautverschiebung (zweite Lautverschiebung) und einigen anderen lautlichen und morphologischen Unterschieden, während die Ost-West-Einteilung nach unterschiedlichen Merkmalen der Dialekte erfolgt. Der erste Teil der Hochdeutschen Lautverschiebung [harte Verschlusslaute - stimmlose Plosive wurden entweder zu Reibelauten (Frikativen) oder einer Kombination von Verschluss- und Reibelaut (Affrikaten), also p zu f/pf; t zu s/tz, k zu ch/kch; z. B. Ap zu Affe, Appel zu Apfel usw.] wurde für die Einteilung des deutschen Sprachgebiets besonders wichtig: Er wurde im Oberdeutschen vollständig, im Mitteldeutschen teilweise und in den niederdeutschen Dialekten gar nicht durchgeführt (Ammon 2008, 158f). Die so genannte Benrather Linie ist die bekannteste Isoglosse (Grenze zwischen 
zwei Ausprägungen eines sprachlichen Merkmals), welche die Grenze zwischen Nieder- und Mitteldeutsch auffächert. Sie ist insofern wichtig, als die deutsche Standardsprache im Wesentlichen auf den mittel- und oberdeutschen Dialekten beruht, die man terminologisch auch als „Hochdeutsch“ zusammenfasst. Anders verhält es sich mit „Niederdeutsch“, das im Jahr 1999 durch die europäische Charta der Regional- und Minderheitensprachen - linguistisch nicht unumstritten - als Regionalsprache anerkannt wurde, aber über keine Standardvarietät verfügt und vom Sprachgebiet her im Wesentlichen auch vom Standarddeutschen überdacht wird. Beim Terminus „Hochdeutsch“ schwingt auch eine Bewertung und soziale Zuordnung mit. Im vorliegenden Kontext wird vorwiegend der Terminus „Standarddeutsch" (s. u.) verwendet. Eine allgemeine Beschreibung von grammatischen Strukturen, Wortschatz und orthographischen Merkmalen der deutschen Sprache findet sich bei Ammon 2008 (162-169). Auf der Ebene der Standardsprache wird die deutsche Sprache im Übrigen als besonders varianten- und varietätenreich charakterisiert.

In Deutschland ist Deutsch die Amtssprache. Deutsch ist in dieser Funktion zwar nicht in der Verfassung verankert, aber z. B. in $\$ 23$ Verwaltungsverfahrensgesetz als solche festgelegt. Von den ca. 82,2 Mio. EinwohnerInnen im Jahr 2015 waren 8,7 Mio. AusländerInnen. 91\% der EinwohnerInnen besaßen die deutsche Staatsbürgerschaft (Statistisches Bundesamt 2017). Für die Verleihung der deutschen Staatsbürgerschaft sind Kenntnisse der deutschen Sprache nachzuweisen (auf dem Niveau A 2+/B1 des Gemeinsamen europäischen Referenzrahmens für Sprachen, GER). Im Unterschied zu Österreich und der Schweiz hat die deutsche Sprache in der Bundesrepublik Deutschland keine ausgeprägte nationalsymbolische Funktion. Die regionale sprachliche Differenzierung der sprachlichen Variation innerhalb Deutschlands erfolgt in sechs Regionen (Deutschland-Nordost/DeutschlandNordwest/Mittelost/Mittelwest/Südost/Südwest, Ammon et al. 2004). Was das Verhältnis von Standard zu Dialekt betrifft, spricht man von „Dialektschwundgebieten " im Norden (Ammon 2006, 1768) und einem Dialekt-Standard-Kontinuum im mittel- und süddeutschen Raum (wie auch in Österreich und Südtirol): In der Privatsphäre wird eher der Dialekt verwendet, in öffentlichen Sprachsituationen und schriftlich in der Regel eher Standard, wobei der Dialektgebrauch zum Teil schichtspezifisch verteilt ist. Auch die Standardsprache ist durch den Akzent regional gekennzeichnet. Neuere Forschungen nehmen überregionale Sprechstandards an und versuchen diese empirisch zu erfassen (s. u.).

In der Schweiz sind von den 26 Kantonen der offiziell viersprachigen Schweiz 17 deutschsprachig, drei zweisprachig (Deutsch, Französisch) und einer dreisprachig (Deutsch, Italienisch, Rätoromanisch). Die deutsche Sprache ist nach der Bundesverfassung der Schweizerischen Eidgenossenschaft von 1999 eine der vier „Landessprachen“ (neben Französisch, Italienisch, Rätoromanisch, Artikel 4) und eine von drei „Amtssprachen“ (neben Französisch und Italienisch, Artikel 70), das Rätoromanische hat eine eingeschränkte Rolle als Amtssprache. Nach 
der Volkszählung von 2000 waren 63,7\% der Wohnbevölkerung und 72,5\% der StaatsbürgerInnen deutschsprachig (Französisch: 21,0 \%; Italienisch: 4,3 \%; Rätoromanisch: o,6 \%; Anderssprachige: 1,6 \%; Lüdi/Werlen 2005, 8). Für den Erwerb der Staatsbürgerschaft durch Einbürgerung ist der Nachweis von Kenntnissen der Sprache des jeweiligen Landesteils erforderlich - die konkrete Umsetzung regeln die Gemeinden. Die Standardvarietät des Schweizerhochdeutsch fungiert „nur als sekundäres Nationalsymbol“" (Ammon 2006, 1767) - die zentrale identitätsbildende Rolle übernimmt in der Schweiz der jeweilige Dialekt des Schweizerdeutschen (Schwyzerdüütsch). Das deutschsprachige Gebiet der Schweiz ist von einer ausgeprägten Diglossie zwischen Dialekt und Standardvarietät gekennzeichnet: Es gibt keinen fließenden Übergang von der einen Sprachform zur anderen. Der Dialektgebrauch herrscht im Mündlichen in allen sozialen Schichten vor, die Standardvarietät bleibt auf einige wenige eher förmliche Domänen beschränkt (z. B. Predigt, Uni-Vorlesung, überregionale Rundfunknachrichten). Der Dialekt ist die Sprachform der Nähe, der Standard die Sprache der Distanz. Laut Volkszählung 2000 sprachen 90,8 \% der DeutschschweizerInnen in der Familie Dialekt, $91 \%$ aller Erwerbstätigen benutzten ihn im Beruf, davon rund $45 \%$ ausschließlich (Haas 2006, 1778), und es benutzten 6,8 \% Hochdeutsch, 37,5\% Schweizerdeutsch und 55,2\% Schweizerdeutsch und Hochdeutsch als Schulsprache (Lüdi/Werlen 2005, 84). Dabei ist das Schweizerdeutsch stark regional differenziert - es existiert keine „Ausgleichsmundart", die als überregionaler Dialekt fungieren könnte (Haas, 2006, 1779), obwohl sich eine gewisse Annäherung der Dialekte feststellen lässt, wie ExpertInnen meinen.

In Liechtenstein ist Deutsch solo-offizielle Amtssprache und Nationalsprache, ohne dass es eine Verankerung in der Verfassung gäbe. Von den ca. 33.00o EinwohnerInnen sind $34 \%$ AusländerInnen, davon ein Teil wiederum deutschsprachig. Die Standardsprache ist vom Standarddeutsch der Schweiz beeinflusst, was sich z. B. bei Besonderheiten im Wortschatz zeigt. Auch die Diglossie zwischen Standard und Dialekt ist ähnlich jener in der Schweiz.

In Südtirol ist Deutsch die regionale staatliche Amtssprache der Autonomen Provinz Bozen-Südtirol. Bei der Volkszählung 2001 gehörten 69,41 \% der deutschsprachigen Bevölkerung, 26,06\% der italienischsprachigen und 4,53\% der ladinischsprachigen Bevölkerung an (Landesinstitut für Statistik, 2012). Einflüsse des Italienischen zeigen sich v. a. in der amtlichen Terminologie und in Form von Entlehnungen (z. B. Hydrauliker für Installateur) und Lehnübersetzungen. Die Standardsprache ist die Form der schriftlichen Kommunikation und bei formellen mündlichen Anlässen. Privat wird vor allem Dialekt gesprochen, wobei eher eine diglossale Situation festzustellen ist als ein Dialekt-Standard-Kontinuum.

In Luxemburg ist Deutsch eine nationale staatliche Amtssprache neben Französisch und Letzeburgisch - Letzteres hat die Funktion einer Nationalsprache (Ammon 2006, 1765). Deutsch ist Einschulungssprache und neben Französisch Schulsprache. Die Standardsprache ist nahe dem Letzeburgischen, das durch 
Ausbau aus einem moselfränkischen Dialekt entstanden ist. Einflüsse in Form von Entlehnungen gibt es v. a. aus dem Französischen. In Ostbelgien schließlich ist Deutsch regionale staatliche Amtssprache der „Deutschsprachigen Gemeinschaft“, die Teil der Wallonie ist. Rund 71.00o SprecherInnen des Deutschen machen 1,1\% der Bevölkerung Belgiens aus. Mündlich und schriftlich herrscht der Standard vor, teilweise auch in der Privatsphäre; es gibt ein Dialekt-Standard-Kontinuum und Einflüsse der französischen Sprache.

Thema der vorliegenden Publikation ist die Variation der deutschen Sprache in Österreich - daher folgt nach diesem Exkurs ein Abriss zur Rolle der deutschen Sprache in Österreich.

\subsubsection{Die deutsche Sprache in Österreich}

In Österreich ist Deutsch (im Sinn der im Alltag vorwiegend verwendeten Sprache $^{2}$ ) die „Umgangssprache“ der überwiegenden Mehrheit der Bevölkerung, und die sprachenrechtlichen Rahmenbedingungen sichern der deutschen Sprache eine besondere Stellung zu: Sie ist nach Artikel 8, Absatz (1) der Bundesverfassung von 1920 die Staatssprache: „Die deutsche Sprache ist, unbeschadet der den sprachlichen Minderheiten bundesgesetzlich eingeräumten Rechte, die Staatssprache der Republik." Weitere gesetzliche Regelungen, die die deutsche Sprache betreffen, finden sich im Schulrecht: Deutsch ist (mit Ausnahme der Regelungen für die autochthonen Minderheiten) die vom Gesetz festgelegte Unterrichtssprache ( $\$ 16$ Abs. 1 Schulunterrichtsgesetz SchUG). Weiters finden sich im Zuwanderungsrecht und im Staatsbürgerschaftsrecht relevante Bestimmungen: Sowohl für die österreichische Staatsbürgerschaft als auch für einen dauerhaften Aufenthalt von Angehörigen aus so genannten Drittländern ist der Nachweis von Kenntnissen der deutschen Sprache auf dem Niveau B1 des GER erforderlich. Die spezifisch österreichische Varietät des Deutschen ist im Übrigen durch das „Protokoll Nr. 10 über die Verwendung spezifisch österreichischer Ausdrücke der deutschen Sprache im Rahmen der Europäischen Union“ rechtlich verankert. Darin wird festgehalten, dass die 23 im Protokoll aufgelisteten spezifisch österreichischen Ausdrücke der deutschen Sprache (z. B. Faschiertes, Marille oder Topfen) „den gleichen Status [haben] und mit der gleichen Rechtswirkung verwendet werden [dürfen], wie die in Deutschland verwendeten entsprechenden Ausdrücke“ (vgl. de Cillia 1995; 1997; 1998, 78 ff; 2006).

2 Die bis 2001 im Abstand von zehn Jahren durchgeführten Volkszählungen in Österreich fragten nach der „Umgangssprache“ in diesem Sinn: „Geben Sie bitte jene Sprache (auch mehrere Sprachen) an, die Sie gewöhnlich im privaten Bereich (Familie, Verwandte, Freunde usw.) sprechen. Fremdsprachenkenntnisse sind hier nicht anzugeben. Bei Personen, die (noch) nicht sprechen können, wird die in ihrer Familie gesprochene Umgangssprache angeführt" (Statistik Austria 2002). 
Die letzte Volkszählung, die die Umgangssprachen der Bevölkerung erhoben hat, fand 2001 statt - jüngere, für ganz Österreich repräsentative Daten gibt es nicht. 2001 gaben ca. 88,6\% der Wohnbevölkerung an, (ausschließlich) Deutsch als Umgangssprache zu sprechen, 8,6\% Deutsch und eine andere Sprache und 2,8\% ausschließlich nicht Deutsch (vgl. de Cillia/Wodak 2006). Betrachtet man nur die österreichischen StaatsbürgerInnen, so ergibt sich, dass die Deutschsprachigen 95,5\% ausmachen. Aktuellere Daten gibt es nur für die Gruppe der 25- bis 64-jährigen (das waren zum Zeitpunkt der Erhebung 4.685.30o Personen), die im Rahmen der repräsentativen AES(Adult Education Survey)-Studie 2011/12 erhoben wurden und bei der nach den Erst- und Zweitsprachen gefragt wurde, wobei Mehrfachangaben möglich waren. In dieser Studie gaben 84,1 \% der Befragten an, Deutsch zu sprechen ${ }^{3}$ (Statistik Austria: Erwachsenenbildungserhebung 2011/2012 [AES], 45).

Der symbolische Wert der österreichischen Varietät der deutschen Sprache ist im Rahmen der nationalen Identitätskonstruktionen relativ hoch zu bewerten (vgl. de Cillia 2012; 2015). Hinweis darauf ist etwa die Tatsache, dass bereits 1951 ein Österreichisches Wörterbuch als eigenes, in Österreich gültiges Nachschlagwerk zur Dokumentation der sprachlichen Unabhängigkeit von Deutschland erstellt wurde. Darüber hinaus ist noch einmal auf das oben erwähnte Protokoll hinzuweisen (siehe dazu de Cillia 1995, 1997, 2006).

Die deutsche Standardsprache wird in der schriftlichen Kommunikation sowie bei formellen mündlichen Anlässen (wie Vorlesungen, Predigt, Rundfunknachrichten) verwendet, während in der privaten Kommunikation eher dialektale Varietäten und Umgangssprache zum Einsatz kommen. Es existiert ein informeller Standard, der umso ausgeprägter ist, je informeller die Situation ist (Ammon et. al. 2004). Daneben ist die sprachliche Situation im Süddeutschen durch ein Dialekt-Standard-Kontinuum gekennzeichnet - Letzteres gilt im alemannischen Vorarlberg nur beschränkt. Regional wird der Sprachgebrauch z. T. im Standard, v. a. aber in der Umgangssprache noch nach ostösterreichisch, westösterreichisch, südostösterreichisch und österreichische Mitte differenziert (Ammon et. al 2004, XXXVII) - im vorliegenden Projekt werden fünf Regionen angenommen (siehe Kap. 2.5).

3 Die weiteren Sprachen waren Türkisch (5,1\%), Serbisch (3,5\%), Englisch (2,8\%), Kroatisch (2,4\%), Bosnisch (2,1\%), Rumänisch (1,3\%), Polnisch (1,1\%), Russisch ( $0,7 \%)$, Ungarisch ( $0,7 \%)$, Tschechisch/Slowakisch (o,6\%), Slowenisch (o,4\%), Arabisch (0,4\%), Italienisch (o,4\%) und Spanisch $(0,3 \%)$. 


\subsection{Sprachliche Variation und deutsche Sprache}

Die deutsche Sprache wird - wie schon erwähnt - als eine an Varianten und an Varietäten besonders reiche Sprache beschrieben. So etwa von Barbour/Stevenson (1998) in ihrem Buch „Variation im Deutschen“: „Das Deutsche ist wahrscheinlich die vielgestaltigste Sprache Europas“ (11). Als Grund dafür wird häufig die völlig andere historische Entwicklung des Deutschen im dezentralen deutschsprachigen Raum ins Treffen geführt, im Vergleich etwa zum Englischen mit dem Zentrum London, zum Französischen mit der Île de France, Italien mit der Toskana und Florenz als Zentrum oder Spanien mit Kastilien und Burgos (Protze 2001, 505). Löfler (2005) stellt fest, die „deutsche Gemeinsprache“ sei „ein Kunstprodukt und nicht die Sprache eines politischen und kulturellen Mittelpunktes eines Hofes oder einer Hauptstadt“ (25).

Unter sprachlichen Varietäten verstehen Dittmar/Schmidt-Regener $(2001,521)$ „funktional voneinander geschiedene, konstitutive Subsysteme des Gesamtsystems einer Sprache“. Diese seien „theoretisch idealisierte Konstrukte, die inventarisieren, welche Realisierungen von Sprache in Abhängigkeit von der Sprachgebrauchssituation systematisch zu erwarten und als solche auf allen Ebenen des Sprachsystems beschreibbar sind (Phonologie, Grammatik, Lexik).“

Dabei kann man nach extralinguistischen Variablen zahlreiche unterschiedliche Varietäten unterscheiden: nach geographischen, diatopischen (räumlichen) Kriterien Dialekte, nach diastratischen, schichtspezifischen Kriterien Soziolekte, nach staatlichen/regionalen Kriterien plurizentrische oder pluriareale Varietäten, nach funktionalen Kriterien Fachsprachen, nach situationellen Kriterien Register, nach dem Geschlecht genderspezifische Variation oder nach dem Alter z. B. "Jugendsprache“.

Eine Abgrenzung der Varietäten voneinander ist schwierig. Die Variation auf der horizontalen Ebene kann letztlich als ein Kontinuum mit Kern- und Übergangszonen modelliert werden, deren Kernzonen von einem gewissen Ausmaß an Stabilität und Homogenität gekennzeichnet sind und die man als Standardvarietät, Umgangssprache(n) und Dialekt(e) bezeichnet. Dabei wird die Standardsprache auch als „Hochsprache“, „Schriftsprache“ oder „Gemeinsprache“ bezeichnet. Die Standardsprache ist der eine Extrempunkt eines Kontinuums. Das Kontinuum von lokalen über regionale Dialekte bis hin zu diesem Standard wird häufig als „Nonstandard“ oder, wertender, als „Substandard“ bezeichnet.

Im schulischen Kontext und bei der Vermittlung der Bildungssprache, in dem sich die vorliegende Forschungsarbeit positioniert, geht es in erster Linie um die Vermittlung der Standardvarietäten, zumindest was die Vorgaben in Lehrplänen und Lehrmaterialien angeht. Aber man muss sich dessen bewusst sein, dass die Grenzen zwischen den Varietäten fließend sind und dass das Ziel

4 Wie komplex eine Modellierung der Varietäten sein kann, zeigt eine Graphik bei Löffler 2005, 19. 
schulischen Sprachenunterrichts letztlich darin besteht, eine kommunikativ und situativ adäquate Verwendungskompetenz der jeweiligen Varietäten zu vermitteln (s. u.).

Unter einer Standardvarietät wird nach Dittmar/Schmidt-Regener (2001) „das Subsystem einer Sprache verstanden, dessen Normen den höchsten Verbindlichkeitsgrad für alle Angehörigen einer politisch definierten Kommunikationsgemeinschaft besitzen, da sie in Regelwerken kodifiziert und deshalb präskriptiv sind." Die Standardsprache werde geschrieben, besitze überregionale Reichweite und Gültigkeit, werde vorzugsweise in institutionellen Kontexten und offiziellen Kommunikationssituationen benutzt und erscheine in der Alltagssprache niemals in ihrer idealtypisch kodifizierten Norm (521f). Und weiter: „Standardsprache oder Standardvarietät ist die Bezeichnung für eine kodifizierte Sprache, die ihre Verbindlichkeit als offizielle Nationalsprache eines Staates erhält und in der Regel prestigebesetzt ist“ (a. a. O., 525). Die wertende Bezeichnung „Hochsprache“ werde in diesem Zusammenhang als Synonym verwendet.

Die (korrekten) Normen einer Standardsprache werden nach Ammon (2005) von so genannten „normsetzenden Instanzen“ gesetzt. Das Setzen von Normen mache diese gültig, und die Gültigkeit der Normen erlaube oder gebiete es Normautoritäten, von „Normsubjekten“ die Normbefolgung zu verlangen. Normen von Nonstandardvarietäten würden informell gesetzt (Ammon 2005, 32). Als solche normsetzenden Instanzen führt Ammon ModellsprecherInnen oder -schreiberInnen an, z. B. NachrichtensprecherInnen in Massenmedien, SchauspielerInnen oder SchriftstellerInnen. Auch Modelltexte fallen in diese Kategorie. Eine zweite Instanz sei der „Sprachkodex“ ${ }^{\text {5 }}$. Kodizes, Wörterbücher, Regelbücher und Grammatiken seien autoritative Nachschlagwerke für den "korrekten, also standardsprachlichen Gebrauch“. Diese Kodizes müssten nicht unbedingt präskriptiv sein, heute sei eher ein "deskriptiver Bescheidenheitsgestus" typisch (35). Die dritte standardsetzende Instanz seien die „Sprachexperten“, sprachwissenschaftliche Fachleute, und die vierte Instanz seien „Sprachnormautoritäten“, d.h. alle Personen, „die über ausreichende Macht verfügen [...], um das Sprachhandeln anderer Personen (der Normsubjekte) zu korrigieren.“ (36). Solche „Sprachnormautoritäten“ sind nach Ammon primär LehrerInnen, AmtsvorsteherInnen, VerlagslektorInnen oder RedakteurInnen. Für den Sprachstandard sei es typisch, dass die Hierarchie der Normautoritäten letztlich bis zum/zur staatlichen Souverän/in hinaufreiche.

5 An anderer Stelle bezeichnet Ammon mit Kodex , alle Beschreibungen der betreffenden Sprache, auf die sich Amtspersonen (z. B. Lehrer oder Beamte und Angestellte in staatlichen Ämtern und Behörden) rechtens beziehen können, um die von ihnen verwendeten oder vorgeschriebenen Sprachformen als korrekt zu legitimieren“. Er schränkt jedoch ein, dass die ausdrückliche Legitimation einer Sprachform durch den linguistischen Kodex keine notwendige Bedingung für die Zugehörigkeit einer Sprachform zur Standardvarietät ist, denn kein linguistischer Kodex könne die Standardvarietät einer Sprache, die sich ständig weiterentwickelt, vollständig beschreiben (Ammon 1991, 19). 
Daher würden sich die Gültigkeitsbereiche der Normen mit den Staatsgrenzen (z. B. Österreich) decken (39).

In Modellierungen der regionalen, diatopischen Variation des Deutschen wird nach Spiekermann $(2010,346)$ traditionell zwischen drei Ebenen unterschieden, auf denen man Varietäten nach ihrer kommunikativen Reichweite ansiedeln kann. Die Standardsprache gelte als die Varietät mit der größten kommunikativen Reichweite. Sie umfasst das gesamte Sprachgebiet. Den Dialekten werde nur eine kleinräumige Kommunikationswirkung zugebilligt. Und zwischen den beiden gebe es eine Gruppe von Varietäten mit einer mittleren Kommunikationsreichweite, „die ,Umgangssprachen“ oder besser Regionalsprachen“. Dabei werde in Süddeutschland und Österreich ein Kontinuum regionaler Varietäten angenommen, in der Schweiz und Teilen Norddeutschlands werde von einem Nebeneinander von Dialekt und Standard (Diglossie) ausgegangen. Zwischen den Polen Standard (als idealisierte, im Alltag nicht realisierte Ausdrucksform) und Dialekt (mit kleinräumiger Verbreitung) nimmt Spiekermann regionale Standards und (dem Dialekt näherstehende) Regionalsprachen an. Im dialektnahen Bereich unterscheidet Dittmar (1997) noch stärker zwischen lokalen, regionalen, städtischen und überregionalen Varietäten (179). „Regionalsprachen“ nach Spiekermann „umfassen alle Varietäten, die im Kontinuum regionaler Varietäten [...] zwischen den Dialekten und den Standardvarietäten anzusetzen sind“ (Spiekermann 2010, 346). Gerade sie seien durch Tendenzen der De-Standardisierung und des Dialektabbaus großen Veränderungen unterworfen. Sie würden - je nach Standard- und Dialektnähe - ein breites Spektrum von Varietäten abdecken.

Auch Löffler (2005) geht „aus praktischen Gründen“ zunächst vom „alten einfachen Dreiermodell“ aus, der Trias „Hochsprache - Umgangssprache - Mundart“. Dem entspricht moderner ausgedrückt: Standardsprache - Substandardsprache Dialekt oder Standard - Substandard - Nonstandard (11). Ein Gegenbegriff zum Standard sei der Dialekt, der andere die „Umgangssprache“, der vielleicht nur ein Unterbegriff von Standard sei. Umgangssprache sei eine Art „Ausgleichsvarietät“ zwischen Hochsprache und Dialekt, die zwar deutliche regionale Färbung, jedoch keine extremen Dialektismen aufweise, sie sei „teilweise überregional, teilweise normiert und kodifiziert, eher mündlich, aber auch schriftlich - und nur teilweise für den (Ausländer-)Unterricht geeignet, allenfalls auf der Fortgeschrittenenstufe“ (a.a. O., 18). Ammon (2005, 35) nennt die Markierung eines als „umgangssprachlich“ bezeichneten sprachlichen Elements „berüchtigt“, weil nicht klar sei, ob damit eine weitere Normebene zwischen Standard und Nonstandard, ein Grenzfall des Standards gemeint sei oder eine Stilschicht innerhalb des Standards (kolloquialer Standard) ${ }^{6}$. Und der Terminus habe mindestens noch

6 Auch Löffler (2005) führt diese Bedeutung von Umgangssprache im Sinn einer „Stilschicht, die für informellere, privatere Situationen angemessener erscheint" als die Hochsprache, an: Es entspreche dem Englischen colloquial speech (Löffler 2005, 18). 
eine dritte Bedeutung: „das im alltäglichen Umgang sprachlich Übliche“ (a. a. O., 35), im Sinn von Alltagssprache.

Für Österreich unterscheidet Wiesinger $(1985,2010)$ vier Ebenen, wobei er zweierlei Dialektebenen einbezieht. Er teilt das mündliche Variationsspektrum in die als Dialekt und Standard bezeichneten äußeren Pole und eine als Umgangssprache bezeichnete, auf das „Hochdeutsche“ gerichtete Umgangssprache ein (2010, 636). Für Steinegger $(1998,32)$ ist die Umgangssprache das „Ausgleichsprodukt zwischen standardsprachlichen und dialektalen Varietäten“. In allen oben angeführten Modellierungen zeigt sich, dass vor allem die Varietät, die als "Umgangssprache" bezeichnet wird, äußerst vage und schwer zu operationalisieren ist. Das hat sich letztlich auch im vorliegenden Projekt gezeigt, in dem wir aber, wie Löffler, „aus praktischen Gründen“ zunächst vom „alten einfachen Dreiermodell“ ausgegangen sind (siehe Kap. 2.5).

\subsection{Konzeptualisierungen der Variation im Standarddeutschen}

Trotz Standardisierung und Kodifizierung geht man davon aus, dass auch die Variation in der Standardsprache systematisch regional oder abhängig von staatlichen Grenzen geprägt ist. Das Ende der monozentrischen Ära, die von einer geographischen Lokalisierbarkeit der „besten“ Standardsprache ausging, wird häufig in Zusammenhang mit einer Podiumsdiskussion auf der Internationalen Deutschlehrertagung IDT in Bern 1986 gebracht (vgl. von Polenz 1987). Die beiden heute am häufigsten verwendeten Modellierungen dieser Variation im Bereich der Standardsprache - es geht explizit nicht um Nonstandardvarietäten - sind das plurizentrische und das pluriareale Konzept, die im Folgenden charakterisiert werden sollen. Das vorliegende Projekt hatte das Ziel, primär den von stark normativen Rahmenbedingungen geprägten Bereich der schulischen Bildungssprache zu untersuchen - ein Bereich, für dessen konzeptuelle Einbettung die Plurizentrik geeigneter erscheint. Dieser Zugang wird im Folgenden ausführlicher dargestellt.

\subsubsection{Plurizentrik}

Die Termini „plurizentrische“ bzw. auch „polyzentrische“ Sprachen wurden vermutlich vom US-amerikanischen Soziolinguisten Willam A. Stewart zuerst verwendet, von Heinz Kloss (1978) für die Beschreibung sprachlicher Variation eingeführt, und später vor allem von Michael Clyne weiter verbreitet. Auf der anderen Seite wurden ähnliche Konzepte, verbunden mit den Begriffen nationaler

7 Bei den bis 2001 in Österreich üblichen Volkszählungen wurde z. B. nach der „Umgangssprache“ in diesem Sinn gefragt. 
Varianten/Varietäten, in der russischen Sprachwissenschaft seit den 1950er-Jahren entwickelt, wobei vor allem und vielleicht nicht zufällig die 1934 aus Österreich geflüchtete Jüdin Elise Riesel bei der Übertragung derartiger Konzepte auf die deutsche Sprache eine wichtige Rolle spielte. Eine ausführliche Darstellung der Begriffsgeschichte findet sich bei Ammon 1995 (42 ff, siehe auch Ammon 1998, $331 \mathrm{ff}$ ). Dieses Konzept der plurizentrischen Sprachen wurde v. a. von Clyne (1992, 1995, 2005) und Ammon (Ammon 1995, 2005, Ammon et al. 2004, Ammon/ Bickel/Lenz 2016) ausdifferenziert. Eine ausführliche Darstellung der aktuellen Forschungslage findet sich bei Schmidlin 2011 und Kellermeier-Rehbein 2014. Das Konzept der Plurizentrik geht davon aus, dass es von staatlichen Grenzen beeinflusste (nationale) Varietäten des Deutschen gibt und in so genannten Halbzentren (Südtirol, Liechtenstein, Luxemburg und Belgien) Besonderheiten der deutschen Sprache zu finden sind. Die zweite Auflage des Variantenwörterbuchs (Ammon/Bickel/Lenz 2016) unterscheidet noch drei Viertelzentren: Rumänien, Namibia und die Mennoniten-Gemeinden in Mexiko (XXVI).

Die plurizentrische Auffassung von der deutschen Sprache bedeutet, dass sprachliche Besonderheiten der Zentren des Deutschen nicht als Abweichungen von einer übergreifenden deutschen Standardsprache gelten, sondern als gleichberechtigt nebeneinander bestehende standardsprachliche Ausprägungen des Deutschen. (XLI)

Eine Grundlage dafür seien die Nationalstaaten, die eine wichtige Rolle bei der Standardisierung von Sprachen spielten, v. a. in Verwaltung, im Rechtswesen, in Bildungsinstitutionen, aber auch in Verlagen und in den Medien. Das Modell der Plurizentrik trägt, um es mit Schmidlin $(2017,45)$ zu formulieren, dem Umstand Rechnung, ,dass Standardsprachen überall dort, wo sie National- oder Amtssprachen sind, aufgrund politisch-historischer Eigenentwicklung der betreffenden Gebiete Besonderheiten aufweisen". Sowohl die Verknüpfung von Staat und Sprache (siehe auch bei Dittmar, s. o.) wie auch die für das Deutsche charakteristische Tatsache, dass es kein historisches Zentrum der Sprachnormierung und Standardisierung gegeben hat, legen es nahe, vom Deutschen als einer plurizentrischen Sprache zu sprechen. Diese Sicht der Dinge ist wohl nicht zufällig in den letzten Jahrzehnten in den Vordergrund gerückt. Durch die historische Entwicklung nach 1945, in der das Gebiet der deutschsprachigen Bevölkerung auf vier (später nach dem Ende der DDR drei) große Staaten aufgeteilt wurde, hat sich zunehmend die Einsicht durchgesetzt, dass es sich hier um unterschiedliche Varietäten ein und derselben Sprache handelt. So formulierte Peter von Polenz 1988: „Auch in der Geschichte der deutschen Sprache ist das Zeitalter der perfektionierten monomanen Standardisierung heute wohl zu Ende“ (v. Polenz 1988, 216). Für die mittlerweile breite Anerkennung dieser Konzeptualisierung spricht die Tatsache, dass es ein einführendes Lehr- und Studienbuch dafür gibt: „Plurizentrik. Eine Einführung in die nationalen Varietäten des Deutschen" (Kellermeier-Rehbein 
2014) und eben ein von diesem Konzept ausgehendes Wörterbuch, das mittlerweile in zweiter Auflage erschienen ist (Ammon/Bickel/Lenz 2016). Das Modell der plurizentrischen und plurinationalen Sprachen verfügt über eine ausdifferenzierte Theorie und Terminologie.

Ulrich Ammon hat in seinem Standardwerk „Die deutsche Sprache in Österreich, Deutschland und der Schweiz. Das Problem der nationalen Varietäten“ (Ammon 1995) das Konzept für den deutschsprachigen Raum ausgearbeitet. Er schlägt folgende Terminologie vor: Eine Sprache, die über mindestens zwei Standardvarietäten in verschiedenen Zentren verfügt (wie z. B. das Englische, Französische, Spanische, Arabische und eben Deutsche), wird "plurizentrische Sprache“ genannt. Unter einer „plurinationalen“ Sprache sei eine plurizentrische Sprache zu verstehen, zu deren Zentren mindestens zwei Nationen zählen. Ammon (1998, 314f) hält die Unterscheidung zwischen staatlichem und nationalem Zentrum für zweckmäßig, da Staat und Nation nicht in jedem Fall kongruent sein müssen (Schottland und Wales seien nations, würden aber keine eigenen states bilden) ${ }^{8}$. Dabei wird von Vollzentren gesprochen, wenn die standardsprachlichen Besonderheiten in eigenen Nachschlagwerken festgehalten und autorisiert sind (wie z. B. in Deutschland $=$ D, Österreich $=\mathrm{A}$, der Schweiz $=\mathrm{CH}$ ). Beim Fehlen eines richtigen Zentrums der Standardisierung spricht Ammon von „nationalen Halbzentren", z. B. im Fall des Deutschen in Liechtenstein (LIE), Luxemburg (LUX), Ostbelgien (BELG) und Südtirol (STIR). Ammon/Bickel/Lenz 2016 führen noch die oben erwähnten Viertelzentren an. Clyne 2005 trifft eine andere Differenzierung: Er spricht von "full centres of a pluricentric language (e.g. Britain, Germany)“, die eigene endonormative Standards haben, und „semi-centres (e.g. Australia, Austria)", die zum Teil exonormativen, zum Teil endonormativen Standards folgen, sowie von „rudimentary centres (e. g. Liechtenstein)“, die all ihre Normen von außen übernehmen (Clyne 2005, 298). Auch Kellermeier-Rehbein $(2014,29)$ schlägt - neben den Halbzentren - Viertelzentren vor, die über eine eigene Standardvarietät verfügen würden, aber keinen amtlichen Status hätten. Als Beispiele werden von Kellermeier-Rehbein Namibia und die Regionen mit deutschsprachiger Bevölkerung in Rumänien (Siebenbürgen) angeführt.

Mit „nationaler Variable“ bezeichnet Ammon „eine Menge einander entsprechender einzelner Sprachformen, die in verschiedenen Nationen gelten", also die unterschiedlichen gleichbedeutenden „Varianten“ für ein und dieselbe Sache. Beispiel: Ein „Tacker“ in Deutschland ist eine „Klammermaschine“ in Österreich und ein „Bostitch“ in der Schweiz (Ammon 2005, 30). Oder ein „Eissalon“ in Österreich ist ein „Eiscafé" oder eine „Eisdiele“ in Deutschland, in der Schweiz fehlen derartige Lokale überhaupt. Um ein Beispiel von Michael Clyne für das Englische anzuführen: „pavement" im britischen Englisch entspricht „sidewalk"

8 Dementsprechend könnte man staatsspezifische und nationale Varietäten/Varianten unterscheiden (Ammon 1998, 315). 
im amerikanischen und "footpath"im australischen Englisch (Clyne 2005, 296). Variablen können auch innerhalb einer Nation variieren, z. B. wenn eine Variante nur in Ostösterreich vorkommt, oder auch nationsübergreifend sein. Mit „spezifischen Varianten“ werden in diesem Fall diejenigen Formen bezeichnet, die in ihrer Verwendung auf eine Nation beschränkt sind (wie etwa die drei obigen Beispiele oder die „Befehlsausgabe“ beim österreichischen Militär). Unspezifische Varianten kommen auch darüber hinaus vor, z. B. der Blumenkohl in der Schweiz und Deutschland (gegenüber dem Karfiol in Österreich). Die nationalen Varianten Österreichs werden in der Regel als Austriazismen bezeichnet, die des Schweizer Deutsch als Helvetismen - der Ausdruck „Teutonismus“ (VWB 2016, XLII) für das deutsche Deutsch ist umstritten - alternativ wird in Fachkreisen auch „Deutschlandismus“ verwendet. Kellermeier-Rehbein verwendet „Teutonismus“ (Kellermeier-Rehbein 2014, 37). In der vorliegenden Arbeit verwenden wir den von uns als neutraler empfundenen Terminus Deutschlandismus, der auch Ammon (1998, 315) als „vertretbar“ erscheint. ${ }^{9}$ Für Varianten, die im gesamten deutschsprachigen Raum üblich sind, verwenden wir den Terminus „gemeindeutsch“.

Zwischen den unterschiedlichen Varietäten plurizentrischer Sprachen herrschen meist asymmetrische Verhältnisse - Clyne spricht von $\mathrm{D}$ (ominanten) und A(nderen) Nationalvarietäten („D(ominant) and $\mathrm{O}$ (ther) varieties“, Clyne 2005, 297). Das Deutsch, das in Deutschland gesprochen wird, ist für Clyne die Varietät der dominierenden, der „D-Nation“, das Deutsch Österreichs hingegen die Varietät der anderen, einer so genannten „A-Nation“. Clyne $(1995,22)$ charakterisiert die bestehende Asymmetrie zwischen diesen beiden Varietäten und deren Nationen folgendermaßen: Für D-Nationen sei es schwierig, das Konzept der Plurizentrizität zu begreifen. Unterschiede zwischen nationalen Varietäten würden als trivial abgetan. D-Nationen würden dazu tendieren, aufgrund der Überlappung linguistischer Faktoren nationale Varietät mit regionaler Varietät gleichzusetzen, ohne die Funktion, den Status und den symbolischen Charakter nationaler Varietäten zu verstehen. D-Nationen würden im Allgemeinen ihre nationale Varietät als den normgebenden Standard betrachten und sich selbst dabei als Hüter dieser Standardnorm sehen. Die nationalen Varietäten der A-Nationen würden dabei als abweichend, „non-standard“ und exotisch, charmant und etwas veraltet angesehen. Die kulturellen Eliten der A-Nationen würden dazu tendieren, sich an den Normen der D-Nationen zu orientieren. Die Normen der A-Nationen würden als weniger streng als die der D-Nationen gelten. Ein Anpassen an die Varietät der jeweils anderen Nation finde meist in Richtung der Norm der D-Nation statt, wenn SprecherInnen verschiedener nationaler Varietäten zusammentreffen, z. B. in Arbeitskreisen, Konferenzen oder bei Begegnungen im touristischen Bereich in Drittländern. D-Nationen hätten auch bessere Ressourcen als A-Nationen, um

9 Als Alternativen wurden auch Germanismus, Bundesgermanismus oder Germanizismus vorgeschlagen. 
ihre Varietät im Fremdsprachenunterricht zu exportieren. D-Nationen hätten weiters bessere Instrumente zur Kodifizierung der Sprache, da sich die meisten Verlage für Grammatikwerke und Wörterbücher im Land der D-Nation befinden. Die Annahme, Diversität existiere nur in der gesprochenen Sprache, sei vor allem in D-Nationen beheimatet. In manchen Fällen seien SprecherInnen der D-Nationen nicht mit der nationalen Varietät der A-Nation vertraut oder verstünden sie nicht. Clyne $(1995,23)$ verweist als mögliche Erklärung für das bestehende asymmetrische Verhältnis zwischen der Varietät Deutschlands und der Varietät Österreichs auf eine semantische Verwechslung der unterschiedlichen Bedeutungen von „deutsch“: einerseits ,deutsch“ als Adjektiv zu Deutschland, andererseits als Bezeichnung der Sprache:

The first is a sematic confusion between deutsch/'German' pertaining to Germany, and deutsch/'German' pertaining to the pluricentric language. This is due to the fact that the states which unified under Prussia in 1871 chose the name Deutschland although there were some German-language areas outside the new nation-state. In the AustroHungarian empire, the word 'German' or 'German-Austrian' was used to distinguish German speakers from the other ethnolinguistic groups. (Clyne 1995, 23)

Dies wäre, so Clyne weiter, von der (unter Deutschen) weitverbreiteten Annahme begleitet, dass ihre Varietät überlegen ist.

Bei Ammon 1995 (484-494) finden sich insgesamt zwölf auf die Varietäten des Deutschen bezogene „verbreitete Asymmetrien“: (1) die verbreitete Vorstellung von der sprachlichen Dominanz des deutschen Zentrums, (2) die Meinung, Austriazismen und Helvetismen seien weniger korrektes Deutsch, und ihre geringere Bekanntheit außerhalb der eigenen Zentren, (3) das Stereotyp von den deutschen Schnellschwätzern, (4) die größere Funktionsbreite des deutschen Standarddeutsch, (5) die zusätzliche Überschätzung der Funktionsbreite des deutschen Standarddeutsch, (6) der größere Umfang des deutschen Sprachkodex, (7) das höhere Prestige von Deutschlands Sprachkodex im Vergleich zu den Sprachkodizes Österreichs und der Schweiz, (8) die reine Binnenkodifizierung des deutschen Standarddeutsch gegenüber der partiellen Außenkodifizierung des österreichischen und des schweizerischen Standarddeutsch, (9) die größere Normtoleranz des Sprachkodex Deutschlands und der deutschen Sprachnormautoritäten, (10) die rein soziale Kritik an den eigenen Sprachnormen in Deutschland gegenüber der nationalen Kritik in Österreich und der Schweiz, (11) das mangelnde Bewusstsein von den Teutonismen und ihre fehlende Darstellung und (12) der stärkere Variantenimport aus Deutschland als aus der Schweiz und aus Österreich.

Besonders hervorgehoben wird von einigen AutorInnen eine geringere Loyalität in der A-Nation gegenüber der eigenen Varietät. So spricht Michael Clyne $(1995,31)$ von „linguistic cringe“, einem sprachlichen Unterlegenheitsbewusstsein (zit. nach Schmidlin 2011, 226). Auch bei Ammon/Bickel/Lenz 2016 findet sich der 
Hinweis auf einen „gewissen sprachlichen Minderwertigkeitskomplex“(XLIV). Schmid 1990 spricht auch von einem "kollektiven Minderwertigkeitskomplex“ und meint: „Ein gewisses hündisches Verhalten österreichischerseits muss da allerdings schon konstatiert werden. " (32) Vor allem Muhr hat im Zusammenhang mit Spracheinstellungen wiederholt darauf hingewiesen (z. B. Muhr 1982, 1989, Muhr 2005b), dass bei SprecherInnen nicht-dominanter Varietäten ein sprachlicher Minderwertigkeitskomplex entstehen könne, also in unserem Kontext bei ÖsterreicherInnen und SchweizerInnen. Das sei Folge eines weitverbreiteten Unwissens bezüglich der sprachlichen und kommunikativen Eigenschaften der eigenen Varietät, was wiederum zur Abwertung und Vermeidung der sprachlichen Merkmale dieser eigenen Varietät führe. Ein Effekt davon sei eine Art „sprachliche Schizophrenie“ (Muhr 2005b, 18).

Der Schweizer Linguist Joachim Scharloth (2005) berichtet von einem Projekt, das diese Annahmen bestätigt. In diesem Projekt wurde das Sprachbewusstsein der SchweizerInnen untersucht, z. B. ob sie der Meinung sind, dass es mehrere gleichberechtigte Normen in der deutschen Standardsprache gibt. Als Methode wurde dabei neben einer direkten Befragung der subjective-evaluation-test verwendet, der auf indirekte Weise Spracheinstellungen erhebt. Dabei wurden den ProbandInnen 96 Beispielsätze vorgelegt, die dahingehend bewertet werden mussten, ob sie Standardformen darstellen. Die Beispielsätze enthielten eine Mischung von deutschen Standards, Schweizer Standard, überregionalem Substandard und Dialektformen. Die Sätze wurden akustisch präsentiert, und zwar zum Teil von einem deutschen Sprecher und zum Teil von einem Schweizer Sprecher mit schweizerischem Akzent gesprochen. Neben einer Reihe anderer interessanter Ergebnisse (z. B. dass $58 \%$ der DeutschschweizerInnen ihre E-Mails teilweise in der dialektalen Varietät des Schweizerdeutsch verfassen, und $75 \%$ ihre SMS, Scharloth 2005, 24), zeigte sich v. a. eine geringe Sprachloyalität der DeutschschweizerInnen ihrer eigenen Standardvarietät gegenüber: $79 \%$ der Befragten erklärten, Hochdeutsch sei für die SchweizerInnen die erste Fremdsprache. In einem weiteren Test, in dem sie gebeten wurden, deutsch(ländisch)e und Schweizer Standardformen zu bewerten, haben die ProbandInnen in 60 und $70 \%$ der Fälle die Schweizer Varianten als schlechtes oder fehlerhaftes Standarddeutsch beurteilt (Scharloth 2005, 39), also z. B. folgende Sätze: „Der Pöstler macht sich jeden Morgen um sieben auf seine Tour“; „Die Beiz ist hübsch eingerichtet“ oder „Gestern fuhr ich mit dem Tram ins Krankenhaus" (a.a.O., 30). Dieses Ergebnis ist umso erstaunlicher, als bei der expliziten direkten Befragung mit dem Fragebogen die Mehrheit (58\%) der Meinung war, man solle schweizerhochdeutsche Formen pflegen (Scharloth 2005, 30). Resümee: „Deutschschweizer haben offenbar ein sprachliches Inferioritätsbewusstsein gegenüber Deutschen und neigen daher dazu, deren Sprachproduktion als besseres Standarddeutsch gelten zu lassen“" (a. a. O., 33). Schmidlin (2011) weist allerdings darauf hin, dass die von Scharloth gewählten Varianten zum Teil Grenzfälle des Standards sind. 
Letztlich handelt es sich bei diesem Phänomen um eine Frage der Sprachloyalität. Daher sei hier näher darauf eingegangen. Das Konzept der Sprachloyalität, in unserem Fall sozusagen die „Varietätenloyalität“, wird in der Soziolinguistik verwendet, um „das bewusste oder unbewusste Verhältnis einer Sprechergruppe $\mathrm{zu}$ ihrer Muttersprache" zu charakterisieren (Schmidlin 2011, 219). Eine positive Einstellung gegenüber der eigenen Sprache sowie die Identifikation mit der eigenen Sprache (z. B. in Minderheitensituationen) bzw. der eigenen Sprachvarietät gegenüber (z. B. beim Wechsel von dialektalen zu standardsprachlichen Varietäten (vgl. Hartig 1987) ist wichtig für den Spracherhalt. So wird Sprachloyalität als besonders wichtiger soziopsychologischer Faktor für das Überleben von Sprachen in Situationen, in denen sie von "Sprachtod“ bedroht sind, eingeschätzt (Dressler/de Cillia 2006, Brenzinger 1997, Fishman 1964). In der germanistischen Linguistik findet sich das Konzept bei Löffler $(2016,60)$, wonach Sprachzugehörigkeit durch Sprachloyalität konstituiert werde. Die SprecherInnen müssten sich zur Sprachgemeinschaft zugehörig fühlen und die Bereitschaft zeigen, „sich den pragmatischen und sprachlichen Regeln der Gemeinschaft dieser Sprache anzuschließen“.

Schmidlin (2011) hat unseres Wissens nach als erste die Loyalität gegenüber Sprachvarietäten, im konkreten Fall der staatsbezogenen Varietäten des Deutschen, untersucht (219-233), der Begriff der „Variantenloyalität“ sei ihr zufolge noch nicht belegbar. In ihrer Studie erforscht Schmidlin zum ersten Mal empirisch die Loyalität gegenüber den eigenen Varianten im plurizentrischen/ pluriarealen deutschsprachigen Raum. Insgesamt stellt sie einen signifikanten Einfluss der regionalen Herkunft auf die Loyalität gegenüber Eigenvarianten fest. Danach weisen Gewährspersonen aus den Regionen Deutschland-Nordost, Deutschland-Nordwest, Deutschland-Mittelwest und Deutschland-Mittelost die höchsten Loyalitätswerte auf. Gewährspersonen (GP) aus den südlichen Regionen würden hingegen mehr Fremdvarianten wählen als jene aus den übrigen Regionen. Und trotz der typologisch-dialektalen Verwandtschaft der alemannisch geprägten Regionen Österreich-West, Deutschland-Südwest und der Schweiz würden hier die Gewährspersonen aus Deutschland-Südwest und Österreich-West signifikant häufiger die Eigenvarianten wählen als die aus der Schweiz. Schmidlin wertet dies als Hinweis darauf, dass dieses Sprachverhalten eher nationalvarietätenspezifisch als regionalvarietätenspezifisch geprägt sei (226). Auch was die „phonologische Variantenloyalität" betrifft, stellt Schmidlin (2011, 233) fest:

Die nationale Grenze scheint somit wiederum als stärkere sprachpragmatische Isoglosse als die dialektologische Homogenität; im alemannischen Sprachraum, der sich innerhalb des deutschen Sprachgebiets auf die Schweiz, süddeutsche Regionen und Österreich-West erstreckt, können sich GP je nach Nationalität unterschiedlich verhalten. 
Auch im vorliegenden Projekt waren einige Items der Befragungen der Frage gewidmet, wie die ProbandInnen gegenüber der österreichischen Varietät eingestellt sind (vgl. Kap. 6.4).

Das plurizentrische Modell hat neben dem auf diesem Konzept basierenden Variantenwörterbuch (Ammon et al. 2004, Ammon/Bickel/Lenz 2016) eine Reihe empirisch fundierter Arbeiten zur Folge gehabt (z. B. Markhardt 2005, Ransmayr 2006, Hägi 2006, Schmidlin 2011, Wissik 2014), ein Lehrbuch hervorgebracht (Kellermeier-Rehbein 2014) und zur 2010 gegründeten, internationalen „Working Group on Non-dominant Varieties of Pluricentric Languages ${ }^{\text {"10 }}$ geführt. Insofern kann die Plurizentrik trotz Kritik von unterschiedlichen Seiten als eine wissenschaftlich anerkannte, adäquate Modellierung der staatlich basierten regionalen Variation der deutschen Standardsprache betrachtet werden. Im plurizentrisch konzipierten Variantenwörterbuch werden aber durchaus auch länderübergreifende und regionale Phänomene dokumentiert und für den bundesdeutschen Raum sechs, für Österreich vier regionale Untergliederungen angenommen. Hier könnte man auch von „regionaler Plurizentrizität“ sprechen (vgl. Reiffenstein 2001, 88). Das für eine solche kleinräumigere Gliederung des deutschen Sprachraums in der Regel verwendete Modell ist das der Pluriarealität, das kein Gegenmodell zur Plurizentrik darstellen muss, sondern als komplementär angesehen werden kann.

\subsubsection{Pluriarealität}

Das pluriareale Konzept (vgl. Ammon 1998) wurde zunächst in den 1990erJahren - manchmal recht vehement (vgl. Scheuringer 1996, vgl. auch die sich auf Scheuringer berufenden Seifter \& Seifter 2015) - als eine konzeptuelle und sprachenpolitische Gegenposition formuliert, wobei bisher (noch) keine weitergehende theoretische Ausarbeitung bzw. kein systematisches Begriffsinventar ähnlich dem des plurizentrischen Ansatzes zur Analyse vorliegt. Gemeinsam ist den VertreterInnen des pluriarealen Konzepts die Ablehnung des plurizentrischen Ansatzes, dem häufig unterstellt wird, „,ideologisch“ zu sein (vgl. z. B. Wolf 1994, Putz 2002, Glauninger 2007, Dürscheid/Elspass/Ziegler 2015, Seifter \& Seifter $\left.2015^{11}\right)$. Dieser Zugang existiert im Übrigen unseres Wissens nur im Bereich der germanistischen Linguistik, in der laut Eichinger $(2005,2)$ ein „terminologischer Kleinkrieg“ stattfinde - ein ähnlicher Ansatz für die englische, spanische oder französische Sprache ist uns nicht bekannt. Dollinger (in Druck, 5) stellt zum Terminus „pluri-areal“ fest, er sei “virtually unknown outside of German linguistics

$10 \mathrm{http} / / /$ www.pluricentriclanguages.org/about-us/ndv-working-group (18. 12. 2018).

11 Eine genauere diskursanalytische Betrachtung der wissenschaftstheoretischen Konzepte und Ideologiebegriffe, die manchen dieser Publikationen zugrunde liegen, wäre eine interessante epistemologische Frage. In unserem Kontext würde das allerdings zu weit führen. 
but central to many if not most dialectological approaches of German today". Das mag mit der Geschlossenheit des deutschsprachigen Gebiets zusammenhängen, in dem - im Unterschied zu anderen großen Sprachen - die Staaten, in denen die betreffende Sprache Mehrheitssprache ist, Nachbarstaaten sind. In bestimmten Fällen mögen auch Konzepte einer deutschen Kultur- und Sprachnation dahinterstehen, denen zufolge das Abgrenzungskriterium einer Nation die Muttersprache ist. Danach wäre die überwiegende Mehrheit der ÖsterreicherInnen ebenso wie der DeutschschweizerInnen und der SüdtirolerInnen Deutsche. ${ }^{12}$

Norbert Richard Wolf (1994, siehe auch 2012) etwa schlägt den Begriff „pluriareal“ vor, "nicht zuletzt auch deshalb, weil Staatsgrenzen mitnichten auch Sprachgrenzen sind“ (2012, 499). In einer Besprechung von Pollak (1992) meint Wolf, dass Pollak die ,Ideologie von der staatsnationalen Varietät perpetuiere, die schon zur Schaffung des ÖWB geführt habe und die auch heute noch in offiziellen Kreisen der Kulturbürokratie gerne gepflegt werde (Wolf 1994, 75). Dieses ÖWB sei „ein merkwürdiges Produkt restaurativer österreichischer Kulturpolitik nach dem Zweiten Weltkrieg" (a. a. O., 67). Vertreter des pluriarealen Konzepts gehen also davon aus, dass eine Konzeptualisierung des Deutschen als "pluriareale"Sprache adäquater sei als die plurizentrische Konzeption, indem sie auf „durch dialektale Großräume bestimmte Areale“ abstellt (Pohl 1997, 69). Sie verweisen vor allem auf standardsprachliche Unterschiede innerhalb Deutschlands zwischen Norden und Süden und innerhalb Österreichs zwischen Osten und Westen. Und sie betonen des Weiteren die zahlreichen grenzüberschreitenden Gemeinsamkeiten, z. B. Übereinstimmungen zwischen Süddeutschland, Österreich und der Schweiz oder zwischen Westösterreich und Südostdeutschland oder auch zwischen Vorarlberg, Liechtenstein und der Schweiz. Die nicht mit den staatlichen Grenzen übereinstimmenden regionalen Unterschiede im Standarddeutschen werden bei diesem Zugang als wichtiger erachtet als die mit staatlichen/nationalen Grenzen kongruierenden Unterschiede.

Scheuringer 1996 vertritt die Auffassung, dass „der Terminus plurizentrisch den arealen Mustern des deutschen Sprachgebiets nicht gerecht werden kann“ (151):

... jene, die den Terminus weiterhin verwenden, [unterliegen] entweder der Suggestivkraft des nun einmal eingeführten Terminus oder - und dies nehme ich an - sie handeln so wider besseres Wissen (150). ${ }^{13}$

12 So meint der Wiener Historiker Lothar Höbelt, die Zuordnungen „deutsch“ und „österreichisch“ würden einander nicht ausschließen, sie seien vielmehr unterschiedliche Komponenten der Identität. Österreich sei heute „deutscher als je in seiner Geschichte“ (Höbelt 1994, 341).

13 Fachkollegen wird hier sozusagen unterstellt, unlauter, nämlich wider besseres Wissen, zu handeln. Der Beitrag enthält auch andere für die Textsorte „wissenschaftlicher Zeitschriftenartikel“ unübliche Passagen. So wird Wodak 1994 unterstellt, ,im Stile dumpfer Boulevardblätter mit Sätzen wie ,Wir sind nicht Duden-Land“ Hetze [zu] betreiben“. (152). 
Das Deutsche sei eine „pluriareale Sprache“. Plurizentrisch suggeriere, es gebe national und staatlich einheitliche Varietäten des Deutschen in relativ strikter Abgrenzung voneinander. Wichtig sei aber vor allem „die Arealität, also die Räumlichkeit des Deutschen“, die sich in den verschiedenartigsten Mustern äußere, von denen die Staatlichkeit nur eines sei, die in den Bereich einer „Randgröße“ verwiesen wird. Andere „Räumlichkeiten“ seien wesentlich häufiger wie die, die einen „altbayerisch-österreichischen Raum“ oder solche, die einen „gesamtsüddeutschen Raum“ ergeben. Und „geradezu überwältigend dominant“ sei eben die „Ein-Räumlichkeit“ (a.a. O., 152), die die Grundlage einer funktionierenden Hochsprache sei. Staatsgrenzenüberschreitende Gemeinsamkeiten einerseits bzw. innerstaatliche Differenzierungen andererseits werden hiermit also als wichtiger eingeschätzt als staatsbezogene sprachliche Gemeinsamkeiten im Sinn des plurizentrischen Konzepts. ${ }^{14}$ In seinem Beitrag betont Scheuringer weiter, dass er grundsätzlich antinational und antinationalistisch eingestellt sei. Er glaube „an den Primat natürlicher Räume“, darunter auch „Sprachräume“, die er als natürlicher empfinde als Staaten oder Nationen. So gesehen seien Deutschland, Österreich oder die Schweiz „willkürliche Gebilde“ (a.a.O., 151). Die Debatte rund ums österreichische Deutsch sieht er als „Stellvertreterdebatte“. 1945 sei in Österreich „die Lüge als Prinzip der Geschichtsbetrachtung“ etabliert worden. Die dafür notwendige Abkehr von den eigenen anderthalb Jahrtausenden deutscher Geschichte habe auch die Sprache erfasst (a. a. O., 148). Man habe in mehr als fünfzig Jahren offizieller Geschichtsverfälschung das Kind mit dem Bad ausgeschüttet, „nämlich Österreichs Geschichte in und mit Deutschland im Jahrtausend davor, damit auch Österreichs demographische und sprachliche Geschichte“.15 Darauf, dass Scheuringer noch 1985 in einer Publikation von staatsbezogener Variation des Deutschen ausging, sei hier nur kurz hingewiesen: „Being a nation with a long tradition of statehood, Austria has developed a specific variety of German in the same way as English shows different varieties in different parts of the world.“ Und etwas weiter unten: „On the whole, the concept of an Austrian variety of

14 Eine Diskussion dieser Frage findet sich übrigens schon bei Ammon 1995, 505 ff.: „Region und Nation als varietätsprägende Kräfte“.

15 Nach Brückmüller existieren zwei Abstammungsmythen zu den ÖsterreicherInnen: Einerseits existiert die Vorstellung, die heutigen ÖsterreicherInnen seien vor allem deutscher Abstammung. Das Gegenteil davon sei der multikulturelle Mythos (Bruckmüller 1994, 142). Dass es wenig Sinn macht, derartige genealogische Spekulationen anzustellen, begründet er mit der Tatsache, dass sich die Vorfahren eines Individuums in jeder Generation quadratisch vermehren. Die definitive Herausbildung eines eigenen österreichischen Nationalbewusstseins wird von manchen AutorInnen schon in der Zeit der nationalsozialistischen Herrschaft angesetzt (etwa von Kreissler 1984 und Bruckmüller 1996, 348-353, vgl. auch Wodak et al 1998, 111-117). Dieses eigene österreichische Nationalbewusstsein hat sich ab den 196oer-Jahren zu einer „österreichischen nationalen Identität“ verdichtet. $47 \%$ sahen bei einer Befragung im Jahr 1964 Österreich als Nation, 66 \% im Jahr 1970 und 80 \% im Jahr 1993 (Bruckmüller 1994, 15). 
standard German is generally accepted (for Austrian German cf. Reiffenstein

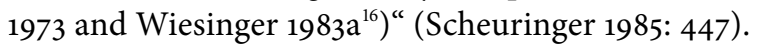

Einen empirisch fundierten pluriarealen Zugang zur Beschreibung regionaler Variation der deutschen Sprache in Deutschland wählte Nina Berend $(2005)^{17}$. Sie hat regionale Gebrauchsstandards untersucht - also Varietäten, die über ein hohes Prestige verfügen und sowohl im informellen, als auch im formellen Kontext gebraucht werden. Die Autorin positioniert diese regionalen Gebrauchsstandards unter dem kodifizierten Standard, aber über den Dialekten und Umgangssprachen. Sie beschreibt damit den „deutschen Sprechstandard“ (ähnlich dem von Durrell eingeforderten ,colloquial German', ,allemand populaire‘, Durrell 2003) und unterscheidet vier sprechsprachliche regionale Gebrauchsstandards: norddeutscher Sprechstandard, mitteldeutscher Sprechstandard, Südwest-Sprechstandard, Südost-Sprechstandard. Als sprechsprachliche linguistische Varianten seien beispielhaft angeführt: <eine> wird realisiert als „eine - ne - a/e“; <nicht> wird realisiert als „nicht - nich - net“ oder die e-Apokope in der 1. Pers. Sg. des Verbs (find ich, sag ich, mach ich etc.) wird in ihrem Material zu 98,8\% realisiert (Berend 2005, $148 \mathrm{ff}$ ). Einen ähnlichen, in dem Fall alemannischen, Regionalstandard bestätigen die Ergebnisse von Spiekermann (2008, siehe auch Spiekermann $2005^{18}$ ).

Mittlerweile hat sich im Rahmen detaillierter empirischer linguistischer Analysen durch die Weiterentwicklung der Korpuslinguistik eine neue Ausprägung des pluriarealen Ansatzes entwickelt, der zwar, soweit Publikationen vorliegen, theoretisch (noch) nicht so entwickelt und terminologisch nicht so ausdifferenziert ist wie die plurizentrische Theorie, aber Befunde für eine pluriareale Konzeptualisierung des Deutschen vorlegt (s. Elspaß \& Niehaus 2014, Dürscheid, Elspaß \& Ziegler 2015). Man geht von einem „pluriarealen Ansatz aus, der grundsätzlich die regionalen Unterschiede innerhalb der deutschsprachigen Länder ernst nimmt“ (Dürscheid, Elspaß \& Ziegler 2015, 211) und führt drei Argumente gegen den „Plurinationalitäts-Ansatz“ an ${ }^{19}$ : Erstens ein sprachpolitisches Argument: Das Ende der DDR hätte gezeigt, wie fragil der plurinationale Ansatz sei, denn das DDR-Deutsch hätte sich über Nacht in Luft aufgelöst. Dann ein variationslinguistisches Argument: Vermeintlich nationale Varianten würden häufig

16 Scheuringer bezieht sich hier auf: Wiesinger, Peter (1983): Sprachschichten und Sprachgebrauch in Österreich. In: Zeitschrift für Germanistik 4, 184-195. Und: Reiffenstein, Ingo (1973): Österreichisches Deutsch. In: Adolf Haslinger (Hrsg.): Deutsch heute. München: Hueber, 19-26.

17 In der Beantwortung einer Anfrage von Anna Golovko vom 19. 9. 2013 zur Frage des pluriarealen Ansatzes, die diese im Rahmen einer BA-Arbeit an Berend gerichtet hatte, meinte ein Mitarbeiter von Nina Berend, Ralf Knöbl, dazu: „Unseres Erachtens ist es richtig, dem sicher wichtigen Konzept der Plurizentrizität das der Pluriarealität entgegen- bzw. vielmehr danebenzustellen."

18 Er beschreibt den gesprochenen südwestdeutschen Standard als Beispiel einer regionalen Standardvarietät. Solche regionalen Standards würden die nichtregionale Standardsprache unterschichten, ohne diese zu verdrängen (Spiekermann 2005, 100).

19 "der in gewisser Weise eine neue Ideologie bildet“ (211) - auch hier kommt man ohne IdeologieVorwurf nicht aus, was immer auch unter Ideologie zu verstehen ist. 
politisch-nationale Grenzen überschreiten. Und schließlich auch ein perzeptionslinguistisches Argument: Spracheinstellungserhebungen bei linguistischen Laien würden zeigen, dass der von LinguistInnen geschaffene Gegenstand „nationale Varietät" in deren Wahrnehmung (noch) nicht angekommen sei $(211 \mathrm{f})^{20}$. Daher habe sich das Projekt Variantengrammatik nicht auf „vermeintlich ,nationale Varianten" festgelegt, sondern ein Korpus aus allen Gebieten des geschlossenen deutschsprachigen Raums erstellt und vorläufig nach Sektoren untergliedert wie im Variantenwörterbuch ${ }^{21}$, und nach Bedarf könnten diese Sektoren dann als Ergebnis der empirischen Analyse zu größeren Gebieten zusammengefasst werden (212). Empirische Daten sollten, wie es heißt, die Grundlage für die Beantwortung der Frage sein, ob das Deutsche in höherem Maße eine plurinationale oder eine pluriareale Sprache sei. Das Projektteam gebe „dem neutraleren Terminus ,pluriareales Deutsch den Vorzug“ (213). Ziel des Projekts sei es, ausgehend von einem Korpus aus den Regionalteilen von 690 Onlinezeitungen im Umfang von über 640 Millionen Wortformen die arealen Unterschiede in der Grammatik der deutschen Standardsprache systematisch zu erforschen und später in einem Handbuch zu dokumentieren. Dabei wird ein striktes Standardkonzept angewandt - Grenzfälle des Standards sind nicht vorgesehen - Phänomene sind entweder Standard oder Non-Standard $(215)^{22}$. Weiters wird davon ausgegangen, dass es sich in den meisten Fällen von Variation nicht um ,absolute, sondern relative Varianten“ (218) handelt, d.h., dass in den meisten Fällen Varianten nebeneinander vorkommen, die man nach Mehrheits- und Minderheitsvarianten unterteilen könne, also dass z. B. in einem Areal A die Variante $\mathrm{X}$ zu $70 \%$ und die Variante Y zu $30 \%$ vorkomme. Bei Dürscheid/Elpaß/Ziegler (2015) finden sich Fallanalysen von vier Varianten in einem Pilotkorpus, die den Zugang illustrieren. Als Beispiel sei die prädikative Verwendung von "n-jährig“ (z. B. „Der Chronist wird 8o-jährig“ im Sinn von „80 Jahre alt") angeführt, bei der von der Hypothese ausgegangen wird, dass diese fast nur im Schweizer Korpus vorkommt. Die Pilotanalyse deutet darauf hin, dass „die prädikative Verwendung des Adjektivs in Bezug auf das Alter von Menschen in der österreichischen und deutschen Standardvarietät nicht möglich zu sein scheint" (224). Abschließend

20 Als Beleg dafür werden die Arbeiten von Pfrehm (2007) angeführt, die allerdings genau das Gegenteil belegen, wie eine Anfrage beim Autor bestätigt hat - siehe Kap. 2.4.3.

21 Es handelt sich dabei um Deutschland Nordwest, Deutschland Nordost, Deutschland Mittelwest, Deutschland Mittelost, Deutschland Südwest, Deutschland Südost, Österreich West, Österreich Mitte, Österreich Südost, Österreich Ost, Schweiz, Ostbelgien, Liechtenstein, Luxemburg, Südtirol. Die Zusammenfassung von Vorarlberg und Tirol zu einem Sektor „Westösterreich“ ist angesichts des pluriarealen Zugangs allerdings ungenau.

22 Kritisch angemerkt sei hier, dass Standard-Sprachgebrauch auf den Gebrauch in online zugänglichen Regionalzeitungen reduziert wird. Und inwiefern bzw. ob ein derart rigider Normbegriff zur Erfassung des einen Endes des im Prinzip ein Kontinuum zwischen Standard und Dialekt darstellenden Sprachgebrauchs (vgl. Löffler 2005) beschreibungs- und erklärungsadäquat ist, wäre zu hinterfragen. 
wird in der hier berichteten Publikation, die bei den empirischen Fällen interessanterweise doch auf drei staatsbezogene Varietäten in Deutschland, Österreich und der Schweiz referiert, festgehalten: „Unser Projekt beschreitet in Bezug auf die verschiedenen Standardvarietäten in den deutschsprachigen Ländern und Regionen einen konsequent deskriptiven Weg" (323f). Man werde vielleicht dazu beitragen können, „ein klares und empirisch fundiertes Bild der grammatischen Gebrauchsstandards im Deutschen zu liefern" $(233)^{23}$.

Niehaus (2017), der in Übereinstimmung mit dem Projekt Variantengrammatik davon ausgeht, „dass es sich bei der deutschen Sprache nicht um eine plurizentrische, sondern vielmehr um eine pluriareale Sprache handelt“ (Niehaus 2017, 62), nennt als Vorteile des pluriarealen Ansatzes die „höhere theoretische Gewichtung relativer Varianten“, „die methodische Offenheit bezüglich der Stärke arealer Grenzen“, die „vergleichsweise hohe Flexibilität des Korpusdesigns“ und „generell die Anpassungsfähigkeit an die Empirie“ (Niehaus 2017, 85). Das Argument der relativen Varianz als Vorteil des pluriarealen Modells wird an vier Beispielen illustriert (der Variation der Pluralbildung von Balkon [Balkone - Balkons]; der Genusvariation von (E-)Mail, der Variation bei der Adverbienbildung wie durchweg/durchwegs; der Variation in der Trennbarkeit von Verben am Beispiel widerspiegeln), die zeigen, dass relative Varianten in der Grammatik keine Randerscheinung seien und dass ,grammatische Varianten in vielen Fällen Relativität statt Absolutheit zeigen" (81). Zumindest für die Grammatik dürfe anzuzweifeln sein, ob absolute Varianten in der Mehrheit seien und ob es legitim sei, nationalarealen Varianten theoretisch so viel Raum in der Charakterisierung von Varietäten zu gewähren, wie das im plurizentrischen Modell der Fall sei. Ausgehend von der (nicht zutreffenden, die Verf.) Annahme, der plurizentrische Zugang würde nur Mehrheitsvarianten im Auge haben, sieht Niehaus für den didaktischen Umgang mit Variation durch den plurizentrischen Zugang die Gefahr, „dass ohne empirische Grundlage ein sprachmonomaner Variantenabbau, von oben' auf eine einheitliche nationale Norm hin betrieben wird“ (84). Dass der plurizentrische Zugang mit der Unterscheidung zwischen "spezifischen“ und „unspezifischen“ Varianten sehr wohl ein Konzept für die Phänomene grenzübergreifender Variation hat, sei hier jedenfalls erwähnt. ${ }^{24}$

Ein weiterer Zugang, welcher der Plurizentrik gegenüber in der Form eines „plurinationalen Ansatzes“ kritisch eingestellt ist, findet sich bei Glauninger (2007). Ausgangspunkt ist die Frage, ob ein ,national“ determiniertes Modell eine adäquate

23 Angemerkt sei hier auch, dass natürlich auch andere Ansätze wie der plurizentrische Ansatz empirisch arbeiten und um deskriptive Adäquatheit bemüht sind; und dass es u. E. theorielose Empirie nicht gibt: Jeder Zugang zur außersprachlichen Realität ist nur durch sprachliche Konzepte und theoretische Verallgemeinerungen möglich.

24 Der Titel des Aufsatzes von Niehaus 2017 „Die Begrenztheit plurizentrischer Grenzen [...]“ entbehrt im Übrigen nicht einer gewissen herablassenden Abwertung einer anderen wissenschaftlichen Position. 
theoretische Basis zur Erforschung und Beschreibung des Deutschen sein könne, und zwar unter im Vergleich zu den 1990er-Jahren vom Verfasser angenommenen „dramatisch veränderten außersprachlichen/geopolitischen Verhältnissen, nämlich in einem sich in atemberaubender Geschwindigkeit ,supranational' formierenden makrosozialen bzw. -ökonomischen Kontext, den man über kurz oder lang wohl ,postnational' bezeichnen wird können“ (Glauninger 2007, 1). Neben einer aufgrund der historischen Entwicklung angenommenen „Sonderstellung des Deutschen", das nie eine monozentrische Phase durchlaufen habe, die für andere plurizentrische Sprachen postuliert werde, sei das Deutsche „permanent ,plurizentrisch' geprägt" worden (a.a.O., 2). Warum deshalb die Annahme staatsspezifischer Varietäten theoretisch nicht adäquat sein sollte, geht aus der Argumentation allerdings nicht klar hervor. Als zweites Gegenargument wird die „Inadäquatheit einer ,staatsnationalen' respektive, nationalstaatlichen' Konzeption für die ,plurizentrische Theorienbildung im Hinblick auf die deutsche Sprache“ (a.a. O., 4) ins Treffen geführt. Es fehle im deutschsprachigen Raum mit Ausnahme der Schweiz „ein im kollektiven Bewusstsein der Bevölkerung (historisch) stabil verankertes voluntativ-konstitutionelles Verständnis von ,Nation“. Denn die Staatengebilde der BRD und Österreichs würden „Produkte einer konstitutionell nachgerade atypischen, bis in die jüngere und jüngste Vergangenheit nur im Einvernehmen mit den Siegermächten der beiden Weltkriege möglichen Genese“ darstellen. Der „(voluntativ-konstitutionelle), Nations'-Begriff“" sei inadäquat. Und den Vertretern des Modells einer plurinationalen Sprache könne man „einen besonders schwerwiegenden Vorwurf nicht ersparen“: (a.a. O., 4) Sie negierten bzw. unterschätzten die aufgrund der „Katastrophe des Nationalsozialismus“ beobachtbare Stigmatisierung bzw. Tabuisierung alles „Nationalen“ im deutschsprachigen Raum, meint Glauniger. Auch hier wird unser Erachtens nach nicht klar, inwiefern das ein Argument gegen eine staatsbezogene Modellierung von sprachlicher Variation sein kann oder soll. Festzustellen ist allerdings, dass die reiche wissenschaftliche Literatur zur österreichischen Nation und zu österreichischen Identitätskonstruktionen sehr wohl die Genese eines ausgeprägten Nationalbewusstseins im Sinn einer Staatsnation und Konsensualnation zumindest in der Zweiten Republik nach 1945 ausführlich dokumentiert (z. B. Heer 1981, Kreissler 1984, Bruckmüller 1996, Wodak et al 1998; 2009, de Cillia/Reisigl/Wodak 1999, de Cillia/Wodak 2006, 2009, vgl. auch Fußnote 15 oben). Glauninger schreibt in der Folge „Teilen der Forschung bzw. Publizistik“ „sozialsymbolisch verbrämte ideologische Wunschbilder“ zu, die in Widerspruch zur ökonomisch-politischen Realität stünden. Die „zunehmend supranationale außersprachliche Wirklichkeit" würde ein national determiniertes Beschreibungsmodell des Deutschen endgültig ad absurdum führen. Für die Linguistik ergebe sich die Aufgabenstellung, mit einer zeitgemäßen, „vielleicht ,supra'- bzw. ,postnational' orientierten Theorie“ die komplexe Heterogenität des Deutschen zu beschreiben (a.a. O., 6). Dazu könnte man an das „,bereits elaborierte“ pluriareale Modell anknüpfen. 
Ähnlich argumentiert Joachim Herrgen (2015), wenn er meint, die Ablösung des Monozentrismus durch ein Plurizentrismus-Konzept sei in der Mitte des 20. Jahrhunderts deskriptiv adäquat gewesen. „Das 21. Jahrhundert hingegen ist durch die lingualen Auswirkungen einer neuen Medienrevolution geprägt. Diese resultieren unter anderem in der Ausbildung transnationaler Oralisierungsnormen“ (Herrgen 2015, 157). An anderer Stelle ist von „supranationalen“ Bewertungsmustern die Rede. Für das Deutsche habe „eine neue Phase der Standardnorm" begonnen (156). Als empirischer Befund wird im betreffenden Text eine perzeptionslinguistische Untersuchung zur „Oralisierungsnorm“ des Standardsprache in Deutschland, Österreich und der Schweiz berichtet, in der drei sehr standardnahe Texte geschulter professioneller Nachrichtensprecher (ARD, ORF, DRS) und als intendierte Standardsprache einzustufende Texte junger AkademikerInnen aus Trostberg (Deutschland), Feldkirchen (Österreich) ${ }^{25}$ und Sattel (Schweiz) Studierenden in Fribourg, Wien und Marburg zur Beurteilung präsentiert wurden. Ergebnis: „Die Beurteiler aus Deutschland, Österreich und der Schweiz sind sich völlig einig, dass die deutsche Probe diejenige ist, die dem standardsprachlichen Pol am nächsten kommt“" (155), wobei in Österreich auch die österreichische Sprachprobe als „,reines Hochdeutsch“ akzeptiert wurde. Die Schweizer Sprachprobe werde hingegen auch von den Schweizer ProbandInnen nicht als „reiner Standard“ klassifiziert. Diese empirischen Daten können natürlich problemlos im Rahmen einer Theorie der Plurizentrik interpretiert werden, worauf auch der Verfasser hinweist (Asymmetrie zwischen D- und A-Varietäten; linguistic cringe, vgl. Kap. 2.4.1). Herrgen schließt daraus jedoch, dass „für das Deutsche eine neue Phase der Standardnorm begonnen hat" (156), da die entscheidenden Medien sich immer stärker entnationalisierten. Die neuen Medien, insbesondere das Internet operierten global statt national. Unter diskursanalytischem Blick fällt auch in diesem Text auf, dass FachkollegInnen „nationale Engführungen“ (142) unterstellt werden, dass die Diskussion v. a. in Österreich mit einer „eher politisch als linguistisch motivierten Verbitterung " geführt werde (a. a. O.), und dass "nationalistische Argumentationen“ in dem Zusammenhang völlig fehl am Platz seien.

Die offene Infragestellung einer österreichischen Staatsnation findet sich bei Seifter \& Seifter (2015), die sich auf Seiten der Pluriarealisten positionieren (66). Auf die im Duktus sehr polemische und in mehrerer Hinsicht für einen wissenschaftlichen Text grenzwertige Arbeit näher einzugehen, ist hier nicht der Ort. ${ }^{26}$

25 Welches der zumindest drei Feldkirchen in Österreich (in Kärnten, an der Donau, bei Graz) gemeint ist, wird nicht präzisiert.

26 Das beginnt damit, dass der Beitrag in keiner Weise auf den Vortrag, auf den sich der Titel bezieht, eingeht. Bei der Konstruktion eines Feindbilds „Plurizentriker“ werden mehrere AutorInnen völlig undifferenziert in einen Topf geworfen. VertreterInnen anderer wissenschaftlichen Konzepte werden abwertend abqualifiziert, ihnen werden unlautere Motive unterstellt (So findet sich der Ausdruck „Lügen“ in einem Zitat von Scheuringer 2016, 381.), in Umkehrstrategie 
Nur so viel sei berichtet: Die Autoren sind der Ansicht, das „österreichische Deutsch“ sei eine „Erfindung, die nur im historischen wie gesellschaftlichen Kontext des Nachkriegsösterreich erklärt werden" könne (65). Das österreichische Deutsch sei ein (sprach-)politisches Konstrukt, um „die Etablierung und Akzep$\operatorname{tanz}$ der ,österreichischen Nation' auf der sprachlichen Ebene zu legitimieren bzw. zu erhöhen“ (a. a. O.). Es sei auch falsch, von einem „österreichischen Volk“ zu sprechen. Und Jörg Haiders Ausspruch, wonach „die österreichische Nation eine Mißgeburt gewesen ist, eine ideologische Mißgeburt", wird als eine "vielleicht überspitzte[n], aber an sich nachvollziehbare[n] Aussage" zitiert (a.a. O., 86). Es sei bedauerlich, „dass sich die Deutschen seit Auschwitz nicht mehr zusammengehörig fühlen dürfen und neue, vollkommen haltlose Nationalismen Platz griffen, die eben auch auf die Linguistik Auswirkungen hatten“ (a.a. O.). Das lässt - wie auch andere Passagen im Text - u. E. keine andere Interpretation als die der Annahme einer großdeutschen Sprachnation zu.

\subsubsection{Plurizentrisch - Pluriareal?}

Auffällig ist bei allen pluriarealen Ansätzen, dass dieser Zugang im Vergleich zum plurizentrischen Ansatz theoretisch derzeit noch weniger elaboriert ist (wie etwa die Offenlegung grundlegender axiomatischer Annahmen und Hypothesen über den Zusammenhang von sprachlichen und außersprachlichen Variablen, Ausarbeitung eines klar definierten terminologischen Instrumentariums wie bei Ammon 1995, methodische Zugangsweisen).

Relativ scharf kritisiert das Dollinger (2016), der feststellt, dass viele ForscherInnen der "German dialectology" von einer "One Standard German Hypothesis“ als zugrundeliegender Annahme ausgehen: „,Pluri-areal' says nothing more than that the German language is subject to regional variation, which extends across national boundaries“. Und: „Proponents of ,pluriareality are proponents of a monocentric approach to Standard German" (Dollinger in Druck, 40). Er ortet einen theorielosen empirischen Zugang („based on naive, a-theoretical positivism“, 48). Aber: „The idea of description without theory is impossible“(Dollinger in Druck, 39). Letztlich liege dem Ansatz „the One German Axiom or the One Standard German Axiom“" zugrunde (26).

Vor allem von den GegnerInnen des plurizentrischen Konzepts wurde die Diskussion um die beiden Konzepte zum Teil sehr emotional und polemisch geführt (s.u.). So ist da z. B., wie schon erwähnt, von der „,Ideologie‘ von der

des den Text prägenden aggressiven Tonfalls wird der Gegenposition unterstellt, larmoyant zu sein etc. Erstaunlich ist, dass ein derartiger Beitrag in einer - zugegeben in der Fachwelt kaum bekannten und wohl auch nicht peer reviewten - Zeitschrift „Beiträge zur Fremdsprachenvermittlung" aufgenommen wurde. 
staatsnationalen Varietät, [...] die auch heute noch in offiziellen Kreisen der Kulturbürokratie gerne gepflegt“ werde, die Rede (Wolf 1994, 75); von „zusätzliche[r] Aufoktroyierung sprachlicher Besonderheiten durch staatliche Zentralstellen" (Scheuringer 1988: 66; vgl. auch Fußnote 13 oben). Unserer Meinung nach handelt es sich beim pluriarealen Konzept letztlich nur um eine andere Konzeptualisierung der sprachlichen Variation innerhalb der deutschen Sprache, deren Beschreibungs- und Erklärungsadäquatheit vor dem Hintergrund unterschiedlicher Annahmen über Sprache als soziales Phänomen beurteilet werden muss und die mit der plurizentrischen Konzeptualisierung durchaus vereinbar ist, sodass sich das pluriareale und das plurizentrische Konzept komplementär ergänzen können. Zum „areallinguistischen“ Einwand, wonach die nationalen Varietäten bereits sehr unterschiedlich seien, sei mit Schmidlin $(2017,47)$ festgehalten: „Im plurizentrischen Konzept haben beide Variationstypen Platz.“ Apodiktische, ausschließende Formulierungen wie: „Das Projekt ,Variantengrammatik' geht davon aus, dass es sich bei der deutschen Sprache nicht um eine plurizentrische, sondern vielmehr um eine pluriareale Sprache handelt" (Niehaus 2017, 62. Herv. im Original) halten wir im Kontext dieser wissenschaftlichen Diskussion nicht für zielführend.

Auch das jeweilige Erkenntnisinteresse und die jeweils gewählte Analyseebene spielen u. E. in den entsprechenden Debatten eine ausschlaggebende Rolle: Steht die präskriptive, kodifizierte Norm im Vordergrund, mag der plurizentrische, staatsraumbezogene Zugang adäquat $\operatorname{sein}^{27}$. So z. B., wenn es wie bei Moosmüller (2015) um die „Bestimmung der Standardaussprache in Österreich" und der Untersuchung der Akzeptanz bestimmter Aussprachevarianten bei österreichischen ProbandInnen geht, auch weil die national organisierten elektronischen Medien dafür eine Rolle spielen. Oder um es mit Spiekermann (2010) zu formulieren: „dass - im Fall des Deutschen - die genormte und kodifizierte Standardsprache in mehreren Staaten Amtssprache ist und die Staaten jeweils eigenverantwortlich darüber entscheiden, was innerhalb des Staatsgebiets als Standard anzusehen ist" (349). Auch Auer (2013, 21) geht von der deutschen Sprache als plurizentrischer Sprache aus sowie von drei verschiedenen Normen im Standarddeutschen in Deutschland, Österreich und der deutschsprachigen Schweiz.

Ebner $(2014,8)$ weist auf die Tatsache hin, dass trotz der historischen Sprachräume sprachliche Ausprägungen aufgrund der Staatsgrenzen im Rahmen des österreichischen Staatsgebiets entstanden sind, „weil die Verwaltungssprache vielfach auf die Gemeinsprache ausstrahlt und die Medien sprachlich bestimmend

27 Ulrich Ammon betonte bei einer Diskussion nach einem Vortrag bei der Internationalen Deutschlehrertagung 2017 in Fribourg $(\mathrm{CH})$, bei der eine der AutorInnen anwesend war, dass das plurizentrische Konzept eben genau im normativen Kontext griffig und auch zur Anwendung auf dieser Ebene gedacht sei. 
sind“. Ist das Interesse auf die (regionalen) Gebrauchsnormen und Gebrauchsstandards gerichtet (etwa wie bei Berend 2005 oder im Projekt Variantengrammatik), mag sich ein pluriarealer Zugang als adäquater erweisen. Dass der pluriareale Zugang gerade für das Deutsche fruchtbar sein kann, erklärt Spiekermann (2010) aus der föderalistischen Tradition der deutschsprachigen Staaten und den im Vergleich zu anderen europäischen Ländern relativ großen Unterschieden in den Ausprägungen der Dialekte (350). Andererseits schreibt auch Scheuringer, ein Vertreter des pluriarealen Ansatzes: „Und doch ist, österreichisches Deutsch eine feste Größe, die sich auf allen Ebenen der Sprache manifestiert, österreichische Spezifika, so genannte ,Austriazismen', aufweist.“ (Scheuringer 2001, 95) Dabei sei das, was österreichisches Deutsch vom Deutschen in den anderen deutschsprachigen Nationen und Regionen unterscheide, primär in Österreichs langer Eigenstaatlichkeit begründet (a. a. O., 96).

Auf die Vereinbarkeit der beiden Ansätze hat schon Ammon 1998 hingewiesen, für den „eine nach Blickwinkeln differenzierte Charakterisierung des Deutschen möglich“ ist, „etwa derart, daß aus der Sicht bestimmter Personen oder Gruppen das Deutsche eher eine plurinationale und aus der Sicht anderer Personen oder Gruppen eher eine pluriareale Sprache ist“. Je nach Perspektive bzw. Funktion wäre die eine oder die andere Zugangsweise angemessener (Ammon 1998, 320). Das Projekt des Variantenwörterbuchs (Ammon et al 2004, Ammon/Bickel/Lenz 2016) trat im Übrigen mit dem Anspruch an, „sowohl der Plurinationalität als auch der Pluriarealität des Deutschen gerecht zu werden“ (Ammon 1998, 320). Das Variantenwörterbuch zeigt in seiner Differenziertheit der Beschreibung des Lexikons der deutschen Sprache auf, dass die Konzepte einander nicht widersprechen. Und mit der Unterscheidung spezifischer und unspezifischer nationaler Varianten ist ein Begriffsinstrumentarium zur Hand, das bei grenzüberschreitender Variation differenzieren kann und sozusagen beide Perspektiven integriert.

VertreterInnen einer plurizentrischen Konzeptualisierung der deutschen Sprache zweifeln staatsgrenzenüberschreitende Gemeinsamkeiten und innerstaatliche Variation - auch auf standardnaher Ebene - nicht an. Sie verweisen aber darauf, dass ein und dieselbe Variante in einem Land als Standard und in einem anderen als Substandard gelten kann. Auch Auer $(2013,24)$ weist darauf hin, dass grenzüberschreitende sprachliche Varianten in den jeweiligen Staaten unterschiedlichen Status und unterschiedliche Normativität genießen: „The difference is that in Austria and Switzerland, they may constitute the only norm, while in Germany, they are merely alternants of the norm (lexicon) or even violations of it (phonetics, some parts of syntax)." Dollinger (2016), der die Relevanz der staatlichen Organisation für sprachliche Variation betont und die Rolle der politischen Grenze zwischen Kanada und den USA für die Plurizentrizität des Englischen mit der Grenze zwischen (Ober-)Österreich und Bayern vergleicht, bemerkt in Bezug auf eine Studie von Scheuringer (1990), dass die historische 
und letztlich dialektologische Zugangsweise des pluriarealen Zugangs der zeitgenössischen sprachlichen Realität im Bereich der Standardsprachen nicht gerecht werde: „Treating international borders linguistically as merely regional boundaries, as proposed by Scheuringer in the Austrian context, does not do justice to the linguistic realities of the past 200 years in neither the Austrian nor the Canadian context" (Dollinger 2017, 98).

Eine von James Pfrehm im Rahmen seiner Dissertation (Pfrehm 2007, 2011b) durchgeführte Spracheinstellungserhebung in Österreich und Deutschland untermauert mit empirischen Argumenten die Plausibilität des plurizentrischen Konzepts. Er ging der Frage nach, wie deutsche $(n=102)$ MuttersprachlerInnen aus zwei Großregionen, Bayern $(\mathrm{n}=57)$ und Nord-/Mitteldeutschland $(\mathrm{n}=45)$, und österreichische $(\mathrm{n}=115)$ MuttersprachlerInnen ${ }^{28}$ die Verwendungshäufigkeit und Standardsprachlichkeit von 36 lexikalischen Items bewerten. Die 36 Items, die in den Quellen „Österreichisches Wörterbuch“, „Wie sagt man in Österreich?“, „Variantenwörterbuch des Deutschen“ und „Duden - Deutsches Universalwörterbuch" als standardsprachlich markiert sind, standen dabei für die „österreichisch-deutsche“ („Austrian Standard German“, $\mathrm{n}=18^{29}$ ) bzw. die "deutsch-deutsche“ („German Standard German“, $\mathrm{n}=18^{30}$ ) geschriebene Standardsprache. Alle lexikalischen Items wurden in einem schriftlichen Fragebogen in einer durch das Zufallsprinzip ermittelten Abfolge präsentiert und von den deutschen und österreichischen TeilnehmerInnen auf zwei Likert-Skalen ${ }^{31}$ bewertet. In der Auswertung wurden die „Nationalität" und „regionale Herkunft" der Befragten und die Lexem-Eigenschaften „Austriazismus" und „Teutonismus“ („Deutschlandismus") berücksichtigt. Die empirisch gewonnenen quantitativen Daten wurden darüber hinaus statistisch analysiert und auf ihre Signifikanz überprüft. Als ein Beispiel sei das Wort „heuer" genannt. Die durchschnittlichen Bewertungen der österreichischen, bayrischen und nord-/mitteldeutschen ProbandInnen bezüglich der Verwendungshäufigkeit und der Standardsprachlichkeit von „heuer“ zeigen, dass zwar in der Verwendungshäufigkeit kein großer Unterschied zwischen ÖsterreicherInnen und Bayern, sehr wohl aber zu den Nord-/Mitteldeutschen bestand, dass jedoch bei der Standardsprachlichkeit ein

28 Die geographische Einteilung der ProbandInnen erfolgte nach deren jeweiliger Antwort auf die Frage "Wo sind Sie aufgewachsen?"

29 Marille, Fisolen, Kren, Schmankerl, Kukuruz, Karfiol, Faschiertes, Türschnalle, Sackerl, Schuhband, Stiege, Putzerei, Greißler, Realkanzlei, heuer, Wimmerl, Jänner, Gspusi.

30 Aprikose, grüne Bohnen, Meerrettich, Leckerbissen, Mais, Blumenkohl, Hackfleisch, Türklinke, Tüte [z. B. für Essen], Schnürsenkel, Treppe, chemische Reinigung, Lebensmittelhändler, Immobilienbüro, dieses Jahr, Pickel [im Gesicht], Januar, Liebesverhältnis.

31 Die Skalen umfassten die Werte 1 bis 4 . Bei der Frage nach der Verwendungshäufigkeit: $1=$ „Das Wort würde ich beim Schreiben eines Aufsatzes bestimmt nicht verwenden“ und $4=$ "Das Wort würde ich beim Schreiben eines Aufsatzes ohne zu zögern verwenden“; bei der Frage nach der Standardsprachlichkeit: 1 = ,umgangssprachlich/nicht standardsprachlich“ und 4 = "standardsprachlich“. 
statistisch hochsignifikanter Unterschied zwischen ÖsterreicherInnen einerseits und Bayern und Nord-/Mitteldeutschen andererseits feststellbar war: Nur Erstere beurteilen dieses Lexem als standardsprachlich. So könne man laut Pfrehm bei solchen Wörtern wie „heuer“ von einem Schibboleth für die österreichische Varietät des Deutschen sprechen. Pfrehm liefert klare empirische Indizien dafür, dass es Wörter gibt, die zwar von MuttersprachlerInnen der deutschen Sprache in verschiedenen Sprachgebieten in ähnlicher Weise verwendet, jedoch im Hinblick auf die Standardsprachlichkeit anders beurteilt werden, und unterstützt so die plurizentrische Konzeptualisierung der deutschen Sprache. Auch Scheuringer (2001) weist darauf hin, dass es Wörter gibt, die in Bayern und Österreich „,in unterschiedlicher Weise bewertet" werden. „Während sie in Österreich problemlos Teil der deutschen Standardsprache sind, gelten sie in Bayern in der Regel als Nonstandard, nur selten sind sie ,teilstandardfähig' wie (seltener) Kren oder (häufiger) Topfen, Quark““ (109, Herv. im Original).

$\mathrm{Zu}$ ähnlichen Ergebnissen kommt Mohn (2017), der in seiner Dissertation eine Untersuchung der Aussprache von Nachrichtensprechern und Nachrichtensprecherinnen des Bayrischen Rundfunks und des ORF durchgeführt hat. Dabei stellte sich zunächst schon heraus, dass BR-SprecherInnen sehr oft nicht aus Bayern kommen und eine außerbayerische Sprechausbildung haben, also eine areallinguistisch heterogene Gruppe darstellen - ein Hinweis auf die staatsbezogene Organisation des Mediums. Norddeutsche NachrichtensprecherInnen wären in Österreich nicht vorstellbar. Mohn hat daher „zugereiste“ NachrichtensprecherInnen nicht berücksichtigt. Trotzdem zeige sich neben einigen Gemeinsamkeiten, dass die Aussprache der ORF-SprecherInnen deutlich von BR-SprecherInnen abweiche, die Aussprache von ORF-SprecherInnen relativ einheitlich sei und dass es eine deutlich erkennbare „österreichische Sprechkonvention" gebe. In Bayern sei die Aussprache, obwohl sie auch hier bei einigen Variablen von der „bundesdeutschen Standardlautung“ abweiche, näher an dieser "bundesdeutschen Standardlautung " als im ORF. Das bedeutet, dass bei BR-SprecherInnen eine deutlich stärkere Orientierung am Aussprache-Duden vorliegt (Mohn, in Druck).

Auch die Ergebnisse der Spracheinstellungsforschung von Ender/Kaiser (2009) liefern Indizien dafür, dass staatliche Grenzen eine Rolle für Sprachverwendung und Spracheinstellungen spielen. Sie vergleichen den Stellenwert von Dialekt und Standard im österreichischen und Schweizer Alltag, wobei in Österreich das durch das Dialekt-Standard-Kontinuum geprägte bairische Sprachgebiet und das - ähnlich wie in der Schweiz - durch eine diglossale Situation gekennzeichnete alemannischsprachige Vorarlberg getrennt betrachtet werden und Letzteres wiederum mit der Situation in der Schweiz verglichen wird. Für den vorliegenden Kontext sind die konstatierten Unterschiede zwischen Vorarlberger und Schweizer SprecherInnen von Interesse: Erstere betrachten ihren Dialekt als weiter vom Standard entfernt als die Schweizer SprecherInnen 
( $47 \%$ gegenüber $32 \%$ bezeichnen den Unterschied zwischen ihrem Dialekt und dem Standard als ,groß‘, 278). Für Vorarlberg werden „einige deutliche Sonderentwicklungen durch die Ausrichtung auf Gesamtösterreich" festgestellt. Es gäbe eine positivere Einstellung zum Hochdeutschen: „Im Sprachbewusstsein der befragten Vorarlberger/-innen hat die Standardsprache keinesfalls den Status einer Fremdsprache, wie dies immerhin mehr als ein Drittel der Schweizer/-innen von sich aussagen" (a. a. O., 291). Die gemeinsame diskursive und mediale Öffentlichkeit innerhalb eines Staates und die national organisierte schulische und mediale Sozialisation sind hier mögliche Erklärungen für diese unterschiedlichen Spracheinstellungen.

Die Arbeit von Peter (2015) zum Textbewertungsverhalten von SprecherInnen in Deutschland, Österreich und der Schweiz weist in dieselbe Richtung. Er hatte jeweils 50 Studierenden der Universitäten Bern, Innsbruck und Siegen Ganztexte (drei kurze von Studierenden in Innsbruck erstellte Zusammenfassungen von längeren populärwissenschaftlichen Zeitschriftenartikeln) zur Textbewertung und Korrektur vorgelegt und sie gebeten, sprachliche Auffälligkeiten zu markieren und in Hinblick auf den Schweregrad der Sprachnormverletzung zu bewerten. Als Resultat wurde u. a. festgestellt,

dass sich die Normvorstellungen von Sprecherinnen und Sprechern in Österreich, Deutschland und der Schweiz nicht nur grundsätzlich voneinander unterscheiden, sondern dass die Normvorstellungen vor allem in Hinblick auf unterschiedliche Ebenen wie Varietätenwahl, Varietätenprestige oder Normerfüllung jeweils unterschiedlich ausgeprägt sind (139).

Österreichische, und noch deutlicher Schweizer SprecherInnen zeichneten sich im Vergleich zu SprecherInnen der bundesdeutschen Varietät durch „eine erhöhte Sensibilität insbesondere für kleinräumige und formbezogene Phänomene" aus.

Regula Schmidlin (2011) hat in ihrer Habilitationsschrift untersucht, „inwiefern die Plurizentrik der deutschen Standardsprache in den Köpfen der deutschsprachigen Sprecher repräsentiert ist" (281) und hat dazu 908 Gewährspersonen (GP) aus allen Regionen des deutschsprachigen Raums mittels Internetfragebogen befragt. Auch ihr, an mehreren Daten ausgemachter Befund ist, dass Landesgrenzen als „Variantenloyalitätsgrenze “ für Spracheinstellungen und Sprachloyalität wichtig sind, und das zeige sich auch an der „binnennationalen Einheit der GP aus Österreich und Deutschland“ (282). Auch bei der Kenntnis von Varianten zeige sich "der Effekt der Landesgrenze als kognitive und sich daraus ergebende sprachpragmatische Grenze“ (282). So hätten GP aus Deutschland-Südost für den spezifischen Austriazismus keine höheren Kenntnis- und Gebrauchswerte angegeben als die GP aus den übrigen Regionen Deutschlands. Schließlich würden auch die GP aus Deutschland-Süd die Varianten mit Geltungsareal Österreich, Schweiz und Deutschland-Süd als dialektaler einschätzen als die GP aus allen 
übrigen Regionen. „Erneut zeigt sich die Nationalgrenze bei den Einstellungen von GP aus D als wirksamer als Dialektgrenzen.“ (284) Letzterer Befund ist dem von Pfrehm ähnlich. Dabei würden die GP aus Österreich die Standardsprachlichkeit von Varianten am höchsten einschätzen. Das bestätigt wiederum Ammon (2000a): „Ein verhältnismäßig großer Teil der Bevölkerung (Österreichs, Anm.d. Verf.) scheint sich der Existenz eigener Nationalvarianten bewusst zu sein“ (518). Die Frage nach dem besten Hochdeutsch ergab im Übrigen ein ähnliches Ergebnis: Nur $25 \%$ aus Österreich, aber $42 \%$ aus der Schweiz und $55 \%$ aus Deutschland sehen das beste geschriebene Hochdeutsch in Deutschland (Schmidlin 2011, 287). Auch wenn Schmidlin das Plurizentrizitätsbewusstsein generell als schwach ausgeprägt einschätzt („dass die in der Linguistik und Lexikographie akzeptierte Plurizentrik des Deutschen mehrheitlich nicht bis in die Köpfe der Sprecherinnen und Sprecher vorgedrungen zu sein scheint“, 296), zeigt es sich so gesehen am ehesten in Österreich.

Um zur Frage „Plurizentrik - Pluriarealität“ zurückzukommen: Schmidlin hebt als bemerkenswerten Befund ihrer Arbeit „die Wichtigkeit der Landesgrenzen als pragmatische und kognitive Grenze" hervor (297). Diese sei im Zusammenhang mit den Standardvarietäten des Deutschen „erstmals in dieser Deutlichkeit und erstmals auf einer breiten empirischen Basis nachgewiesen“ worden (a.a.O). „Die Plurizentrik ist kein Phantom, sondern eine Variationsebene, deren Existenz in öffentlichen, breit rezipierten Texten nachgewiesen werden kann. Sie mag in den Köpfen der Sprecher nicht als Konzept existieren, wird aber praktiziert.“ (300) An anderer Stelle hält Schmidlin fest, die Plurizentrik sei „so lange kein Widerspruch zur Pluriarealität, als man nicht nur nationale Varianten, sondern auch regionale Varianten berücksichtigt, deren Geltungsareale über nationale Grenzen hinausgehen" (Schmidlin 2011, 4).

Schmidlin/Wyss/Davies (2017), die zwischen ,einer stärker der Dialektologie verpflichteten pluriarealen Modellierung einerseits und der soziolinguistischinstitutionell argumentierenden plurizentrischen Modellierung andererseits “ (16) unterscheiden, stellen sich die Frage, welches Gewicht der nationalen Zugehörigkeit bei der Kategorisierung der Varietäten der deutschen Standardsprache beigemessen werden könne. Auch wenn Nationalismen zu Recht hinterfragt würden und ihre Überwindung gefordert werde: „Die soziokulturelle Wirkung nationalterritorialer politischer Grenzen bei der Beschreibung von Sprachvariation zu negieren, wäre unseres Erachtens aber der falsche Weg“ (a.a.O., 16). Auch eine plurizentrische Modellierung der Sprachvariation könne Überlappungen von Merkmalen entlang verschiedener Kontaktzonen darstellen, und die nationale Variationsdimension könne als eine Spielart neben anderen Variationsdimensionen verstanden werden (16).

Im Kontext der vorliegenden Forschungsarbeit ist es uns wichtig, festzuhalten, dass wir es mit sprachpolitischen und sprachsoziologischen Phänomenen zu tun haben, mit Kodifizierung, Normsetzung und staatlichen Instanzen, die diese 
Normierung letztlich überwachen. Die Nationalstaaten haben eine zentrale Rolle gespielt bei der Herausbildung von sprachlichen Standards - für die Verwaltung, das Rechtswesen und sonstige staatliche Institutionen, sowie im Verlagswesen und in den Medien. Vor allem: Das Schulwesen ist in Österreich gesamtstaatlich organisiert. Das ist ein Grund, warum im vorliegenden Projekt der plurizentrische Ansatz gewählt wurde, ohne dass - wie der empirische Teil zeigen wird - innerösterreichische Variation außer Acht gelassen worden wäre. Die Ergebnisse der Befragung zur Verwendung von Austriazismen/Deutschlandismen haben auch gezeigt, dass einerseits die Kodifizierung des österreichischen Deutsch lückenhaft ist und dass es auch eine dynamische altersabhängige Entwicklung im Sprachgebrauch unter dem Einfluss der Medien gibt, sodass kodifizierte Norm und Gebrauchsnorm auseinanderdriften.

\subsection{Sprachliche Variation der deutschen Sprache in Österreich}

Überträgt man das oben angeführte Modell von sprachlicher Variation auf die Sprachsituation in Österreich, dann wird meist zwischen österreichischem Standarddeutsch/Hochdeutsch, einer großräumigeren Umgangssprache und Dialekt unterschieden. Das führt zu einer drei- bzw. vierstufigen „Polyglossie“: Basisdialekt, Verkehrsdialekt, Umgangssprache und Standardsprache (Steinegger 1998, Wiesinger 1985). Das Kontinuum zwischen Dialekt und Standard („Hochdeutsch“) kann man nach Wiesinger in vier Ebenen einteilen: örtliche Basisdialekte, regionale Verkehrsdialekte, eine dem Hochdeutschen angenäherte Umgangssprache, die keine auffälligen Dialektmerkmale mehr enthält, und „Hochdeutsch“ (Wiesinger 2010, 363). Der Basisdialekt weise dabei die geringste Verbreitung auf, werde von alteingesessenen DorfbewohnerInnen in Gesprächen untereinander und in Gesprächen mit jüngeren Familienangehörigen verwendet. Beim Verkehrsdialekt handle es sich um eine mehr oder weniger dialektal beeinflusste Alltagssprache, wobei die Stärke der Beeinflussung von regionalen und sozialen Faktoren abhängig zu sein scheine. Die Beherrschung der Standardvarietät stehe wiederum in direktem Zusammenhang mit ihrer Definition: Werde Standard als überregional gesprochene Alltagssprache verstanden (im Sinn eines Gebrauchsstandards, Anm. d. Verf.), dann sei die Gruppe derer, die sie nicht oder mangelhaft beherrschen, relativ klein. Werde der Standard nach einer schriftlichen Norm definiert, sei das Gegenteil der Fall.

Eine andere Modellierung als die mit der Trias „Standard - Umgangssprache - Dialekt" besteht darin, die österreichische Sprachsituation als eine Diglossie zu fassen, wie dies Muhr vorschlägt $(2013,1997){ }^{32}$ Demnach weise der

32 Rudolf Muhr schreibt Österreichisches Deutsch im Übrigen mit großem Anfangsbuchstaben, da er österreichisches Deutsch als Eigennamen interpretiert (Muhr 2013). 
Sprachgebrauch der ÖsterreicherInnen alle Merkmale einer komplexen Diglossie auf, in der im Alltag und in nähesprachlichen Situationen (ähnlich wie in der Schweiz) regionale oder großregionale Varietäten des österreichischen Deutsch verwendet werden. In formellen Situationen würden Varianten des formalen, schriftbasierten Sprechstandards gebraucht. Je nach beruflicher oder privater Situation komme es zu einem regelmäßigen Wechsel zwischen diesen Varietäten. Dem liege eine komplexe Kompetenz der SprecherInnen zugrunde, und es sei aufgrund der linguistischen Distanz zwischen den Varietäten von einem bilingualen Sprachverhalten auszugehen. Muhr spricht von nähesprachlichen und distanzsprachlichen Varietäten, die als kommunikative und nicht als linguistische Standards wirken.

Die Untersuchung von Ender/Kaiser hat allerdings gezeigt, dass die „Umgangssprache" als Varietät zwischen Standard und Dialekt in Österreich - im Unterschied zur Schweiz - von den SprecherInnen als wichtig wahrgenommen wird (Ender/Kaiser 2009), was ein Argument für die Annahme eines dreistufigen Modells ist, auch wenn die Konzeptualisierung der Umgangssprache schwierig ist (vgl. 2.3). Im vorliegenden Projekt ist zunächst - der zentralen Fragestellung gemäß - die Standardvarietät im Fokus gestanden, die das von der Schule anzustrebende bildungssprachliche Register darstellt. Aber sowohl bei der Analyse der relevanten Dokumente (Lehrpläne, Studienpläne, Lehrbücher) als auch bei der Befragung war es wichtig, die gesamte Bandbreite der Sprachverwendung des Deutschen in Österreich im schulischen Kontext zu thematisieren (s. u.) und zu erfragen, wie mit dem Varietätenspektrum des Deutschen in Österreich umgegangen wird. Dabei sind wir von der oben dargestellten dreistufigen Modellierung des Dialekt-Standard-Kontinuums ausgegangen. Es zeigte sich, dass alle drei Varietäten - unterschiedlich gewichtet bei LehrerInnen und SchülerInnen eine wichtige Rolle bei der Wahrnehmung der sprachlichen Situation und beim Sprachgebrauch in Österreich spielen (siehe Kap. 6.1).

Bei der Beschreibung der sprachlichen Situation in Österreich bezieht sich die Bezeichnung „österreichisches Deutsch“ in der Regel auf die Standardsprache (Wiesinger 2010, 360; Ebner 2009; vgl. auch Clyne 2005, 296 $6^{33}$ ). Wenn alle schriftlichen und mündlichen Varietäten der deutschen Sprache gemeint sind, wird das mit „Deutsch in Österreich“ bezeichnet.

Wenn von Deutsch in Österreich oder der deutschen Sprache in Österreich die Rede ist, sind prinzipiell alle sprachlichen Ausprägungen der deutschen Sprache in Österreich gemeint [...]. Österreichisches Deutsch meint hingegen die Standardvarietät des Deutschen in Österreich, wozu sowohl innerösterreichische regionale Ausprägungen gehören, sofern sie standardsprachlich sind, als auch Ausprägungen, die mit

33 Er verweist darauf, dass manche „national variety“ dafür verwenden, „to include all varieties used in a nation", z. B. Muhr (296). 
den Nachbarlandschaften in Deutschland, der Schweiz oder Südtirol übereinstimmen. (Ebner 2009, 442, Herv. im Original)

Was die innerösterreichische Variation betrifft, werden wie schon erwähnt in der Regel vier Sprachlandschaften/Regionen unterschieden (Ammon et al. 2004, Ammon/Bickel/Lenz 2016, Ebner 2009, 448): Ostösterreich, Mitte, Südostösterreich und Westösterreich. Vorarlberg und der Tiroler Bezirk Reutte haben dabei wegen der alemannischen Dialektbasis eine Sonderstellung, weshalb wir in der vorliegenden Studie fünf Regionen angenommen und zwischen Tirol und Vorarlberg unterschieden haben (s.u.). ${ }^{34}$

In der Praxis verschwimmen freilich die Grenzen zwischen dem österreichischen Deutsch im engeren Sinn und Deutsch in Österreich, v. a. im Sprachgebrauch von LaiInnen, für die österreichisches Deutsch häufig mit allen sprachlichen Erscheinungen Österreichs verbunden ist (siehe auch die Ergebnisse unserer Befragung, Kap. 6). Die österreichischen Spezifika beziehen sich dabei auf alle Sprachebenen (von der phonetisch-phonologischen, orthographischen Ebene über Morphologie, Syntax, Semantik bis hin zur Pragmatik). Was den Wortschatz betrifft, sind in Ebner (2009) ca. 8.00o Wörter enthalten, womit nach Wiesinger Austriazismen etwa $3 \%$ vom gesamtdeutschen Wortschatz ausmachen (2010, 360). Spezifische Varianten würden nur einen kleinen Teil davon bilden, beim größeren Teil handle es sich um unspezifische Varianten (361). Es gäbe länderübergreifende oberdeutsche Varianten in Süddeutschland, Österreich und der Schweiz, westoberdeutsche Varianten in Südwestdeutschland, der Schweiz, Liechtenstein und Vorarlberg und ostoberdeutsche Varianten in Bayern und Österreich. ${ }^{35}$ Auf der anderen Seite werden auch innerösterreichische Unterschiede in der Literatur immer wieder genannt - Varianten, die auf Teilgebiete Österreichs beschränkt sind, vor allem im Wortschatz sind West-Ost-Unterschiede relevant (Wiesinger 2010, 361, Ebner 2009, 448).

Beschreibungen des österreichischen Deutsch bzw. eine Darstellung der Besonderheiten der deutschen Sprache in Österreich finden sich in zahlreichen Publikationen von Wiesinger (z. B. 1988b, 2006/2008/2014, 2010), Ammon (1995), Ebner (2008, 2012), Scheuringer (2001), Dokumentationen des Lexikons in den Differenzwörterbüchern (Ebner 2009) und - indirekt - im Variantenwörterbuch (Ammon et al. 2004 bzw. Ammon/Bickel/Lenz 2016). Eine offizielle Stelle, in deren Aufgabenbereich die Sprachenpolitik und damit auch die Korpusplanung des österreichischen Deutsch fallen könnte, existiert nicht ${ }^{36}$. Der das ÖWB

34 Da weder im Bezirk Reutte noch im westlichen Salzburg Daten erhoben wurden, ist im vorliegenden Datenmaterial vereinfacht von „Tirol“ und „Vorarlberg“ die Rede.

35 Inwieweit die jeweiligen Varianten in den einzelnen Ländern auch als Standard empfunden werden, müsste u. E. empirisch geklärt werden (vgl. die Arbeiten von Pfrehm).

36 Auch Wiesinger $(2014,191)$ stellt fest, es mangle, in Österreich an einer nach innen betriebenen Sprachpolitik", und da sei besonders im Deutschunterricht an den Schulen anzusetzen. 
herausgebende, seit 1772 existierende Österreichische Bundesverlag ist überdies seit seiner Privatisierung im Jahr 2002 Teil einer bundesdeutschen Verlagsgruppe. Auch die Österreichische Akademie der Wissenschaften erklärte sich bis vor wenigen Jahren nicht dafür zuständig. 2012 wurde jedoch von Jutta Ransmayr an der ÖAW in Kooperation mit der Austria Presse Agentur ein neues, umfassendes Datenkorpus, das AMC (Austrian Media Corpus) geplant, angebahnt und ins Leben gerufen, um eine breite empirische Datenbasis zur Erforschung des österreichischen Deutsch zu schaffen. Das Austrian Media Corpus (AMC) ist eine digitale Datensammlung (mehr als 10,5 Milliarden Tokens mit Stand 31. 12. 2017) sämtlicher österreichischer Medientexte und APA-Meldungen über einen Zeitraum von rund drei Jahrzehnten. Das Korpus ist partiell exhaustiv und in seiner Zusammensetzung insofern einzigartig, als es praktisch die gesamte Printmedienlandschaft eines Landes über einen langen Zeitraum abdeckt. Durch das AMC ist es erstmals möglich, auf einer großen, empirisch fundierten Basis zum österreichischen Standarddeutsch zu forschen. Das AMC durfte als erste große lexikographische Anwendung als Datenbasis dem österreichischen Teil des Variantenwörterbuchs NEU zugrunde liegen. Auch die 43. Auflage des Österreichischen Wörterbuchs wurde durch das AMC unterstützt (vgl. dazu Ransmayr/ Mörth/Durco 2017). Seit 2015 existiert auch eine eigene Abteilung im ACDH, die sich mit der Variation und dem Wandel des Deutschen in Österreich beschäftigt.

Insgesamt ist es noch relativ schwierig, den Kodex des österreichischen Deutsch zu benennen. Ammon (1995, $137 \mathrm{ff}$ ) gibt als Bestandteil des Binnenkodex des österreichischen Deutsch in erster Linie das ÖWB und das darauf basierende kleinere Wörterbuch für den Schulgebrauch an sowie für die Aussprache einen vom ORF (1987) herausgegebenen Lernbehelf „Sprache und Sprechen in Hörfunk und Fernsehen“ (2016 in der 3. überarbeiteten Auflage erschienen: „Über Sprache und Sprechen im ORF“). Auch Ebner (1969, 1980, letzte, 4. völlig überarbeitete Auflage 2009) kann, obwohl im Duden-Verlag erschienen, zum Binnenkodex gezählt werden. Ansonsten werden diverse Duden-Wörterbücher zum Kodex gezählt, quasi als Beiträge der Außenkodifizierung zum österreichischen Deutsch, wie etwa das österreichische Schulwörterbuch von Duden (Duden 2008). Einen offiziell von einer Institution der Republik Österreich initiierten Kodex gibt es heute nicht (mehr), da wie erwähnt auch das ÖWB in einem Verlag erscheint, der Teil der bundesdeutschen Klett-Gruppe ist. Als Kodex könnte man trotzdem das Österreichische Wörterbuch, Ebner 2009, die Publikationen von Wiesinger (1988a, b, 2014, zur Aussprache 2009; Muhr 2007 - kritisch dazu jedoch Hirschfeld 2009) bezeichnen. Die Ergebnisse des Projekts Variantengrammatik werden weitere Beiträge zum österreichischen Deutsch liefern. Für das vorliegende Projekt wurde vor allem - sowohl bei der Dokumentenanalyse als auch bei der Erstellung der entsprechenden Fragebogen-Items - auf das ÖWB, auf Ebner 2009, die Beiträge in Wiesinger 1988a, Wiesinger 2006a, Ammon 1995, Ammon et al. 2004, fallweise auf Muhr 2000 und den Duden 2008 zurückgegriffen. 
Die Forschungslage ist dadurch gekennzeichnet, dass eine umfassende exakte Beschreibung der tatsächlichen Sprachvariation in Österreich bislang aussteht und man auf wenig empirische Forschungen zurückgreifen kann. Eine realitätsnahe Modellierung und genaue empirische Beschreibung des heimischen Sprachgebrauchs, welche die gesamte Variationsbreite des Sprachverhaltens im Dialekt-Standard-Kontinuum erfasst und variationslinguistisch begründet, stellt ein wichtiges Desiderat dar. Einen Beitrag dazu wird - so kann man annehmen der Spezialforschungsbereich „Deutsch in Österreich“ (DiÖ) ${ }^{37}$ leisten.

Über die konkrete Varietätenvielfalt im Mündlichen gibt die Arbeit von Steinegger (1998) Auskunft, der Sprachgebrauch und Sprachbeurteilung in Österreich und Südtirol mit einer Fragebogenerhebung $(n=1464)$ 1984/85 und 1991 untersucht hat. Steinegger stellt zum einen ein deutliches Stadt-Land-Gefälle beim Gebrauch der Standardsprache bzw. der Umgangssprache und des Dialekts fest. Zum anderen konnte er zeigen, dass das Sprachverhalten der Befragten abhängig von der Situation und vom Gesprächspartner variiert: Mit Familienmitgliedern (Kinder ausgenommen) werde eher Dialekt bevorzugt, bei weniger bekannten Personen schwanke der Sprachgebrauch zwischen Umgangssprache, Dialekt und Hochsprache. Mit dem Formalitätsgrad der Situationen (im Gespräch mit Lehrern, Ämtern, Ärzten, sonstigen ,Unbekannten') sinke der Dialektgebrauch und ein Übergang zu Umgangssprache oder Hochsprache finde statt (Steinegger 1998, 105 f). Beim gewünschten Sprachgebrauch mit Kindern plädiere knapp die Hälfte für die Verwendung der Hochsprache, der Rest schwanke zwischen Umgangssprache und Dialekt. Bezogen auf die eigene Schulzeit fiel es etwas mehr als der Hälfte der von Steinegger befragten Personen leicht, Hochdeutsch im Unterricht zu sprechen. Rund ein Drittel gab an, keine besonderen Probleme mit dem Hochdeutschen im Unterricht gehabt zu haben, während es jedoch rund 12 \% schwergefallen sei, in der Schule Hochdeutsch verwenden zu müssen.

Wiesinger (2010), der die Arbeiten von Steinegger einbezieht, stellt fest, dass „die österreichisch geprägte Standardsprache [...] in erster Linie in nur wenigen Situationen des öffentlichen Lebens wie Rundfunk, Fernsehen, Kirche und Schule als offiziöse Sprachform und das mit phono-stilistischen Abstufungen gebraucht" werde, und bloß eine kleine, besonders städtische Bildungsschicht spreche sie auch als Alltagssprache (363). Die mündliche Variation sei abhängig von Variablen wie sozialer Stellung, Beruf, Mobilität, Wohnort, Generationszugehörigkeit, Geschlecht etc. Bei Ammon/Bickel/Lenz 2016 wird zwischen zwei Formen des Standards in Österreich unterschieden, der Sprache der Schriftlichkeit und jener mündlichen Sprechakte, die als öffentlich und/oder formell gelten (z. B. Ansprachen, Predigten, Nachrichten), und einer informellen Variante, die sich in wenigen Merkmalen vom formellen Standard unterscheide. Diese

37 Siehe https://dioe.at/ (9.1. 2019). 
Variante sei umso ausgeprägter, je deutlicher dialogisch Äußerungen angelegt seien. Sie werde in öffentlichen Debatten, von FernsehmoderatorInnen, aber auch in privaten Gesprächen in sozial und bildungsmäßig "gehobenen“ Kreisen im urbanen Raum, v. a. in Ostösterreich, verwendet. (XLV). Die beiden Varietäten der Standardsprache beherrschten auch den Schulunterricht.

In der 1984/85 und 1991 durchgeführten Umfrage von Steinegger (Steinegger 1998; vgl. Wiesinger 2014, 87-101), auf die Wiesinger (2010) Bezug nimmt, gaben $79 \%$ der Befragten an, Dialektsprecher zu sein. 50 \% gaben den Dialekt, $45 \%$ die Umgangssprache und $5 \%$ Hochdeutsch als ihre durchschnittliche Alltagssprache an. Dabei lagen in den Dörfern die Werte bei 62:35:3, bei Großstädtern wurde die Umgangssprache am häufigsten genannt: 27:65:8. Was das schichtspezifische Sprachverhalten angeht, nimmt Wiesinger zufolge „der Dialekt von einer unteren über eine mittlere zu einer höheren Sozialschicht zugunsten der höheren Varietäten im Gesamtdurchschnitt von 76:23:1, 47:49:4 und 35:56:9 ab" (Wiesinger 2010, 363). Beim situationsspezifischen Sprachverhalten sei eine Abnahme der Verwendung des Dialekts mit zunehmender Distanz zum Gesprächspartner zu beobachten, von der Domäne Familie und der Kommunikation mit dem Partner über den Einkauf und den Arbeitsplatz bis hin zum Sprachverhalten vor einem Amt (a. a.O). Laut Wiesinger lasse sich aber zunehmend beobachten, dass auch in offiziösen Situation immer mehr der Dialekt um sich greife, und auch die Schule habe „sich im Unterricht zunehmend auf die Umgangssprache als mündliche Konversationsform eingependelt" (364).

Mit der Frage, welche Sprachform die ÖsterreicherInnen am ehesten als „österreichisches Hochdeutsch" empfinden, hat sich schon Moosmüller (1991) in ihrer soziolinguistischen Untersuchung über Hochsprache und Dialekt in Österreich (1991) befasst. Erhoben wurde dabei auch, wie die ÖsterreicherInnen zu ihrer eigenen Varietät stehen. Moosmüller stellt in diesem Zusammenhang bezüglich des "gehobenen Deutsch“ in Österreich fest, dass die Existenz einer selbständigen österreichischen Hochsprache durchaus im Sprachbewusstsein der ÖsterreicherInnen verankert ist und die Befragten die nationale Einheit und Eigenständigkeit der Republik mit dieser sprachlichen Frage in Verbindung setzen (Moosmüller 1991, 16). Was die Frage angeht, welche Sprachform die ÖsterreicherInnen am ehesten als „österreichisches Hochdeutsch“ empfinden, identifiziert Moosmüller (1991, 23 ff) zwei ausschlaggebende Faktoren: nämlich eine regionale und eine soziale Zuordnung, die entscheidend mitbestimmen, welche Varietät in Österreich als überregionaler, hochsprachlich angesiedelter Standard anerkannt wird. Der Studie zufolge werden Varietäten, die einer Landesregion Österreichs (aber nicht Wien) zugeordnet werden, als „nicht für ganz Österreich gültig" empfunden. Die Befragten aus den Bundesländern siedelten eine österreichische Hochsprache eindeutig in Wien an, und auch in Wien werde das "gehobene Wienerisch“ mit der österreichischen Hochsprache gleichgesetzt. Unter Miteinbeziehung des Faktors der sozialen Zuordnung meint Moosmüller (1991, 29): 
Wird für die Herausarbeitung dessen, was in Österreich als hochsprachlich angesehen wird, die Zuerkennung einer Varietät als überregional sowie deren Zuordnung zu einem sozial hohen Status herangezogen, so werden in erster Linie die Sprecher oberer sozialer Schichten aus Salzburg und Wien für eine Standardsprache als geeignet anerkannt.

Diese Ergebnisse sind im Übrigen von Steinegger (1998) bestätigt worden, in dessen Studie auf die Frage, wo in Österreich ein besonders schönes Hochdeutsch gesprochen werde, Wien und Salzburg an oberster Stelle genannt werden $(355,377 \mathrm{f})$.

\subsection{Sprachnorm und Sprachenunterricht}

Das vorliegende Buch befasst sich mit der Rolle der deutschen Sprache im schulischen Kontext, im Speziellen als Unterrichts- und Bildungssprache und im Unterrichtsfach Deutsch. Deshalb seien vorweg auch einige Bemerkungen zur Frage der Sprachnormen dem Ergebnisbericht vorausgeschickt. Dabei kann hier nicht eine eingehende Auseinandersetzung mit der Frage der Norm, nach Peyer/ Portmann/Brütsch/Gallmann/Lindauer/Linke/Nussbaumer/Looser und Sieber (1996) einem der „Schmuddelkinder“ der Linguistik, geleistet werden, die mit Recht feststellen, dass der Normbegriff äußerst schillernd und „das Begriffswirrwarr im Bereich ,Sprache und Norm' kaum zu bewältigen“ sei (Peyer et al. 1996, 10). Es folgt daher lediglich eine kurze Diskussion von Normkonzepten, insofern sie für die Fragestellung unseres Projekts von Relevanz sind.

Mit Sprachnorm meint man zunächst „das sprachlich Korrekte, Richtige“ (Ammon 200ob, 664), so wie es z. B. durch grammatische Hand- und Wörterbücher und durch den Kodex für die deutsche Standardsprache fest- und vorgeschrieben oder zumindest empfohlen ist und durch Bildungseinrichtungen (v. a. Schulen und Hochschulen), Medien u. Ä. verbreitet und z. T. überwacht wird. Normverstöße führen zu sozialen Sanktionen, z. B. zu schlechten Noten in der Schule oder zu Geringschätzung des Gesprächspartners/der Gesprächspartnerin bis hin zum Kommunikationsabbruch. Ein weiterer Normbegriff bezieht jedoch auch Nonstandardvarietäten mit ein. Ammon (200ob) zufolge sei die Gleichsetzung von Sprachnorm und Standardvarietät irreführend, denn auch Nonstandardvarietäten wie Dialekte würden über Sprachnormen verfügen und könnten korrekt oder inkorrekt gesprochen werden (a.a. O., 665).

Kleineidam (1986) unterscheidet zwischen einem „engeren“ und einem „weiteren“ Sprachnormbegriff. Der engere Normbegriff entspreche dem Paradigma der strukturellen Linguistik, die sich auf die Beschreibung eines als homogen angenommenen Sprachsystems oder eines idealisierten SprecherHörers konzentriere (a.a.O., 58). „Sprachliche Normen sind nach diesem Begriff auf die grammatische Wohlgeformtheit und Korrektheit sprachlicher Formen und Konstruktionen bezogen“ (58, Herv. im Original). Man könne 
diesen Normbegriff auch „systemlinguistischen“ Normbegriff nennen. Der „weitere“ Normbegriff sehe die Sprache im Gesamtzusammenhang sozialer Interaktion. Sprachliche Normen seien danach eine Teilmenge von sozialen Normen, Vorschriften und Erwartungen, die mit der Verwendungsabsicht und der Verwendungssituation von sprachlichen Äußerungen in Zusammenhang stünden (a.a. O., 59). Die sprachlichen Normen seien hier auf die kommunikative Angemessenheit von sprachlichen Äußerungen bezogen. Dieser zweite Normbegriff sei einer soziolinguistisch ausgerichteten Sprachwissenschaft verpflichtet, die die Homogenitätsannahme der Systemlinguistik aufgebe. Man könne ihn daher auch „soziolinguistischen Normbegriff“ nennen (a. a. O., 60). Diese Dichotomie entspreche einer Differenzierung von "Systemnorm" und „Verwendungsnorm“: „Systemnormen sind situationsunabhängige sprachliche Normen, während Verwendungsnormen situationsabhängige sprachliche Normen darstellen" (6o, Herv. im Original). Kleineidam verwendet dafür schließlich den Terminus „kommunikative Norm“ (61).

Bei Sprachnormen geht es auch um die Unterscheidung zwischen impliziten oder expliziten verbindlichen Regeln. Laut Bussman (1990) beruhen Sprachnormen entweder implizit auf einem Konsens der SprecherInnen oder sie sind explizit festgesetzt und legitimiert durch Kriterien wie Verbreitung, Alter, Strukturgemäßheit und Zweckmäßigkeit - das sei die „präskriptive Norm“. Dabei sei die Abgrenzung von Sprachnormen und Regeln des Sprachsystems methodisch schwierig, da die Existenz impliziter Sprachnormen nur aus der Regelhaftigkeit des Sprachgebrauchs erschlossen werden könne - die so genannte „deskriptive“ Norm, die auch als Gebrauchsnorm bezeichnet wird (Bussmann 1990, 710).

Dem (eher didaktisch orientierten) präskriptiven Normbegriff steht also die (eher linguistisch orientierte) deskriptive Sprachnorm, die Gebrauchsnorm gegenüber. Sie zielt auf den tatsächlich vorkommenden Sprachgebrauch ab und umfasst somit auch Formen eines Standards, die zwar präskriptiv „falsch“, in der Praxis aber weit verbreitet sind. Weiter gefasst meint die Gebrauchsnorm das allen Mitgliedern einer Sprachgemeinschaft verfügbare Regelsystem einer Sprache oder von sprachlichen Varietäten, auch von Nonstandardvarietäten.

Normen können sich auf alle sprachlichen Bereiche beziehen, von der Morphologie und Syntax, die im Fokus der Systemlinguistik stehen, bis hin zur Pragmatik, deren Regeln weniger explizit und selten in einem Kodex festgelegt sind. So verstanden sind sprachliche Normen unterschiedlich verbindlich, viele sind nicht schriftlich notiert, und sie unterliegen wie alle sozialen Normen dem sprachlichen und gesellschaftlichen Wandel. Dabei gibt es außer im Bereich der Rechtschreibung mit dem Rat für deutsche Rechtschreibung ${ }^{38}$ keine Normierungsverfahren

38 „Der Rat für deutsche Rechtschreibung ist ein zwischenstaatliches Gremium, das vonseiten der staatlichen Stellen damit betraut wurde, die Einheitlichkeit der Rechtschreibung im deutschen Sprachraum zu bewahren und die Rechtschreibung auf der Grundlage des orthografischen 
oder -instanzen. Dass Nachschlagwerke wie das Österreichische Wörterbuch oder der Duden auch für Fragen außerhalb der Rechtschreibung als maßgeblich gelten, ist eine Konvention, die aber höchstens im Bereich von Schule und Amtsstellen festgeschrieben werden kann. Dass sich die meisten Menschen in einer Sprachgemeinschaft dennoch an sprachliche Normen halten, verweist auf den klar sozialen, großteils verbindlichen Charakter sprachlicher Normen.

Die Normen der Standardvarietäten sind von sehr hoher Verbindlichkeit. Sie sind kodifiziert - es gibt für sie Nachschlagwerke für den „korrekten“ Gebrauch, sie haben amtlichen Status, sie werden in der Schule förmlich gelehrt, sie sind oft auch gesetzlich verankert (z. B. in der Verfassung, in Lehrplänen) und ihre Einhaltung wird von „Sprachnormautoritäten“ kontrolliert (siehe oben, Ammon 2005). Für die Durchsetzung oder Aufrechterhaltung dieser Normen sorgen, wie schon oben ausgeführt, so genannte normsetzende Instanzen (Ammon 2005, $32 \mathrm{ff})$ wie ModellsprecherInnen/Modelltexte; BerufssprecherInnen, -schreiberInnen (z. B. NachrichtensprecherInnen, SchriftstellerInnen); Sprachkodices (Nachschlagwerke, Wörterbücher, Grammatiken); SprachexpertInnen (sprachwissenschaftliche Fachleute) und eben Sprachnormautoritäten, die von Berufs wegen Texte korrigieren (dürfen) (LehrerInnen, Amtsvorstände, LektorInnen, RedakteurInnen etc.).

Aus den obigen Ausführungen geht hervor, dass u. E. im schulischen Kontext nur ein situativer bzw. ein kommunikativer Normbegriff sinnvoll ist: Mit der Entwicklung von Soziolinguistik und Pragmalinguistik und der so genannten "pragmatischen Wende" in der Sprachwissenschaft seit den 196oer-Jahren, die sich auch mit sprachlicher Variation außerhalb des Standards und mit der Beziehung zwischen dem Sprachsystem und den SprachverwenderInnen befasst hat, wurde ein flexibleres Normverständnis modelliert. Sprache wird hier als Sprachhandeln verstanden. Demnach verfügen kompetente SprecherInnen einer Sprache über die so genannte kommunikative Kompetenz (Habermas 1971, Hymes 1972, Searle 1971, Austin 1972), die sie in möglichst vielen unterschiedlichen Situationen sprachlich handlungsfähig macht. Nach dem Motto, frei nach Fishman: Wer spricht wie/ welche "Sprache" mit wem und wann in welcher Situation/unter welchen sozialen Umständen über welchen Inhalt/welches Thema mit welchen Absichten und Konsequenzen? (Fishman 1975). Dieser Konzeptualisierung von Sprache entspricht der erwähnte situative Normbegriff, der die Beherrschung von unterschiedlichen Registern der Standardsprache, aber auch dialektaler oder umgangssprachlicher Varietäten je nach Situation, Thema, GesprächspartnerIn und anderen Faktoren umfasst. In der Familie spricht man anders als bei einem Vortrag, mit den

Regelwerks im unerlässlichen Umfang weiterzuentwickeln. Der Rat ist somit die maßgebende Instanz in Fragen der deutschen Rechtschreibung und gibt als solche mit dem amtlichen Regelwerk das Referenzwerk für die deutsche Rechtschreibung heraus. " http://www.rechtschreibrat. com/der-rat/ueber-den-rat/ (18.12.2018). 
MechanikerInnen anders als mit den ÄrztInnen, und die DeutschlehrerInnen verwenden eine andere Varietät, wenn sie über das klassische Balladenjahr vortragen, als wenn sie SchülerInnen maßregeln oder disziplinieren. Ein derartig flexibles Normverständnis entspricht wohl eher dem tatsächlichen Sprachgebrauch als ein starr an der präskriptiven Norm orientiertes Konzept.

Aufgabe des schulischen Sprachunterrichts ist es, neben der grammatisch korrekten systemlinguistischen Norm auch die kommunikativen Sprachnormen im weiteren Sinn und daher auch Varietätenkompetenz zu vermitteln. Dabei ist der Deutschunterricht an Schulen nicht nur Deutsch-als-Muttersprache-Unterricht, sondern auch Unterricht in der Bildungssprache, der der Entwicklung allgemeiner Sprachkompetenzen dient, und außerdem Deutsch-als-Zweitsprache-, Deutschals-Drittsprache-Unterricht. Im Schuljahr 2014/15 betrug etwa der Prozentsatz von SchülerInnen mit anderen Erstsprachen als Deutsch an den allgemein bildenden österreichischen Pflichtschulen 27,4 \%, den Volksschulen 27,6 \%, in den AHS 17,1 \% (BMB 2016). Dabei wird die Bildungssprache Deutsch natürlich nicht nur im Deutschunterricht vermittelt, sondern in allen Fächern, denn jeder Unterricht, auch der Physik-, Mathematik- oder Musikunterricht, ist Sprachenunterricht (vgl. de Cillia 2013 und das Konzept des Curriculum Mehrsprachigkeit, Reich/ Krumm 2013).

Auf die Frage, welche Varietät unterrichtet werden soll, ist die Antwort von Vertretern der Schulbehörden häufig: „Unterrichtssprache ist Hochdeutsch!“, d. h., die Standardsprache soll in der Schule die alleinige oder zumindest vorherrschende Varietät sein. Die sprachliche Realität ist aber wesentlich komplexer (s. o.), auch an den Schulen, wie schon eine Erhebung von Eva-Maria Rastner aus $1997 \mathrm{zu}$ Österreich gezeigt hat (Rastner 1997).

Rastner hat eine Umfrage zum Umgang mit den Varietäten Hochsprache Umgangssprache - Dialekt und deren Verwendung unter DeutschlehrerInnen in acht Bundesländern ( $n=24$, Vorarlberg wurde nicht erfasst) durchgeführt. Herausgekommen sind deutliche regionale Unterschiede: Während man im Nordosten Österreichs (Wien, NÖ, nördliches Burgenland) einer "gepflegten“ Umgangssprache bzw. dem Standard das höchste Prestige zuspricht, ist es Rastners Daten zufolge im Süden und Westen (Steiermark, Kärnten, Oberösterreich, Salzburg, Tirol) so, dass hier der gepflegten Umgangssprache in offiziellen Gesprächssituationen der Vorzug gegeben wird, in informellen Situationen jedoch der Dialekt als selbstbewusster Ausdruck regionaler Zugehörigkeit eingesetzt wird. Die Befragten diagnostizierten auch, dass nur ein geringer Prozentsatz der Kinder die Standardsprache in den Unterricht als Herkunftssprache mitbringe und dass dialektsprechende Jugendliche häufiger Schwierigkeiten beim Erlernen des Standards hätten. Was die Bewertung der Varietäten durch die Gesellschaft betrifft, sei die Aussage eines Lehrers aus Kärnten zitiert: „Dialekt ist wie ein Arbeitsgewand, mieft, ist beschmutzt, abgenützt, hat Farbkleckse; Umgangssprache ist legere, bequeme Alltagskleidung von Leuten, die aus dem Ärgsten 
herausen (sic!) sind, Standardsprache: korrekte, betont saubere Kleidung“ (Lehrer aus Kärnten, Rastner 1997, 82). Die Daten unseres Projekts, über das das vorliegende Buch berichtet, sollten helfen, diese Befunde zu ergänzen. Ergebnisse finden sich in Kap. 6.2.

Rastner 1997 schlägt vor, dass in einem „aufklärerischen Sprachunterricht“ auch umgangssprachliche und dialektale Varietäten ihren Platz haben sollten und dass es um ein funktional richtiges Einsetzen von Sprachvarietäten entsprechend der Kommunikationssituation geht.

Die Aufhebung der Dichotomie „richtiges/falsches“ bzw. „gutes/schlechtes“ Deutsch zugunsten einer "situationsangemessenen/situationsunangemessenen“ Sprachverwendung ist ein wichtiger Beitrag zur Kultivierung eines toleranten Umgangs mit Sprache, den Schule und Gesellschaft von heute dringend brauchen. (Rastner 1997, 93)

Welche Norm, welche Varietät in welcher Situation, in welcher Textsorte in Bezug auf welches Thema, welchen Inhalt adäquat ist, das müssen LehrerInnen als zentrale normsetzende Instanzen letztlich immer wieder in ihrer Unterrichts- und Korrekturpraxis entscheiden. Ziele eines derartigen Sprachenunterrichts sollten die Sensibilisierung für die unterschiedlichen Formen innersprachlicher Mehrsprachigkeit und für unterschiedliche situative Normen sein, die Realisierung flexibler, situativer Normen und die Entwicklung von Normtoleranz bzw. Akzep$\tan z$ unterschiedlicher Normen.

Der heimische Schulunterricht sollte im Idealfall ein sprachkritisches Bewusstsein für die vielschichtige Sprachsituation in Österreich vermitteln, damit die SchülerInnen zu einem selbstbewussten Umgang mit der österreichischen Varietät finden und sich dessen bewusst werden, dass die staatlichen Varietäten der deutschen Sprache gleichwertig sind. Befunde aus der Forschung zu Beginn unseres Projekts wiesen darauf hin, dass an Österreichs Schulen die Gleichwertigkeit der Varietäten in der bildungssprachlichen Praxis nicht "gelebt" wird (Ammon 1995, Legenstein 2008, Heinrich 2010). Das war unter anderem der Anlass für die Durchführung des im vorliegenden Buch dokumentierten Projekts.

Auch unsere als Vorstudie durchgeführte Interviewerhebung bei elf ExpertInnen für Deutsch-Didaktik ${ }^{39}$ führte zu einer ähnlichen Vorab-Einschätzung, etwa durch Werner Wintersteiner: „Varietätenfragen finden im Unterricht kaum Niederschlag. "Norbert Griesmayer stellte fest, dass letztendlich nur die individuellen Normvorstellungen der Lehrperson ausschlaggebend seien, denn nirgends sei festgelegt, wo das ,richtige“ Deutsch zu finden wäre: „Die Sprachauffassung in Lehrplänen [ist eine] relativ nebulose, die sich damit zufrieden gibt, dass so etwas wie ,richtiges' Deutsch existiert."

39 Für die Liste der ExpertInnen siehe Fußnote 40. 
Eine wichtige Fragestellung des hier dargestellten Forschungsprojekts war daher, wie Lehrpläne, Studienpläne und Lehrbücher Fragen sprachlicher Variation und Norm handhaben, wie es um den Umgang mit Varietäten im Unterricht steht und welche Spracheinstellungen unter LehrerInnen und SchülerInnen vorherrschen.

\subsection{Forschungslage zum österreichischen Deutsch als Unterrichtssprache und ExpertInnenbefragung}

Um zu Projektbeginn den Status Quo der Forschung zum Kontext unserer Fragestellung zu erheben, wurde in einer (eingangs bereits erwähnten) Vorstudie in einem ersten Schritt die vorliegende wissenschaftliche Literatur gesichtet. Da speziell zur Frage „Varietäten des Deutschen und Deutsch-als-Muttersprache(DaM)Unterricht“ die Quellenlage sehr überschaubar war, wurden zur Erfassung des ganzen Spektrums der neuesten Forschung und der aktuellen Praxis zusätzlich Leitfadeninterviews mit elf ausgewiesenen ExpertInnen aus universitären sowie außeruniversitären Institutionen durchgeführt, die sich in Forschung und Lehre mit den Bereichen Deutschunterricht und Deutschdidaktik (DaM, DaZ) sowie mit Varietätenlinguistik beschäftigen. ${ }^{40}$ Ihre Fachmeinungen sowie der Stand der wissenschaftlichen Literatur zu Projektbeginn wurden in die Formulierung von Annahmen und Entwicklung der Forschungsfragen miteinbezogen und werden im Folgenden dargestellt.

Wie in Kap. 2.4 ausführlich dargelegt und begründet, gehen wir - entsprechend der diesbezüglichen Fachliteratur - davon aus, dass auf der standard- bzw. bildungssprachlichen Ebene im normativen Kontext von Schule und Unterricht die deutsche Sprache am zutreffendsten mit dem plurizentrischen Konzept beschrieben werden kann. Diese Konzeptualisierung, die mittlerweile als eine der etablierten Konzeptualisierungsmöglichkeiten in der Fachdiskussion gilt, war allerdings (zu Projektbeginn) noch eher unsystematisch bis gar nicht in der

40 Folgende ExpertInnen wurden vor Projektbeginn befragt: Univ.-Prof. Mag. Dr. Klaus-Börge Boeckmann: Deutsch als Fremdsprache, Institut für Germanistik, Universität Wien; Dr. Jakob Ebner: Lexikograph, Dudenredaktion; Dr. Manuela Glaboniat: Deutschdidaktik, Institut für Germanistik, Universität Klagenfurt; OR Dr. Norbert Griesmayer: Fachdidaktik, Neuere deutsche Literatur, Institut für Germanistik, Universität Wien; Univ.-Prof. Mag. Dr. Stefan Krammer: Fachdidaktik, Neuere Deutsche Literatur, Institut für Germanistik, Universität Wien; Dr. Wolfgang Moser: Österreichisches Sprachenzentrum Graz; Ass.-Prof. Mag. Dr. Rudolf Muhr: Forschungsstelle Österreichisches Deutsch, Institut für Erziehungs- und Bildungswissenschaften, Universität Graz; Univ.-Prof. Dr. Annemarie Saxalber-Tetter: stellvertretende Leiterin des AECC, Universität Klagenfurt; Dr. Robert Saxer, Institut für Germanistik, Universität Klagenfurt, Leiter des Vereins „Deutsch in Österreich. Fremdsprachenkurse“; em. Univ.-Prof. Dr. Peter Wiesinger: Sprachwissenschaft, Institut für Germanistik, Universität Wien; Univ.Prof. Dr. Werner Wintersteiner: Leiter des AECC (Österreichisches Kompetenzzentrum für Deutschdidaktik), Universität Klagenfurt. 
DaM-/DaF-Unterrichtspraxis wiederzufinden: Wie Untersuchungen zu Spracheinstellungen unter Deutschlehrenden und -lernenden im In- und Ausland gezeigt haben (Ammon 1995, de Cillia 1997a, Markhardt 2005, Ransmayr 2005, 2006), wird das österreichische Deutsch häufig nicht als Standardvarietät wahrgenommen, sondern wird vielfach als Substandard-Varietät eingestuft (Ransmayr 2005: 374). Hägi (2006) hat in ihrer Untersuchung gängiger DaF-Lehrwerke gezeigt, dass die Umsetzung des plurizentrischen Konzepts in den Lehrbüchern meist noch unzulänglich erfolgt war. Am Beginn unserer Untersuchung lag - bis auf eine Fallstudie über DaM-Lehrwerke in der Sekundarstufe I (Heinrich 2010) - noch keine umfassende Lehrwerksanalyse der gängigsten Lehrwerke für den DeutschMuttersprachenunterricht in Österreich vor.

In den ExpertInnen-Interviews unserer Vorstudie fand sich unisono die Einschätzung, dass in den österreichischen LehrerInnenbildungsinstitutionen (Universitäten und Pädagogische Hochschulen) die österreichische Varietät als Bildungssprache nur eine geringe oder gar keine ausgewiesene Rolle spielen dürfte. Im Laufe ihrer Ausbildung würden die angehenden LehrerInnen mit dem Thema der österreichischen Standardsprache und den Varietäten der deutschen Sprache (außer in den Fächern DaF und DaZ) wohl kaum in Berührung kommen, hieß es, was zur Vermutung geführt hat, dass an Österreichs Schulen nur selten Fachwissen über die Plurizentrik des Deutschen vorhanden sein dürfte.

Was Normverständnis und Korrekturverhalten unter PädagogInnen betrifft, zeigt die Fallstudie von Heinrich (2010), dass österreichische LehrerInnen tendenziell Austriazismen zugunsten bundesdeutscher Varianten korrigieren. Es fanden sich keine weitergehenden wissenschaftlichen Untersuchungen über die Normtoleranz und das Korrekturverhalten österreichischer LehrerInnen in Bezug auf Austriazismen/Deutschlandismen/Helvetismen. In einer Pilotstudie aus dem Jahr 1995 (Ammon 1995, 423-445) unter österreichischen, deutschen und Schweizer LehrerInnen hat Ammon die Tendenz zur stärkeren Korrektur fremdnationaler Varianten festgestellt und eine deutlich geringere Toleranz gegenüber Austriazismen und Helvetismen beobachtet als gegenüber Deutschlandismen. Ammon deutet dies als eine eher schwach ausgeprägte Sprachloyalität österreichischer und Schweizer LehrerInnen gegenüber der eigenen Varietät und ortet einen Zusammenhang zwischen dem Korrekturverhalten und der Kenntnis nationaler Varietäten. Ähnliche Zusammenhänge stellt Ransmayr unter Universitätslehrenden für Deutsch im nicht-deutschsprachigen Ausland fest (Ransmayr 2005, 236). Auch in einer Pilotstudie von Legenstein $(2008,113)$ finden sich Hinweise auf eine Exonormorientierung unter österreichischen PädagogInnen: Demnach würden österreichische DeutschlehrerInnen bei der Textkorrektur im Zweifelsfall vielfach den Duden (und nicht das ÖWB) verwenden und generell einen normunsicheren Eindruck machen.

Eine weitere Frage für die Bestandsaufnahme war die nach der Berücksichtigung der Thematik der staatsspezifischen Varietäten, insbesondere des österreichischen Deutsch, und generell der soziolinguistischen Variation in den Lehrplänen 
und im Unterricht. Die diesbezügliche Einschätzung der ExpertInnen deutete darauf hin, dass Varietätenfragen im Unterricht kaum Niederschlag finden dürften, obwohl gerade im Fach Deutsch die LehrerInnen besonders viel Reflexionswissen bräuchten, darin waren sich die befragten ExpertInnen einig.

Was die Spracheinstellungen der ÖsterreicherInnen betrifft, so zeigten die ExpertInneninterviews und die Fachliteratur (Steinegger 1998, Muhr 2005b, Legenstein 2008, de Cillia 2009), dass unter der österreichischen Bevölkerung zwar ein Bewusstsein für das „Anderssein“ der österreichischen Varietät im Vergleich zur Varietät Deutschlands besteht. Darüber hinaus sei aber wenig Wissen über die österreichische Standardvarietät vorhanden. Muhrs (1989) Untersuchungen zufolge hält ein Großteil der ÖsterreicherInnen das österreichische Deutsch für einen Dialekt. Unsere ExpertInnen wiesen einheitlich darauf hin, dass es in Österreich sprachliche Unklarheiten hinsichtlich Standard/Hochsprache und Dialekt gäbe. Auch diskursanalytische Befunde deuteten darauf hin, dass fehlende alltagssprachliche Konzepte von einer flexiblen, plurizentrischen Norm von Sprache/ $\mathrm{n}$ zu einer Verwirrung in Bezug auf die Zuordnung österreichischer Sprachvarietäten führen (de Cillia 2009).

Sprachliche Unsicherheit führt Muhr zufolge zu mangelnder Sprachloyalität der eigenen Varietät gegenüber, zu sprachlichen Minderwertigkeitsgefühlen und letztlich zu sprachlichem Identitätsverlust (2005b, 18). Ähnlich argumentierten die meisten befragten ExpertInnen, wenn es um den Zusammenhang zwischen innerer Mehrsprachigkeit und Identität geht, und um die Fähigkeit, die Rollen von Standard und Dialekt zu ordnen. Da im vorschulischen und schulischen Bereich kaum bewusster und reflektierter Umgang mit den soziolinguistischen Varietäten, insbesondere mit dem Dialekt, stattfinde, werde Dialekt häufig als defizitäre Sprachform empfunden, was sich wiederum negativ auf das sprachliche Selbstwertgefühl auswirke. Schließlich war für uns noch der Aspekt der Kodifizierung des österreichischen Standarddeutsch relevant. Einen zentralen Kodexbestandteil für Deutsch als Unterrichtssprache stellt das Österreichische Wörterbuch (ÖWB) dar. Welche Stellung das ÖWB in den österreichischen Schulen genießt, ob es als Referenzwerk von LehrerInnen und SchülerInnen herangezogen wird und inwiefern es über die Gratisschulbuchaktion seinen Weg in die österreichischen Haushalte gefunden hat, waren allesamt Aspekte, über die es zu Projektbeginn keine Informationen gab.

\subsubsection{Forschungslücken/Forschungsfragen}

Nach der Bestandsaufnahme durch Literatur und ExpertInnen-Interviews konnten wir eine Reihe von Forschungslücken identifizieren:

$\mathrm{Zu}$ Projektbeginn lag keine umfassende Analyse des Aspekts der sprachlichen Variation in den Lehr- und Studienplänen bzw. der PädagogInnenausbildung vor. 
Was die Lehrpläne für österreichische Schulen betrifft, sollten die Lehrpläne der in Frage kommenden Schultypen systematisch dahingehend analysiert werden, ob sprachliche Variation (plurizentrische/pluriareale und andere soziolinguistische Variation) darin Berücksichtigung findet. Die Bestandsaufnahme und die ExpertInnen-Befragung legten den Schluss nahe, dass die gängigen Lehrwerke für den DaF-/DaZ-Unterricht weitgehend monozentrisch konzipiert sind. Dasselbe wird für den DaM-Bereich vermutet. Eine Analyse der gängigen Lehrwerke sollte in dieser Frage Klarheit bringen. Über die Einstellungen österreichischer LehrerInnen und SchülerInnen zum österreichischen Deutsch und anderen standardsprachlichen Varietäten des Deutschen, über ihr diesbezügliches Wissen sowie über das Korrekturverhalten der Lehrkräfte lagen abgesehen von Pilotstudien (Ammon 1995, Legenstein 2008, Heinrich 2010) ebenfalls keine umfassenden Untersuchungen vor. Es gab auch keine Daten darüber, welche Nachschlagwerke tatsächlich von Lernenden und Lehrenden verwendet werden und welche Bedeutung insbesondere das ÖWB in österreichischen Schulen hat.

Von dieser Ausgangslage aus wurden zentrale Annahmen und Fragestellungen für das Forschungsprojekt formuliert. 


\section{Forschungsfragen und Untersuchungsdesign}

\subsection{Zentrale Fragestellungen}

Aus der Sichtung der Literatur und den Interviews mit den ExpertInnen ließen sich zu Beginn des Projekts eine Reihe von Annahmen ableiten: Die erste Annahme war, dass in den Deutschlehrplänen für österreichische Schulen, ebenso in gängigen Lehrwerken für den DaM-Unterricht, zum Themenkomplex Sprachreflexion und Sprachbewusstsein in Bezug auf soziolinguistische, insbesondere staatsspezifische Variation wenig explizite Informationen zu finden sein würden. Wir konnten weiters davon ausgehen, dass DeutschlehrerInnen kaum Materialien und Publikationen zum österreichischen Deutsch und standardsprachlicher Variation zur Verfügung stehen. Ob und inwieweit sprachliche Variation im Unterricht thematisiert wird, dürfte in erster Linie von den Interessen und der Kompetenz der individuellen Lehrkraft und den Ressourcen, die ihr zur Verfügung stehen, abhängen. Insgesamt dürfte „sprachliche Variation“ in der Praxis des schulischen Unterrichts wenig thematisiert werden.

Bei den Studienplänen der Ausbildungsinstitutionen für angehende PädagogInnen (Universitäten, Pädagogische Hochschulen, BAKIP ${ }^{1}$ ) war zu vermuten, dass staatsspezifische bzw. soziolinguistische Variation im Allgemeinen nur unsystematisch Berücksichtigung finden. Es war daher anzunehmen, dass LehramtskandidatInnen im Lauf ihrer Ausbildung weder ausreichende Grundkenntnisse über nationale Varietäten des Deutschen noch über den Umgang mit österreichischem Deutsch und anderen deutschen Standardvarietäten erhalten.

Was konkret das Wissen und die Einstellungen von LehrerInnen und SchülerInnen zur staatsspezifischen plurizentrischen Variation betrifft, wurde angenommen, dass wenig Wissen um die Unterschiede zwischen den Standardvarietäten des Deutschen, also etwa zwischen dem österreichischen Standarddeutsch und dem deutschen Standarddeutsch, vorhanden ist und dass Unsicherheit in Bezug auf die eigene Varietät des Deutschen sowie Unklarheit über die Existenz eines österreichischen Standarddeutsch besteht bzw. diese Varietät als umgangssprachlich oder dialektal beurteilt wird. Dabei finden sich v. a. in der Literatur Hinweise auf einen Widerspruch zwischen der zentralen Bedeutung der eigenen Varietät für nationale Identitätskonstruktionen und der linguistischen Bewertung sowie auf eine Art sprachlichen Minderwertigkeitsgefühls.

1 Bildungsanstalt für Kindergartenpädagogik. Heute heißen diese Schulen zur Ausbildung von KindergartenpädagogInnen BAfEP (Bundesbildungsanstalt für Elementarpädagogik). 
Ausgehend von diesen Annahmen wurden die folgenden forschungsleitenden Fragen formuliert:

Fragen zum Bereich Lehrpläne, Lehrmaterial und Unterricht an den Schulen (Volksschule, Hauptschule/Neue Mittelschule, AHS ${ }^{2}$, BAKIP):

- Ist der systematische Umgang mit sprachlicher Variation (Standard, Umgangssprache, Dialekt bzw. auch staatsspezifische Standardvariation) in den österreichischen Lehrplänen verankert?

- Wie werden Fragen sprachlicher Normen in den Lehrplänen thematisiert? Welche Sprachauffassung liegt den Lehrplänen diesbezüglich zugrunde?

- Sind das Konzept der Plurizentrik oder andere Konzeptualisierungen des Deutschen implizit oder explizit in den Lehrplänen verankert?

- Welche Sprachkonzepte und Normauffassung werden in den am häufigsten verwendeten Lehrwerken der Volksschule, Sekundarstufe I und II transportiert?

- Auf welche Nachschlagwerke greifen österreichische LehrerInnen und SchülerInnen zurück?

- Welche Materialien und Publikationen über österreichisches Deutsch und die Standardvarietäten des Deutschen stehen LehrerInnen zur Verfügung?

- Wird sprachliche Variation im Deutschen in der Praxis des Deutschunterrichts thematisiert?

Fragen zur LehrerInnenaus- und -fortbildung in den Studienplänen der Pädagogischen Hochschulen und der Universitäten:

- Ist der Umgang mit staats-/regionsspezifischen Normen ein Thema in den Lehramtscurricula der PHs und Universitäten?

- Wird der Umgang mit Dialekt/Umgangssprache/Standardsprache in der fachdidaktischen LehrerInnenausbildung berücksichtigt?

- Gibt es implizite oder explizite Hinweise auf die Plurizentrik oder andere Formen der Konzeptualisierung des Deutschen in den Studienplänen?

Fragen zur Spracheinstellung und zum Sprachwissen über länderspezifische Standardvariation sowie zur Konzeptualisierung des Deutschen bei LehrerInnen und SchülerInnen:

- Welche Einstellungen gegenüber dem österreichischen Deutsch finden sich bei österreichischen LehrerInnen (Volksschule, Sekundarstufe I und II) und SchülerInnen der gymnasialen Oberstufe?

- Ist das Konzept der Plurizentrik unter LehrerInnen und SchülerInnen der Sekundarstufe II bekannt?

2 Allgemeinbildende höhere Schule (Gymnasium, Realgymnasium). 
- Wie konzeptualisieren die beiden Gruppen sprachliche Variation bzw. an welchen Sprachnorm-Konzepten (plurizentrisch/pluriareal/monozentrisch) orientieren sich die LehrerInnen und SchülerInnen?

- Bestätigen sich unter den LehrerInnen und SchülerInnen die Einschätzungen der Fachliteratur, dass ÖsterreicherInnen eine geringe „Sprachloyalität“ gegenüber der eigenen Varietät aufweisen? Wie wird die eigene Varietät im Vergleich zum deutschen Standarddeutsch eingeschätzt?

- Bestätigen sich Hinweise aus Pilotstudien, dass österreichische LehrerInnen exonorm-orientiert korrigieren?

- Gibt es bei den LehrerInnen Zusammenhänge zwischen ihrer Spracheinstellung und außersprachlichen Variablen (Gender, Alter, Dienstjahre, Schultyp, Lehramtsausbildung an Universität oder Pädagogischer Hochschule, das Bundesland, in dem sie unterrichten, ihre Fächerkombination, Bekanntheit des plurizentrischen Konzepts)?

- Gibt es bei den SchülerInnen Zusammenhänge zwischen ihrer Spracheinstellung und außersprachlichen Variablen (Gender, Alter, Bundesland, Muttersprache(n), Erstsprache, Sprachen der Eltern, aufgewachsen innerhalb/außerhalb Österreichs, längere Auslandsaufenthalte, weitere gelernte Sprachen, außerordentliche/r Schüler/in, Bekanntheit des plurizentrischen Konzepts, Interesse am österreichischen Deutsch, TV-Konsum in der Kindheit, derzeitiger TVund Radiokonsum)?

\subsection{Untersuchungsdesign}

Für die Erhebung des Datenmaterials wurden im Sinn der Daten- und Methodentriangulation ${ }^{3}$ (des „Ergänzungsmodells der Triangulation“, vgl. Meyring 2001, Fielding/Schreier 2001, Vetter 2008) mehrere unterschiedliche Korpora mit verschiedenen Methoden erhoben, um ein möglichst differenziertes Bild des Forschungsgegenstands zu gewinnen, eine Erweiterung der Erkenntnismöglichkeiten durch mehrfache Perspektiven zu erreichen und alle Ebenen und Aspekte der Frage „Variation im Deutschunterricht“ zu erfassen.

Zunächst wurden unterrichtsrelevante bzw. handlungsleitende Dokumente gesichtet: Nach einer eingehenden Untersuchung der zu Projektbeginn gültigen schulischen Deutschlehrpläne und der Studienpläne für die Lehramtsausbildung im Fach Deutsch für Volksschul-, Hauptschul- und GymnasiallehrerInnen sowie einer exemplarischen Analyse von Lehrveranstaltungsbeschreibungen des Sommersemesters 2012 und des Wintersemesters 2012/13 an österreichischen

3 Häufig werden vier Formen von Triangulation unterschieden: Daten-Triangulation, methodologische Triangulation, Investigator-Triangulation, Theorien-Triangulation (Flick 2007, 310) die beiden letzteren Formen sind u. E. für die vorliegende Fragestellung nicht relevant. 
Pädagogischen Hochschulen und Universitäten wurden die drei meistverwendeten Lehrbuchserien der jeweils 4. Stufe Volksschule, Sekundarstufe I und II ${ }^{4}$ analysiert ${ }^{5}$.

Ausgehend von den Ergebnissen der Dokumentenanalyse wurden Fragebögen für eine österreichweite quantitative Befragung entwickelt. Eine solche schriftliche Befragung mittels Fragebogen bietet sich vor allem bei geographisch stark verstreuten Adressaten an (Schlobinski 1996, 40). Die Fragebogenerhebung zielte darauf ab, neben den bereits durch die Dokumentenanalyse gewonnenen Erkenntnissen noch Daten über sprachbezogene Einstellungen und Kenntnisse der LehrerInnen und SchülerInnen zu erheben.

Die Ergebnisse dieser schriftlichen LehrerInnen- und SchülerInnenBefragungen stellten ein großes Korpus dar, das noch durch qualitative Leitfadeninterviews mit Lehrpersonen in ganz Österreich und zwei Gruppendiskussionen (eine mit LehrerInnen, eine mit SchülerInnen) ergänzt wurde. Die Durchführung von Interviews mit den LehrerInnen war erforderlich, um vertiefende Daten über Einstellungen, Meinungen und (mit Einschränkungen) auch über das sprachliche Verhalten der Befragten zu den untersuchungsrelevanten Fragen zu gewinnen. Das Leitfadeninterview als zielgerichtetes Gespräch stellt eine der wichtigsten sprachwissenschaftlichen Methoden dar, um die Wahrnehmung und Interpretation von Sachverhalten durch Individuen zu ermitteln. Es wird unter anderem für die Erhebung des Varietätenverständnisses der Lehrenden und ihrer subjektiven Theorien von Sprachnormen verwendet (vgl. Schlobinski 1996, 45 ff; Friedrichs 1997).

Die Gruppendiskussion wird als Methode zur Erfassung von Meinungen und Einstellungen einzelner DiskussionsteilnehmerInnen im halböffentlichen Diskurs eingesetzt, die es auf verhältnismäßig ökonomische Weise ermöglicht, Informationen von einer relativ großen Anzahl von Personen zu erhalten. Gleichzeitig ermöglicht sie, die gemeinsame diskursive Konstruktion von Konzepten wie sprachlicher Normen zu dokumentieren (vgl. Lamnek 1989, 121; Wodak/de Cillia/ Reisigl/Liebhart/Hofstätter/Kargl 1998, 315 ff).

Den Abschluss der empirischen Erhebungen bildeten die teilnehmenden Unterrichtsbeobachtungen, die - im Unterschied zur Befragung - eine direkte Beobachtung des Sprachverhaltens der Testpersonen möglich machten und die Perspektive der Selbstwahrnehmung der befragten Lehrenden und Lernenden (Schlobinski 1996, 50 ff; Atteslander 2000, 98 ff) ergänzen konnten.

4 Funkelsteine, Sprachlichter und Lilos (Grundstufe/4. Schulstufe), Treffpunkt Deutsch, Deutschstunde und Ganz klar Deutsch (Sekundarstufe I/8.Schulstufe), Aktion Sprache, Das Sprachbuch und Klartext Deutsch (Sekundarstufe II/11. und 12. Schulstufe).

5 Eine detaillierte Beschreibung der Dokumentenanalyse sowie der einzelnen Datensätze findet sich im Kapitel 4. 
Die Datenauswertung bestand einerseits aus einer inhaltlichen bzw. einer gesprächs- und diskursanalytischen Interpretation der erhobenen Quellentexte (Lehrpläne, Studienpläne, Lehrveranstaltungen in den Institutionen der LehrerInnenausbildung und Lehrbücher), der transkribierten Leitfadeninterviews, der Gruppendiskussionen sowie der Notizen aus den teilnehmenden Unterrichtsbeobachtungen, und andererseits aus einer statistischen Auswertung der aus den schriftlichen Befragungen gewonnenen quantitativen Daten mit dem Programm SPSS (Statistical Package for the Social Sciences).

Bei der statistischen Auswertung der quantitativen Daten der Fragebogenerhebung wurden folgende Verfahren angewandt:

Mithilfe von Verfahren der deskriptiven Statistik, wie Häufigkeits- und Kreuztabellen bzw. statistischen Kennzahlen wie Mittelwert, Median und Standardabweichung, wurde in einem ersten Schritt das Antwortverhalten der Befragten dargestellt. Ziel dabei war es, allgemeine Aussagen über die Einstellungen zum untersuchten Thema abzubilden.

Für die Untersuchung von verschiedenen Gruppenunterschieden (z. B. Alter, Region, Geschlecht etc.) wurden Testverfahren aus dem Bereich der inferentiellen Statistik angewendet, um Schlüsse auf die Grundgesamtheit ziehen zu können. Bei allen durchgeführten Tests wurde mit einem Signifikanzniveau von 0,05 (5\%) gearbeitet. Je nach Datenniveaus der untersuchten Variablen fiel die Entscheidung auf einen der folgenden Tests:

\section{Chi-Quadrat-Test}

Für den Vergleich von nominalen Variablen zwischen verschiedenen Gruppen (z. B. Ja/Nein-Fragen) wurde der Chi-Quadrat-Test verwendet. Dieser ermöglicht auf Basis einer Darstellung in einer Kreuztabelle die Untersuchung von Gruppenunterschieden. Voraussetzung für die sinnvolle Interpretation dieses Tests ist allerdings, dass die einzelnen Zellen der Kreuztabelle mit einer ausreichenden Anzahl an Fällen befüllt sind. Ausreichend heißt in diesem Fall, dass die erwarteten Häufigkeiten mindestens den Wert 5 erreichen sollten. War diese Bedingung in mehr als $20 \%$ der Zellen nicht erfüllt, wurde der Test nicht interpretiert.

\section{Nichtparametrische Testverfahren}

Bei ordinalem Datenniveau der abhängigen Variablen wurde auf Testverfahren aus dem Bereich der nichtparametrischen Tests zurückgegriffen. Die Grundlage dieser Tests ist die Bildung von Rangplätzen, die zwischen verschiedenen Untersuchungsgruppen verglichen werden können. Die konkrete Auswahl eines Verfahrens erfolgte hier auf Basis der Anzahl der zu vergleichenden Gruppen. Wurden nur zwei Gruppen miteinander verglichen (z. B. Geschlecht) kam der Mann-Whitney-U-Test zur Anwendung. Wurde ein Vergleich von mehr als zwei Gruppen angestellt (z. B. Regionen), wurde der Kruskal-Wallis-Test zur Berechnung von signifikanten Unterschieden herangezogen. 


\section{Mittelwertsvergleiche}

Die Mittelwertsvergleichstests kamen dann zur Anwendung, wenn die abhängige Variable ein metrisches Datenniveau aufwies. Eine weitere Voraussetzung für die Anwendung dieser Tests ist, dass die untersuchte Variable einer Normalverteilung folgt. War diese Bedingung nicht erfüllt, wurde auf ein Testverfahren aus dem Bereich der nichtparametrischen Tests zurückgegriffen, die auch bei schiefen Verteilungen eingesetzt werden können.

Bei gegebenen Bedingungen für einen Mittelwertsvergleich war wiederum die Gruppenanzahl entscheidend für die Auswahl des Tests. Beim Vergleich von zwei Untersuchungsgruppen (z. B. Geschlecht) kam der T-Test für unabhängige Stichproben zum Einsatz. Bei mehr als zwei zu untersuchenden Gruppen (z. B. Region) wurde hingegen die Varianzanalyse verwendet.

Die folgende Tabelle zeigt eine Übersicht über die Datenerhebungs- und Auswertungsmethoden der empirischen Erhebungen:

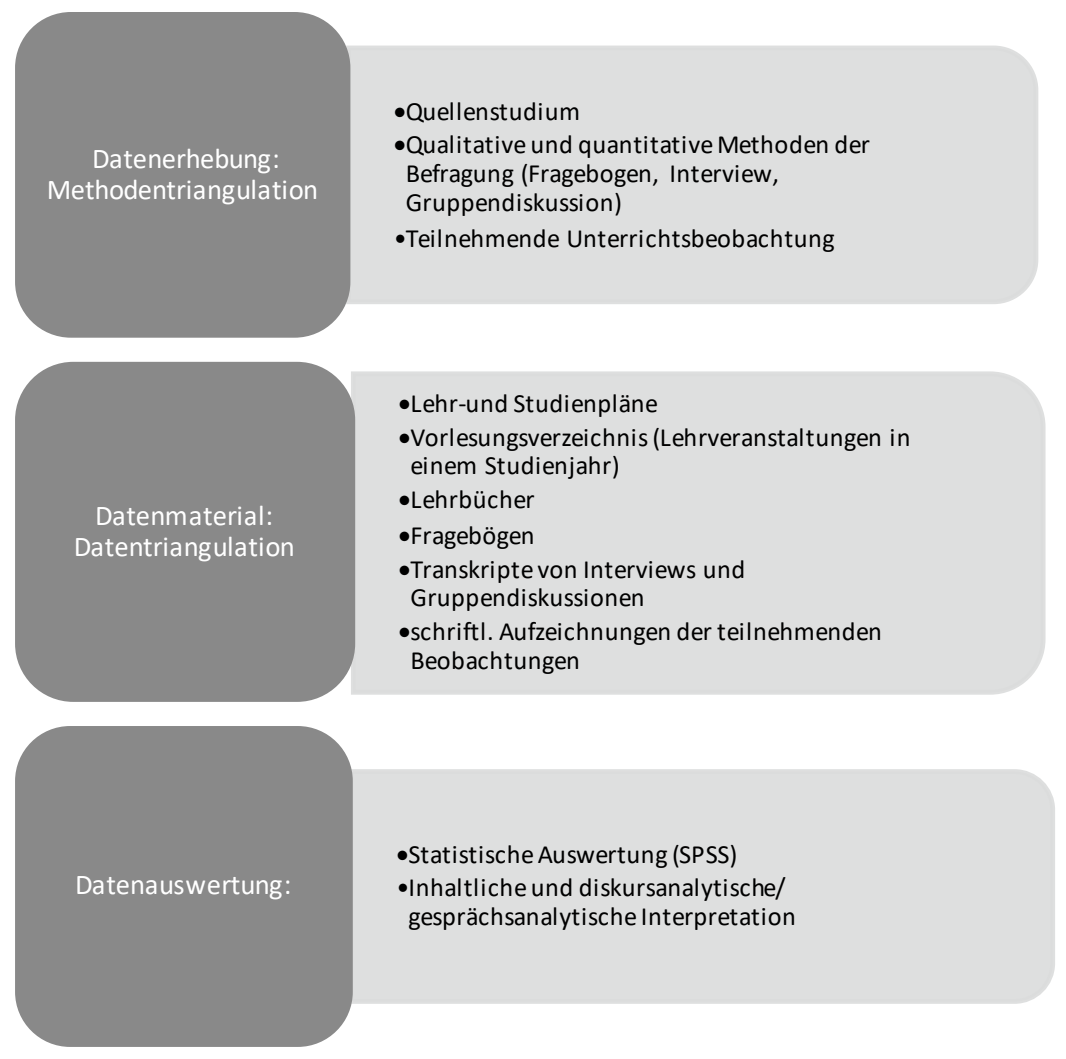

Tab. 1: Empirische Erhebung 
In der folgenden Tabelle sind die Datensätze, die im Projekt ausgewertet wurden, im Detail angeführt:

\begin{tabular}{|c|c|}
\hline Lehrpläne für Deutsch ${ }^{6}$ & Grundstufe, Sekundarstufe I und II \\
\hline Studienpläne ${ }^{7}$ & $\begin{array}{l}\text { (LehrerInnenausbildung Deutsch) der Universitäten und } \\
\text { Pädagogischen Hochschulen }\end{array}$ \\
\hline Vorlesungsverzeichnis & $\begin{array}{l}\text { Lehrveranstaltungen exemplarisch an PHs und Universitäten } \\
\text { im Studienjahr 2012/2013 aus der LehrerInnenausbildung für } \\
\text { Deutsch }\end{array}$ \\
\hline Deutschlehrbücher & $\begin{array}{l}\text { die je drei meistverwendeten Lehrbuchserien der Grundstufe, } \\
\text { Sekundarstufe I und II im Jahr } 2012\end{array}$ \\
\hline Fragebögen & $\begin{array}{l}\text { LehrerInnen in allen Bundesländern (GS, Sek. I+II), n = 164: } \\
20,7 \% \text { männlich, } 79,3 \% \text { weiblich, Alter: } 22-63 \text { Jahre, } \\
\text { Unterrichtsfächer: } 86 \% \text { Deutsch/Deutsch+1 anderes Fach, } \\
14 \% \text { andere Fächer } \\
\text { SchülerInnen in allen Bundesländern (AHS und BHS Sek. II), } \\
\text { n = 1253: } 42,4 \% \text { männlich/57,6 \% weiblich, Alter: } 13-25 \text { Jahre }\end{array}$ \\
\hline Interviews & 21 mit LehrerInnen aller Schultypen aller Bundesländer \\
\hline Teiln. Beobachtung & je eine Schulstunde in sieben Schulklassen \\
\hline Gruppendiskussion & $\begin{array}{l}\text { eine LehrerInnengruppe (neun Lehrende an } \\
\text { versch. Schultypen), eine SchülerInnengruppe (elf } \\
\text { Oberstufen-SchülerInnen) }\end{array}$ \\
\hline
\end{tabular}

Tab. 2: Analysierte Datensätze

Nach Abschluss der Analyse und Auswertung der einzelnen Datensätze wurden diese im Sinn der Datentriangulierung aufeinander bezogen und auf Übereinstimmungen, Widersprüche und offene Fragen hin abgeglichen (vgl. Wodak/ Krzyzanowski 2008, Friedrichs 1997, Lamnek 1989, Dörnyei 2007).

Im Folgenden werden die einzelnen Korpora beschrieben, bevor die Ergebnisse der Dokumentenanalyse (Lehrpläne, Studienpläne, Lehrbücher) vorgestellt werden. Danach folgen die Ergebnisse der empirischen Erhebung an Schulen (Fragebogenerhebung bei LehrerInnen und SchülerInnen, Interviews mit LehrerInnen, Gruppendiskussionen und teilnehmende Unterrichtsbeobachtung).

6 Es wurden die jeweils zu Projektbeginn 2012 gültigen Fassungen der Lehrpläne analysiert. Stichprobenartige Überprüfungen haben ergeben, dass sich bis zur Drucklegung kaum Änderungen im Wortlaut der betreffenden Passagen ergeben haben.

7 Stand 2012. 


\title{
4 Analyse von unterrichtsrelevanten Dokumenten
}

\author{
(Lehrpläne, Studienpläne, Lehrbücher)
}

Zunächst wurden die zum Zeitpunkt des Projektbeginns (2012) gültigen Lehrpläne ${ }^{1}$ für die Volksschule, die Sekundarstufe I (Hauptschule, Neue Mittelschule [NMS], Allgemeinbildende höhere Schule [AHS] Unterstufe), die Sekundarstufe II (AHS Oberstufe Pflichtfach Deutsch, Wahlpflichtfach Deutsch und DaZ), der BAKIP (Bundeslehranstalt für Kindergartenpädagogik) und der „Bildungsplan-Anteil Sprache des Bildungsplans für elementare Bildungseinrichtungen" untersucht. Danach folgte eine Analyse der Studienpläne für Deutsch und der Lehrveranstaltungen des Sommersemesters 2012 und des Wintersemesters 2012/13 in den Institutionen der LehrerInnenbildung.

\subsection{Deutschlehrpläne}

Ausgehend von unserer Annahme, dass Sprachreflexion und Sprachbewusstsein hinsichtlich staatsspezifischer und anderer soziolinguistischer Variation in den Lehrplänen für österreichische Schulen nicht eindeutig festgehalten sind, haben wir nach Sichtung der zum Thema Lehrplananalyse bereits vorhandenen Literatur (u. a. Griesmayer 2004, Legenstein 2008, Heinrich 2010) einen Analysekatalog entwickelt, um folgende Forschungsfragen beantworten zu können:

- Ist sprachliche Variation (Standard, Umgangssprache, Dialekt bzw. auch nationale Standardvariation) als Lehrziel in den Lehrplänen verankert?

- Sind Fragen sprachlicher Normen als Lehrziel in den Lehrplänen verankert? Welche Sprachauffassung liegt den Lehrplänen diesbezüglich zugrunde?

- Sind das Konzept der Plurizentrik oder andere areale Konzeptualisierungen des Deutschen implizit oder explizit in den Lehrplänen verankert?

- Wird auf Nachschlagwerke Bezug genommen?

Die Ergebnisse der Lehrplananalyse ergaben über die verschiedenen Schulstufen hinweg ein relativ homogenes Bild.

1 Folgende Lehrpläne für das Fach Deutsch an österreichischen Schulen wurden im Rahmen dieses Projekts analysiert: Die Lehrpläne für die Volksschulen (VS, Stand: Juni 2003), für die Hauptschulen (HS, Stand: Juli 2010) und allgemein bildenden höheren Schulen (AHS Sekundarstufe I, Stand: Juli 2010), die Neuen Mittelschulen (NMS, Stand: Mai 2012) und die AHS Oberstufe, Sekundarstufe II (Fach Deutsch, Wahlpflichtfach Deutsch und DaZ, Stand: 2008). 
Im Lehrplan der Volksschule für das Fach Deutsch finden sich weder Bezüge zum österreichischen Deutsch oder anderen Standardvarietäten der deutschen Sprache noch zur Thematik der Plurizentrik. Nicht-standardsprachliche Varietäten finden insofern Erwähnung, als die Begriffe „Mundart“ und „regionale Umgangssprache“ im Kontext Sprechen/richtige Satzmuster bzw. Erweiterung der Sprachfähigkeit erwähnt werden: „Üben von Satzmustern, die häufig fehlerhaft verwendet werden, insbesondere Fehlformen, die sich aus dem Unterschied zwischen Mundart- und Standardsprache ergeben. “ (Lehrstoff Grundstufe I, S. 4) Oder: „Der Übergang von der Mundart oder der regionalen Umgangssprache zur Standardsprache soll sich ohne Bruch vollziehen. " (Didaktische Grundsätze/allgemein, S. 24)

Der Standardsprache wird im Volksschul-Lehrplan sehr große Bedeutung beigemessen: Zwar wird fast ausschließlich im mündlichen Kontext, jedoch durchgängig auf allen Schulstufen der Grundstufe I und II immer wieder auf die Standardsprache verwiesen, wie im folgenden Beispiel:

Im Einzelnen geht es darum, die individuelle Sprache des Kindes zur Standardsprache zu erweitern. [...] wesentliche Aufgabe des Teilbereichs Sprechen ist es, die Bereitschaft und Fähigkeit der Schüler zur mündlichen Kommunikation allmählich zu erhöhen und sie zu einem möglichst sicheren Gebrauch der Standardsprache zu führen. (Bildungs- und Lehraufgabe/allgemein, S. 1)

Bei den didaktischen Grundsätzen/Sprechen/allgemein (S. 24) wird darauf hingewiesen, dass „das Hinführen zu den Formen der Standardsprache keinesfalls unter Leistungsdruck erfolgen“ soll, um „sprachliche Hemmungen zu vermeiden bzw. abzubauen“. Dass der Standardsprache eine Norm zugrunde liegt, geht aus dem Lehrplan der Volksschule implizit hervor. Allerdings gibt es keine konkreten Verweise darauf, wie diese Norm definiert ist, zumeist bezieht sich der Normverweis auf das Rechtschreiben. Obwohl der Begriff „normgerecht“vielfach verwendet wird, existieren keinerlei Empfehlungen oder Richtlinien hinsichtlich der „Zielnorm“: „Aufgabe des Rechtschreibunterrichtes ist es, die Schüler zu normgerechtem Schreiben zu motivieren.“ (Bildungs- und Lehraufgabe/ Rechtschreiben/allgemein, S. 2); „Sicherung des normgerechten Gebrauchs von Zeitwort, Namenwort und Eigenschaftswort" (Sprechen/4. Schulstufe, S. 14); „das Bemühen um normgerechtes Schreiben erhalten und festigen“ (Rechtschreiben/3. Schulstufe, S. 19).

Die Lehrpläne der AHS Unterstufe, der NMS und der HS gleichen einander im Prinzip, bis auf kleine, für diese Untersuchung nicht relevante Unterschiede. Alle drei Lehrpläne haben gemein, dass weder das österreichische Deutsch noch das plurizentrische Konzept Erwähnung finden. Der Umgang mit Varietäten und den dafür notwendigen Begrifflichkeiten ist unsystematisch; es ist von Standardsprache, Herkunftssprache und Muttersprache die Rede, diese jedoch werden nicht näher definiert: 
Sprechen verlangt von den Schülerinnen und Schülern, sich in zunehmendem Maß auf die jeweilige Sprechsituation einzustellen und dabei auch unterschiedliche Leistungen von Standardsprache und Herkunftssprachen zu erfahren. In geeigneten Gesprächs(Partner-, Kleingruppen-, Klassengespräch ...) und Redeformen (spontanes, vorbereitetes und textgebundenes Sprechen) sollen die Schülerinnen und Schüler die Wirkungsweise verschiedener verbaler und nonverbaler Ausdrucksmittel erleben. (Lehrplan der Sekundarstufe I, S. 2)

Es bleibt unklar, ob hier mit „Herkunftssprache“ auch dialektale bzw. umgangssprachliche oder andere Varietäten der deutschen Sprache gemeint sind oder andere Erstsprachen. Im Abschnitt „Besondere didaktische Grundsätze, wenn Deutsch Zweitsprache ist" ist von der deutschen Standardsprache die Rede - ein Hinweis darauf, dass auch den Sek. I-Deutschlehrplänen eine eher monozentrische Sichtweise zugrunde liegt: „Aussprache und Intonation sollen der deutschen Standardsprache möglichst nahe kommen. Neben der Lautsicherheit ist ein in Rhythmus, Melodieführung und Sprechtempo möglichst natürlicher Sprechton anzustreben." (Lehrplan der Sekundarstufe I, S.3)

Ist in den Lehrplänen von Normen die Rede - meist werden die Ausdrücke „Sprach- und Schreibnormen“, „Sprach- und Schreibrichtigkeit“, „richtig“, und „sprachrichtig“ gebraucht -, dann bleibt es unklar, worauf sich die erwähnten Kriterien beziehen. Im Abschnitt „Besondere didaktische Grundsätze, wenn Deutsch Zweitsprache ist “ wird beispielsweise vorgeschrieben, dass Schülerinnen und Schüler „bestimmte Sprachnormen“ einzuhalten haben, was eine sehr vage und eigentlich sehr unbestimmte Anweisung ist; ähnlich verhält es sich mit „der richtigen Aussprache“, die im selben Kapitel thematisiert wird. Es wird an keiner Stelle erläutert, welche Norm gemeint ist; so bleibt es letztlich den Lehrenden überlassen, diese Normen nach eigenem Ermessen zu definieren:

Die Schulung der mündlichen Ausdrucksfähigkeit (einschließlich der richtigen Aussprache und Intonation) soll einen Schwerpunkt im Unterricht von Deutsch als Zweitsprache bilden. Der Spracherwerb ist dabei als Prozess zu verstehen, in dessen Verlauf die Schülerinnen und Schüler befähigt werden, sprachliche Mittel selbstständig einzusetzen und bestimmte Sprachnormen einzuhalten. (Lehrplan der Sekundarstufe I, S. 3)

Die einzige Form der Konkretisierung der normrelevanten Zielvorgaben ist eine Empfehlung im Teil „Besondere didaktische Grundsätze, wenn Deutsch Zweitsprache ist“, wonach den SchülerInnen Nachschlagetechniken in „österreichischen und zweisprachigen Wörterbüchern“ beizubringen sind. Welche Wörterbücher konkret gemeint sind, bleibt unklar.

Die Analyse der Lehrpläne von AHS Unterstufe, NMS und HS führt zum Ergebnis, dass zwar von Varietäten und Normen die Rede ist, diese jedoch nicht näher definiert werden und es somit unklar bleibt, worauf sie sich genau beziehen. 
Immer wieder wird die Standardsprache genannt, die jedoch nicht weiter konkretisiert wird und keinen näheren Bezug zum Kodex erfährt. Das österreichische Deutsch wird nicht erwähnt, genauso verhält es sich mit der staatsspezifischen Variation und mit Hinweisen auf bestimmte Konzeptualisierungen.

Der Lehrplan für den Gegenstand Deutsch AHS Oberstufe thematisiert als einziger Lehrplan explizit - an einer Stelle - die „österreichische Standardsprache“: „[Die SchülerInnen sollen] verschiedene sprachliche Register einschließlich der - österreichischen - Standardsprache beherrschen“ (Lehrstoff/mündliche Kompetenz/Sprechsituationen und Sprechanlässe/7. und 8. Klasse, S. 3). Abgesehen von diesem Hinweis werden im Sekundarstufe-II-Deutschlehrplan neben dem österreichischen Deutsch keine anderen nationalen Standardvarietäten genannt oder Konzeptualisierungen unternommen. Auch der Umgang mit nichtstandardsprachlichen Varietäten wird nicht näher erläutert. Auf die Standardsprache wird an anderer Stelle zwar Bezug genommen, ohne jedoch näher auszuführen, worauf sich die „sprachlichen Standards“ beziehen: „[Die Lernenden sollen] befähigt werden, schriftlich und mündlich sowie in Form medialer Präsentation Texte zu produzieren, die den sprachlichen Standards und den situativen Anforderungen entsprechen." (Bildungs- und Lehraufgabe, S. 1) Wenn Varietäten thematisiert werden, geschieht dies im Kontext von innerer und äußerer Mehrsprachigkeit im Zusammenhang mit Sprachreflexion, wie in folgender Passage:

Die Identifizierung des eigenen Sprechens und damit die Reflexion der eigenen Rolle und Identität schaffen auch Platz für die Akzeptanz und das Verstehen anderen Sprechens und sind tragende Elemente für den Umgang mit Sprachvarietäten und Mehrsprachigkeit. (Bildungs- und Lehraufgabe/Beiträge zu den Bildungsbereichen, S. 1; Ähnliches findet sich bei: Didaktische Grundsätze/Sprachreflexion, S. 3)

Auf Normen wird im Lehrplan der Sekundarstufe II in vielerlei Bereichen hingewiesen, sei es im Bereich „Schreibprozess“, „Sprachreflexion“, „Rechtschreiben“ oder auch "grammatische Phänomene“, wo sich mehrfach Begriffe wie „Schreibnormen“ oder „Normenwandel“ finden, bzw. von „Sprachrichtigkeit“ und „Schreibrichtigkeit“ die Rede ist. Als Lehrziele werden etwa genannt: „Insbesondere sollen die Schülerinnen und Schüler [...] befähigt werden, sich zwischen Normen und Abweichungen zu orientieren." (Bildungs- und Lehraufgabe, S. 1)

Aber auch im Sekundarstufe-II-Lehrplan bleibt die Frage offen, welche Norm umgesetzt werden soll - und es werden keine Verweise auf das Österreichische Wörterbuch oder ein anderes Nachschlagwerk gemacht. Letztlich bleibt es dem Ermessen des Lehrenden überlassen, sowohl die Norm als auch den "Standard“ für den eigenen Unterricht festzulegen.

Im Lehrplan für das Wahlpflichtfach Deutsch der gymnasialen Oberstufe bleiben Varietäten (nationale oder soziolinguistische), Standard(s), Norm(en) oder ein Kodex gänzlich unerwähnt. Der DaZ-Lehrplan für die Sekundarstufe II seinerseits 
beschäftigt sich mit Normen, normativer Sprachrichtigkeit und mit der Standardsprache, indem es beispielsweise heißt, dass , auch auf die normative Sprachrichtigkeit im Sinn eines unterstützenden Sprachtrainings Deutsch besonderer Wert zu legen“ ist (Didaktische Grundsätze, S. 1) und die SchülerInnen „schrittweise unter besonderer Beachtung der Regelhaftigkeit die Standardsprache gewandt schriftlich und mündlich einsetzen können." (Bildungs- und Lehraufgabe, S. 1) Letztlich werden die groben Zielvorgaben und Erwartungen aber ebenfalls nicht näher beschrieben und operationalisierbar gemacht.

Ein ähnliches Bild ergibt im Übrigen der Lehrplan der Bildungsanstalt für Kindergartenpädagogik BAKIP (jetzt BAfEP). Der Bildungsplan-Anteil zur sprachlichen Förderung in elementaren Bildungseinrichtungen legt großen Wert auf Respekt gegenüber und Förderung von Erstsprachen von Kindern, was sowohl andere Erstsprachen als Deutsch, als auch Dialekt und Umgangssprache betrifft. Konzepte zur Beschreibung sprachlicher Variation und zum Umgang damit werden auch hier nicht näher thematisiert.

Zusammenfassend kann festgehalten werden, dass den Deutschlehrplänen der österreichischen Schulen kein klares Konzept hinsichtlich der Konzeptualisierung räumlicher Variation des Deutschen zugrunde liegt, da weder die Plurizentrik noch andere Konzepte genannt werden.

In allen Lehrplänen der Volksschule, Sekundarstufe I und Sekundarstufe II werden zwar Varietäten (Dialekt, in der VS v. a. Mundart; Umgangssprache) thematisiert, allerdings eher unsystematisch, und die Standardsprache wird meist im Sinn einer deutschen Standardsprache in den Lehrplänen erwähnt. Einzig der Oberstufenlehrplan nennt an einer Stelle explizit die österreichische Standardsprache des Deutschen. Dieser Hinweis wird jedoch anschließend nicht näher erläutert bzw. es wird an keiner anderen Stelle des Lehrplans daran angeknüpft. Alle Lehrpläne haben gemeinsam, dass es mangels konkreter Bezüge dem Ermessen der einzelnen LehrerInnen obliegt, wie sie in der Praxis mit Fragen zu Normen, Standard und Varietäten umgehen.

Somit bieten weder der Oberstufenlehrplan noch die anderen Lehrpläne DeutschlehrerInnen ein theoretisches oder praktisches Orientierungsgerüst für den Umgang mit Varietäten.

\subsection{Studienpläne für die Ausbildung von DeutschlehrerInnen}

Ausgehend von unserer Hypothese, dass nationale/staatsspezifische bzw. soziolinguistische Varietäten in den Studienplänen der Ausbildungsinstitutionen für angehende PädagogInnen kaum Berücksichtigung finden, haben wir die Studienpläne der LehrerInnenausbildung in Hinblick auf folgende Leitfragen näher in den Blick genommen:

- Ist der Umgang mit staatsspezifischen Normen ein Thema in den Curricula der PHs und Universitäten? 
- Wie wird der Umgang mit Dialekt/Umgangssprache/Standardsprache in der fachdidaktischen LehrerInnenausbildung berücksichtigt?

- Gibt es implizite oder explizite Hinweise auf bestimmte Konzeptualisierungen des Deutschen in den Studienplänen?

Folgende Curricula lagen dabei der Untersuchung zugrunde:

$\begin{array}{lll}\text { PH Wien } & \text { Studienplan für Volksschulen } & 2008 / 09 \\ \text { KPH Wien/Krems } & \text { Studienplan für Volksschulen } & 2008 \\ \text { PH Oberösterreich } & \text { Studienplan für Volksschulen } & 2009 \\ \text { PH Steiermark } & \text { Studienplan für Volksschulen } & 2011 \\ \text { PH Kärnten } & \text { Studienplan für Volksschulen } & 2012 \\ \text { PH Salzburg } & \text { Studienplan für Volksschulen } & 2009 \\ \text { PH Tirol } & \text { Studienplan für Volksschulen } & 2010 \\ \text { PH Wien } & \text { Studienplan für Hauptschulen } & 2009 \\ \text { KPH Wien/Krems } & \text { Studienplan für Hauptschulen } & 2008 \\ \text { PH Oberösterreich } & \text { Studienplan für Hauptschulen } & 2009 \\ \text { PH Steiermark } & \text { Studienplan für Hauptschulen } & 2012 \\ \text { PH Kärnten } & \text { Studienplan für Hauptschulen } & 2012 \\ \text { PH Salzburg } & \text { Studienplan für Hauptschulen } & 2009 \\ \text { PH Tirol } & \text { Studienplan für Hauptschulen } & 2010 \\ \text { Universität Wien } & \text { Studienplan } & 2002 \\ \text { Universität Graz } & \text { Studienplan } & 2011 \\ \text { Universität Innsbruck } & \text { Studienplan } & 2001 \\ \text { Universität Salzburg } & \text { Studienplan } & 2011 \\ \text { Universität Klagenfurt } & \text { Studienplan } & 2011 \\ \text { Curriculum Mehrsprachigkeit } & & 2011 \\ & & \end{array}$

$\mathrm{PH}=$ Pädagogische Hochschule; $\mathrm{KPH}=$ Kirchliche Pädagogische Hochschule

In sämtlichen von uns analysierten Curricula für die Volksschullehrer-Ausbildung wird sprachliche Variation nicht thematisiert. In einzelnen HauptschulCurricula finden sich Termini wie „österreichisches Deutsch“ und „Varietäten des Deutschen“ (Curriculum HS der PH Salzburg), wobei sich letztere auf Nonstandardvarietäten beziehen. Ebenfalls im Hauptschul-Curriculum der PH Salzburg wird die „Problematik von Dialekt (Herkunftssprache) und Standard“ erwähnt.

In den Curricula für den Studiengang Hauptschule der PH Kärnten und PH Tirol wird auf die „Varietäten der deutschen Sprache“, auf „innere Mehrsprachigkeit“ und auf „deutsche Sprachvarietäten“ explizit hingewiesen; gleichzeitig finden 
sich in den Curricula aber auch Textstellen, die darauf hindeuten, dass nur von der Existenz einer einzigen Standardsprache ausgegangen wird.

Was die Uni-Studienpläne betrifft, finden „österreichisches Deutsch“ oder Konzeptualisierungsmöglichkeiten des Deutschen in den Lehramtsstudienplänen der Universitäten Wien, Graz, Innsbruck, Klagenfurt und Salzburg zwar keine explizite Erwähnung, im Grazer Studienplan wird jedoch explizit auf nationale Varietäten hingewiesen, in den anderen Studienplänen gibt es dazu implizite Hinweise.

Der Umgang mit divergierenden nationalen Normen und mit Dialekt/Umgangssprache/Standardsprache wird in der LehrerInnenaus- und -fortbildung an den Universitäten unterschiedlich gehandhabt. Die Thematik stellt aber auf jeden Fall keinen Schwerpunkt in der Ausbildung dar. Als Erweiterung der Studienplananalyse wurden im Rahmen dieser Untersuchung über zwei Semester hin die Lehrveranstaltungen der Universitäten Wien, Graz, Klagenfurt, Salzburg und Innsbruck sowie der PHs Wien, Oberösterreich, Steiermark, Kärnten, Salzburg, Tirol und der KPH Wien/Krems dahingehend untersucht, ob Lehrveranstaltungen zu Plurizentrik bzw. zu österreichischem Deutsch angeboten werden. Unter die Lupe genommen wurden jeweils die beiden aktuellsten Semester, je nach Verfügbarkeit im Internet entweder die Sommer- und Wintersemester 2012 (Wien, Innsbruck), oder das Studienjahr 2012/2013 (Graz, Klagenfurt, Salzburg), wobei kein Anspruch auf Vollständigkeit erhoben wird. Der Aufwand einer Recherche hinsichtlich der Zuordnung der Lehrveranstaltungen zu Wahlfächern oder Pflichtfächern sowie zu Lehramtsfächern wäre im Rahmen dieser Untersuchung zu groß gewesen.

Die Analyse der Lehrveranstaltungen ergab, dass österreichisches Deutsch und das plurizentrische Konzept in einzelnen Lehrveranstaltungen thematisiert werden. So etwa in einer einführenden Übung an der Universität Wien zum Thema „Sprache: Regionale Standardsprachen“. Auch an den Universitäten Graz („Sprache und Gesellschaft in Österreich III - Diskurs und Pragmatik des Österreichischen Deutsch“), Klagenfurt („Österreichisches Deutsch - zugleich Einführung in die Varietätenlinguistik“) und Salzburg („Mehrsprachigkeit und Gesellschaft“) fanden sich im Erhebungszeitraum derartige Lehrveranstaltungen. Bei der Mehrheit der Lehrveranstaltungstitel und Kursbeschreibungen war dies jedoch nicht der Fall. Viele Lehrveranstaltungen haben sich vielmehr mit der empirischen Erforschung von Dialekt beschäftigt; eine nicht wirklich nachvollziehbare Schwerpunktsetzung angesichts der Tatsache, dass für die zukünftigen Deutschlehrenden die Frage des zu unterrichtenden Standards der Bildungssprache zentral ist.

Insgesamt ist festzustellen, dass sich im Lehrveranstaltungsangebot der Pädagogischen Hochschulen und Universitäten - so wie in den Studienplänen - nur vereinzelt etwas zur staatsspezifischen Variation auf der standardsprachlichen Ebene findet, und seltener etwas zur Plurizentrik des Deutschen. Bei den entsprechenden Kursen handelt es sich meist nicht um Pflichtlehrveranstaltungen, sodass Lehramtsstudierende der Germanistik mit dem Thema der Standardvarietäten und Konzeptualisierungsmöglichkeiten nicht zwangsläufig in Berührung kommen. 


\subsection{Deutsch-Lehrwerke}

Um unsere zu Projektbeginn formulierten Annahmen zu überprüfen, wonach den DeutschlehrerInnen kaum Materialien und Publikationen über die Merkmale des österreichischen Deutsch sowie allgemein über standardsprachliche Variation zur Verfügung stehen und dass die gängigen Lehrwerke für den DaM-Bereich monozentrisch konzipiert sind, wurden österreichische Lehrwerke untersucht.

$\mathrm{Da}$ es uns am wahrscheinlichsten schien, dass plurizentrische sprachliche Variation in höheren Schulstufen thematisiert wird, wurden die Lehrbücher, Übungsbücher und LehrerInnenhandbücher der ausgewählten Serien für die 4., die 8. und die 11./12. Schulstufe analysiert, und zwar die drei in Österreichs Schulen zu Projektbeginn am häufigsten verwendeten Deutsch-Lehrbuchserien der genannten Schulstufen.

Dabei handelte es sich um folgende Lehrwerke: Für die Grundstufe waren es Funkelsteine 4. Schulstufe von Gerlinde Fürnstahl, Verlag E. DORNER: Sprachbuch (Schulbuchnummer 105 242) (11. Aufl. 2012), Arbeitsblätter (SBNr. 105 243) (11. Aufl. 2011), Material für Lehrerinnen und Lehrer (4. Aufl. 2012); Sprachlichter 4. Schulstufe von Christa Blumenschein, Veritas Verlag: Basisteile $1+2$ (SBNr. 155 198), Serviceteil (2. Aufl. 2012); Lilos Sprachbuch 4. Schulstufe von Herbert Puchta und Renate Welsh, Helbling Verlag: Sprachbuch - Basisteil (SBNr. 120 747) (4. Aufl. 2011), Sprachbuch - Übungsheft (SBNr. 120 748) (3. Aufl. 2007), Lesewelt - Lesebuch (SBNr. 120 746) (2. Aufl. 2006), Handbuch für Lehrerinnen und Lehrer.

Für die Sekundarstufe I waren es Treffpunkt Deutsch 4 (8. Schulstufe) von Ursula Figl, Gudrun Natter und Stefan Schäfer, öbv: Sprachbuch (SBNr. 155 134), Arbeitsheft (SBNr. 155 130), Leseheft (SBNr. 155 132), Begleitheft für Lehrerinnen und Lehrer (1. Aufl. 2007); Deutschstunde 4 (8. Schulstufe) von Wolfgang Pramper, Helmut Hammerschmid, Stefan Hochwind, Elisabeth Nömair, Veritas Verlag: Basisteil Standard (SBNr. 145 110), Basisteil Plus (SBNr. 145 106), Serviceteil für LehrerInnen (3. Aufl. 2012); ganz klar: Deutsch 4 (8. Schulstufe) von Wolfgang Gruber, Gertraud Hilger, Jugend \& Volk: Arbeitsbuch (SBNr. 130 041), Arbeitsbuch leicht inkl Beiheft „Fit und kompetent“ (SBNr. 155 463), Übungsbuch A (SBNr. 130 329) und B (SBNr. 130 330), Lehrerbegleitheft (1. Aufl. 2011).

In der Sekundarstufe II wurden folgende Lehrwerke analysiert: Aktion Sprache 3/4 (11./12. Schulstufe) von Eva und Gerald Rainer, Veritas Verlag: Sprachbuch (SBNr. 125 221), Lehrerbegleitheft; Das Sprachbuch 3 bis zur Matura (11./12. Schulstufe) von Christian und Ulrike Schacherreiter, Veritas Verlag: Sprachbuch (SBNr. 150 255), Serviceteil für LehrerInnen (2. Aufl. 2012); klar_Deutsch 7/8 (11./12. Schulstufe) von Gertraud Hilger, Maria Kiener, unter Mitarbeit von Elisabeth Baumgartner, Jugend \& Volk: Sprachbuch (SBNr. 150 550), Begleitheft für Lehrerinnen und Lehrer, (1. Aufl. 2011).

Diese Lehrwerke wurden auf die explizite und implizite Erwähnung nationaler Varietäten hin untersucht. Die Fragen zur expliziten Erwähnung waren: Werden 
Varietäten der deutschen Sprache ausdrücklich erwähnt? Wenn ja, welche Ebenen werden angesprochen? Werden in den Begleitmaterialien für LehrerInnen Vorschläge zum Umgang mit Varietäten oder Dialekten gemacht? Wird österreichisches Deutsch thematisiert und wenn ja, wie wird österreichisches Deutsch dargestellt? Werden Wörterbücher empfohlen? Gibt es Texte, die von ihrer Varietät her als bundesdeutsche, österreichische oder Schweizer Texte explizit deklariert werden? Gibt es in LehrerInnenhandbüchern zusätzliche Informationen zu Unterschieden zwischen den Standardvarietäten, etwa auf Ebene der Grammatik, der Orthographie oder des Wortschatzes?

Folgende Fragen an das Material wurden zur impliziten Thematisierung von Varietäten gestellt: Kommen in den Lehrbüchern spezifische und unspezifische Varianten vor (Austriazismen, Deutschlandismen, Helvetismen)? Gibt es österreichische/deutsche/Schweizer Texte, die nicht explizit als solche deklariert sind? Werden Austriazismen den Deutschlandismen gegenüber als gleichwertig dargestellt?

Weitere zentrale Fragen bei der Analyse waren: In welchem Umfang wird standardsprachliche Variation und dabei auch österreichisches Deutsch thematisiert? Welches Normkonzept wird in der Lehrwerkserie transportiert?

Als Referenzwerke für den Kodex zum österreichischen Deutsch dienten uns bei der Analyse das Österreichische Wörterbuch (2012), Ebner 2008 und Ebner 2009, das Variantenwörterbuch (Ammon et al. 2004) und das große österreichische Schulwörterbuch aus dem Duden-Verlag (Duden 2008). Fallweise wurde auch auf Wiesinger 1988b, zur Morphologie auf Tatzreiter 1988 zurückgegriffen. Dabei wurden in die Liste der in den Lehrwerkserien gefundenen Varianten sowohl spezifische als auch unspezifische Austriazismen/Deutschlandismen aufgenommen. Die relativ mangelhafte Kodifizierung des österreichischen Deutsch (vgl. auch Ender/Kaiser 2009, 268) machte sich bei der Analyse dahingehend bemerkbar, dass einige Varianten nicht eindeutig zugeordnet werden konnten. Diese Zweifelsfälle wurden im Projektteam besprochen; einige wurden entsprechend der Kompetenz der ProjektmitarbeiterInnen als L1-SprecherInnen des österreichischen Deutsch zugeordnet, einige blieben jedoch offen.

Unsere Analyse der einzelnen Lehrbücher im kurzen Überblick:

\section{Volksschule}

Im Volksschullehrbuch „Funkelsteine“ findet in keinem Teil der Serie eine Auseinandersetzung mit standardsprachlicher Variation des Deutschen oder mit österreichischem Deutsch statt - weder explizit noch implizit. Österreichisches Deutsch wird nicht thematisiert, es kommen aber sehr wohl Austriazismen und Deutschlandismen in den Textbeispielen und Übungen vor. Diese bleiben unkommentiert - bewusste Sprachbetrachtung hinsichtlich nationaler Varianten anhand von konkreten Beispielen scheint in der Serie „Funkelsteine“ - in Analogie zum Lehrplan der Volksschule - nicht vorgesehen zu sein. Zur Beschreibung 
der Registerebenen verwendet das Lehrwerk ausschließlich die Begriffe „Standardsprache“ und „Mundart“: Für SchülerInnen oder LehrerInnen gibt es dazu jedoch keine nähere Erläuterung, auch nicht im LehrerInnen-Serviceteil. Die österreichische Standardvarietät wird nicht erwähnt, auch nicht unter einer anderen Bezeichnung. Zum Umgang mit Varietäten werden in keinem Lehrbuchteil Vorschläge gemacht.

Was die implizite Abbildung der Varietäten betrifft, kommen in den Lehrbuchtexten eine Reihe von Austriazismen und Deutschlandismen (und keine Helvetismen) vor, die bis auf eine Ausnahme unmarkiert und unkommentiert bleiben. Dies erscheint bei den Austriazismen nicht weiter ungewöhnlich, da Begriffe wie heuer, Jause, Fasching oder Kasten dem Alltagssprachgebrauch der Kinder entsprechen und keiner Erklärung bedürfen. Einige Deutschlandismen jedoch entsprechen nicht unbedingt dem üblichen Sprachgebrauch der SchülerInnen, wie z. B. Geschirr von Hand abwaschen, durchgucken oder Nachtisch. Sämtliche Deutschlandismen werden in den drei Lehrbuchteilen nicht kommentiert oder erläutert. In den Übungsteilen, die der Sprachbetrachtung zugeordnet sind, wäre jedoch Gelegenheit gewesen, die sprachliche Variation der deutschen Sprache in Österreich im Vergleich zu Deutschland anhand der im Text vorkommenden Varianten mit einfachen Mitteln spielerisch und exemplarisch zu thematisieren, um so erste Schritte zu einem geschärften Sprachbewusstsein zu unternehmen. Das Lehrbuch greift diese Gelegenheiten nicht auf und klammert die Thematik der staatsspezifischen und regionalen Variation in der deutschen Sprache aus. An spezifischen und unspezifischen Austriazismen finden sich in „Funkelsteine“ z. B.: Fasching, Servus, zwicken, Leibchen, Sessel, (den Salamander) angreifen (in der Bedeutung von berühren), jausnen, Schularbeit, Deutsch-Schularbeit, auf Urlaub, Kasten, Lade, Drehsessel, herrichten, Jause, Topfen, Schlag, dag (Dekagramm), Faschiertes, Zungenzerfitzler, Gurkerl, fernschauen, Palatschinken, Geschirrspüler, Jausenbrot, Semmel, auf das Gebäck vergessen, Matura, Adventzeit, Gendarm, Jänner, Weckerl, Turnzeug, Sessel, Kaiserschmarren, Brösel. Beispiele für spezifische und unspezifische Deutschlandismen z. B.: durchgucken, gucken, Mütze, Stühle, zu Bett gehen, Nachtisch, Gardinen, Spülmaschine, anfassen, Geschirr von Hand abwaschen oder Imbissstand.

Auch in der Serie „Sprachlichter" für die 4. Schulstufe findet standardsprachliche Variation weder implizit noch explizit Erwähnung. Außerdem ist, wie im Folgenden näher erläutert wird, im LehrerInnenhandbuch mehrmals von der Standardsprache die Rede. Als Termini für die Beschreibung der Varietäten finden sich „Dialekt“, „Mundart“ und (im LehrerInnenhandbuch) „Standardsprache“. Die SchülerInnen werden dazu aufgefordert, sich mit Dialekt und Mundart auseinanderzusetzen („Suche in Büchern oder im Internet ein Gedicht und lies es in Deinem Dialekt vor! Welche Mundart wird in Deinem Bundesland oder in Deiner Umgebung gesprochen?“, Basisteil 1, Sprachlichter 4. Schulstufe, S. 77). Und es 
finden sich Gedichte, die im Dialekt verfasst sind, z. B. von Christine Nöstlinger: „Vua lauta Aungsd“ und Eugen Stadelmann: „Dr Funko“.

Im LehrerInnenhandbuch finden sich Lerninhalte wie „Sprachfähigkeiten erweitern und an der Standardsprache orientiert sprechen [...], Sprachrichtigkeit, Sätze erweitern“, „Mundart/Dialekt mit Hochsprache vergleichen“ (LehrerInnenhandbuch, Sprachlichter 4. Schulstufe, S. 2), „Einsicht in Sprache: Unterschiede Dialekt - Standardsprache kennenlernen“" (a. a. O., S. 16). Es gibt allerdings keine näheren Erläuterungen zu Dialekt oder Standardsprache. Auffallend ist, dass in diesem LehrerInnenhandbuch im Abschnitt „Bildungsstandards im Kompetenzbereich Rechtschreiben“ folgende drei Punkte genannt werden: einen begrenzten Wortschatz normgerecht schreiben; Regelungen für normgerechtes Schreiben kennen und anwenden; für normgerechtes Schreiben Rechtschreibstrategien und Arbeitstechniken anwenden. Der Begriff „normgerecht" findet sich auch in den österreichischen Lehr- und Studienplänen wiederholt, und an keiner Stelle gibt es dazu nähere Erklärungen.

Was die standardsprachliche Variation betrifft, werden im Basisteil 2 die SchülerInnen dazu aufgefordert, für mündliche Erzählungen das Perfekt zu verwenden, für schriftliche Erzählungen jedoch die Mitvergangenheit. („Wenn ich etwas Vergangenes mündlich erzähle, verwende ich die lange Zeitform Vergangenheit, z. B. Ich bin ... gegangen, ich habe ... erlebt. Ich weiß schon: Wenn ich etwas Vergangenes aufschreibe oder schriftlich berichte, verwende ich die kurze Zeitform Mitvergangenheit, z. B. ich ging...", Basisteil 2, Sprachlichter 4. Schulstufe, S. 41). Die meisten spezifischen und unspezifischen Varianten, die im Lehrwerk vorkommen, sind auf der lexikalischen Ebene zu finden. Dabei nehmen naturgemäß spezifische und unspezifische Austriazismen den größten Teil ein (z. B. Bub, Federschachtel, Schlagobers, Palatschinken, Semmel, Samstag, Vanillekipferl, Beistrich, (Erdbeer-) bzw. (Himbeer-)Marmelade, in der Früh, das Keks, Jause, Tixo). Es finden sich aber auch einige spezifische und unspezifische Deutschlandismen (z. B. Wörtertreppe, Blech- bzw. Konservenbüchse, lecker, Kissen, Kerzenbüchse). Spezifische Helvetismen kommen nicht vor.

Auch in keinem der analysierten Teile der Serie "Lilos" findet die Thematik standardsprachliche Variation oder „österreichisches Deutsch“ Erwähnung weder auf expliziter noch impliziter Ebene. In vielen Texten der Lehrbuchserie sind spezifische und unspezifische Austriazismen und Deutschlandismen vorzufinden, die allesamt unkommentiert bleiben, was auch hier bei alltäglichen Austriazismen naheliegend erscheint, bei manchen Deutschlandismen jedoch geboten wäre. Betrachtet man die Texte aus einem plurizentrischen Blickwinkel, so fällt auf, dass mehrmals deutsche und österreichische Varianten eines Wortes (z. B. Karneval/Fasching) im selben Text in unmittelbarer Nähe stehen. Gerade hier werden Gelegenheiten „verschenkt“, die man dazu nützen könnte, die regionale Variation der deutschen Sprache mit einfachen Mitteln anzuschneiden und VolksschülerInnen für sprachliche Variation zu sensibilisieren. 
An spezifischen und unspezifischen Austriazismen finden sich z. B. Grüß Gott, Bub, Fasching, Plastiksackerl, Zündholzschachtel, Blumenkisterl, Gehsteig, Kuvert, Jause, zerbröseln, heuer, Jause, Kassa, sind gestanden, Faschingsumzug, Faschingsfest, Faschingsparty, Sessel, Zündholz, auf das Lernen vergessen, auf Urlaub fahren, Zuckerl, bin gesessen, Kasten, Polster, auf einen Bauernhof fahren, in der Früh, Jänner, Semmel, sich verkühlen, Schinkenkipferl, Obers, auf den Fußballplatz gehen, sich anpatzen, Knödel, Eismandln, grauslich, hat er vergessen gehabt, in den er gesteckt ist, zum Einer den Zweier, der Dreier, der Nuller, die Viecher, Matura. Häufig kommen auch unspezifische und spezifische Deutschlandismen vor: Guten Tag, Kindchen, Schornstein, Karneval, Milchkarton, Streichhölzer, hat gesessen, Tschüss dann, Aprikose, Bürgersteig, Tabakladen, guckte, Karamellbonbons, Kasse, Stuhl, Junge, Flur, Mütze, Grütze, zur Schule gehen, auspusten, Apfelsine, Erdbeerkonfitüre, das Konfitüremachen, sich Schlittschuhe leihen, die 8 (Ziffer), Laden, Brotkrumen, die Augen zusammenkneifen, Vordertreppe, anblaffen, Blumenkohl.

\section{Sekundarstufe 1}

Im Lehrwerk „Treffpunkt Deutsch 4" wird an einer Stelle die „österreichische Standardsprache“ explizit erwähnt (Sprachbuch, S. 107). Was die Terminologie betrifft, werden im Lehrbuch Dialekt, Umgangssprache, Standardsprache und Jugendsprache kurz angerissen. Es gibt kurze Erklärungen zu den jeweiligen Termini, wobei für die verschiedenen Varietäten der Terminus „Variante“ gebraucht wird: „Dialekte sind regionale Varianten einer Sprache. So spricht man in Wien anders als in Villach oder in Bregenz, in Berlin anders als in Hamburg und in Bern anders als in Basel.“ (Sprachbuch „Treffpunkt Deutsch 4“, S. 169) An einer Stelle wird eine Übung zur Unterscheidung von Standard, Umgangssprache und Dialekt angeboten, wobei die Umgangssprache definiert wird als „eine Art mündlichen Standards; Umgangssprache ist gekennzeichnet durch salopperen Stil und Dialekt-Einsprengsel.“ (a.a.O.) Den Definitionen von Standardsprache ist kein Hinweis auf einen plurizentrischen Ansatz zu entnehmen; vielmehr ist aufgrund der wiederholt verwendeten Formulierung „die Standardsprache“ auf eine monozentrische Sichtweise zu schließen.

Im Rahmen der Erklärungen zu Dialekt, Umgangssprache und Standardsprache wird auch eine Empfehlung zum Umgang mit diesen Varietäten im Sinn einer situativen Norm gegeben: „Bei offiziellen Anlässen, bei deiner schriftlichen und mündlichen Bewerbung solltest du möglichst wenig umgangssprachliche Ausdrücke verwenden und nicht im Dialekt sprechen." (a.a. O., S. 139) An einer anderen Stelle ist jedoch auch von der österreichischen Standardsprache die Rede: „Nenne aus dem Artikel ,lieb' und/oder aus deinem eigenen Wortschatz drei Wörter, die heute in der österreichischen Standardsprache als veraltet gelten“" (a.a.O., S. 107; Hervorhebung durch die Verf.). 
Im Arbeitsheft zu Treffpunkt Deutsch 4 wird auf regionale Unterschiede bezüglich des Gebrauchs von Perfekt und Präteritum hingewiesen; die Erläuterungen deuten auf die Möglichkeit einer pluriarealen Sichtweise hin, weniger auf einen plurizentrischen Ansatz: „Das Präteritum wird im oberdeutschen Sprachraum (Österreich, Schweiz, Süddeutschland) immer durch Formen im Perfekt ersetzt" (Arbeitsheft „Treffpunkt Deutsch 4“, S. 27).

Spezifische und unspezifische Austriazismen (z. B. Bursch, Beistrich, Bankomatkarte, HAK, Zweier, Turnsaal, Hausübung, Grüß Gott, Melanzani, Erdäpfel, Du bist gestanden, sie waren gestanden, er wird gestanden sein, Schularbeit) stellen auch hier naturgemäß den Großteil der in den Lehr-, Übungs- und LehrerInnenhandbüchern der 8. Schulstufe vorkommenden nationalen Varianten dar. Spezifische und unspezifische Deutschlandismen (z. B. Brötchentüte, Brötchen, Ranzen, Junge(n), Schuljahr, benutzen) finden sich ebenfalls, spezifische Helvetismen nicht.

Plurizentrik ist auch in "Deutschstunde 4"kein Thema - weder in den beiden Teilen Basisteil Standard und Basisteil Plus noch im Serviceteil. Es gibt jedoch sehr wohl den Versuch, sprachliche Variation innerhalb der deutschen Sprache auf durchaus unterhaltsame und humorvolle Art und Weise darzustellen. Allerdings fokussiert dieses Kapitel indirekt auf eine Zuordnung „österreichisches Deutsch $=$ anders $=$ Nonstandard“ im Vergleich zum Deutsch Deutschlands (s.u.).

Auf der expliziten Ebene werden die Termini „plurizentrisch“, „Varietät“, „österreichisches Deutsch" nicht erwähnt, obwohl es ein Kapitel zu Sprachbetrachtung und Sprachvariation gibt, das sich für die Thematisierung des österreichischen Deutsch als eine Varietät der plurizentrischen Sprache Deutsch eignen würde, nämlich das Kapitel „Die verschiedenen Sprachebenen“ im Basisteil Plus auf S. $149 \mathrm{ff}$ (im Basisteil Standard auf S. $143 \mathrm{ff}$ ). In beiden Teilen werden die Begriffe "Dialekt", „Umgangssprache“ und "Standardsprache“ erläutert, auch die Begriffe „Slang, Fachsprache und Jugendsprache“ finden Erwähnung, konkrete Vorschläge zum Umgang mit Varietäten oder mit Dialekt werden allerdings nicht gemacht.

Wie schon erwähnt, macht das Lehrwerk an einer Stelle den Versuch, die Thematik ,sprachliche Unterschiede zwischen Österreich und Deutschland“ zu behandeln und explizit auf Unterschiede der deutschen und österreichischen Varietäten einzugehen. Dabei kommt es aber zu einer eher problematischen Vermischung der Sprachebenen, indem anhand von Wortbeispielen (Verteilung von "Mädchen" und "Junge" in deutschsprachigen Mundartgebieten) die dialektale mit der standardsprachlichen Ebene vermischt wird, wobei bei diesem konkreten Beispiel „Bub“ gar nicht vorkommt bzw. suggeriert wird, dass Bub wie Bua ein reines Dialektwort ist. An anderer Stelle werden deutsche und österreichische Ausdrücke einander kontrastiv gegenübergestellt. Auch da fehlt eine klare Trennung zwischen der dialektalen und der standardsprachlichen Ebene beim österreichischen Deutsch, und manche österreichischen Ausdrücke, die als Gegenstücke zu deutschen standardsprachlichen Ausdrücken angeführt werden, sind eher der Umgangssprache oder dem Dialekt zuzuordnen. Die Anweisung lautet 
beispielsweise: „Versuche einem Deutschen das ,österreichische Deutsch ' Schritt für Schritt beizubringen“. Als Beispiele für Doubletten finden sich u. a. „Gschlader“ „ungenießbares Getränk“; „Gstätten“ - „ungepflegtes Grundstück“; „Gschrapp“ „Kind“; „Dippel“ - „Beule“ und „Jauckerl“ - „Injektion“; diese fünf Beispiele sind als dialektal oder Grenzfälle des Standard einzuordnen. Auch „Hutsche“ - „Schaukel“, „sich einhaun“ - „sich einschmeicheln“, „matschkern“ - „sich beschweren“ finden sich in diesem Teil (Basisteil Plus, S. 152). Die Variation wird dabei nicht mit Unterschieden zwischen österreichischer Standardvarietät und deutschem Deutsch erklärt, sondern mit der Opposition „auf gut Österreichisch“ versus - „heißt auf gut Deutsch“ (Basisteil Plus, S. 152). Das Lehrwerk „Deutschstunde 4 “thematisiert zwar die staatsspezifische Variation in expliziter Form, erweckt dabei allerdings den Eindruck von einem „lustigen“ österreichischen Deutsch, das im Gegensatz zum bundesdeutschen Deutsch eine Fülle von non-standardsprachlichen Ausdrücken aufweist. Auch der Serviceteil liefert keine Hintergrundinformationen rund ums österreichische Deutsch oder um die Plurizentrik.

In vielen Texten dieser Lehrbuchserie sind spezifische und unspezifische Austriazismen/Deutschlandismen/Helvetismen vorzufinden, die allesamt unkommentiert bleiben, was - und das sei hier wiederholt - bei aus der Alltagssprache stammenden Austriazismen (z. B. Schularbeit, einen Dreier/Vierer ..., heuer, Kopfpolster, Matura, Zuckerl, Semmel, Beilagen, Fallergänzung, Umstandsergänzung, Artergänzung, Beistrich, das geht mich echt an, einen Fleck bekommen, jem. angreifen (= Deutschland, Schweiz anfassen), Gendarm, Schlawiner, Tuchent, Paradeiser, Kehrricht, Corner, Goal, Dreiradler, Plastiksack, Skihaube, patzen, Pickerl, Briefträger, Kasten, Hausverstand, sich niederlegen) naheliegend erscheint, sich jedoch bei manchen spezifischen und unspezifischen Deutschlandismen, die den SchülerInnen zwar sicherlich rezeptiv bekannt sind, aber deren Einordnung unklar sein könnte, anbieten würde (z. B. pusten, Imbissbude, Mütze, Selbstbedienungsladen, Ladenbesitzer, Laden, Kippe, Gardine, Kommasetzung, eine SMS schreiben, Hefe, Bollerwagen, erkältet, Junge, zur Schule gehen, eine Zwei würfeln, Stuhl, Postbote).

Die plurizentrische Variation der deutschen Sprache und österreichisches Deutsch kommen auch in keinem der analysierten Serienteile des dritten Lehrwerks der Sekundarstufe I „ganz klar: Deutsch“zur Diskussion. Im Beiheft „Fit und kompetent “ist von einer Standardsprache die Rede, was auf eine monozentrische Sichtweise hindeutet. Abgesehen davon beschäftigt sich „ganz klar: Deutsch 4 "zwar mit soziolinguistischer Variation (Dialekt, Umgangssprache, Standardsprache), klammert aber den Bereich der standardsprachlichen Variation im Deutschen aus. Auch der Serviceteil liefert keine Hintergrundinformationen oder Verweise auf den Umgang mit diesem Thema.

Das Lehrbuch bespricht relativ ausführlich die Frage der Varietäten (Dialekt, Mundart, Umgangssprache, Standardsprache, Jugendsprache, Fachsprachen oder Gruppensprachen/Soziolekte). So sollen in einer Übung die SchülerInnen 
den Begriffen „Standardsprache“, „Umgangssprache“, „Jargon/Soziolekt“, und "Mundart/Dialekt" verschiedene Definitionen zuordnen, und so mit den Termini vertraut werden. Erneut ist von „der Standardsprache“ die Rede, während in wertender Form von "den schönen ländlichen Dialekten“ gesprochen wird (Fit und kompetent, S. 20). Das Lehrwerk liefert aber keine Informationen zu den Unterschieden zwischen dem österreichischen, dem Schweizer und dem deutschen Standarddeutsch.

In einem Teil des Lehrbuchs wird in einem Rechtschreibkapitel „Übersicht über Doppelschreibungen“ darauf hingewiesen, dass Wörter auf zwei verschiedene Weisen geschrieben werden können; dass diese orthographische Unterscheidung in manchen Fällen (Sauce-Soße, Spaghetti-Spagetti, ganz klar: Deutsch leicht 4, S. 164) auch auf regionale Besonderheiten zurückzuführen ist, wird nicht erwähnt: Weiters existiert im Übungsbuch A ein vager Hinweis auf verschiedene Varietäten der deutschen Sprache: Die SchülerInnen werden aufgefordert, im Wörterbuch nachzuschauen: „In den Texten wird für ,schummeln' das Wort ,mogeln' verwendet. Schau im Wörterbuch nach, woher das Wort kommt." (Übungsbuch A, ganz klar: Deutsch 4, S. 2) Es wird allerdings nicht expliziert, dass es sich hier um eine Variante der deutschen Standardvarietät des Deutschen handelt.

In einer Übung wird die österreichische Standardvarietät implizit erwähnt, indem die SchülerInnen Beispiele aus einer Liste auswählen sollen, die entweder gesprochener oder geschriebener Sprache zugeordnet werden können; in der Auswahlliste sind u. a. „Präteritum als Erzählzeit“ und „Perfekt als Erzählzeit“ zu finden (Fit und kompetent, S. 25). Im Lösungsteil wird das Perfekt als Erzählzeit der gesprochenen Sprache und das Präteritum als Erzählzeit der geschriebenen Sprache zugeordnet (a. a. O., S. 34).

An spezifischen und unspezifischen Austriazismen finden sich: Beistrich, Hausübung, Schularbeit, in die Schule gehen, Verkühlung, Geschäft, das E-Mail, einen Vierer/Einser, bei einer Schularbeit schwindeln, Bub, Spital, Kuvert, Semmel, Artergänzung, ein Jaukerl bekommen, Ärztekammer, Bub, Beistrich, AMS/Arbeitsmarktservice, ÖNORM, Beilage, NMS, AHS, Zweier, zu Allerheiligen, Samstag, Verkühlung, in der Früh, im Vorhinein, Vierer, Schullandwoche, Bankomat, Fünfer, Zwölfer, Sechser, Neuner, Hunderter, Zwanziger, Zehner, Schularbeit, Samstags-, sekkieren, Polizze, deppert, tramhapert, Gstanzl. An spezifischen und unspezifischen Deutschlandismen: Klassenarbeit, Spickfächer, Mogelhäufigkeit, Mogeln, Komma, Junge, zum Bäcker laufen, einen Eckball schießen, zur Schule gehen, schrankartig, Bonbon, Kaffeetüten, Januar, heute Morgen, jdn. ausschelten, eine Null (Ziffer), Spagetti, mogeln, Schuljahr, Komma, benutzen, Kohle (für: Geld).

\section{Sekundarstufe 2}

Das Oberstufenlehrwerk „Aktion Sprache“ befasst sich im Buch der 7./8. Klassen eingehend mit Sprachvarietäten und widmet diesem Thema ein eigenes Subkapitel. Das Buch präsentiert eine Vielzahl an literarischen Texten, Zeitungstexten, 
Essays usw. Häufig gibt es zu den AutorInnen dieser Texte aber keine näheren biographischen Informationen, sodass die SchülerInnen auch bei zeitgenössischen Texten, die eine Reihe an Deutschlandismen (oder vereinzelt auch Helvetismen) enthalten, keine Möglichkeit haben, die einzelnen Texte und die darin verwendeten Varianten einem Land bzw. einer Region oder Varietät zuzuordnen. Auch das LehrerInnenbegleitheft liefert dazu keine weiteren Informationen - weder in Hinsicht auf die Biographien der AutorInnen noch in Hinsicht auf die sprachliche Varianz.

Österreichisches Deutsch wird im Kapitel, das sich mit Sprachvarietäten befasst, nicht thematisiert, ebenso wenig wie deutsches Standarddeutsch oder Schweizer Standarddeutsch. Der Zugang zur Thematik sprachliche Variation innerhalb der deutschen Sprache bleibt auf die Einteilung in die Kategorien Dialekt, Umgangssprache, Standardsprache beschränkt. Anhand einer Karte aus dem „Wortatlas der deutschen Umgangssprachen“ von Jürgen Eichhoff wird versucht, darzustellen, „welche Unterschiede im Wortschatz deutschsprachiger Länder bestehen“ (Sprachbuch „Aktion Sprache 7/8“, S. 140). Die verwendete Karte bildet regionale Varianz anhand der unterschiedlichen Bezeichnungen für „Frikadelle“ ab. Der Ansatz der Darstellung sprachlicher Variation entspricht also einem pluriarealen Zugang. An dieser Stelle hätte eine logische Weiterverfolgung oder Ergänzung des Themas stattfinden können bis hin zu den standardsprachlichen Unterschieden im Deutschen auf staatlicher Ebene und die damit in Verbindung stehenden Konzepte der Pluriarealität und Plurizentrizität. Auch die Begriffe „österreichisches Deutsch“, „deutsches“ und „Schweizer Standarddeutsch“ wären in diesem Kapitel sinnvoll einzubetten gewesen. Diese Konzeptualisierung findet sich jedoch weder im Sprachbuch noch im LehrerInnenbegleitheft, obwohl variationslinguistische Fragen ausführlich dargestellt und die Termini „Varietät“, „Dialekt“, Umgangssprache“ oder „Standardsprache“ abgehandelt werden.

Das Buch verweist auch darauf, dass die weit verbreitete Sichtweise, der ländliche oder städtische Dialekt wäre eine minderwertige Varietät einer Sprache und werde von Personen mit niederem Status gesprochen, der sprachwissenschaftlichen Sichtweise widerspricht, „denn aus sprachwissenschaftlicher Sicht spricht jeder Mensch einen Dialekt, kein Dialekt ist dem anderen überlegen“ (Sprachbuch, S. 141).

In vielen Texten der Lehrbuchserie „Aktion Sprache“ sind spezifische und unspezifische Austriazismen (Matura, Professor, Schularbeit, heurige, weiters, Cornerfahne, Maturant, Kasten, Maturaarbeit, maturieren, Aber sehens ..., eh, Fetzendepperte, Pfui gack, bin gestanden, war gegenübergestanden, Grüß Gott, Mistkübel, sind unterschreiben gewesen, schriftliche Erzählform Perfekt, gnädige Frau, der Papa, Glasluster, Stutzen), besonders viele spezifische und unspezifische Deutschlandismen (so 'ne Art, Schlafanzugshosen, Sahnetorte, Mann stürzt vom Bau, 'ne große Rolle, Flammenmale, auf den Backen, Jungen, ging zum Schulhof, ADAC-Pannendienst, ein Kasten Bier, Stuhl, Stuhlbein, Erziehungsurlaub, Erziehungsurlauber, jemandem ins Gesicht fassen, fegen, Schulranzen, 
„Kultus und Unterricht“, Examensarbeit, Müllkippe, gucken, außen vorlassen, bislang, dröge, Metzgerei, sich drängeln, Laden, Kasse, Abitur, Metzgersladen, Trambahn, Klassenarbeit, Januar, hacken auf mir rum, war nett gewesen, Postbote, Laken, zusammenlesen, Bommeln, Abiturnoten, Klassenlehrer, Biochemie belegen, 20 Pfund verlieren, platt, Brotzeittrachtenjankerbazi, Leute ans Lesen kriegen, haben gestanden, (Erdbeeren mit) Sahne, Mütze, eine SMS, schreibend laufen sie durch die Stadt, eine Mail, am Sonntagmorgen) und auch einige Helvetismen (in Blust, Militärdienst, Spannteppich) vorzufinden, die bis auf drei Fälle unkommentiert bleiben.

Im LehrerInnenhandbuch der Serie "Sprachbuch" für die Schulstufen 11/12 wird in Zusammenhang mit dem Thema "Politische Reden halten“ der Begriff „Nationalsprache“ gebraucht. Auch die Begriffe Variantenreichtum und Sprachvarietäten werden verwendet: „Der Code dürfte im nationalen politischen Rahmen meistens die Nationalsprache sein, allerdings in ihrem Variantenreichtum (Sprachvarietäten). Dialektausdrücke und eine mundartliche Färbung müssen nicht schlecht sein." (Serviceteil, Sprachbuch 7/8, S. 23) Wie schon im Sprachbuch finden sich auch im LehrerInnenhandbuch keine Verweise auf die österreichische Standardvarietät der deutschen Sprache oder andere staatsspezifische Variation.

Von „korrekter Standardsprache“ ist nur einmal im Zusammenhang mit wissenschaftlichem Arbeiten im Sprachbuch die Rede (Sprachbuch 7/8, S. 170). Auffallend ist außerdem, dass im Zusammenhang mit Mindmapping zum Thema "Schreibunterricht“ der Begriff „Schreibrichtigkeit“ verwendet wird; hier wie an anderen Stellen zeigen sich intertextuelle Bezüge zwischen den österreichischen Lehrplänen und diesem Lehrbuch (a. a. O., S. 66).

Im Kapitel „Einen Text vortragen“ findet sich ein Hinweis auf Unterschiede innerhalb des deutschen Sprachraums; hier wird näher auf die Artikulation eingegangen: „In unserem Sprachraum neigen wir dazu, die harten und die weichen Konsonanten (b-d, g-k, d-t) nicht zu unterscheiden und das , $\mathrm{r}^{\text {' }} \mathrm{zu}$ verschlucken. " (a.a.O, S. 55) - ein hier möglicher Verweis auf die Plurizentrik findet jedoch nicht statt, und was mit ,unserem Sprachraum“ gemeint ist, wird nicht erläutert. Interessanterweise haben wir in diesem Lehrwerk keine Deutschlandismen und Helvetismen gefunden, an Austriazismen fanden sich Artikel+Eigenname, Bub, Landeshauptmann, Primar, BMUKK, Landesschulrat, Stadtschulrat, BMBWK, Wiener Schnitzel, Gamsbarthut, Bezirkshauptmannschaft, Beistrich, Hausübung.

Sowohl im Sprachbuch als auch im LehrerInnenhandbuch der letzten hier analysierten Lehrwerkserie „klar_Deutsch“ werden das österreichische Deutsch und das plurizentrische Konzept nicht thematisiert, weder implizit noch explizit. Häufig wird die Formulierung „die Standardsprache“ verwendet; andere Varietäten werden nicht angesprochen.

Im Kapitel „Sprache, Sprachbetrachtung und Stil - die wichtigsten Regeln, die häufigsten Fehler" wird das Präteritum als Erzählzeit genannt; dass in Österreich zumindest in der mündlichen Sprache das Perfekt als Erzählzeit verwendet wird, 
wird nicht erwähnt. Dafür wird auf die als „falsch“ bezeichnete Verwendung des Plusquamperfekts in Deutschland hingewiesen, was immerhin als impliziter Verweis auf die Arealität auf der Nonstandardebene gesehen werden kann: „Zeitenfolge (Tempusverwendung): Erzählzeit Präteritum - Vorzeitigkeit Plusquamperfekt: In Deutschland wird umgangssprachlich zunehmend das Plusquamperfekt anstelle des Perfekts verwendet - das ist falsch!" (Sprachbuch, klar_Deutsch 7/8, S. 29). Der einzige im Sprachbuch der Serie „klar_Deutsch“vorkommende unspezifische Deutschlandismus ist das Wort „Anlagen“, an Austriazismen fanden sich: benützen, Beistrich, AMS, Maturant/in, AMS, Maturazeugnis, Beilagen.

\subsection{Zusammenfassung der Lehrwerksanalysen}

Bezugnehmend auf die anfangs gestellten Forschungsfragen lässt sich feststellen, dass in fast allen untersuchten Lehrwerken weder das österreichische Deutsch noch andere nationale Varietäten der deutschen Sprache explizit thematisiert werden. Auch das plurizentrische Konzept oder andere Konzepte wie das der Pluriarealität finden keine Erwähnung. Nur im Sprachbuch der Serie „Treffpunkt Deutsch" für die 8. Schulstufe ist einmal die Rede von der österreichischen Standardsprache; da an anderen Stellen desselben Sprachbuchs aber von der Existenz nur einer Standardsprache ausgegangen wird, ist nicht anzunehmen, dass dem Sprachbuch eine plurizentrische Sichtweise zugrunde liegt. Implizit wird das österreichische Deutsch ein paar Mal erwähnt: Einmal ist vom „oberdeutschen Sprachraum“ (Arbeitsheft, „Treffpunkt Deutsch“) die Rede, einmal von „unserem Sprachraum“ (Sprachbuch, „Sprachbuch“). Zur Darstellung des österreichischen Deutsch findet sich dementsprechend nicht viel: Im Sprachbuch der Serie „Aktion Sprache“ werden anhand einer Karte lexikalische Unterschiede zwischen den deutschsprachigen Ländern skizziert, und im Basisteil Plus der Serie „Deutschstunde“ gibt es ein Kapitel zum Thema ,Sprachvergleich Österreich - Deutschland', das jedoch keine sachliche Information bietet und die Standardebene mit der Dialektebene vermischt.

Normbegriffe wie „sprachrichtig“ und „normgerecht“ kommen in einigen der analysierten Lehrwerke und LehrerInnenhandbücher ebenso vor wie in den Lehr- und Studienplänen, und auch hier unkommentiert. Diese Parallelität und die Intertextualität zwischen diesen Dokumenten fällt auf und ist auch nachvollziehbar, weil Lehrmittel in Österreich von einer Lehrbuchkommission approbiert werden müssen, die sich wiederum an den Lehrplänen orientiert.

Was die Verwendung von nationalen Varianten betrifft, ist festzustellen, dass der Großteil der in den Lehrwerken vorkommenden Austriazismen und Deutschlandismen lexikalische Varianten sind - Helvetismen kommen kaum vor. Die Varianten sind nicht als solche markiert. Woher die VerfasserInnen einzelner Texte stammen, wird an manchen Stellen expliziert; meist bleibt es für die SchülerInnen 
jedoch unklar, ob es sich um Texte von bundesdeutschen, österreichischen oder Schweizer AutorInnen handelt. Klare Verweise auf Wörterbücher gibt es, ebenso wie in den österreichischen Lehrplänen, nicht, lediglich allgemeine Empfehlungen, die SchülerInnen mögen im Wörterbuch nachschlagen.

In keinem der analysierten Lehrwerke wird die Varietätenfrage metasprachlich thematisiert. Termini wie „plurizentrisch“, „pluriareal“, „plurinational“, ,österreichisches Deutsch“, „Schweizer Deutsch“, „, deutsch(ländisch)es Deutsch“, „Austriazismen“, „Helvetismus“, „Deutschlandismus/Teutonismus“ werden nicht verwendet, und zwar weder in den für die SchülerInnen bestimmten Materialien noch in den LehrerInnenbegleitheften. Andere Aspekte der Variation der deutschen Sprache (Standard vs. Dialekt/Mundart; Jugendsprache; Fachsprache u. Ä.) - werden dagegen lehrplangemäß immer wieder thematisiert. Überall dort wäre eine Thematisierung unterschiedlicher Konzeptualisierungen des Deutschen sachlich logisch und sinnvoll gewesen - sie findet jedoch nicht statt. Auch dort, wo es im Kontext von Mediensprache angebracht wäre, Einflüsse des deutschen Deutsch auf das österreichische Deutsch via Kabel-TV und andere moderne Medien zu thematisieren, fehlen die gebotenen Querverweise. Insgesamt lässt die Analyse der oben genannten Lehr- und Übungsbücher bzw. LehrerInnenhandbücher vermuten, dass diesen Lehrwerken eine monozentrische Einstellung zugrunde liegt. An den Stellen, an denen man auf das österreichische Deutsch hinweisen hätte können und auf verschiedene Konzeptualisierungsmöglichkeiten (z. B. die Plurizentrizität - auf der Ebene der Standard- und Bildungssprache), wurde diese Möglichkeit nicht aufgegriffen. Die SchülerInnen werden dadurch für das österreichische Deutsch nicht sensibilisiert, obwohl diese staatliche Variation der deutschen Sprache für nationale Identitätskonstruktionen der ÖsterreicherInnen und für Identitätspolitik eine wichtige Rolle spielt. ${ }^{2}$

Zusätzliche Informationen zu Grammatik, Orthographie oder Wortschatz des österreichischen Deutsch gibt es weder in den Lehrbüchern noch in den LehrerInnenhandbüchern. Vorschläge zum Umgang mit Varietäten oder Dialekten gibt es in den analysierten Lehrwerken nur andeutungsweise. Begriffe wie Standardsprache, Umgangssprache, Dialekt, Jugendsprache etc. werden nicht immer ausreichend erklärt.

In den analysierten Lehrwerken gibt es viele Texte österreichischer und deutscher Herkunft, jedoch deutlich weniger Texte von Schweizer AutorInnen. ${ }^{3}$ Keiner dieser Texte ist explizit als deutscher, österreichischer oder Schweizer Text

2 Mögliche Anlässe zur Sprachreflexion über die Rolle der Sprachvarietäten für nationale Identitätskonstruktionen könnten das Protokoll Nr. 10 oder die Auseinandersetzung mit der EU um die Bezeichnung „Marmelade“ sein (vgl. de Cillia 2006).

3 Gerade Texte von Schweizer AutorInnen böten Gelegenheit, auch Helvetismen zu thematisieren, wie Peter Bichsels „Ein Tisch ist ein Tisch“ oder Dürrenmatts „Romulus der Große“ mit folgender Passage: „ROMULUS: Das Morgenessen. DIENER: Eure Exzellenz, es heisst das Frühstück. ROMULUS: Das Morgenessen. Was in meinem Hause klassisches Latein ist, bestimme ich." 
ausgewiesen; äußerst selten wurde jedoch eine Kurzbiographie des Autors/der Autorin beigefügt. In keinem der untersuchten Lehrwerke finden sich Verweise auf das Österreichische Wörterbuch oder andere Nachschlagwerke wie z. B. das Variantenwörterbuch, auf den Kodex des österreichischen Deutsch. Auch hier bestätigt sich letztlich unsere Annahme, dass die Konzeption des Deutschen als plurizentrische Sprache in den Lehrwerken entweder nur eine marginale oder überhaupt keine Rolle spielt. ${ }^{4}$

Die analysierten Schulbücher sind im Übrigen durchgängig als Bücher für Deutsch als Muttersprache konzipiert. Die Tatsache, dass Deutsch für einen großen Teil (ca. ein Viertel) der SchülerInnen Zweitsprache ist, kommt nicht zum Tragen, und auch der bildungssprachliche Aspekt wird nicht explizit thematisiert. So entsteht der Eindruck, dass sich auch die Entwicklung der Sprachdidaktik in den letzten 10 bis 15 Jahren in Richtung eines integrativen Sprach/en/unterrichts (vgl. de Cillia 2013, Reich/Krumm 2013) zum Zeitpunkt unserer Erhebung noch nicht in den Lehrwerken niederschlägt.

\subsection{Zusammenfassung der Dokumentenanalyse}

Die hier vorgestellte Untersuchung stellte sich hinsichtlich der unterrichtsrelevanten Basisdokumente für den Deutschunterricht (Lehrpläne, Studienpläne, Schulbücher) zunächst folgende Fragen: Wie wird mit sprachlicher Variation an der österreichischen Schule auf der Ebene der Lehrpläne, Studienpläne, Lehrbücher umgegangen? Wie kommen die Standardvarietät des österreichischen Deutsch und andere Varietäten in diesen Dokumenten vor? Welche Normkonzepte in Bezug auf die Standardsprache (plurizentrisch/pluriareal/ monozentrisch) liegen ihnen zugrunde? Und wie wird mit Nonstandardvarietäten umgegangen?

Die Behandlung der soziolinguistischen Varietäten Standard, Umgangssprache, Dialekt erfolgt nicht systematisch. Wenn es um die Norm geht, wird häufig von Norm, richtig, sprachrichtig gesprochen, werden „bestimmte Normen“ genannt, die aber völlig unbestimmt bleiben. In keinem der analysierten Dokumente wird explizit Bezug genommen, schon gar nicht auf eine Kodifizierung der deutschen

4 Seit Projektende sind einige neue Lehrwerke erschienen. In einzelnen finden sich mittlerweile gut und differenziert gestaltete Lehrinhalte zum österreichischen Deutsch und zur Plurizentrik des Deutschen. Dies könnte unter Umständen auch mit der relativ starken Medienresonanz auf unser Projekt sowie mit Maßnahmen des BMBWF zusammenhängen: Zum einen fanden regelmäßige LehrerInnen- und MultiplikatorInnenfortbildungen in Kooperation mit unserem Projekt ab Projektbeginn statt - wie etwa 2012 das erste thematische Bundesseminar „Welches Deutsch an Schulen?" (PH Steiermark). Zum anderen wurde ein Informations- und Materialheft für DeutschlehrerInnen („Österreichisches Deutsch als Unterrichts- und Bildungssprache“) vom jetzigen BMBWF herausgegeben. 
Sprache in Österreich. Sowohl in Lehrplänen als auch Studienplänen, Lehrbüchern und in ausgewählten Lehrveranstaltungen werden tendenziell monozentrische Normkonzepte vertreten. Das Konzept und der Terminus der Plurizentrik kommen in keinem einzigen Dokument vor (weder in Lehrplänen, noch in Studienplänen, noch in Lehrwerken, noch in LehrerInnenhandbüchern, und nur vereinzelt in Lehrveranstaltungsbeschreibungen).

Letztlich muss man davon ausgehen, dass die Entwicklung der Varietätenlinguistik in den letzten Jahrzehnten und die Entwicklung der Theorie der plurizentrischen Sprachen von der DaM-Didaktik nicht gleichermaßen wie in der DaF-Didaktik rezipiert wurde und dass daher auch den meisten AutorInnen der Lehrpläne und Studienpläne, aber auch den LehrbuchautorInnen die Diskussion um die areale Konzeptualisierung der deutschen Sprache, plurizentrische und pluriareale Zugänge und staatsbezogene Variation nicht bekannt sind. Am ehesten dürfte das noch bei LehrveranstaltungsleiterInnen der Fall sein, wo sich in einer nicht repräsentativen und punktuellen Erhebung des Angebots zweier Semester - einige Beispiele derartiger variationslinguistischer Zugänge gezeigt haben. Aber so gesehen kann diese Konzeptualisierung auch in den Lehrplänen, Studienplänen und Lehrwerken keine Berücksichtigung finden. Die Ergebnisse unserer Fragebogenerhebung zur Bekanntheit der Plurizentrik bei Lehrpersonen (siehe Kap. 5.1) weisen im Übrigen in dieselbe Richtung.

Unsere Befunde decken sich mit denen einer Studie in Deutschland. Davies (2017), die in Nordrhein-Westfalen untersuchte, inwiefern das Plurizentrik-Modell den Lehrenden bekannt ist und sich auf die Praxis auswirkt, hat festgestellt, dass das Konzept in den Lehrplänen nicht vorkommt und keine Relevanz für die Lehrenden besitzt. Wyss (2017) zeigt, dass bei den DeutschlehrerInnen sehr vage Standardkonzepte vorhanden sind, die sich auch in den Curricula finden.

Als Schlussfolgerung aus unserem Befund können wir uns Schmidlin/Wyss/ Davies (2017) anschließen, die feststellen, dass es wünschenswert wäre,

die Frage der Standardvarietäten im internationalen deutschsprachigen Raum in der Ausbildung von DeutschlehrerInnen stärker mitzuberücksichtigen und insbesondere auch bei der Schulbuchkonzeption (für alle Stufen und Schultypen) im Blick zu haben. (Schmidlin/Wyss/Davies 2017, 18) 


\title{
5 Empirische Erhebung bei Lehrerlnnen und Schülerlnnen an österreichischen Schulen
}

\author{
Beschreibung der Daten
}

Um die Spracheinstellungen von LehrerInnen und SchülerInnen gegenüber dem österreichischen Deutsch und den Varietäten des Deutschen sowie die Rolle des österreichischen Deutsch in der Unterrichtspraxis zu erheben, haben wir einerseits eine großangelegte Fragebogenerhebung in ganz Österreich unter LehrerInnen und SchülerInnen, andererseits 21 vertiefende Interviews mit Lehrkräften in acht Bundesländern sowie je eine Gruppendiskussion mit SchülerInnen und LehrerInnen durchgeführt. Ergänzt wurden die Befragungsdaten mit teilnehmender Unterrichtsbeobachtung.

Zunächst sollen hier die einzelnen Teilkorpora beschrieben werden, und anschließend werden in Kapitel 6 die Ergebnisse der Teilerhebungen dargestellt.

\subsection{Fragebogenerhebung}

Die Fragebogenerhebung wurde in den Monaten September und Oktober 2013 österreichweit durchgeführt. Insgesamt wurden 1.253 SchülerInnen der AHS-Oberstufe an 28 Schulen in ganz Österreich (2-4 Schulen pro Bundesland), sowie 164 LehrerInnen an 56 Schulen verschiedener Schultypen (VS, HS/NMS, AHS Sekundarstufe I und II, BHS) aus allen Bundesländern mit schriftlichen Fragebögen befragt.

Die inhaltlichen Fragestellungen der Fragebögen haben sich auf die in Kap. 3.1 näher ausgeführten Themenfelder bezogen. Zum einen ging es im Fragebogen um die Konzeptualisierung der sprachlichen Variation im deutschsprachigen Raum und innerhalb Österreichs, zum anderen um die Spracheinstellungen von LehrerInnen und SchülerInnen gegenüber den nationalen Varietäten des Deutschen und speziell gegenüber dem österreichischen Deutsch. Ein weiterer Teil der Befragung befasste sich mit der Varietätenverwendung durch LehrerInnen und SchülerInnen innerhalb und außerhalb der Schule. Außerdem war von Interesse, welche Vorstellungen konkret mit dem österreichischen Deutsch assoziiert werden. Eine wichtige, immer wieder in der Literatur formulierte Hypothese (Muhr 1989, 2005b; Moser 1999; Clyne 1995) ist die, dass ÖsterreicherInnen eine geringe Sprachloyalität gegenüber der eigenen Varietät aufweisen und die eigene Varietät nicht als gleichwertig mit dem deutschen Deutsch betrachten bzw. nicht als gleichermaßen korrekt wie dieses empfinden - ja, dass österreichische LehrerInnen sogar exonorm-orientiert korrigieren würden. Eine Reihe von Items im LehrerInnen- und SchülerInnen-Fragebogen (aber auch in den Interviews 
und Gruppendiskussionen) haben sich diesen Fragestellungen gewidmet. Auch die Frage nach dem österreichischen Deutsch und einem allfälligen Zusammenhang mit nationalen Identitätskonstruktionen wurde in diesem Kontext gestellt.

Der LehrerInnen-Fragebogen enthielt insgesamt 65 Items, der SchülerInnenFragebogen 47 Items, wobei sich die Fragen zu einem großen Teil deckten, bei den Lehrpersonen aber einige zusätzliche Fragen gestellt wurden. Insbesondere wurde den LehrerInnen ein Musteraufsatz vorgelegt, der Austriazismen und Deutschlandismen enthielt und den sie gebeten wurden zu korrigieren, um das mutmaßlich exonorm-orientierte Korrekturverhalten zu überprüfen.

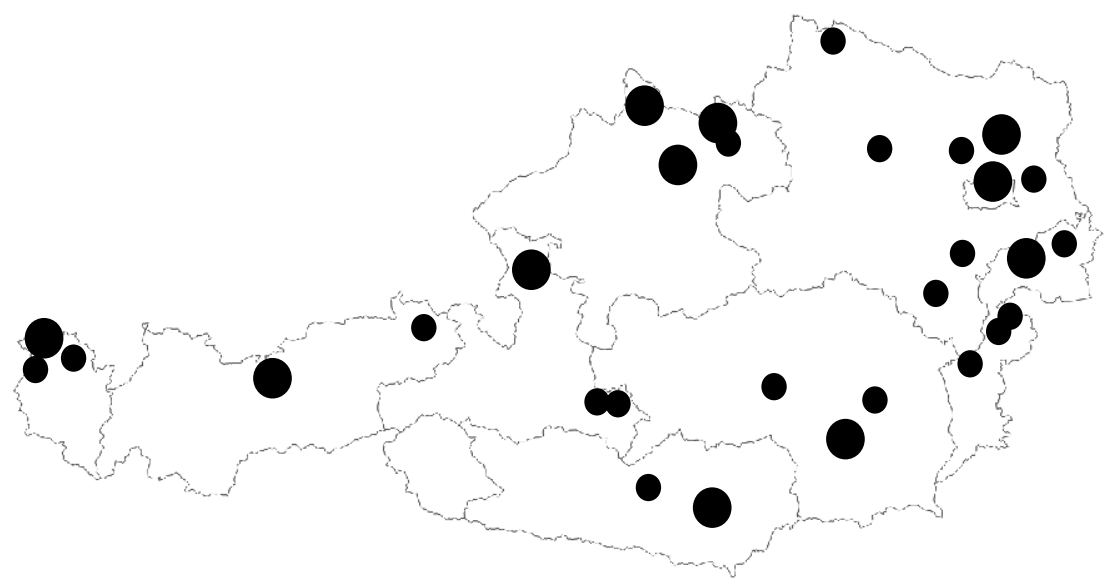

Abb. 1: Erhebungsstandorte der Fragebogenerhebungen unter LehrerInnen und SchülerInnen (Legende: Kleine Punkte = Erhebung an einer Schule pro Ort/Stadt; große Punkte = Erhebung an 2-7 Schulen pro Ort/Stadt) ${ }^{1}$

Im Detail wurde jedes Themenfeld nach Möglichkeit mithilfe verschiedener Fragestellungen beleuchtet. Was die Konzeptualisierung der sprachlichen Variation im deutschsprachigen Raum und innerhalb Österreichs betrifft, wurde eingangs folgende Frage gestellt: „Wie würden Sie die Sprache, die die Mehrheit der Österreicher/innen als Muttersprache spricht, nennen?" Dann wollten wir wissen, ob man Deutsch als einheitliche Sprache mit einer einzigen standardsprachlichen (hochdeutschen) Form wahrnimmt, die in allen deutschsprachigen Ländern gilt, oder als Sprache mit Unterschieden in der Standardsprache (im Hochdeutschen) zwischen den einzelnen Ländern. Eine weitere Frage im Konzeptualisierungsblock war, was nach Meinung der ProbandInnen österreichisches Deutsch ist, wobei hier bei möglicher Mehrfachnennung drei Antwortoptionen vorgegeben waren: „das, was man in österreichischen TV- und Radionachrichten spricht“; „das, was

1 Quelle: http://d-maps.com/carte.php?num_car=17719\&lang=de (18.12. 2018). Punkte wurden von den AutorInnen eingefügt. Eine Auflistung aller Erhebungsstandorte findet sich im Anhang. 
man in Österreich im Alltag spricht“ (Umgangssprache) und „die verschiedenen Dialekte in Österreich“. Weiters wurde nach Unterschieden zwischen der deutschen Sprache in Österreich und in Deutschland bzw. der Schweiz gefragt, ob es ein österreichisches Standarddeutsch (Hochdeutsch) gäbe und ob dieses für genauso korrekt gehalten würde wie das in Deutschland gängige Standarddeutsch. An anderer Stelle im Fragebogen wurde noch einmal etwas differenzierter nach der Korrektheit des österreichischen Deutsch gefragt, und zwar eingebettet in Fragen, die inhaltliche Analogien zum Englischen und Französischen hergestellt haben („Wie sehr würden Sie den folgenden Aussagen zustimmen: Britisches Englisch ist korrekter als amerikanisches Englisch/Deutsches Deutsch ist korrekter als österreichisches Deutsch/Das Französisch in Frankreich ist korrekter als das Französisch in der Schweiz.").

Schließlich wurde nach der Bedeutung regionaler Sprachunterschiede innerhalb Österreichs (z. B. zwischen Ost- und Westösterreich) gefragt, ob es ein überregionales österreichisches Standarddeutsch gebe, das sich vom Deutsch Deutschlands in manchen Bereichen klar unterscheidet, und ob Österreich und Süddeutschland sprachlich mehr gemeinsam hätten als Nord- und Süddeutschland. Mit Polaritätsprofilen wurde erhoben, wie das in Österreich/in Deutschland/in der Schweiz gesprochene Standarddeutsch auf die ProbandInnen wirkt.

Anschließend wurden die LehrerInnen gebeten, einen fiktiven Aufsatz eines 11-jährigen Schülers zu korrigieren und dabei Fehler und stilistisch unpassende Ausdrücke anzuzeichnen. Außerdem wurden den ProbandInnen 30 Sätze mit Doubletten von Austriazismen bzw. Deutschlandismen und west- bzw. ostösterreichischen Varianten vorgelegt, bevor sie anzugeben hatten, welche sie eher verwenden würden.

In einem weiteren Item wurden LehrerInnen und SchülerInnen danach gefragt, ob sie sich sprachlich anpassen würden, wenn Sie mit einem/er deutschen Gesprächspartner/in sprechen. Weiter unten im Fragebogen wurde gefragt, wie stark sich das in Österreich gesprochene Deutsch von dem in Deutschland und in der Schweiz gesprochenen Deutsch bzw. das im Osten Österreichs gesprochene Deutsch von dem im Westen Österreichs gesprochenen Deutsch unterscheide. Es wurde erhoben, ob es nach Meinung der ProbandInnen ein besonders gutes Deutsch gäbe und wenn ja, wo es gesprochen werde. Eine Reihe von Items zielte darauf ab, ob die Verwendung von Deutschlandismen in bestimmten Situationen (bei den Nachrichten, beim Einkaufen, auf Speisekarten, Tschüss beim Verabschieden) stören würde, welche Bedeutung das österreichische Deutsch für persönliche Identitätskonstruktionen habe, ob der Sprachgebrauch in Österreich durch deutsche Fernsehsender beeinflusst werde, und schließlich, ob das Thema „Österreichisches Deutsch und die verschiedenen Varietäten der deutschen Sprache" für ein wichtiges Thema im Deutschunterricht gehalten würde.

Ein anderer Teil des Fragebogens befasste sich damit, wie LehrerInnen und SchülerInnen die Verwendung unterschiedlicher Varietäten (Standard, Umgangssprache, 
Dialekt) selbst wahrnehmen, und zwar nicht nur in der Schule, sondern auch im Kontext Familie/Freundschaft. Die LehrerInnen wurden auch gefragt, mit welchen Herkunftssprachen bzw. Varietäten der SchülerInnen sie im Unterricht konfrontiert seien, ob ihrer Erfahrung nach die Herkunftssprachen bzw. Varietäten der SchülerInnen den Erwerb der Standardsprache beeinflussen, ob ihnen Unterrichtsmaterialien, die sprachliche Variation (z. B. Dialekte, Jugendsprache, österreichisches Deutsch etc.) thematisieren, zur Verfügung stünden, welche Lehrbücher sie im Deutschunterricht verwenden und ob österreichisches Deutsch im Unterricht oder im Gespräch mit KollegInnen ein Thema sei. Es wurde auch nachgefragt, ob die Lehrpersonen im Deutschunterricht unterschiedliche Sprachformen (Dialekt/Umgangssprache/Standardsprache) thematisieren, welche/s Nachschlagwerk/e bei Fragen der Norm und Sprachrichtigkeit herangezogen würden, um bei ihrer Korrekturarbeit in Zweifelsfällen die Sprachrichtigkeit von Schülertexten zu beurteilen, ob es bei der Korrektur manchmal Zweifelsfälle gäbe, die nicht mithilfe von Nachschlagwerken geklärt werden können, und schließlich, welche Nachschlagwerke und Internetseiten sie ihren SchülerInnen zum Nachschlagen in sprachlichen Fragen (Rechtschreibung und Grammatik) empfehlen würden.

Eine Frage befasste sich damit, wie die LehrerInnen überwiegend mit Ihren SchülerInnen in bestimmten Unterrichtssituationen (Vortrag, Arbeitsauftrag, Disziplinäres, Organisatorisches) sprechen und ob bzw. in welchen Situationen die SchülerInnen Dialekt verwenden würden. Weiters wurde erhoben, ob die befragten LehrerInnen Deutsch als Zweitsprache unterrichten oder nicht, welche Lehrbücher/Materialien im DaZ-Unterricht verwendet würden, ob in den DaZ-Lehrbüchern und in den DaZ-Materialien österreichisches Deutsch oder soziolinguistische Variation (Dialekt/Umgangssprache/Standardsprache) des Deutschen thematisiert würde, ob das Konzept des integrativen Sprachunterrichts, wonach jeder Unterricht Sprachunterricht ist (d. h. auch der Unterricht in nichtsprachlichen Fächern wie Physik oder Biologie) bekannt sei und das im Auftrag des Bildungsministeriums entwickelte „Curriculum Mehrsprachigkeit“.

Darüber hinaus wurden persönliche Daten wie Geschlecht, Alter, Geburtsort, Ausbildungsinstitution, Ort des Aufwachsens, Auslandsaufenthalte, Schulform und Bundesland der Schule, Dauer der Unterrichtserfahrung, Studienfächer und Unterrichtsfächer und höchster Abschluss in der Ausbildung erhoben, ob im Studium DaF/DaZ-Lehrveranstaltungen besucht wurden, ob Deutsch als Bildungssprache in der Ausbildung und ob sprachliche Variation ein Thema gewesen bzw. ausreichend thematisiert worden sei. Es wurde danach gefragt, in welcher Varietät/Sprache mit KollegInnen, SchülerInnen und in der Familie kommuniziert würde. Abschließend wurde nach dem Medienverhalten gefragt, ob österreichisches Deutsch für die ProbandInnen ein wichtiges Thema sei und ihnen das Konzept der plurizentrischen Sprachen bekannt sei, und wenn ja, woher. 
Der Fragebogen der SchülerInnen unterschied sich zunächst dadurch, dass dort der Korrekturtext nicht enthalten war. Die Fragen zur Ausbildung und zum Unterricht der LehrerInnen waren naturgemäß nicht enthalten. Bei den persönlichen Daten wurde noch nach der/den Muttersprache/n der SchülerInnen und der Eltern und der Erstsprache gefragt, ob Deutsch die Muttersprache war, und wenn nicht, wo Deutsch erlernt worden war, ob der Dialekt dabei ein Problem gewesen sei und ob der/die Betreffende ein/e außerordentliche/r SchülerIn gewesen sei. Es wurde nach dem aktuellen Fernsehverhalten und den TV-Gewohnheiten in der Kindheit gefragt, nach regelmäßig gehörten Radiosendern und nach dem Lieblingsbuch, ob österreichisches Deutsch ein interessantes Thema sei und ob das Konzept der Plurizentrik bekannt sei. Schließlich wollten wir noch wissen, in welcher Varietät/Sprache vorwiegend mit LehrerInnen, FreundInnen und unterschiedlichen Familienmitgliedern (Mutter, Vater, Geschwister, Großeltern) gesprochen würde.

Die Ergebnisse wurden deskriptiv und inferenzstatistisch (Berechnung von Korrelationen, Mittelwertvergleiche mit chi2-Tests, T-Tests, U-Tests, KruskalWallis-Tests) ausgewertet (für die statistischen Testverfahren siehe Kap. 3.2).

\subsection{Fragebogenerhebung: Stichprobe der LehrerInnen}

In der österreichweiten Fragebogenerhebung unter 164 DeutschlehrerInnen wurden, wie schon erwähnt, Lehrkräfte aller Schultypen zu ihrer Spracheinstellung gegenüber dem österreichischen Standarddeutsch und anderen Varietäten des österreichischen Deutsch befragt. Auch die Konzeptualisierung der deutschen Sprache und die Rolle der standardsprachlichen Variation des Deutschen im Deutschunterricht waren Gegenstand der Befragung.

Im Fragebogen für die Lehrpersonen wurden die folgenden sprachexternen Variablen erhoben: das Geschlecht, das Alter, die Anzahl der Dienstjahre, die Schulform und die Region ${ }^{2}$, in der sie unterrichteten. Weiters wurde nach den Studienfächern und den tatsächlich unterrichteten Unterrichtsfächern gefragt, auch nach dem Ort der Ausbildung (PädAk bzw. PH oder Universität) und danach, ob den LehrerInnen das plurizentrische Konzept bekannt war.

Rund vier Fünftel des Samples waren Lehrerinnen (79,3\%), etwa ein Fünftel Lehrer $(20,7 \%)$. Hinsichtlich ihrer Altersstruktur wurden die Lehrenden in vier Gruppen zusammengefasst: 22-31 Jahre, 32-41 Jahre, 42-51 Jahre und 52-63 Jahre.

2 Die österreichischen Bundesländer wurden für die Auswertung zu folgenden Kategorien zusammengefasst: 1. Ost (Wien, Niederösterreich und Burgenland), 2. Mitte (Oberösterreich und Salzburg), 3. Südost (Steiermark und Kärnten), 4. Tirol und 5. Vorarlberg (vgl. Kap. 2.5). Zunächst wurden Tirol und Vorarlberg probehalber zu der Kategorie „West“ zusammengefasst; es stellte sich jedoch heraus, dass das Antwortverhalten der beiden Bundesländer stark voneinander abweicht; daher wurden sie getrennt voneinander ausgewertet. 
Die Graphik zeigt, dass die LehrerInnen im Alter von 22-63 Jahren streuten, wobei mehr als zwei Drittel (70\%) über 41 Jahre alt waren und die Unter-41-jährigen ein schwaches Drittel des Samples (29,5\%) ausmachten:

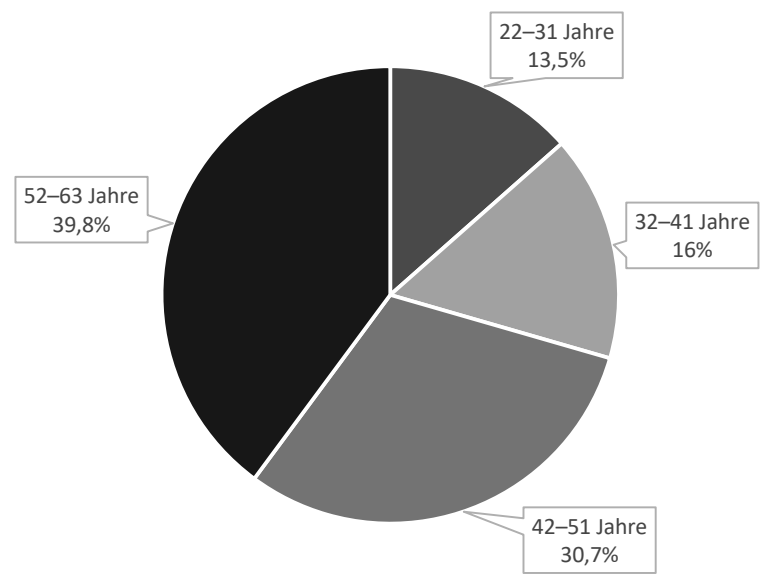

Abb. 2: Alter der befragten Lehrerlnnen (in \%)

Da unterschiedliche Spracheinstellungen auch mit verschiedenen Schultypen in Zusammenhang gebracht werden können, wurden LehrerInnen der Volksschule, der Hauptschule/Neuen Mittelschule sowie der AHS (Unterstufe und Oberstufe), BHS und BAKIP ${ }^{3}$ befragt. Nach ihrer Unterrichtstätigkeit zum Zeitpunkt der Befragung verteilte sich das Sample folgendermaßen: 33 \% Volksschule, $13 \%$ Hauptschule/Neue Mittelschule, 54 \% AHS/BHS/BAKIP.

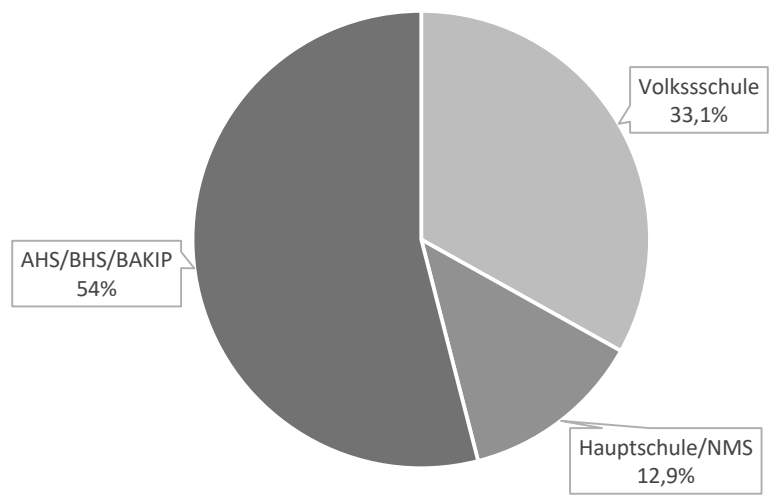

Abb. 3: Schulformen der Lehrerlnnen (in \%)

3 Der betreffende Schultyp heißt mittlerweile BAfEP - Bundesanstalt für Elementarpädagogik. 
Auch Unterschiede hinsichtlich des Zusammenhangs zwischen der Spracheinstellung und der Anzahl der Dienstjahre sollten überprüft werden. Nach Anzahl ihrer Dienstjahre wurden die LehrerInnen in vier Gruppen zusammengefasst (o-10 Dienstjahre, 11-2o Dienstjahre, 21-30 Dienstjahre und 31-40 Dienstjahre) mit dem Ergebnis, dass sich das Sample nach Dienstjahren relativ ausgewogen verteilt hat:

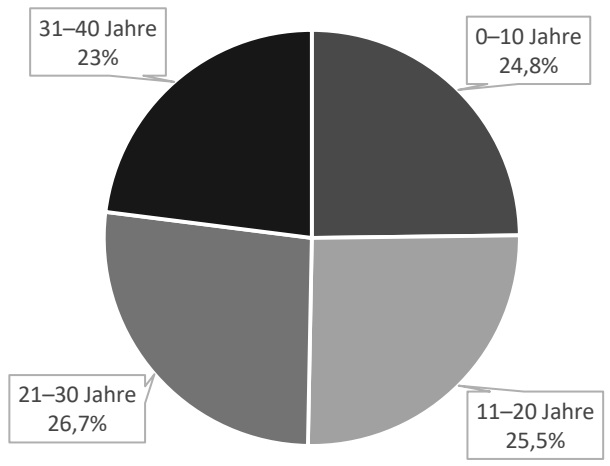

Abb. 4: Dienstjahre der LehrerInnen (in \%)

Um die LehrerInnen nicht nur nach Alter und Dienstjahren, sondern auch nach Regionen statistisch miteinander vergleichen zu können, wurde die Befragung in allen österreichischen Bundesländern durchgeführt. Die Beteiligung der Schulen und LehrerInnen in den neun Bundesländern fiel dabei unterschiedlich aus. Nach den Dienstorten gegliedert, verteilte sich die Stichprobe der befragten LehrerInnen folgendermaßen:

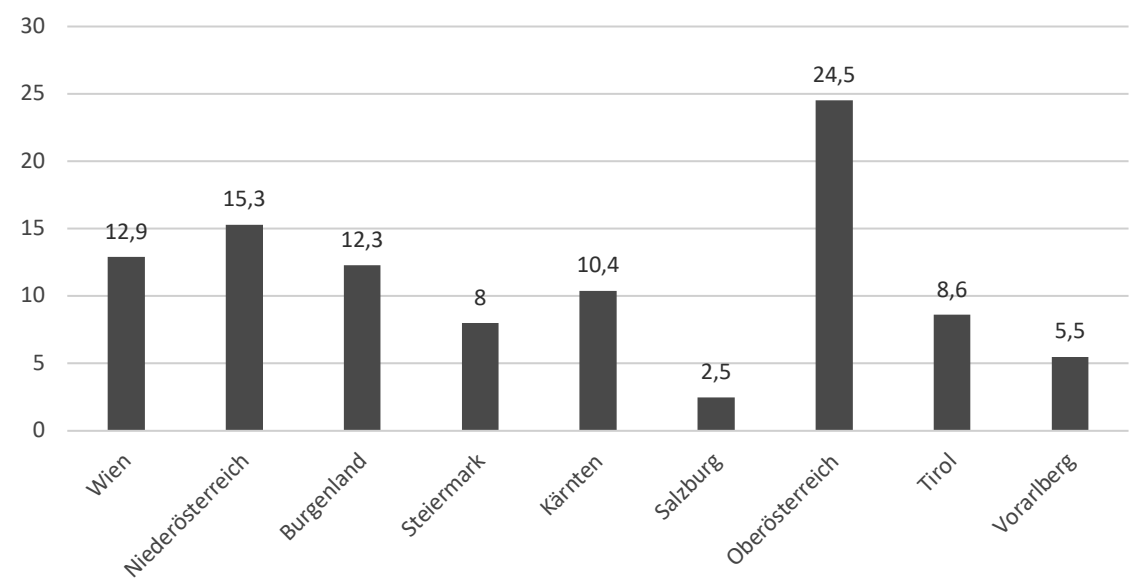

Abb. 5: Dienstort nach Bundesländern (in \%) 
Innerhalb der neun Bundesländer wurden die LehrerInnen nochmals nach den schon erwähnten fünf Subkategorien zusammengefasst: (1) Ost (Wien, Niederösterreich, Burgenland), (2) Südost (Steiermark, Kärnten), (3) Mitte (Oberösterreich, Salzburg), (4) Tirol, (5) Vorarlberg:

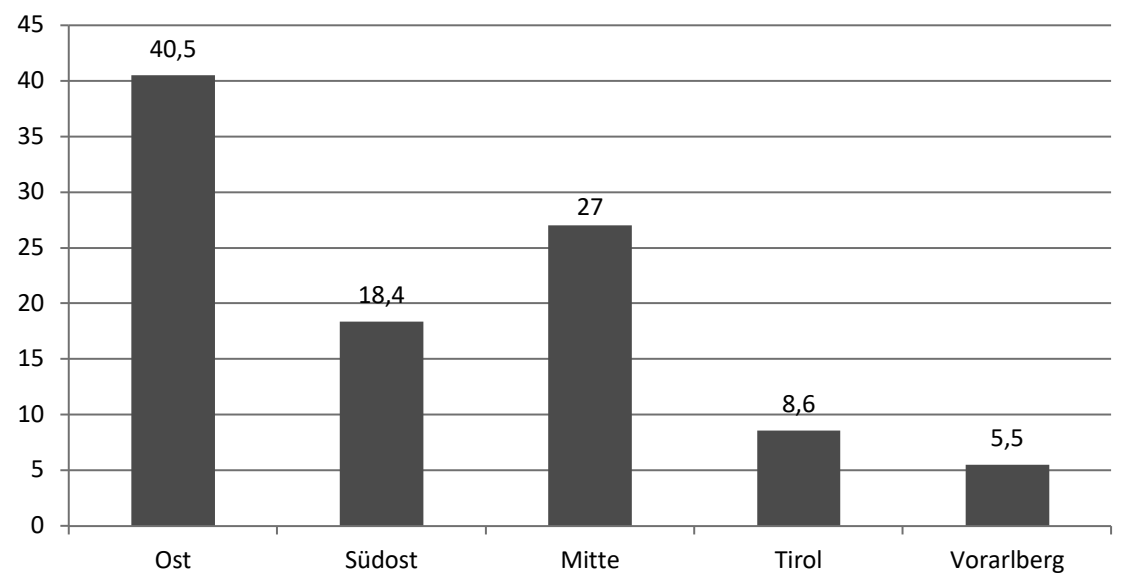

Abb. 6: Dienstort nach Regionen (in \%)

Ihre LehrerInnenausbildung hatten $53,4 \%$ der befragten LehrerInnen an einer Universität absolviert, $46,6 \%$ an einer Pädagogischen Hochschule (früher PädAk).

Da im Bereich der Sekundarstufe davon auszugehen war, dass es unter den befragten Lehrenden viele verschiedene Kombinationen der weiteren Unterrichtsfächer neben Deutsch gibt und sich diese unterschiedlichen Fächerkombinationen möglicherweise in verschiedenen Spracheinstellungen niederschlagen würden, wurden die Lehrpersonen auch nach ihren weiteren Unterrichtsfächern gefragt. Danach wurden die LehrerInnen in vier Gruppen zusammengefasst: (1) jene, die Deutsch in Kombination mit einem weiteren Sprachfach unterrichteten, (2) jene, die Deutsch in Kombination mit einem nichtsprachlichen Fach unterrichteten, (3) jene, die nur ein anderes Fach, aber nicht Deutsch unterrichteten (4) und jene, die nur Deutsch und kein weiteres Fach unterrichteten. Die Gruppierung ergab folgendes Bild: 59,3\% unterrichteten Deutsch in Kombination mit einem nichtsprachlichen Fach, ein gutes Fünftel $(22,1 \%)$ Deutsch in Kombination mit einer zweiten Sprache, und 4,7 \% ausschließlich Deutsch. Der Rest (13,9\%) unterrichtete andere Fächer. 


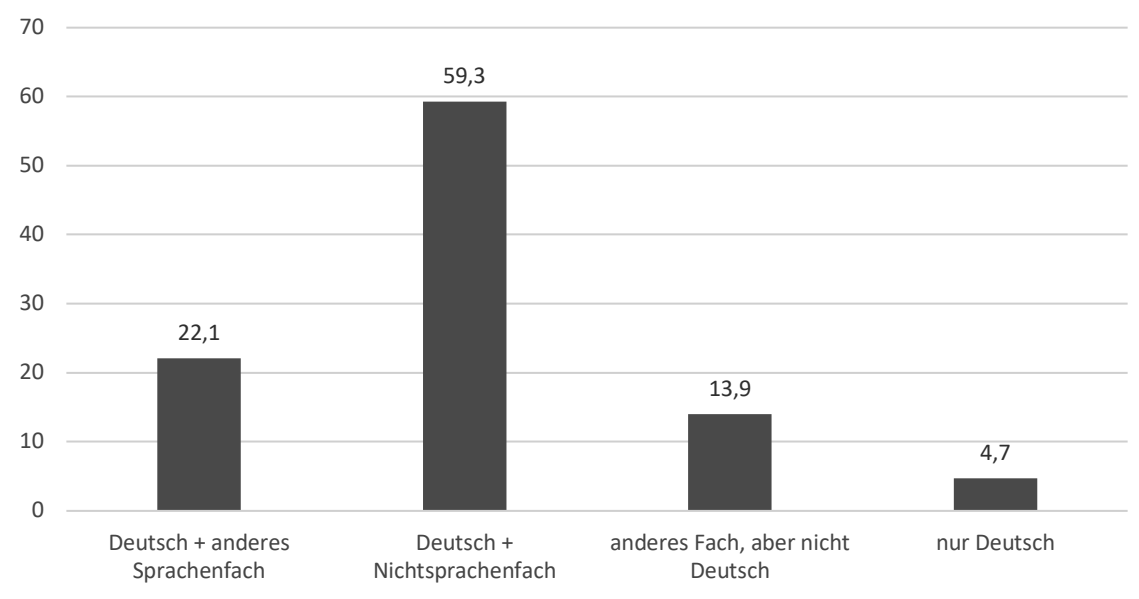

Abb. 7: Unterrichtsfächer (in \%)

Schließlich war auch von Interesse, ob es zwischen dem höchsten Ausbildungsabschluss der LehrerInnen und ihren Spracheinstellungen Zusammenhänge gibt. Danach ließen sich die befragten LehrerInnen in vier Gruppen einteilen: (1) DiplompädagogInnen, (2) Magister/Magistra, (3) Doktor/Doktorin und (4) noch in Ausbildung befindlich. In Prozentwerten ausgedrückt verteilte sich die Anzahl der Befragten folgendermaßen: 2,7\% befanden sich noch in der Ausbildung, 41,6\% hatten den Abschluss DiplompädagogIn, 48,3\% das Magisterium und $7,4 \%$ ein Doktorat.

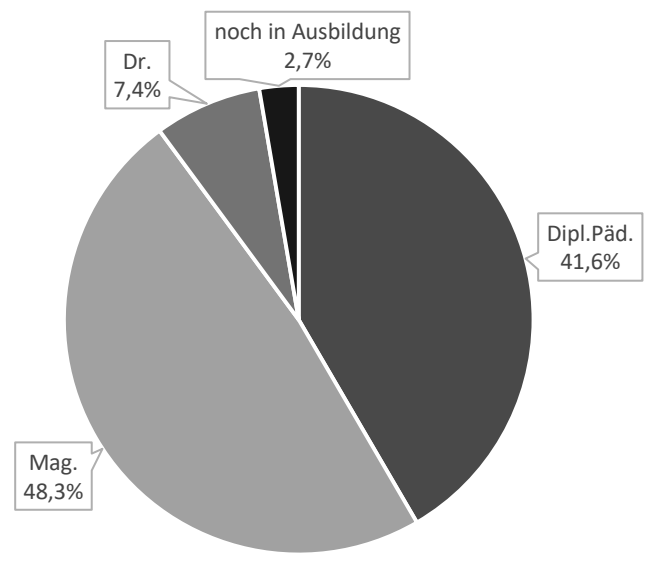

Abb. 8: Höchster Ausbildungsabschluss der Lehrerlnnen (in \%) 
Eine Reihe von Erhebungsfragen beschäftigte sich mit Hintergründen zur Unterrichtspraxis und zur Ausbildung der LehrerInnen sowie mit ihrer Einstellung gegenüber dem österreichischen Deutsch als unterrichtsrelevantem Thema. Die Frage, ob die LehrerInnen das Thema „Österreichisches Deutsch und die verschiedenen Varietäten der deutschen Sprache“ für ein wichtiges Thema im Deutschunterricht halten - mit den Antwortmöglichkeiten sehr wichtig/wichtig/ wenig wichtig/gar nicht wichtig - ergab folgendes Bild: Für rund zwei Drittel der LehrerInnen ist das österreichische Deutsch im Unterricht ein sehr wichtiges bzw. wichtiges Thema (13,5\% bzw. 52,1\%), für ein knappes Drittel (31,3\%) ist es wenig wichtig, und nur 3,1\% der LehrerInnen gaben an, dass für sie das Thema „Österreichisches Deutsch“ im Deutschunterricht unwichtig ist. Innerhalb der Gruppe der LehrerInnen gab es keine signifikanten Unterschiede.

Um ein Bild von der Vielfalt der sprachlichen Varietäten an österreichischen Schulen zu erhalten, mit denen LehrerInnen im heimischen Unterrichtsalltag zu tun haben, wurden die LehrerInnen gebeten anzugeben, mit welchen Varietäten sie im Unterricht konfrontiert sind (Dialekte, Umgangssprache, österreichisch geprägtes Standarddeutsch, bundesdeutsch geprägtes Standarddeutsch, schweizerisch geprägtes Standarddeutsch, andere Herkunftssprachen als Deutsch). Dabei ergaben sich signifikante Unterschiede nach mehreren außersprachlichen Variablen. Die Ergebnisse finden sich in Kapitel 6.5. Im Fragebogen wurden die Lehrenden auch dazu befragt, ob ihnen Unterrichtsmaterialien zur Verfügung stehen, die sprachliche Variation (z. B. Dialekte, Jugendsprache, österreichisches Deutsch etc.) thematisieren. Auffallend ist hier die relativ hohe Anzahl an LehrerInnen, die gar nicht wussten, ob ihnen solche Unterrichtsmaterialien zur Verfügung stehen. Unter den VS-LehrerInnen war die Anzahl der „Weiß nicht“-Antworten mit 27,8 \% am höchsten. Die Hälfte der VS-LehrerInnen gab an, dass ihnen kein Material zur sprachlichen Variation zur Verfügung stehe. HS/NMS- und AHS-/Sek.-II-LehrerInnen unterscheiden sich hier nur geringfügig. $45 \%$ der HS/NMS-LehrerInnen und 48,2 \% der AHS-/Sek.-II-LehrerInnen gaben an, dass ihnen solche Unterlagen zur Verfügung stehen. Lehrende mit der Fächerkombination Deutsch + Fremdsprache gaben zu rund drei Viertel an, dass ihnen einschlägiges Unterrichtsmaterial zur Verfügung stehe, während LehrerInnen mit der Kombination Deutsch + keine Fremdsprache dies nur zu $43 \%$ angegeben haben.

Ein signifikanter Zusammenhang hat sich zwischen der Kenntnis des plurizentrischen Konzepts und dem Zugriff auf einschlägiges Unterrichtsmaterial aufgetan: Diejenigen LehrerInnen, die das Konzept der Plurizentrik kannten, gaben zu rund zwei Drittel auch an, solches Material zu haben - möglicherweise sind sie stärker für das Thema Variation sensibilisiert. Von den Lehrenden, denen das plurizentrische Konzept kein Begriff war, gaben nur $44 \%$ an, dass sie über Unterrichtsmaterial zur sprachlichen Variation verfügen. 
Die Frage, ob österreichisches Deutsch im Unterricht oder im Gespräch mit KollegInnen sehr häufig/häufig/selten/nie ein Thema ist, wurde den LehrerInnen ebenfalls gestellt. Die Ergebnisse zeigen, dass sich Gespräche im Kollegenkreis selten bis nie um das österreichische Deutsch drehen (insgesamt rund $92 \%$ ).

Dass österreichisches Deutsch im Unterricht sehr häufig oder häufig ein Thema ist, gab knapp ein Fünftel der Befragten an (19\%), wobei hier signifikante Unterschiede nach Schulformen feststellbar waren $(\mathrm{p}=0,010)$. Bei der überwiegenden Mehrheit kommt es allerdings selten oder nie im Unterricht zur Sprache (zusammen rund $78 \%$ ). Auch wenn man berücksichtigt, dass $14 \%$ der LehrerInnen im Sample keine DeutschlehrerInnen waren, ist das ein hoher Prozentsatz. Bei der genaueren Aufschlüsselung nach Schulformen zeigte sich, dass österreichisches Deutsch mit 27,4\% der Nennungen von AHS-/Sek.-II-LehrerInnen häufig im Unterricht thematisiert wird. Bei der Angabe „selten“ deckten sich die drei Schulform-Gruppen im Wesentlichen (zwischen $50 \%$ und $57 \%$ ). Am häufigsten gaben VS-LehrerInnen an, dass österreichisches Deutsch für sie im Unterricht „nie“ ein Thema ist:

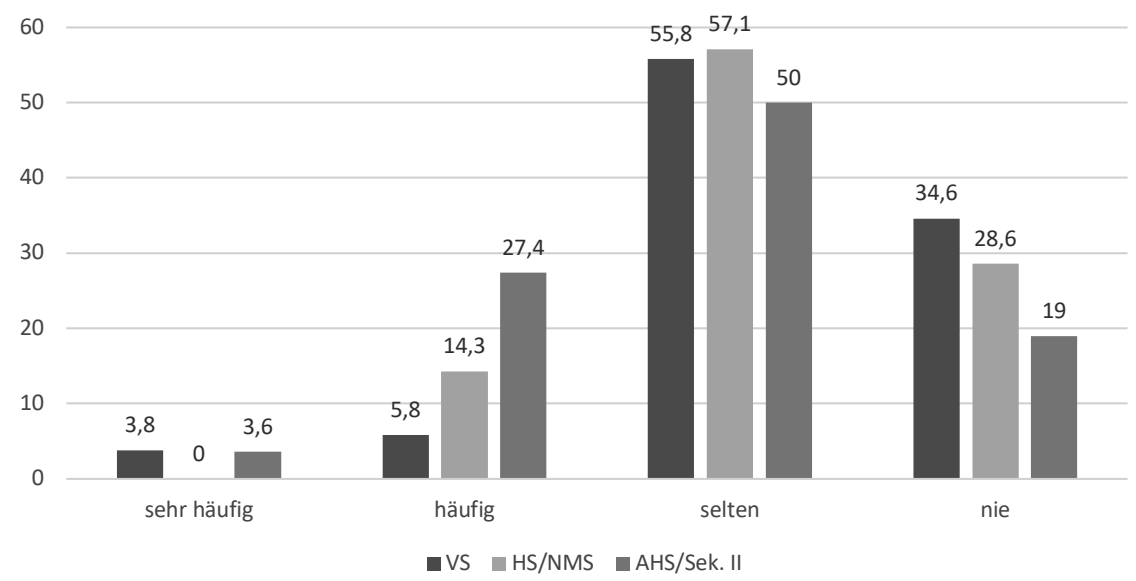

Abb. 9: Österreichisches Deutsch: Thema im Unterricht (Antworten Lehrerlnnen) (in \%)

Auch zum verwendeten Kodex bei der Korrekturarbeit für Fragen der Norm und Sprachrichtigkeit wurden die Lehrpersonen befragt, wobei mehrere Angaben gemacht werden konnten. Knapp $70 \%$ der LehrerInnen geben an, das Österreichische Wörterbuch zu verwenden, gefolgt vom Duden mit rund $63 \%$ (siehe Abb. 10 auf S. 101).

Innerhalb der Gruppe der LehrerInnen ließen sich statistisch signifikante Unterschiede hinsichtlich der Verwendung des Österreichischen Wörterbuchs (ÖWB) oder des Duden nach Alter $(\mathrm{p}=\mathrm{o,023})$, Dienstjahren $(\mathrm{p}=0,004)$ und PlurizentrikKenntnis $(\mathrm{p}=0,045)$ feststellen: Je älter die Befragten waren, desto eher wurde das 
ÖWB als Nachschlagwerk bei der Textkorrektur genannt, umso jünger, desto eher findet der Duden/Online-Duden Verwendung. Auch die Verbindung zwischen der Anzahl der Dienstjahre und der Verwendung von Nachschlagwerken ist eindeutig: Je mehr Dienstjahre jemand hat, desto häufiger kommt das ÖWB zum Einsatz. Jene, denen das plurizentrische Konzept bekannt ist, gaben stärker an, den Duden zu verwenden. Werden auch die Mehrfachantworten (ÖWB und Duden) berücksichtigt, zeigte sich: Je weniger Dienstjahre Lehrende hatten, desto mehr zogen sie ausschließlich den Duden für ihre Korrekturarbeit heran, und umso weniger die Kombination aus ÖWB und Duden.

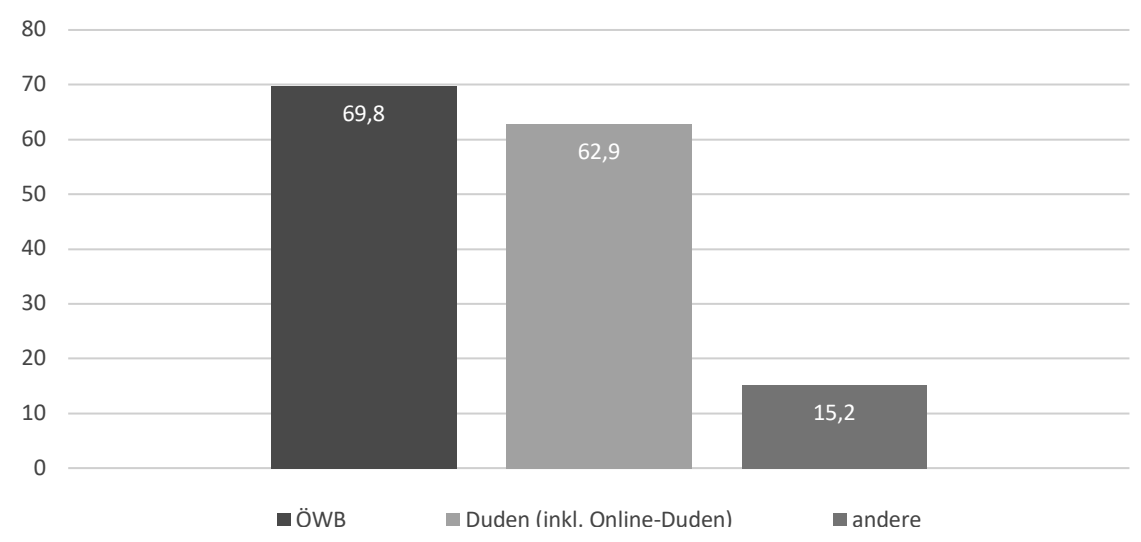

Abb. 10: Verwendete Nachschlagwerke (Antworten Lehrerlnnen) (in \%)

In einer offenen Frage wurden die DeutschlehrerInnen danach gefragt, welche Nachschlagwerke und Internetseiten sie ihren SchülerInnen zum Nachschlagen in sprachlichen Fragen (Rechtschreibung und Grammatik) empfehlen. Dabei war besonders auffallend, dass die Mehrheit der Männer (72 \%) den Duden empfiehlt, während dies von den weiblichen Lehrkräften nur rund $43 \%$ tun ( $p=0,005)$.

Von Interesse war auch, wie viele der DeutschlehrerInnen auch Deutsch als Zweitsprache (DaZ) unterrichteten, ob DaZ- oder DaF-Lehrveranstaltungen im Rahmen der LehrerInnenausbildung der PädagogInnen angeboten wurden und ob diese von den DeutschlehrerInnen besucht wurden. 14,1\% der DeutschlehrerInnen unterrichteten neben den regulären Deutschstunden auch DaZ, von denen wiederum 38,7\% angaben, dass in ihrer Lehramtsausbildung DaF- und DaZ-Lehrveranstaltungen angeboten worden wären, und etwas weniger, nämlich $34,4 \%$, dass sie solche Lehrveranstaltungen auch besucht haben. Bei der Frage nach den im DaF/DaZ-Unterricht verwendeten Lehrbüchern wurde eine große Bandbreite an Werken genannt, wobei allerdings keines besonders hervorsticht.

Wie haben diejenigen, die DaZ unterrichten, auf die Frage reagiert, ob in den DaZ-Lehrbüchern und in den DaZ-Materialien österreichisches Deutsch oder soziolinguistische Variationen (Dialekt/Umgangssprache/Standardsprache) des 
Deutschen behandelt werden? 47,1\% der DaZ-Unterrichtenden waren der Meinung, dass österreichisches Deutsch in den DaZ-Lehrmitteln thematisiert wird, $52,9 \%$ sahen dies nicht so. Soziolinguistische Variation wird nach Einschätzung der DaZ-LehrerInnen in DaZ-Lehrmitteln kaum thematisiert: Nur 18,8 \% gaben an, dass diese Variation in den Lehrmitteln für DaZ berücksichtigt wird: Mehr als vier Fünftel $(81,2 \%)$ meinten, dass sie dort keinen Niederschlag findet.

Danach gefragt, was sie vom Konzept des integrativen Sprachunterrichts halten, demzufolge jeder Unterricht Sprachunterricht ist, reagierten die LehrerInnen überwiegend positiv: Mehr als $86 \%$ der Befragten stimmten dem integrativen Konzept stark oder sehr stark zu. Rund $14 \%$ konnten dem Ansatz wenig oder nichts abgewinnen. Viele, die hinter dem Konzept stehen, sehen den Ansatz in der Unterrichtspraxis aber noch als kaum verwirklicht an. Fast $60 \%$ der Befragten zufolge werde das integrative Konzept in ihrem Arbeitsumfeld wenig oder gar nicht umgesetzt.

In den Einzelinterviews (siehe Kap. 5.4) wurde den LehrerInnen die Frage gestellt, inwieweit sie das Gefühl haben, dass sich auch andere KollegInnen (die nicht Deutsch unterrichten) für den Bildungssprachenunterricht zuständig fühlen, wie es das integrative Konzept von Sprachunterricht vorsieht. Die meisten Befragten gaben an, dass sich ihre KollegInnen nicht oder kaum zuständig fühlen würden, wie das folgende Beispiel demonstriert:

Also i hab oft das Gefühl, dass vieles auf uns einfach abgeschoben wird, und wenns dann darum geht, Referate zu halten oder zu präsentieren, dann kommt oft die Meldung: „Wieso habts Ihr des no nit ausführlich besprochen?“ Und: „Können die kein Exzerpt schreiben?"“, also das wird oft einfach so gerne auf uns abgewälzt, und - - ja. Ma hat manchmal das Gefühl, ma könnte ENDLOS arbeiten.

Was die LehrerInnenausbildung angeht, ist die Wahrnehmung der Befragten eindeutig: $86 \%$ waren der Ansicht, dass sprachliche Variation in der LehrerInnenausbildung zu wenig oder gar nicht thematisiert wird (siehe Abb. 11 auf S. 103).

Die befragten VolksschullehrerInnen haben die Themen „Deutsch als Unterrichts- und Bildungssprache“ sowie „sprachliche Variation im Deutschen“ in ihrer Ausbildung signifikant am stärksten wahrgenommen $(\mathrm{p}=\mathrm{o}, \mathrm{ooo})$, gefolgt von HS/NMS- und schließlich von AHS-/Sek.-II-LehrerInnen. Frauen gaben dabei signifikant häufiger als Männer an, dass von diesen beiden Themenbereichen in ihrer Ausbildung die Rede gewesen sei $(\mathrm{p}=0,008)$. Jüngere LehrerInnen (bis 31 Jahre) gaben überhaupt am häufigsten an, dass Deutsch als Unterrichtssprache in ihrer Ausbildung thematisiert wurde $(\mathrm{p}=0,002)$ - ein Hinweis auf mögliche Änderungen in der Ausbildung in letzter Zeit.

Auf die Frage, ob sprachliche Variation in der LehrerInnenausbildung ausreichend thematisiert wurde, antworteten rund $70 \%$ der LehrerInnen, dass die Thematisierung in ihrer Ausbildung nicht ausreichend stattgefunden hat (siehe Abb. 12 auf S. 103). 


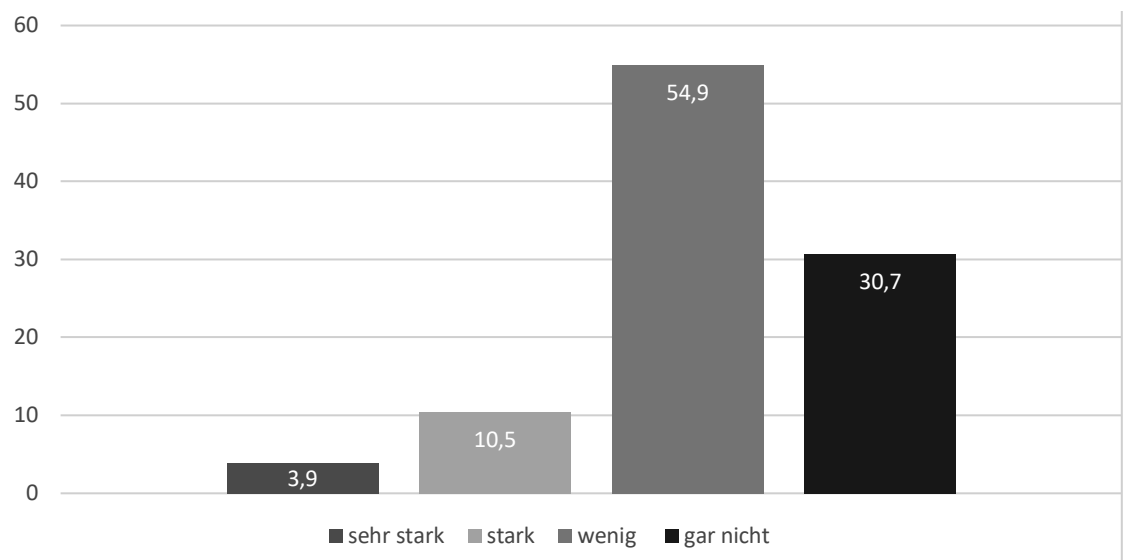

Abb. 11: War sprachliche Variation (Standardvarietäten/Umgangssprache/Dialekt) ein Thema in Ihrer Ausbildung? (in \%)

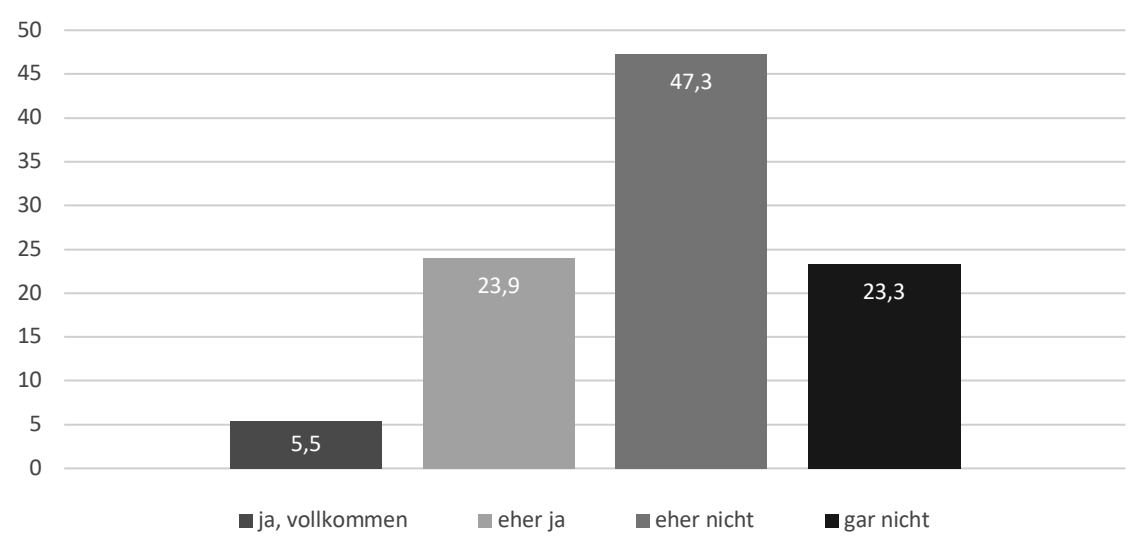

Abb. 12: Wurde sprachliche Variation in der Ausbildung ausreichend thematisiert? (in \%)

Da Lehrveranstaltungen zu sprachlicher Variation keine Pflichtveranstaltungen sind, dürften viele LehrerInnen mit dem Thema nicht wirklich in Berührung kommen. Ganz explizit wurde am Ende des Fragebogens auch abgefragt, ob den LehrerInnen das Konzept der Plurizentrik bekannt ist. Dies war nur bei 14,7\% der PädagogInnen der Fall. 


\subsection{Fragebogenerhebung: Stichprobe der SchülerInnen}

Nach Abschluss der Fragebogenerhebung lagen vollständige Daten von 1.253 SchülerInnen vor ${ }^{4}$. An sprachexternen Variablen wurden das Geschlecht, das Alter und die Wohnregion erhoben. Es wurde nach einem längeren Auslandsaufenthalt gefragt, nach der/den Muttersprache/n und der/den Muttersprache/n der Eltern, nach der Anzahl zusätzlich gelernter Sprachen und danach, ob das plurizentrische Konzept bekannt war.

42,4 \% der befragten SchülerInnen waren männlich und 57,6 \% weiblich. Das entspricht in etwa den Zahlen für ganz Österreich. Die Schulstatistik ergab im Jahr 2013/14 eine Geschlechterverteilung von 57,2 \% weiblichen und 42,8 \% männlichen SchülerInnen an der AHS-Oberstufe sowie 50,1 \% Schülerinnen bzw. 49,9\% Schülern an der BHS (Statistik Austria 2015, 25).

$16,4 \%$ der SchülerInnen waren zum Zeitpunkt der Fragebogenerhebung 13-14 Jahre alt, $22 \% 15$ Jahre, 27,2 \% 16 Jahre, 19,2\% 17 Jahre, 9,6\% 18 Jahre und $5,6 \% 19$ bis 25 Jahre alt:

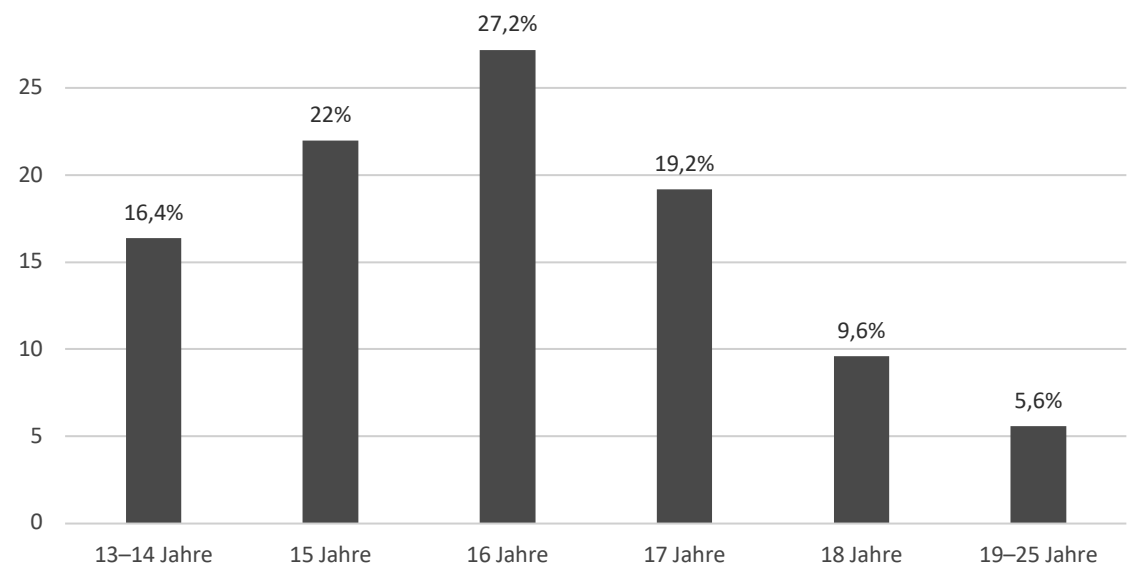

Abb. 13: Altersverteilung SchülerInnen (in \%)

Was die regionale Verteilung betrifft, besuchten 38,8\% der befragten SchülerInnen zum Zeitpunkt der Fragebogenerhebung eine Schule im Osten Österreichs (Wien, NÖ, Burgenland), $22 \%$ in OÖ und Salzburg, 22,9\% in der Steiermark und in Kärnten, 9,7\% in Tirol und 6,6 \% in Vorarlberg: ${ }^{5}$

4 Die Daten von elf SchülerInnen waren unvollständig, daher wurden diese Fragebögen in der Auswertung nicht berücksichtigt.

5 Aufgrund der Komplexität der Angaben zur Herkunft der SchülerInnen und dem damit verbundenen großen Aufwand, alle Angaben zu kategorisieren, wurden nicht die Geburtsorte der SchülerInnen ausgewertet, sondern die Schulstandorte. 


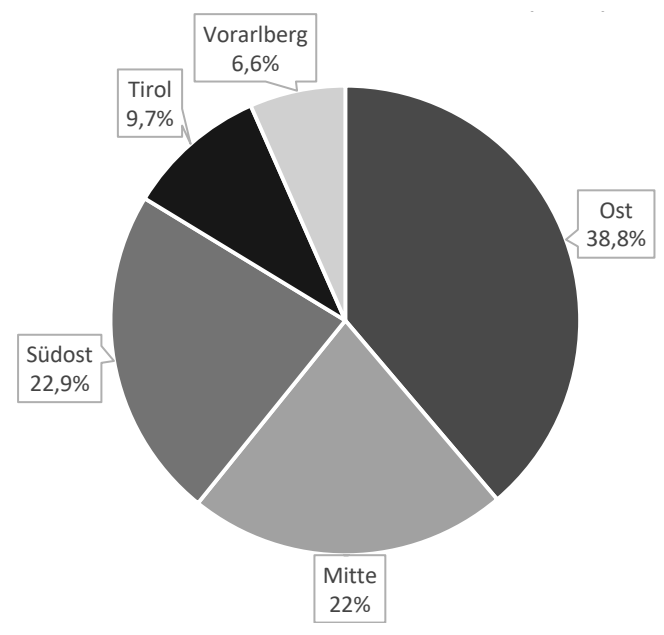

Abb. 14: Schulstandorte der befragten Schülerlnnen (in \%)

Über $90 \%$ der befragten SchülerInnen haben ihr ganzes Leben in Österreich verbracht. Von denjenigen, die eine längere Zeit im Ausland gelebt haben, sind die meisten ( $8,9 \%$ ) im Ausland aufgewachsen bzw. haben in den ersten sechs Lebensjahren im Ausland gelebt. Im Alter von 7-10 Jahren waren nur noch 4,9\% der jetzt in Österreich lebenden ProbandInnen im Ausland, im Alter von 11-14 Jahren nur mehr 2,2 \%. Lediglich 1,7\% der befragten SchülerInnen sind erst nach Vollendung ihres 15. Lebensjahres nach Österreich gekommen.

Um einen Eindruck von der Mehrsprachigkeit in den Schulen zu bekommen, wurden auch die Muttersprachen ${ }^{6}$ der SchülerInnen erhoben. Auf die Frage: „Was ist/sind deine Muttersprache/n? (Mehrfachnennungen möglich)"gaben $85,3 \%$ der SchülerInnen Deutsch als Muttersprache an, 3,4 \% Bosnisch/Kroatisch/Serbisch, 2,5\% Türkisch, und 16,6\% andere (siehe Abb. 15 auf S. 106).

Als weitere Muttersprachen wurden genannt: Albanisch (0,9\%), Arabisch $(1,1 \%)$, Bengalisch $(0,1 \%)$, Bulgarisch $(0,3 \%)$, Burmesisch (o,1\%), Chinesisch (o,7 \%), Dänisch (o,1\%), Dari (o,2 \%), Englisch (1,4\%), Filipino (o,1\%), Finnisch (o,1\%), Französisch (o,4\%), Friaulisch (o,1\%), Georgisch (o,2\%), Griechisch $(0,2 \%)$, Hindi $(0,2 \%)$, Holländisch $(0,2 \%)$, Italienisch $(0,7 \%)$, Japanisch $(0,1 \%)$, Koreanisch (o,2 \%), Kurdisch (o,6\%), Lingála (o,1\%), Luxemburgisch (o,1\%), Mazedonisch (o,1\%), Niederländisch (o,1\%), Nord-Ossetisch (o,1\%), Persisch (o,1 \%), Polnisch (1\%), Portugiesisch (o,1\%), Rumänisch (o,7\%), Russisch (o,5\%), Schwedisch (o,1\%), Slowakisch (o,2\%), Slowenisch (1,8\%), Spanisch (0,3\%), Tagalog (o,1\%), Tschechisch (o,1\%), Tschetschenisch (o,2\%), Ukrainisch (o,1\%),

6 Zur Verwendung des Terminus „Muttersprache“ siehe die Fußnote 1 in Kap. 1. 
Ungarisch (1,1\%) und Urdu (o,1\%). $2 \%$ der SchülerInnen nannten Dialekte als Muttersprache, 1,4 \% „Österreichisch“, ein/e Schüler/in „österreichisches Deutsch“.7

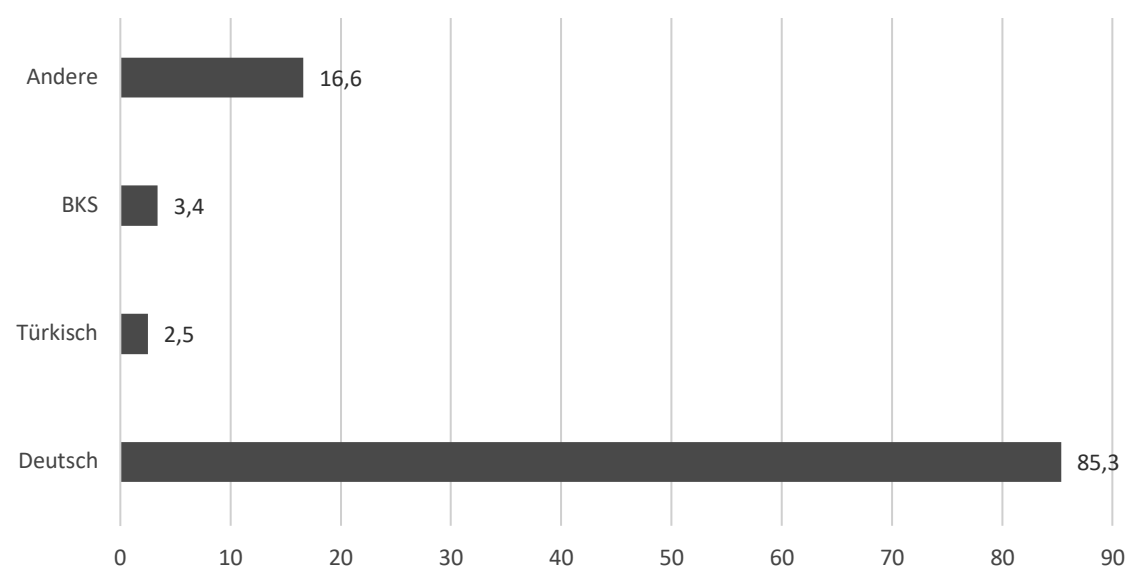

Abb. 15: Muttersprachen Schülerlnnen (in \%) ${ }^{8}$

Aufgrund der Vielfalt und der großen Zahl der angegebenen Sprachen wurden zur Erleichterung der Ergebnisauswertung drei Kategorien gebildet: (1) SchülerInnen mit nur Deutsch als Muttersprache, (2) SchülerInnen mit Deutsch und einer weiteren Sprache als Muttersprachen und (3) SchülerInnen mit ausschließlich nichtdeutschen Muttersprachen.

$78 \%$ der SchülerInnen gaben als ihre Muttersprache nur Deutsch an, 7,3\% verstanden sich als zwei-/mehrsprachig mit Deutsch und einer anderen Sprache und 14,7\% gaben ausschließlich andere Sprachen an (siehe Abb. 16 auf S. 108).

Auch nach der Erstsprache der SchülerInnen wurde gefragt. Bei der Auswertung der Antworten wurden dieselben Kategorien gebildet wie bei den Muttersprachen: 84,5\% der SchülerInnen gaben als ihre Erstsprache nur Deutsch an, 1,6 \% Deutsch und eine andere Sprache und 13,9\% nur andere Sprachen. Die Unterschiede in den Angaben zu den "Muttersprachen“ könnten darauf hinweisen, dass ein Teil der mehrsprachigen SchülerInnen Deutsch in ihrem Sprachrepertoire höher bewertet als die zuerst gelernte Muttersprache ( $78,1 \%$ für Deutsch als Muttersprache vs. $84,5 \%$ als Erstsprache).

7 Die Statistik des Bildungsministeriums für den muttersprachlichen Unterricht ergab für das Schuljahr 2012/13 folgende Sprachen: Albanisch (2.379), Arabisch (1.075), BKS (10.778), Bulgarisch (168), Chinesisch (77), Dari (101), Französisch (46), Italienisch (8), Kurdisch/Kurmanci (64), Kurdisch/ Zazaki (36), Pashto (41), Persisch (392), Polnisch (851), Portugiesisch (94), Romanes (128), Rumänisch (239), Russisch (336), Slowakisch (152), Slowenisch (23), Somali (32), Spanisch (129), Tschechisch (67), Tschetschenisch (428), Türkisch (14.911), Ungarisch (202), gesamt (32.757), BMBF 2014b: Tabelle 6a.

8 Da Mehrfachnennungen im Sinn von mehrsprachigen SchülerInnen möglich waren, ergibt die Summe mehr als $100 \%$. 
Deutsch und eine andere Sprache

nur andere Sprachen

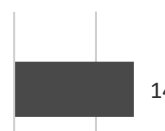

nur Deutsch

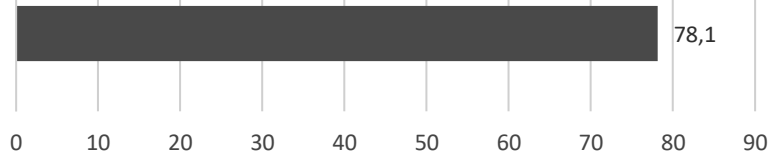

Abb. 16: Muttersprachen SchülerInnen mit Kombinationsnennungen (in \%)

Die Ergebnisse entsprechen in etwa den von der Statistik Austria ${ }^{9}$ erhobenen Zahlen, wonach der Anteil nichtdeutschsprachiger SchülerInnen im Jahr 2013 in der AHS bei $15 \%$ und in der BHS bei $14 \%$ lag.

Bei der Frage nach den Muttersprachen der Eltern gaben 76,9\% der befragten SchülerInnen Deutsch an, 12,5\% als Muttersprache beider Eltern die gleiche nichtdeutsche Sprache. Die Eltern von 1,4 \% der SchülerInnen haben jeweils unterschiedliche nichtdeutsche Sprachen als Muttersprache. 9,2\% der SchülerInnen haben einen Elternteil, der mit Deutsch und einen, der mit einer anderen Sprache aufgewachsen ist.

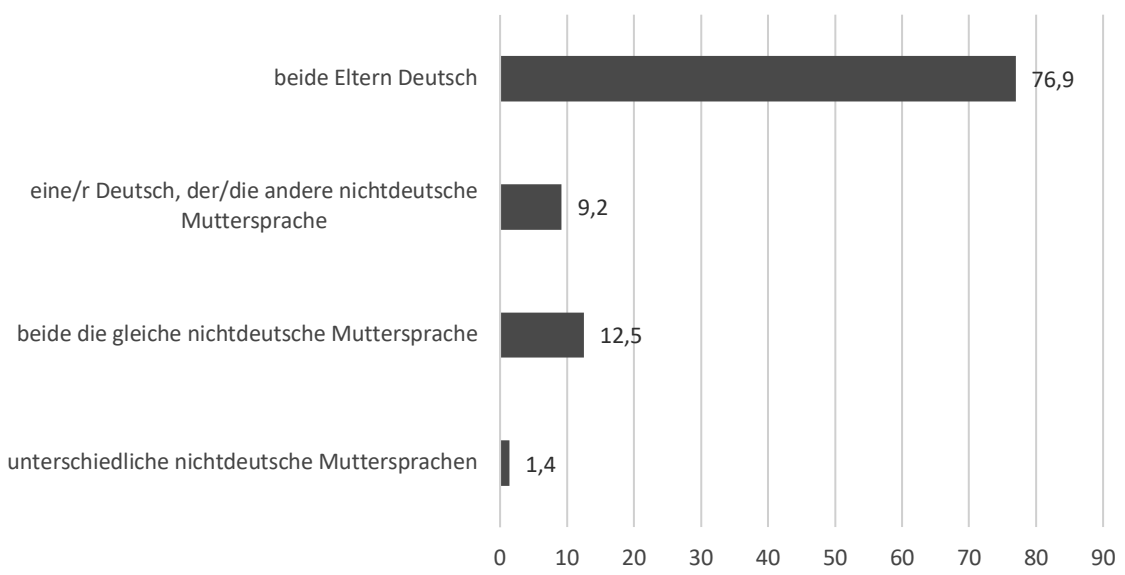

Abb. 17: Muttersprachen Eltern (in \%)

9 Statistik Austria: migration\&integration.zahlen.daten.indikatoren. 2013. 
Vergleicht man die Angaben zu den Muttersprachen der SchülerInnen mit denen zu den Muttersprachen der Eltern, so zeigt sich, dass bei ca. drei Viertel des Samples Eltern und SchülerInnen deutschsprachig sind, und dass bei ca. der Hälfte der zweisprachigen Familien (4,2\%) die Minderheitensprache nicht an die nächste Generation weitergegeben werden dürfte.

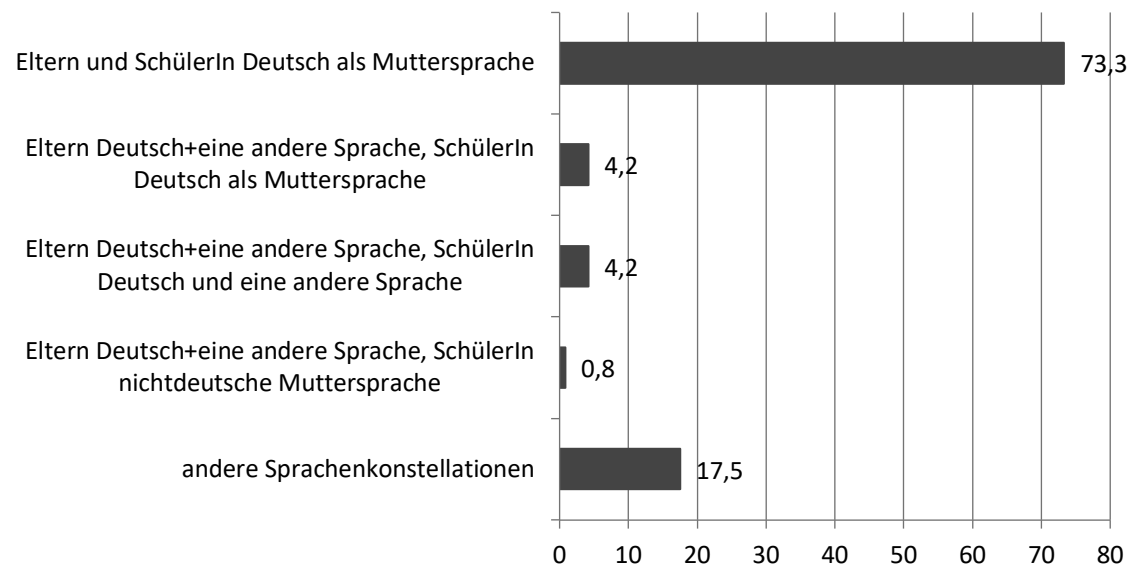

Abb. 18: Muttersprache SchülerInnen - Muttersprache Eltern (in \%)

Auf die Frage, wo die SchülerInnen, die zum Teil im Ausland aufgewachsen sind, Deutsch gelernt haben, gaben $58,7 \%$ an, dass sie im österreichischen Kindergarten Deutsch gelernt haben, $16,3 \%$ in der Volksschule, 14,5 \% in weiterführenden Schulen (HS/NMS/AHS). Lediglich 1,7\% haben ihren Angaben zufolge Deutsch bereits im Ausland gelernt. Und 91,7\% der bilingualen SchülerInnen haben Deutsch im Rahmen des regulären Deutschunterrichts gelernt, nur 8,3\% in einem eigenen Deutschkurs. 31,1 \% der SchülerInnen mit nichtdeutscher Muttersprache waren bei ihrem Schuleintritt in Österreich außerordentliche/r Schüler/in.

Auf die Frage nach weiteren in der Schule gelernten Sprachen wurden folgende Sprachen angegeben: Albanisch, Altgriechisch, Arabisch, Aserbaidschanisch, Chinesisch, Deutsch, Englisch, Französisch, Griechisch, Hochdeutsch, Italienisch, Koreanisch, Latein, Polnisch, Russisch, Serbisch/Kroatisch/Bosnisch, Schwedisch, Slowenisch, Spanisch, Tschechisch, Ukrainisch, Ungarisch und Urdu. Das weist darauf hin, dass auch im DaZ- und im muttersprachlichen Unterricht gelernte Sprachen als Fremdsprachen angegeben wurden.

Folgende Sprachen wurden von den befragten SchülerInnen eigenen Angaben zufolge zusätzlich außerhalb der Schule gelernt: Ägyptisch, Albanisch, Arabisch, Aramäisch, Chinesisch, Dänisch, Dari, Deutsch, Englisch, Faröisch, Filipino, Finnisch, Französisch, Friaulisch, Griechisch, Hindi, Igbo, Isländisch, Italienisch, Japanisch, Klingonisch, Koptisch, Koreanisch, Kurdisch, Latein, Niederländisch, Nord-Ossetisch, Norwegisch, Pashto, Polnisch, Portugiesisch, Rumänisch, Russisch, 
Schwedisch, Schweizer Deutsch, Serbisch/Kroatisch/Bosnisch, Slowakisch, Slowenisch, Spanisch, Thai, Tschechisch, Türkisch und Ungarisch. Insgesamt zeigen unsere Daten eine beachtliche Vielfalt in den individuellen Sprachrepertoires der SchülerInnen.

Da sich in der Literatur immer wieder Annahmen finden, wonach ein Zusammenhang zwischen Medienkonsum und der Übernahme von Deutschlandismen besteht (z. B. Muhr 2003, Ebner 2008), wurden die SchülerInnen auch nach ihren Fernsehgewohnheiten sowie nach ihrem liebsten Kinderbuch gefragt.

Die SchülerInnen sollten bei der Frage zu ihrem Fernsehverhalten zunächst die TV-Kanäle angeben, auf denen sie derzeit häufig fernsehen („TV aktuell“), und anschließend jene, auf denen sie in ihrer Kindheit häufig ferngesehen haben („TV Kindheit“).

Auf die Frage „Auf welchen TV-Kanälen siehst du häufig fern?“ („TV aktuell“) wurden sowohl bundesdeutsche als auch österreichische TV-Sender genannt, wobei deutsche Sender insgesamt häufiger genannt wurden: $67 \%$ der SchülerInnen nannten Pro7, 49,2 \% ORF 1, 28,7 \% RTL, 21,3 \% ORF, 19,4 \% Sat1, 12,9\% ATV, 11,8 \% RTL2, 11,4 \% Puls 4 und 10 \% VOX. ${ }^{10}$ Zusammengefasst sieht die große Mehrheit der befragten SchülerInnen, nämlich 72,3\%, sowohl auf deutschen als auch auf österreichischen TV-Kanälen fern, 18,7\% nur auf deutschen, 7,8 \% nur auf österreichischen und 1,2\% nur auf anderssprachigen (siehe Abb. 19 auf S. 110).

Die Auswertung der Ergebnisse zum aktuellen TV-Verhalten ergab nach Bundesländern aufgeschlüsselt zwar keine signifikanten Unterschiede, dennoch ist unter denjenigen SchülerInnen, die angaben, entweder nur auf deutschen oder nur auf österreichischen TV-Kanälen fernzusehen, ein leichtes Ost-WestGefälle feststellbar: Am häufigsten gaben SchülerInnen aus Ostösterreich an, nur auf österreichischen TV-Kanälen fernzusehen $(8,7 \%)$, gefolgt von SchülerInnen aus südöstlichen $(8,3 \%)$ und mittleren Bundesländern (7,6\%). Vorarlberger und Tiroler SchülerInnen sehen eigenen Angaben zufolge am wenigsten nur auf österreichischen Kanälen fern (3,8 bzw. 6,1\%) (siehe Abb. 20 auf S. 110).

10 Die befragten SchülerInnen nannten noch weitere Sender: 8,6 \% Dmax, 6,4 \% Viva, 5 \% Sixx, jeweils 4,6\% Kabel 1 und ZDF, 4,3\% Servus TV, 2,9\% N24, 2,8 \% Sky, jeweils 2,6\% Arte und ARD, 2,1\% Nickelodeon, jeweils $2 \%$ MTV und Comedy Central, 1,9\% Eurosport, 1,8 \% NTV, 1,7\% SUPER RTL, 1,3\% BBC, 1,1\% 3sat, 0,7\% GOTV, 0,6\% CNN, 0,5\% KIKA, jeweils 0,4\% National Geographic und Discovery Channel, jeweils o,3\% türkische Sender, das Erste, BR und Phoenix, jeweils 0,2 \% Disney Channel und SF, jeweils 0,15\% NDR, Schweizer Sender, Euro News, ATV+, ABC, Bibel TV, und RAI, sowie jeweils o,1 \% Al Jazeera, NBC, TV 5, französische Sender, amerikanische Sender, Astro-TV, SWR, SFR und kroatische Sender. 


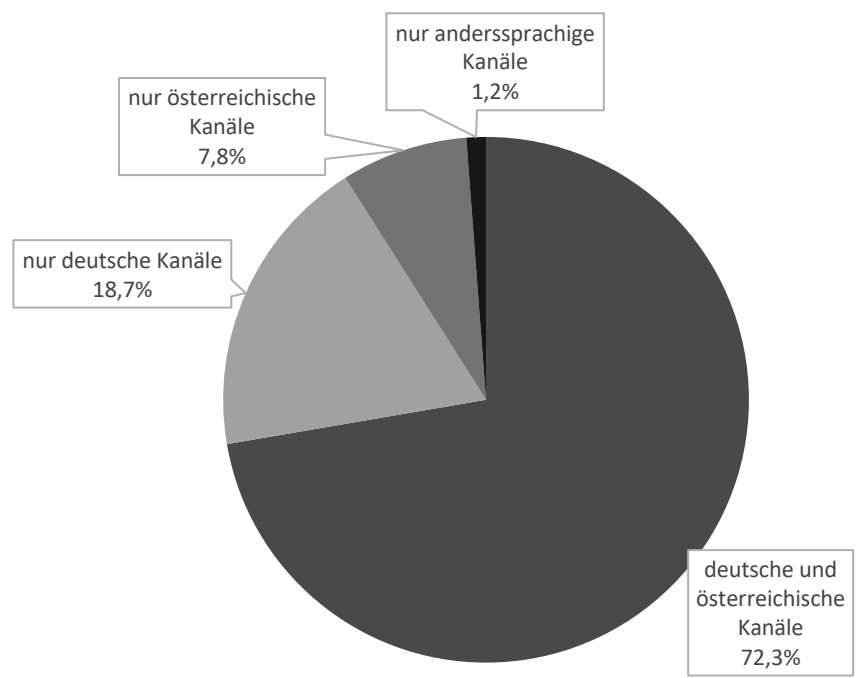

Abb. 19: TV-Kanäle aktuell: SchülerInnen (in \%)

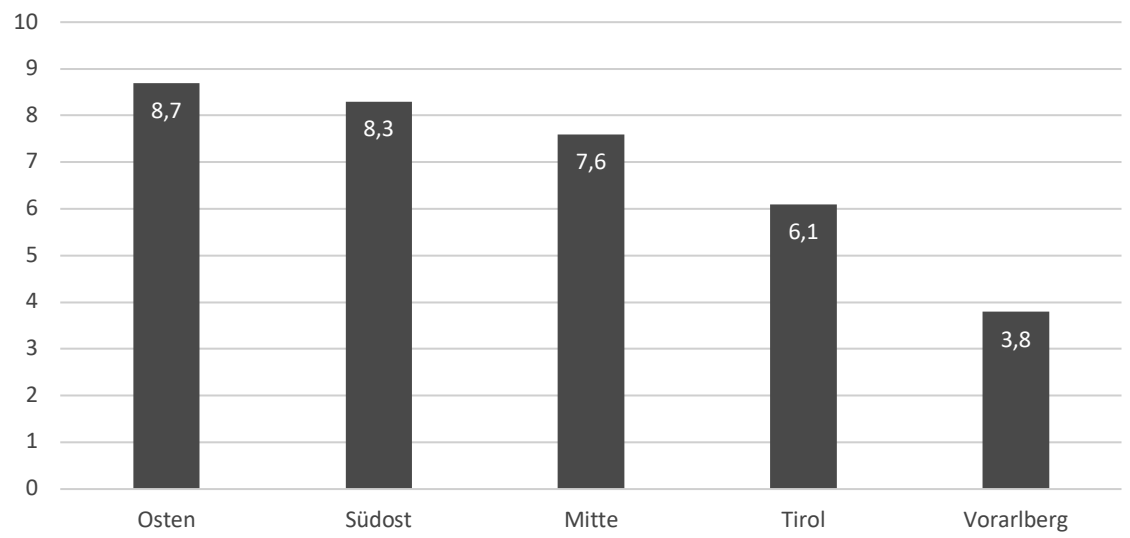

Abb. 20: Nur österreichische TV-Kanäle nach Region („TV aktuell“ Schülerlnnen) (in \%)

Was die deutschen Fernsehsender angeht, zeigt sich ein etwas anderes Bild: Die Gruppe der jugendlichen Zuseher, die nur deutsche Sender einschalten würden, war in Tirol mit 22,8 \% am größten, gefolgt von der Region Südost (22,3\%) und Vorarlberg (20\%), während in der Region Mitte der entsprechende Prozentsatz weit geringer war $(12,5 \%)$. 


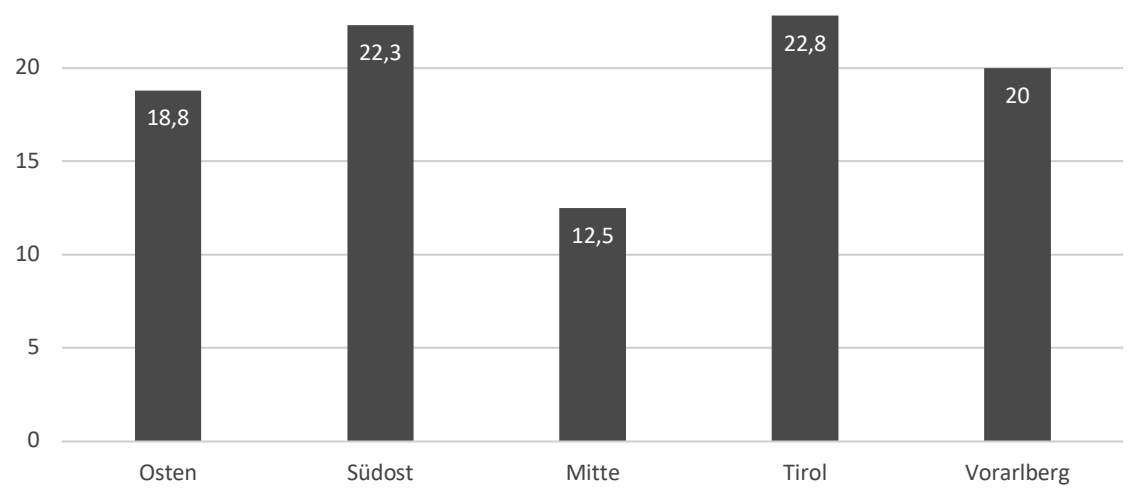

Abb. 21: Nur deutsche TV-Kanäle nach Region („TV aktuell“ SchülerInnen) (in \%)

Auf die Frage nach den Sendern, auf denen die SchülerInnen in ihrer Kindheit ferngesehen haben, wurden folgende Sender hauptsächlich genannt: Super RTL (61,9\%), KIKA (48,6\%), ORF 1 (26,9\%), Nickelodeon (25,2\%), ORF (11,2 \%), RTL $(3,6 \%)$, RTL2 $(3,3 \%)$, Pro7 (2,9\%), Disney Channel $(1,2 \%) .^{11}$

Zusammengefasst waren mit großem Abstand die wichtigsten TV-Sender in der Kindheit in dieser Reihenfolge Super RTL, KIKA, ORF 1 und Nickelodeon, d.h., die SchülerInnen haben ihren Angaben nach in ihrer Kindheit großteils auf deutschen Sendern ferngesehen: $56,9 \%$ der SchülerInnen geben an, nur auf deutschen TV-Kanälen ferngesehen zu haben, 32,1 \% auf deutschen und österreichischen, 9,8\% nur auf österreichischen und 1,2 \% nur auf anderssprachigen Sendern (siehe Abb. 22 auf S. 112).

Wertet man die Antworten auf die Frage nach der TV-Kanalpräferenz in Kindheitstagen nach der Variable Region aus, ergeben sich hochsignifikante Unterschiede ( $\mathrm{p}=\mathrm{o}, \mathrm{oo4})$ : Die meisten SchülerInnen, die angaben, nur auf österreichischen TV-Kanälen ferngesehen zu haben, kommen aus Ostösterreich (12,7\%), gefolgt von SchülerInnen aus südöstlichen Bundesländern (11\%). In den restlichen Bundesländern waren es nur etwas über $6 \%$.

11 An weiteren Sendern wurden genannt: Toggo (o,9\%), jeweils o,8\% Cartoon Network und ZDF, jeweils o,6 \% Viva und Kabel 1, 0,5\% Konfetti TV, 0,45\% ATV, jeweils 0,3\% arte, Junior TV, Dmax, 0,4 \% MTV, 0,35\% ARD, jeweils o,2 \% sat 1, N24 und ntv, jeweils 0,15\% Arabische Sender, Bayrischer Rundfunk, CNN, das Erste, JetX, Minimax, Nickelodeon Austria, Okidoki TV, pakistanischer Sender, Pockito TV, Comedy Central, französische Sender und Premiere, und jeweils 0,1 \% 3sat, afghanischer Kanal, ägyptischer Kanal, Animal Planet, Assi TV, ATV+, Baby TV, Bang Bang, CBR, CC, CN, Discovery Channel, Disney Playhouse, Eurosport, Gufo, Mediaset, National Geographic, NDR, Phönix, polnischer Sender, Pro 7 Austria, Puls 4, RAI, RIRA, RT, serbischer Kanal, SF 1, Sky, Sky Cinemagic, Sky Junior, Slo 1, spanische Kanäle, ungarischer Kanal und VOX. 


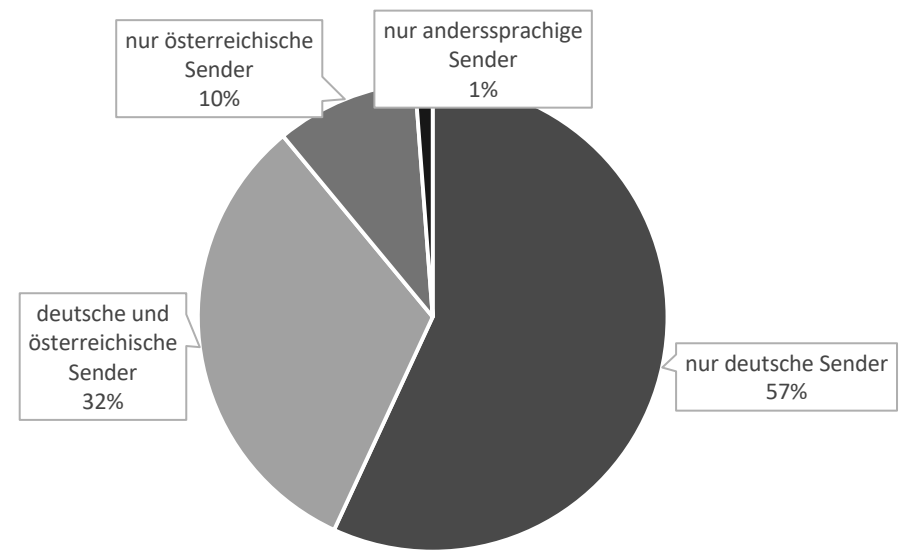

Abb. 22: Bevorzugte TV-Kanäle Kindheit (SchülerInnen) (in \%)

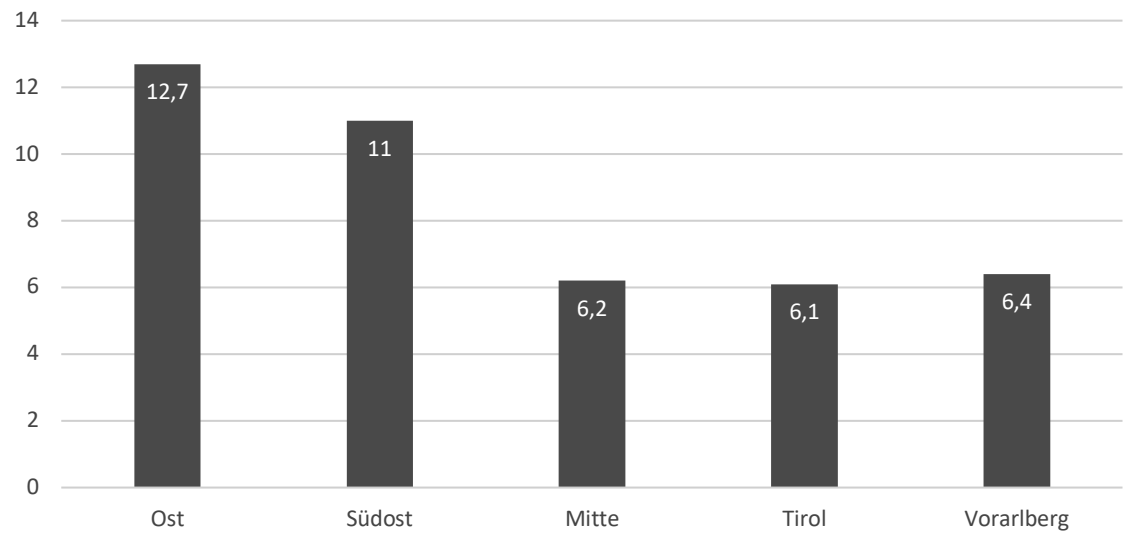

Abb. 23: Nur österreichische TV-Kanäle („TV Kindheit“) nach Region (SchülerInnen) (in \%)

Nur deutsche Kanäle wurden vor allem von Vorarlberger und Tiroler SchülerInnen in deren Kindheit konsumiert (67,9\% bzw. 66,1\%), deutlich weniger häufig war dies mit rund $50 \%$ im Osten der Fall (siehe Abb. 24 auf S. 113).

Um einem weiteren sprachexternen Faktor auf den Grund zu gehen, der den Sprachgebrauch der SchülerInnen beeinflusst haben könnte, wurde nach dem liebsten Kinderbuch gefragt. Am häufigsten wurden ganz allgemein „Märchenbücher“ angeführt (12,4\%), am zweithäufigsten „Die kleine Raupe Nimmersatt“ (10,4\%). Weitere, von den SchülerInnen relativ häufig genannte (nicht-österreichische) Lieblingskinderbücher bzw. LieblingsautorInnen waren u. a.: „Harry Potter“ (5,6\%), „Der Regenbogenfisch“ (4,8\%), Gebrüder Grimm (4,8\%), Otfried Preußler (4,8\%), „Pippi Langstrumpf“ (4,5\%) bzw. Astrid Lindgren (3,2\%) und „Max \& Moritz“ 
$(3,4 \%)$. Auffallend war, dass relativ wenige österreichische AutorInnen genannt wurden - diese wenigen österreichischen AutorInnen dafür umso häufiger: Mira Lobe (7,2\%), Christine Nöstlinger (4,5\%), Thomas Brezina (4,3\%) und Franz Karl Ginzkey $(0,9 \%)^{12}$.

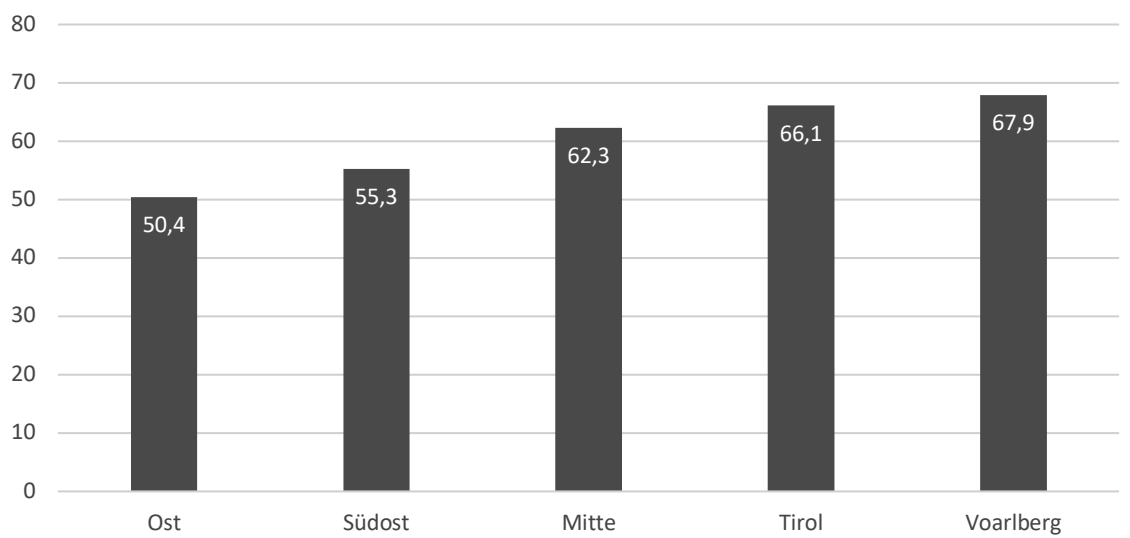

Abb. 24: Nur deutsche TV-Kanäle Kindheit nach Region (SchülerInnen) (in \%)

Wie die Lehrpersonen wurden auch die SchülerInnen danach gefragt, welche Nachschlagwerke sie in Normfragen normalerweise verwenden. Das mit Abstand am häufigsten $(48,4 \%)$ genannte Nachschlagwerk ist der Duden. Nur 28,2 \% der SchülerInnen nannten das Österreichische Wörterbuch. Dieses Ergebnis könnte auf eine tendenzielle Exonorm-Orientiertheit hinweisen. 38,3 \% führten Internetquellen an. Das große österreichische Schulwörterbuch aus dem Duden-Verlag wurde nur von $0,5 \%$ der SchülerInnen genannt. Eine andere Erklärungsmöglichkeit ist, dass viele SchülerInnen das Internet nutzen. Das ÖWB ist im Internet nicht verfügbar, der Duden jedoch sehr wohl (siehe Abb. 25 auf S. 114).

Hochsignifikante Unterschiede tun sich bei der Verwendung des Duden und des ÖWB zwischen den Regionen ( $\mathrm{p}=0,000)$ auf: Der Duden wurde in Vorarlberg am häufigsten genannt $(64,3 \%)$, gefolgt von den mittleren Bundesländern $(56,5 \%)$ und Tirol $(52,5 \%)$. In den südöstlichen und östlichen Bundesländern wurde der Duden von weniger als der Hälfte der ProbandInnen angegeben (45,7 \% bzw. 41,8\%). Das ÖWB wird hingegen mit Abstand am häufigsten im Osten (37,3\%)

12 Die SchülerInnen nannten noch weitere Bücher: 4,1 \% „Weißt du eigentlich, wie lieb ich dich hab?“, jeweils 2,2\% Hexe Lilli und Petterson und Findus, 1,8 \% „Die drei Fragezeichen???“, jeweils 0,6\% „Das Dschungelbuch“, Disney, und Winnie Pooh, jeweils 1,4\% die Bibel, „Der kleine Prinz“ und Felix, 1,3\% „Struwwelpeter“, jeweils 1,1\% Conni und Erich Kästner, o,9\% „Eragon“, jeweils o,7 \% der Grüffelo, fünf Freunde, Heidi, „Herr der Ringe“, Sternenschweif, 0,5\% Janosch, jeweils 0,4\% Cinderella, „Die wilden Kerle“ und Michael Ende, und o,2 \% Nils Holgerson. 
und Südosten (30,8\%) von den SchülerInnen genannt, gefolgt von der Region Mitte mit 21,6\%. In Tirol waren es 17,2 \% der ProbandInnen, die das ÖWB angaben, während Vorarlberg bei der ÖWB-Verwendung mit 4,8 \% der SchülerInnen das Schlusslicht bildet.

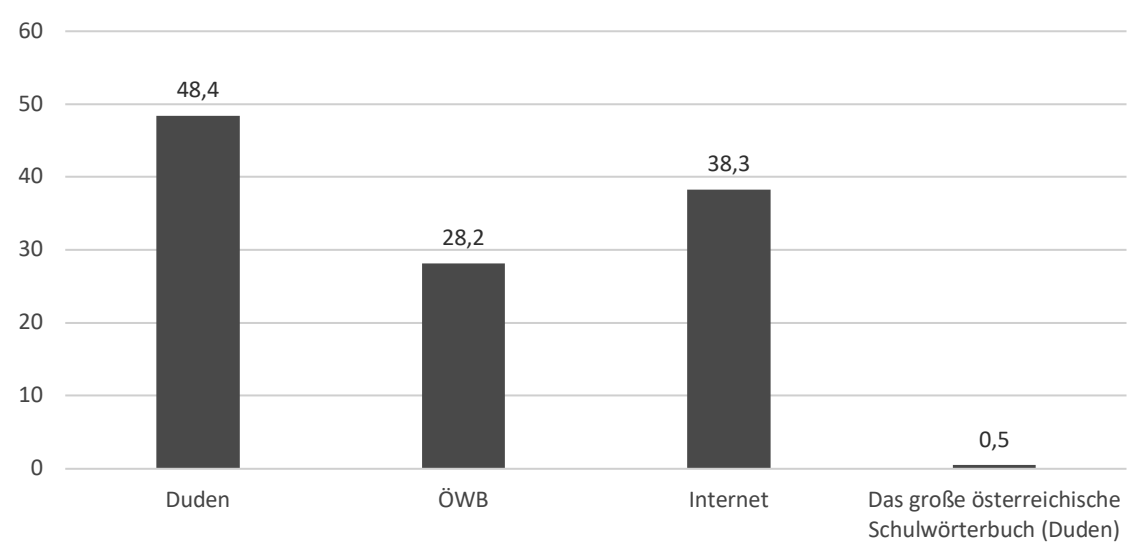

Abb. 25: Nachschlagwerke Schülerlnnen (in \%)

Auch zwischen der Anzahl der (von den SchülerInnen) in der Schule erlernten Sprachen und der ÖWB-Verwendung besteht ein signifikanter Zusammenhang $(\mathrm{p}=0,012)$ : Von denjenigen SchülerInnen, die zum Befragungszeitpunkt nur eine Sprache lernten, gaben nur $20 \%$ an, das ÖWB zu benutzen. Von den SchülerInnen, die zwei Fremdsprachen lernten, waren es 25,9\% und am häufigsten wurde die ÖWB-Verwendung von LernerInnen von drei oder mehr Sprachen angegeben $(31,4 \%)$. Hochsignifikant war die Antwortverteilung auch in Abhängigkeit von der Frage, ob die SchülerInnen bereits vom plurizentrischen Konzept gehört hatten oder nicht ( $\mathrm{p}=0,000)$ : Rund $8 \%$ der SchülerInnen gaben an, das Konzept zu kennen, im Gegensatz zu fast $92 \%$, denen ihrer Angabe nach dieses Konzept gänzlich unbekannt war. Von jenen SchülerInnen, die noch nie vom plurizentrischen Konzept gehört hatten, gaben rund $27 \%$ an, (auch) das ÖWB zu benutzen. Unter denjenigen, die bereits vom plurizentrischen Konzept gehört hatten, waren es signifikant mehr, nämlich 44,6\%. Schließlich gab es auch signifikante Unterschiede, was die Internetnutzung und die ÖWB-Verwendung nach Geschlecht ( $\mathrm{p}=0,049)$ angeht: Während $32 \%$ der Schülerinnen angaben, das ÖWB zu nutzen, machten dies nur 23,7\% der Schüler. Umgekehrt nutzen 21,5\% der männlichen Schüler eigenen Angaben zufolge das Internet, während das nur $17,1 \%$ der Schülerinnen tun. 


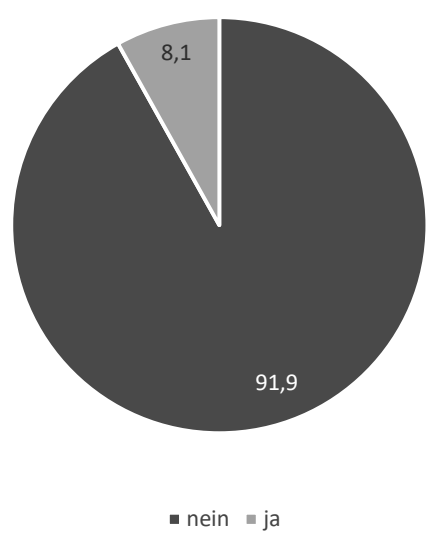

Abb. 26: Plurizentrisches Konzept bekannt (Schülerlnnen) (in \%)

\subsection{Interviews mit LehrerInnen}

Als Ergänzung zu den durch die Fragebogenerhebung gewonnenen Daten wurden vom 19. September bis zum 9. Dezember 2013 Interviews mit 21 LehrerInnen in ganz Österreich (außer Kärnten ${ }^{13}$ ) durchgeführt. Ziel war es, eine differenzierte qualitative Ergänzung zu den Ergebnissen der Fragebogenerhebung zu erhalten, wobei die folgenden Themen den Schwerpunkt des Forschungsinteresses bildeten:

- das eigene Sprachverhalten und das der SchülerInnen

- das Korrekturverhalten der LehrerInnen (schriftlich/mündlich)

- Spracheinstellungen der LehrerInnen gegenüber den Varietäten des Deutschen

- Norm, an der sich die Lehrenden orientieren

- Thematisierung von österreichischem Deutsch und sprachlicher Variation im Unterricht

- Zuständigkeit anderer KollegInnen für den Bildungssprachenunterricht und Förderung von Kindern mit anderen Erstsprachen als Deutsch

Von den insgesamt 21 LehrerInnen waren 13 weiblich und 8 männlich. Das Alter der interviewten Personen lag zum Zeitpunkt der Befragung zwischen 29 und 61 Jahren. 16 der befragten LehrerInnen unterrichteten an einer AHS, drei an einer BHS, eine Lehrerin an einer Volksschule und eine an einer Hauptschule.

Die Schulen, an denen die besagten LehrerInnen zum Zeitpunkt der Befragung unterrichteten, waren mit Ausnahme von Kärnten über alle Bundesländer verstreut. Ihre Ausbildung hatten die interviewten Lehrkräfte an den Universitäten Wien,

13 Aus organisatorischen Gründen konnten keine Interviews mit Lehrpersonen aus Kärnten durchgeführt werden. 
Graz, Salzburg und Innsbruck bzw. an der PH Linz und der PH Wien absolviert. Alle LehrerInnen, bis auf die erwähnte Volksschullehrerin, unterrichteten zum Zeitpunkt der Befragung Deutsch in Kombination mit mindestens einem anderen Fach, wobei die teilnehmenden LehrerInnen zusätzlich zum Unterrichtsfach Deutsch folgende Fächer angegeben haben: Geschichte, Geographie, Psychologie/ Philosophie, „Schultheater“, Französisch, Englisch, Ethik, Kommunikation Sprachen und Kulturen, Lerntechnik, Sport, Biologie, technisches Werken und Latein.

Die Unterrichtserfahrung der Befragten reichte von wenigen Wochen bis hin zu einer Zeitspanne von 36 Jahren, wobei die Lehrerin mit der kürzesten Unterrichtserfahrung eine Ausnahme darstellte. Alle anderen hatten zum Zeitpunkt der Befragung bereits mindestens 10 Jahre lang unterrichtet.

Alle Gespräche fanden in der jeweiligen Schule der Interviewten statt. Meist nach dem Unterricht - entweder in einem leeren Raum oder im Lehrerzimmer. Nur ein Lehrer wurde aus zeitlichen Gründen während des Unterrichts im Klassenzimmer interviewt; die SchülerInnen beschäftigten sich währenddessen mit einer schriftlichen Aufgabe.

Sämtliche Interviews wurden nach HIAT (Halbinterpretative Arbeitstranskription) transkribiert. Die nach HIAT verschrifteten Passagen wurden anschließend für diese Publikation lesbarer gemacht und vereinfacht. Die für die Auswertung der Daten relevanten Passagen werden im Kapitel 6 auszugsweise dargestellt. Die Verschriftungskonventionen der Transkripte der Interviews befinden sich im Anhang.

\subsection{Gruppendiskussionen}

Zweck der beiden Gruppendiskussionen war es einerseits, als Ergänzung zum Fragebogen in einigen wichtigen Punkten qualitative Zusatzdaten zu erheben, und andererseits, eine Art kommunikative Validierung der ersten Ergebnisse zu erhalten und damit die Interpretation der Daten aus dem Fragebogen und der Dokumentenanalyse zu vertiefen bzw. zu triangulieren. Die Gruppendiskussionen zielten darauf ab, Informationen zur Konzeptualisierung der sprachlichen Variation in Österreich zu bekommen, mit den LehrerInnen über die Themen „Standardvariation im Deutschen“, „Umgangssprache“, „Dialekt“ und „österreichisches Deutsch“ zu sprechen sowie die zum Zeitpunkt der Diskussionen bereits vorliegenden vorläufigen Ergebnisse der Fragebogenerhebung zu diskutieren. Weitere Fragestellungen der Gruppendiskussionen waren: die Einstellungen gegenüber dem österreichischen Deutsch, generelle Beobachtungen zum Sprachverhalten in der Schule, das Normverständnis und Korrekturverhalten der LehrerInnen, mögliche Erklärungen für die Unterschiede im Sprachgebrauch zwischen LehrerInnen und SchülerInnen, die in der Fragebogenerhebung festgestellten altersspezifischen Angaben zur Variantenverwendung und schließlich der Zusammenhang von Sprache und (nationalen) Identitätskonstruktionen. 
Die Gruppendiskussion mit den SchülerInnen fand am 5. Juni 2014 an einem Gymnasium in Wien in zwei Unterrichtsstunden statt und dauerte ca. 90 Minuten. 11 Schülerinnen und 3 Schüler zwischen 17 und 19 Jahren nahmen daran teil. 12 von ihnen waren in Wien geboren und hatten die österreichische Staatsbürgerschaft, ein Schüler war in Mazedonien geboren und mazedonischer Staatsbürger, eine in Kanada geborene Austausch-Schülerin war kanadische Staatsbürgerin. Die Frage nach dem Geburtsland der Eltern zeigt - ebenso wie die nach den Muttersprachen und danach, wie lange sie schon in Österreich lebten - dass ein Teil der SchülerInnen aus der zweiten Zuwanderergeneration stammen. Mit Ausnahme des mazedonischen Schülers und der kanadischen Austausch-Schülerin lebten alle seit ihrer Geburt in Österreich, und niemand von ihnen hatte bis zum Zeitpunkt der Diskussion eine längere Zeit im Ausland verbracht. Als Geburtsland der Eltern wurde in acht Fällen Österreich (auch Wien und Burgenland) angegeben, je einmal Bosnien/Kroatien, Bosnien/Syrien, Kosovo, Mazedonien und Türkei. Als Muttersprachen gaben die SchülerInnen acht Mal Deutsch an (davon einmal österreichisches Deutsch), einmal Albanisch, einmal Englisch, einmal Kroatisch, einmal Mazedonisch, einmal Serbokroatisch; einmal fand sich die Doppelnennung Türkisch/Kurdisch. Interessant ist, dass so eine vermutlich frühe Zweisprachigkeit nur einmal und nicht in Kombination mit Deutsch vorkam - und dass ein/e Schüler/in die in den Herkunftsländern nicht mehr übliche Selbstbezeichnung Serbokroatisch verwendete. Nach anderen erlernten Sprachen gefragt, nannten die TeilnehmerInnen v. a. Englisch und Französisch (je 10 mal), je einmal Deutsch und österreichisches Deutsch, Albanisch, Arabisch und Kroatisch/Bosnisch.

Die Gruppendiskussion mit den LehrerInnen fand am 16. Juni 2014 nachmittags in einer pädagogischen Fortbildungsinstitution in Graz statt und dauerte 90 Minuten. Neun Lehrerinnen und ein Lehrer nahmen daran teil. Die jüngste Teilnehmerin war 38 Jahre alt, drei TeilnehmerInnen waren zwischen 39 und 50 und sieben Lehrkräfte über 50 Jahre alt. Alle LehrerInnen waren österreichische StaatsbürgerInnen, in Österreich geboren und bis auf eine Person in der Steiermark aufgewachsen. Einige TeilnehmerInnen machten genauere regionale Angaben (Graz, Oststeiermark, Südweststeiermark, Deutschlandsberg, Ligist, Fürstenfeld), die ihnen im Hinblick auf ihre eigene Sprachbiographie und ihren Kontakt zu Nonstandardvarietäten wichtig waren, wie der Verlauf der Diskussion zeigte. Ein/e Teilnehmer/in hatte in der Kindheit einen Wohnortwechsel von Fürstenfeld nach Frankfurt am Main und dann nach Linz erlebt und beschrieb das so: „Ich bin, jo, sprachlich sehr/ in verschiedenen, ah, Gebieten sozialisiert worden." $(\mathrm{M} 1,0135)^{14}$

14 Die Verschriftungskonventionen der Transkripte der Interviews und Gruppendiskussionen befinden sich im Anhang. INT = Interviewer, $\mathrm{F}=$ weibliche Sprecherin, $\mathrm{M}=$ männlicher Sprecher; bei den SprecherInnen in den Interviews wurden auch die Bundesländer angegeben (Wien: W, Burgenland: B, Niederösterreich: N, Oberösterreich: O, Steiermark: St, Kärnten: K, Salzburg: Sa, Tirol: T, Vorarlberg: V). Sie wurden nach zeitlicher Abfolge der Interviews im jeweiligen Bundesland durchnummeriert, Beispiel: FSt3: weibl. Sprecherin aus der Steiermark, 
Vier TeilnehmerInnen konnten auf längere Auslandsaufenthalte verweisen (2 Jahre in Frankreich und 9 Jahre als LehrerIn in der Türkei; 2 Jahre in Frankreich; 2 Jahre Berlin während des Studiums; 1 Jahr in Irland), wobei auch hier wiederum die Auswirkungen auf die Sprachbiographie, den individuellen Sprachgebrauch und der Kontakt mit der bundesdeutschen Varietät thematisiert wurden. Alle gaben Deutsch als Muttersprache an, drei explizit österreichisches Deutsch. Als weitere Sprachkenntnisse wurden Englisch (8), Französisch (7), Italienisch (3) und Slowenisch und Türkisch (je 1) angegeben. Ein/e Teilnehmer/in war bereits in Pension (sie hatte an einer AHS, an der PH und der Uni unterrichtet), eine unterrichtete an der VS, fünf an einer BHS (HAK, HAS, HLW, BAKIP ${ }^{15}$ ), drei an einer AHS. Als Unterrichtsfächer wurden interkulturelles Lernen und Kombinationen von Deutsch mit Englisch (2), Französisch (2), Geographie, Psychologie und Philosophie, katholische Religion und Deutsch als Fremdsprache angegeben. Die TeilnehmerInnen unterrichteten zum Zeitpunkt der Diskussion in Graz, Bruck/ Mur, Mureck, Liezen, Fohnsdorf und in Weiz - also nicht nur in der Landeshauptstadt, in der die Gruppendiskussion stattfand.

\subsection{Teilnehmende Beobachtungen}

Um feststellen zu können, inwieweit sich die Ergebnisse der Fragebogenerhebungen und der Interviews mit dem decken, was tatsächlich im Unterricht beobachtbar ist, wurden an fünf Schulen in sieben Schulklassen teilnehmende Unterrichtsbeobachtungen durchgeführt. Diese Beobachtungen dienten dazu, unsere Ergebnisse aus den Fragebögen und Interviews zu den Themenbereichen Sprachverwendung und Variation durch eine zusätzliche empirische Erhebung abzustützen. Aus budgetären und zeitlichen Gründen war es nicht möglich, eine noch größere Anzahl an Beobachtungen durchzuführen. Die durchgeführten Unterrichtsbeobachtungen können den sprachlichen Alltag in Österreichs Schulen daher nur ansatzweise abbilden.

Aufgrund von Schwierigkeiten bei der Bewilligung von Kameras und Diktiergeräten bei der Erhebung konnten die Beobachtungen nur schriftlich festgehalten und nicht durch Tonaufnahmen bzw. Videos dokumentiert werden, was im Sinn einer möglichst objektivierten Auswertung wünschenswert gewesen wäre. In jedem Fall ist bis zu einem gewissen Grad auch das ,Beobachterparadoxon' zu

3. InterviewpartnerIn in diesem Bundesland. In den Gruppendiskussionen bedeutet MO Moderator, F weibliche Sprecherin, M männlicher Sprecher. Die SprecherInnen wurden nach zeitlicher Abfolge der Sprechbeiträge durchnummeriert, Beispiel: F2 = weibliche Sprecherin, meldete sich als zweite zu Wort. Eventuelle Zahlen danach verweisen auf die entsprechende Stelle im Transkript. Beispiel: F2, 892 = zweite Sprecherin, Fläche 892.

15 HAK $=$ Handelsakademie, HAS $=$ Handelsschule, HLW = Höhere Lehranstalt für wirtschaftliche Berufe, BAKIP = Bundesanstalt für Kindergartenpädagogik. 
berücksichtigen: Es ist durchaus möglich, dass die SchülerInnen aufgrund der Anwesenheit der Beobachterin stärker dazu tendiert haben, Standard zu sprechen, als sie dies normalerweise tun würden. ${ }^{16}$

Insgesamt wurden sieben teilnehmende Beobachtungen (je eine Unterrichtseinheit) im Zeitraum von 8. bis 23. Mai 2014 durchgeführt:

- eine Klasse (27 SchülerInnen) an einer AHS in Wien (11. Schulstufe)

- eine Klasse (20 SchülerInnen) an einer privaten VS in Wien (2. Schulstufe)

- zwei Klassen an einer BHS (je 28) in Niederösterreich (jeweils 11. Schulstufe)

- eine Klasse (25 SchülerInnen) an einer zweisprachigen AHS in einem städtischen Gebiet Kärntens (8. Schulstufe)

- zwei Klassen (je 19 SchülerInnen) an einer VS in einem städtischen Gebiet Vorarlbergs (jeweils 2. Schulstufe)

Die sieben Lehrpersonen waren zwischen 25 und 65 Jahre alt. Fünf LehrerInnen sprachen der Beobachterin zufolge während der gesamten Unterrichtsstunde Standard mit leichten regionalen Färbungen, zwei sprachen hingegen nur beim Vortrag Standard, sonst aber Umgangssprache bzw. Dialekt. Auf der anderen Seite wurden ca. 140 SchülerInnen beobachtet. Der Anteil der SchülerInnen mit anderen Erstsprachen als Deutsch lag nach Auskunft der Lehrpersonen in vier Schulen bei ca. $60 \%$. Die Muttersprachen der SchülerInnen waren nach Auskunft der LehrerInnen neben Deutsch noch Slowenisch, Italienisch, Türkisch, Bosnisch, Hindi, Mazedonisch, Dari, Englisch, Arabisch, Georgisch, Chinesisch, Französisch, Koreanisch, Tschechisch und Ungarisch.

Die Beobachtungen liefen folgendermaßen ab: Die Beobachterin war ab dem Läuten bis kurz nach Ende der Unterrichtsstunde in der Klasse anwesend. Sie wurde den SchülerInnen gleich zu Beginn kurz vorgestellt, anschließend lief der Unterricht an. Zeitgleich begann die teilnehmende Beobachterin mit der Beobachtung, die mit dem Läuten zum Schluss der Unterrichtsstunde beendet wurde. Der Fokus richtete sich darauf, wer welche Varietät in welcher Situation wem gegenüber verwendet hatte. Sämtliche Äußerungen der Lehrpersonen und SchülerInnen wurden handschriftlich auf einem Raster mit einer fünfstufigen Skala von Dialekt über Umgangssprache bis hin zur Standardsprache eingestuft; zusätzlich wurden Notizen über besondere Vorkommnisse gemacht. Weiters wurde vermerkt, wer die jeweilige Äußerung wem gegenüber zu welchem Zweck in welcher Situation getätigt hatte (z. B. Schüler richtet im Plenum an den Lehrer eine Frage etc.).

Im Folgenden wird eine beispielhafte Kurzbeschreibung einer teilnehmenden Beobachtung in einer 4. Klasse AHS (8. Schulstufe) in Kärnten dargestellt:

16 Die beobachteten SchülerInnen und LehrerInnen wussten allerdings nicht, dass ihr sprachliches Verhalten beobachtet wird. Ihnen wurde davor nicht mitgeteilt, worum es bei der Hospitation genau geht. 
Die Beobachterin war, gemeinsam mit der Lehrperson, bereits kurz vor dem Läuten zum Unterrichtsbeginn in der Klasse anwesend und wurde den SchülerInnen zu Stundenbeginn kurz vorgestellt. Die Beobachterin nahm im hinteren Teil der Klasse Platz und machte über die Äußerungen von Lehrkraft und SchülerInnen Notizen in einem dafür vorbereiteten Raster, indem die jeweilige Art der Äußerung und die Unterrichtshandlung bzw. Kommunikationssituation, in der sie getätigt wurde, vermerkt wurde. So erfolgte die Begrüßung der SchülerInnen durch die Lehrerin beispielsweise in der Standardsprache; eine nicht-stoffbezogene Frage der Lehrerin an einen Schüler („Wo host Du Deine Sochn?") jedoch in der Umgangssprache.

Die Beobachterin blieb, um den Unterricht nicht zu stören, zunächst im hinteren Teil der Klasse auf einem Sessel sitzen. Die Inhalte des Unterrichts waren zwei Referate zu den Themen „Junk Food“ und „Parfum“, anschließend an jedes Referat wurden durch die Lehrkraft und die MitschülerInnen Fragen gestellt. Danach wurden Fragebögen zu den Referaten ausgeteilt, die die SchülerInnen in kleinen Gruppen gemeinsam beantworteten, während die Lehrkraft vorne stand und die SchülerInnen bei der Arbeit beobachtete. Zuweilen ging sie durch die Reihen und hörte einzelnen Gruppen zu.

Hierbei stand die Beobachterin auf und ging zwischen den Gruppen herum, um zuzuhören und Daten zur Sprachverwendung in das Beobachtungsraster eintragen zu können. Bei den Gesprächen über die Beantwortung der Fragen sprachen die SchülerInnen, soweit es hörbar war, oft Umgangssprache. Bei den Referaten und im stoffbezogenen SchülerInnen-LehrerInnen-Gespräch sprachen die SchülerInnen meist Standarddeutsch, wobei dieses teilweise etwas bemüht klang und eine minimale kärntnerische Färbung aufwies. Nicht standardsprachliche Äußerungen betrafen meist nicht stoff- bzw. themenbezogene Gespräche. Die Lehrerin sprach im Unterricht fast ausschließlich Standarddeutsch, nur nicht stoffbezogene Äußerungen waren dialektal/umgangssprachlich gefärbt. Je formeller also die Situation, desto größer schien das Bemühen um die Verwendung der Standardsprache. Bei organisatorischen Gesprächen wurde Umgangssprache gesprochen, die ungezwungen wirkte, z. B. „Fongst oba heite on!“, oder „Wos i letzte Stund vagesn hob ..... Während der Stunde füllte die Beobachterin das Beobachtungsraster aus: Für jede Äußerung, die getätigt wurde, wurde ein Kreuz auf einer 5-stufigen Skala (wobei 1 für stark dialektal, 3 für umgangssprachlich und 5 für standardsprachlich stand) gemacht sowie Sozialform, Art und Funktion der jeweiligen Äußerung vermerkt. Nach Möglichkeit wurden auffällige (z. B. vom normalen Sprachgebrauch der jeweiligen Person abweichende) Äußerungen aufgeschrieben. Zusätzlich wurden der Ablauf der Stunde, die Anzahl, das Alter, die Herkunft und die Muttersprachen der SchülerInnen, sowie Schulform, Schulstufe und besondere pädagogische Konzepte der Klasse notiert und weiters das Setting kurz beschrieben. Nach dem Ende der Unterrichtsstunde verließ die Beobachterin die Klasse. 


\section{Ergebnisse der empirischen Erhebung an Schulen}

Im folgenden Teil werden die Ergebnisse der Fragebogenerhebung der LehrerInnen und SchülerInnen zu den einzelnen Dimensionen unserer Analyse dargestellt, wobei auf Befragungsergebnisse bzw. Unterschiede innerhalb der Gruppen nur dann näher eingegangen wird, wenn diese aussagekräftig sind - wenn also z. B. SchülerInnen ein signifikant unterschiedliches Antwortverhalten aufweisen, wenn das Alter der Lehrpersonen eine Rolle spielt, deren Ausbildungsinstitution, der Schultyp, an dem sie unterrichten, oder die Fächerkombination, wenn die Region das Antwortverhalten beeinflusst oder die Erstsprachen der SchülerInnen bzw. deren individuelles Sprachrepertoire oder deren (Fremd-)Sprachenkenntnisse. Die hier angeführten Faktoren sind auch die, die in der statistischen Auswertung am häufigsten relevant waren. Die Daten der quantitativen Fragebogenerhebung werden dabei dort, wo es sinnvoll schien, durch die qualitativen Daten ergänzt und in der Interpretation aufeinander bezogen.

\subsection{Konzeptualisierung der Variation des Deutschen in Österreich}

Ein erster Fragenblock der Fragebogenerhebung betraf die Konzeptualisierung der deutschen Sprache in Österreich, d.h. die Vorstellungen, die die Befragten von der Variation der deutschen Sprache in Österreich und der länderübergreifenden Variation der deutschen Sprache insgesamt haben. So sollte erhoben werden, wie die Mehrheitssprache in Österreich benannt wird und mit welchen Varietäten das „österreichische Deutsch" am ehesten assoziiert wird. Außerdem sollte erfragt werden, ob (implizit) eher monozentrische oder eher plurizentrische Konzepte vorhanden sind, inwieweit das Konzept der Plurizentrik bekannt ist, wie die innerösterreichische und wie die Staatsgrenzen übergreifende Variation eingeschätzt wird und auf welchen Ebenen bzw. wie die Unterschiede zwischen dem Standarddeutsch in Österreich, in Deutschland und der Schweiz wahrgenommen werden.

\subsubsection{Wie wird die Mehrheitssprache in Österreich benannt?}

$\mathrm{Zu}$ Beginn wurde zunächst eine offene Frage (mit der Möglichkeit von Mehrfachnennungen) gestellt: „Wie würden Sie die Sprache, die die Mehrheit der Österreicher/ innen als Muttersprache spricht, nennen?" Hier fielen die Antworten relativ eindeutig aus: Allen voran wurden „Deutsch“ (mit 51,6 \% der LehrerInnen und 49,1 \% der SchülerInnen) und „österreichisches Deutsch“ (mit 19,6\% der LehrerInnen und $11,2 \%$ der SchülerInnen) genannt. Nur $6 \%$ der LehrerInnen und 9,9\% der 
SchülerInnen nannten spontan „Österreichisch“ - eine Bezeichnung, die in den Medien häufig verwendet wird ${ }^{1}$. „Dialekt“ wurde von 5,2 \% der LehrerInnen und 9,1\% der SchülerInnen genannt.

Weitere Antworten mit geringen Prozentzahlen waren „Deutsch mit österreichischer Färbung“ (5,2\% der LehrerInnen und 3,3\% der SchülerInnen), die Antwort „Deutsch mit Dialekt/Mundart“ kam von 4,6\% der LehrerInnen und $12,3 \%$ der SchülerInnen. Ebenfalls genannt wurde von 2,6 \% der LehrerInnen und 1,3 \% der SchülerInnen „Deutsch und Österreichisch“. Sämtliche andere Antworten wurden unter „Anderes“ zusammengefasst (6,2\% der LehrerInnen und 3,8\% der SchülerInnen).

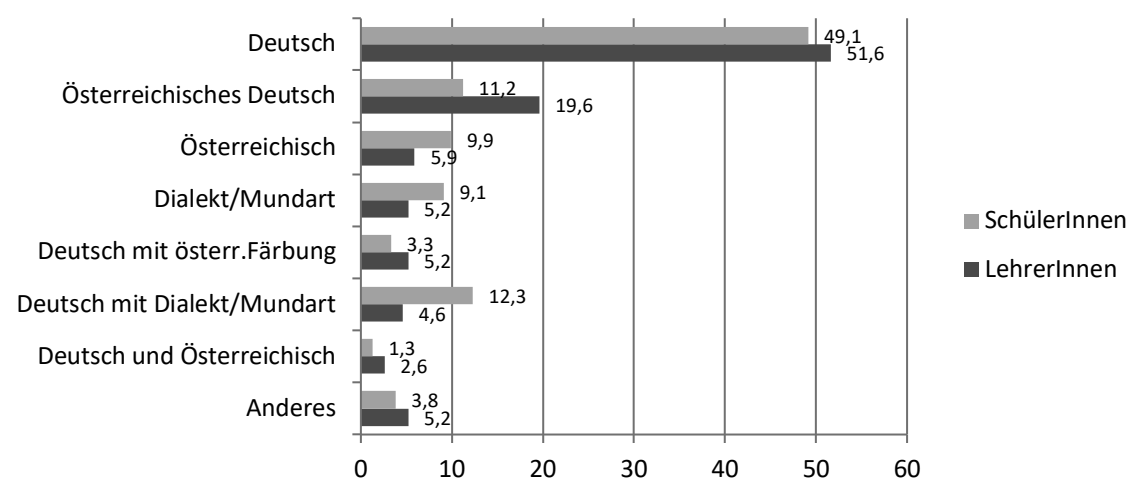

Abb. 27: Wie wird die Mehrheitssprache in Österreich benannt (LehrerInnen/SchülerInnen)

Signifikante Unterschiede konnten innerhalb der Gruppe der Lehrenden nach Alter $(\mathrm{p}=\mathrm{o}, \mathrm{ooo})$, Schulform $(\mathrm{p}=\mathrm{o}, \mathrm{00})$ und dem Ausbildungsort $(\mathrm{PH} / \mathrm{Uni})(\mathrm{p}=$ o,002) festgestellt werden sowie abhängig davon, ob das Konzept der Plurizentrik bekannt war oder nicht $(\mathrm{p}=0,017)$. VS-LehrerInnen gaben „Deutsch“ mit $56 \%$ am häufigsten an, gefolgt von AHS-/Sek.-II-LehrerInnen (50,6\%) und HS/ NMS-LehrerInnen (47,6\%). Auffallend ist, dass „österreichisches Deutsch“ von VS-LehrerInnen kaum angegeben wurde (4\%), während es wesentlich häufiger von AHS-/Sek.-II- bzw. HS/NMS-LehrerInnen (27,2 \% bzw. 28,6\%) genannt wurde. Hinsichtlich der Angabe „Dialekt“ als Bezeichnung für die Sprache, die die Mehrheit der ÖsterreicherInnen spricht, fällt vor allem auf, dass dieser am stärksten von den VS-LehrerInnen angegeben wurde (14\%), von den LehrerInnen der anderen Schultypen jedoch so gut wie gar nicht. Nach Ausbildungsort unterscheiden sich

1 Zum Beispiel im DialogForum des ORF „Wie lecker ist Österreichisch?“ am Dienstag, 15. März 2016 im Radiokulturhaus und in den darauf Bezug nehmenden Zeitungsberichten, oder in einem Artikel zum vorliegenden Projekt in der Neuen Kronen Zeitung am 8. 2. 2014 „Österreichisch schreiben dürfen. [...]“ 
die LehrerInnen vor allem hinsichtlich der Wahl der Bezeichnung „österreichisches Deutsch“: Diese fand sich mehr als doppelt so oft bei UniversitätsabsolventInnen $(27,5 \%)$ wie bei PH-AbsolventInnen (11,1\%). Auch nach dem Alter zeigten sich deutliche Unterschiede. Je älter die Befragten, umso eher gaben sie „Deutsch“ als Bezeichnung für die Sprache an, die die Mehrheit der ÖsterreicherInnen als Muttersprache spricht. Wobei hier der Unterschied zwischen der jüngsten und der ältesten LehrerInnen-Gruppe besonders eklatant ist: $28,6 \%$ bei den jüngsten und $55,9 \%$ bei den ältesten LehrerInnen-Gruppen wählten bei dieser Frage die Bezeichnung „Deutsch“. Die jüngsten LehrerInnen gaben mit fast $30 \%$ auch die Bezeichnung „Dialekt“ an, während dies die beiden ältesten Gruppen überhaupt nicht gemacht haben (o \%). Die Ergebnisse sind nach Regionen betrachtet zwar nicht signifikant, aber es zeigt sich doch eine interessante Tendenz: Die Bezeichnung „österreichisches Deutsch“ wurde besonders häufig von Befragten aus der Region Südost gewählt (37\%), die Bezeichnung „Deutsch“ am stärksten in den westlichen Bundesländern Tirol (69,2 \%) sowie in Vorarlberg $(71,4 \%)$. Die Werte für „Deutsch“ liegen in den anderen Teilen Österreichs zwischen $44 \%$ und $51 \%$ : 50 \% in der Region Ost, 51,2 \% in der Region Mitte und 44,4 \% in der Region Südost. „Österreichisch" wurde in Tirol und Vorarlberg von niemandem angegeben.

Innerhalb der Gruppe der SchülerInnen zeigten sich bei dieser Frage signifikante Unterschiede danach, in welcher Region sie die Schule besuchen $(\mathrm{p}=$ $0,000)$. Aber auch das Geschlecht $(p=0,004)$ und die Muttersprache $(p=0,001)$ hatten einen Einfluss auf das Antwortverhalten. So nannten am häufigsten (6o \%) SchülerInnen aus dem Osten des Landes „Deutsch“ als die Sprache, die die Mehrheit der ÖstereicherInnen als Muttersprache spricht. Tiroler SchülerInnen nannten hingegen „Deutsch“ deutlich weniger oft $(41,2 \%)$, auch in Vorarlberg wird „Deutsch“ nur von $36 \%$ der SchülerInnen genannt. „Deutsch“ ist zwar auch die von Tiroler und Vorarlberger SchülerInnen die am häufigsten genannte Antwort, jedoch werden hier deutlich öfter als im Osten „Deutsch mit Dialekt/Mundart“ (Tirol: 26,9\%, Vorarlberg: 19,5\%, Osten: 7,2 \%) bzw. nur „Dialekt/Mundart“ (Tirol: $16 \%$, Vorarlberg: 14,6\%, Osten: 2,6 \%) genannt. 17,5\% der SchülerInnen aus Salzburg und Oberösterreich nannten Dialekt bzw. 13,4\% Deutsch mit Dialekt. Österreichisches Deutsch wird relativ am häufigsten in den östlichen Bundesländern angegeben (13,4\%), gefolgt von Kärnten und der Steiermark (12,9\%). In den westlichen Bundesländern wird österreichisches Deutsch stärker mit dem "Dialekt" assoziiert, der im alltäglichen Sprachgebrauch grundsätzlich eine wichtigere Rolle spielt als im Osten, der offensichtlich stärker standardorientiert ist (siehe Abb. 28 und 29 auf S. 124).

Auch der sprachliche Hintergrund der SchülerInnen spielte eine Rolle: SchülerInnen mit einer anderen Muttersprache als Deutsch geben am häufigsten „Deutsch“ an (62\%), SchülerInnen mit Deutsch als Muttersprache deutlich seltener (47,2\%), hingegen nannten sie häufiger Dialekt/Mundart (13,6\%) als SchülerInnen mit nichtdeutscher Muttersprache $(5,5 \%)(\mathrm{p}=0,001)$. 
70

60

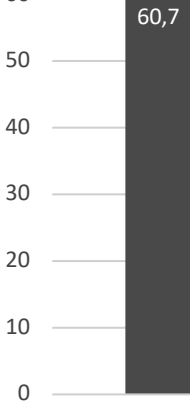

Ost

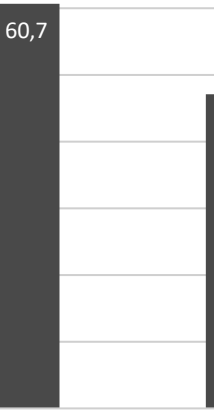

Südost

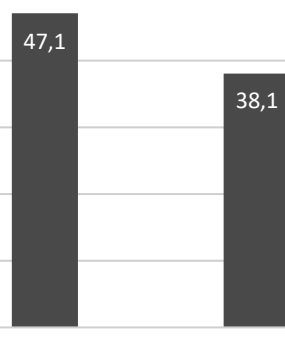

Mitte

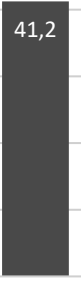

Tirol
39

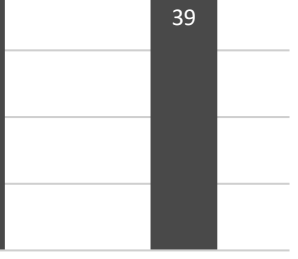

Vorarlberg

Abb. 28: SchülerInnen-Antworten nach Regionen: Sprache, die in Österreich gesprochen wird: Deutsch (in \%)

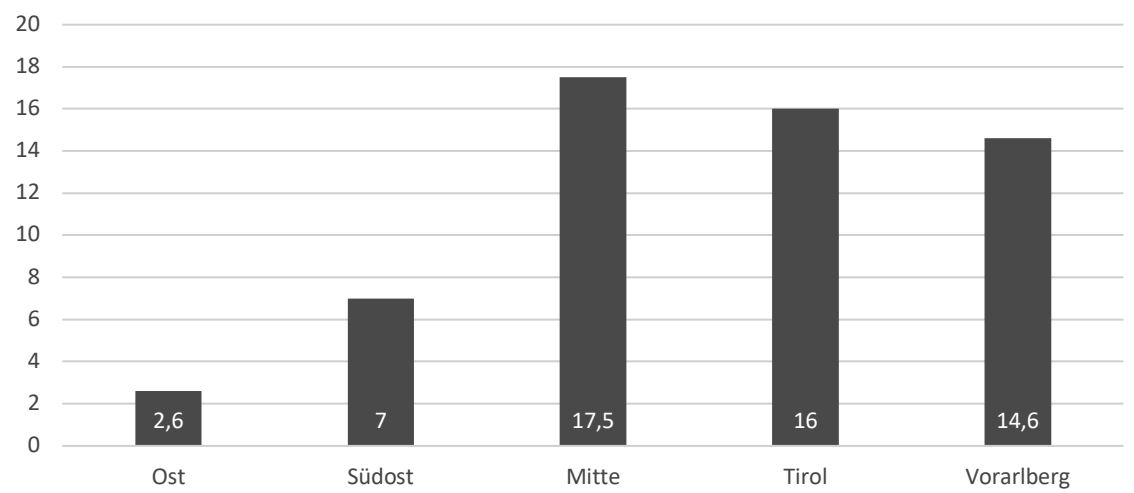

Abb. 29: Schülerlnnen-Antworten nach Bundesländern: Sprache, die in Österreich gesprochen wird: Dialekt/Mundart (in \%)

Auch nach Geschlecht unterscheiden sich die Antworten signifikant ( $p=0,004)$ : So geben 51,7\% der Schülerinnen „Deutsch“ an, jedoch nur 45,6\% der Schüler. Diese geben allerdings „Österreichisch“ häufiger an (12\%) als Schülerinnen (8,3\%). Auch österreichisches Deutsch wird von Schülern öfter (12,9\%) genannt als von Schülerinnen (10\%). Schülerinnen geben Dialekt/Mundart öfter an (10\%) als Schüler (7,8\%). Letztere tendieren hier also eher dazu, bei der Benennung der Mehrheitssprache in Österreich den österreichischen Aspekt zu betonen. 


\subsubsection{Mit welchen Varietäten wird österreichisches Deutsch assoziiert?}

Wie aus der Literatur geht auch aus unseren Daten hervor, dass nicht nur die Standardsprache, sondern alle anderen Varietäten im Alltag der ÖsterreicherInnen und damit auch in der Schule eine wichtige Rolle spielen (vgl. auch Ender/ Kaiser 2009, Rastner 1997, Neuland 2006, Steiner 2008). Eine weitere Frage zur Konzeptualisierung der deutschen Sprache in Österreich versuchte daher der Frage nachzugehen, ob die LehrerInnen und SchülerInnen mit „österreichischem Deutsch" am ehesten Standard, Umgangssprache oder Dialekt verbinden. Auf die Frage „Was ist Ihrer Meinung nach österreichisches Deutsch?“" waren folgende Antwortmöglichkeiten vorgegeben: „das, was man in österreichischen TV- und Radionachrichten spricht; das, was man in Österreich im Alltag spricht (Umgangssprache); die verschiedenen Dialekte in Österreich; sonstiges" (Mehrfachnennungen waren möglich).

Die Ergebnisse zu dieser Frage bestätigen, dass LehrerInnen im Grunde alles, was in Österreich gesprochen wird, unter „österreichischem Deutsch“ subsumieren. Da Mehrfachnennungen möglich waren, gab es durchaus auch einige Kombinationsantworten. Im Wesentlichen wurde aber klar, dass unter den LehrerInnen in erster Linie nicht die standardnahe Mediensprache mit österreichischem Deutsch assoziiert wird (47,6\%), sondern vielmehr die Umgangssprache (69,5\%), und mit einer etwas geringeren Häufigkeit als die standardnahe Mediensprache wurden die verschiedenen Dialekte genannt $(43,9 \%)$.

Die SchülerInnen verbinden österreichisches Deutsch ebenso mehrheitlich mit Umgangssprache, aber in Gegensatz zu den LehrerInnen auch stark mit Dialekt: $72,5 \%$ der SchülerInnen wählten „das, was man in Österreich im Alltag spricht“ (Umgangssprache), 70,2 \% wählten „die verschiedenen Dialekte in Österreich“. Die standardnahe Varietät der TV-und Radionachrichten wurde nur von 20,5\% der SchülerInnen gewählt. Knapp 78 \% der SchülerInnen gaben nur Umgangssprache oder Dialekt oder beides in Kombination an und wählten die standardnahe Varietät gar nicht (siehe Abb. 30 auf S. 126).

Auf sprachwissenschaftlicher Ebene wird österreichisches Deutsch meist mit „österreichischem Standarddeutsch“ gleichgesetzt (siehe Kapitel 2.5). Dies ist unter den LehrerInnen und SchülerInnen jedoch nur bedingt der Fall: Sehr häufig wurden sowohl Umgangssprache als auch Dialekt unter den Begriff „österreichisches Deutsch“ eingeordnet, wobei die standardnahe Mediensprache ausgeklammert bleibt. Österreichisches Deutsch wird offenbar als eine Kombination aus Umgangssprache, Dialekt und Standardsprache empfunden, wobei letztere v. a. bei den SchülerInnen nicht die größte Bedeutung hat und erst an dritter Stelle genannt wird.

Sieht man sich Unterschiede innerhalb der Gruppe der LehrerInnen an, dann zeigt sich, dass vor allem AHS-/Sek.-II-LehrerInnen (60,2\%) meinten, dass die standardnahe Mediensprache österreichisches Deutsch ist. Diese Ansicht vertraten 
aber nur 37,0 \% der VS-LehrerInnen und 23,8\% der HS/NMS-LehrerInnen ( $\mathrm{p}=$ 0,002). Auch der Ausbildungsort (PH oder Universität) der LehrerInnen ist hier relevant: Die überwiegende Mehrheit der LehrerInnen (60,9\%), die ihre Ausbildung an der Universität absolviert haben, war in unserem Sample der Meinung, dass das, was man in österreichischen TV - und Radionachrichten spricht, österreichisches Deutsch sei, während nur knapp ein Drittel der LehrerInnen (32,9\%), die ihre Ausbildung an einer Pädagogischen Hochschule gemacht haben, dieser Meinung waren $(\mathrm{p}=\mathrm{o,000})$ - ein Hinweis auf eine stärkere Standardorientiertheit der Universitäten.

das, was man in österreichischen TVund Radionachrichten spricht

das, was man in Österreich im Alltag spricht (Umgangssprache)

die verschiedenen Dialekte in Österreich

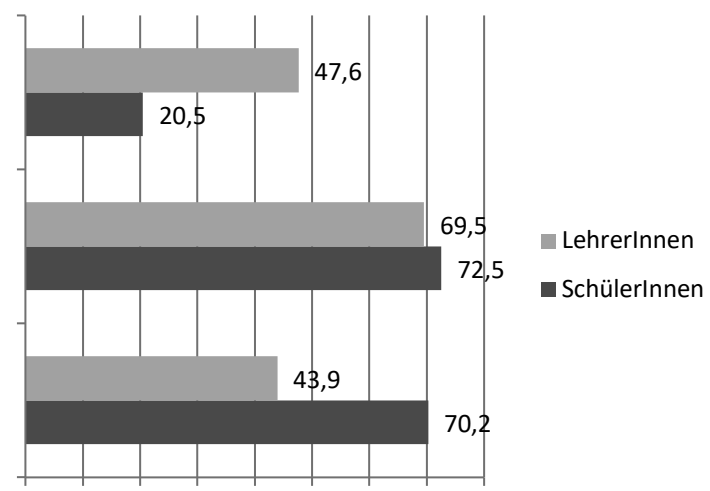

Abb. 30: Varietät, die mit österreichischem Deutsch verbunden wird (in \%)

Bei der Einschätzung des österreichischen Deutsch als Umgangssprache verläuft eine Grenze zwischen der jüngeren und älteren LehrerInnengruppe: Die Gruppe der 42-51-jährigen (74,0 \%) und die Gruppe der 52-63-jährigen $(81,5 \%)$ meinten wesentlich häufiger als die Gruppen der jüngeren LehrerInnen, dass die Umgangssprache österreichisches Deutsch sei $(\mathrm{p}=0,001)$. Ähnliche Ergebnisse liegen vor, wenn man die Daten zum Dialekt nach der Anzahl der Dienstjahre aufschlüsselt $(p=0,001)$. Dass die verschiedenen Dialekte in Österreich als österreichisches Deutsch zu bezeichnen sind, meinen allen voran die jüngsten LehrerInnen (22-32-jährige) mit 63,6\%, die insgesamt signifikant stärker am Dialekt orientiert sind als an der Umgangssprache $(\mathrm{p}=0,015)$. Auch nach der Schulform gibt es Unterschiede bei der Einschätzung des österreichischen Deutsch als die Summe der heimischen Dialekte ( $\mathrm{p}=0,006)$ : VS-LehrerInnen sehen am stärksten die verschiedenen Dialekte in Österreich als österreichisches Deutsch $(61,1 \%)$, während das die HS/NMS- und die AHS/BHS/BAKIP-LehrerInnen nur zu einem Drittel tun. Innerhalb der Gruppe der SchülerInnen gab es nach folgenden Variablen signifikante Unterschiede: in den Angaben bezüglich Umgangssprache nach dem Bundesland bzw. der Region $(\mathrm{p}=\mathrm{o}, 017)$ und der Muttersprache $(\mathrm{p}=0,000)$ bzw. der Muttersprache der Eltern $(\mathrm{p}=\mathrm{o}, \mathrm{ooo})$. 
Die Umgangssprache wird am häufigsten von SchülerInnen in den mittleren Bundesländern (Oberösterreich und Salzburg: 77,6\%) und SchülerInnen in südöstlichen Bundesländern (Steiermark und Kärnten: 76,6\%) genannt, gefolgt von SchülerInnen aus Tirol ( $71,3 \%)$ und aus den östlichen Bundesländern (69\%). Am wenigsten oft wurde diese Antwort von SchülerInnen aus Vorarlberg angekreuzt $(64,3 \%)(p=0,017)$. Letzteres könnte damit zu tun haben, dass in Vorarlberg - ähnlich wie in der Schweiz - die Umgangssprache eine deutliche geringere Bedeutung hat, weil dort eine Situation der Diglossie vorherrscht (vgl. Ender/Kaiser 2009, 286).

Diejenigen SchülerInnen, die nur Deutsch als Muttersprache haben, nannten die Umgangssprache signifikant häufiger $(75,5 \%)$ als solche, die nur mit anderen Sprachen als Deutsch aufgewachsen sind $(58,2 \%)(p=0,000)$.

Zusammenfassend kann also festgestellt werden, dass sich im Bewusstsein der heimischen LehrerInnen und SchülerInnen das österreichische Deutsch als eine Kombination aus Umgangssprache, Dialekt und Standard zusammensetzt, wobei die standardnahe Mediensprache, die in den österreichischen TV- und Radionachrichten zum Einsatz kommt, erst in dritter Linie mit österreichischem Deutsch gleichgesetzt wird.

Das führt zu einem generellen Befund, den die qualitativen Daten aus der Gruppendiskussion und den Interviews im Prinzip bestätigt haben: Das österreichische Standarddeutsch wird nicht primär mit dem „österreichischen Deutsch“ assoziiert. In der Gruppendiskussion der SchülerInnen wurde diese Thematik ausführlich diskutiert. Auf die Frage, was österreichisches Deutsch für sie bedeute, erwähnten die befragten SchülerInnen explizit Dialekt und Mundart sowie bestimmte Austriazismen („nicht unbedingt nur Dialekt und Mundart, [...], sondern auch ähm heimische Begriffe “ [F2]); genannt werden Sackerl, Schnackerl, Semmel; , die Dialekte und die Ausdrücke die's einfach beim deutschen Deutsch jetzt nicht so gibt“ (F4), „für mich ist österreichisches Deutsch hauptsächlich Dialekt“ (F6). Mehrmals wurde eine Opposition zwischen österreichischem Deutsch und „Hochdeutsch“ konstruiert („,es klingt einfach sympathischer als Hochdeutsch“, [F8]). Letztlich wurden in erster Linie umgangssprachliche und nähesprachliche Varietäten mit dem österreichischen Deutsch assoziiert.

Das zeigten auch die Prädikationen, die dem österreichischen Deutsch zugeschrieben wurden: Die SchülerInnen assoziierten es teilweise mit Begriffen wie „Familie“ und „Nähe“ und nannten es „familiär“. Vorgebracht wurden auch Bemerkungen wie: „,ich finds auch familiärer als das deutsche Deutsch“ (F10) oder ,ich finde österreichisches Deutsch ist persönlicher als deutsches Deutsch“ (F11). Mehrfach wurde dem österreichischen Deutsch eine nonformelle Note zugeschrieben: "es is auch viel lässiger - chilliger" (F9), ,also in Österreich locker“ (F10), „für mich ist österreichisches Deutsch einfach nicht so - so hochgestochen wie das deutsche Deutsch“ (F5). Auch mit den Begriffen „Tradition und Kultur" wurde die heimische Varietät gedanklich in Verbindung gebracht: „( $(\mathrm{xxxx})$ Ich seh da zum Beispiel ein österreichisches Ehepaar ((lacht)) also im Dirndlanzug" (M3). 
Demgegenüber wurde das deutsche Deutsch - von den SchülerInnen manchmal auch „Hochdeutsch“ genannt - mit Attributen versehen wie „klugscheißermäßig“, „,klingt immer so förmlich“, „die Deutschen sprechen so/ also sie sind so str/ streng“ (F10), ,ich find die Deutschen versuchen einfach immer deutlich zu reden“ (F9), und es wurde mehrmals behauptet: „oft/ verstehen einen die Deutschen nicht" (F1O, was umgekehrt nicht der Fall sei).

Eine Nachfrage in der LehrerInnen-Gruppendiskussion zu den Ergebnissen der Fragebogenerhebung bei den SchülerInnen, wonach rund $70 \%$ der SchülerInnen österreichisches Deutsch mit umgangssprachlichen und dialektalen Varietäten verbinden, aber nur $20 \%$ mit der standardnahen Sprache von Radio und TV, bestätigte auch die Fragebogenergebnisse. „Österreichisches Deutsch, das is klar, dass da eher/ das was charakteristisch is für Österreich, ..., die Dialekte zum Beispiel.“ (M1) Auf die Rückfrage des Moderators „Das heißt, Standardsprache oder Hochsprache sind nicht so charakteristisch wie die Dialekte?" antwortete ein anderer Teilnehmer mit „Genau“.

In weiterer Folge wurde in der Diskussion der SchülerInnen von ein paar TeilnehmerInnen ins Treffen geführt, dass die Sprache im österreichischen Radio (im Sender Ö3) Umgangssprache sei, und bei den Regionalsendern, z. B. Radio Steiermark oder Radio Burgenland - sogar Dialekt. Auch das folgende Statement bestätigte den Gesamteindruck, dass SchülerInnen in erster Linie nonstandardsprachliche Varietäten mit dem österreichischen Deutsch assoziieren.

Also für mich ist österreichisches Deutsch, halt wie ich schon vorher gesagt hab, viel charakteristischer, also wären das eher für mich ahm die Dialekte oder eher das Lässigere für mich, wie zum Beispiel „Ich hab gsehn“. Und dass man nicht sagt, „ich hab g/ habE gesehEN“, sondern ,ich hab gsehn“. (F1)

Die Diskussion zur Konzeptualisierung sprachlicher Variation in der Gruppe der SchülerInnen bestätigt, abschließend zusammengefasst, den Eindruck, dass eher nähesprachliche Varietäten und mündliche Sprachformen mit österreichischem Deutsch assoziiert werden, wobei die Grenzen zwischen den Varietäten so wie zwischen Umgangssprache und Dialekt fließend sind und die beiden immer wieder gleichgesetzt werden. Den SchülerInnen fehlt auch die Begrifflichkeit, um differenziert über Variation zu sprechen - dieser Befund durchzieht von der Dokumentenanalyse an als roter Faden sämtliche Ergebnisse. Dies weist auf die Bringschuld des Schulsystems hin, differenzierte Terminologie und Wahrnehmung in diesem Zusammenhang zu vermitteln.

Ähnliche Befunde haben die Interviews mit den LehrerInnen ergeben. Ein Vorarlberger Lehrer (MV1) betonte für sein Bundesland:

Unsere Enkulturation ist eine dialektale. Nicht eine österreichische DeutschGeschichte, ... der Dialekt ... hat ... auch einen ganz anderen Stellenwert ... wer was 
auf sich hält, spricht Dialekt, und wer das nicht kann, ist halt nicht dabei. Ganz einfach. $(\mathrm{xxxx})$ Wenn man hier dazugehören will, ist Dialekt entscheidend.

Vor allem die Gruppendiskussionen haben gezeigt, dass es letztlich für die SprecherInnen sehr schwierig ist, die Variation des Deutschen in Österreich und das Dialekt-Standard-Kontinuum zu konzeptualisieren, wie folgende Äußerung in der Gruppendiskussion der SchülerInnen zeigt: „Ja es is irgendwie schwer da eine Linie zu ziehen und zu sagen, das is jetzt Hochdeutsch und das is Umgangssprache, weil es bewegt sich immer irgendwie dazwischen, finde ich." (F2)

In der LehrerInnen-Gruppe entstand bei der Nachfrage, wie es denn zu interpretieren sei, dass den Fragebogenergebnissen zufolge die meisten LehrerInnen beim österreichischen Deutsch in erster Linie an die Umgangssprache denken, eine lange Diskussion. Einigen TeilnehmerInnen war relativ klar, dass alle heimischen Varietäten das österreichische Deutsch ausmachen. Andere waren sich aber nicht sicher, was denn unter dem österreichischen Deutsch zu verstehen sei, wobei die entsprechenden Stellungnahmen von vielen Unterbrechungen und Turnwechseln, von Hesitationsphänomenen, Satzbrüchen und Stocken gekennzeichnet waren. Dieser Umstand belegt, dass hier sprachliche Unsicherheiten bestehen. Diese Passage sei hier ausführlicher berichtet:

Für F7 (405ff) war es zunächst relativ klar:

Najo, es ist alles österreichisches Deutsch. Jede Variante, na? Ob jetzt in einem, was i net, a/ aso tiefste Umgangssprache mehr oder weniger (orientiert), tiefster Dialekt, oder geHOBene Umgangssprache, w:ie, w:/ jo, im Alltag häufig oder auch in den, (in den) Medien mit den typisch/ mit der typischen Betonung und den typisch österreichischen Wörtern. (Gibts) anfoch verschiedene Abstufungen.

F2 stimmte dem zu und führte als literarisches Beispiel Handkes „Immer noch Sturm“ an, also literarische „Hochsprache“, in die eben auch Dialekt einfließe, „also, des is net abzugrenzen genau, daher ist für mich SCHON, ah, die Vielfalt da." (434).

Auch für M1 (476 ff), der die Situation mit dem Irischen in Irland verglich, wo er ein Jahr gelebt hat, und dem von $\mathrm{F}_{2}$ und $\mathrm{F}_{3}$ während seines Turns zugestimmt wurde, waren alle drei typische Ausprägungen des österreichischen Deutsch: „Und olle drei Varianten ergeben das österreichische Deutsch.“

Also, für mich ist Deutsch eindeutig der Überbegriff und die drei - verschiedenen Ebenen san die Unterbegriffe. Standardsprache, Umgangssprache, Dialekt. (Zustimmung F2: ,JA. Genau. Genau.‘; F3: ,Ja. Des is genau des, ja') [...] und des unterscheidet sich vom Schweizer Deutsch und vom deutschen Deutsch, vom deutschländischen Deutsch. Aber, - für MICH müsste man dazu sagen, was ist österreichisches Standarddeutsch, net? Oder Standardsprache. Für mich ist des a eigene Variante des österreichischen Deutsch. (F2: „Do stimm i dir zu.“) Und olle drei Varianten ergeben das österreichische Deutsch. 
Wie schon gesagt, stimmten $\mathrm{F}_{2}$ und $\mathrm{F}_{3}$ dem zu, F3 z. B. (496f): „Des wäre jetzt, was alles Österreicher sprechen, so quasi. Oder gesammelt."

F2 (567 ff) ergriff dann selbst das Wort und unterstrich ihre Auffassung:

Tschuldigung. Ah, also für mi wärs a totale VerARMung, wenn ma das österreichische Deutsch auf eine dieser DREI Säulen reduzieren würde, weil Sprache eben, ah, a lebendiger Prozess is, der sich ständig verändert, ... eine innere Mehrsprachigkeit gibts jo net nur bei uns, sondern auch in anderen Sprachen. [...] Und das österreichische Deutsch versteh ich schon als VIELSCHICHTIG ..., in verschiedenen Färbungen. Und das is ja a Reichtum. Das ist für mi der Reichtum, dass es so vielfältige Möglichkeiten gibt.

$\mathrm{F}_{3}$ (445), die prinzipiell auch einem Konzept zustimmte, wonach alle Varietäten als österreichisches Deutsch zu verstehen sind, meinte aber relativierend:

... dass ich SCHON a bisschen unterscheide zwischen, aso, für MICH jetzt da dieses österreichische Deutsch als ein Deutsch, das ich überall verstehe, und einen Dialekt als SpeZIALausführung einer Sprache, wenn ich zum Beispiel nach Tirol komme, und DIE sich bemühen, ein österreichisches Deutsch zu sprechen, dann komm ich mit. Wenn die untereinander ihren Dialekt sprechen, dann kann ICH NICHT MEHR MIT.

Auch F9 war sich nicht sicher, und $\mathrm{F}_{4}$ forderte dann (vom Moderator) eine Definition ein, der den Ball zurückspielte:

F9 (458): „Ist es die Sprache, die die Österreicherinnen und Österreicher sprechen? Und des geht vom Dialekt über die Umgangssprache, oder ist das österreichische Deutsch das kodifizierte Deutsch, was man in dem schönen Wörterbuch findet?“

F4 (465): „Ja. Deshalb/ Genau. Deshalb war jo meine Frage. Wie wirds definiert? Was ist die Definition? Ja? (lachend)“

Und als der Moderator meinte: „Ich frag Sie. Ja? Wie Sies definieren. Es geht genau um eines, wir wollens einfach WISSEN von Ihnen, ja?" meinte F4 (469ff), für sie sei es [das österreichische Deutsch] der Standard: „Ja, für $\underline{\text { m:ich }}$, also für MI:CH PERSÖNLICH is es, ah, differiert. Der Dialekt ist der Dialekt und die Österreich/ also, des österreichische DEUTSCH, diese STANDARDsprache, sozusagen, des is für mich eine eigene Sprache. Im Gegenzug zum Dialekt.“ (Kommentar F2, 472: „Na! (Glaub i net, na!) ((gemurmelt, kopfschüttelnd))“

Die Satzbrüche in den obigen Textpassagen, die beobachteten Hesitationen und der Gebrauch relativierender Partikel dokumentieren, dass für einen Teil der DiskutantInnen Unsicherheit bei der Konzeptualisierung des österreichischen Deutsch besteht. In der ganzen Diskussion kam zum Vorschein, dass etlichen TeilnehmerInnen nicht ganz klar war, was unter dem österreichischen Deutsch zu verstehen ist. Sie bemühten sich in der Diskussion, eine Definition für das „österreichische 
Deutsch“ zu konstruieren. Mit anderen Worten: Den diskutierenden LehrerInnen, die ja auch eine normsetzende Instanz darstellen, war mehrheitlich selbst nicht klar, was österreichisches Deutsch für sie bedeutet. Ein schlagendes Argument dafür, sich stärker mit der Variation des Deutschen in Österreich in der LehrerInnenausbildung zu befassen, weil Lehrpersonen ja die Sprachnormen über Textkorrekturen und Bewertungen von SchülerInnentexten und von mündlichen Äußerungen an die nächste Generation weitergeben.

In diesem Kontext wurden die LehrerInnen in den Interviews auch gefragt, wie sie ihren SchülerInnen den Unterschied zwischen Standard, Umgangssprache und Dialekt erklären. Auch hier kam unter den Lehrenden ein recht großes Maß an begrifflicher Unsicherheit zum Vorschein, wie an den wiederholten Wortabbrüchen in den Interviewaussagen unschwer zu erkennen ist. Die Befragten leisteten auch auffallend viel Formulierungsarbeit, darüber hinaus wurden viele relativierende Wörter und abschwächende Partikel und Formulierungen wie „eher“, „irgendwie“, „vielleicht“ oder „würde ich sagen“ verwendet.

Auf die Frage „Wie erklären Sie Ihren Schülern den Unterschied zwischen Dialekt, Standard und Umgangssprache?" antwortete eine Lehrerin aus dem Burgenland (FB2), die sich sichtlich schwertat, die Thematik in Worte zu fassen:

- - Hm. (lacht) Mmh - - Najo. - - Puh. Des is schwierig. Najo umgangs/ umgangssprachliche/ also do würd i eher sogn is es vom Wortschatz her Wortebene, und ahm der Dialekt spiegelt si wie gesagt eher auf der hm - Wie sogt ma do. Also einfach vom/von der Aussprache und so, mehr in der Aussprache vielleicht wider als/als auf der lexikalen.

Ein Lehrer aus Salzburg (MSa3) räumte ein, dass die Differenzierung für ihn ein „schwieriges Thema“ sei:

Mündlich erzählen im Perfekt sozusagen, das wär dann schon fast ein Kennzeichen für Umgangssprache. Ahm da muss ma a bissl aufpassen wahrscheinlich. Weils für uns ja üblich is. Aber ahm das is auch das was ich/was mir auffällt, dass dieser Übergang eben so fließend is bei uns und dass man das ganz schwer trennen kann, ja? Aber wenn man ihnen den Unterschied zwischen Vortrag und Gespräch klor macht, dann/dann merken die Schüler dann auch ahm wo der Unterschied is. Aber des is a schwieriges Thema, net?

Auch eine Lehrerin aus Tirol (FT1) gab zu, dass sie sich oft selbst nicht im Klaren sei:

Standardsprache [ist] überhaupt ..., ah so a gewisse Norm ah norm/normative Form ah - - die also so wirklich die überregionale Kommunikation ermöglicht und/ und ah ... gewisse Verbindlichkeit äh - - in den Regeln ah hat, - ja. So a normative ah Sache, und die Umgangssprache, die, jo, do gibt's jo ah irgendwie/do bin i mir selber oft net so gonz ah klar, ja? Es is ... a bisl a ... verschwommener Begriff, ja. Die bildet sich ja 
hauptsächlich ... in Städten heraus, wo die dialektalen Formen a bissl obgschliffen werden und im Alltags-ah Gebrauch, oder ah (a) gewisse soziale Komponente, (die) is sicher a damit verknüpft, aber das is wirklich so der Bereich, wo/wo i sogn muass, jo, wo fängt sie an, wo hört sie auf?

Die angeführten Interviewpassagen bestätigen im Prinzip die Ergebnisse unserer Dokumentenanalyse: Die Vermittlung der sprachlichen Variation ist ein pädagogisch-didaktisches Problem, weil sprachliche Variation im allgemeinen und plurizentrische Variation im Besonderen in der LehrerInnenausbildung zu wenig oder gar nicht thematisiert werden. Schließlich bieten auch die Lehrwerke, wie deren Analyse gezeigt hat, hier wenig Hilfe und Unterstützung (siehe Kap. 4.3).

\subsubsection{Unterschiede im Deutschen aus der Perspektive von LehrerInnen und SchülerInnen}

Dass Lehrende und SchülerInnen sprachliche Unterschiede zwischen den deutschsprachigen Ländern - Österreich, Deutschland und Schweiz - nicht gleichermaßen differenziert wahrnehmen, zeigen die Antworten auf zwei weitere Fragen. Danach gefragt, ob es ihrer Meinung nach Unterschiede zwischen der deutschen Sprache in Österreich und in Deutschland gibt, waren sich LehrerInnen und SchülerInnen über die Existenz solcher Unterschiede zunächst fast vollständig einig (LehrerInnen $100 \%$, SchülerInnen 98,2 \%, Abb. 31). Gleichermaßen herrschte weitestgehender Konsens darüber, dass es zwischen der Schweiz und Österreich ebenfalls sprachliche Unterschiede gibt (LehrerInnen 99,4\%, SchülerInnen 98,7\%). Auch die Wahrnehmung der Ausspracheunterschiede zwischen Österreich und Deutschland bzw. Österreich und der Schweiz war unter LehrerInnen und SchülerInnen ähnlich stark ausgeprägt ( $97 \mathrm{zu} 93 \%$ bzw. 99,4 zu 97,6\%).

Wurde jedoch nach Unterschieden im „Wortschatz“, in der „Grammatik“ und beim „Kommunikationsverhalten in Gesprächen“ (Pragmatik) gefragt, gab es beim Antwortverhalten der LehrerInnen und SchülerInnen signifikante Diskrepanzen: Unterschiede in der Lexik zwischen Österreich und Deutschland sahen fast $90 \%$ der LehrerInnen, aber nur rund $69 \%$ der SchülerInnen ( $p=0,000$ ). Knapp 60\% der LehrerInnen gaben an, dass es grammatikalische Unterschiede zwischen Österreich und Deutschland gibt, während es unter den SchülerInnen nur halb so viele waren, nämlich $30 \%(\mathrm{p}=0,000)$. Auch bei der Einschätzung der Unterschiede im Gesprächsverhalten gab es zwischen den LehrerInnen und SchülerInnen deutliche Abweichungen: 55,5\% der LehrerInnen meinten, dass es Unterschiede im Kommunikationsverhalten zwischen ÖsterreicherInnen und Deutschen gibt. In der Gruppe der SchülerInnen waren nur 46,4\% dieser Ansicht $(\mathrm{p}=0,029)$. 
In Bezug auf die sprachlichen Eigenheiten der Schweiz lagen die LehrerInnen und SchülerInnen ebenfalls weit auseinander: Während fast $93 \%$ der LehrerInnen Wortschatzunterschiede zwischen Österreich und der Schweiz sehen, tun dies unter den SchülerInnen nur 64\% $(\mathrm{p}=$ o,ooo). Auf der Grammatik-Ebene sahen mehr als die Hälfte der LehrerInnen sprachliche Unterschiede zwischen der Schweiz und Österreich (52,4\%), doch mit 24,4\% nicht einmal ein Viertel der SchülerInnen $(\mathrm{p}=0,000)$. Und was die Pragmatik angeht, werden von $42,1 \%$ der LehrerInnen und 32,6\% der SchülerInnen Unterschiede wahrgenommen $(\mathrm{p}=$ o,016). Diese unterschiedlichen Einschätzungen von LehrerInnen und SchülerInnen können zweifellos zum Teil damit erklärt werden, dass SchülerInnen als linguistische Laien mit diesen Themen wenig vertraut sind.

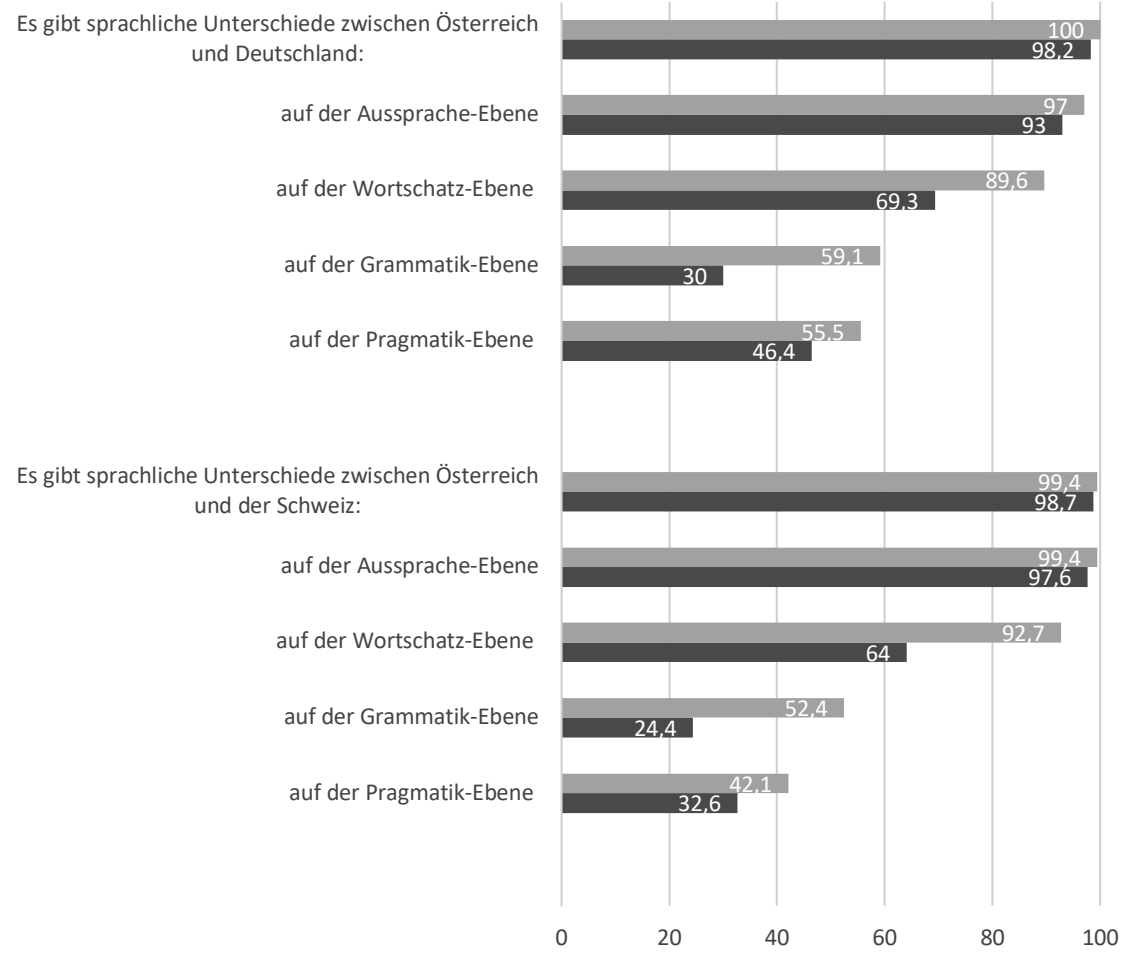

Abb. 31: Sprachliche Unterschiede zwischen Österreich, Deutschland und der Schweiz: LehrerInnen-SchülerInnen-Vergleich (in \%)

In einer weiteren Frage wurden LehrerInnen und SchülerInnen dazu befragt, inwiefern sich auf einer 4-stufigen Skala in einer mündlichen, formellen Situation das in Österreich gesprochene Deutsch zum einen von dem in Deutschland und zum anderen von dem in der Schweiz gesprochenen Deutsch unterscheiden 
würde. Beide Gruppen, sowohl SchülerInnen als auch LehrerInnen, empfanden die Unterschiede zwischen Österreich und Deutschland im mündlichen, formellen Kontext mit insgesamt je rund zwei Drittel als sehr stark oder stark. Die beiden Gruppen unterschieden sich hinsichtlich der Graduierung der Antwort „sehr stark/stark“ signifikant voneinander $(\mathrm{p}=0,005)$ - LehrerInnen wählten die Antwort „sehr stark“ weniger häufig.

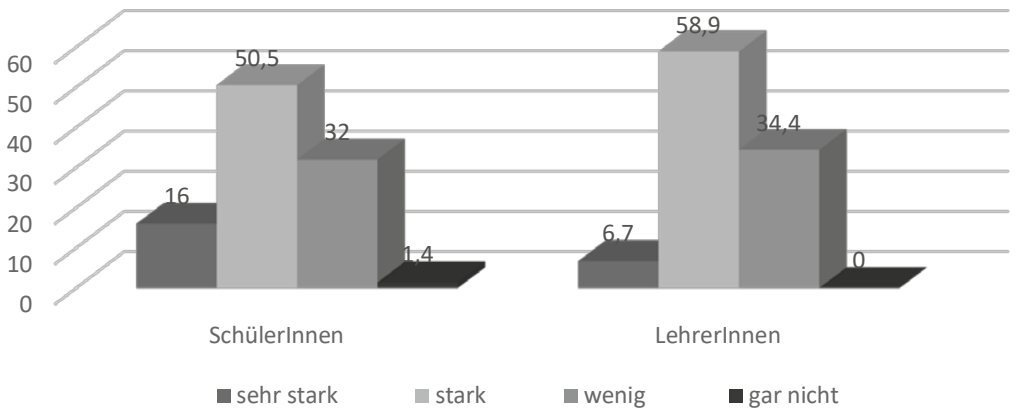

Abb. 32: Das in Österreich gesprochene Deutsch unterscheidet sich von dem in Deutschland gesprochenen Deutsch in einer mündlichen, formellen Situation ... (in \%)

Innerhalb der Gruppe der LehrerInnen konnten keine Unterschiede nach externen Faktoren festgestellt werden. Innerhalb der Gruppe der SchülerInnen ergaben sich signifikante Unterschiede nach dem Geschlecht $(p=0,005)$. Schülerinnen gaben häufiger an, einen sehr starken (17\% Schülerinnen vs. 14,4\% Schüler) oder starken (52,6\% Schülerinnen vs. 47,8 \% Schüler) Unterschied zwischen dem in Österreich und dem in Deutschland gesprochenen Deutsch in einer mündlichen formellen Situation herauszuhören. Demzufolge nehmen Schülerinnen sprachliche Unterschiede zwischen Österreich und Deutschland stärker wahr als Schüler. Im Übrigen nahmen diejenigen SchülerInnen, die vor der Befragung nur im Ausland aufgewachsen sind, die Unterschiede zwischen dem österreichischen und dem deutschen Deutsch stärker wahr $(88,9 \%$ sehr stark oder stark) als SchülerInnen, die nie längere Zeit im Ausland verbracht hatten $(66,5 \%$ sehr stark oder stark) $(\mathrm{p}=0,020)$. Was die Unterschiede zur Schweiz betrifft, sind diese sowohl von den LehrerInnen als auch den SchülerInnen zu fast $90 \%$ als sehr stark/stark empfunden worden (siehe Abb. 33 auf S. 135).

Auch hier konnten innerhalb der Gruppe der LehrerInnen keine Unterschiede im Antwortverhalten festgestellt werden, während die SchülerInnen im Osten die Unterschiede zum Schweizer Deutsch zu 90,5\% als sehr stark oder stark angegeben haben. In den anderen Bundesländern lag der Anteil derer, die Unterschiede sahen, jeweils knapp über $80 \%(p=0,000)$. SchülerInnen mit nichtdeutscher Muttersprache empfanden den Unterschied zwischen dem Schweizer und dem österreichischen Deutsch häufiger als sehr stark oder stark (88,7\%) als SchülerInnen mit ausschließlich Deutsch als Muttersprache $(84,2 \%)(p=0,035)$. 


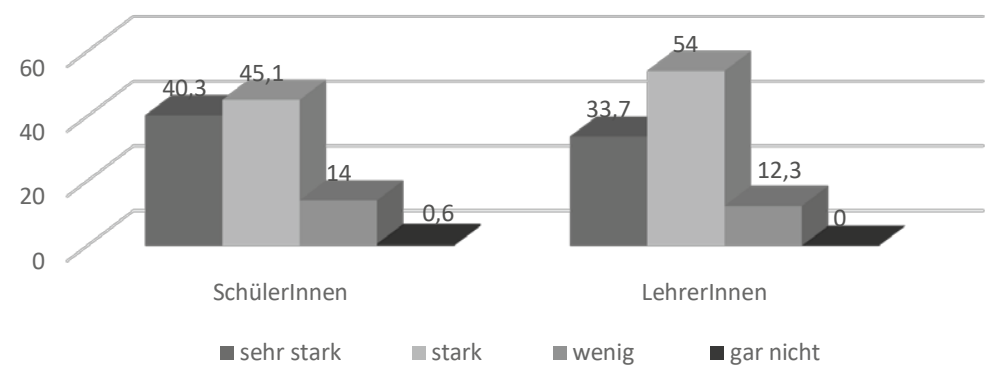

Abb. 33: Das in Österreich gesprochene Deutsch unterscheidet sich von dem in der Schweiz gesprochenen Deutsch in einer mündlichen, formellen Situation ... (in \%)

In diesem Fragenkomplex wurde auch erhoben, in welchem Ausmaß die befragten LehrerInnen und SchülerInnen Unterschiede zwischen dem im Osten und im Westen Österreichs gesprochenen Deutsch in einer formellen, mündlichen Situation wahrnehmen. Etwas mehr als die Hälfte der Befragten nahm die sprachlichen Unterschiede zwischen Ost- und Westösterreich eher stark wahr, während etwas weniger als die Hälfte diesbezüglich kaum Unterschiede wahrgenommen hat.

Die Anteile jener SchülerInnen und LehrerInnen, die die sprachlichen OstWest-Unterschiede sehr stark oder stark empfinden, sind in etwa gleich hoch (je knapp 60\%). Rund $40 \%$ der LehrerInnen und SchülerInnen nahmen hingegen diese Unterschiede auf der formellen Ebene wenig bis gar nicht wahr:

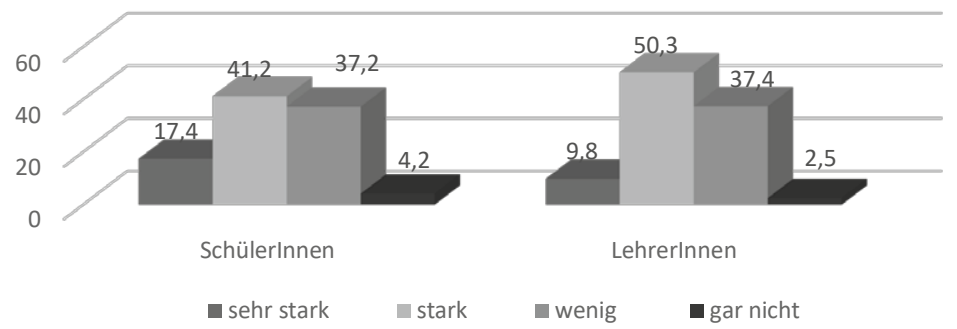

Abb. 34: Das im Osten Österreichs gesprochene Deutsch unterscheidet sich von dem im Westen Österreichs gesprochenen Deutsch in einer mündlichen, formellen Situation ... (in \%)

Schüler gaben indes häufiger $(21,1 \%)$ als Schülerinnen $(14,4 \%)$ an, einen sehr starken sprachlichen Unterschied zwischen Ost- und Westösterreich wahrzunehmen ( $\mathrm{p}=0,002)$. Am stärksten werden solche sprachlichen Unterschiede in Vorarlberg wahrgenommen (67,8\% sehr stark oder stark), in Tirol sowie im Osten Österreichs dagegen etwas weniger deutlich: Hier kreuzten jeweils knapp über $60 \%$ der SchülerInnen „sehr stark“ oder „stark“ an. Die SchülerInnen aus der Region Mitte gaben zu 56,4\% an, dass die Unterschiede sehr stark oder stark seien, die aus den südöstlichen Bundesländern zu $50 \%(p=0,001)$ : 


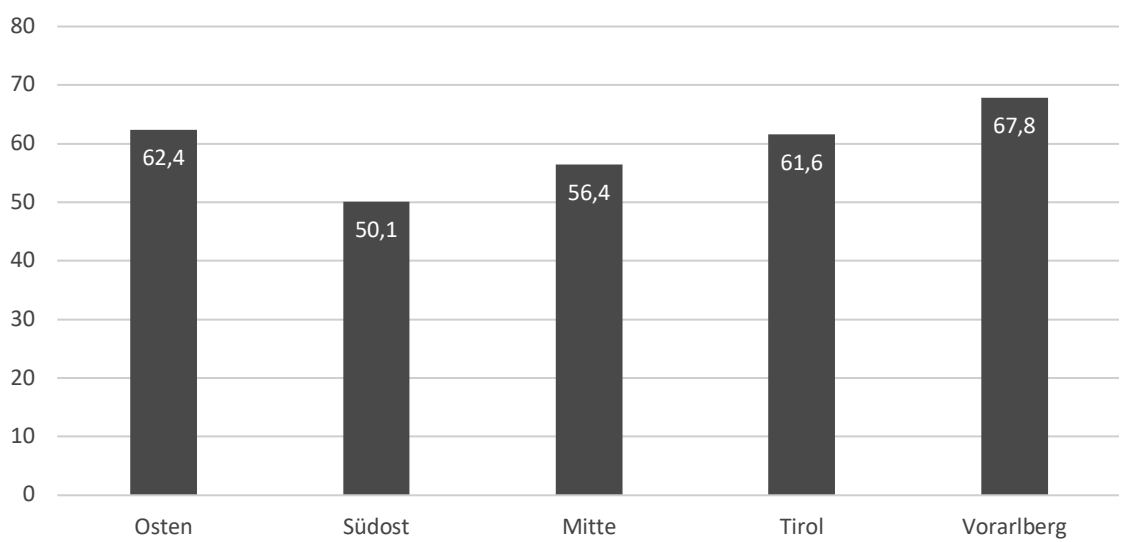

Abb. 35: Wahrgenommene Unterschiede zwischen Ost- und Westösterreich (sehr stark/stark)

SchülerInnen, deren Muttersprache ausschließlich Deutsch ist, gaben öfter an, dass der Unterschied zwischen dem österreichischen Deutsch im Osten und dem im Westen des Landes stark oder sehr stark sei (60,5\%), während der Prozentsatz jener, deren Muttersprache nicht Deutsch ist, deutlich kleiner war $(49,1 \%)(\mathrm{p}=$ o,o03). Insgesamt sind diese Ergebnisse nicht erstaunlich, da sowohl LehrerInnen als auch SchülerInnen österreichisches Deutsch in erster Linie mit dialektalen und umgangssprachlichen Varietäten assoziieren. Fragt man nach der Standardsprache, bekommt man andere Ergebnisse (s. u. Kap. 6.1.4).

\subsubsection{Deutsch als plurizentrische Sprache?}

Um einen Eindruck davon zu gewinnen, wie LehrerInnen und SchülerInnen die deutsche Standardsprache konzeptualisieren, wurden sie gefragt, ob Deutsch als „einheitliche Sprache mit einer einzigen standardsprachlichen (hochdeutschen) Form, die in allen deutschsprachigen Ländern gilt", oder als eine „Sprache mit Unterschieden in der Standardsprache (im Hochdeutschen) zwischen den einzelnen Ländern" zu sehen ist. $90 \%$ der LehrerInnen und 79,2 \% der SchülerInnen wählten hier die zweite Antwort (siehe Abb. 36 auf S. 137).

Signifikante Unterschiede innerhalb der Gruppe der LehrerInnen zeigen sich abhängig vom jeweiligen Ausbildungsort ( $\mathrm{PH} /$ Universität, $\mathrm{p}=0,009$ ). LehrerInnen mit Universitätsausbildung waren nahezu einstimmig (95,4\%) der Ansicht, dass Deutsch eine Sprache mit Unterschieden in der Standardsprache (im Hochdeutschen) zwischen den einzelnen Ländern ist. Diese Meinung war unter den LehrerInnen, die ihre Ausbildung an einer Pädagogischen Hochschule bzw. PädAk gemacht hatten, ebenfalls stark ausgeprägt, aber mit $82,9 \%$ nicht ganz so stark. 


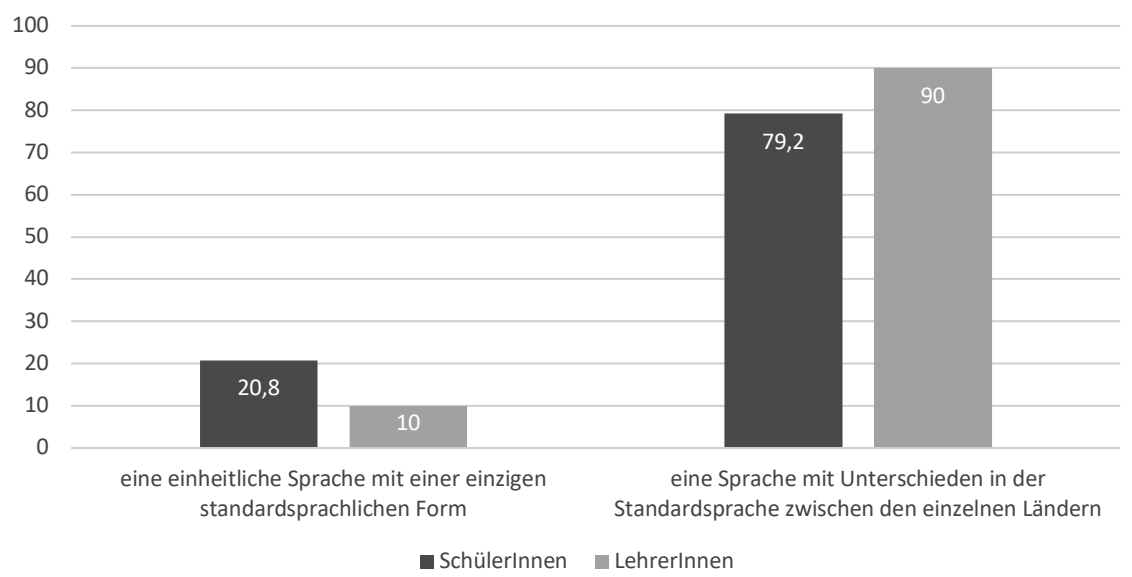

Abb. 36: Deutsch ist ... (in \%)

Innerhalb der Gruppe der SchülerInnen konnten signifikante Unterschiede je nach Region des Schulstandortes festgestellt werden ( $\mathrm{p}=0,037)$, sowie Zusammenhänge mit der Anzahl der Sprachen, die die SchülerInnen in der Schule gelernt hatten $(\mathrm{p}=$ o,o22). Von SchülerInnen der Regionen Mitte $(81,8 \%)$ und Südost $(81,6 \%)$ wurde am häufigsten die Antwortmöglichkeit gewählt, dass Deutsch eine Sprache mit Unterschieden in der Standardsprache zwischen den einzelnen Ländern ist. Die relativ größte Zustimmung zur alternativen Antwort kam von SchülerInnen aus Vorarlberg: 33 \% haben der Aussage zugestimmt, dass Deutsch eine einheitliche Sprache mit nur einer standardsprachlichen Form ist. In Tirol waren es nur $20,7 \%$, in Ostösterreich 21,4\%, in den anderen Bundesländern jeweils rund $18 \%$. Die Antworten der Vorarlberger SchülerInnen wichen damit signifikant von denen in anderen Bundesländern ab. SchülerInnen aus Vorarlberg sind im Vergleich zu ihren KollegInnen aus den anderen österreichischen Regionen relativ öfter monozentrisch ausgerichtet, aber nichtsdestotrotz mit einer deutlichen Mehrheit insgesamt plurizentrisch eingestellt.

Vorarlberg verhält sich hier so wie in anderen Spracheinstellungsfragen etwas anders als das restliche Österreich (vgl. auch Ender/Kaiser 2009). Die Statistik brachte auch das folgende Ergebnis: Je mehr Sprachen die SchülerInnen lernten bzw. gelernt hatten, desto häufiger meinten sie, dass Deutsch eine Sprache mit Unterschieden in der Standardsprache sei. Mehrsprachige SchülerInnen tendieren demnach - bewusst oder unbewusst - zu einer plurizentrischen Einstellung.

Eine weitere Frage, die darauf abzielte, die Vorstellungen von der deutschen Sprache in Österreich zu erfassen, lautete: „Glauben Sie, dass es ein österreichisches Standarddeutsch (Hochdeutsch) gibt?" Darauf antworteten 80,2 \% der LehrerInnen und 59,4 \% der SchülerInnen mit „Ja“, 12,1 \% der LehrerInnen und 24\% der SchülerInnen mit Nein und 7,8\% der LehrerInnen bzw. 16,6\% der SchülerInnen mit „Weiß nicht“. 


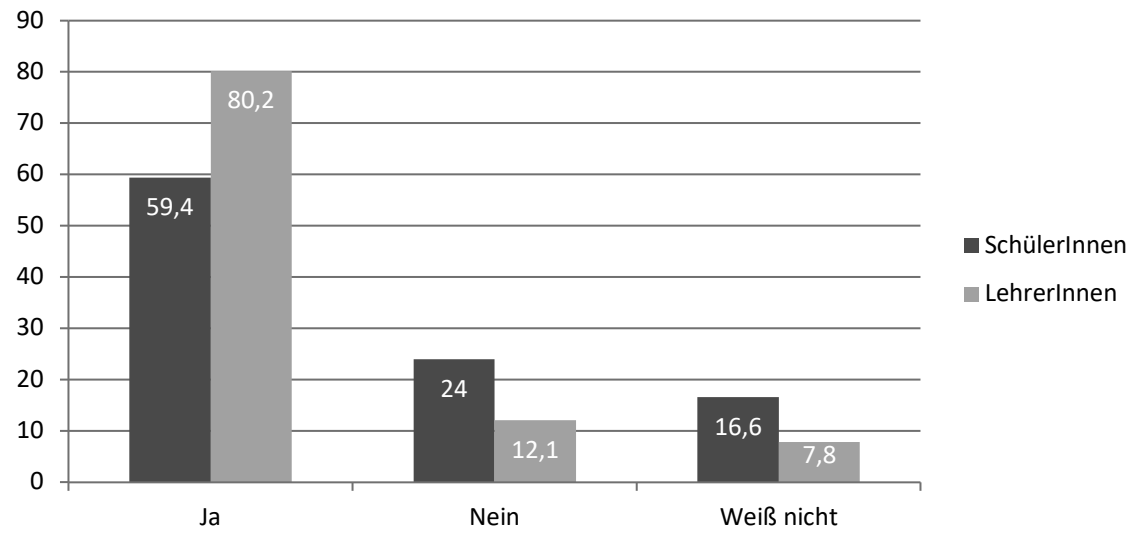

Abb. 37: Glauben Sie, dass es ein österreichisches Standarddeutsch (Hochdeutsch) gibt? (in \%)

Dieses Ergebnis legt ebenso wie die Antworten auf die vorhergehende FragebogenFrage nahe, dass die heimischen LehrerInnen und SchülerInnen mehrheitlich eine plurizentrische Sicht auf die deutsche Sprache haben. Und das, obwohl auf die explizite Nachfrage nur 14,7\% der LehrerInnen und nur 8,1\% der SchülerInnen angegeben haben, dass ihnen das Konzept der plurizentrischen Sprachen bekannt sei.

Innerhalb der Gruppe der LehrerInnen zeigten sich beim Antwortverhalten keine signifikanten Unterschiede, innerhalb der Gruppe der SchülerInnen gibt es jedoch wiederum signifikante Unterschiede nach der Region ( $p=0,000)$ und abhängig davon, wie viele Sprachen gelernt wurden $(\mathrm{p}=0,010)$ und welche Muttersprachen die SchülerInnen $(\mathrm{p}=0,010)$ und welche Muttersprachen deren Eltern hatten $(\mathrm{p}=0,003)$.

Die größte Zustimmung fand die Aussage, dass es ein österreichisches Standarddeutsch gibt, unter den befragten SchülerInnen aus dem Osten Österreichs $(66,5 \%)$. In Tirol (41,3\%) und in Vorarlberg (47,6\%) fand diese Aussage die geringste Zustimmung - knapp weniger als die Hälfte stimmten hier zu. Die Regionen Mitte und Südost liegen im Mittelfeld (59,6 \% bzw. 58,2 \%) (siehe Abb. 38 auf S. 139).

Auch die Anzahl der erlernten Fremdsprachen dürfte einen Einfluss auf die jeweilige Meinung zum heimischen Standarddeutsch haben: Je mehr Sprachen die SchülerInnen erlernt hatten, desto eher stimmten sie der Behauptung zu, dass es ein österreichisches Standarddeutsch gibt: 62,5\% der SchülerInnen, die drei oder mehr Sprachen gelernt hatten, stimmten zu, jedoch nur 54,4\% der SchülerInnen, die nur eine weitere Sprache gelernt hatten. Letztere entschieden sich hingegen am häufigsten für die Antwort „weiß nicht“. Dies spricht dafür, dass durch das Erlernen von Sprachen die Sprachensensibilisierung steigt. Weiters wird das Antwortverhalten der SchülerInnen davon beeinflusst, mit welcher Muttersprache sie aufgewachsen sind. Die größte Unsicherheit bezüglich der Frage, ob es ein 
österreichisches Standarddeutsch gibt, gab es unter denjenigen SchülerInnen, die mit einer anderen Muttersprache als Deutsch aufgewachsen sind: nur 55,2 \% dieser Gruppe stimmten der Frage zu, 26,8 \% gaben an, es nicht zu wissen. Die größte Zustimmung kam mit 64,8 \% aus der Gruppe der SchülerInnen, die mit Deutsch und einer anderen Sprache aufgewachsen waren.

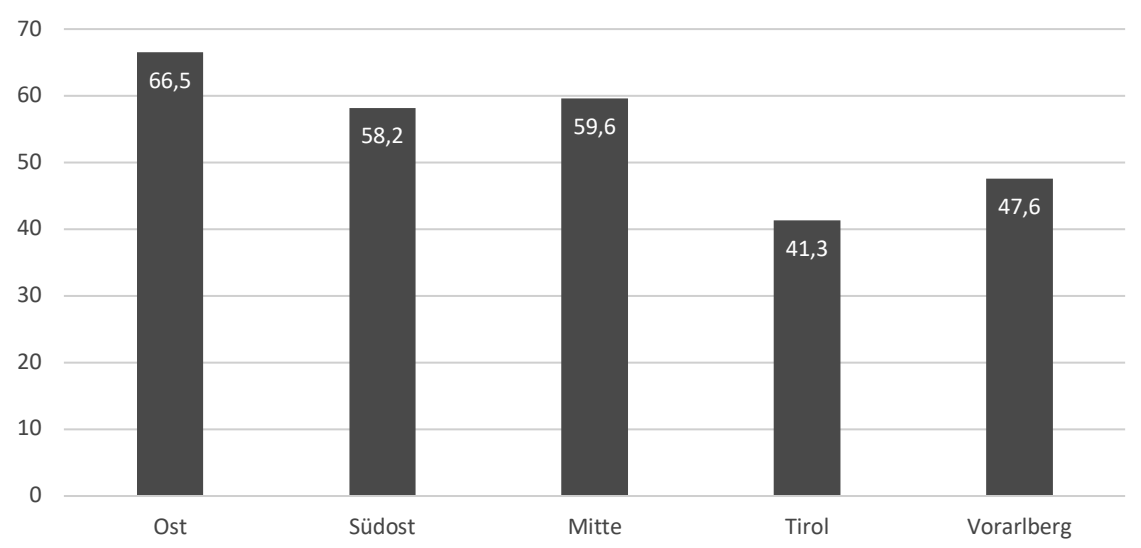

Abb. 38: Ja, es gibt ein österreichisches Standarddeutsch (nach Bundesländern; Schülerlnnen) (in \%)

In der Literatur findet sich die Behauptung, dass man aufgrund von großen sprachlichen Unterschieden zwischen Ostösterreich und Westösterreich nicht von einem österreichischen Deutsch sprechen könne bzw. dass der österreichische Standard der „Sprachwirklichkeit und Sprachidentität der Westösterreicher keinesfalls gerecht“ werde (Putz 2002 ${ }^{2}$, 52). Daher wurde in unserer Untersuchung explizit danach gefragt. Obwohl etwas mehr als die Hälfte der Befragten der Ansicht war, dass es auf der mündlichen Ebene in der Standardsprache starke oder sehr starke Unterschiede zwischen Ost- und Westösterreich gibt, wurde die Behauptung „Innerhalb Österreichs gibt es zu große regionale sprachliche Unterschiede (z. B. zwischen Ostund Westösterreich), als dass es ein eigenes österreichisches Standarddeutsch geben kann" von 81,6\% der LehrerInnen und von 57,4\% der SchülerInnen abgelehnt. D.h.: Die häufig geäußerte Behauptung, man könne wegen zu großer regionaler Unterschiede nicht von einem österreichischen Standarddeutsch sprechen, findet keine Bestätigung in unserem Sample, v. a. nicht unter LehrerInnen. Österreichische LehrerInnen konzeptualisieren die deutsche Sprache mehrheitlich als plurizentrisch. Die regionale Plurizentrik/Pluriarealität steht an zweiter Stelle.

2 In dem relativ polemischen Text von Putz findet sich als Zwischenüberschrift „Michael Ebners Wie sagt man in Österreich?“" (67). Dass in einer peer reviewten Zeitschrift ein derartiger Fehler durchgeht, ist doch erstaunlich. Denn der Doyen der österreichischen Lexikographie heißt natürlich Jakob Ebner. 


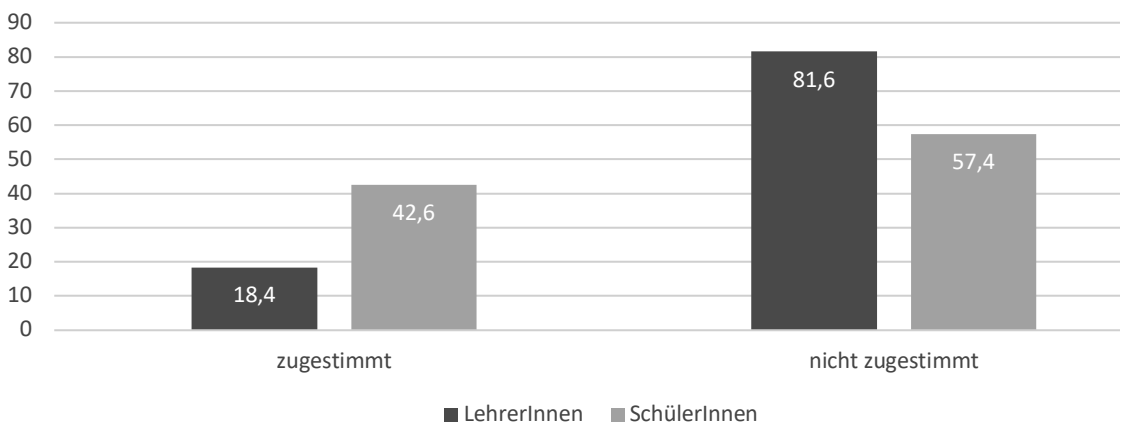

Abb. 39: Innerhalb Österreichs gibt es zu große regionale sprachliche Unterschiede (z. B. zwischen Ost- und Westösterreich), als dass es ein eigenes österreichisches Standarddeutsch geben kann. (in \%)

Unterschiede innerhalb der Gruppe der LehrerInnen fanden sich einerseits bei der Schulform, an der die LehrerInnen unterrichten $(\mathrm{p}=0,011)$ und der Ausbildungsinstitution (PH/Uni $\mathrm{p}=0,007)$ sowie abhängig von der Bekanntheit des Konzepts der Plurizentrik $(\mathrm{p}=0,040)$ : Dass es ,innerhalb Österreichs zu große regionale sprachliche Unterschiede gibt (z. B. zwischen Ost- und Westösterreich), als dass es ein eigenes österreichisches Standarddeutsch geben kann", meinten am (relativ) stärksten die VS-LehrerInnen (30,2\%). Deutlich weniger Zustimmung kam von den beiden anderen LehrerInnen-Gruppen (AHS 10,2 \%, HS/NMS 19\%). Nur $10,3 \%$ der Lehrpersonen, die ein Universitätsstudium absolviert haben, stimmten zu; bei AbsolventInnen einer PH/PädAk waren es 26,7\%. Ist Lehrenden das plurizentrische Konzept bekannt, dann lehnen sie zu fast $100 \%$ die Aussage ab, dass es innerhalb Österreichs zu große regionale sprachliche Unterschiede gibt (z. B. zwischen Ost- und Westösterreich), als dass es ein eigenes österreichisches Standarddeutsch geben kann.

Signifikante Unterschiede innerhalb der Gruppe der SchülerInnen gab es abhängig vom Geschlecht $(\mathrm{p}=0,010)$, der Region $(\mathrm{p}=0,001)$ und der Muttersprachen $(\mathrm{p}=0,004)$. Der Aussage, dass es ,innerhalb Österreichs zu große regionale sprachliche Unterschiede gibt (z. B. zwischen Ost- und Westösterreich), als dass es ein eigenes österreichisches Standarddeutsch geben kann", stimmen wesentlich mehr männliche (46,8 \%) als weibliche (39,4\%) SchülerInnen zu. Männliche Schüler sind offenbar weniger stark von der Existenz eines eigenen österreichischen Standarddeutsch überzeugt als weibliche. Im Regionenvergleich gab es dazu wie erwartet mehr Zustimmung unter SchülerInnen aus Vorarlberg (56,6\%) und Tirol $(55,4 \%)$ als in der Region Mitte (38,8 \%) und Südost (38,5\%) und Ost (41,6\%). Beim Vergleich der SchülerInnen mit deutscher oder nichtdeutscher Muttersprache zeigte sich, dass diejenigen, die mit einer nichtdeutschen Muttersprache aufgewachsen waren, der Aussage, es gäbe zu große regionale Unterschiede innerhalb 
Österreichs, um von einem österreichischen Deutsch sprechen zu können, am stärksten zustimmen (53,6 \%) - im Gegensatz zu SchülerInnen, die nur Deutsch als Muttersprache hatten (40,4\%). Diese Diskrepanz könnte sich damit erklären lassen, dass SchülerInnen mit nichtdeutscher Muttersprache ungewohnte sprachliche Färbungen stärker auffallen.

Der im selben Fragenkomplex vorgelegten Aussage „Es gibt ein überregionales österreichisches Standarddeutsch, das sich vom Deutsch Deutschlands in manchen Bereichen klar unterscheidet" stimmten 50,4\% der SchülerInnen und $77,3 \%$ der LehrerInnen zu. Also nur die Hälfte der SchülerInnen stimmte bei dieser Kontrollfrage bezüglich der überregionalen österreichischen Standardvarietät zu (im Vergleich zu drei Vierteln der LehrerInnen) - sie nehmen möglicherweise die regionalen Unterschiede als wichtiger wahr im Vergleich zu den Lehrkräften, bzw. sie tun sich schwer damit, standardsprachliche Variation konzeptionell einzuordnen. Dies deckt sich mit dem Befund Schmidlins (2013, 23), wonach von linguistischen Laien die Plurizentrik von Standardsprachen oft für dialektale Variation gehalten wird.

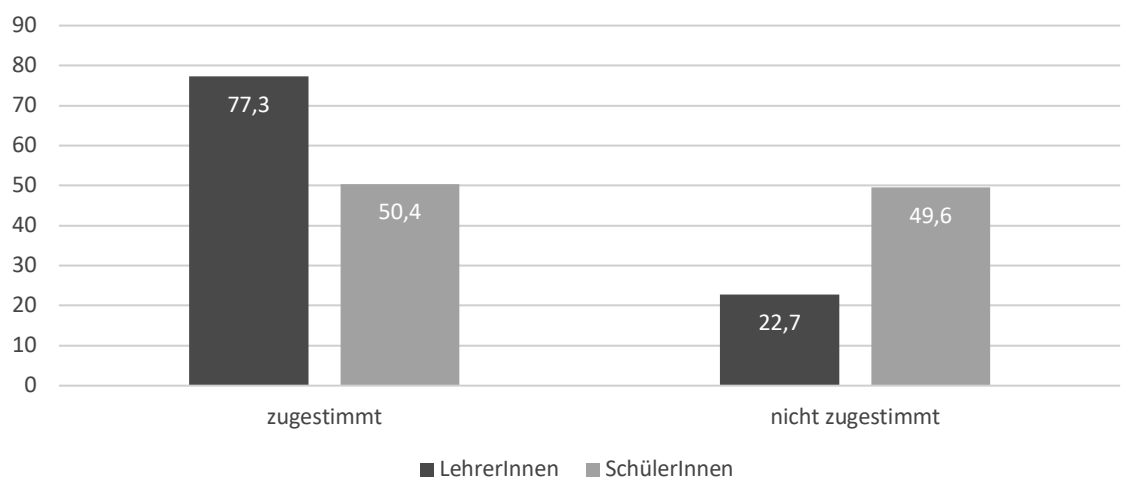

Abb. 40: Es gibt ein überregionales österreichisches Standarddeutsch, das sich vom Deutsch Deutschlands in manchen Bereichen klar unterscheidet. (in \%)

Signifikante Unterschiede innerhalb der Gruppe der LehrerInnen finden sich auch hier abhängig von der Schulform und davon, ob das plurizentrische Konzept bekannt ist. Der Aussage in Abb. 40 stimmten vor allem AHS-/Sek.-II-LehrerInnen $\mathrm{zu}(85,2 \%)$, HS/NMS- und VS-LehrerInnen lagen bei $66,5 \%$ bzw. $67,9 \%(\mathrm{p}=0,028)$. Von denjenigen LehrerInnen, denen das plurizentrische Konzept bekannt war, stimmten $96 \%$ dieser Aussage zu; unter den LehrerInnen, die das plurizentrische Konzept nicht gekannt haben, waren es lediglich $74,1 \%(\mathrm{p}=0,016)$.

Bei den SchülerInnen fanden sich Unterschiede nach dem Geschlecht und nach Region und den Muttersprachen. 54,4\% der weiblichen und im Gegensatz dazu nur 44,8\% der männlichen SchülerInnen stimmen der Aussage zu, es gäbe ein überregionales österreichisches Standarddeutsch $(p=0,001) \mathrm{mit}$ 
Unterschieden zum deutschen Standarddeutsch. Schülerinnen stimmen also, wie schon der obige Befund gezeigt hat, der Annahme eines überregionalen österreichischen Standarddeutsch deutlich stärker zu als Schüler. Die größte Zustimmung kam von SchülerInnen aus dem Osten Österreichs (57,1\%), die wenigste von SchülerInnen aus Tirol $(33,1 \%)$ und aus Vorarlberg $(34,9 \%)$ ( $\mathrm{p}=$ o,0oo). SchülerInnen im Osten haben ein ausgeprägteres Bewusstsein für ein eigenes österreichisches Deutsch als SchülerInnen der westlichen Bundesländer, bei denen nur eine Minderheit der Annahme eines überregionalen österreichischen Standarddeutsch zustimmt. Weiters glaubten 52,4\% der befragten SchülerInnen mit Deutsch als Muttersprache, dass es ein überregionales österreichisches Standarddeutsch gibt. Unter den SchülerInnen mit nichtdeutscher Muttersprache waren es nur noch $42,5 \%(p=0,032)$. Dies ist im Übrigen eine immer wiederkehrende Tendenz in unseren Daten, dass SchülerInnen mit einer anderen Muttersprache als Deutsch weniger stark plurizentrisch orientiert sind als deutsche MuttersprachlerInnen in Österreich.

In diesem Fragenblock wurde auch die Zustimmung zur Aussage: „Österreich und Süddeutschland haben sprachlich mehr gemeinsam als Nord- und Süddeutschland“ abgefragt, wobei hier bewusst nicht nach der Standardsprache gefragt wurde. 55,5\% der SchülerInnen und 76,1\% der LehrerInnen stimmten dieser Aussage zu.

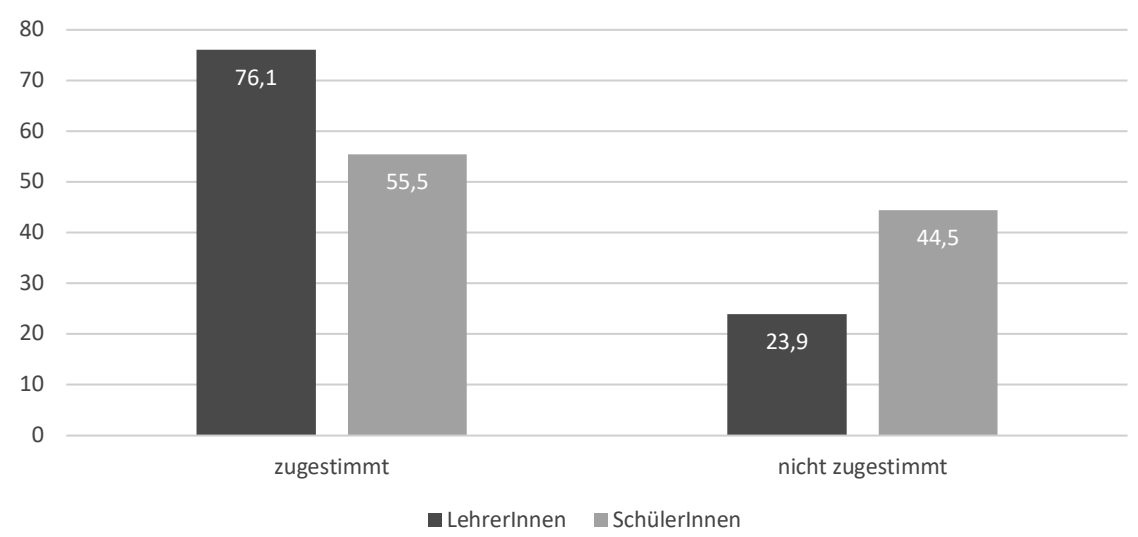

Abb. 41: Österreich und Süddeutschland haben sprachlich mehr gemeinsam als Nord- und Süddeutschland. (in \%)

Der Aussage, dass Österreich und Süddeutschland sprachlich mehr gemeinsam haben als Nord- und Süddeutschland, stimmten unter den LehrerInnen Männer signifikant $(\mathrm{p}=0,020)$ stärker $\mathrm{zu}(91,2 \%)$ als Frauen $(72,1 \%)$. Innerhalb der Gruppe der SchülerInnen ergaben sich Unterschiede nach dem Geschlecht und der Region. 
$66 \%$ der männlichen, aber nur 48,3\% der weiblichen SchülerInnen waren der Ansicht, dass es mehr Gemeinsamkeiten zwischen Österreich und Süddeutschland gibt als zwischen Nord- und Süddeutschland $(\mathrm{p}=\mathrm{o}, \mathrm{ooo})$. Die stärkste Zustimmung unter den SchülerInnen zu dieser Aussage gibt es in der Region Mitte, gefolgt von Tirol (57,9\%), was sich durch die sprachliche und geographische Nähe zu Bayern erklären lässt. Am schwächsten war die Zustimmung im Osten $(49,7 \%)$ und in Vorarlberg $(51,8 \%)(\mathrm{p}=0,002)$ - in letzterem wohl wegen der sprachlichen Nähe zur allemannischsprachigen Schweiz.

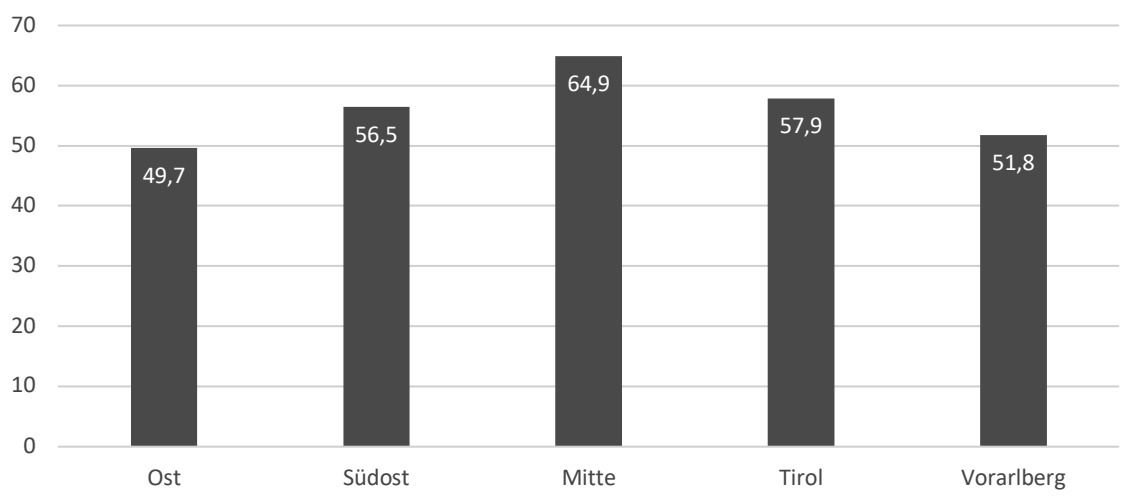

Abb. 42: Schülerlnnen: Österreich und Süddeutschland haben sprachlich mehr gemeinsam als Nord- und Süddeutschland. (in \%)

Um abschließend auf die sprachlichen Ost-West-Unterschiede innerhalb Österreichs zurückzukommen: Von einem kleinen Teil (knapp ein Fünftel) der befragten LehrerInnen bzw. SprachnormexpertInnen wird das Konzept eines überdachenden österreichischen Standarddeutsch in Frage gestellt. Für die überwiegende Mehrheit (rund $80 \%$ ) gibt es jedoch ein eigenes österreichisches Standarddeutsch. Bei den SchülerInnen fällt die Zustimmung deutlich geringer aus. Mehr als $40 \%$ stellen eine österreichische Standardvarietät in Frage.

Die qualitativen Daten aus unseren Interviews haben die Ergebnisse der quantitativen Erhebung im Prinzip bestätigt. Gleichzeitig brachten sie aber eine differenziertere Sicht. Alle in den Interviews befragten LehrerInnen nahmen sprachliche Unterschiede zwischen Ost- und Westösterreich auf der Dialektebene wahr. Die meisten Lehrenden sahen jedoch auch auf standardsprachlicher Ebene Unterschiede.

Stark betont wurden die Unterschiede von einem Lehrer aus Vorarlberg (MV1). Die folgenden Ausschnitte aus dem Interview mit ihm bestätigen die Ergebnisse aus der Fragebogenerhebung, durch welche immer wieder ersichtlich wird, dass das Antwortverhalten in Vorarlberg oft ganz anders ist als in Tirol bzw. im übrigen Österreich: 
Also wir sprechen nicht österreichisch, wir sprechen Dialekt. Also auch unser Deutsch ist nicht österreichisches Deutsch (die Leute) ... Leiberl ist ihnen/das ist kein Begriff. [...] (und wir) bekommen mit, dass wir eine Minderheit sind, sprachlich, sag ich jetzt einmal. Also sie sind sich nicht - ident mit den Schweizern, sondern irgendwie in einer Zwischenposition, (xxx) wirklich originell. Also das ist eben diese Zugehörigkeit zu/ zu Österreich, no na net, ungebrochen und/und auch völlig unbestritten, ah und auf der anderen Seite aber die sprachliche Nähe zur Schweiz, (die uns allerdings) / das ist nicht unser Land. Und aus dem Grund diese komische Zwischenposition. [...]

Jaja, aber das ist in Vorarlberg natürlich (lacht)/wir sind ja wirklich Grenzland, wir sind/wir sind de facto Deutsche und sind Schweizer und sind Österreicher, halt alles in Einem, und das ist/von uns ist Deutschland wirklich sehr nah! Also es is (xxx)/also für uns is München < deutlich näher als Wien (lachend) $>$ !

Folgende Ausschnitte aus dem Interview mit einer Tiroler Lehrerin (FT2) thematisieren ebenfalls Unterschiede zwischen Ost- und Westösterreich:

Also I hob zum Beispiel praktisch keine Schüler mehr, die den Ausdruck „Obers“ selber verwenden. In Tirol hast des a nit Obers, des is also für uns ein ostösterreichisches Wort, des hast Rahm. Es gibt Süßrahm und Sauerrahm. Net? Und in der Schweiz is Rahm a ganz normales Wort. Aber bei uns, ... die Schüler assoziieren das inzwischen nur mehr mit Sauerrahm. Für Süßrahm sogns Sahne.

Die folgende Lehrende aus Tirol (FT1) thematisierte den Einfluss des Fremdenverkehrs auf die Verwendung der Küchensprache.

Die üblichen Dinge im Vokabular ... also Paradeiser, Kartoffel, Marille und so weiter, das is in Tirol nicht so ah relevant, weil in Tirol hat man IMMER Tomate gsogt. Jo? Und IMMER Kartoffel und net Erdäpfel/ah Erdapfel. [...] aber in/Tirol is a sehr stoak da der Einfluss des Fremdenverkehrs [...] alle Speisekarten sind natürlich bundesdeutsch. Da kriegt man halt tatsächlich Aprikosenmarmelade, Sahne und so weiter, klarerweise. Und des is a den/den Schülern net wirklich bewusst, dass sie a Sahne sogn ganz //ah natürlicherweise, und "Obers" is für sie scho fast a bisl fremd eben. Das is Ostösterreich, ja? Also des sind so verschiedene Einflüsse, die ma do feststellen kann.

Um die Ergebnisse dieses Subkapitels zusammenzufassen: Auf die Frage, wie sie die Mehrheitssprache in Österreich benennen, antwortete die große Mehrheit der Befragten mit dem Wort „Deutsch“. „Österreichisches Deutsch“ wurde immerhin von 19,6\% der LehrerInnen und 11,2\% der SchülerInnen genannt. Dabei wird das gesprochene Deutsch in Österreich in erster Linie mit umgangssprachlichen und dialektalen Varietäten assoziiert, erst an dritter Stelle folgt die standardnahe Varietät der Mediensprache. Was die staatsübergreifende Variation des Deutschen betrifft, ist bei einer Mehrheit der Befragten intuitiv ein plurizentrisches 
Verständnis vorhanden, auch wenn das Konzept nur einer kleinen Minderheit bekannt ist. Und was die innerösterreichische Variation betrifft, sind sich die Befragten durchaus der regionalen Unterschiede bewusst, sie werden aber mehrheitlich nicht als so relevant eingeschätzt, dass dadurch ein gemeinsames überdachendes österreichisches Standarddeutsch in Frage gestellt würde. Bei den SchülerInnen ist die Zustimmung allerdings deutlich geringer als bei den LehrerInnen. Die Ergebnisse zeigten bei den Sprachnormautoritäten - also den LehrerInnen - eine stärkere Standard- und Plurizentrikorientierung. Unterschiede in den Spracheinstellungen innerhalb der LehrerInnen zeigen sich v. a. abhängig vom Alter, der Ausbildungsinstitution und der Schulform, bei den SchülerInnen sind sie abhängig von der Region, dem Geschlecht, der Erstsprache und der Mehrsprachigkeit/den (Fremd-)Sprachenkenntnissen.

\subsection{Spracheinstellungen gegenüber den Varietäten des Deutschen}

\subsubsection{Korrektheit des österreichischen Deutsch}

Mit Blick auf die Einstellungen zur und die „Sprachloyalität" gegenüber der eigenen Varietät (vgl. Schmidlin 2011, 219; vgl. Kap. 5.1) und den in der Literatur behaupteten Minderwertigkeitskomplex der ÖsterreicherInnen, wonach diese ihre eigene Varietät des Deutschen nicht für gleichwertig halten würden, haben wir auch nach der Korrektheit des österreichischen Deutsch gefragt. Die konkrete Frage war: „Halten Sie das Standarddeutsch (Hochdeutsch), das in Österreich verwendet wird, für genauso korrekt wie das in Deutschland?"

Zunächst haben wir auf diese einfache Frage eine sozial erwünschte, ,politisch korrekte" Antwort erhalten: Von der überwiegenden Mehrheit der LehrerInnen und auch der Mehrheit der SchülerInnen ( $86 \%$ bzw. 67,7 \%) kam die Antwort „Ja“. Nur 8,5\% der Lehrenden und 22,7\% der SchülerInnen antworteten mit „Nein“, während sich ein kleiner Teil der LehrerInnen und SchülerInnen (5,5\% bzw. 9,6 \%) diesbezüglich unsicher zeigte („weiß nicht“) (siehe Abb. 43 auf S. 146).

Die Antworten auf eine Kontrollfrage weiter unten im Fragebogen lieferten jedoch ein abweichendes Ergebnis. In dieser Frage hatten die LehrerInnen und SchülerInnen die Möglichkeit, ihre Einstellung zur Korrektheit des österreichischen Deutsch auf einer vierstufigen Skala differenzierter einzuordnen. Eingebettet in den plurizentrischen Vergleichskontext (Englisch in Großbritannien/ USA, Französisch in Frankreich/in der Schweiz) sollten die Befragten angeben, wie sehr sie der folgenden Aussage zustimmen oder sie ablehnen: „Deutsches Deutsch ist korrekter als österreichisches Deutsch“. Das überraschende Resultat: Nur 44,1\% der LehrerInnen lehnten diese Aussage dezidiert ab, und insgesamt 16,1\% der LehrerInnen stimmten der Aussage sogar sehr stark oder stark zu. D. h., viele der befragten LehrerInnen hielten das deutsche Deutsch für (etwas) 
korrekter (siehe Abb. 44 und 45 auf S. 147). Dieses Ergebnis war bemerkenswert, da bei der Frage zuvor (siehe Abb. 43) noch $86 \%$ der LehrerInnen angegeben hatten, dass sie das österreichische Standarddeutsch für genauso korrekt wie das deutsche Standarddeutsch halten. Durch die Kontrollfrage hatte sich die Zahl der LehrerInnen, die der eigenen Varietät gegenüber sehr loyal eingestellten waren, also fast halbiert. Erklären lassen sich die unterschiedlichen Ergebnisse mit der Zahl der LehrerInnen, die das österreichische Deutsch zwar tendenziell für gleichermaßen korrekt wie deutschländisches Deutsch hielten, aber letztlich offenbar doch kleine Zweifel hatten. Diese Gruppe der „unsicheren“ LehrerInnen finden wir in dem relativ großen Teil der LehrerInnen (knapp 40\%), die dieser Aussage zwar nur „wenig“ zugestimmt, sie aber auch nicht abgelehnt haben, was auf eine ambivalente Spracheinstellung zum österreichischen Deutsch hindeutet.

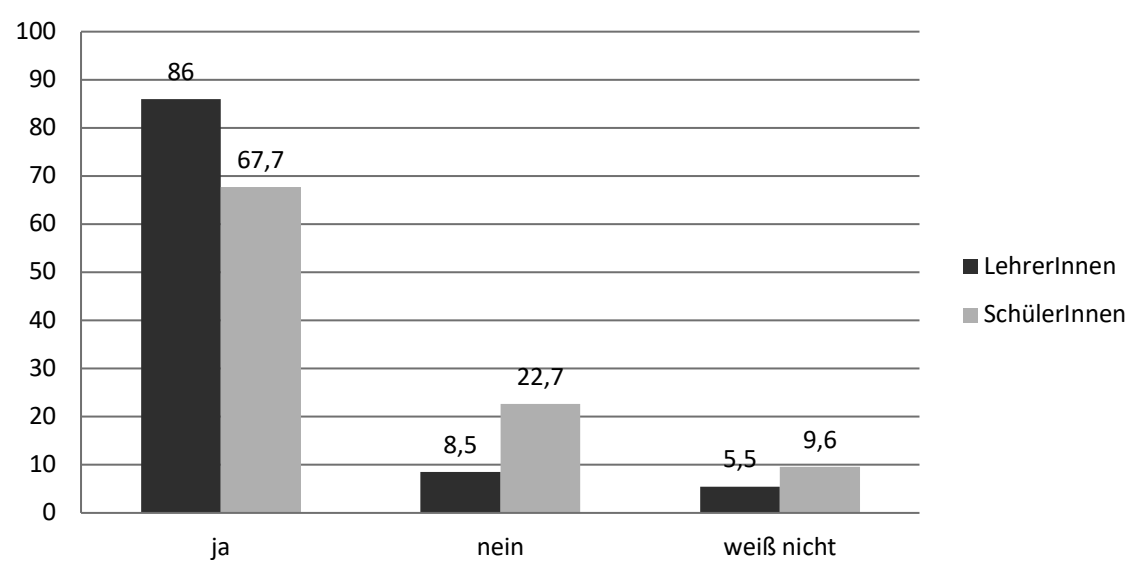

Abb. 43: Österreichisches Deutsch gleich korrekt wie deutsches Deutsch? - LehrerInnen/ SchülerInnen (in \%)

Spannend ist in diesem Zusammenhang auch das Ergebnis hinsichtlich der Korrektheit von britischem und amerikanischem Englisch: Weniger als ein Drittel der LehrerInnen hielt amerikanisches Englisch für gleichermaßen korrekt wie britisches Englisch, und fast $70 \%$ der österreichischen LehrerInnen stimmten der Aussage, dass britisches Englisch korrekter sei als amerikanisches Englisch, mehr oder weniger zu (siehe Abb. 44 auf S. 147).

Bei der „Korrektheitsfrage“ fielen die Ergebnisse bei den SchülerInnen etwas „österreichskeptischer“ aus als bei den LehrerInnen. Der Anteil jener, die die Aussage „Deutsches Deutsch ist korrekter als österreichisches Deutsch“klar ablehnten, war bei den SchülerInnen deutlich geringer als bei den LehrerInnen. Gleichermaßen war die Anzahl jener SchülerInnen, die der Aussage „sehr stark“ oder „stark“ zustimmten, deutlich höher als bei den LehrerInnen. Sie verteilten sich bei ihren Antworten relativ gleichmäßig in drei fast gleich große Gruppen: Ein 
Drittel (33,3\%) stimmte der Aussage "sehr stark“ oder „stark“ zu, ein knappes Drittel (31,9\%) lehnte die Aussage ab, und ein gutes Drittel (34,8\%) umfasste die Gruppe der „unsicheren“ SchülerInnen, die der Aussage „wenig“ zustimmten.

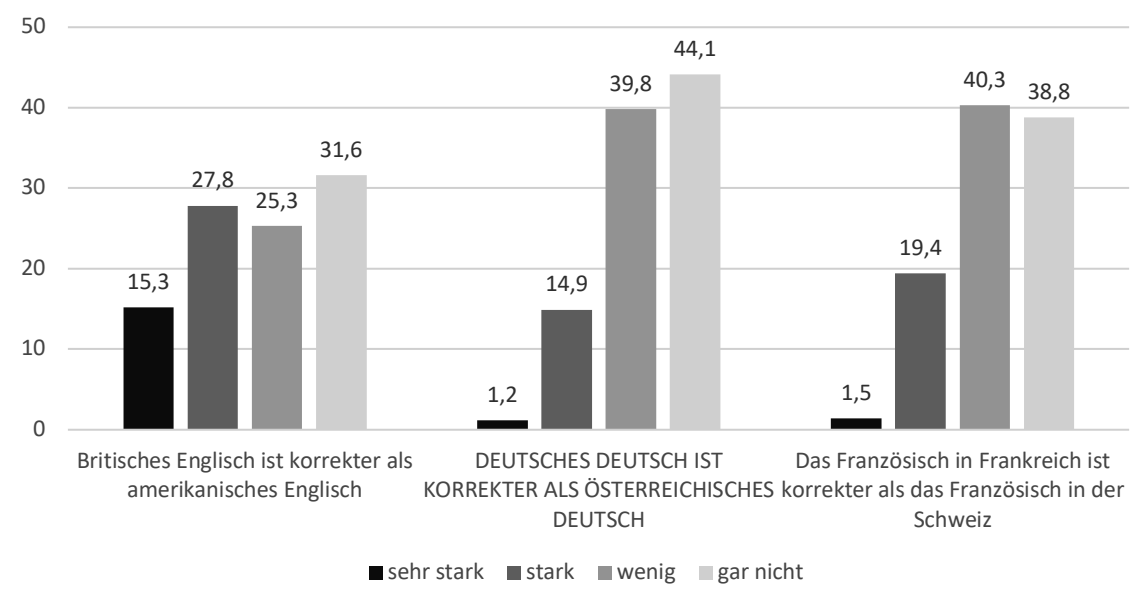

Abb. 44: LehrerInnen: Deutsches Deutsch korrekter? (in \%)

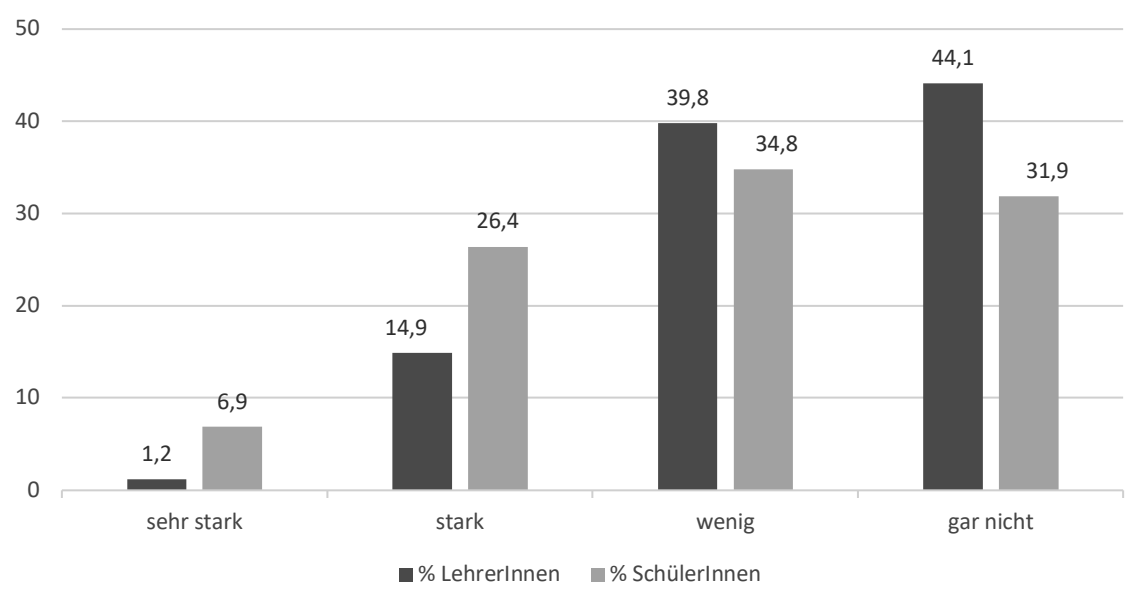

Abb. 45: Deutsches Deutsch korrekter? Lehrerlnnen/Schülerlnnen (in \%)

Die TeilnehmerInnen der Gruppendiskussionen wurden mit den widersprüchlichen Ergebnissen der beiden oben genannten Fragen konfrontiert, und in beiden Gruppen gab es lebhafte Diskussionen dazu, die eine tiefsitzende Ambivalenz gegenüber dem österreichischen Deutsch bestätigten. So meinte eine Schülerin: „Also grammatisch würd ich fast sagen, dass die Österreicher inkorrekt sind, aber sonst eigentlich gar nicht.“ (F1) Und eine andere $\left(\mathrm{F}_{3}\right)$ : 
Also ich stimm dem schon: teilweise zu, weil ichs einfach so seh, dass sich die Deutschen einfach mehr an das Geschriebene halten, für mich hörts sich halt so an. Und was die F1 schon gesagt hat, dass die Österreicher einfach so daherreden und nicht wirklich auf die Grammatik oder so etwas achten ... da hab ich halt bei den Deutschen schon mehr das Gefühl, dass sie mehr - ähm - wirklich nach der Schrift so reden. ${ }^{3}$

In der Gruppendiskussion der LehrerInnen wurde das Ergebnis der Fragebogenerhebung, wonach für nur knapp $45 \%$ der LehrerInnen das deutsche Deutsch nicht korrekter als das österreichische Deutsch ist, ausführlich diskutiert.

Die Diskussion drehte sich primär um Erklärungsversuche dafür, warum das österreichische Deutsch von vielen DeutschlehrerInnen und SchülerInnen als nicht gleichwertig wahrgenommen wird. Durchgängig wurden ein "geringes Selbstbewusstsein“, ein „Minderwertigkeitskomplex“, „, gewisse Unterwürfigkeit“ (F2) und mangelndes Nationalbewusstsein auf österreichischer Seite diagnostiziert. Unterm Strich konnten anhand der gesammelten Bemerkungen Phänomene der Asymmetrie zwischen der A- und D-Varietät festgestellt werden: „die (die SprecherInnen des deutschen Deutsch) ham/ glauben sie ham das nicht nötig; wo wir weniger ah Selbstbewusstsein haben“, wie eine Lehrerin (F8) meinte.

Um ein möglichst differenziertes Bild der vorhandenen Spracheinstellungen zu erhalten, wurden sowohl die SchülerInnen als auch die LehrerInnen danach gefragt, ob es ihrer Meinung nach ein besonders gutes Deutsch gebe und wo dieses zu finden sei. Hier ergaben sich signifikante Unterschiede $(\mathrm{p}=0,018)$ : Während $32,1 \%$ der SchülerInnen der Meinung waren, dass es ein besonders gutes Deutsch gibt, war das nur bei 22,7\% der Lehrpersonen der Fall.

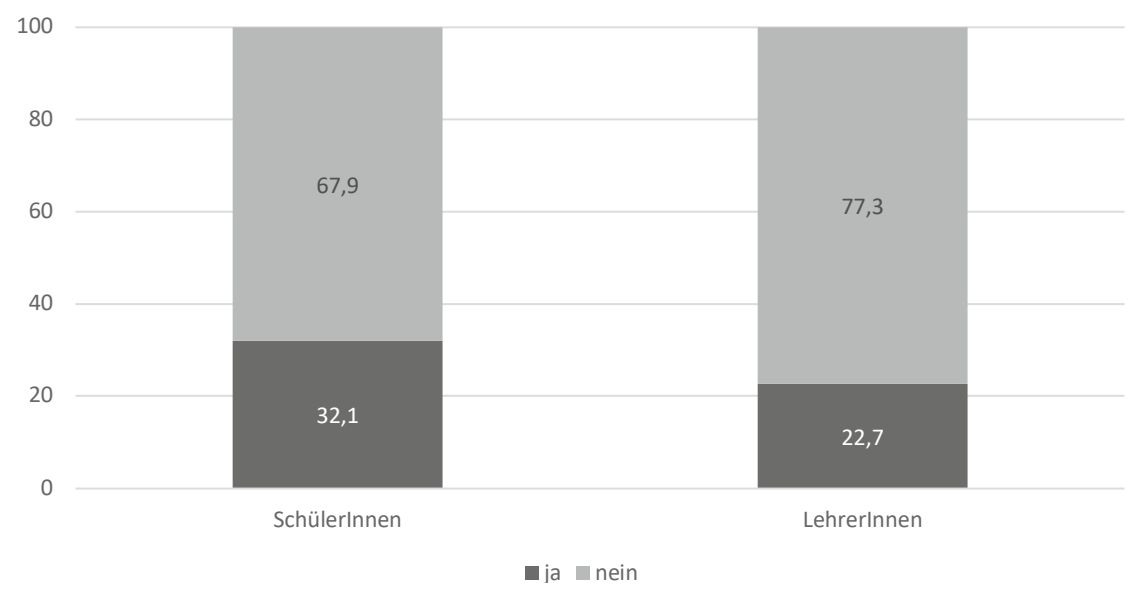

Abb. 46: Gibt es Ihrer Meinung nach ein besonders gutes Deutsch? (in \%)

3 Kursivsetzungen dienen als Hervorhebung und verweisen auf im Textteil erwähnte inhaltliche Argumente. 
Aussagekräftige und signifikante Auffassungsunterschiede ( $\mathrm{p}=0,003)$ zwischen LehrerInnen und SchülerInnen gab es auch, als danach gefragt wurde, ob man das besonders gute Deutsch regional lokalisieren könne. In der Gruppe derer, die meinten, dass es ein besonders gutes Deutsch gibt (32,1\%), waren die SchülerInnen mit knapper Mehrheit der Ansicht, dass das besonders gute Deutsch in Österreich zu finden sei, und nur knapp ein Drittel davon verortete das besonders gute Deutsch in Deutschland. Unter den Lehrenden sah die Sache etwas anders aus: Nur knapp ein Fünftel derjenigen, für die ein besonders gutes Deutsch existiert, meinte, dass dieses in Österreich zu finden sei. Rund $40 \%$ dieser LehrerInnenGruppe verorteten es in Deutschland.

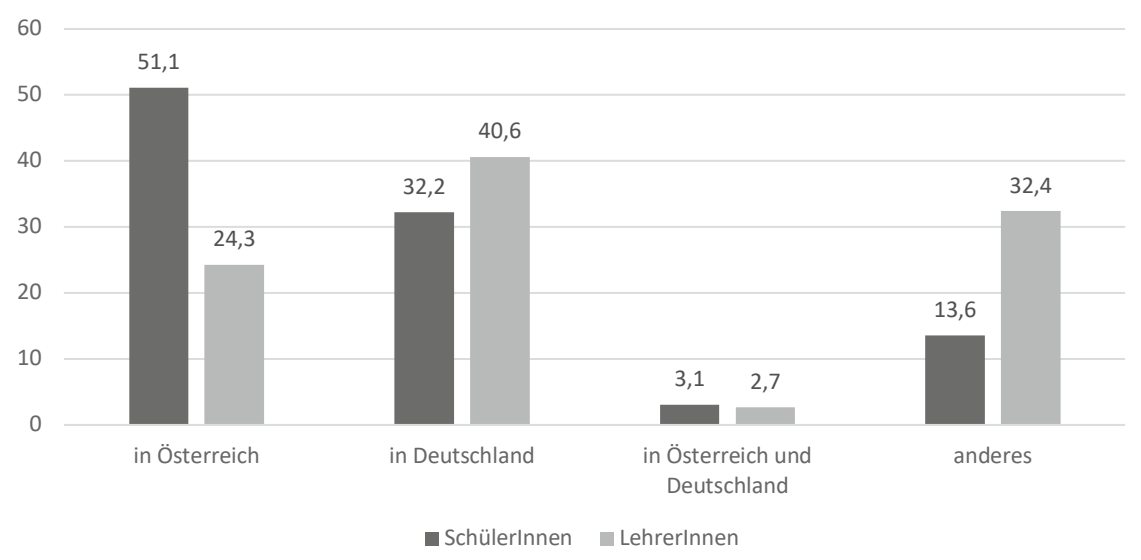

Abb. 47: Wo (in welchem Land/in welcher Region) wird besonders gutes Deutsch gesprochen? (in \%)

Wegen der auch in der Literatur (Clyne 1995, Muhr 1987, Ammon 1995) erwähnten geringen „Loyalität“ der ÖsterreicherInnen gegenüber der eigenen Varietät wurden die ProbandInnen in der Fragebogenerhebung auch danach gefragt, was ihrer Meinung nach passieren würde, wenn sie als österreichische SprecherInnen mit deutschen SprecherInnen zusammentreffen. Verschiedene Szenarien waren als Antwortmöglichkeiten vorgesehen: Der Proband/die Probandin würde sich anpassen; der/die deutsche/r Sprechpartner/in passt seine/ihre Sprache an; beide passen sich an; keiner passt sich an; nichts davon trifft zu. Wie Abb. 48 zeigt, meinte rund die Hälfte der befragten LehrerInnen und SchülerInnen (54,1 \% bzw. 46,4 \%), dass sie sich an die/den deutsche/n Gesprächspartner/in anpassen würden. Etwa ein Viertel meinte, beide würden sich einander sprachlich anpassen (LehrerInnen $23,9 \%$, SchülerInnen $27,6 \%$ ), und etwas weniger, dass sich keiner anpassen würde (17\% bzw. 18,1\%). Allerdings rechnete kaum jemand damit, dass sich der/ die deutsche Gesprächspartner/in anpasst (LehrerInnen o \%, SchülerInnen 1,6 \%). 


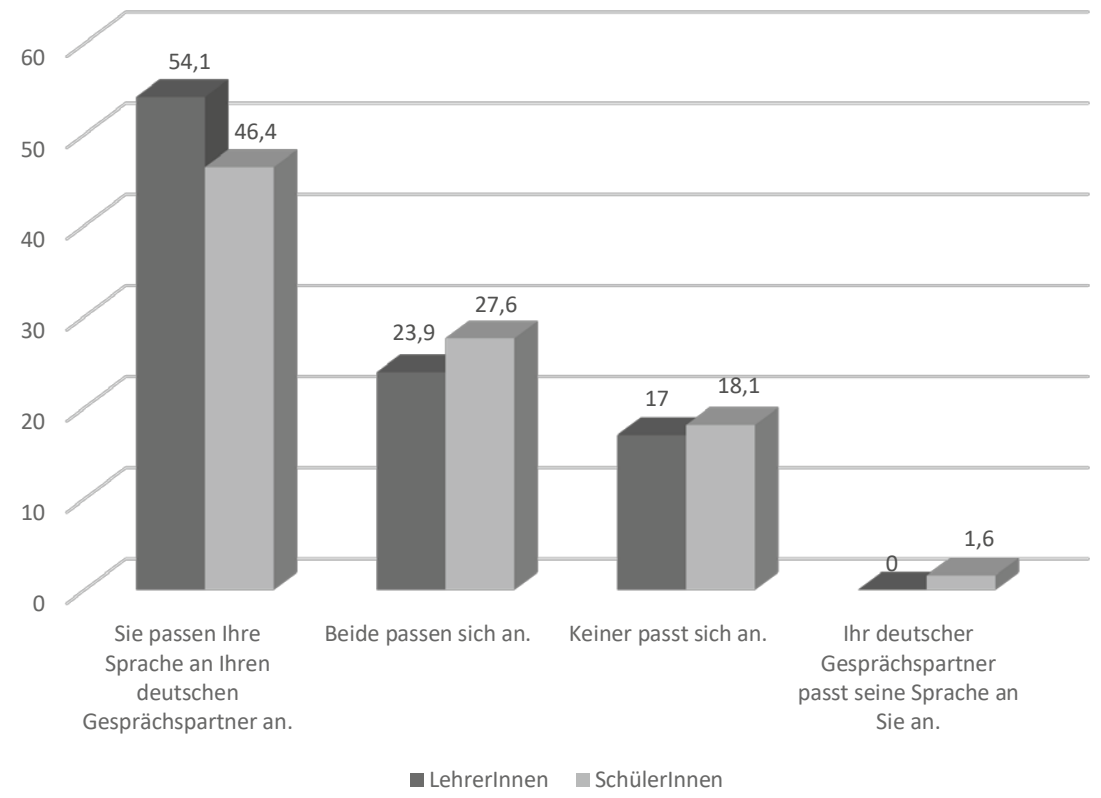

Abb. 48: Was wäre eher der Fall, wenn Sie mit einem/er deutschen Gesprächspartner/in sprechen? (in \%)

Auf die Nachfrage in der Gruppendiskussion, wie die LehrerInnen das Ergebnis interpretieren, dass mehr als die Hälfte der LehrerInnen angeben, sie würden sich sprachlich einem/ $\mathrm{r}$ deutschen Gesprächspartner/in anpassen, aber keiner davon ausgeht, dass sich die deutschen GesprächspartnerInnen anpassen, wurde lakonisch zustimmend kommentiert: „Durchaus“ (F7); „Fehlende Flexibilität. (lachend) Ja. Des hat schon Selbstbewusstsein. Ja.“ (F2) Diese Flexibilität im Umgang mit Varietäten, deren Fehlen „den Deutschen“ in den Gruppendiskussionen zugeschrieben wird, ist in Form der Registervariabilität etwas sehr Typisches für Österreich und hat wohl auch mit dem in der Literatur beschriebenen Dialekt-Standard-Kontinuum zu tun. Dass ÖsterreicherInnen davon ausgehen, dass sich deutsche GesprächspartnerInnen nicht anpassen, kann wohl auch dahingehend interpretiert werden, dass österreichische SprecherInnen von deutschen GesprächspartnerInnen gar keine Anpassung erwarten (können). DialektsprecherInnen können meist problemlos in eine Standardvarietät switchen - StandardsprecherInnen können jedoch nicht gleichermaßen in eine dialektale Varietät wechseln. Untersuchungen (Ammon 1995, Ransmayr 2005, Markhardt 2005) haben gezeigt, dass Deutsche weniger über die Merkmale des österreichischen Standarddeutsch wissen als ÖsterreicherInnen über deutsches Deutsch - was wiederum verdeutlicht, dass ein Anpassen von deutscher Seite schwerfallen muss. Dass deutsches Deutsch entgegen der sprachlichen Realität von zahlreichen LehrerInnen und SchülerInnen mit Standarddeutsch gleichgesetzt 
wird, und ebenso Dialektgleichsetzungen mit dem österreichischen Deutsch vorkommen, waren ebenfalls Ergebnisse der Gruppendiskussionen:

Über die eigenen Schüler sagte ein Lehrer, dass „sie eben der Meinung sind, Standardsprache ist deutschländisches Deutsch" $\left(\mathrm{M}_{1}\right)$. Eine Lehrerin $\left(\mathrm{F}_{5}\right)$ berichtete von einer Erfahrung mit Studierenden, die das österreichische Deutsch mit Dialekt gleichsetzten:

Ich habe das letztes Jahr das erste Mal mit Studierenden an der Pädagogischen Hochschule in Graz gemacht [...] Ah, sie ham das österreichische Deutsch SOFORT mit dem Dialekt/sehr häufig gleichgesetzt, nicht einmal Umgangssprache, sondern Dialekt. Und des war für sie/ : ah, für die meisten nicht gleichwertig.

Eine andere Teilnehmerin berichtete von einer Erfahrung mit einer Freundin: "Und ich hobs auch des erste Mal persönlich erlebt, von einer Freundin in Frankreich, die gsogt hot, na, das österreichische Deutsch ist furchtbar. Als Österreicherin.“

In den Diskussionsbeiträgen wurden die möglichen Ursachen für diese Phänomene gesucht, und zwar auf den verschiedenen Ebenen der sprachlichen Analyse, wobei hier die Grenze fließend war zwischen der Interpretation, warum andere das österreichische Deutsch für weniger korrekt halten, und der Selbsteinschätzung des Sprechers/ der Sprecherin, warum er/sie die angeführten Merkmale des österreichischen und deutschländischen Deutsch für zutreffend hält. Genannt wurde zum einen die Aussprache: „Ah, und i glaub des hängt mit der Lautung, mit der Aussprache zusammen“, wie ein Lehrer (M1) in der Gruppendiskussion meinte. Zum anderen wurde deutschen SprecherInnen mehrfach zugeschrieben, sich eher an die Grammatik zu halten als österreichische SprecherInnen dies täten. Eine Lehrerin (F8) erklärte beispielsweise:

\begin{abstract}
Ah, ich glaub des/ das hat ein, ein wenig auch damit zu tun, dass es manchmal zu beobachten ist, dass, äh, Menschen in Deutschland, äh, sich eventuell ein: w:enig stärker an die Grammatikregeln halten. Also, ich denk jetzt an Satzbau, a Nebensatz, der mit WEIL beginnt (( $F_{7}$ lacht, nickt $)$ ), ich glaub, dass ein Österreicher oder eine Österreicherin den eher folsch baut. Sozusogn nicht grammatikalisch korrekt.
\end{abstract}

Die häufigsten Eigenschaften, die zugunsten deutscher SprecherInnen ins Treffen geführt wurden, waren ihre angebliche mündliche Eloquenz, ihre ,argumentative Eloquenz“ und ganz pragmatische Merkmale: „Die“ (Deutschen) würden zielgerichteter reden, ihr Habitus und die Körpersprache seien überzeugender, und sie würden die Dinge auf den Punkt bringen. So meinte etwa eine Lehrerin ( $\left.\mathrm{F}_{4}\right)$ : „I bin der Meinung, dass - die mündlich korrekter sind als wir mit unserem österreichischen Deutsch. Sie sind treffender in den: in den: Aussagen." Eine andere (F1) schloss sich dem später an: „Ja, aso, i würd mi unbedingt da, der/ ersten Wortmeldung nu anschließen, ich glaube, dass das mit der Eloquenz in der Mündlichkeit vielleicht zusammenhängen könnte." Eine weitere Teilnehmerin (F9) sprach von 
einer „argumentativen Eloquenz“, die bis in die Körpersprache hineinreichen würde. Im österreichischen Deutsch dagegen würde man „herumreden“ und eine „argumentative Weichheit" an den Tag legen. Eine andere Teilnehmerin ( $\left.\mathrm{F}_{3}\right)$ beschrieb das österreichische Deutsch als vorsichtiger, es sei „südländischer“. Bei vielen Wortmeldungen in der Gruppendiskussion zu diesem Thema fielen Satzbrüche, Hesitationen, zögerliche Antwortmuster und Relativierungen auf, die Unklarheit und Unsicherheit ausdrücken, wohl auch, weil sich die erwähnten Äußerungen häufig an der Grenze oder jenseits der Grenze von sprachideologischen Stereotypen bewegt haben.

Was in den Gruppendiskussionen häufig angesprochen wurde - nämlich dass ÖsterreicherInnen die Standardsprache nicht so gut beherrschen würden wie Deutsche - haben viele LehrerInnen auf direkte Nachfrage („Manche meinen, dass Österreicher nicht so gut Deutsch sprechen können wie Deutsche. Würden Sie dem zustimmen?") in den Einzelinterviews allerdings zurückgewiesen:

Diese Meinung sei „lächerlich“, meinte eine Lehrerin aus Niederösterreich (FN2). Eine Tiroler Lehrerin (FT2) war entrüstet: „Ah - ich halte das für eine/für eine unanständige Aussage ... dass die Deutschen ihr Deutsch für DAS Deutsch halten, is das Problem der Deutschen und nicht der Österreicher", meinte sie lachend. Und die Sache sei „gepaart mit einer gewissen deutschen Überheblichkeit", wie eine oberösterreichische Lehrerin bemerkte.

Für einen Lehrer aus der Steiermark (MSt2) lag die Wurzel des Problems darin begründet, dass eine nationale Varietät zur supranationalen Norm erhoben wird: „Oida. Ein alter Hut. Sie [die Deutschen] sprechen anders Deutsch. Wenn i allerdings das Bundesdeutsche zur Norm erhebe, dann hab i Probleme, weil dann weicht das Österreichische ab."

Aber einige LehrerInnen stimmten der in der Gruppendiskussion mehrfach geäußerten Annahme, dass Deutsche besser Deutsch sprechen würden als ÖsterreicherInnen, auch in den Interviews zu. Ein Lehrer aus Salzburg sprach von „sprachlich schwächeren“ österreichischen SprecherInnen:

Wenn ma sich Debatten im deutschen Parlament anhört, und Debatten im ÖSTERREICHISCHEN Parlament anhört, stellt man Unterschiede fest, ja? [...] Das heißt, das deutsche Deutsch klingt natürlich unglaublich eloquent, überzeugend, selbstsicher [...] Das ÖSTERREICHISCHE is meistens doch sprachlich schwächer. Sprachlich schwächer, weniger überzeugend.

Ein anderer Lehrer aus Salzburg (MSa4) postulierte ebenfalls eine höhere Eloquenz der deutschländischen SprecherInnen:

Hörn Sie sich mal ein Interview eines deutschen Fußballers und eines österreichischen Fußballers an. Ahm - - also ich spreche nich vom Burchtheaterdeutsch, aber ahm die Eloquenz in der mündlichen Sprache is sicher ahm bei Bundesdeutschen im Schnitt wahrscheinlich besser ausgeprägt als bei uns. 
Die beiden folgenden Auszüge veranschaulichen auf treffende Weise, dass viele Lehrende - wie weiter oben bereits angesprochen - ein ambivalentes Verhältnis zur eigenen Varietät haben, das auch in den Interviews zum Ausdruck kommt: Sie haben in unserer Untersuchung das deutschländische Deutsch zwar nicht für überlegen gehalten, aber dennoch gewisse Mängel angesprochen, die dem österreichischen Deutsch ihrer Ansicht nach anhaften würden. Eine Lehrerin aus Wien (FW2) meinte, das deutschländische Deutsch sei etwa durch mehr Klarheit beim Satzbau und der Zeitenverwendung gekennzeichnet:

Das [...] heißt nicht, dass die Deutschen besser Deutsch sprechen, es kommt manchmal ah straffer oder korrekter ah zur Information, da ah hab ich schon den Eindruck, dass ah ganz allgemein ah gesagt, ah, das österreichische Deutsch ein bisschen - verschachtelter is, ja? Ahm, aber es hat alles seine Berechtigung.

Den angeblich großen Unterschied, den es in punkto Eloquenz zwischen ÖsterreicherInnen und Deutschen gäbe, kommentierte eine Lehrerin aus Niederösterreich $(\mathrm{FN} 1)$ so:

Naja, die Deutschen haben das Glück, dass ihr Dialekt zufällig zur Standardsprache erklärt worden is. Sog i a meine Schüler. Sie brauchen si net genieren, das is so ein großer Sprachraum, da gibt's viele Varietäten und die ostmitteldeutsche is hoit gewählt worden ois Standardsprache und insofern wirken die Deutschen dann eloquenter wenn sie des womit sie aufwochsn donn a natürlicherweise onwenden. Und unsaans klingt immer wie so a Bauer.

\subsubsection{Einstellungen gegenüber dem österreichischen, deutschen und Schweizer Standarddeutsch: Polaritätsprofile}

Zum Vorschein kamen solche stereotypen Einstellungen auch bei den Polaritätsprofilen, die zur kontrastiven Einschätzung des mündlichen österreichischen, deutschen und Schweizer Standarddeutsch erhoben wurden und die exemplarisch für die Spracheinstellungen der LehrerInnen gegenüber diesen drei Varietäten des Deutschen waren. Das Deutsch Deutschlands wurde darin tendenziell als „korrekter“, „gebildeter“ und „direkter“ empfunden. Deutlich positivere Werte gab es für das österreichische Deutsch bei den Merkmalen „sympathisch“, „vertraut“, gemütlich“, „melodisch“, „,weich“ und „natürlich“. Das Schweizer Deutsch schnitt durchwegs deutlich schlechter ab als das österreichische Deutsch. Die befragten LehrerInnen empfanden die Schweizer Varietät als „langsamer" und „fremder“. Im Vergleich zum österreichischen und zum deutschländischen Deutsch kamen Schweizer SprecherInnen als „schlampiger“, „ungebildeter“ und „unhöflicher“ weg. 


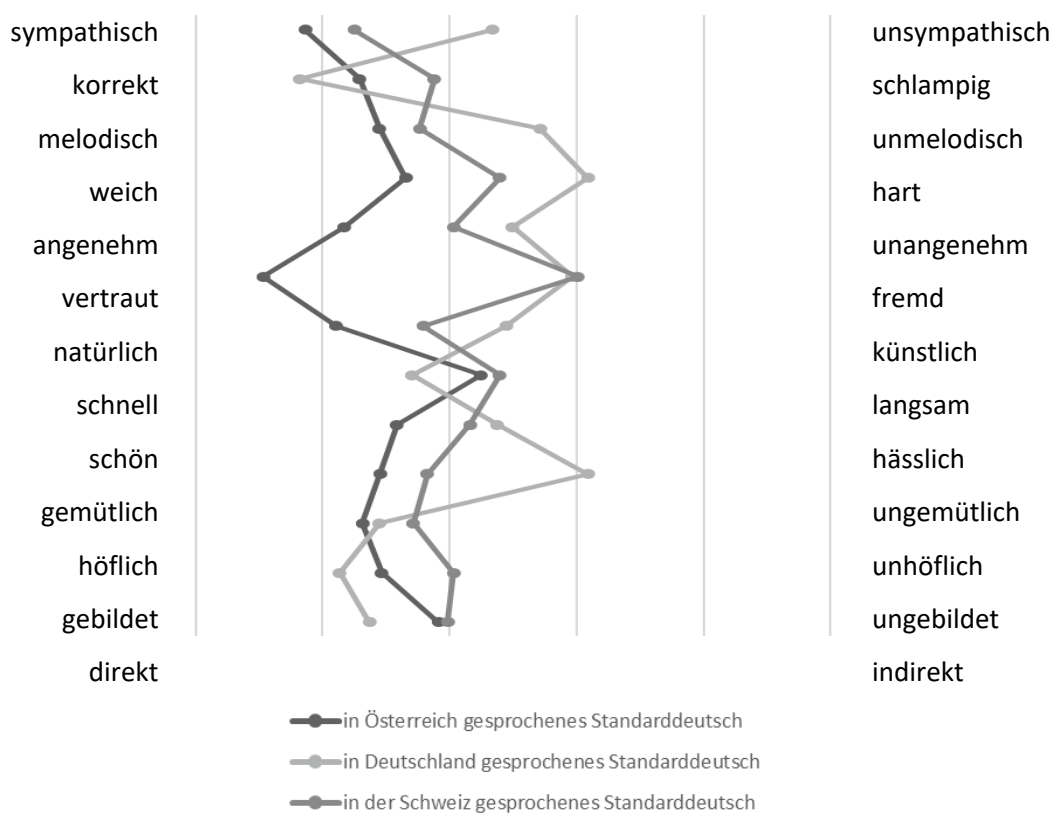

Abb. 49: LehrerInnen: Wie wirkt in Österreich, in Deutschland und in der Schweiz gesprochenes Standarddeutsch auf Sie?
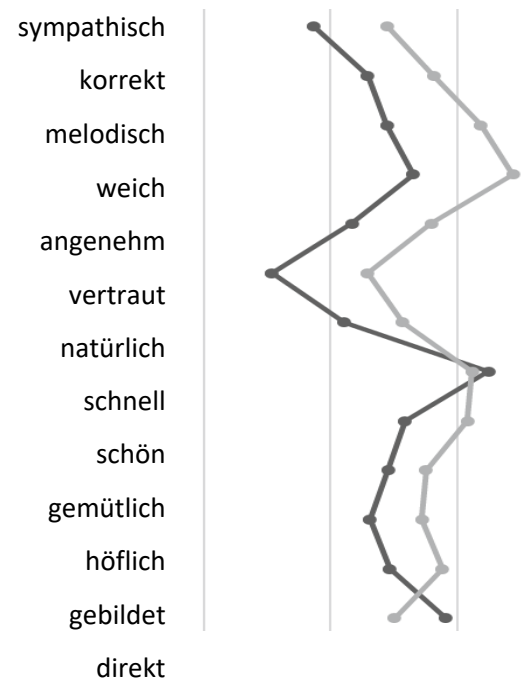

unsympathisch

schlampig

unmelodisch

hart

unangenehm

fremd

künstlich

langsam

hässlich

ungemütlich

unhöflich

ungebildet

indirekt

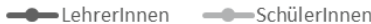

Abb. 50: LehrerInnen/SchülerInnen: Wie wirkt in Österreich gesprochenes Standarddeutsch auf Sie? 
Vergleicht man die LehrerInnen mit den SchülerInnen hinsichtlich ihrer Spracheinstellung gegenüber dem österreichischen Deutsch, so wird deutlich erkennbar, dass sich die SchülerInnen bei ihren Einschätzungen etwas vorsichtiger verhielten, indem sie bei fast allen Items stärker zur Mitte tendierten.

\subsubsection{Sprache-Identität}

Bei einer weiteren Reihe von Fragen konnten die Befragten auf einer 4-stufigen Skala (sehr stark/stark/wenig/gar nicht) angeben, wie wichtig ihnen die Verwendung des österreichischen Deutsch bzw. von Austriazismen ist. Die folgenden Situationen wurden vorgegeben:

- In einer Fernsehsendung über österreichisches Deutsch sagte eine Frau in einem Interview: „Österreichisches Deutsch: Das ist das, wo ich mich wohlfühle, es gehört zu meiner Identität." Inwiefern trifft diese Aussage auch auf Sie zu?

- Wenn auf Speisekarten österreichischer Restaurants Wörter wie Rinderbraten, Quarktasche oder Schorle stehen - stört Sie das?

- Wenn Sie beim Einkaufen auf Produkten Wörter wie Brötchen oder Blumenkohl lesen - stört Sie das?

- Wenn ein/e deutsche/r Nachrichtensprecher/in die österreichischen Nachrichten verlesen würde - würde Sie das stören?

- Meinen Sie, dass über die deutschen Fernsehsender der Sprachgebrauch in Österreich beeinflusst wird?

- Wenn Sie Tschüss hören - stört Sie das?

Abb. 51 zeigt zunächst die Antworten der Befragten auf die erste Frage, ob die Befragten österreichisches Deutsch mit ihrer Identität in Verbindung bringen. SchülerInnen und LehrerInnen gaben überwiegend an, dass diese Aussage sehr stark oder stark auf sie zutreffe - sie waren sich also relativ einig, dass das österreichische Deutsch ein wichtiger Teil ihrer sprachlichen Identität sei (siehe Abb. 51 auf S. 156).

Ob und wie sehr LehrerInnen und SchülerInnen Begriffe wie Rinderbraten, Quarktasche oder Schorle auf österreichischen Speisekarten als störend empfinden, wurde in einer weiteren Frage erhoben - mit hochsignifikanten Unterschieden zwischen der Gruppe der LehrerInnen und SchülerInnen $(p=0,000)$. Während Deutschlandismen in Speisekarten (z. B. Rinderbraten, Quarktasche, Schorle) die SchülerInnen etwa zur Hälfte ziemlich kalt gelassen haben, reagierten die LehrerInnen in dieser Hinsicht anders: Rund drei Viertel gaben an, dass die genannten Bezeichnungen für Speisen/Getränke sie stark oder sehr stark stören würden. 


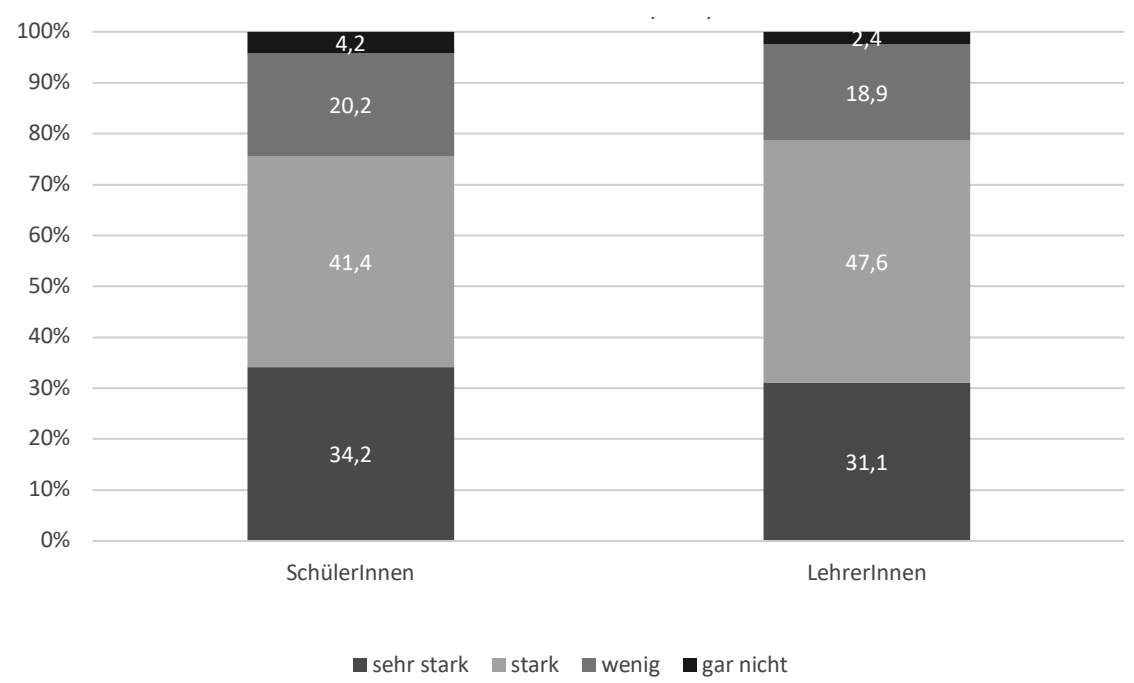

Abb. 51: Österreichisches Deutsch: Das ist das, wo ich mich wohlfühle, es gehört zu meiner Identität. Inwiefern trifft diese Aussage auch auf Sie zu? (in \%)

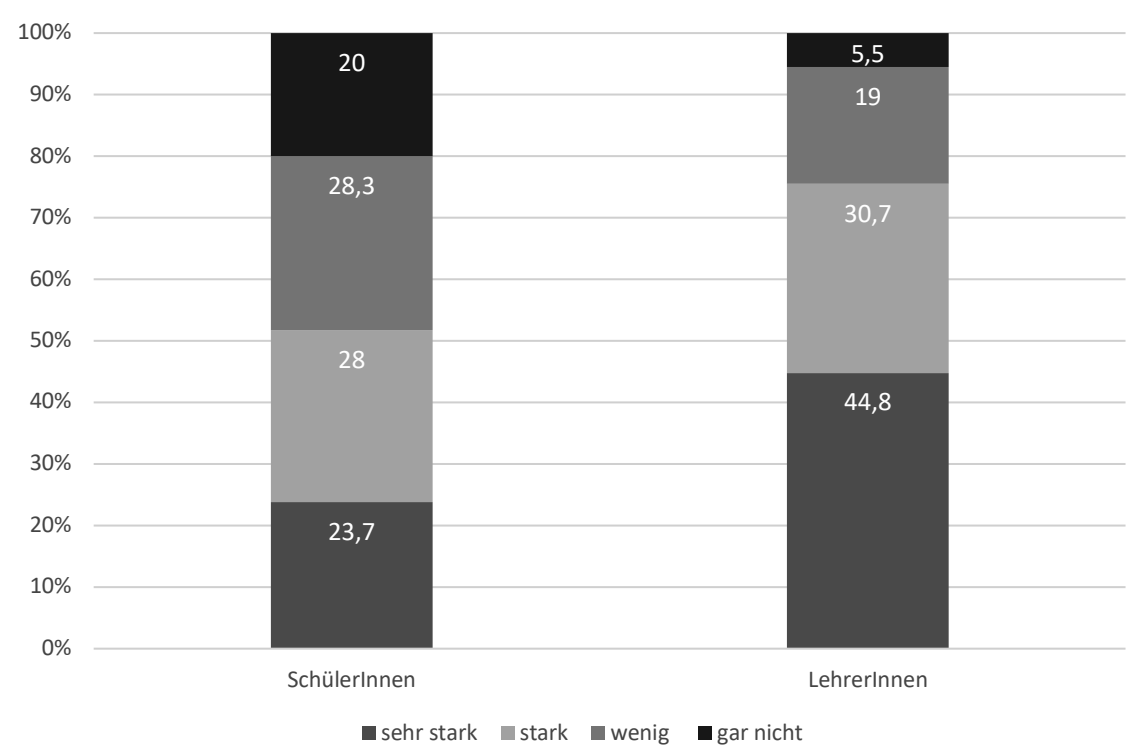

Abb. 52: Wenn auf Speisekarten österreichischer Restaurants Wörter wie Rinderbraten, Quarktasche oder Schorle stehen - stört Sie das? (in \%)

Auch bei der Frage nach Deutschlandismen im Handel kamen hochsignifikante Unterschiede zwischen SchülerInnen und LehrerInnen $(\mathrm{p}=0,000)$ zum Vorschein: Begriffe wie Blumenkohl oder Brötchen störten Lehrende beim Einkaufen 
wesentlich stärker (gesamt rund $48 \%$ sehr stark oder stark) als SchülerInnen (nur rund $25 \%$ sehr stark oder stark). Rund drei Viertel der SchülerInnen nahmen Deutschlandismen in Lebensmittelgeschäften als ,wenig bis gar nicht störend“ wahr, bei den LehrerInnen war es knapp die Hälfte:

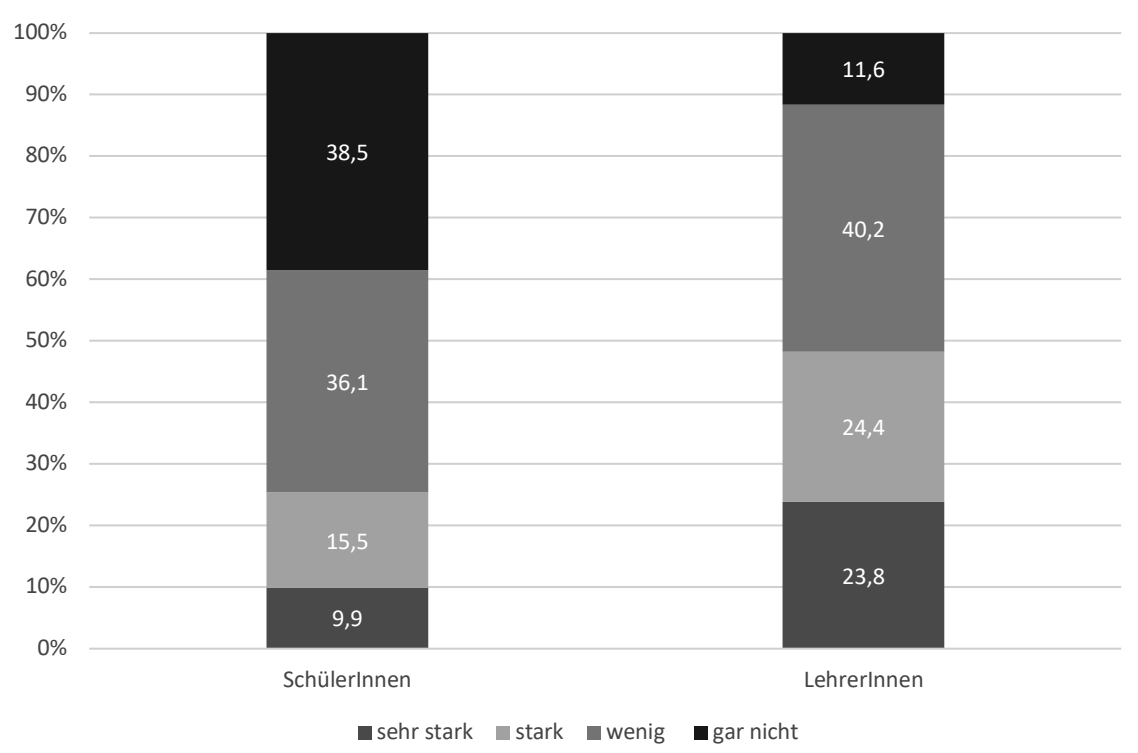

Abb. 53: Wenn Sie beim Einkaufen auf Produkten Wörter wie Brötchen oder Blumenkohl lesen stört Sie das? (in \%)

Ob ein deutscher Nachrichtensprecher beim Verlesen österreichischer Nachrichten als störend empfunden würde, sollte eine weitere Frage in diesem Block klären.

In den Antworten wurden erneut hochsignifikante LehrerInnen-SchülerInnenUnterschiede $(\mathrm{p}=0,000)$ erkennbar: Die Zahl jener LehrerInnen, die das in irgendeiner Form stören würde - von sehr stark über stark bis wenig - ist insgesamt deutlich höher (79,6\%) als bei den SchülerInnen (67,5\%). Umgekehrt würde es ein Drittel der SchülerInnen nicht stören - diese Zahl ist bei den LehrerInnen mit rund $20 \%$ deutlich geringer (siehe Abb. 54 auf S. 158).

Wir haben auch gefragt, ob nach Meinung der ProbandInnen der Sprachgebrauch in Österreich durch deutsche Fernsehsender beeinflusst werde. Auch hier sind hochsignifikante Unterschiede zwischen der LehrerInnen- und der SchülerInnengruppe $(\mathrm{p}=\mathrm{o}, \mathrm{ooo})$ zu verzeichnen: Rund zwei Drittel der befragten LehrerInnen waren der Meinung, dass eine starke Beeinflussung vorliegt. Diese Ansicht wurde dagegen nur von ca. $40 \%$ der SchülerInnen geteilt. Der unterschiedliche Wahrnehmungsgrad zwischen LehrerInnen und SchülerInnen könnte daher kommen, dass DeutschlehrerInnen als professionelle SprachbeobachterInnen über eine geschärfte Wahrnehmung verfügen und viele SchülerInnen 
als Teil der „YouTube-Generation“ deutsche Idiome bereits stärker internalisiert haben, Unterschiede daher auch nicht immer wahrnehmen bzw. „einsickernde“ Sprachelemente als weniger störend empfinden.

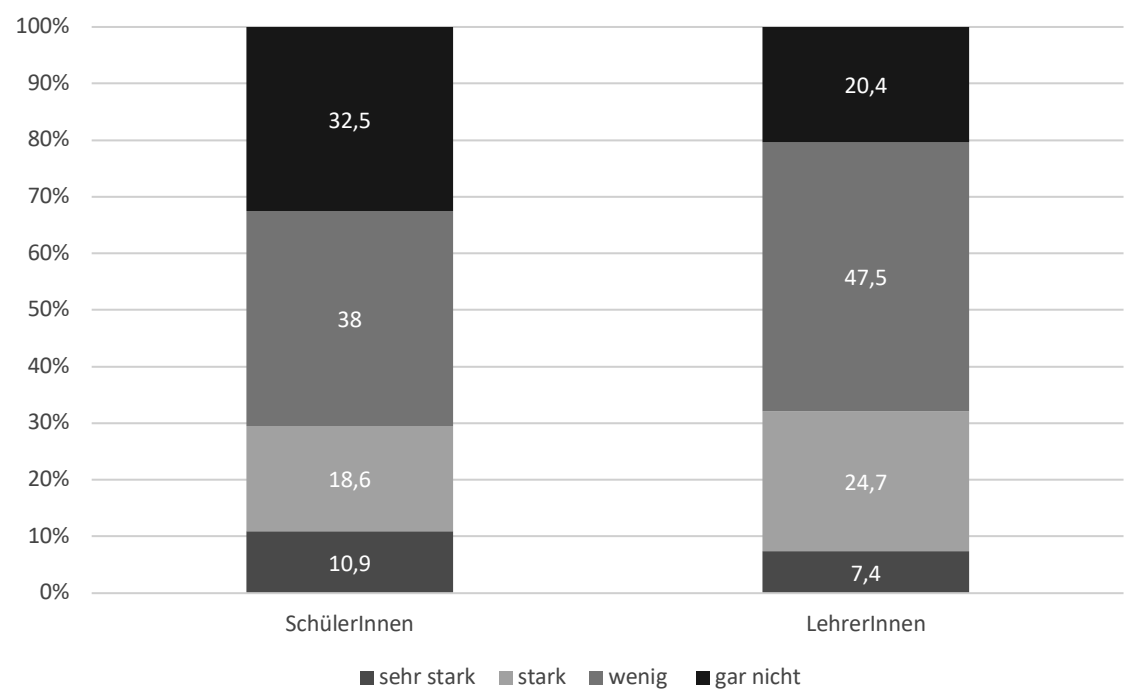

Abb. 54: Wenn ein/e deutsche/r Nachrichtensprecher/in die österreichischen Nachrichten verlesen würde - würde Sie das stören? (in \%)

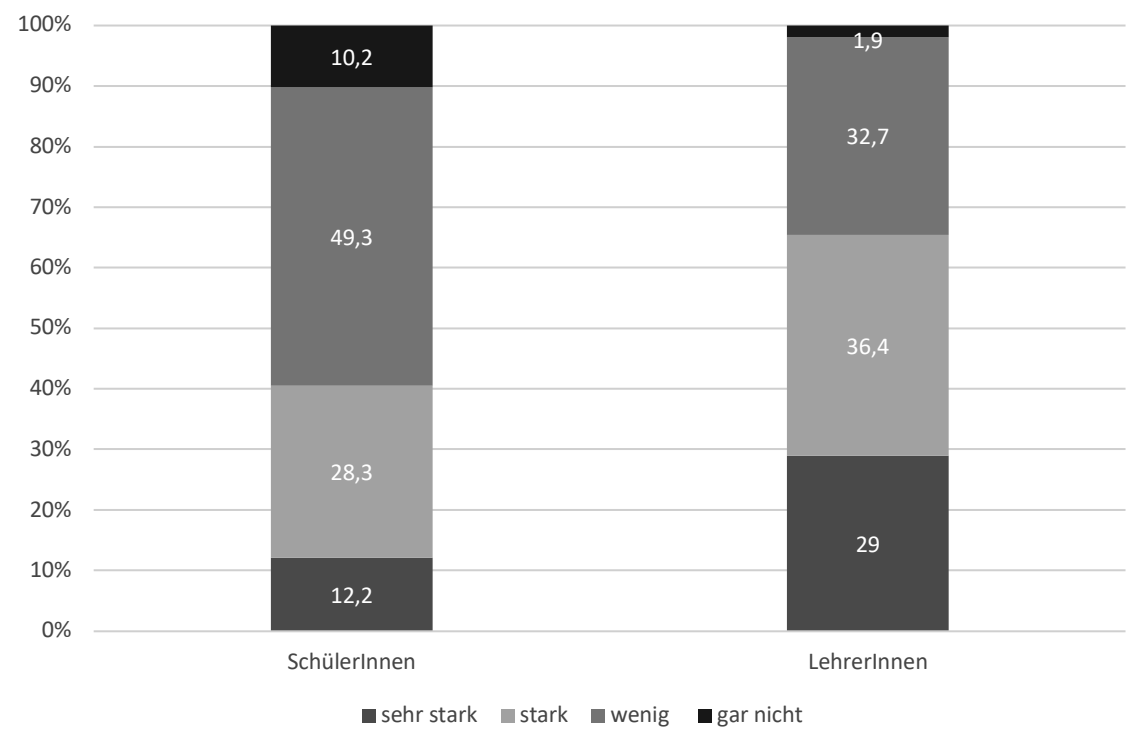

Abb. 55: Meinen Sie, dass über die deutschen Fernsehsender der Sprachgebrauch in Österreich beeinflusst wird? (in \%) 
Die letzte Frage zum Themenblock „Einstellungen zu Austriazismen/Deutschlandismen“ befasste sich mit der Akzeptanz der Abschiedsgrußformel Tschüss. Als hochfrequente Verabschiedung musste das mittlerweile weitverbreitete „Tschüss“ in diesem Block zu „Sprache und Identität“ separat betrachtet werden.

Sowohl die LehrerInnen als auch die SchülerInnen störte der Gruß Tschüss wenig bis gar nicht (insgesamt 14,7 \% bzw. 4,2 \% stört es). Trotzdem gab es wiederum hochsignifikante Unterschiede zwischen den LehrerInnen und den SchülerInnen ( $\mathrm{p}=\mathrm{o}, \mathrm{ooo})$ : Deutlich weniger LehrerInnen als SchülerInnen gaben an, dass sie Tschüss als Verabschiedung "gar nicht“ störe. Die Zahl jener, die Tschüss gar nicht stört, war auch unter den Lehrenden mit 51,8\% relativ hoch - unter den SchülerInnen war sie mit $84 \%$ noch wesentlich höher. Auf die Verwendung von „Tschüss“ wird weiter unten (Kap. 6.4) noch ausführlicher eingegangen.

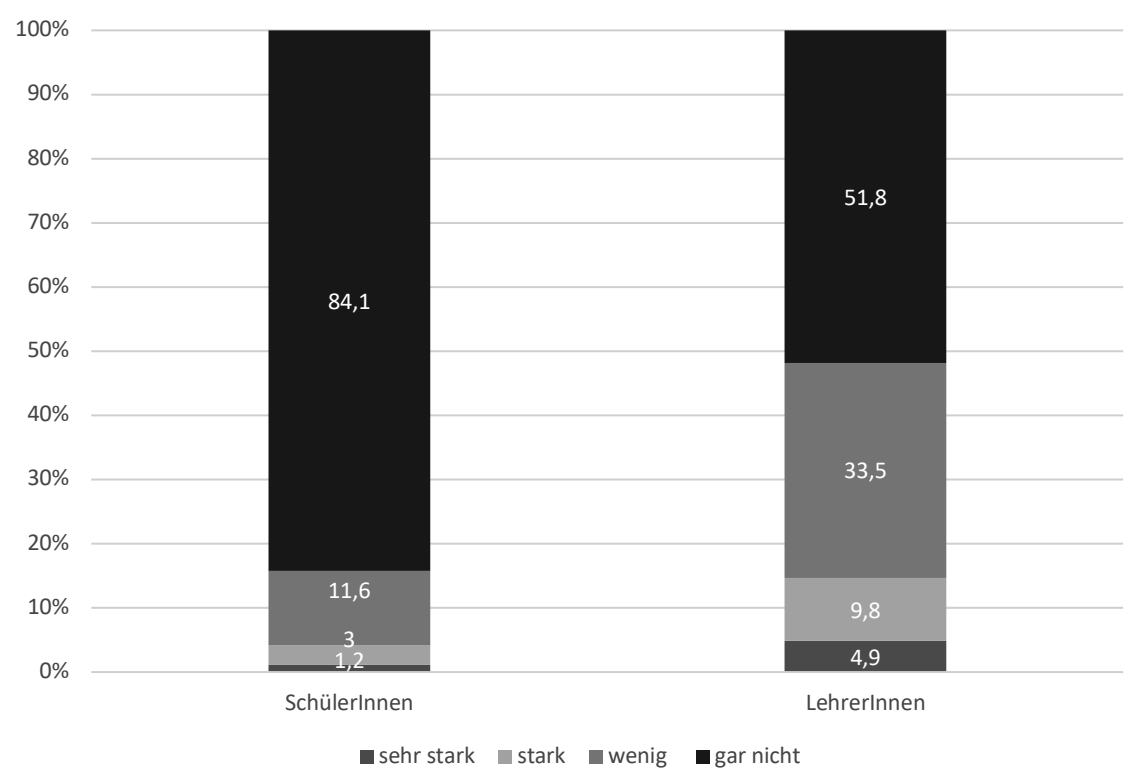

Abb. 56: Wenn Sie Tschüss hören - stört Sie das? (in \%)

Insgesamt zeigen die Antworten unserer ProbandInnen auf unsere Fragen zwar, dass sie österreichisches Deutsch als einen wichtigen Teil ihrer sprachlichen Identität wahrnehmen, dass aber andererseits eine gewisse Unaufgeregtheit im Zusammenhang mit der Verwendung von Deutschlandismen v. a. bei den Schülerinnen in Gastronomie und Handel vorhanden ist - die LehrerInnen stört das in diesen Kontexten deutlich mehr. Auch ein deutscher Nachrichtensprecher würde signifikant mehr LehrerInnen als SchülerInnen irritieren - insgesamt bei beiden Gruppen deutlich mehr als die Hälfte. Und auch den Einfluss des deutschen Fernsehens auf den Sprachgebrauch in Österreich schätzen LehrerInnen 
deutlich höher ein. Die Verwendung des Grußes „Tschüss“ stört in beiden Gruppen die Mehrheit der Befragten gar nicht, und auch hier ist die Akzeptanz bei den SchülerInnen noch deutlich größer.

Über den Zusammenhang von österreichischem Deutsch und persönlichen Identitätskonstruktionen sagten auch die Gruppendiskussionen einiges aus, was im Folgenden berichtet werden soll.

\section{Sprache und Identität in den Gruppendiskussionen}

Die Frage des Zusammenhangs von Sprache und (nationaler) Identität wurde auch in den beiden Gruppendiskussionen gestellt: „Für Ihr Selbstverständnis als ÖsterreicherIn (die so genannte österreichische Identität) - spielt die Sprache und speziell das Deutsch in Österreich eine Rolle dafür/ hat das eine Bedeutung dafür?“ Die qualitativen Daten waren hier als Ergänzung zu den Fragebogenergebnissen besonders interessant, weil sie stärker ins Detail gehen.

In beiden Gruppen wurde die Frage ausführlich diskutiert. Etwas mehr als die Hälfte der SchülerInnen hat der „Sprache“ eine Rolle für die nationale Identitätskonstruktion zugeschrieben, wie die folgenden Statements dokumentieren. F2 (1104): „Ja, für mich ist das genauso wichtig, wie heimisches Essen zum Beispiel. Oder ein Dirndl“. Und F1 (1143) erklärte:

Mir is Sprache auch sehr wichtig, auch wenn ich jetzt nicht im Dialekt daherred, es is ganz einfach Teil von dem Unterschied, ob deutscher Akzent oder ob österreichischer Akzent. --- Ich find, das gehört einfach dazu, zu/ So wies Wiener Schnitzel zu Österreich ghört, die Weißwurst zu Deutschland.

F4 (1163) meinte:

Ja für mich persönlich is es schon auch die Sprache, und jetzt unbedingt nicht nur der Akzent, [...] sondern einfach die Dialekte oder einfach das, was es nur in Österreich gibt, is für mich wichtig, dass ich mich hier zu Hause fühl, weils für mich anders is, sowas zu hören, das gibts einfach nirgend wo anders und das is für mich halt Heimat oder so.

Auch der aus der Türkei stammende $\mathrm{M}_{3}$, der im Sommer mit der Familie immer in die Türkei auf Urlaub fliegt, stellte fest: „Nach einer Zeit vermisst man schon irgendwie das Wienerische. Aber auch das österreichische Deutsch“ (1163).

$\mathrm{F}_{3}$ (1106 ff) hat „der Sprache“ wiederum keine Bedeutung beigemessen: „Bei mir ist das ein bissl anders, ich fühl mich trotzdem als Österreicherin, auch wenn ich nicht Erdäpfel und Paradeiser sag, deswegen seh ich mich trotzdem als Österreicherin, obwohl ich das nicht sag." F9 stimmte ihr zu, und brachte den Begriff „Heimat" ins Spiel (1125): „Also ich glaub einfach, die Heimat ist da, wo man sich am wohlsten fühlt und wo man sagt, ja ich bin jetzt - Österreicher, und das hat 
nichts mit der Sprache zu tun." F5, die in Kroatien geboren ist, nahm das „Heimatmotiv" auf und meinte: „Wenn ich in Kroatien bin, sag ich immer, ich will nach Hause, und wenn ich zu Hause sage, mein ich immer - Österreich. Also ist Österreich die: Heimat. Es ist halt, da bin ich zu Hause" (1128).

$\mathrm{F}_{3}$ (1155) fokussierte auch darauf, dass Identitätskonstruktionen als ÖsterreicherIn individuell sind: „Jeder [muss] für sich selber wissen, durch was er sich als Österreicher sieht. Manche halt über die Sprache, und manche dadurch, dass sie gern Schnitzel essen, was weiß ich.“

Auch M1 (1110) vertrat einen ähnlich konstruktivistischen Identitätsbegriff:

Ich komm aus Österreich, deshalb bin ich Österreicher. So würd ich das sagen [...] da ist für mich nix anderes ausschlaggebend, wie man jetzt spricht und ah, trotzdem bin ich Österreicher, weil ich hier geboren bin.

In der Gruppe der LehrerInnen wurde der Bezug zur eigenen nationalen und auch regionalen Identität von Beginn an hergestellt - ohne dass die Frage zunächst explizit gestellt wurde. Skeptische Positionen fanden sich hier nicht: „Ähm, österreichisches Deutsch bedeutet für mich SCHON einen Teil meiner Identität. Und auch der Sprachidentität meiner Schülerinnen und Schüler." (F2, o69) Oder F3, die zwei Jahre in Berlin studiert hatte und dort auch als Österreicherin identifiziert wurde („Ich weiß noch, dass meine Wortmeldungen am BeGINN, wie ich dort war, immer für, ahm, ErHEITERung gesorgt haben“), meinte (o83): „F:ür $\mathrm{MICH}$, ähm, bedeutet das österreichische Deutsch, ahm - eindeutig eine sprachliche Identifizierung.“ Und etwas später: „Und i glaub, das macht für MICH das österreichische Deutsch aus. Also, dass ma sich/ ja, - hier beheimatet fühlt."

F5 (o133 ff), die auch eine Auslandserfahrung ins Treffen führte, meinte: „Meine persönliche SPRACHE ist das österreichische Deutsch. A:hm, und ... des erste BeWUSSTwerden ist vielleicht durch Arbeitssituationen in der Schweiz stärker geworden. "Die Erfahrung, dass man sich des Eigenen erst dann besonders bewusst wird, wenn man sich im Ausland aufhält, ist ein bei Identitätsdiskussionen immer wiederkehrendes Motiv, das sich z. B. in früheren Projekten zur diskursiven Konstruktion nationaler Identität gezeigt hat (Wodak et al. 1998, 2009, de Cillia/Wodak 2009). Neben $F_{2}$ und $F_{5}$ erzählte auch $F_{4}$ (o114) - deren Tochter ,im deutschen Raum" arbeitet - von ähnlichen Erfahrungen, wenn sie ihre Tochter besucht: „Da spür ich genau dasselbe wie sie das in Berlin erlebt hat. Aso, net so, dass ma belächelt wird, sondern, jaja, des is halt so. Des is halt österreichisch. So auf die Art.“

F9 (0343) bezog sich auf eine Wortmeldung von F1: „Im gleichen Augenblick wie du (an F1 gerichtet) Identität gsogt host, hab ich auch Identität damit verbunden." Und erzählte dann sehr emotional, dass sie ein ganz konkretes Bild mit dem österreichischen Deutsch verbinde, das mit ihrer schulischen Sozialisation zusammenhängt, nämlich die in grünem Leinen gebundene Ausgabe des ÖWB der 1960er-Jahre, das ihr ihre Lehrerin ans Herz gelegt hatte: 
Ich hab so das Bild vor mir von diesem GRÜNEN ÖSTERREICHISCHEN WÖRTERBUCH. Und i hab des dann, wie ich mich damit beschäftigt hab, verzweifelt gesucht. I hab gehofft, dass i das nu net weggehaut hab, habs aber nicht mehr gefunden, zu meinem Bedauern, ah, [...] da die Lehrerin gesagt hat, das ist das (((langsamer, jedes Wort betont)) ÖSTERreichische Wörterbuch. Das ist EUER Wörterbuch. Wir in Österreich haben das Österreichische Wörterbuch [...] ahm/ wenn i meine Identität beschreiben würde als Österreicherin an Artefakten, dann ist es dieses grüne Wörterbuch [...] (F3 nickte währenddessen heftig). ${ }^{4}$

F8 (0334) meinte daraufhin in einem Rückgriff auf den schulischen Kontext, dass es wichtig sei, das österreichische Deutsch zu erhalten:

Und wos die SCHULE anbelangt, denk i ma, ist es ganz wichtig, a:h, diese österreichische Sprachvariante aufrechtzuerhalten und laut aufzuschreien, wenn a Schülerin oder ein Schüler beim Referat Chirurg (((Schirurg)) sagt. Was passiert durch die vielen, ah: „Synchronisationen“ (Zustimmung von F3 durch heftiges Nicken, „oder Schemie“).

\subsubsection{Zusammenfassung der Ergebnisse zu den Einstellungen gegenüber den Varietäten des Deutschen unter LehrerInnen und SchülerInnen}

Zusammenfassend lässt sich über die Ergebnisse der Fragebogenerhebung zu den Spracheinstellungen unter LehrerInnen und SchülerInnen Folgendes festhalten:

Zur Frage der Korrektheit des Standarddeutsch in Österreich erhielten wir zunächst von der überwiegenden Mehrheit der Befragten die Antwort, sie hielten das österreichische Standarddeutsch für genauso korrekt wie das Standarddeutsch in Deutschland. Eine ähnliche, jedoch in einem anderen Kontext gestellte Kontrollfrage weiter unten im Fragebogen ergab ein abweichendes Ergebnis: hier war der Prozentsatz der „sprachloyal“ eingestellten Befragten deutlich geringer.

Durch die anschließend geführten Gruppendiskussionen wurde diese ambivalente und widersprüchliche Einstellung gegenüber der eigenen Varietät bestätigt; als Erklärung dafür wurde unter anderem der auch in der Literatur immer wieder erwähnte Minderwertigkeitskomplex der österreichischen SprecherInnen genannt.

4 Sie nennt übrigens noch ein zweites identitäres Versatzstück der 1950er-/1960er-Jahre: „und des zweite waren die Fahnderl, die ma in der Volksschule gemalt ham, und dann immer beim Fenster an/ am Fensterbankl hinauf haben, ja. Das ist für mich, sozusagen, meine österreichische Sozialisation." 
Um einen weiteren Aspekt der in der Literatur (Clyne 1995, Muhr 1987, Ammon 1995) genannten geringen „Loyalität“ der eigenen Varietät gegenüber zu beleuchten, wurden die ProbandInnen in der Fragebogenerhebung auch danach gefragt, was ihrer Meinung nach der Fall wäre, wenn österreichische SprecherInnen mit deutschen GesprächspartnerInnen zusammentreffen. Rund die Hälfte der befragten LehrerInnen und SchülerInnen gab an, dass sich die österreichischen GesprächspartnerInnen den deutschen GesprächspartnerInnen sprachlich anpassen würden. Auch dieses Ergebnis wurde durch die Gruppendiskussionen bestätigt.

Die Gruppendiskussionen zeigten weiters, dass deutsches Deutsch oft mit Standarddeutsch gleichgesetzt wird, und Dialektgleichsetzungen mit dem österreichischen Deutsch häufig vorkommen. Als mögliche Ursachen dafür wurden von den TeilnehmerInnen der Gruppendiskussionen die Aussprache und mündliche Eloquenz der Deutschen genannt. Außerdem wurde die Meinung geäußert, deutschländische SprecherInnen würden sich eher an die Grammatik halten als österreichische. In den Einzelinterviews wiederum lehnten die meisten LehrerInnen die Aussage, dass „ÖsterreicherInnen weniger gut Standardsprache sprechen könnten als Deutsche“, auf direkte Nachfrage ab.

Stereotype Einstellungen ergaben weiters die im Fragebogen erhobenen Polaritätsprofile zur Einschätzung von mündlichem österreichischem, deutschländischem und Schweizer Deutsch. Das Deutsch Deutschlands wurde darin tendenziell als „korrekter“, „gebildeter“ und „direkter“ bewertet; das österreichische Deutsch hingegen stärker ,sympathisch“, „vertraut“, „gemütlich“, , melodisch“, ,weich“ und „natürlich“. Das Schweizer Deutsch schnitt v. a. bei den Merkmalen „schlampig“, „fremd“, „ungebildet“ und „unhöflich“ am schlechtesten ab.

Sowohl die SchülerInnen als auch die LehrerInnen wurden danach gefragt, ob es ihrer Meinung nach ein besonders gutes Deutsch gebe, und wo dies zu finden sei. Während etwa ein Drittel der SchülerInnen der Meinung war, dass es ein besonders gutes Deutsch gäbe, war das nur bei etwa einem Fünftel der Lehrpersonen der Fall. Diejenigen SchülerInnen, die meinten, dass es ein besonders gutes Deutsch gäbe, waren knapp mehrheitlich der Ansicht, dass dieses in Österreich zu finden sei. Unter den betreffenden Lehrenden war nur knapp ein Fünftel dieser Ansicht.

In einer weiteren Fragenreihe sollten die Befragten für den Zusammenhang von österreichischem Deutsch und Identität relevante Situationen beurteilen. So wurden die ProbandInnen etwa danach gefragt, ob es sie stören würde, wenn ein deutscher Nachrichtensprecher österreichische Nachrichten verlesen würde, oder wenn sie in österreichischen Speisekarten oder beim Einkaufen auf Deutschlandismen stoßen würden. Obendrein wurde gefragt, ob die Befragten österreichisches Deutsch mit ihrer Identität in Verbindung brächten, ob sie die Abschiedsgrußformel „Tschüss“ störe, und ob der Sprachgebrauch in Österreich ihrer Meinung nach durch deutsche Fernsehsender beeinflusst werde. 
Dabei zeigte sich, dass die Befragten österreichisches Deutsch als wichtigen Bestandteil ihrer Identitätskonstruktionen wahrnehmen, auch wenn die Verwendung von Deutschlandismen in bestimmten Kontexten v. a. von den SchülerInnen eher gelassen gesehen wird. Auch eine gewisse Diskrepanz zwischen der wichtigen Bedeutung und der emotionalen Besetztheit des österreichischen Deutsch für Identitätskonstruktionen (Gruppendiskussion v. a. der LehrerInnen, vgl. auch de Cillia 1998, 2006, 2015, Wodak/de Cillia 2006), und dem ambivalenten und widersprüchlichen Umgang mit der eigenen Varietät spiegelt sich in unseren Daten wider. Im Zusammenhang mit Spracheinstellungen gibt es in der Literatur auch Hinweise auf eine Exonormorientierung von PädagogInnen bei der Korrektur von Schülertexten (Ammon 1995, Heinrich 2010, Legenstein 2008). Darauf soll im folgenden Kapitel näher eingegangen werden.

\subsection{Korrekturverhalten}

\subsubsection{Normen und Korrektur bei schriftlicher Kommunikation im Deutschunterricht}

In der einschlägigen Forschungsliteratur findet sich die Annahme, dass österreichische DeutschlehrerInnen dazu tendieren, exonorm-orientiert zu korrigieren. Auf das Vorhandensein solcher Asymmetrien zwischen sprachlichen Varietäten haben schon Clyne (1995, 22 f.) und Ammon (1995, 448 ff) hingewiesen. In Ammons Pilotstudie kam heraus, dass fremdnationale Varianten von den in seiner Studie befragten LehrerInnen negativer bewertet wurden als eigennationale Varianten, Deutschlandismen aber insgesamt positiver beurteilt wurden als Austriazismen oder Helvetismen. Einige DeutschlehrerInnen aus der Schweiz und aus Österreich korrigierten im Rahmen der Studie eigene nationale Varianten zugunsten gemeindeutscher Alternativen (Ammon 1995, 446, 480). Ammon $(1995,480)$ und auch Muhr $(1995,96)$ führen ein derartiges Korrekturverhalten darauf zurück, dass die betreffenden Lehrkräfte mit der eigenen Varietät bzw. den sprachlichen Verhältnissen im eigenen Land nicht vertraut sind, weil das Thema der nationalen Varietäten in der Ausbildung der DeutschlehrerInnen keine große Rolle spielt.

Auch Heinrich (2010) kommt zu ähnlichen Ergebnissen: Obwohl die von ihr befragten Lehrpersonen angaben, dass das österreichische Deutsch für sie einen hohen Stellenwert hätte, wurde der in der Erhebung vorgelegte Aufsatz von den meisten TeilnehmerInnen über weite Strecken hin als umgangssprachlich gewertet. Auch in Heinrichs Untersuchung war beim Korrigierverhalten der Testpersonen die Tendenz erkennbar, Austriazismen zugunsten bundesdeutscher Varianten zu korrigieren. Die vorhandenen Studien legen nahe, dass es einen engen Zusammenhang zwischen Sprachwissen, Varietätenbewusstheit und Korrekturverhalten gibt. 
Da keine weiteren Arbeiten zum Korrekturverhalten österreichischer Lehrender vorliegen, haben wir dazu im Rahmen unserer Fragebogenerhebung eine eigene Untersuchung durchgeführt. Der LehrerInnen-Fragebogen enthielt einen (prinzipiell fehlerfreien) fiktiven Schüleraufsatz, in den eine Reihe von standardsprachlichen regionsspezifischen und nationalen Varianten eingebaut worden waren, um den lehrerseitigen Umgang mit plurizentrischer und regionaler Varianz im Deutschen beim Korrigieren von Schülerarbeiten analysieren zu können. Verwendet wurden dabei sowohl spezifische als auch unspezifische Austriazismen bzw. Deutschlandismen. ${ }^{5}$

Um eine möglichst große Bandbreite an Unterschieden zwischen den Varietäten des österreichischen Deutsch und des deutschländischen Deutsch abzutesten, wurden sowohl Varianten der lexikalischen Ebene, als auch der grammatischen sowie der morphologischen Ebene in diese Aufgabe eingebaut, die gemäß der verwendeten Kodices ${ }^{6}$ als standardsprachlich gelten. Grundlage dafür waren in erster Linie das Variantenwörterbuch (2004), aber auch das Österreichische Wörterbuch, Jakob Ebners Arbeiten und der Duden.

Unter den im Aufsatz eingebauten Begriffen fanden sich Varianten, die laut Variantenwörterbuch als in Deutschland bzw. in großen Teilen Deutschlands, aber als nicht in Österreich gebräuchlich ausgewiesen sind. Es handelt sich hierbei um so genannte spezifische Deutschlandismen: Leibgericht, Aprikosenkonfitüre, Sahne, Plätzchen, fegen, Mülleimer, Backwerk, Schlachter. Als unspezifische Austriazismen (in Österreich und Südost-Deutschland standardsprachlich gebräuchlich) verzeichnet das Variantenwörterbuch heuer, in der Früh, Küchenkastl (Grenzfall des Standards), Hendl, Wimmerl, Stutzen, fladern (Grenzfall des Standards), sekkieren, das Eck. Daneben gab es im Aufsatz noch Varianten, die das Variantenwörterbuch als „ausschließlich in Österreich gebräuchlich“ anführt, also spezifische Austriazismen: Schularbeit, Palatschinke, Powidl, Sackerl, Greißler, Vogerlsalat.

Weitere im Aufsatz enthaltene lexikalische Varianten, die im Variantenwörterbuch nicht explizit als staats- oder regionsspezifisch ausgewiesen sind, aber dennoch von manchen SprachexpertInnen und sprachlich versierten Laien instinktiv einem Land zugeordnet werden, waren Postbote, Jungs, Ski laufen, im Voraus und aufder Uni. Auch Tomate - ein gemeindeutscher Ausdruck, für den in Ost-Österreich synonym auch das Wort Paradeiser gebräuchlich ist - war Teil des Aufsatzes.

5 Ammon $(1997,4 \mathrm{f})$ unterscheidet zwischen spezifischen und unspezifischen Varianten. Spezifische sind Ammon zufolge Varianten, die ausschließlich in einem Zentrum vorkommen (z. B. Schularbeit: A); unspezifische sind solche, die in zwei von drei Zentren gebräuchlich sind (z. B. Karfiol: A/CH). Vgl. Kapitel 2.4.1.

6 Folgende Referenzwerke wurden herangezogen: Variantenwörterbuch (2004); Jakob Ebner (2009): Wie sagt man in Österreich; Jakob Ebner (2008): Österreichisches Deutsch; Duden (25. Aufl.); Österreichisches Wörterbuch (41. Aufl.); Peter Wiesinger (2014): Das österreichische Deutsch in Gegenwart und Geschichte; Robert Sedlaczek (2004): Das österreichische Deutsch. 
Andere im Aufsatz enthaltene Varianten betrafen auf der grammatischen Ebene Unterschiede beim Genus-Gebrauch ( $a m E c k / A^{7}$; eine Eins $/ D^{8}$ ), beim Präpositionsgebrauch (zur Schule gehen $/ D^{9}$, an Weihnachten ${ }^{10} / D$ ), bei der Verwendung des Fugenmorphems (Schweinebraten $/ D^{11}$ ) und bei der Perfektbildung (habe gestanden $/ D^{12}$ ). Als weiteres grammatikalisches Phänomen wurde eine Variante der Verbstellung im Nebensatz eingebaut, die als Zusatzvariante in Österreich üblich ist, aber nicht in Deutschland: die Verbstellung bei dreiteiligen Verben im Nebensatz nach dem Schema obwohl ich der Mama helfen hätte sollen. Weiters war im Aufsatz die durchgängige Verwendung des Perfekts als schriftliche Erzählzeit im Erlebnisaufsatz enthalten sowie der Gebrauch des bestimmten Artikels vor Vornamen und Verwandtschaftsbezeichnungen (die Mama, die Oma, der Wolfgang) und vor Nachnamen (die Frau Meier).

Die Aufgabe für die LehrerInnen bestand darin, den folgenden Schüleraufsatz unter Beachtung der entsprechenden Anweisungen zu korrigieren:

Bitte lesen Sie den folgenden Aufsatz eines 11-jährigen Schülers durch. Gibt es Ausdrücke bzw. Formulierungen, die Sie in Hinblick auf normative Sprachrichtigkeit und Ausdruck korrigieren würden? Wenn ja, dann kennzeichnen Sie diese bitte folgendermaßen:

Burchgestrichen $=$ Fehler

Wellenlinie $=$ stilistisch unpassender Ausdruck, aber nicht falsch

Bitte führen Sie Verbesserungsvorschläge sowie jegliche andere Kommentare im Korrekturrand an:

\section{Meine Weihnachtsferien}

Die letzten Weihnachtsferien waren sehr schön, weil ich nicht zur Schule gehen musste. Am Vormittag habe ich immer am Eck auf den Postboten gewartet, weil ich schon im Voraus wissen wollte, welche Geschenke ich heuer bekommen würde. Da ich auf die letzte Schularbeit eine Eins bekommen hatte, erwartete ich mir besonders viele. Am Nachmittag bin ich meistens mit anderen Jungs Ski laufen gegangen, obwohl ich eigentlich der Mama helfen hätte sollen. Danach hat mir die Oma oft mein Leibgericht gemacht: Palatschinken mit Aprikosenkonfitüre oder Powidl und Sahne.

An Weihnachten habe ich schon in der Früh in der Küche gestanden, um Mama dabei zu helfen, Plätzchen zu backen. Danach habe ich alle Küchenkastln ausgewischt, den Boden gefegt und den Mülleimer hinausgestellt. Dann habe ich noch schnell der Frau Meier ein Sackerl mit Backwerk und Zuckerln gebracht, bevor ich zum Schlachter gehen

7 An der Ecke/D.

8 Ein Einser/A.

9 In die Schule gehen/A.

$10 \mathrm{Zu}$ Weihnachten/A.

11 Schweinsbraten/A.

12 Bin gestanden/A. 
musste, um den Schweinebraten abzuholen und beim Greißler Vogerlsalat, Tomaten und ein Hendl zu kaufen.

Leider habe ich nicht so viele Geschenke bekommen, vielleicht weil ich den Wolfgang, meinen Bruder, immer wegen seiner Wimmerln sekkiert habe, und außerdem habe ich inm einmal die Stutzen gefladert, während er gerade auf der Uni war.

Was bei der Durchsicht der Korrekturen zu allererst ins Auge gestochen ist, war die enorme Bandbreite bei der Einschätzung der normativen Sprachrichtigkeit und der stilistischen Angemessenheit: Bei einer Textlänge von knapp 200 Wörtern wurden von einzelnen LehrerInnen bis zu 14 Normverletzungen markiert, also „Fehler“ angestrichen - in einem im Prinzip „fehlerfreien“ Text. Nimmt man die „Fehler“ und die unterwellten Ausdrücke zusammen, so haben einige Lehrkräfte sogar bis zu $25 \mathrm{Mal}$ den Rotstift angesetzt. Andere LehrerInnen hingegen machten keine einzige Korrektur.

Alles in allem haben die LehrerInnen moderat korrigiert: Der Mittelwert an angestrichenen Normverletzungen lag bei drei „Fehlern“, eine Wellenlinie wurde durchschnittlich neun Mal gesetzt. Insgesamt wurden im Schnitt 8 \% der Austriazismen und $5 \%$ der enthaltenen Deutschlandismen als Fehler korrigiert. Mit einer Welle versahen die LehrerInnen durchschnittlich $17 \%$ der Austriazismen und fast doppelt so viele Deutschlandismen, nämlich rund $33 \%$ :

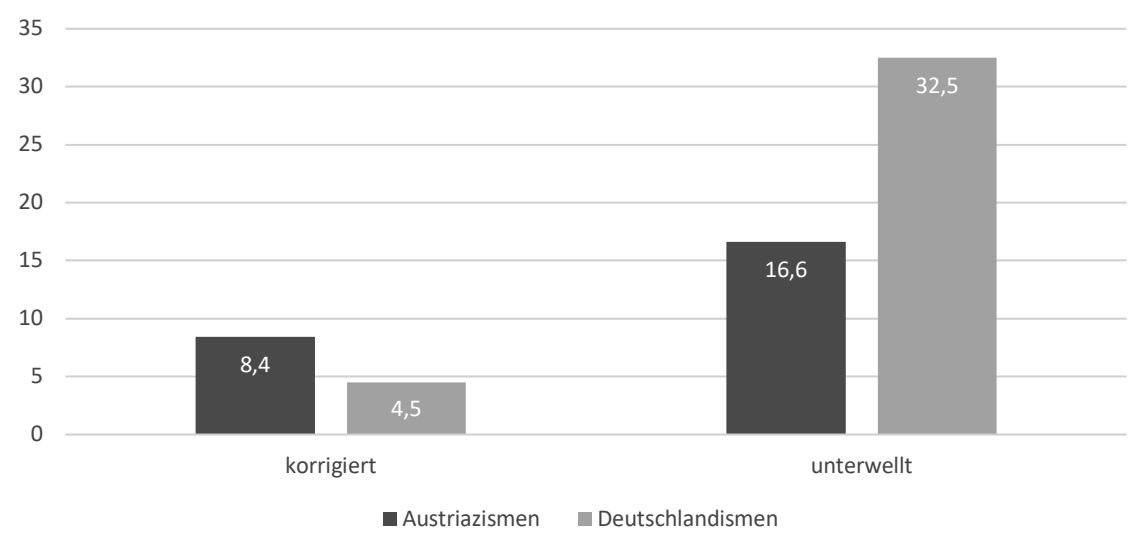

Abb. 57: Austriazismen und Deutschlandismen: korrigiert und unterwellt im Durchschnitt (in \%)

Der folgende Abschnitt zeigt eine Übersicht der am häufigsten von den LehrerInnen angestrichenen Ausdrücke - unterwellt oder korrigiert (Abb. 58). Auffällig ist, dass viele der Deutschlandismen zwar nicht ausgebessert, aber mit entsprechenden Anmerkungen im Korrekturrand unterwellt wurden. 


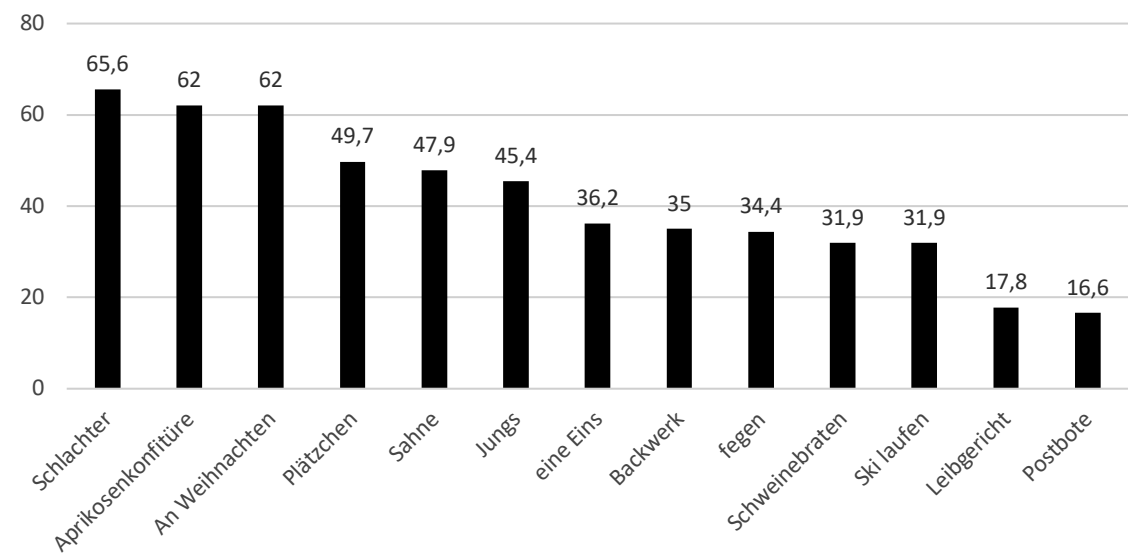

Abb. 58: Deutschlandismen: am häufigsten korrigiert oder unterwellt (in \%)

Welche Korrekturen oder Anmerkungen haben die obigen Deutschlandismen hervorgerufen? Die folgende Tabelle zeigt, mit welchen Verbesserungsvorschlägen und Kommentaren die LehrerInnen am häufigsten reagiert haben:

\author{
Deutschlandismen Korrekturvorschläge/Anmerkungen \\ Jungs \\ „deutsches Deutsch“; Buben; Burschen \\ Aprikosenkonfitüre \\ „deutsch“; das passt nicht zusammen!; Marillenmarmelade \\ Sahne \\ „deutsch“; A Schlagobers; Schlag; Obers; Schlagrahm; „das passt \\ nicht zusammen!"; inzwischen schon quasi selbstverständlich \\ An Weihnachten \\ Zu Weihnachten; Am Hl. Abend; am Weihnachtstag; Ausdruck!; \\ dte. Version \\ Plätzchen \\ „deutsch“; Ö: Kekse \\ Schlachter \\ „deutsch“; Fleischhauer; Ö: Fleischer; Metzger; Fleischhacker
}

Tab. 3: Korrekturvorschläge/Anmerkungen zu Deutschlandismen

Wenn wir nun unseren Blick auf die Austriazismen richten, die von den LehrerInnen im Aufsatz besonders häufig angestrichen wurden, dann fällt auf, dass die beiden Spitzenreiter unter den Meistkorrigierten jene waren, die laut Variantenwörterbuch als Grenzfall des Standards einzuordnen sind (fladern, Küchenkastl) trotz einer der Mündlichkeit nahen, narrativen Textsorte. Die folgende Abbildung gibt einen Überblick über die am häufigsten von den LehrerInnen markierten Austriazismen (korrigiert oder unterwellt): 


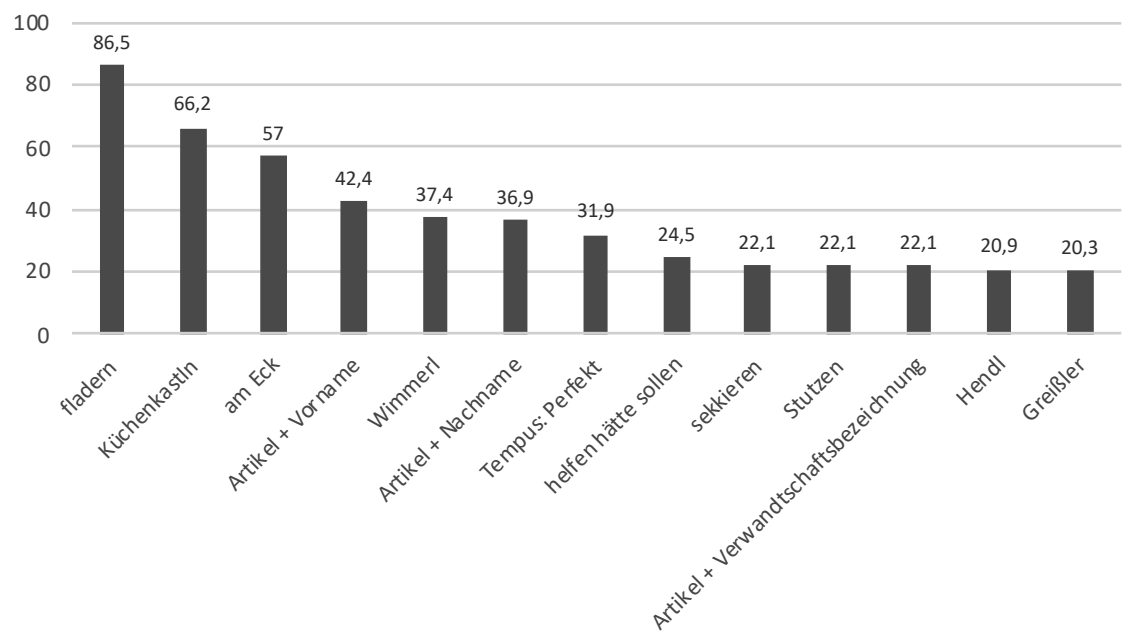

Abb. 59: Austriazismen: am häufigsten korrigiert oder unterwellt (in \%)

Zu einigen der in Abb. 59 dargestellten Austriazismen haben die LehrerInnen folgende Korrekturvorschläge bzw. Anmerkungen in den Korrekturrand geschrieben:

\begin{tabular}{|c|c|}
\hline Austriazismen & Korrekturvorschläge/Anmerkungen \\
\hline am Eck & an der Ecke; Mundart; ugs. \\
\hline Küchenkastln & $\begin{array}{l}\text {-kästchen; -regale; Küchenkästen; Schränke; Küchenkasteln; } \\
\text { Küchenkasterln; Mundart; zu umgangssprachlich }\end{array}$ \\
\hline Wimmerl & ugs.; Hautunreinheiten; Mitesser; Mundart; Pickel; Akne \\
\hline gefladert & $\begin{array}{l}\text { Ausdruck; Dialektausdruck; genommen/weggenommen; gestohlen; } \\
\text { stibitzt; Mundart; ugs.; zu umgangssprachlich }\end{array}$ \\
\hline Greißler & Dorfladen; Lebensmittelgeschäft; Kaufmann \\
\hline Hendl & Hähnchen; Hendel; Henderl; Huhn; Mundart!; ugs. \\
\hline
\end{tabular}

Tab. 4: Korrekturvorschläge/Anmerkungen zu Austriazismen

Aus den Korrekturvorschlägen in Tab. 4 geht hervor, dass die Austriazismen am Eck, Küchenkastl, Wimmerl, fladern, Greißler und Hendl von einem Teil der DeutschlehrerInnen korrigiert und durch gemeindeutsche oder deutschländische Varianten ersetzt bzw. stilistisch kommentiert wurden - damit also von diesen LehrerInnen als nicht standardsprachlich eingestuft wurden. Obwohl es sich beim Erlebnisaufsatz um eine der Mündlichkeit nahe Textsorte handelt, deuten die Ergebnisse darauf hin, dass bei einem großen Teil der DeutschlehrerInnen relativ rigide Normvorstellungen vorhanden sind - etwas, worauf auch Steinig/Huneke und Neuland/ Peschel hinweisen (Steinig/Huneke 2015, 129; Neuland/Peschel 2013, 63 ff.). 
Weitere häufige lehrerseitige Anmerkungen und Textkorrekturen betrafen Artikel vor Eigennamen. Hier fiel auf, dass vor einem Vornamen stehende Artikel (z. B. „der Wolfgang“) etwa doppelt so häufig korrigiert wurden wie jene, die in Kombination mit einer Verwandtschaftsbezeichnung verwendet wurden (z. B. „die Oma“).

Der vorgelegte Aufsatz ist im Perfekt erzählt. Diese Verwendung des Perfekt als Erzählzeit war ebenfalls vereinzelt Gegenstand der Korrektur. Das Perfekt, das in Österreich - wie ein Blick in die Kinderliteratur bestätigt ${ }^{13}$ - auch im Schriftlichen als Erzählzeit gebräuchlich ist, wurde von mehr als zwei Drittel der Lehrenden akzeptiert. 31,9\% der LehrerInnen haben jedoch die Perfektformen im Testaufsatz nicht unmarkiert stehen lassen.

Analysiert man die Korrekturen von Deutschlandismen und Austriazismen auf Basis der Lehrerkommentare, sind zwei Korrekturmotive nicht zu übersehen: Lehrerseitig wurden Deutschlandismen wegen ihrer - zugespitzt formuliert - „zu deutschen Natur“ angestrichen. Bei den Austriazismen war es hingegen so, dass die LehrerInnen an den betreffenden Ausdrücken Anstoß nahmen, weil diese als nicht „standardsprachlich genug“ empfunden wurden.

Besonders auffällig war, dass sich die LehrerInnen beim Unterwellen von Ausdrücken je nach Alter, Schulform und Unterrichtsort abweichend verhalten haben. Beim Vergleich der LehrerInnen-Korrekturen nach Altersgruppen gab es signifikante Unterschiede $(\mathrm{p}=0,019)$ beim Umgang mit Deutschlandismen. Wie das folgende Diagramm darlegt, wurden von der jüngsten LehrerInnengruppe rund $19 \%$ der Deutschlandismen mit einer Welle markiert, während die älteste LehrerInnengruppe fast doppelt so viele Deutschlandismen unterwellt hatte. Hinsichtlich der unterwellten Austriazismen zeigte sich die Tendenz, dass die jüngste LehrerInnengruppe etwas mehr Austriazismen unterwellt hatte (18,8\% der Austriazismen unterwellt) als die älteste LehrerInnengruppe (15,2 \% der Austriazismen unterwellt). In Kombination betrachtet deutet dies darauf hin, dass jüngere LehrerInnen Deutschlandismen eher akzeptieren als ältere LehrerInnen.

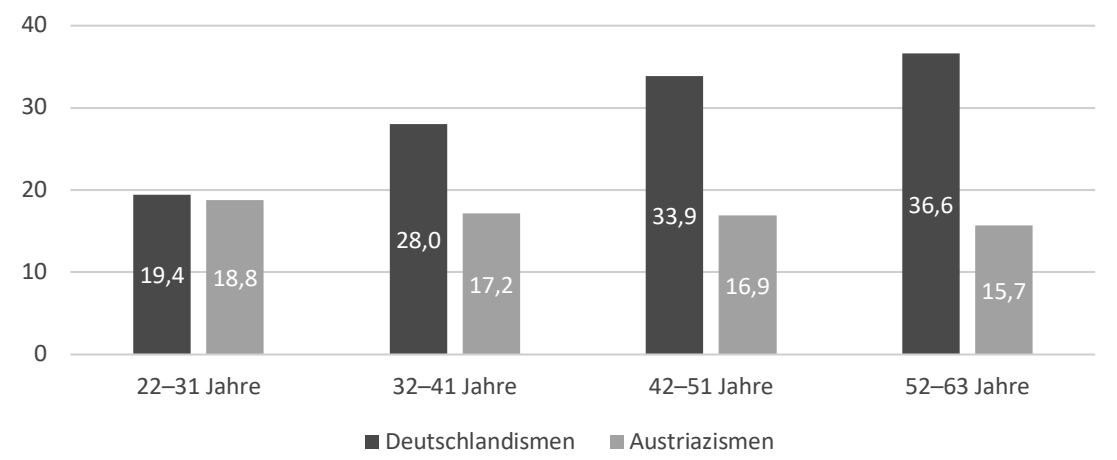

Abb. 60: Unterwellt: Unterschiede zwischen den Altersgruppen (in \%)

13 Z. B. Christine Nöstlinger: Geschichten vom Franz. 
Beim Umgang mit Deutschlandismen ergaben sich auch abhängig von der Schulform signifikante Unterschiede $(\mathrm{p}=\mathrm{o}, 048)$. LehrerInnen der AHS und Sek. II unterwellten von allen LehrerInnen die meisten Deutschlandismen. Sie markierten rund $36 \%$ der Deutschlandismen mit einer Welle. Die VolksschullehrerInnen unterwellten etwa $32 \%$ der Deutschlandismen, die LehrerInnen der HS/NMS rund $22 \%$. HS- und NMS-LehrerInnen unterwellten somit nicht nur die wenigsten Deutschlandismen, sondern auch die wenigsten Austriazismen: Nur knapp $12 \%$ der Austriazismen im Text wurden von den HS/NMS-LehrerInnen unterwellt (dafür wurden Austriazismen umso häufiger von den HS/NMS-LehrerInnen als falsch angestrichen - deutlich häufiger als von anderen LehrerInnen, wie weiter unten näher beschrieben wird). Von VS- und AHS-/Sek.-II-LehrerInnen wurden etwa $17 \%$ der Austriazismen mit einer Welle markiert. HS/NMS-LehrerInnen korrigierten rigoroser als die anderen Gruppen, wenn es um richtig/falsch ging.

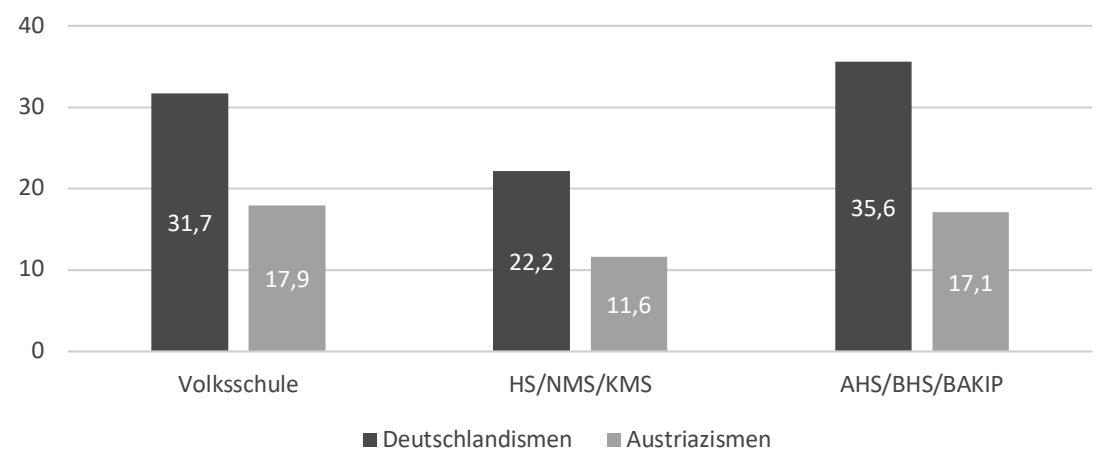

Abb. 61: Unterwellt: Unterschiede zwischen den Schulformen (in \%)

Auch der Unterrichtsort der LehrerInnen wirkte sich auf das Korrekturverhalten aus, wobei die Diskrepanz beim Unterwellen von Varianten zwischen den östlichen Bundesländern (Wien, Niederösterreich und Burgenland) und Vorarlberg am markantesten war. Hochsignifikant $(p=0,001)$ waren die Unterschiede zwischen den Bundesländern beim Unterwellen von Austriazismen: Während im Osten Österreichs durchschnittlich $17 \%$ der Austriazismen unterwellt wurden, waren es in Vorarlberg rund $42 \%$. Umgekehrt unterwellten DeutschlehrerInnen aus Ostösterreich durchschnittlich $38 \%$ der Deutschlandismen, in Vorarlberg wurden dagegen nur halb so viele Deutschlandismen (ca. 19\%) unterwellt: ein Indiz dafür, dass das Standarddeutsch in Vorarlberg als dem deutschen Standarddeutsch relativ nahe empfunden wird (siehe Abb. 62 auf S. 172).

Wenn es darum ging, ob eine Lehrkraft Austriazismen bzw. Deutschlandismen nicht nur unterwellte, sondern als falsch empfand und dementsprechend als falsch korrigierte, zeigten sich ebenfalls signifikante Regionsunterschiede ( $p=0,012$ bzw. o,016). Lehrkräfte aus der Region Mitte waren in jeder Hinsicht am (relativ 
gesehen) „korrigier-eifrigsten“, denn sie strichen sowohl die meisten Deutschlandismen als auch die meisten Austriazismen an. Vorarlberger LehrerInnen korrigierten fast ebenso viele Austriazismen wie die Region-Mitte-KollegInnen, aber von allen Lehrkräften markierten sie mit Abstand die wenigsten Deutschlandismen als falsch - eine ähnliche Tendenz wie beim Unterwellen von Deutschlandismen. LehrerInnen aus Südostösterreich sowie ihre KollegInnen aus Tirol waren mit dem Rotstift am zurückhaltendsten: Sie markierten nur vereinzelte Austriazismen, und noch weniger Deutschlandismen als „falsch“:

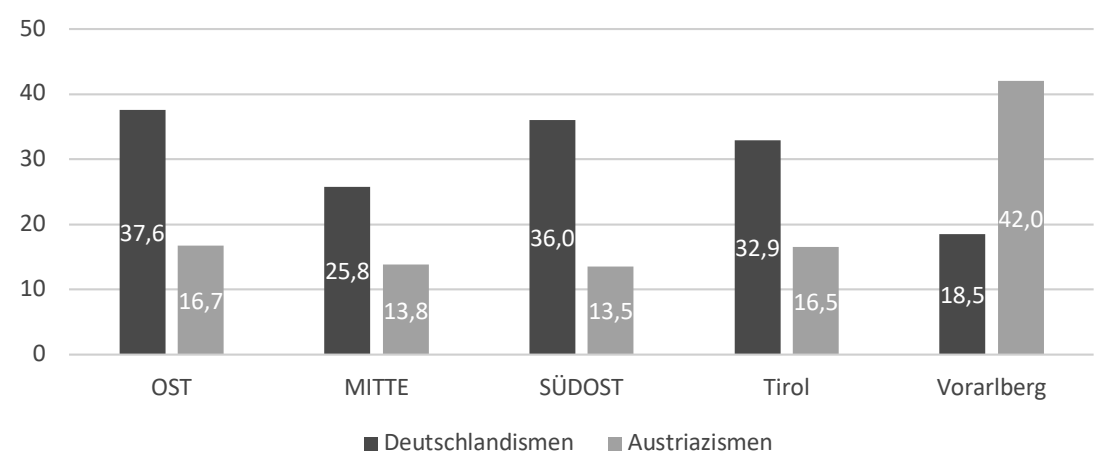

Abb. 62: Unterwellt: Unterschiede zwischen den Regionen (in \%)

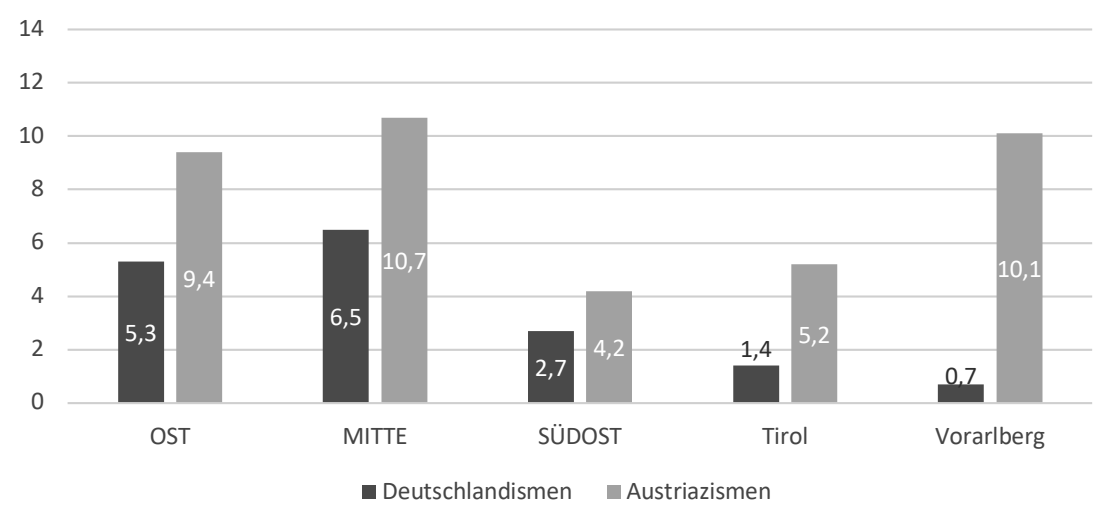

Abb. 63: Korrigiert: Unterschiede zwischen den Regionen (in \%)

Beim Korrekturverhalten kamen darüber hinaus auch signifikante Unterschiede nach den Variablen Alter und Schulform zum Vorschein. Hochsignifikant sind die Unterschiede zwischen den Altersgruppen der LehrerInnen hinsichtlich der Korrektur von Deutschlandismen $(\mathrm{p}=\mathrm{o}, 016)$. Die wenigsten Deutschlandismen wurden von den beiden jüngeren Altersgruppen (also LehrerInnen im Alter von 
22 bis 41 Jahren) als falsch markiert, während vor allem die Gruppe der 42-51-jährigen LehrerInnen deutlich mehr Deutschlandismen als falsch wertete, nämlich rund $7 \%$. Umgekehrt waren es wiederum - ähnlich wie bei den unterwellten Ausdrücken - die jüngsten LehrerInnen (22-31 Jahre), welche die meisten Austriazismen als falsch korrigierten (10,1\%):

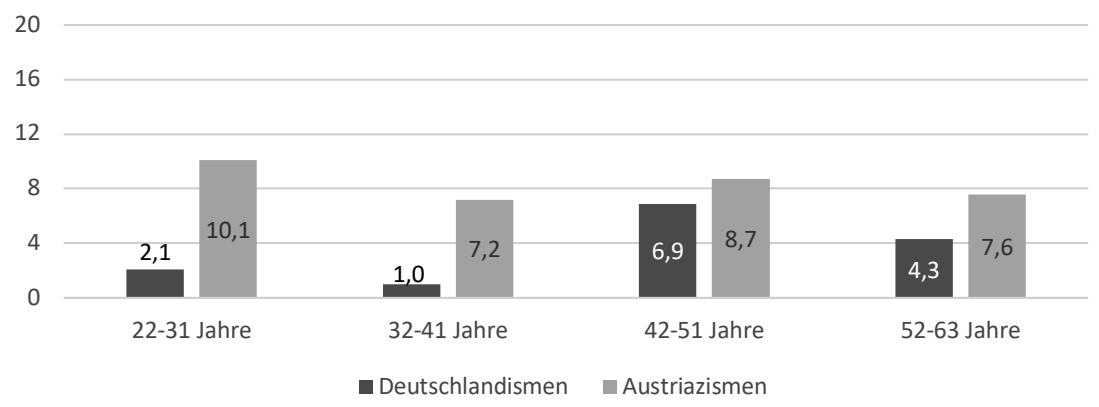

Abb. 64: Korrigiert: Unterschiede nach Altersgruppen (in \%)

Betrachtet man die Ergebnisse nach Schulform, werden eklatante Unterschiede zwischen dem Korrigieren und dem Unterwellen sichtbar $(\mathrm{p}=0,034)$. Geht es um richtig oder falsch, haben die LehrerInnen der HS/NMS mit Abstand die meisten Austriazismen (13,9\%) angestrichen. Von den LehrerInnen der Volksschule und AHS/Sek. II wurden jeweils nur etwa halb so viele Austriazismen als falsch markiert:

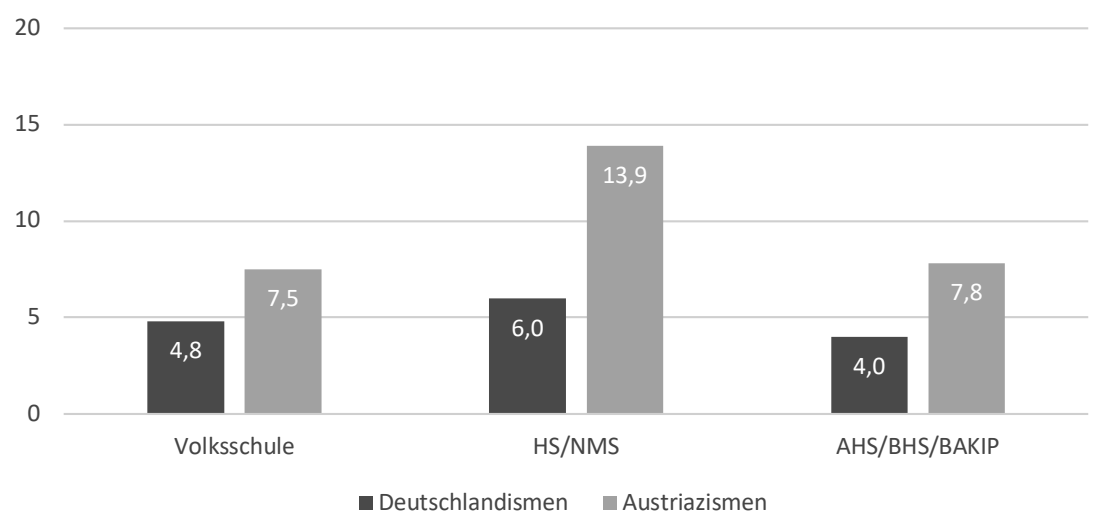

Abb. 65: Korrigiert: Unterschiede zwischen den Schulformen (in \%)

Manche LehrerInnen führten im Fragebogen unter dem Aufsatz zusätzliche Bemerkungen an, um transparent zu machen, warum etwas korrigiert wurde. Ein Beispiel liefert der folgende Kommentar: 
Beim Korrigieren würde ich die Wörter, die eher in Deutschland benutzt werden, akzeptieren, aber am Rand ihre Herkunft und hauptsächliche Verwendung dazuschreiben, FALSCH ist nur „habe gestanden“, das heißt etwas ganz Anderes. (z. B. „Gesteh, Schurke!“).

Das Thema „Textkorrektur und Umgang mit sprachlichen Varianten“ war auch Gegenstand der LehrerInnen-Interviews. Dabei waren die Meinungen unter den interviewten LehrerInnen sehr vielfältig. Augenscheinlich war dies beispielsweise bei der Frage des Präteritums oder des Perfekts als Erzählzeit im Fragebogen. Es haben zwar nur etwa 40 \% der LehrerInnen im Fragebogen das Perfekt korrigiert, aber in den Interviews gab die Mehrheit der LehrerInnen an, das Präteritum als Erzählzeit zu bevorzugen. Vereinzelt wurde darauf bestanden, wie das folgende Beispiel einer Lehrerin aus Oberösterreich (FO1) zeigt:

Ahm da sind wir, also auch meine Kollegen sehr streng. Also während in der mündlichen Sprache so ziemlich alles erlaubt ist, wird in der schriftlichen Sprache AUSDRÜCKLICH Präteritum verlangt.

Die Verwendung eines Artikels vor Eigennamen sowie einige Austriazismen wurden von manchen LehrerInnen ebenfalls als Fehler markiert oder zumindest unterwellt. Auf die Frage, wie man beispielsweise mit Ausdrücken wie „das Leiberl“ oder "gestern bin ich mit dem Franz ins Kino gegangen“ umgehen solle, erklärte eine Lehrerin aus Tirol (FT4), dass sie in schriftlichen Erzähltexten hinsichtlich der Verwendung von Präteritum oder Perfekt „durchgängig das Präteritum verlangen“ und „das Leiberl“ unterwellen würde. Eine Lehrerin aus Wien (FW3) wiederum bewertete die Artikelverwendung vor Vornamen („mit dem Franz“) als Grammatikfehler, der folglich ausgebessert werden müsse.

Andere LehrerInnen waren nicht dieser Meinung. Für sie waren das Perfekt als Erzählzeit, die Artikel vor Eigennamen oder Varianten wie „Leiberl“ keine Fehler, wie ein Lehrer aus Salzburg (MSa3) im Interview betonte. Er verwies darauf, dass SchülerInnen häufig nach der Klassenlektüre von Christine Nöstlinger das Perfekt und den Artikel vor Namen übernehmen würden. Es komme auf die Textsorte („erzählender Text oder argumentativer Text“) an und „wenn Kinder fabulieren sollen, ahm dann wird das eher akzeptabel sein“. Grundsätzlich, so der Salzburger Deutschlehrer, sei der Gebrauch von Austriazismen kein Problem, solange es sich um akzeptierte und auch normierte Austriazismen handle.

Aus den Interviews für unsere Untersuchung kann man eine wichtige Schlussfolgerung ableiten: Viele LehrerInnen sind unsicher, was als Fehler zu gelten hat und was nicht. Das belegen die häufigen Relativierungen und die rhetorischen Widersprüche, die sich in den Statements zur Benotungsthematik finden. Die folgende Interviewpassage mit einer Lehrerin aus der Steiermark (FSt1) unterstreicht, wie schwer LehrerInnen der Umgang mit bestimmten Phänomenen des österreichischen Deutsch fallen kann: 
Mit DEM Franz - - - Najo, wenns zum Erzähltext passt, aber natürlich würd ichs anstreichen und des besprechen. Oder benennen, dass des ah - - umgangssprachlich is, aber wenn das in den/in den Kontext dieses Aufsatzes passt, dann schon.

Dass die Verwendung von Präteritum und Perfekt in schriftlichen Erzähltexten eine Quelle der Unsicherheit bildet, wurde von einer burgenländischen Lehrerin (FB2) deutlich gemacht: „Na, des is überhaupt a schwierige Frage, würd ich sagen, weil des ja net SO festgelegt is, ja? Also es gibt schon diese Zeitenfolge, aber so/ so richtig festgelegt findet ma des ja net, ja?"

Dieser Kommentar bringt uns dazu, dass in der Fachliteratur häufig darauf verwiesen wird, dass die empirische Beschreibung des österreichischen Deutsch als unzureichend einzustufen ist. Diese Kodifizierung stellt LehrerInnen in der Praxis vor die schwierige Situation, dass sie bei der Korrektur bis zu einem gewissen Grad auf sich allein gestellt sind und Normfragen nach persönlichem Ermessen entscheiden müssen. Die folgende Interviewpassage mit einer Wiener Lehrerin (FW1) illustriert recht anschaulich, wie subjektiv der Korrekturprozess in der Praxis gehandhabt wird:

Was ich SCHON angestrichen hab, ist diese konsequente Verwendung des Perfekt ... das streich ich SCHON an, das üben wir aber auch sehr intensiv, aber zum Beispiel äh DER Franz, das schon auch, der Mama hab ich geholfen, die Oma, die Frau Meier, der Wolfgang, mein Bruder, also das streich ich AUCH an, ah was ich NICHT anstreich sind so TYPISCH österreichische Ausdrücke wie „Greißler“ und ah „Wimmerl“, „Stutzen“, „GEFLADERT“ allerdings schon. Des ghört für mich dann eigentlich schon in den Dialekt hinein.

So wie dieser Lehrerin dürfte es vielen anderen Lehrkräften auch gehen: Die Entscheidung darüber, was Dialekt ist und was nicht, wird beim Verbessern von Texten intuitiv getroffen und im Zweifelsfall mitunter wieder relativiert. Auffällig ist weiters, dass die zitierte Lehrerin „typisch österreichische Ausdrücke“ mit ebenso österreichischen Phänomenen wie Artikelgebrauch vor Eigennamen und die Verwendung des Perfekt kontrastiert hat. Diese Unterscheidung macht deutlich, dass Deutschlehrende beim Umgang mit der österreichischen Varietät große Unsicherheiten haben, was für den Deutschunterricht besonders problematisch ist.

Denn DeutschlehrerInnen sind normsetzende Instanzen (vgl. Kap. 2.6). Obwohl den LehrerInnen damit bei der Vermittlung des österreichischen Deutsch als Bildungssprache eine prägende Rolle zukommt, müssen sie beim kontinuierlichen Urteilen über Korrektheit und sprachliche Angemessenheit ohne einen verbindlichen normativen Maßstab zurechtkommen.

Erkennbar wurde diese Problematik auch in einer langen Passage der LehrerInnen-Gruppendiskussion, die sich konkret um die Akzeptanz des Perfekts anstelle des Präteritums als schriftlicher Erzählzeit im österreichischen Deutsch gedreht hat. Zur qualitativen Illustration der Normfrage sei die betreffende Passage in der Gesprächsrunde hier etwas ausführlicher dargestellt. 
Der Moderator stellte mit Verweis auf die Autorin Christine Nöstlinger, die das Perfekt als Erzählzeit verwendet, folgende Zwischenfrage: „Ist des mündlich/ schriftlich/ ist des schriftlich akzeptabel? Is des falsch, oder? Wie sehen Sie des? Die Verwendung - des Perfekts statt des Präteritums als Erzählzeit?" Daraufhin entstand eine sehr lebhafte Diskussion. Die Gruppe war sich zunächst einig, dass das Präteritum in der Sekundarstufe II als Norm eingefordert werden müsse, dass in der Volksschule das Perfekt akzeptiert werden solle und dass die SchülerInnen in der Volksschule und Sekundarstufe I an das Präteritum herangeführt werden müssten. In weiterer Folge wurde dann aber die Normsetzung grundsätzlich hinterfragt, was letztlich zu einer prinzipiellen Infragestellung der Norm und zu einem Plädoyer für ein flexibles Normkonzept führte.

Eine der Deutschlehrerinnen brachte das Problem schon zu Beginn auf den Punkt:

Wir sehen ja des alles immer wieder ... aus dem Blickwinkel der Deutschlehrerinnen. A:h, - u:nd, a:h, f:ür bestimmte Textsorten gibts eben diverse Normen, die auch vielleicht nicht unabänderlich sind, ja? A::h, und deshalb wird dann in einem ERLEBNISaufsatz unterwellt oder unterstrichen oder angemerkt, das ist falsch, wenn ein Kind im Perfekt erzählt. Aber ich würds NICHT so als absolit/ absolut und unabänderlich sehen (F8, 598).

Sind bei einer Orientierung am tatsächlichen Sprachgebrauch also textsortenspezifische Normen gefragt und welche Bereiche sind für das österreichische Standarddeutsch im Klassenzimmer-Alltag abzustecken? Auf die Frage des Moderators, ob sie das Perfekt anstreichen würde, antwortete eine Volksschullehrerin (F2, 607): „Niemals.“ ((schüttelt heftig den Kopf)) F4, eine AHS/HS-Lehrerin, pflichtete ihr bei (607): „Nein. Ich würds akzeptieren.“ Aber in der Sekundarstufe II sei die Sachlage anders, gab eine Kollegin zu bedenken $\left(\mathrm{F}_{1}, 614\right)$ :

„In der Sek zwei, da ist es definitiv NICHT möglich, eine Erzählung, also schriftlich narrativ im Perfekt zu sein. Aso, des is narrativ völlig klor, ahm, der derzeitige Stand. Aso keine Frage, dass das nicht unabänderlich ist, aber von den Normen ist es, ä:h, is es/ ähm, würd i sogn/ i was net, ob ihr mir zustimmts, oder?“ Eine AHS-Lehrerin stimmt zu: „Bin i ganz bei dir.“ $(\mathrm{F} 7,619)$

Eine Lehrerin warf ein, dass sie es vor allem dann anstreichen würde, wenn zwischen Perfekt und Präteritum gewechselt wird. Sonst würde sie das Perfekt akzeptieren, erklärte die Lehrerin, die für situative, textsortenabhängige und flexible Normen plädierte (F8, 736):

Ich glaube schon, dass die Normen ja durchaus einen Sinn haben [...] In manchen Verwendungszusammenhängen wird das Perfekt wahrscheinlich gut passen, und do sollt es auch erlaubt sein, denk ich mir, und do sollt man vielleicht manchmal überlegen, ob man, ob man überall diese Regeleinhaltung so streng b/ah, sehen muss. [...] 
wichtig ist, dass man net hin und her switcht und immer wieder wackelt, des is eher das Problem meiner Schüler.

Dem stimmte eine weitere Lehrerin im Prinzip zu, wobei sie sogar so weit ging, die Norm allgemein in Frage zu stellen: „Letztendlich ist mir bewusst geworden, es STEHT DIE: NORM, ähm, Erzählungen sind im Präteritum zu sein. KEIN MENSCH frogt, ob diese Norm überhaupt richtig ist“ (F9, 637). Ein Diskussionsteilnehmer gab dem Recht, relativierte dann aber (M1, 647):

Aso, jo. I muss zugeben, mir gehts genauso. [...] Im Deutschbuch steht, Präteritum ist die schriftliche Erzählform und [...] Perfekt ist die MÜNDLICHE - erlaubt, jo, aber nicht schriftlich. [...] Aso i würds anstreichen, eben, weil für mich diese Norm - verbindlich ist. Ah, - i hob die a ehrlich gsogt nu net hinterfrogt.

Eine weiterere Diskussionsteilnehmerin stellte Normen noch grundlegender in Frage - auch punkto Textaufbau - und erzählte von einer Erfahrung mit einer Unterstufenklasse, in die auch ein Bub mit afrikanischen Wurzeln ging (F6, 647):

Der hat ma Gschichten geschrieben, der hat irgendwo angefangen/ aso die ham so gekreist diese Geschichten, ja? Die ham KAN Höhepunkt gehabt, ja? Also, EHRLICH net. Und i hab mir dann gedacht, ja, der hat diese Norm jetzt net erfüllt, aber es is a gute Gschicht, ja?

In weiterer Folge wurde die „Normfrage“ auf eine noch allgemeinere Ebene gehoben: Man brauche Normen, um sich daran zu orientieren und damit arbeiten zu können, hieß es - und erst, wenn man wisse, dass es sich um eine Norm handle, könne man überhaupt eine Norm brechen:

$\mathrm{Na}$, na, ich, ich, ich denke, das mit den Normen. Ähm, Diese Normenfrage. Ganz prinzipiell mit der Normenfrage. Aber es is halt trotzdem so, aso, äh, zumindest, ahm, seh ich das, dass es LEICHTER ist, sich, ahm, zum Beispiel EINE NORM, eine/ an, an, an EINER Norm, ah, sich zu orientieren, aber diese Norm nicht für absolut ((breitet Hände zustimmend aus)) gültig zu halten. Sondern für änderbar. Aber erst, wenn ich weiß, dass es eine Norm ist, kann ich überhaupt eine Norm brechen, beziehungsweise MIT dieser Norm arbeiten. $\left(\mathrm{F}_{3}, 718\right)$

Eine Diskussionsteilnehmerin plädierte schließlich nochmals für einen flexiblen Normbegriff, indem sie auf den individuellen Spielraum verwies, den LehrerInnen als normsetzende Instanzen ausschöpfen könnten:

Letztendlich sind WIR die Entscheidenden, ... WIR können uns nur bilden und möglichst weit werden und nicht - BLIND Normen v:e:rfolgen, ... wenn ma si genauer 
anschaut, eigentlich frogt, woher kumman sie, w::elcher liebe Gott hat des gsogt, dass ein/ eine Erzählung im Präteritum sein MUSS? (F9, 751)

Diese widersprüchliche Diskussion bildet unseres Erachtens den Alltag der LehrerInnen als normsetzende Instanzen treffend ab: Den heimischen Lehrkräften sind vage Normen vorgegeben (auf den Kodex, der in Österreich eher schwach dokumentiert ist, wird übrigens nie Bezug genommen), die zum Teil in Widerspruch zur Gebrauchsnorm und zur Sprachrealität stehen. An diesen vagen Normen können sich die LehrerInnen nur bis zu einem gewissen Grad orientieren - vielfach müssen sie ihre Rolle als normsetzende Instanz „auf eigene Faust“ wahrnehmen und die schwammigen Normen quasi nach bestem Wissen und Gewissen eigenverantwortlich anwenden. Wie gehen LehrerInnen aber behelfsmäßig mit der regulatorischen Lücke zwischen Gebrauchsnorm und kodifizierter präskriptiver Norm um?

Die Frage, an welchen Normen sich die in den Interviews befragten Lehrpersonen orientieren und welche Kodices sie bei der Korrektur bevorzugen, ergab, dass die Mehrheit der LehrerInnen sowohl im Österreichischen Wörterbuch als auch im Duden nachschlagen. Einige LehrerInnen sagten, dass sie nur den Duden oder nur das Österreichische Wörterbuch benutzen würden, wobei es in etwa gleich viele waren, die das eine oder das andere Nachschlagwerk bevorzugt haben. Manche gaben für ihre Präferenz auch eine Begründung an, wie dieser Lehrer aus Tirol (MT3):

Ah am schnellsten zugänglich is das österreichische Wörterbuch. Des mit hinten den ... ah ... Appendix für die Rechtschreibregeln und so weiter, jo des is/ hat/bei uns hat jeder das österreichische Wörterbuch, das is natürlich als Unterrichtsmaterial dann sehr super.

Eine Lehrerin aus Salzburg (FSa2), die aus Deutschland stammt, favorisierte wiederum den Duden:

Da orientier ich mich eigentlich immer (und) ehrlich gesagt auch (nur) online, ja, weil da is/find ich SUPER, da stehn dann schon immer Synonyme und die genaue BEDEUTUNG und äh irgendwelche grammatikalischen Phänomene, ja.

Auch ein Lehrer aus Salzburg (MSa4) plädierte ganz klar für den Österreich-Duden:

Und da is für mi der Duden irgendwie schon die Norm. Diese ÖSTERREICH-Ausgabe. Die Österreich-Ausgabe des Dudens, an der orientier ich mich jetzt, i man, wenn die Schüler den Duden haben, natürlich is des für mi a die Norm jetzt hier an der Schule. Oba, wenns des österreichische Wörterbuch is, $\mathrm{i}$ hob da jetzt keine/keine Berührungsängste, weder mit dem einen noch mit dem andern. Der Duden is halt größer, dicker, umfassender ... es is halt einfach, der Duden is der Duden. 
Wie auch immer die individuellen Präferenzen der DeutschlehrerInnen aussehen mögen - die Lückenhaftigkeit des Kodex des österreichischen Deutsch und die daraus resultierende Notwendigkeit, in Zweifelsfällen ohne Referenzwerk entscheiden zu müssen, wurden in den Interviews sichtbar. Eine Lehrerin aus der Steiermark (FSt3) nannte Beispiele aus Schülertexten, die bei der Textkorrektur knifflig wären:

[...] ich steh am Fenster, ich steh beim Fenster is so was typisches/bin gegangen AH, ich bin gesessen, ich habe gesessen also/ [...]. Des kommt auch in [Schüler-]Texten vor und dann sitzt man schon dort und überlegt: Mmh. Is das jetz richtig?

Dass ein Lehrer als normsetzende Instanz abseits von simplen Rechtschreib- oder Grammatikfragen letztlich dem eigenen Sprachempfinden vertrauen muss, mit dieser Vorgangsweise aber immer subjektiv agiert, brachte ein Interviewpartner aus dem Burgenland (MB1) auf den Punkt:

Wenns jetzt um Rechtschreibung geht oder ... um den Fall nach einem Pronomen, dann orientiert man sich am/am österreichischen Wörterbuch oder am/am österreichischen Duden. Ansonsten - hält man das für richtig, was man selbst verwendet.

Zusammenfassend kann festgehalten werden, dass beim Korrekturverhalten der Lehrenden in unserem Sample eine Tendenz dazu erkennbar war, bestimmte standardsprachliche österreichische Ausdrücke als Normverletzung zu klassifizieren und daher zu korrigieren - darunter eine Reihe von laut Kodex „einwandfreien“ Begriffen, sowie auch vereinzelt solche, die als „Grenzfälle des Standards“ eingeordnet werden können (der Kodex ist bezüglich der "Grenzfälle des Standards“ uneinheitlich bzw. lückenhaft). Auf das Phänomen der Korrektur von per se richtigen sprachlichen Formen und Ausdrucksweisen, die von den Kodices eigentlich zugelassen werden, weisen auch andere Studien hin (Schmidlin 2017, 51 und Davies 2017, 137), und es wird durch unsere Ergebnisse bestätigt.

Austriazismen wurden eher korrigiert als nur unterwellt, und sie wurden auch signifikant häufiger korrigiert als Deutschlandismen. Was die Korrektur von eigennationalen Varianten betrifft, kommt Gatta $(2017,389)$ zu ähnlichen Ergebnissen. Sie stellt in einer Untersuchung des Korrekturverhaltens von Schweizer LehrerInnen in Hinblick auf Helvetismen fest, dass Schweizer Lehrkräfte Helvetismen häufig korrigieren: Gegenstand der Korrektur sind bei den Schweizer KollegInnen nicht nur lexikalische, sondern vor allem auch syntaktische Varianten, die als Normverletzung markiert werden, wobei die jüngeren eidgenössischen LehrerInnen tendenziell etwas toleranter gegenüber Helvetismen zu sein scheinen. Dies deckt sich jedoch nicht mit unseren Ergebnissen für Österreich, denn im Sample der österreichischen Lehrkräfte sind es vor allem die jüngsten PädagogInnen, die die meisten Austriazismen korrigieren. 
Unter den österreichischen Lehrpersonen der vorliegenden Studie wurden Deutschlandismen weiters zwar häufiger als Austriazismen durch Unterwellen als stilistisch unpassend markiert, aber seltener als falsch korrigiert. Wenn Deutschlandismen korrigiert oder unterwellt wurden, dann waren häufiger ältere LehrerInnen am Werk. Unter jüngeren LehrerInnen war hingegen eine größere Akzeptanz von Deutschlandismen erkennbar. Besonders häufig wurden Austriazismen von LehrerInnen der HS/NMS als nicht normgerecht angestrichen. Die Ergebnisse zeigten auch, dass die LehrerInnen aus Vorarlberg Austriazismen kritischer gegenüberstanden als ihre KollegInnen aus den übrigen Bundesländern, weil sie Austriazismen nicht nur häufiger unterwellten, sondern auch öfter als falsch korrigierten. Was die Akzeptanz von Deutschlandismen in den verschiedenen Regionen Österreichs betrifft, waren es die LehrerInnen aus Ost- und Südösterreich, die in Summe mehr Deutschlandismen mit einer Welle versahen. In den Interviews entstand der Eindruck, dass es hinsichtlich der Normerwartungen in der LehrerInnenschaft große Auffassungsunterschiede und Unsicherheiten gibt. Das belegen die häufigen Relativierungen, die in den Interviews aufgefallen sind. Auch aus der Gruppendiskussion ging hervor, dass unter den Deutschlehrenden ambivalente Normvorstellungen vorherrschen.

\subsubsection{Normen und Korrektur bei mündlicher Kommunikation im Deutschunterricht}

Im Rahmen der Interviews wurden die DeutschlehrerInnen auch dazu befragt, welche Sprachregister sie von ihren SchülerInnen bei mündlicher Kommunikation erwarten. Das Ergebnis war eine sehr große Bandbreite von Meinungen, die von der durchgängigen Standarderwartung bis hin zum relativ situationsabhängigen Einfordern der Standardsprache reichten.

Einig waren sich die befragten LehrerInnen, dass in bestimmten Situationen ausschließlich der Standard adäquat ist und demzufolge eingefordert werden muss. Manche LehrerInnen erwarteten allerdings von ihren SchülerInnen in sämtlichen Kommunikationssituationen im Deutschunterricht durchgängig den Standard. Würden seine SchülerInnen vom Standard abweichen, mache er sie darauf aufmerksam, berichtete ein Lehrer aus Vorarlberg (MV2): „[...] sie versuchen/oder sie fangen dann mit Dialekt an, und (sag ich), na bitte, hochdeutsch!““ Ähnlich äußerte sich ein weiterer Deutschlehrer aus Vorarlberg, der seiner Aussage nach ebenfalls situationsunabhängig sofort korrigiert („Relativ rigoros.“). Einer Lehrerin aus dem Burgenland (FB2) war das Ziel der konsequenten schülerseitigen Standardverwendung ebenfalls ein Anliegen. Ihrer Meinung nach sei es wichtig, die Beherrschung des Standards meistens am Anfang des Schuljahres schon einzuführen: „[...] also wenn ich die Klasse neu bekomm, dass ich dann sag: ,Kannst Du mir das bitte in der Hochsprache wiederholen?' oder halt: ,Kannst Du Dich darauf konzentrieren oder halt - was i net -,in Zukunft das bitte in der Hochsprache formulieren?“ 
Andere LehrerInnen machten es von der Situation abhängig, ob auch Umgangssprache angebracht sein kann, wie die folgende Interviewpassage mit einem steirischen Lehrer (MSt2) verdeutlicht: „Erstens kommts drauf an, was ausgemacht is, zweitens kommts drauf an, äh welche Sprach- oder Kommunikationsfunktion gerade eingesetzt wurde [...]. Wenn sozusagen situationsadäquat ein falsches Register verwendet wurde."

Einer Meinung waren viele LehrerInnen darin, dass man Prüfungssituationen besser aus dem strengen Korsett der Standarderwartung ausnehmen solle, vor allem, wenn es um inhaltliche Antworten geht und nicht um die sprachliche Form, wie ein Lehrer aus Salzburg (MSa3) betonte:

Bei einer Prüfung kommts drauf an sozusagen, wenns um die Form geht, dann muss es natürlich dem entsprechen, wenns in einer Fragebeantwortung im Dialekt passiert, dann is es im Dialekt, es sei denn/ wenns nur um den Inhalt geht, dann is das auch in Ordnung, da wer i net drauf bestehn und die Nervosität net no steigern.

Dass generell viele SchülerInnen massive Schwierigkeiten bei der souveränen Verwendung der Standardsprache hätten, wurde von etlichen LehrerInnen als problematisch eingestuft. Eine Lehrerin aus Tirol (FT2) brachte es unverblümt auf den Punkt: „I man Standard kann man das ja fascht net nennen, was a österreichischer Schüler zambringt, na? (lacht) Aber ich verlange, dass sie sich der Standardsprache möglichst annähern."

Wie führen die befragten Lehrpersonen die Schüler an den „Standard“ heran? Indem sie im Unterricht Dialekt, Umgangssprache und Standard thematisieren und auf Registervariabilität hinweisen - insbesondere auf deren Funktionalität. Um die SchülerInnen langsam über den Aspekt der situativen Angemessenheit an die Standardsprache heranzuführen, bestehen die LehrerInnen eigenen Angaben zufolge im Unterricht nicht immer rigoros auf den Standardgebrauch. Eine Lehrerin aus der Steiermark (FSt1) beschrieb ihre Herangehensweise an das Spannungsfeld zwischen Dialekt und Standard folgendermaßen:

I thematisier des IMMER, und wenn die Schüler sogn: „Derf mas im Dialekt sogn?“, sog i: „Jo freilich, du darfst das im Dialekt sogn“, und ... wenn sie ihre Gedanken bündeln, dürfen sies sogn in der Sprache, in der sie wollen, UND ich sag ihnen gleichzeitig, Ziel is des, dass sie den Standard bis zur Matura hin sprechen können. [...] also NIEMALS (wenn) i mit der Sprache oabeit mit falsch-richtig bewerten. [...] Als Ziel [...], dass man Sprache nur als Spiralcurriculum sehen kann, dass das net „foisch“/,richtig“ gibt, sondern dass es einen Weg gibt, der sie immer weiter hinauf ahm führt, ... schon mit dem Standard als Ziel, aber das Andere hat alles Platz.

Insgesamt geht aus den Fragebogendaten, den Interviews und den Gruppendiskussionen eines klar hervor: Innerhalb der Norm, die jede/r einzelne Lehrer/in 
für sich als gültig und adäquat erachtet, gibt es einen großen Spielraum. Was der eine als einen gängigen Austriazismus erachtet, ist für den anderen zu umgangssprachlich, während der eine oder andere Deutschlandismus einigen LehrerInnen wiederum „zu deutsch“ ist. Bei manchen Lehrkräften gilt der Grundsatz, dass in jeder Unterrichtssituation Standarddeutsch gesprochen werden muss - womit viele SchülerInnen den Beobachtungen der LehrerInnen zufolge überfordert sind. Die Alternative ist, dass Lehrpersonen einen langsamen Weg zum Beherrschen der Standardsprache unter gleichzeitiger Betrachtung der Funktionsbereiche der Umgangssprache und des Dialekts einschlagen. Ergebnisse zur Frage, welche Varietäten von SchülerInnen und LehrerInnen in der Klasse verwendet werden, finden sich in Kap. 6.5.1.

\subsection{Sprachverwendung: Präferenz von Deutschlandismen/ Austriazismen $^{14}$}

Ein weiterer Themenkomplex im Fragebogen beschäftigte sich mit der Frage, wie die Lehrenden und Lernenden einer Reihe von Austriazismen und Deutschlandismen gegenüber eingestellt sind bzw. welche Präferenzen sie bei der Verwendung dieser Ausdrücke haben. Es wurde bewusst eine möglichst vielseitige Auswahl an Varianten getroffen, darunter sowohl solche, von denen wir angenommen haben, dass sie stabile Austriazismen sind (z. B. Jänner, heuer, bin gestanden), als auch andere, bei denen anzunehmen war, dass deren Verwendung zurückgeht (z. B. das SMS, Bub, die Cola). Zusätzlich sollte ausgelotet werden, wie es um den bevorzugten Gebrauch von lexikalischen Varianten aus Ost- bzw. Westösterreich unter LehrerInnen und SchülerInnen nach Selbstauskunft bestellt ist.

Den ProbandInnen wurden 30 Sätze vorgelegt, die Wahlmöglichkeiten zwischen je zwei Varianten enthielten (nach dem Kodex ${ }^{15}$ als spezifische und unspezifische Austriazismen/Deutschlandismen/gemeindeutsche Ausdrücke bzw. als ostösterreichisch/westösterreichisch eingestuft). Die Fragebögen der LehrerInnen und SchülerInnen enthielten in diesem Befragungsteil exakt dieselben 30 Sätze. Die LehrerInnen und SchülerInnen hatten nun anzugeben, welche der zur Wahl stehenden Ausdrücke sie in schriftlicher Kommunikation eher verwenden würden. Lediglich das erste Item, in dem nach Abschiedsgrußformeln gefragt wurde, bezog sich auf den mündlichen Sprachgebrauch, und da waren auch Mehrfachangaben möglich. Insgesamt standen 31 Doubletten (26 Austriazismen/Deutschlandismen, 5 ost- bzw. westösterreichische Varianten) und fünf Grußvarianten zur Auswahl, die hier aufgelistet sind:

14 Vgl. dazu auch de Cillia 2016b.

15 Als Referenzkodex wurden dafür v. a. das ÖWB, das Variantenwörterbuch und Jakob Ebners Arbeiten herangezogen. 


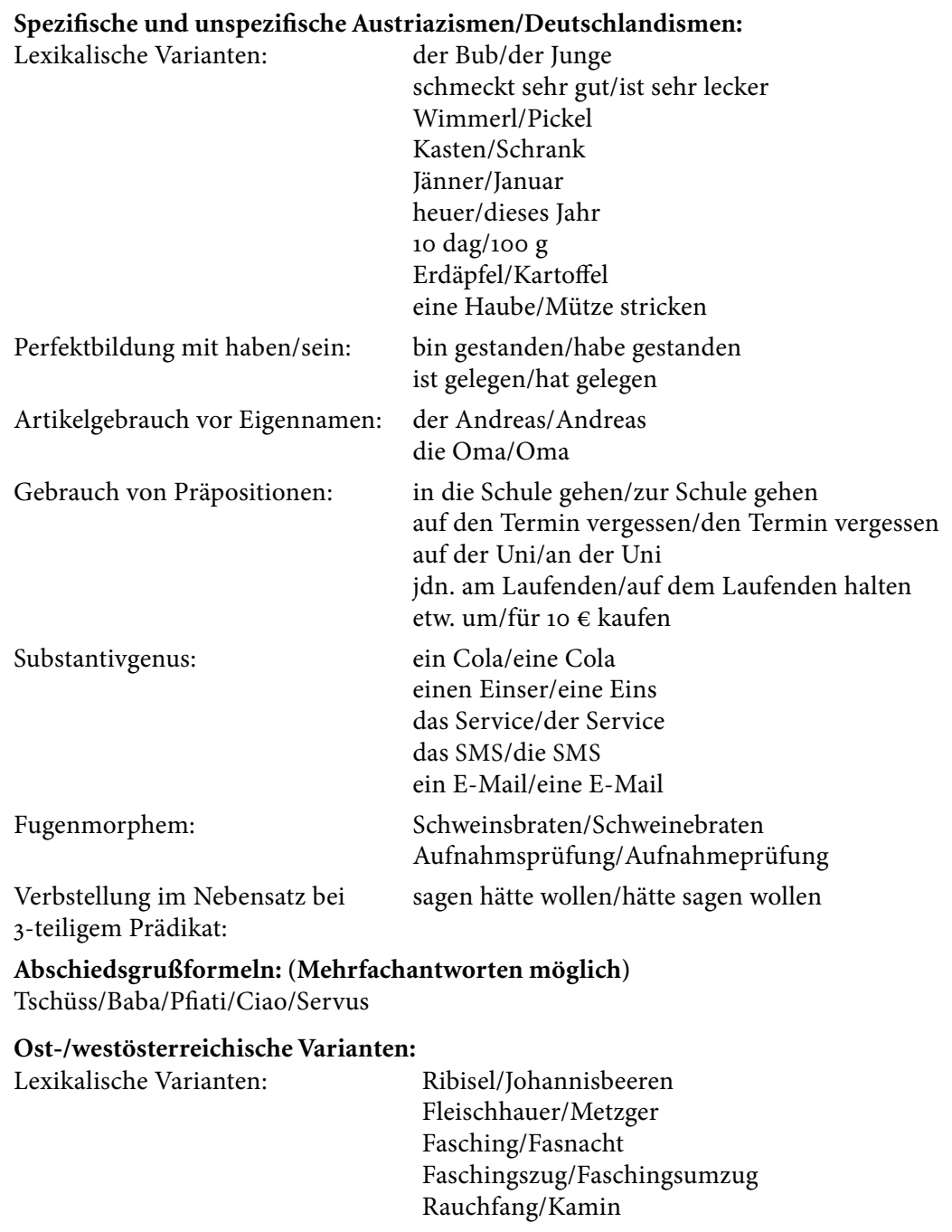

Tab. 5: In den 30 Sätzen enthaltene Varianten zur Auswahl

Im Folgenden werden alle Doubletten und die Häufigkeiten der lehrer- und schülerseitigen Verwendungspräferenzen dargestellt. Anschließend gehen wir auf auffällige Beispiele besonders häufig genannter Austriazismen und Deutschlandismen näher ein.

Bei einem guten Drittel unserer Beispiele wurde von den ProbandInnen eindeutig der Austriazismus vorgezogen bzw. der Deutschlandismus nicht gewählt. Bei einem schwachen Drittel der Beispiele waren die Präferenzen zwischen LehrerInnen und SchülerInnen unterschiedlich: Die Mehrheit der Lehrkräfte bevorzugte die Austriazismen, während die SchülerInnen mehrheitlich die deutschen Varianten bzw. einen gemeindeutschen Ausdruck angaben. Bei einem weiteren Drittel der Beispiele 
gaben sowohl die LehrerInnen als auch SchülerInnen den Deutschlandismen oder einem gemeindeutschen Ausdruck gegenüber den Austriazismen den Vorzug.

Die Doubletten, bei denen sowohl LehrerInnen als auch SchülerInnen mehrheitlich die Austriazismen bzw. einen gemeindeutschen Ausdruck den Deutschlandismen vorgezogen haben, waren bin gestanden - habe gestanden, Jänner - Januar, schmeckt sehr gut - ist sehr lecker, $10 \mathrm{dag}$ - $100 \mathrm{~g}$, ein Einser - eine Eins, Schweinsbraten - Schweinebraten, Kasten - Schrank, heuer - dieses Jahr, Haube - Mütze und in die Schule gehen zur Schule gehen. Abgesehen davon, dass in allen Fällen die Lehrpersonen höhere Werte bei der Austriazismen-Präferenz hatten, ist in dieser Liste auffällig, dass auch von den SchülerInnen mehrheitlich ein Einser gewählt wurde, obwohl Beobachtungen von LehrerInnen zufolge immer mehr SchülerInnen eine Eins verwenden.

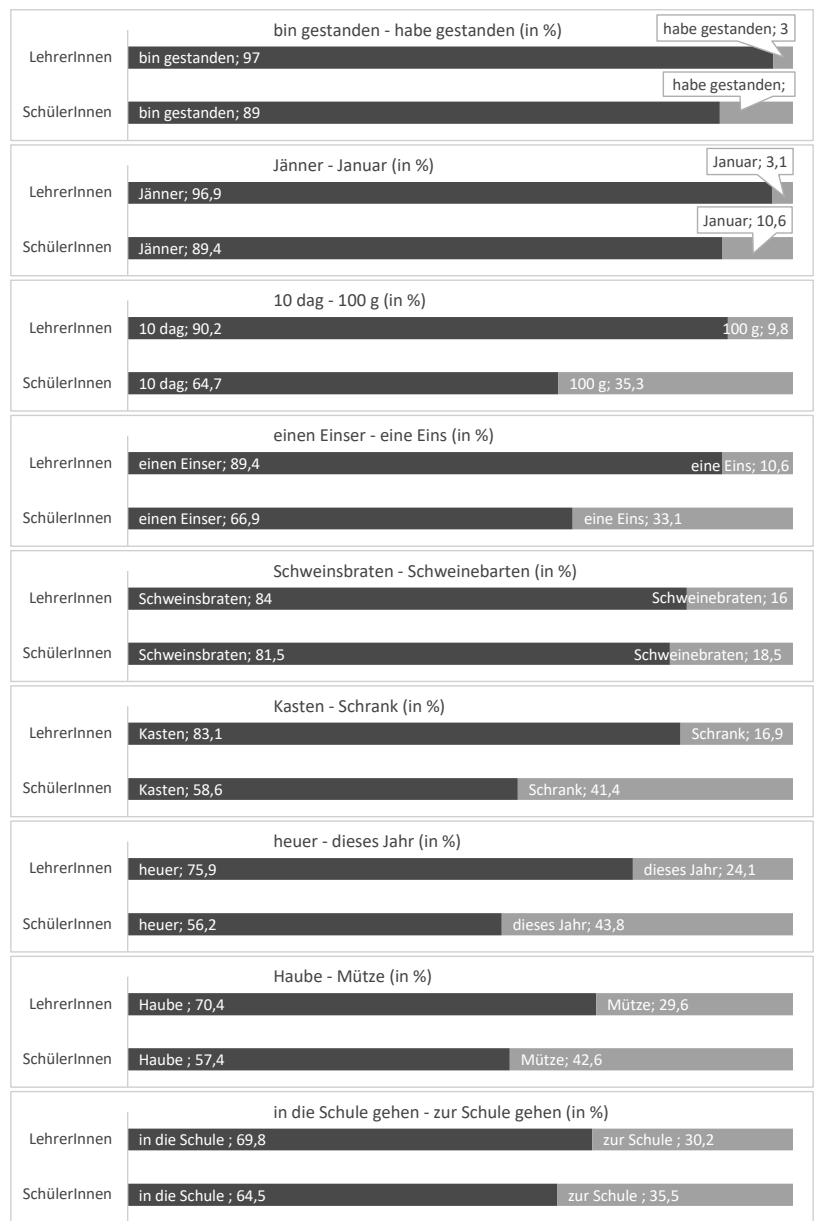

Abb. 66: Lehrerlnnen und SchülerInnen wählen mehrheitlich den Austriazismus oder gemeindeutschen Ausdruck 
Bei den folgenden Doubletten wurden von den LehrerInnen mehrheitlich die Austriazismen gewählt und von den SchülerInnen hingegen die Deutschlandismen bzw. der gemeindeutsche Ausdruck: ein Cola - eine Cola, es ist gelegen - es hat gelegen, Bub - Junge, Aufnahmsprüfung - Aufnahmeprüfung, das E-Mail - die E-Mail, das Service - der Service und Erdäpfel - Kartoffel. Unerwartet bei diesem Ergebnis war, dass die SchülerInnen mehrheitlich es hat gelegen gewählt haben. Erstaunlich war außerdem einerseits die Tatsache, dass die Cola von den SchülerInnen mehrheitlich angegeben wurde, und andererseits die besonders niederen schülerseitigen Werte für die Aufnahmsprüfung, das E-Mail und das Service. In allen genannten Fällen sowie bei Bub - Junge gab es einen großen Unterschied von ca. 30 \% zwischen den LehrerInnen und SchülerInnen.

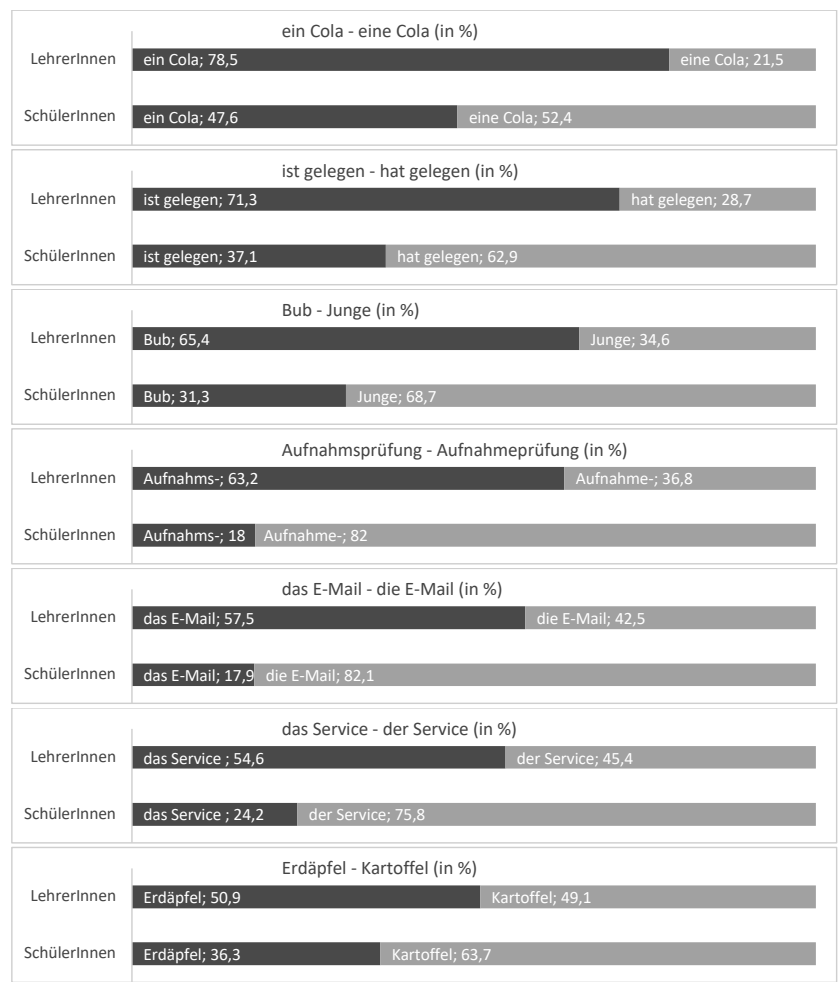

Abb. 67: LehrerInnen wählen mehrheitlich den Austriazismus oder gemeindeutschen Ausdruck, SchülerInnen den Deutschlandismus oder gemeindeutschen Ausdruck

Von beiden Gruppen wurden in den folgenden Fällen mehrheitlich die Deutschlandismen bzw. ein gemeindeutscher Ausdruck als bevorzugte Variante angegeben: Wimmerl - Pickel, das SMS - die SMS, sagen hätte wollen - hätte sagen wollen, die Oma - Oma, auf den Termin vergessen - den Termin vergessen, auf der Uni - an 
der Uni, für $10 €$ kaufen - um $10 €$ kaufen, am Laufenden - aufdem Laufenden und der Andreas - Andreas. Hier fällt auf, dass in einigen Beispielen die SchülerInnen relativ häufiger den Austriazismus gewählt haben (sagen hätte wollen, die Oma, auf der Uni, für $10 €$ kaufen, am Laufenden, der Andreas). Eine mögliche Interpretation dafür ist, dass es sich um Austriazismen handelt, die von den Lehrpersonen eher als umgangssprachlich denn als standardsprachlich beurteilt werden dürften. Auch bei der Doublette Wimmerl - Pickel, auf den Termin vergessen - den Termin vergessen liegt eine derartige Interpretation nahe.

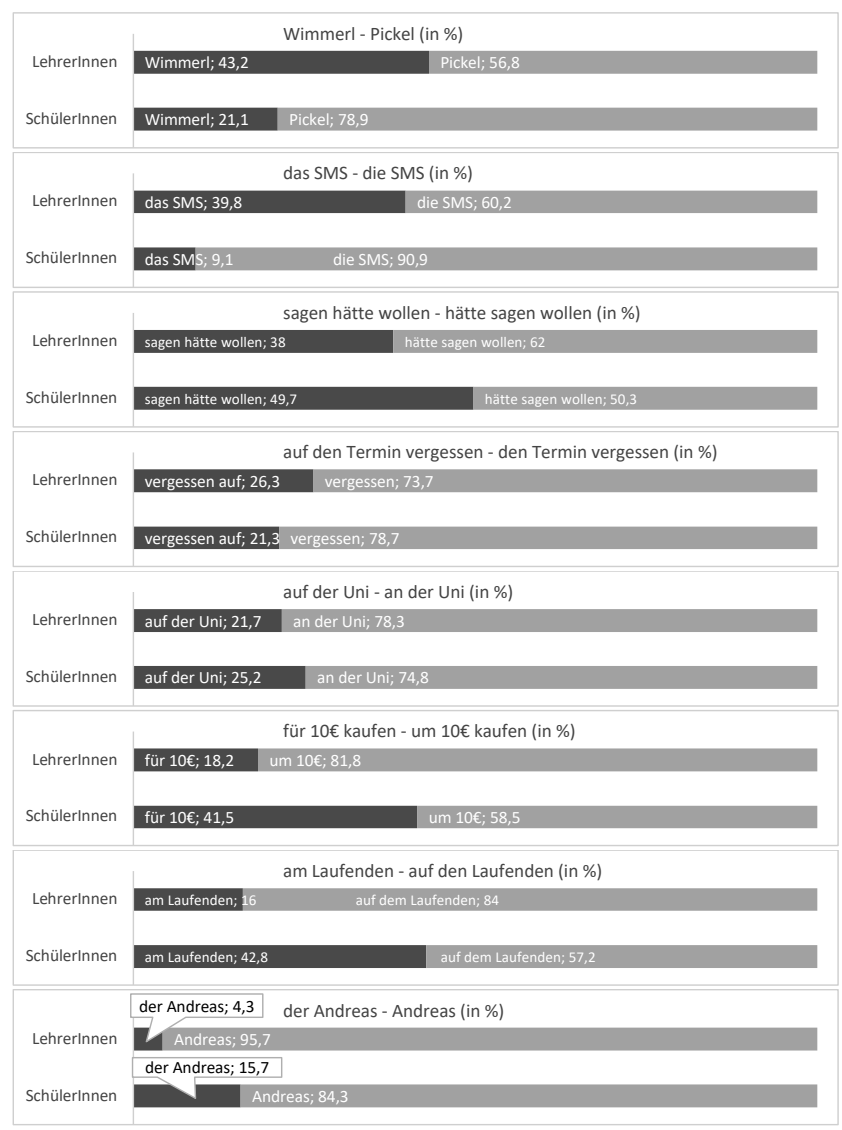

Abb. 68: Lehrerlnnen und SchülerInnen wählen mehrheitlich den Deutschlandismus oder gemeindeutschen Ausdruck

Zur Interpretation sei zunächst festgestellt, dass unsere Liste von Doubletten nur eine kleine Auswahl darstellt. Wollte man eine Studie zur tatsächlichen Sprachverwendung in Österreich und zur Relativität einzelner Varianten durchführen, bräuchte man ein anderes Design (z. B. Befragung ergänzt durch korpuslinguistische Untersuchungen) 
und ein größeres Varianten-Sample. Und wie schon erwähnt, haben wir bewusst eine Reihe von Varianten in die Liste aufgenommen, von denen anzunehmen war, dass sie nicht von allen ProbandInnen als Austriazismus wahrgenommen werden und bei denen wir vermutet haben, dass jüngere ProbandInnen zu den Deutschlandismen tendieren. Interessiert hat uns dabei, wie verlässlich die Angaben im Kodex zum österreichischen Deutsch, der uns bei der Lehrbuchanalyse als lückenhaft aufgefallen war, sind, und ob es altersabhängige Sprachwandeltendenzen gibt, wie sie v.a. immer wieder in Zusammenhang mit dem Medienkonsum angenommen werden.

Im Folgenden soll nun anhand einiger besonders auffälliger Beispiele diesen Fragestellungen nachgegangen werden.

Analog zu den altersspezifischen Unterschieden im Korrekturverhalten bezüglich Austriazismen/Deutschlandismen konnten auch bei der präferenziellen Verwendung von Austriazismen/Deutschlandismen altersbedingte Unterschiede festgestellt werden. $\mathrm{Zu}$ allererst soll hier das divergierende Antwortverhalten von LehrerInnen und SchülerInnen thematisiert werden, bevor wir Bezüge zum unterschiedlichen Antwortverhalten jüngerer und älterer LehrerInnen herstellen wollen. Im vorangehenden Kapitel zeigte sich, dass jüngere LehrerInnen zu einer größeren Akzeptanz von Deutschlandismen tendieren und ältere zu einer stärkeren Variantenloyalität gegenüber Austriazismen neigen. Ein ähnliches Ergebnis ergab sich nun bei den Angaben zur Variantenpräferenz: Nicht nur war die durchschnittliche Zahl an gewählten Deutschlandismen unter den jüngeren Befragten, den SchülerInnen, durchwegs höher als bei den LehrerInnen, sondern SchülerInnen gaben sogar mehr Deutschlandismen (54\%) als Austriazismen (46\%) als ihre bevorzugte Variante an ( $\mathrm{p}=\mathrm{o}, \mathrm{ooo})$. Bei den Lehrenden war die Tendenz umgekehrt: LehrerInnen bevorzugten deutlich mehr Austriazismen (61,2\%) als Deutschlandismen (38,8\%) (p = o,000), was wiederum die Annahme einer stärker ausgeprägten Loyalität der eigenen Varietät gegenüber unter SprachexpertInnen und älteren ProbandInnen bestätigt:

70

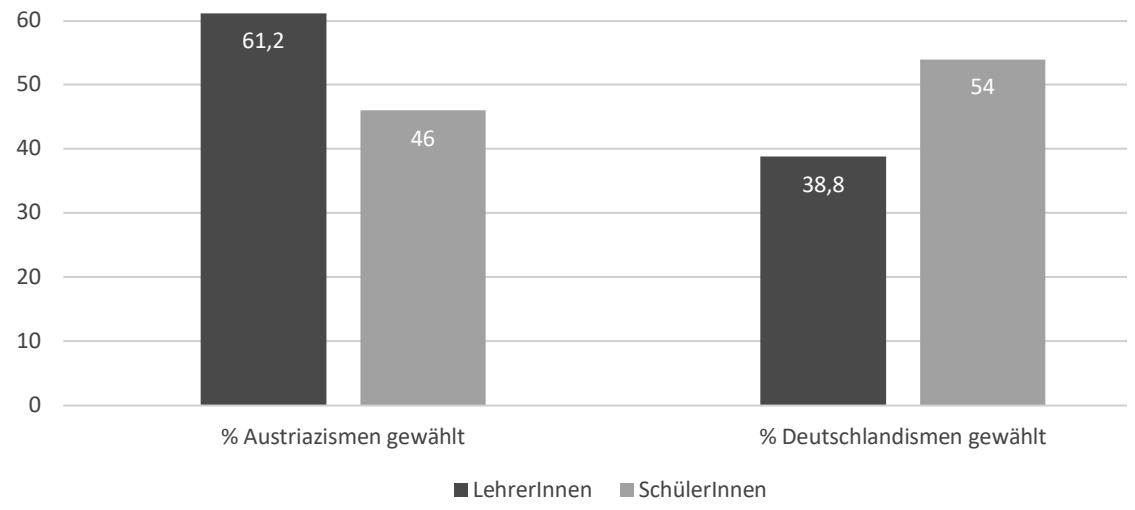

Abb. 69: Antwortverhalten LehrerInnen/SchülerInnen 
Ein Blick auf die Daten zu den einzelnen Begriffen zeigt uns deutlich, bei welchen Varianten sich SchülerInnen und LehrerInnen besonders unterschiedlich verhalten. Die Begriffe eine Cola ( $\mathrm{p}=\mathrm{o}, 0 \mathrm{oo})$, Junge $(\mathrm{p}=\mathrm{o}, \mathrm{0oo})$, eine E-Mail $(\mathrm{p}=\mathrm{o}, \mathrm{ooo})$, Pickel $(\mathrm{p}=\mathrm{o}, \mathrm{Ooo})$ und die SMS ( $\mathrm{p}=\mathrm{o}, \mathrm{Ooo})$ waren jene deutschländischen Varianten, die die SchülerInnen den österreichischen Pendants (ein Cola, Bub, ein E-Mail, Wimmerl, das SMS) am häufigsten vorgezogen haben:

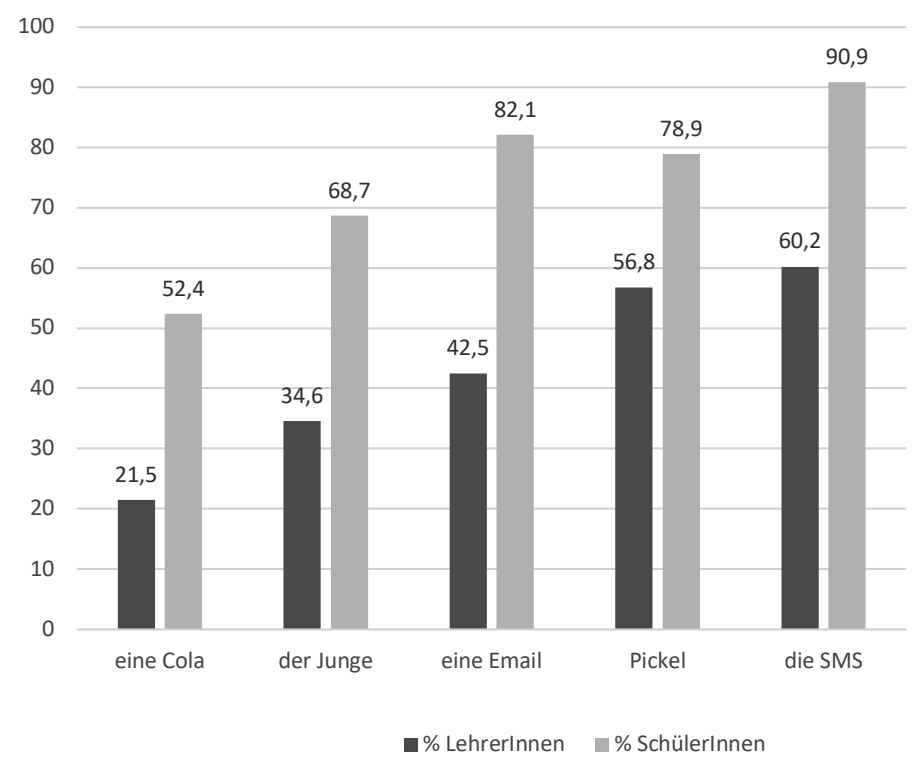

Abb. 70: Am häufigsten bevorzugte Deutschlandismen: LehrerInnen/SchülerInnen (in \%)

Bei den meistgewählten Deutschlandismen war der Unterschied zwischen den SchülerInnen- und LehrerInnenpräferenzen besonders groß. Bei den Begriffen eine Cola, der Junge (statt der Bub) und eine E-Mail gaben die SchülerInnen den deutschländischen Varianten etwa doppelt so oft den Vorzug wie ihre LehrerInnen. Dass beim Begriff SMS fast alle SchülerInnen (90,9\%), aber auch die Mehrheit der LehrerInnen (60,2\%) sich für die SMS und nicht mehr für das SMS entschieden haben, deutet darauf hin, dass sich bei einzelnen Varianten die nach Österreich „importierten“, ursprünglich eher deutschländischen Varianten mittlerweile auch hierzulande etabliert haben. Bei die E-Mail und Pickel ist von einer ähnlichen Entwicklung auszugehen.

Andererseits wurde aber eine Reihe von Austriazismen, die in den 30 Sätzen neben deutschländischen oder gemeindeutschen Gegenstücken zur Wahl standen, von fast allen Lehrenden und auch zu einem sehr hohen Prozentsatz von den SchülerInnen bevorzugt gewählt. Zu den unter den 26 Doubletten am häufigsten bevorzugten und damit punkto Verwendung „stabilen“ Austriazismen 
gehören Jänner $(\mathrm{p}=\mathrm{o}, 002)$, bin gestanden $(\mathrm{p}=\mathrm{o}, \mathrm{001})$, Schweinsbraten $(\mathrm{p}=\mathrm{o}, 430)$,

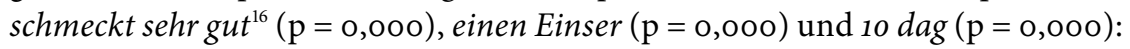

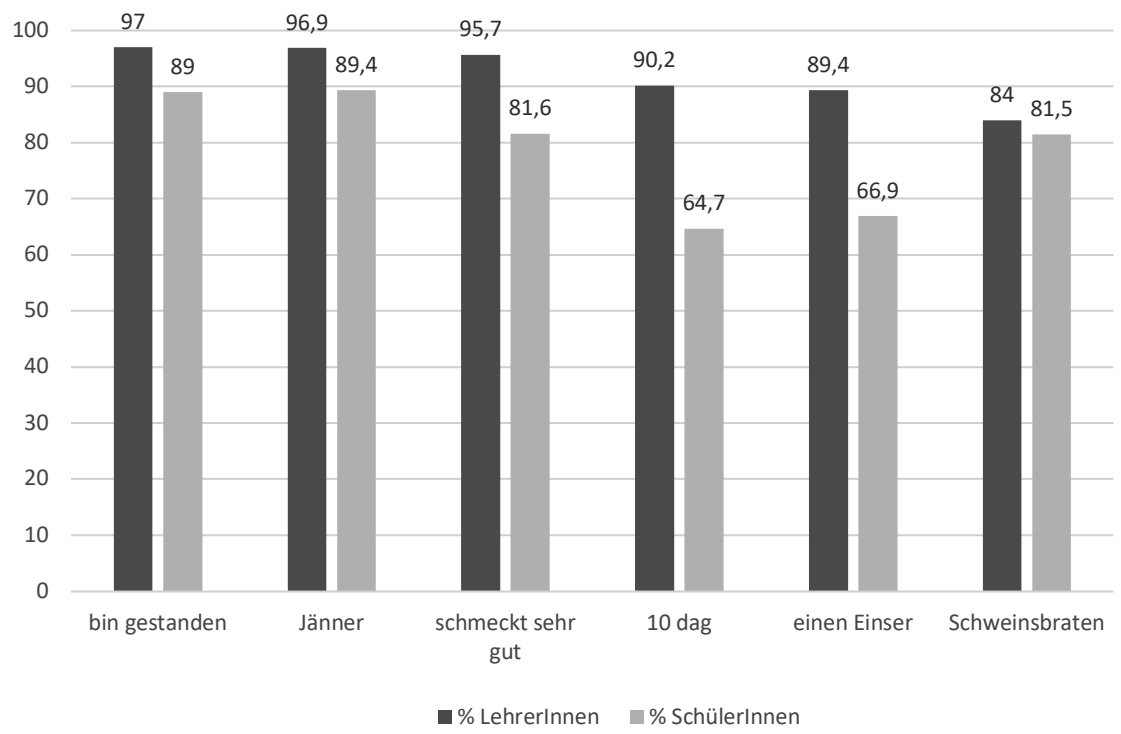

Abb. 71: Am häufigsten bevorzugte Austriazismen: LehrerInnen/SchülerInnen in \%

Was die Verwendung der Grußformeln betrifft, ergab sich folgendes Bild: Am häufigsten wurde von LehrerInnen wie SchülerInnen gleichermaßen das ursprünglich bundesdeutsche Tschüss als bevorzugter Abschiedsgruß angegeben. Bei fast $60 \%$ der LehrerInnen und sogar 80 \% der SchülerInnen war Tschüss ( $\mathrm{p}=0,000)$ beim Abschiedsgruß erste Wahl, gefolgt von Servus $(\mathrm{p}=0,000)$, das noch fast die Hälfte der LehrerInnen verwendet. Unter den SchülerInnen spielen dagegen andere Abschiedsgrußworte als Tschüss eine untergeordnete Rolle. Das bei den LehrerInnen zweitgereihte Servus verwenden eigenen Angaben zufolge nur $22 \%$ der SchülerInnen. Das italienische Ciao ( $\mathrm{p}=0,032)$, das etwa ein Drittel der LehrerInnen als Abschiedsgruß verwendet, erfreute sich mit rund $23 \%$ SchülerInnen-Nennung auch nur mäßiger Beliebtheit bei der Jugend. Das typisch ostösterreichische Baba $(\mathrm{p}=0,000)$ und das ländliche Pfiati ( $\mathrm{p}=0,000$ ) waren jeweils mit rund $10 \%$ die beiden Schlusslichter im Schülergruß-Ranking. Nachdem sich nach Selbstauskunft knapp knapp ein Drittel der LehrerInnen auch mit Pfiati verabschiedet und man von knapp 22 \% der LehrerInnen zum Abschied auch Baba hören kann, wurde - wie beim Spitzenreiter Tschüss - bei diesen beiden Grußformeln nochmals eines klar: Jung und Alt grüßen anders.

16 „Schmeckt sehr gut“ ist zwar kein Austriazismus, was aber bemerkenswert war, war die Tatsache, dass tendenziell NICHT der Deutschlandismus „lecker“, sondern der gemeindeutsche Ausdruck „schmeckt sehr gut“ präferiert wurde. 


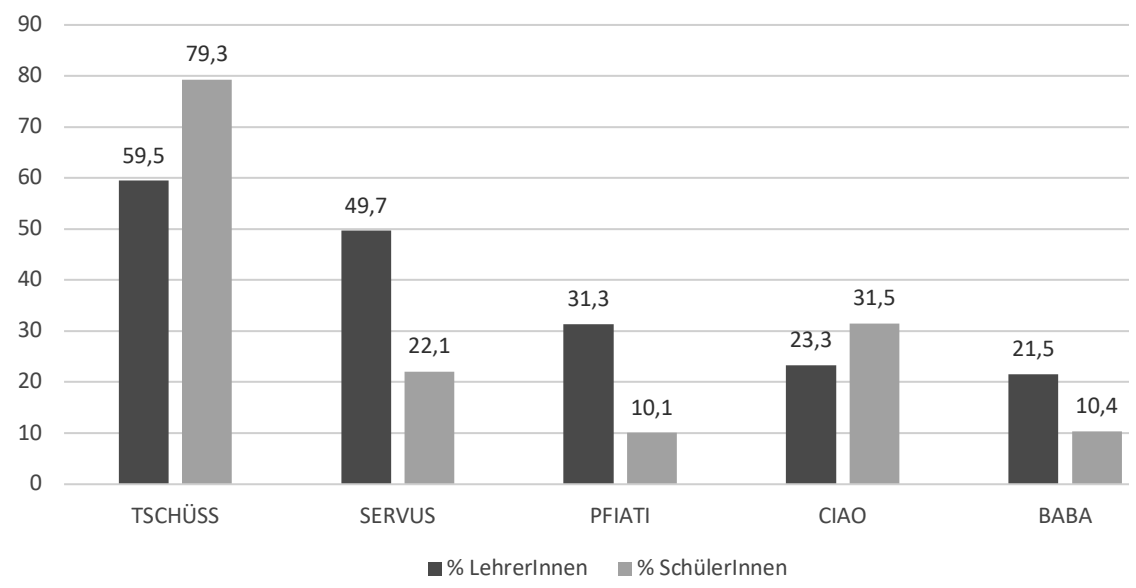

Abb. 72: Mündlicher Abschiedsgruß: LehrerInnen/SchülerInnen (in \%)

Die erstaunliche Diskrepanz zwischen den Präferenzen der SchülerInnen und LehrerInnen legt die Vermutung nahe, dass ein generationsspezifischer Sprachwandel stattfindet. Um dieser Hypothese auf den Grund zu gehen, wurden sämtliche ProbandInnen in drei Alters- bzw. Generationengruppen (Personen bis 21 Jahre, Personen im Alter von 22-40 Jahren, Personen ab 41 Jahre) eingeteilt, um ihr Antwortverhalten nochmals auswerten zu können. In einem weiteren Schritt wurden dann zwei „Generationen“ im Sinn eines relationalen Generationsbegriffs konstruiert, indem die unter 22-Jährigen den über 41-Jährigen gegenübergestellt wurden (Abb. 73). Dabei wurde, wie vermutet, zwischen der jüngsten und der ältesten Altersgruppe die Schere bei einzelnen Verabschiedungsbegriffen noch größer: Unter den über 41-Jährigen waren es nur $50 \%$, die sich für Tschüss als präferierten Abschiedsgruß entschieden haben, während die Prozentwerte in der Gruppe der bis 21-Jährigen mit knapp $80 \%$ in etwa gleich blieben. Ähnlich verhielt es sich mit Servus: Der Anteil der Servus-GrüßerInnen war in der 40+-LehrerInnengruppe mit $60 \%$ höher als bei den Unter-21-Jährigen. Diese Unterschiede sind alle mit $\mathrm{p}=0,000$ hochsignifikant, mit Ausnahme von Ciao $(\mathrm{p}=0,151)$ (siehe Abb. 73 auf S. 191).

Auch bei der Auswertung der weiteren Austriazismen/DeutschlandismenPaare nach Altersgruppen bestätigen sich generationenspezifische Unterschiede: Je älter die ProbandInnen, desto eher wurden Austriazismen bevorzugt, umso jünger, desto eher wurden Deutschlandismen bevorzugt. Austriazismen erfreuten sich unter den älteren LehrerInnen (42-51 Jahre bzw. 52-63 Jahre) der höchsten Beliebtheit, während die Deutschlandismen von den älteren LehrerInnen seltener gewählt wurden. Bei den beiden nächstjüngeren Gruppen schwächte sich diese eindeutige Präferenz der Austriazismen gegenüber den Deutschlandismen sichtlich ab. Bei den jüngsten Befragten war die Sache genau umgekehrt: Die 
13-21-jährigen gaben in 54\% der Wahlmöglichkeiten zwischen Austriazismen und Deutschlandismen den bundesdeutschen Varianten den $\operatorname{Vorzug}^{17}$ (Abb. 74).

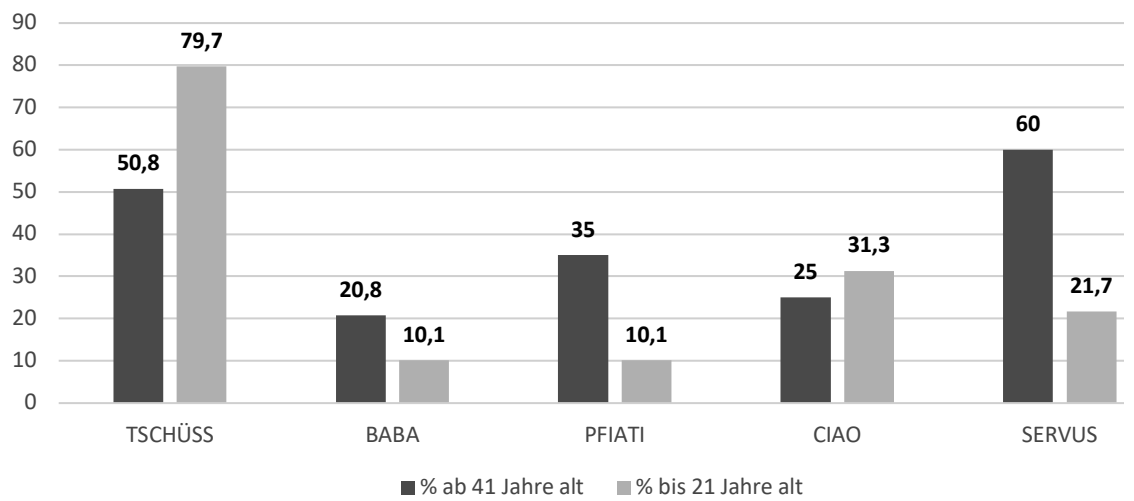

Abb. 73: Mündlicher Abschiedsgruß „Servus“ und „Tschüss“: „Generationen“ (in \%)

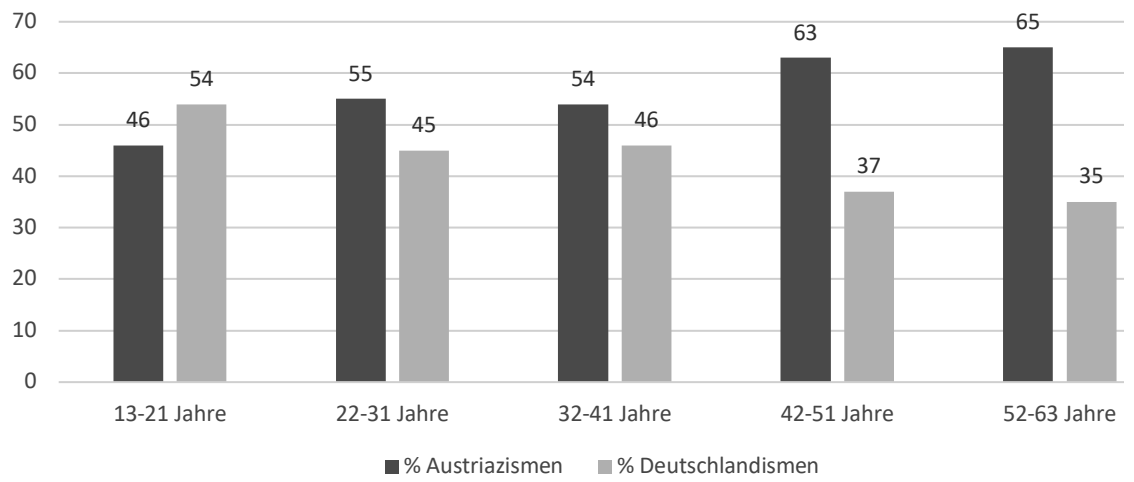

Abb. 74: Austriazismen/Deutschlandismen: Unterschiede zwischen den Altersgruppen (in \%)

Bei den am häufigsten gewählten Deutschlandismen sind die „Generationsunterschiede" am deutlichsten erkennbar. Obwohl mittlerweile fast $60 \%$ der Generation 41+ die SMS verwendet, sind es in der Altersgruppe 13-21 Jahre bereits über $90 \%$. Generationsspezifische Verwendungsunterschiede konnten auch bei den folgenden Begriffen festgestellt werden: die Cola, Junge, die E-Mail, Pickel, die $S M S$, wobei alle Unterschiede hochsignifikant sind $(\mathrm{p}<0,01)$ :

17 Sowohl bei den deutschen als auch den österreichischen Ausdrücken zeigt sich ein hochsignifikanter Unterschied nach Altersgruppen $(\mathrm{p}<\mathrm{o}, 01)$. 


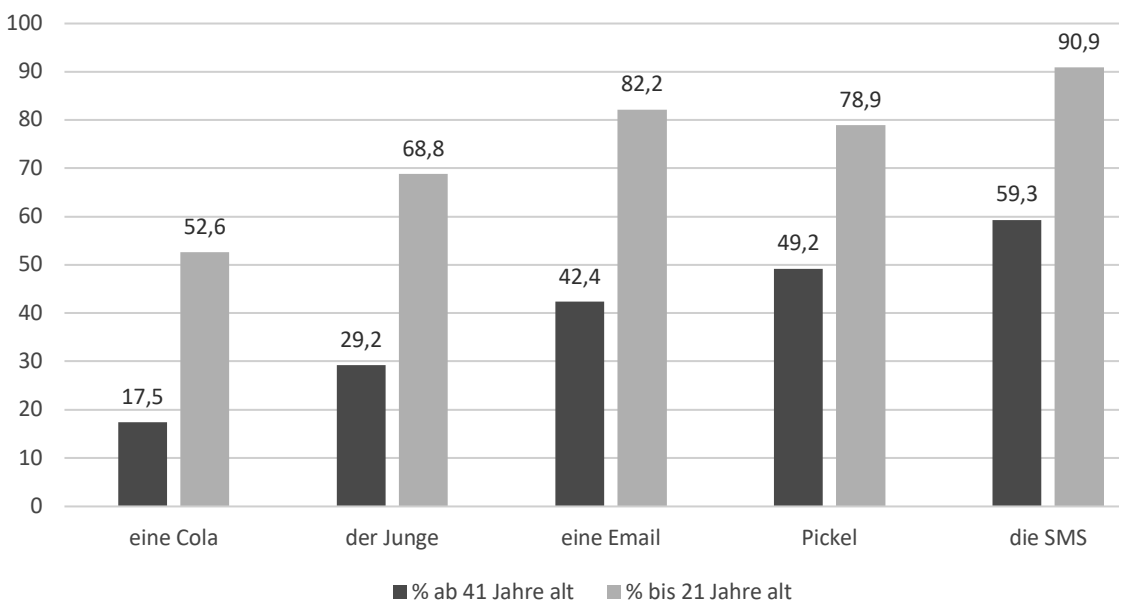

Abb. 75: Am häufigsten gewählte Deutschlandismen: Unterschiede nach „Generationen“ (in \%)

Ebenso ergaben sich generationsabhängige Unterschiede bei der bevorzugten Wahl von Austriazismen, wobei die Schere zwischen der Altersgruppe 41+ und den bis 21-Jährigen nicht so deutlich ausgeprägt war wie bei den Deutschlandismen ${ }^{18}$ :

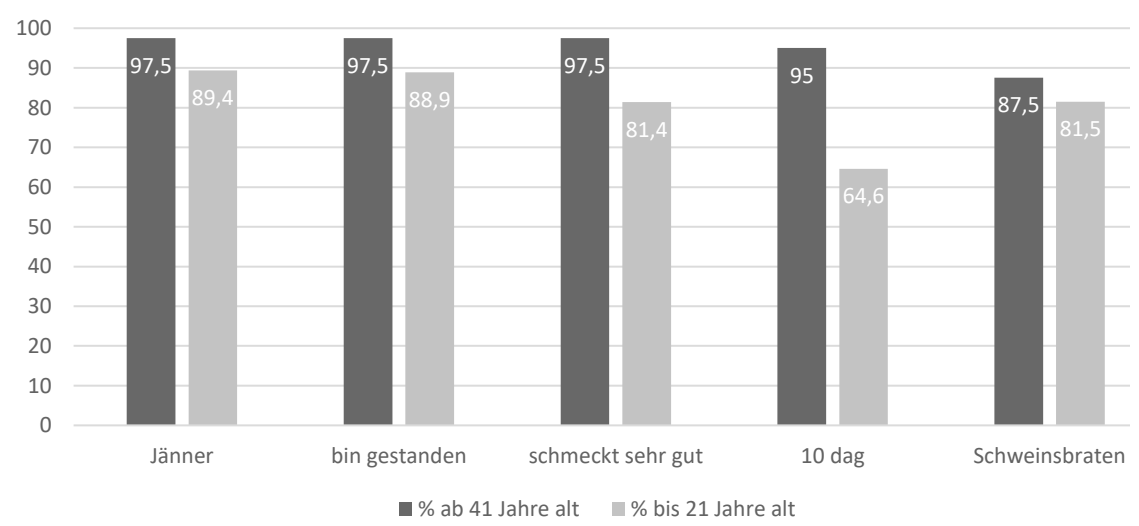

Abb. 76: Am häufigsten gewählte Austriazismen: „Generationsunterschiede“ (in \%)

Auch die regionale Zugehörigkeit spielt eine Rolle bei der Präferenz von Austriazismen und Deutschlandismen, wie das folgende Diagramm zu den LehrerInnen-Ergebnissen zeigt. In beiden Fällen konnten hochsignifikante Unterschiede bei den LehrerInnen der verschiedenen Bundesländern festgestellt werden $(\mathrm{p}=\mathrm{o}, \mathrm{ooo})$. Je weiter wir uns von

18 Jänner: $\mathrm{p}=0,005$, bin gestanden: $\mathrm{p}=0,003$, Schweinsbraten: $\mathrm{p}=0,103$, schmeckt sehr gut $\mathrm{p}=$ $0,000,10$ dag $p=0,000$. 
den östlichen Bundesländern über die Mitte Österreichs nach Westen und schließlich nach Vorarlberg bewegen, umso eher wurden auch Deutschlandismen gewählt. Die lehrerseitige Deutschlandismen-Präferenz nahm von $32 \%$ im Osten auf $58 \%$ in Vorarlberg graduell zu. Mit dem Effekt, dass die befragten LehrerInnen aus Vorarlberg ihren eigenen Angaben zufolge - im Gegensatz zu den PädagogInnen der anderen Bundesländer etwas mehr Deutschlandismen als Austriazismen bevorzugt haben:

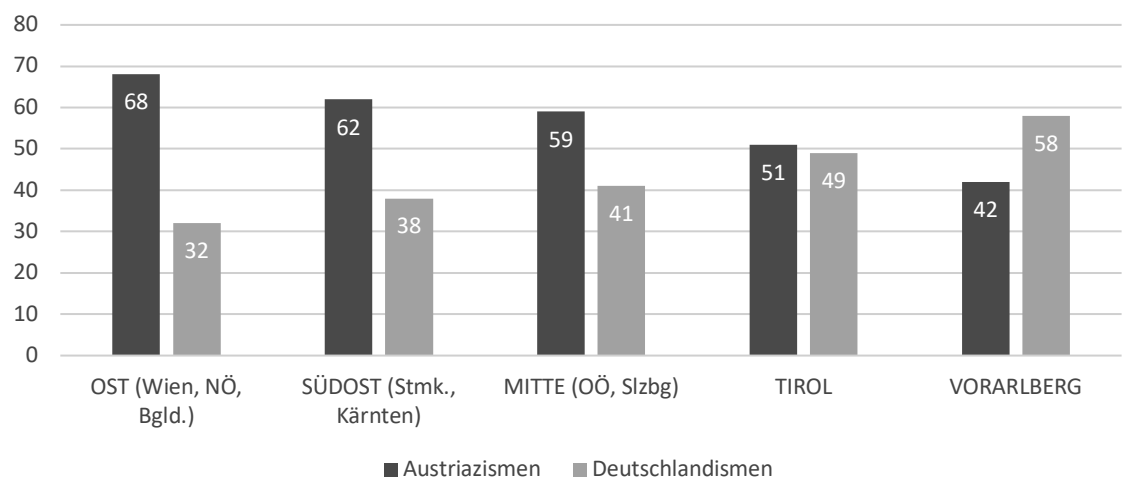

Abb. 77: LehrerInnen: gewählte Austriazismen und Deutschlandismen - Unterschiede zwischen den Regionen (in \%)

Bei den SchülerInnen ergab sich ein gänzlich anderes Bild mit ebenfalls hochsignifikanten Werten $(\mathrm{p}=0,000)$. Zwar nahm auch bei ihnen von Osten nach Westen die Austriazismen-Präferenz ab und die Deutschlandismen-Präferenz $\mathrm{zu}$, jedoch in ganz anderen Ausmaßen. In den östlichen Bundesländern Wien, Niederösterreich und Burgenland hatten die Austriazismen noch ganz knapp die Nase vorn $-51 \%$ bevorzugten Austriazismen, 49 \% bevorzugten Deutschlandismen. Aber bereits ab den Mitte- und Südost-Bundesländern (Oberösterreich, Salzburg, Steiermark, Kärnten) drehten sich die Verhältnisse um: Etwas mehr als die Hälfte der SchülerInnen bevorzugte schon die zur Auswahl stehenden Deutschlandismen. Dieser Trend war in Tirol und Vorarlberg noch stärker ausgeprägt: Tiroler und Vorarlberger SchülerInnen bevorzugten mit $63 \%$ zu $37 \%$ bzw. mit $68 \%$ zu $32 \%$ die Deutschlandismen gegenüber den Austriazismen (siehe Abb. 78 auf S. 194).

Die altersspezifischen Ergebnisse können unser Erachtens zunächst auf zweierlei Art interpretiert werden: Die Daten deuten auf einen altersspezifischen Sprachwandel in der Form hin, dass die jüngere Generation stärker zur Verwendung von Deutschlandismen neigt. Auch innerhalb der Gruppe der LehrerInnen zeigte sich diese Tendenz. Eine andere Interpretation der festgestellten Unterschiede ist, dass LehrerInnen als normsetzende Instanzen im Gegensatz zu SchülerInnen mehr zu Austriazismen tendieren, weil sie sich der Sprache bewusster sind. Die starke Variantenloyalität gegenüber den eigennationalen Varianten, die Schmidlin (2011, 226) in ihrer Untersuchung vor allem unter Gewährspersonen aus Westösterreich 
und dem Südwesten Deutschlands feststellt, konnte in unserem Sample somit nur teilweise bestätigt werden, was möglicherweise durch die Altersverteilung unserer ProbandInnen erklärt werden kann. Schmidlins Ergebnis, dass sich die Landesgrenze „als Variantenloyalitätsgrenze“ zeigt, trifft in unserer Untersuchung nur bedingt $\mathrm{zu}$, wenn die ProbandInnen nach Altersgruppen betrachtet werden. Ein weiterer Grund dafür ist wohl in der Tatsache zu sehen, dass wir bewusst Austriazismen in unserer Liste hatten, von denen angenommen werden konnte, dass ihre relative Verwendung gering ist und dass sie zum Teil schon durch Deutschlandismen ersetzt werden (z. B. Bub/Junge, ein/e E-Mail, ein/e SMS, Tschüss).

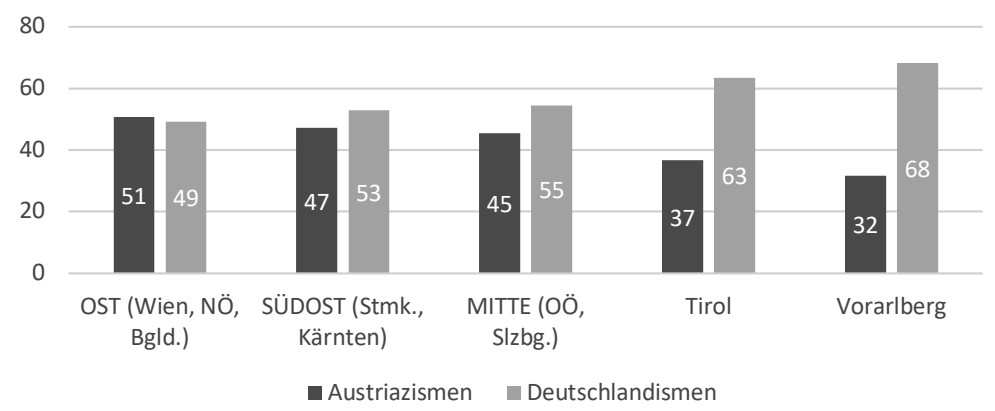

Abb. 78: SchülerInnen: gewählte Austriazismen und Deutschlandismen - Unterschiede zwischen den Regionen (in \%)

Eindeutig bestätigt wird die „Variantenloyalitätsgrenze“ jedoch in einer anderen Arbeit von Konecky (2017), die in einer longitudinalen empirischen Studie (1986-2016) die 26 Varianten-Doubletten, die Grundlage unserer Studie waren, einer korpuslinguistischen Untersuchung mithilfe des Austrian Media Corpus $(\mathrm{AMC})^{19}$ unterzogen hat. Ihre auf die Analyse von Printmedien-Korpora gestützte Untersuchung ergibt weniger Variation in Bezug auf die Verwendung von Deutschlandismen. Sie hatte die Datengrundlage „tatsächliche Sprachverwendung in den Printmedien“, während wir in unserer Befragung als Datengrundlage die Selbsteinschätzung der Variantenverwendung verwendet haben. Jugendliche sind einerseits keine professionellen SchreiberInnen, andererseits gehören die JournalistInnen, aus deren Texten das AMC zusammengesetzt ist, einer anderen Altersgruppe an. So finden sich im AMC 1.400 Belege für das Cola gegenüber 460 für die Cola, und auch im Jahr 2016 dominiert noch eindeutig das Cola (Konecky 2017, 51). Andererseits zeigt ihr longitudinaler Überblick, dass die E-Mail ab Mitte der 1990er-Jahre langsam in der Häufigkeit das E-Mail überholt.

In den Gruppendiskussionen und Interviews haben wir die LehrerInnen und SchülerInnen ersucht, die von uns beobachtete altersspezifische Variantenverwendung zu interpretieren und nach allfälligen Erklärungen zu suchen. Die

19 Zum Austrian Media Corpus (AMC) siehe Kap. 2.5. 
LehrerInnen und SchülerInnen haben dabei übereinstimmend das Medienverhalten als wahrscheinliche Ursache ins Treffen geführt - insbesondere den Konsum von Kabel-TV-Inhalten und bundesdeutsch synchronisierten Filmen. Eine steirische Lehrerin (FSt3) meinte etwa: ,Jo, wenn ma überlegt, ahm mit/oder wieviel Zeit die Jugendlichen mit/vor Medien sitzen und eben im nicht österreichischen Deutsch beschallt werden, dann is das ganz klar, dass der Einfluss sich niederschlägt.“ Ähnlich argumentierte eine Schülerin ( $\left.\mathrm{F}_{2}\right)$ in der Gruppendiskussion:

Ja, ich bin auch der Meinung, dass es vom Alter her abhängt und ähm ... dass viele Schüler in unserem Alter mehr dazu tendieren, deutsches Deutsch zu verwenden aufgrund dessen, weil wir viel mehr deutsches Fernsehen haben und sowas hatte die Generation unserer Lehrer nicht. Einfach diesen wirklichen Kontakt zur deutschen Sprache hat's damals nicht so gegeben wie wir das haben.

Dass sich unter den SchülerInnen vermehrt Sprachformen aus Deutschland durchsetzen, bringt auch eine Lehrerin aus Wien (FW1) mit dem veränderten Fernsehverhalten in Zusammenhang:

Ja beispielsweise, dass ma sagt ... eine Eins/DIE Eins, ja, wir sagen immer DER Einser, ... mittlerweile heißt es schon „ne Eins“. Ich hab ne Eins bekommen. So. Oder „lecker“ is so ein Wort, dass ganz und gar Einzug gehalten hat, ja? ... die Dinge sind alle lecker [...] Ich führ's auf die Fernseher/Fernsehsender zurück.

Durch die Bank haben die DeutschlehrerInnen auf die mediale Dominanz des „bundesrepublikanischen Deutsch“ verwiesen, wie es ein Lehrer aus Vorarlberg im Interview augenzwinkernd genannt hat. Lehrkräfte erzählten davon, dass ihre SchülerInnen die TV-Sprache unreflektiert mit der Zielnorm im Deutschen bzw. mit „richtigem Deutsch“ gleichsetzen und daher die im Fernsehen gehörten Sprachformen imitieren oder Teile davon in den eigenen Sprachgebrauch integrieren würden. Die entsprechenden Code-Switching-Phänomene hat eine Lehrerin aus Niederösterreich ( $\mathrm{FN} 1)$ so umschrieben:

Die Kinder fühlen sie hoit manchmal bemüßigt, a deutsch-deutsche Variante zu verwenden, weil sie glauben, des is hoit richtig, weil sies im Fernsehen immer so hören oder so. Oder, dass hin und wieder Ausdrücke, die ma bei uns net sogt, durch den Einfluss von/hauptsächlich vom Fernsehen ahm dann verwendet werden wie: „Das kann ich voll nicht $a^{\text {“ }} \ldots$ irgendwie reißts an do.

Die Einschätzung, wonach das Fernsehen einen wichtigen Einfluss auf das österreichische Deutsch ausübt, spiegelt sich auch in den Erhebungsdaten wider, die im Rahmen unserer Untersuchung zum Fernsehverhalten der SchülerInnen herausgekommen sind. Sowohl bei der Austriazismenverwendung als auch bei der 
Deutschlandismen-Verwendung unter den SchülerInnen zeigte sich ein statistisch hochsignifikanter Zusammenhang abhängig von der Nutzung bestimmter TV-Kanäle ( $\mathrm{p}=\mathrm{o,00o).} \mathrm{SchülerInnen,} \mathrm{die} \mathrm{angaben,} \mathrm{aktuell} \mathrm{nur} \mathrm{deutsche} \mathrm{Kanäle}$ zu schauen, wählten statistisch signifikant häufiger Deutschlandismen als SchülerInnen, die angaben, nur österreichische Fernsehkanäle zu sehen.

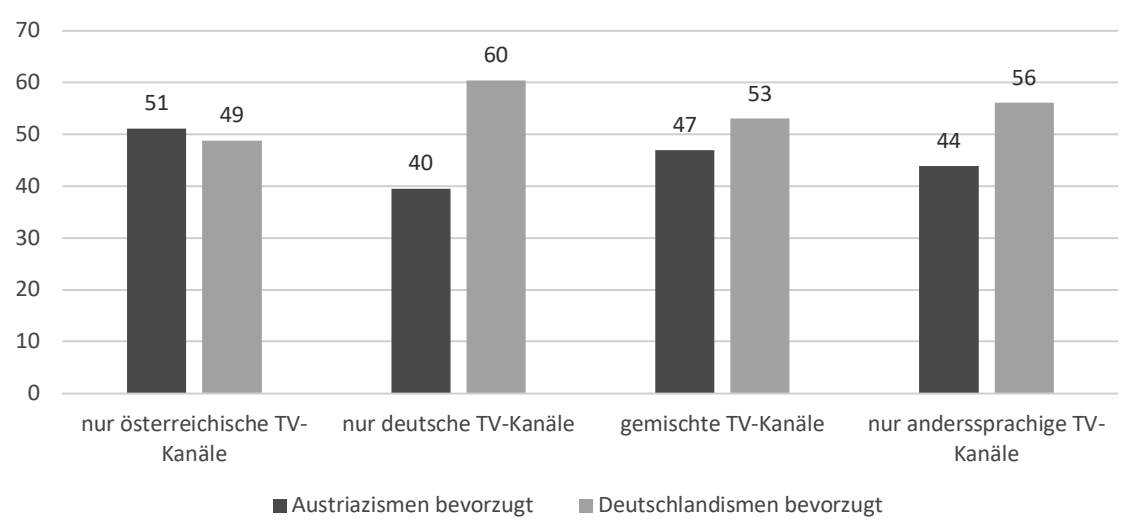

Abb. 79: SchülerInnen: TV-Konsum und Präferenz von Deutschlandismen und Austriazismen (in \%)

Dass sich die altersspezifisch unterschiedliche Verwendung von Austriazismen/ Deutschlandismen nicht konfliktfrei abspielt, zeigen mehrere Passagen in unseren Gruppendiskussionen. Sie bestätigen damit ältere Erhebungen, die am Institut für Sprachwissenschaft der Universität Wien 1995 und 2005 im Rahmen von zwei Projekten zur diskursiven Konstruktion österreichischer Identitäten durchgeführt wurden (Wodak/de Cillia et al. 1998; de Cillia 2009, s. u.). Insbesondere der Gruß Tschüss wurde in diesem Zusammenhang immer wieder thematisiert. So z. B. in einer Gruppendiskussion im Burgenland im Jahr 1995, wo eine Teilnehmerin gemeint hat: $\left(\mathrm{F}_{3}\right)$ „[...] komischaweise es tut mir weh. i find des is a scheußliches Wort“. Worauf eine Burgenlandkroatin so reagiert hat:

(F1) Mir tut's auch sehr weh. Jo: aba mir tut's nicht wegn dem Deutschn weh. Mir tut's wegn dem Kroatischen weh. Wonn i dauernd hör Tschüß und Tschüß und Tschüß. Natürlich unsre Kinda redn mit dir Kroatisch und sogn donn Tschüß. Und wenn dann die Bosnier gekommen sind die Flüchtlingskinda dann hat's geheißn Tschi:s. Is aus dem Tschüß das Tschi:s gwordn. (Wodak/de Cillia et al. 1998, 360)

Im Jahr 2005 berichtete ein Schüler in einer Gruppendiskussion, sein Opa reagiere gereizt darauf, wenn zu ihm jemand Tschüss sage: „Wenn irgendwer zu ihm Tschüss sagt, dann sagt er, das/ das heißt ned Tschüss, oder irgend so was. Das regt mich auf, weil man soll sich verabschieden können wie man will. “ Schülerin ergangen: „Ja, mein Vater regt sich auch auf. Ja, mein Vater sagt das 
auch immer. Ja, er sagt immer das kann/ kannst zu Deinen Freundinnen sagen, aber nicht zu mir“ (de Cillia 2009, 158).

In den Gruppendiskussionen für die vorliegende Untersuchung erzählte eine Schülerin die folgende Geschichte $\left(\mathrm{F}_{3}\right)$ :

Ich weiß nur, da war ich noch kleiner, da war ich mit meiner Mutter einmal beim Arzt und ich hab dann auch irgendwann gsagt „Tschüss“. [...] nein, mit meiner Oma war ich, und die hat dann auch gsagt, du darfst jetzt aber nicht Tschüss zu dem Herren sagen, da musst du schon „Auf Wiedersehen“ sagen, weil das is ja unhöflich.

Das letztgenannte Beispiel ist vor allem deshalb interessant, weil hier auf die Verwendung von Tschüss in der distanzierten Anrede Bezug genommen wird, wie sie in Deutschland durchaus üblich ist, in Österreich aber (noch) nicht.

Trotz des Widerstands der älteren Generation ist Tschüss mittlerweile zum häufigsten Abschiedsgruß geworden. Die vorliegende Untersuchung unterstreicht diesen Befund. In der dazugehörigen Gruppendiskussion der LehrerInnen entwickelte sich in diesem Zusammenhang ein richtiges „Tschüsskonzert“, in dem unterschiedliche Intonationen des Tschüss angesprochen wurden, und die Teilnehmer letztlich eine Austrifizierung des Tschüss diagnostiziert haben.

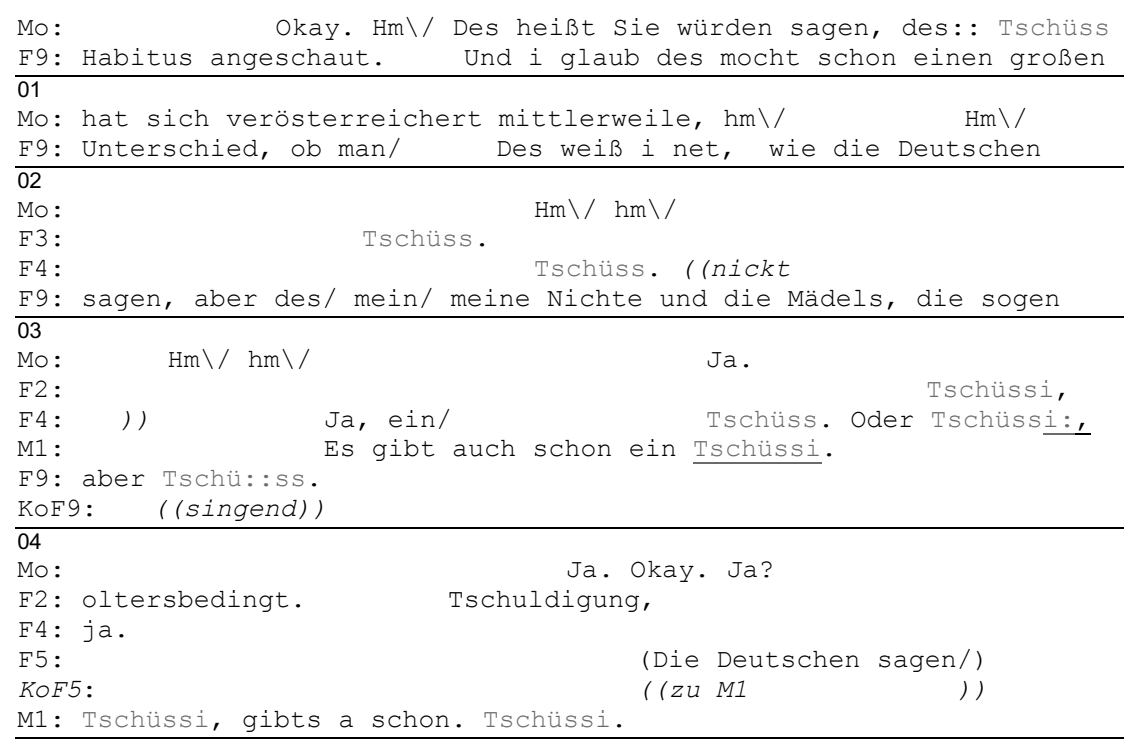

Eine Teilnehmerin ( $\mathrm{F}_{5}$, Alter ca. 45 Jahre) berichtete über die eigene Verwendung des Tschüss: „Ah, des Tschüss fällt mir jetzt nur bei mir persönlich auf, ich sag auch Tschü::ss ((singend)). ((lacht)) Also, net Tschüss. Sondern des/ diese Wellenbewegung." 
Das Wort Tschüss ist ein allgemein sichtbares Anzeichen dafür, dass neben den LehrerInnen vor allem die SchülerInnen mündlich und auch schriftlich zusehends Deutschlandismen verwenden, wie LehrerInnen bemerken. Ein Lehrer aus der Steiermark (MSt2) ortete in diesem Zusammenhang eine Vermischung der Sprachregister:

Ja, ... vor allem in der schriftlichen Variante kommen BUNDESDEUTSCH gesprochene Variationen vor, wie: „mal. Sag mal.“ Mit der Einbildung, dass sei sozusagen gehobenes Schriftdeutsch. Und dann schreib i dazu, das is umgangssprachlich, dann glauben's „Na das is ja DEUTSCH! Das hör ich immer im Fernsehen!“ Also auch sozusagen die Verwechslung zwischen gesprochenem und geschriebenem Deutsch. Und äh/ die Gleichsetzung zwischen gesprochenen oder umgangssprachlichen bundesdeutschen Varianten mit Schriftdeutsch. Des is a Problem.

Dass es wichtig sei, österreichisches Deutsch und deutschländisches Deutsch im Unterricht zu thematisieren, betonten in den Interviews fast alle LehrerInnen. Im In- und Ausland müsse das Bewusstsein für eine existierende österreichische Standardvarietät geschaffen werden, die vom Dialekt abzugrenzen sei, meinte eine Lehrerin aus der Steiermark (FSt1). Sie wies außerdem darauf hin, dass die Suche nach geeigneten Unterrichtsmaterialien zum Sensibilisieren für die Merkmale der deutschen Standardvarietäten nicht immer einfach sei:

Und da war ich auf der Suche nach ah österreichischen Hörtexten [...], aber dieses eine [bundesdeutsche, Anm.] Hörbuch, wos i do am Beginn ghört hob, des hot ma den Magen umgedreht, muaß i gonz ehrlich sogn [...] mich ärgert das schon, wenn ahm/wenn i im Ausland bin, und das österreichische oder meine Standardvariante ... als charmanter Dialekt abgetan wird, das (mag) ich nicht.

Viele der interviewten Lehrkräfte haben eigenen Angaben zufolge beobachtet, dass sich SchülerInnen damit schwer tun, österreichische Standardausdrücke als standardsprachlich und nicht als Substandard-Varianten einzuordnen. Eine Lehrerin aus Niederösterreich ( $\left.\mathrm{FN}_{1}\right)$ schilderte, was sie in diesen Fällen unternimmt:

Do versuch i eben grod anhand dieser Ausdrücke wie „Leiberl“ oder so oder „Bub“ ah dass i eana des hoit erklär, dass ma si do net geniern muaß, sondern (das is) österreichisch. Und des soins bitte verwenden, damits nicht vergessen wird.

Viele Lehrkräfte waren sich einig, dass das schülerseitige Sprachbewusstsein geschärft werden müsse: Indem im Unterricht sprachliche Phänomene des österreichischen Deutsch und des deutschländischen Deutsch explizit reflektiert und diskutiert werden. Dies umso mehr, als sich viele SchülerInnen bewusst oder unbewusst durch ihre Sprache in Österreich verwurzelt fühlen. In diesem Zusammenhang wird auch die Verbindung zwischen Sprachvarietät und Identität von 
einigen Lehrkräften ins Spiel gebracht. Dies veranschaulicht der folgende Interviewauszug mit einem Lehrer aus dem Burgenland (MB1):

Also nach wie vor, glaub ich, is die/is die Identifizierung mit dem österreichischen Deutsch schon groß. Wenn man dann Quark, Aprikosen und Pampelmusen sagt, also das erheitert sie sehr und da sind sie auch der Meinung, dass das - ahm - nicht sein soll, dass diese Begriffe in Österreich Fuß fassen. Also so viel, wie soll ich sagen, Heimatliebe zum österreichischen Deutsch, <wenn man das (lachend) $>$ so bezeichnen darf, bringen sie schon auf.

Ein Aspekt in diesem Teil der Erhebung waren noch Angaben zur Verwendung von fünf Wortdoubletten, die laut Variantenwörterbuch Ost- bzw. Westösterreich zugeordnet werden (Ribisel/Johannisbeeren, Fleischhauer/Metzger, Fasching/ Fastnacht, Faschingszug/Faschingsumzug, Rauchfang/Kamin). Weil der Umfang unserer Untersuchung vorab eingegrenzt war, konnte nur ein sehr kleines Sample dieser Ost-West-Items in die Befragung eingebaut werden, wobei es schwierig war, passende Beispiele zu finden. Die Ergebnisse waren in diesem Bereich nur bedingt aussagekräftig und werden deshalb auch hier nicht ausgeführt.

\subsection{Dialekt - Umgangssprache - Standard? Angaben zum Varietätengebrauch innerhalb und außerhalb der Schule ${ }^{20}$}

Um ein Bild von der gesprochenen Sprachvielfalt im österreichischen Unterrichtsalltag zu bekommen, wurde die lehrerseitige und schülerseitige Varietätenverwendung in der mündlichen Unterrichtskommunikation anhand eines eigenen Fragenblocks in den Fragebögen sowie mithilfe der Interviews mit den LehrerInnen untersucht.

Eine Fragebogen-Frage an die Lehrpersonen lautete: „Mit welchen Herkunftssprachen bzw. Varietäten der Schüler/innen sind Sie im Unterricht konfrontiert?“ Die vorgegebenen Antwort-Optionen waren: „Dialekte“, „Umgangssprache“, „,̈sterreichisch geprägtes Standarddeutsch“, „,bundesdeutsch geprägtes Standarddeutsch“, „schweizerisch geprägtes Standarddeutsch“ und „andere Herkunftssprachen als Deutsch“. Darüber hinaus wollten wir wissen, ob die LehrerInnen häufig, wenig oder gar nicht mit den entsprechenden Varietäten konfrontiert sind (vgl. Abb 80).

Das Ergebnis war: Die Varietät, mit der LehrerInnen in der Schule am häufigsten zu tun hatten, war „Umgangssprache“ (84,7 \% häufig, 15,3\% wenig, o \% gar nicht), gefolgt von „österreichisch geprägtem Standarddeutsch“ (61 \% häufig, 35, 2 \% wenig, 3,8\% gar nicht) und „Dialekten“ (51,2\%; 42,6\%; 6,2\%). „Bundesdeutsch geprägtes Standarddeutsch" wurde von 3,2 \% der PädagogInnen häufig, von 40,8 \% wenig und 56,1 \% gar nicht angeführt, „schweizerisch geprägtes Standarddeutsch“

20 Vgl. dazu auch de Cillia 2018. 
wurde von 3,8 \% wenig wahrgenommen und von 96,2 \% gar nicht, und die Angaben für „,andere Herkunftssprachen als Deutsch“lauteten: 29,7 \% häufig, 58,2 \% wenig und 12,1\% gar nicht. Mit anderen Worten: Alle drei Varietäten des Deutschen in Österreich sind in den Schulen präsent.

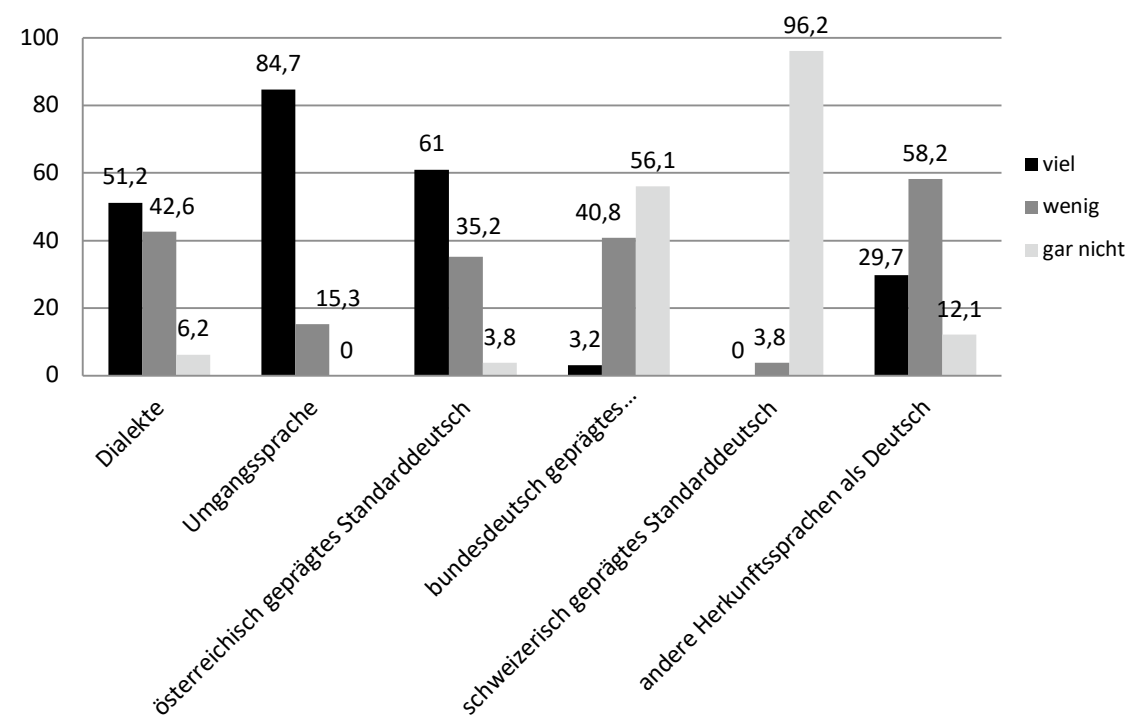

Abb. 80: Herkunftssprachen und Varietäten der Schülerlnnen (in \%)

Schule ist aber nicht gleich Schule - denn beim schulinternen Varietätengebrauch gab es je nach Schultyp ( $\mathrm{p}=0,001)$ und den Regionen/Bundesländern signifikante Unterschiede.

In der HS/NMS $(61,8 \%)$ und in der AHS $(61,9 \%)$ wurde die schülerseitige Dialektverwendung von den Lehrenden am stärksten wahrgenommen, während diesbezügliche Angaben der VolksschullehrerInnen mit nur 29,6\% deutlich geringer waren. In der AHS bzw. Sek. II gaben 67,1\% der Lehrenden an, regelmäßig mit österreichisch geprägtem Standarddeutsch in Kontakt zu stehen, während sie ihren Angaben zufolge mit „anderen Herkunftssprachen“ am wenigsten in Berührung kamen. VS-LehrerInnen gaben hingegen am häufigsten an, es mit anderen Herkunftssprachen zu tun zu haben, was sich durch die zahlreichen Schulkinder aus Zuwandererfamilien erklärt.

Auch zwischen den einzelnen Bundesländern konnten hochsignifikante Unterschiede $(\mathrm{p}=\mathrm{o}, \mathrm{ooo})$ festgestellt werden: Für Westösterreich (Tirol, Vorarlberg) ergab sich aus Sicht der LehrerInnen die stärkste Dialektverwendung durch die SchülerInnen, während eine österreichisch geprägte Standardsprache von LehrerInnen in den östlichen Bundesländern (Wien, Niederösterreich, Burgenland) am stärksten und in den westlichen Bundesländern (Tirol, Vorarlberg) am wenigsten wahrgenommen wurde. 
Die LehrerInnen wurden auch dazu befragt, ob sie in den vier Unterrichtskontexten „Vortrag, Arbeitsauftrag, Disziplinäres, Organisatorisches“ mit den SchülerInnen Standarddeutsch, Umgangssprache oder Dialekt sprechen (Mehrfachnennungen möglich). Die überwiegende Mehrheit der Lehrenden gab an, in allen vier Unterrichtssituationen kaum Dialekt zu verwenden. Vielmehr gaben $85 \%$ bzw. $66 \%$ der LehrerInnen an, dass sie bei einem frontalen Vortrag bzw. beim Erteilen eines Arbeitsauftrags ausschließlich Standardsprache verwenden würden. Bei Disziplinärem und Organisatorischem gaben in etwa gleich viele Lehrende an, Umgangssprache oder Standardsprache zu verwenden - aber kaum Dialekt (siehe Abb. 81). Meist machten die Befragten nur eine Angabe und kaum Mehrfachnennungen.

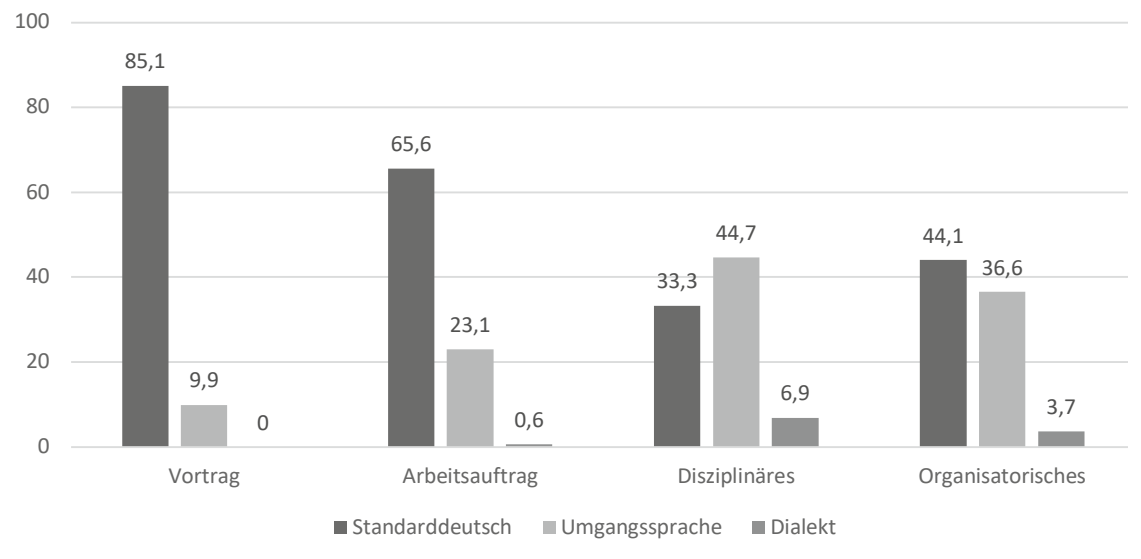

Abb. 81: Sprachverwendung Lehrerlnnen (Selbsteinschätzung) (in \%)

Betrachtet man die Ergebnisse nach Zusammenhängen mit außersprachlichen Variablen, zeigten sich zwar keine signifikanten Unterschiede, aber durchaus interessante Tendenzen: Die Gruppe der jüngsten LehrerInnen (22-31 Jahre) gab mit $27 \%$ am häufigsten von allen Altersgruppen an, in allen Kommunikationssituationen Umgangssprache zu verwenden. Jüngere Lehrkräfte tendieren also stärker zur Umgangssprache. Auch Dialekt wurde im Altersvergleich von der Gruppe der jüngeren LehrerInnen am häufigsten gewählt, im Gegensatz zur Gruppe der älteren LehrerInnen (52-63 Jahre), die am wenigsten Dialekt angaben. Dieses Antwortmuster deckt sich mit der Anzahl der Dienstjahre.

Nach Schulform betrachtet kam heraus, dass HS/NMS-LehrerInnen im Unterricht weniger zum Standard und stärker zur Umgangssprache tendieren als VSund AHS-LehrerInnen. Darüber hinaus zeigte die Auswertung der Daten, dass vor allem von LehrerInnen, die Deutsch und eine andere Sprache unterrichten, die Umgangssprache kaum genannt wurde. 
In den Einzelinterviews gaben die meisten LehrerInnen an - wenn sie auch häufig mit Abschwächungspartikeln („eigentlich“, „ich würde sagen“, „schon“) ihre Aussagen relativierten - dass sie v. a. beim Vortrag Standardsprache verwenden. Manche LehrerInnen deuteten an, dass ihnen sehr wohl bewusst sei, dass fallweises Switchen zwischen Standard und Umgangssprache durchaus vorkommt. Dazu meinte eine Lehrerin aus Wien (FW1): „Im Unterricht versuch ich schon die Standardsprache zu sprechen.“ Ähnlich eine Lehrerin aus Tirol (FT4): „Da versuch i eigentlich die Standardsprache beizubehalten."

Dass viele LehrerInnen in die Umgangssprache oder den Dialekt wechseln, sobald persönliche oder emotionalere Angelegenheiten zur Sprache kommen, ging aus fast allen Interviews hervor. Eine Tiroler Lehrerin (FT2) etwa wechselt bewusst in den Dialekt, wenn sie schimpft: „Ja. Also im Unterricht verwende ich schon gehobene Umgangssprache. Und nur wenns emotional wird, also wenn ich schimpf <zum Beispiel (lachend)>, dann ah is des gonz kloar, doss i des/des im Dialekt [...] moch."

Die in den Antworten auftauchenden Formulierungen deuten aber auch auf eine gewisse Unsicherheit hin. Zur Illustration sei eine Interviewpassage mit einem Vorarlberger Lehrer (MV2) angeführt: „Ja - Standardsprache - - - Deutsch mit natürlich äh österreichischem Akzent würd i amal sagen, - - ja, nicht Umgangssprache, sondern - Hochsprache ist jetzt vielleicht zu hoch gegriffen, aber - ..."

Die wiederkehrenden Wort- und Satzabbrüche, Formulierungsaktivitäten wie „würd i amal sagen“ und „vielleicht“ sowie die Tatsache, dass der Lehrer zwar verschiedenste Varietäten nennt („Standardsprache“, „Deutsch“, „Deutsch mit österreichischem Akzent“, „Umgangssprache“, „Hochsprache“), jedoch alle relativiert und letztendlich zu keinem Schluss kommt, wie er tatsächlich mit seinen SchülerInnen im Unterricht spricht, machen die erwähnte Unsicherheit deutlich erkennbar. Diese Unsicherheit könnte zum Teil aus dem Umstand herrühren, dass die interviewten LehrerInnen möglicherweise davon ausgingen, die sozial erwünschte Antwort auf die Frage nach der im Unterricht verwendeten Varietät sei die „Standardsprache“.

Aus den Interviews geht jedenfalls hervor, dass für die LehrerInnen die Verwendung der Standardsprache im Unterricht oder zumindest im Vortrag das anzustrebende Ziel ist. Eine Dialektverwendung auf Seiten des Lehrers bzw. der Lehrerin im Unterricht wurde in den Interviews zumindest implizit als weniger erwünscht dargestellt. Auf die Frage, welche Varietät sie im Unterricht vorrangig verwendet, meinte etwa eine Wiener Lehrerin (FW3) mit einem fast entschuldigenden Nachsatz: „Hochdeutsch. Aber ... wenn ich mit den Kindern plaudere, dann passiert auch leichter Dialekt.“

Dass die Verwendung der Standardsprache, die offenbar nicht mit österreichischem Deutsch gleichgesetzt wird, im Unterricht zwar erwünscht, aber nicht selbstverständlich ist, geht aus dieser Interviewpassage mit einem Lehrer aus dem Burgenland (MB1) hervor: 
Im Unterricht ist das Deutsch sicher mehr an der - unter Anführungszeichen $<$ Standardsprache (lachend)>, über die man jetzt streiten kann, orientiert, ... im/im Privaten sicher mehr am - wieder unter Anführungszeichen, Österreichischen. Aber man bemüht sich als Lehrer schon, verstärkt im Unterricht ahm Dinge, die man als umgangssprachlich wahrnimmt, nicht SO stark zu verwenden.

Der hier zitierte Lehrer distanziert sich vom Ausdruck „Standardsprache“ durch Lachen und mit den Fingern in der Luft gesetzten „Anführungszeichen“, sowie die Erwähnung, dass man darüber streiten könne. Weiters stellt er den Begriff „Standardsprache“ dem „Österreichischen“ gegenüber. Diesem „Österreichischen“, das er im privaten Umfeld verwendet, stellt er schließlich wieder die Sprache gegenüber, die er im Unterricht verwendet und in der scheinbar umgangssprachliche Elemente vorhanden sind, die er aber gleichzeitig zu vermeiden sucht.

Auch in der Gruppendiskussion der SchülerInnen wurde nach dem Sprachgebrauch im Unterricht gefragt: „Wenn Sie an Ihren Unterricht denken, in welchen Situationen verwenden Ihre LehrerInnen Standardsprache, in welchen Dialekt? Und Sie selbst?" Interessanterweise bezogen sich die Diskussionsbeiträge fast ausschließlich auf die Sprachverwendung der LehrerInnen, die eigene Sprachverwendung wurde von den SchülerInnen kaum thematisiert: „Wenn man Schularbeiten schreibt, dann halt das richtige Deutsch, das/ keine Umgangssprache ist“. (F6). Dabei wird die Persönlichkeit der Lehrperson als wichtiger Einflussfaktor wahrgenommen: LehrerIn X würde „Hochdeutsch“ reden, LehrerIn Y nur im Dialekt, „Manche können auch nicht Hochdeutsch so richtig." (F11). F6 meint:

Ich denk mal, das kommt auch ganz auf den Lehrer an. Ahm da gibts so Lehrer, die benutzen auch so Wörter zum Beispiel statt hässlich schiach. Ahm. Oder statt Bonbon Zuckerl. Sowas halt. Und dann gibts da wieder Lehrer, die benutzen ziemlich hochgestochenes Deutsch.

Zu hören war auch, dass manche LehrerInnen sogar im Unterricht „Dialekt“ sprechen würden, was die kanadische Austauschschülerin als problematisch erlebt hat: „Weil ahm zum Beispiel unser (Unterrichtsfach)Lehrer, ja er redet Dialekt --- und -- ich versteh's fast nicht." (F7). $\mathrm{F}_{3}$ bestätigt, dass der betreffende Lehrer „Dialekt verwendet“. Ob die Lehrperson aus der Stadt oder vom Land kommt, spielt nach Ansicht der SchülerInnen ebenfalls eine Rolle: „Wenn der jetzt irgendwie vom Land, von der Steiermark kommt, und da aufgewachsen ist, is es klar, dass er halt teilweise die Begriffe so sagt, so wie er halt damit aufgewachsen is." (F3). Interessant auch die Wahrnehmung einiger SchülerInnen, die LehrerInnen würden privat Dialekt reden und müssten „sich bemühen“, um „Hochdeutsch“ zu reden, wie im folgenden Beispiel: 
Also bei uns is es halt/ es gibt halt Lehrer, die bemühen sich, dass sie im Unterricht viel Hochdeutsch reden und sehr verständlich reden und wenn man dann privat mit ihnen spricht, persönlich fragt, dass sie dann komplett im Dialekt mit einem reden, das ist schon oft passiert.

Auf die Zwischenfrage des Moderators „Also sie bemühen sich?“

Ja, sie bemühen sich. Ja, das ist mir schon aufgefallen, besonders bei unserer (Unterrichtsfach-) Lehrerin dass sie/ da sie auch vom Land kommt, wirklich oft, dass sie sich konzentrieren muss und ab und zu rutscht ihr was rein, aber sie bemüht sich sehr. Aber ja wir haben auch Lehrer, die halten den kompletten Unterricht durchgehend im Dialekt. (F2)

Im Unterricht haben SchülerInnen auch wahrgenommen, dass die Varietätenverwendung vom Inhalt der Kommunikation abhängt und beispielsweise das Switchen in den Dialekt zum „Schmähführen“ genutzt wird:

Der (Unterrichtsfach-)Lehrer wechselt ganz oft ab. Also es kommt immer ganz aufs Thema drauf an. Er erklärt schon hochdeutsch, damits ja alle verstehen, und dann schiebt er einen Schmäh rein, den aber nur die halbade Klasse versteht, weil der einfach SO im Dialekt ist. ( $\left.\mathrm{F}_{1}\right)$

Wenn von den SchülerInnen hier von „Dialekt“ die Rede ist, dann ist vermutlich eine regional gefärbte, umgangssprachliche Varietät des Deutschen in Österreich gemeint. Umgangssprache als Varietät wird in der metasprachlichen Beschreibung nicht verwendet - es gibt nur Dialekt oder „Hochdeutsch“.

Im Fragebogen wurden die LehrerInnen auch danach befragt, wie sich ihre SchülerInnen in bestimmten Situationen (im LehrerInnen-SchülerInnen-Gespräch, bei Gruppenarbeiten, in Referaten, in den Pausen) ausdrücken. Dabei gaben die LehrerInnen an, dass die Mehrheit der SchülerInnen am häufigsten bei Gruppenarbeiten und in den Pausen Dialekt verwenden (46 \% bzw. 57,4 \%). Im LehrerInnenSchülerInnen-Gespräch beobachten die LehrerInnen offenbar eher weniger Dialekt und noch seltener bei Referaten (siehe Abb. 82 auf S. 205).

Werden diese Ergebnisse nach externen Variablen aufgeschlüsselt, zeigen sich interessante hochsignifikante Unterschiede, so etwa nach Bundesländern $(\mathrm{p}=0,000)$ : Hier sticht Vorarlberg heraus, wo rund $56 \%$ der LehrerInnen angaben, dass fast alle SchülerInnen im LehrerInnen-SchülerInnen-Gespräch Dialekt verwenden. In allen anderen Bundesländern war die Dialektverwendung im LehrerInnen-SchülerInnen-Gespräch wesentlich geringer ausgeprägt: Der Wert lag bei $25 \%$ in Salzburg und Oberösterreich, $21 \%$ in Tirol, $10 \%$ in Kärnten und in der Steiermark, sowie $3 \%$ in Wien, Niederösterreich und Burgenland. 


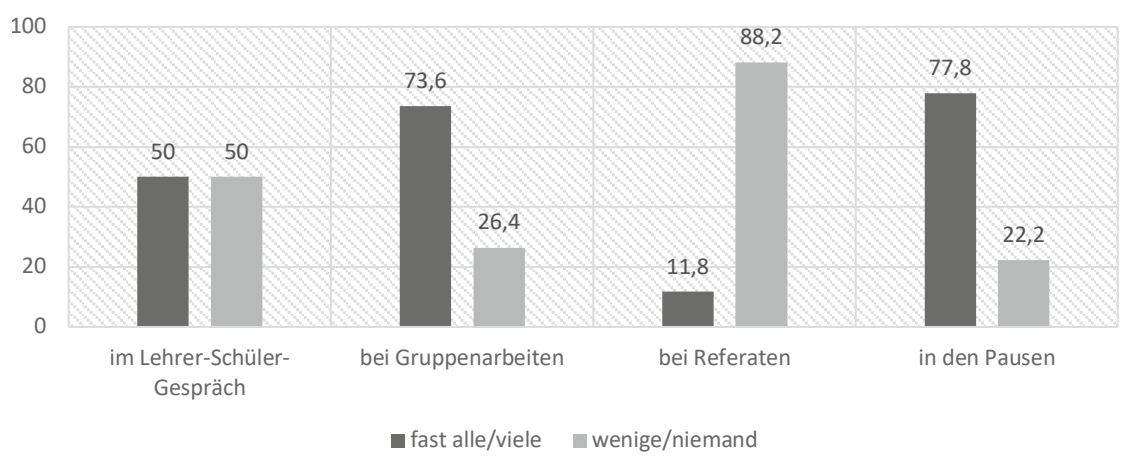

Abb. 82: Dialektverwendung der SchülerInnen in verschiedenen schulischen Situationen (in \%) (nach Einschätzung der LehrerInnen)

Fragt man die LehrerInnen, wie sie mit der schülerseitigen Dialektverwendung in den verschiedenen Unterrichtssituationen umgehen, zeigt sich, dass LehrerInnen in den Pausen und bei Gruppenarbeiten am wenigsten eingreifen und den Dialektgebrauch in diesen Fällen gelten lassen. Am häufigsten korrigiert wird der Dialektgebrauch im LehrerInnen-SchülerInnen-Gespräch und bei Referaten. Insgesamt sagten fast $90 \%$ der LehrerInnen, dass sie den Dialektgebrauch bei einem Referat korrigieren würden oder zumindest mit einem Hinweis einschreiten würden, und mehr als $60 \%$, dass sie dies im LehrerInnen-SchülerInnen-Gespräch tun:

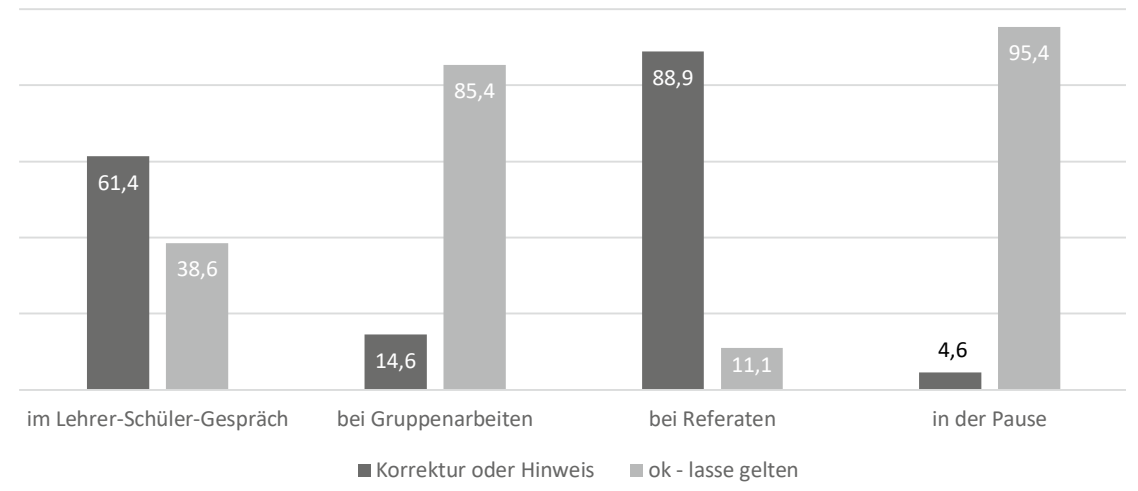

Abb. 83: LehrerInnen-Reaktion auf schülerseitigen Dialektgebrauch: „Korrektur/Hinweis“ oder „lasse gelten“ (in \%)

Die von LehrerInnen häufig beobachtete schülerseitige Präferenz von Dialekt bei Referaten war in der LehrerInnen-Gruppendiskussion auch ein Thema, wie der folgende Auszug veranschaulicht: 
Wenns heißt, ja, es ist ein Referat zu halten [...] dann kicherns herum und fühlen sich lächerlich ode:r, äh, seltsam, ode:r befremdlich, beim zweiten Mal gehts dann schon. Da wird der Schalter umgelegt und dann wird schon versucht, ä::hm::, wirkli in:, in aner gehobenen, ah, Standardsprache, ah/ eben, also, oder gehobenen Umgangssprache zu: sprechen, u::nd, ah, da hab i festgestellt, dass es für die Schüler anfoch a Überwindung/ es ist a - Gewohnheitseffekt auch, a::hm:, wobei, wenns zum Plaudern kommt oder wenns ins Erzählen kommt, sie sofort dann wieder in den Dialekt hineinfallen. In die Sprache einfach, in der sie sich zuhause fühlen. $\left(\mathrm{F}_{7}\right)$

Zusammenfassend kann zur Verwendung von Dialekt, Umgangssprache und Standard im Schulunterricht festgehalten werden, dass das gesamte Spektrum des Dialekt-Standard-Kontinuums bei LehrerInnen und SchülerInnen anzutreffen sein dürfte. Die für Österreich typische Registervariabilität wird von den Lehrenden zum Teil wohl intuitiv, zum Teil auch bewusst ausgeschöpft. Bei den LehrerInnen konnte eine Standardnähe bei bestimmten Teilen der Unterrichtshandlung festgestellt werden wie etwa beim Stoffvortrag und beim Erklären bzw. Erteilen von Arbeitsanweisungen. Bei den Lehrkräften ist eine relativ klare, zum Teil bewusste Trennung von Standard, Umgangssprache und Dialekt je nach Funktionsbereich erkennbar. Bei SchülerInnen verlaufen die Trennlinien bei der Zuordnung und der Verwendung von Standard, Umgangssprache und Dialekt weniger klar. Das ist wohl auch dadurch erklärbar, dass sie noch im Lernprozess der Entwicklung ihrer standardsprachlichen Kompetenz und ihres Varietätenbewusstseins begriffen sind.

Die Ergebnisse von Hochholzer (2004), der u. a. Daten zum Dialektgebrauch an Schulen in Bayern, Nordrhein-Westfalen und Mecklenburg-Vorpommern erhoben hat, ergaben deutlich höhere lehrerseitige Dialektwerte, und zwar nicht nur in Bayern. Danach ist „der Dialekt nach Aussage der Befragten eine feste Größe der sprachlichen Unterrichtsrealität auf Seiten der Deutschlehrer“ (Hochholzer 2004, 327). In Bayern gebe sogar die überwiegende Mehrheit an, Dialekt in verschiedenen Abstufungen auch im Unterricht zu gebrauchen. Zum Teil mögen die unterschiedlichen Ergebnisse daran liegen, dass unsere ProbandInnen zwischen Dialekt und Standardsprache als weitere Option die Umgangssprache angeben konnten - bei Hochholzer ist das Spektrum „Dialekt in verschiedenen Abstufungen“. Aber die Gruppendiskussionen und Interviews weisen auch darauf hin, dass unsere Fragebogenergebnisse die eine oder andere sozial erwünschte Antwort enthalten könnten und dass das gesamte Dialekt-Standard-Kontinuum wohl in allen Unterrichtssituationen eine Rolle spielt.

Auch über die Sprachverwendung im außerschulischen Bereich gaben die Fragebogendaten und Interviews Aufschluss. Die LehrerInnen wurden dazu befragt, welche Varietät(en) sie privat mit einer Auswahl an GesprächspartnerInnen sprechen (KollegInnen, SchülerInnen, eigenen Kindern, PartnerIn, Eltern, Großeltern). Die Mehrheit der mittels Interview befragten LehrerInnen gab an, im privaten Alltag Umgangssprache zu sprechen, viele gaben zusätzlich oder 
ausschließlich Dialekt an, Standarddeutsch sprechen den eigenen Angaben zufolge nur wenige LehrerInnen in der privaten Kommunikation. Genau dasselbe Ergebnis brachte auch die Fragebogenerhebung, wie Abb. 84 zeigt:

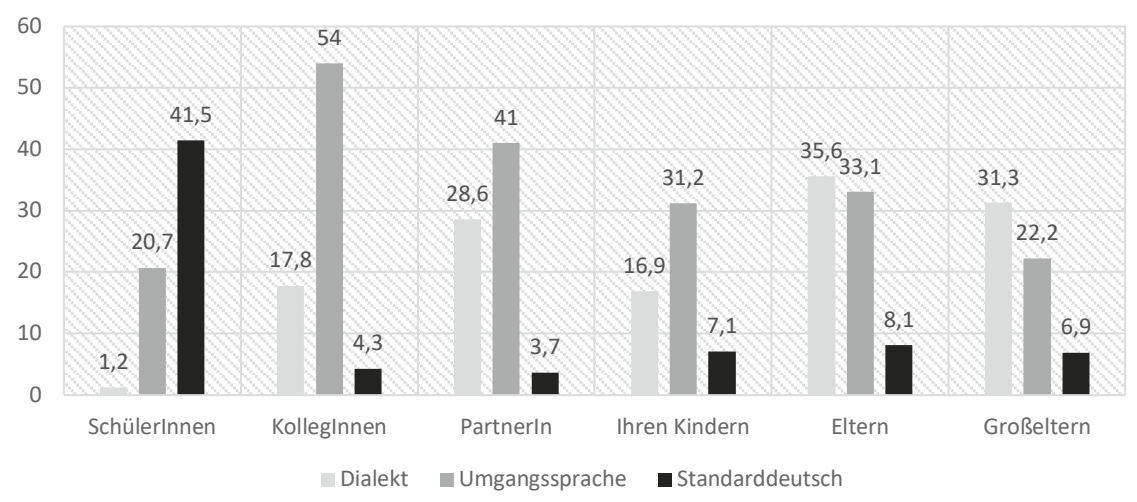

Abb. 84: Lehrerlnnen: Wie sprechen Sie mit wem? (in \%)

Die Lehrpersonen sprechen ihren Angaben im Fragebogen zufolge mit den SchülerInnen hauptsächlich Standardsprache (41,5\%), deutlich weniger Umgangssprache $(20,7 \%)$ und kaum Dialekt (1,2\%); im Gespräch mit den KollegInnen ist die Umgangssprache (54\%) vorherrschend, und auch vom Dialekt (17,8\%) wird wesentlich häufiger Gebrauch gemacht als im Gespräch mit den SchülerInnen. Relativ selten (4,3\%) wird im Gespräch zwischen KollegInnen den Angaben zufolge Standardsprache gesprochen. In familiären Privatgesprächen ist der Gebrauch von Dialekt und Umgangssprache eher ausgewogen, und auch hier wird eher selten die Standardsprache gesprochen.

Die angeführten Ergebnisse sind freilich mit einer Einschränkung zu versehen: Aus den Interwiews ging hervor, dass es für die Befragten häufig schwierig ist, ihren persönlichen „Varietäten-Mix“ im Rahmen des Dialekt-Standard-Kontinuums einzuordnen und die verwendeten Varietäten voneinander abzugrenzen. Aussagen zum persönlichen Sprachgebrauch im privaten Bereich waren in unseren Daten in sich nicht immer konsistent, wie man am folgenden Beispiel einer Lehrerin aus Wien (FW2) sieht. Danach gefragt, wie sie die Sprache bezeichnen würde, die sie in informellen Gesprächssituationen im Alltag, mit Freunden oder Familie spricht, war die spontane Antwort der Lehrkraft „Standardsprache“. Etwas später, im Kontext der Unterscheidung zwischen Standardsprache, Umgangssprache und Dialekt zog die Lehrkraft diese Aussage jedoch zurück: „Ahm. Gut, wenn ich das jetz überleg, dann werd ich wahrscheinlich auch im/ ah in der Kommunikation ah Umgangssprache sprechen, ja?"

Häufig schien es so zu sein, dass die Lehrenden die Varietät, die sie verwenden, nicht klar einordnen konnten, weil sie sich permanent zwischen Umgangssprache, 
Dialekt und Standard bewegen und die Registervariabilität in der Alltagskommunikation voll ausschöpfen. Ein Lehrer aus Salzburg (MSa4) dachte laut über seine Sprachverwendung nach:

In meinem Fall is des a Mischung aus eben schon leicht verschwundenem oberösterreichischen Dialekt, und natürlich als Deutschlehrer is man do irgendwie a (in) einer gewissen Rolle (drinnen) natürlich irgendwie auch im Dialektalen irgendwie verhaftet, irgendwie so mittendrin. Ja? - - Jo, also jetzt/ kein Lungauer Dialekt. Wie man hört ... aber in normalen Gesprächen natürlich mit dialektalen Verwendungen.

Auch eine Lehrerin aus Oberösterreich (FO1) betrachtete ihre Art des Sprechens als einen Mix: „Ahm Umgangssprache. Mit teilweise vielleicht Dialektelementen, aber eher Umgangssprache." Die große Mehrheit aller LehrerInnen gab bei den Interviews an, dass sie außerhalb des Unterrichts mit ihren SchülerInnen Umgangssprache oder Dialekt oder zumindest eine stärker umgangssprachlich gefärbte Standardsprache sprechen würden, wobei Dialekt oft mit Nähe und Standard mit Distanz assoziiert wird. Auch hier zeigt sich wieder das Muster, dass sich LehrerInnen in nähesprachlichen Situationen außerhalb des regulären Unterrichts - wie auch beim emotionalen Schimpfen in der Klasse - etwas vom Standard wegbewegen, wie eine Lehrerin aus Niederösterreich (FN1) beschrieb:

Sobald a Privatsituation is, wird's dialektaler. Und sicher auch Sportwochen und so. Logischerweise, i glaub, das fördert dann ah wie soll i sogn? -- die Zutraulichkeit. Weil des doch a Distanzbarriere is, wenn ma Standard oder halt annähernd an die Standardsprache spricht.

Befunde aus der teilnehmenden Beobachtung ergänzen dieses Bild: Es wird eine gewisse Diskrepanz zwischen Selbsteinschätzung der LehrerInnen („eigentlich fast nie Dialekt“) und beobachtetem Sprachgebrauch festgestellt. Die Beobachtungen bestätigen das Dialekt-Standard-Kontinuum: Die dialektale Varietät werde eher für Disziplinäres und Organisatorisches u. Ä. verwendet, Standardnähe findet sich im Vortrag, im LehrerInneninput und bei Referaten. Ein Protokollausschnitt möge das illustrieren:

Die Lehrerin sprach österreichisches Standarddeutsch mit Tiroler und Vorarlberger Färbung. Umgangssprachliche Äußerungen kamen immer wieder vor, vor allem bei disziplinären Äußerungen und Arbeitsaufträgen, z. B.: „Also jetz fangsch amal an mit einer kleinen Geschichte... “. Ein anderer Lehrer, der kurz hereinkam, um etwas zu holen, sprach die Kinder im Dialekt an. Diese antworteten mit: „Grüß Gott!“ und „Auf Wiedersehen!“

Auch die SchülerInnen wurden gefragt, wie sie mit ihren LehrerInnen sprechen. Was sie am häufigsten im Fragebogen nannten, war die Standardsprache - ihrer eigenen Einschätzung nach verwenden mehr als die Hälfte der Schüler mit ihren 
LehrerInnen Standarddeutsch (52,8 \%), dicht gefolgt von Umgangssprache (49,8\%). Dialekt wurde im Gegensatz dazu wesentlich seltener genannt (17,4\%). Dieser dominierte hingegen klar im privaten Bereich: Über 50 \% der SchülerInnen sprechen nach eigener Einschätzung mit Familie und Freunden Dialekt, über 40 \% nannten in diesem Zusammenhang auch die Umgangssprache, während Standarddeutsch nur von rund $10 \%$ der SchülerInnen für den privaten Bereich angegeben wurde:

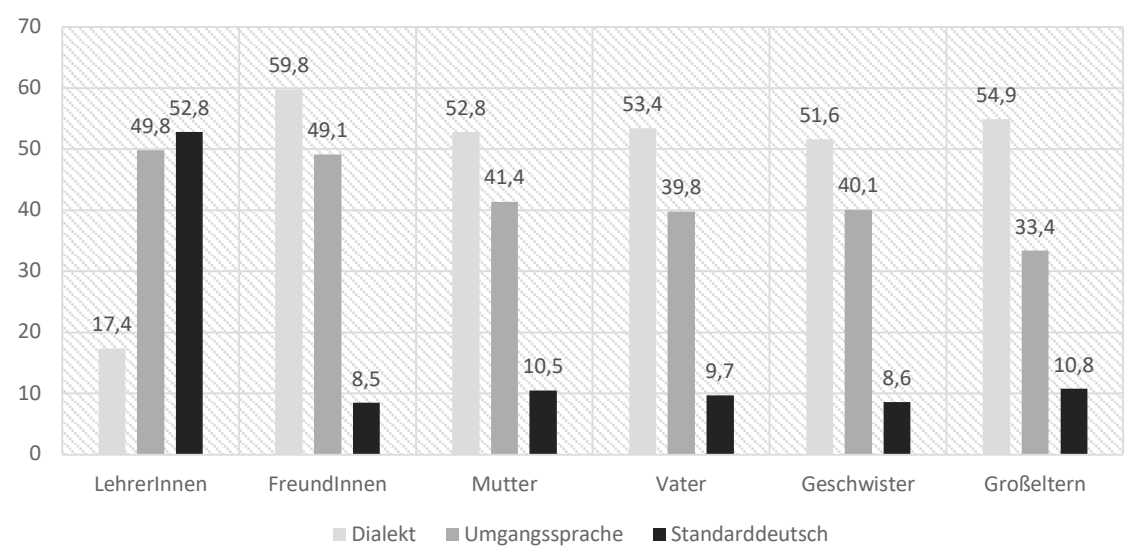

Abb. 85: SchülerInnen: Wie sprichst du mit wem? (in \%)

Auch wenn der Dialekt prima vista unter den SchülerInnen im privaten Bereich sehr präsent zu sein scheint, sagte doch etwa ein Drittel der SchülerInnen von sich, keine DialektsprecherInnen zu sein, wie Abb. 86 zeigt: 31,7\% der befragten SchülerInnen gaben an, weder mit den LehrerInnen noch mit der Familie oder Freunden im Dialekt zu sprechen. Da nur 3,2 \% angaben, ausschließlich Standardsprache zu sprechen, wird wohl unter rund zwei Dritteln der SchülerInnen die Umgangssprache mehr oder wenig vorrangig verwendet werden (siehe Abb. 86 auf S. 210).

Es kann davon ausgegangen werden, dass in den verschiedenen Regionen Österreichs der Varietätengebrauch der SchülerInnen nicht gleich ist. Dem entspricht das Ergebnis, dass sich die Angaben über die Verwendung von Dialekt, Umgangssprache und Standardsprache hochsignifikant $(\mathrm{p}=0,000)$ nach Bundesländern unterscheiden:

So gaben SchülerInnen in den östlichen Bundesländern am häufigsten an, in keiner Situation Dialekt zu sprechen (50,5\%). Dagegen sind ihren eigenen Angaben zufolge nur 3,6 \% der Vorarlberger SchülerInnen keine DialektsprecherInnen, gefolgt von Tirol (16,4\%) und den mittleren Bundesländern (18,7\%). In allen Situationen, also in Gesprächen im Unterricht, mit Familienmitgliedern und mit Freunden, sprechen $31 \%$ der SchülerInnen in Vorarlberg Dialekt, in den mittleren und südöstlichen Bundesländern jeweils $18 \%$ und in Tirol 14,8\%, während es im Osten Österreichs nur 4,3\% sind. 
50

40

44,5

30

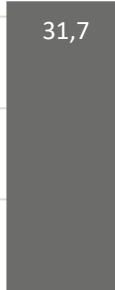

13,2

0

Dialekt

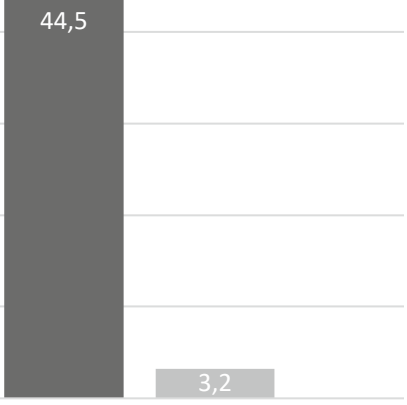

Standarddeutsch

nie immer

Abb. 86: SchülerInnen, die nie/immer Dialekt und Standard sprechen (in \%)

Analysiert man unsere empirischen Daten danach, wer angab, in keiner Situation (Unterricht/Familie/Freundeskreis) Standard zu sprechen, so erhält man mit über 60 \% den höchsten Wert in den mittleren Bundesländern Oberösterreich und Salzburg. Auch in den südöstlichen Bundesländern Kärnten und Steiermark ist dieser Anteil der „Niemals-Standard-SprecherInnen“ relativ hoch mit 58,5\%, ebenso in Tirol mit $50 \%$. Die Anzahl jener SchülerInnen, die angeblich keinen Standard verwenden, war in Vorarlberg mit etwas mehr als einem Drittel und im Osten mit „nur“ rund einem Fünftel der SchülerInnen am niedrigsten, wie in Abb. 87 dargestellt ist:

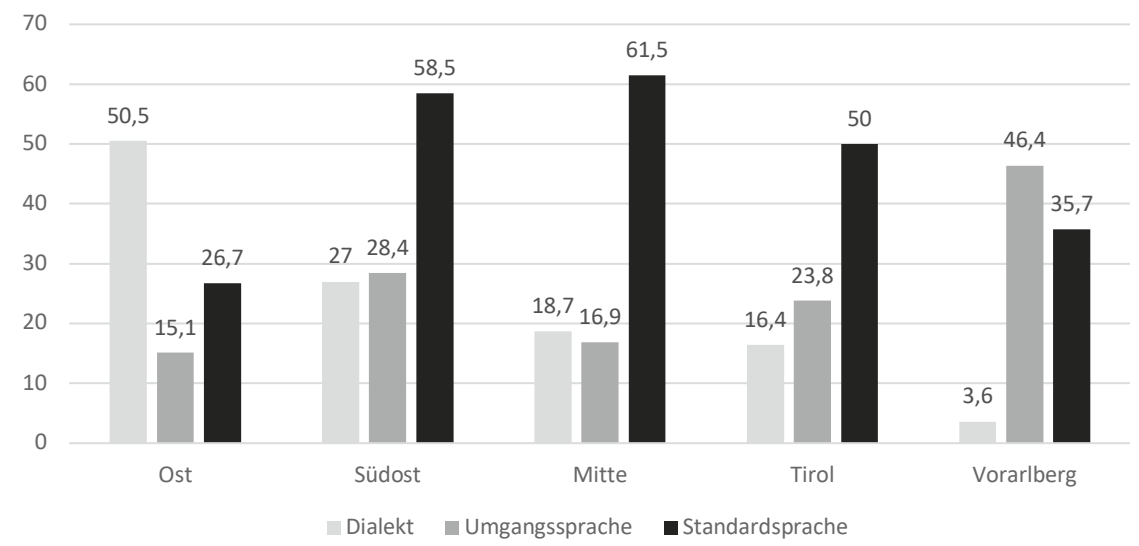

Abb. 87: SchülerInnen: in keiner Situation verwendete Varietäten (nach Region) (in \%)

Dass sie in allen Situationen (Unterricht/Familie/Freundeskreis) Standard sprechen, gaben nur 6,3\% der SchülerInnen in Ostösterreich an. Für Salzburg und 
Oberösterreich lag der entsprechende Wert unter den SchülerInnen bei nur 1,4 \%, in den südöstlichen Bundesländern bei 1,7\%. In Vorarlberg und Tirol gab es praktisch keine ausschließlichen StandardsprecherInnen unter den SchülerInnen.

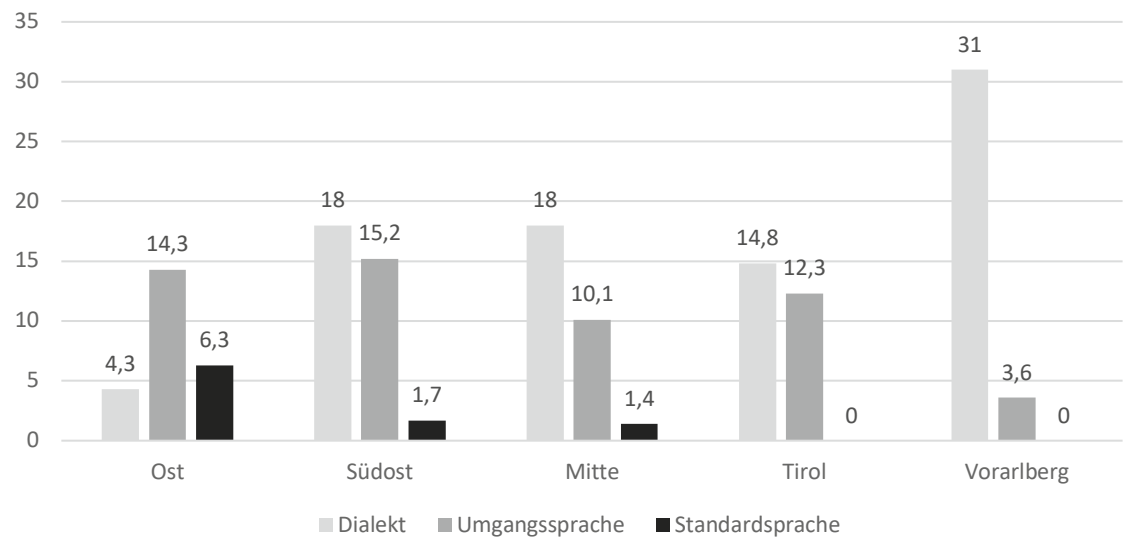

Abb. 88: SchülerInnen: in allen Situationen (Unterricht, Familie, Freundeskreis) verwendete Varietäten (nach Region) (in \%)

Kommen wir zu einer Gesamtbetrachtung der Sprachverwendung von LehrerInnen und SchülerInnen: In den Interviews gaben die LehrerInnen an, dass ihre SchülerInnen im Unterricht meist Standarddeutsch sprechen, wobei es Unterschiede nach Bundesländern gibt: Die meisten LehrerInnen aus Wien, Niederösterreich, Salzburg und Tirol meinten, ihre SchülerInnen sprächen bis auf wenige Ausnahmen Standard oder gehobene Umgangssprache, während die meisten LehrerInnen aus der Steiermark und aus Vorarlberg, sowie eine Lehrerin aus Tirol sagten, ihre SchülerInnen würden häufig Dialekt verwenden. Unter den LehrerInnen aus dem Burgenland gab es unterschiedliche Ansichten dazu: Eine Lehrerin meinte, ihre SchülerInnen verwendeten die Standardsprache, ein Lehrer sagte dagegen, das sei je nach Herkunft der SchülerInnen unterschiedlich.

Die teilnehmenden Beobachtungen haben gezeigt, dass in allen beobachteten Unterrichtsstunden hauptsächlich Standard gesprochen wurde, sowohl von LehrerInnen als auch von SchülerInnen. Zu beobachten war auch, dass LehrerInnen und SchülerInnen die Standardsprache auch gut beherrscht haben, wobei die Standardorientiertheit in Vorarlberg und Kärnten am stärksten zu sein schien. Hier konzentrierten sich die SchülerInnen zum Teil so stark darauf, die Standardsprache korrekt zu verwenden, dass oft kaum regionale Färbung wahrnehmbar war; gleichzeitig klang die Sprache der SchülerInnen dadurch bisweilen etwas künstlich. Das mag einerseits mit der spezifischen diglossalen Situation in Vorarlberg zusammenhängen und mit der Tatsache, dass die Beobachtungen in Kärnten in einer de facto zweisprachigen (slowenisch-deutschen) Schule stattgefunden haben. 
Das für Österreich typische Dialekt-Standard-Kontinuum war in allen Schulen beobachtbar. Die Verwendung der jeweiligen Varietät war jedoch oft situationsabhängig: nicht-standardsprachliche Varietäten wurden in den meisten Fällen entweder beim Tratschen verwendet (SchülerInnen) oder wenn Emotionen im Spiel waren, z. B. Ärger (disziplinäre Äußerungen von LehrerInnen) oder in nicht stoff- bzw. themenbezogenen Gesprächen.

Dasselbe Ergebnis brachten die Interviews, die mit DeutschlehrerInnen geführt wurden: Die meisten von ihnen gaben an, am ehesten bei emotionaleren Angelegenheiten oder außerhalb des Unterrichts bzw. wenn sie gerade nicht vortragen würden, in eine Nonstandardvarietät zu wechseln.

Die Einteilung der Äußerungen in Standard, Umgangssprache und Dialekt erfolgte ausschließlich aufgrund der Wahrnehmung der die Beobachtung durchführenden Person; ein wichtiges Desiderat wäre, solche Beobachtungen mit Videoaufnahmen durchzuführen (wie z. B. Steiner 2008).

\subsubsection{Thematisierung des österreichischen Deutsch im Unterricht}

Inwieweit Deutsch-Lehrkräfte in ihrem Unterricht unterschiedliche Sprachformen (Dialekt/Umgangssprache/Standardsprache) thematisieren und welche Themen Sie dafür zum Anlass nehmen, sollte in einem didaktisch orientierten Fragebogenteil erfragt werden.

Etwa die Hälfte der Lehrenden (49\%) gab an, unterschiedliche Sprachformen häufig oder sehr häufig im Unterricht zu thematisieren, wobei viele unterschiedliche Anlässe genannt wurden, u. a.: aktuelle Pressetexte, Stilübungen, Gedichte und Lieder, Referate, aus Deutschland stammende Lektüre, Entwicklung der deutschen Sprache etc. Bei der anderen Hälfte $(50,9 \%)$ der Lehrpersonen kommt dieses Thema im Unterricht selten bis nie vor.

Signifikante Unterschiede fanden sich dabei nach Geschlecht $(\mathrm{p}=0,011)$, Schulform $(p=0,000)$, Ausbildungsort $(p=0,000)$, Studienfächern $(p=0,050)$ und Unterrichtsfächern $(\mathrm{p}=0,044)$.

So zeigt sich, dass deutlich mehr männliche Lehrkräfte (75\%) unterschiedliche Sprachformen häufig oder sehr häufig im Unterricht angeben zu thematisieren als weibliche (42,2\%) - ein schwer interpretierbarer großer Unterschied zwischen Lehrern und Lehrerinnen in ihren Angaben zur Thematisierung sprachlicher Variation im Unterricht. Und nur 24,1\% der VolksschullehrerInnen gaben an, dieses Thema im Unterricht häufig oder sehr häufig im Unterricht zu besprechen, aber mehr als doppelt so viele LehrerInnen (52,4\%), die an einer HS/NMS unterrichten, und $64,6 \%$ der AHS- bzw. BHS- oder BAKIP-LehrerInnen. Dem entspricht auch, dass deutlich mehr Lehrpersonen, die an einer Universität ausgebildet wurden $(65,3 \%)$, häufig bis sehr häufig unterschiedliche Sprachformen thematisieren als LehrerInnen, die ihre Ausbildung an einer PH absolviert haben (31,5\%). 
Und schließlich gaben LehrerInnen, die Deutsch und eine andere Sprache studiert haben, deutlich öfter $(76,9 \%)$ an, unterschiedliche Sprachformen häufig oder sehr häufig zu thematisieren als solche, die Deutsch und ein anderes Fach (61\%) und solche, die nur ein anderes Fach (nicht Deutsch) studiert haben $(37,5 \%)$ - ein durchaus plausibles Ergebnis.

Dieser Befund, wonach nur die Hälfte der Lehrpersonen unterschiedliche Sprachformen im Unterricht häufig bis sehr häufig thematisiert, deckt sich nicht ganz mit den Ergebnissen der Einzelinterviews: ${ }^{21}$ Hier gab die Mehrheit der befragten LehrerInnen an, das österreichische Deutsch im Unterricht bereits thematisiert zu haben. Nur wenige (wie z. B. die Lehrerin aus Tirol [FT1], die im Folgenden zitiert wird) führten als Grund für die Thematisierung ein fehlendes sprachliches Selbstbewusstsein der österreichischen SprecherInnen an:

Also i denk, man kann durchaus a gewisses Selbstbewusstsein für des österreichische Deutsch entwickeln, und ... net dieses latente Minderwertigkeitsgefühl gegenüber dem bundesdeutschen Standard mit sich herumtragen, sondern es eben als eine durchaus auch legitime Variante sehen.

Die wenigsten Lehrenden, die angaben, österreichisches Deutsch bereits im Unterricht angesprochen zu haben, behandeln dabei die österreichische Standardsprache; öfter handelt es sich bei den Unterrichtseinheiten um Dialekte, wie z. B. ein Lehrer aus dem Burgenland erklärte:

Ahm natürlich, in den Oberstufen is das/is das ganz sicher Thema, [...] wobei ich sagen muss, ahm da geht's mehr immer um die Verwendung des Dialekts. Und nicht um das Österreichische ALS Standardsprache im Vergleich zum Deutschen als Standardsprache, des wird eher weniger thematisiert. (MB1)

Ein Lehrer aus Salzburg (MSa1) sagte, er würde das österreichische Deutsch im Zusammenhang mit Soziolekten thematisieren:

Mmh ja. Aber eher im Zusammenhang eben mit so Soziolekten, also eher in die Richtung, ja, das dann etwas vorkommt. Es is net des große Thema, ahm es is, würd i sogn, ein Randthema, weil ma einfach so viel andere Sachen machen muss.

Auch Zeitmangel wurde in den Interviews als Grund dafür genannt, österreichisches Deutsch nicht zu thematisieren:

21 Das ist insofern nicht verwunderlich, als sich die InterviewpartnerInnen für die Interviews selbst gemeldet haben und vermutlich am Thema besonders interessiert waren. 
Ich glaub, dass die Zeit sehr gering ist für den Deutschunterricht in der Oberstufe und dass die/die Dinge, die zu erledigen sind, von schriftlichen Dingen, über/über Literatur et cetera, ahm so umfassend sind, dass ma afoch gewisse Dinge, die man für nicht so wichtig erachtet, oder für die Reifeprüfung net so wesentlich san, dass man die einfoch weglässt. (MB1)

Eine Lehrerin aus der Steiermark (FSt3) kam durch ein Seminar, das vom Projektteam mitveranstaltet wurde, auf das Thema österreichisches Deutsch und bezieht es aus diesem Grund seither in den Deutschunterricht ein:

Durch die Informationen, die ich bei diesem Seminar erhalten habe, is meine Aufmerksamkeit eben auch auf das österreichische Deutsch gelenkt worden und ah des woa so a Impuls ahm, dass dann auch weiterzugeben und/und klarzumachen auch, das ist jetzt österreichisches Deutsch [...].

Einen Spezialfall bildete auch hier Vorarlberg: Der von dort stammende Lehrer MV1 antwortete auf die Frage, ob er das österreichische Deutsch für ein wichtiges Thema halte, mit Nein, und begründete das wie folgt:

Ich seh die große Wichtigkeit des österreichischen Deutsch in dem Sinn für einen Vorarlberger nicht. [...] in Vorarlberg werden wir diese Sprache nicht sprechen, eh nicht, weil wir Dialekt sprechen. In der $<$ Normalsituation (lachend) $>$ wird sich die Frage nicht stellen, weil da reden wir Dialekt. Und wenn wir miteinander Deutsch reden, dann [...] sind wir wahrscheinlich ein Zwischending zwischen Deutschland und Österreich.

Die meisten LehrerInnen aus den anderen Bundesländern hielten das österreichische Deutsch für ein wichtiges Thema, so wie eine Lehrerin aus Tirol:

Ja, halt ich es schon für ein wichtiges Thema, weil ja: wirs alle < verwenden (lachend) >, und uns eigentlich bewusst verwenden sollten [...] äh - - dass es eben eine unter mehreren Varianten ist, die sehr wohl Existenzberechtigung hat. (FT1)

Im Unterricht einiger LehrerInnen scheint das österreichische Deutsch bis zum Zeitpunkt der Befragung keine große Rolle gespielt zu haben, da sie sich noch nie bewusst damit auseinandergesetzt hatten, wie der folgende Kommentar einer Wiener Lehrerin (FW1) zeigt: „Najo, eigentlich nicht, nachdem ichs kaum mache, na? (lacht) Also man müsste das hinterfragen und sich bewusster machen."

Als weiterer Grund, das österreichische Deutsch nicht zu thematisieren, wurde auch erwähnt, dass in erster Linie die Vorgabe des Lehrplans erfüllt werden müsse - ein Hinweis darauf, dass sprachliche Variation bzw. das österreichische Deutsch und das plurizentrische Konzept im Lehrplan erwähnt werden sollten. 
Ein Lehrer aus dem Burgenland (MB1) erklärte folgendermaßen, warum er das Thema im Unterricht nicht behandelt:

Weils ( 5 sek Pause) viele andere Dinge gibt, die wahrscheinlich in der Rangliste dessen, was man irgendwie halt durchbringen sollte, meines Erachtens weiter vorne kommen, wobei des jetzt net unbedingt meine Meinung is, sondern einfach auch die Vorgabe des Lehrplans, beziehungsweise das, was bei Prüfungen und/und schlussendlich bei der Reifeprüfung gekonnt werden soll. Und da is österreichisches Deutsch als Standardsprache vielleicht amal a Spezialthema von an mündlichen Maturanten, aber mehr net.

Wird das österreichische Deutsch im Unterricht einmal thematisiert, so greifen viele LehrerInnen auf selbst zusammengestelltes Material (oft aus dem Internet) zurück. Einige LehrerInnen lassen die SchülerInnen Dialektgedichte oder Texte von Christine Nöstlinger lesen, ein Lehrer aus Salzburg verwendet Leserbriefe, um seine SchülerInnen für österreichisches Deutsch zu sensibilisieren, und eine Lehrerin aus Tirol lässt ihre SchülerInnen zu diesem Zwecke eigene Texte durchlesen. Der folgende Auszug aus einem Interview mit einer Lehrerin aus Wien (FW1) fasst die von mehreren Lehrpersonen beschriebene Situation zusammen:

Oiso wos MIR fehlt, wären ehrlich gesagt GEEIGNETE Unterrichtsmaterialen, ... in den Büchern is es ja auch stiefmütterlich behandelt, und das Stiefmütterliche zeigt sich einerseits in der Menge, aber andererseits auch in der Art und Weise, es is sehr trocken. Ja? Und mir ist tatsächlich no nix Besseres eingefallen, und daher mach ichs auch so wenig. ... MICH SELBER interessierts schon - - ah und ich wäre dankbar dafür, wenns was Gscheiteres gäbe! (lacht)

Dieses Statement ist ein klarer Hinweis darauf, dass sowohl Bedarf als auch Interesse seitens der LehrerInnen besteht, dass in den Lehrbüchern das Thema „österreichisches Deutsch“ ausführlicher behandelt wird.

Auch in der Gruppendiskussion der LehrerInnen kam zur Sprache, dass es wichtig sei, dass die österreichische Varietät in der Schule thematisiert wird und in den Lehrmaterialien vorkommt. So bedauerte F9, dass das ÖWB keine wichtige Rolle (mehr) spiele, dass es in den neuen Medien nicht berücksichtigt werde, und es auch den "Jugendlichen von heute“ "gar kein Anliegen“ sei. F4 erklärte, warum das ÖWB nicht mehr präsent sei, nämlich weil es in der zweiten Klasse Volksschule die Möglichkeit gebe, ein Wörterbuch auszusuchen und die Lehrpersonen hier altersgemäße Wörterbücher wählen würden:

Warum? Weil das Österreichische Wörterbuch für Volksschulkinder einfach zu umfangreich ist, zu schwer zu lesen. Während der Findefix mit vielen Bildern und so weiter bestückt ist, und das ist, GLAUBE ICH, die Ursache dafür, dass das Österreichische Wörterbuch abhanden kommt. 
Man sollte, so der Ratschlag der Lehrerin, das ÖWB später im Curriculum noch einmal anbieten. M1 (1423) erklärte:

I möchte nur wos ergänzen. Aso, i finds jetzt net wichtig, des [...] im Gesetz festzuschreiben, aber ich würds SEHR wichtig finden, des in der Lehrerausbildung deutlich zu machen, dass es ein österreichisches Deutsch gibt, (Kommentar F3: Hm. Reicht net.) linguistisch fundiert. [...] Es geht darum, [...] wos die Lehrer unterrichten, wie die Lehrer unterrichten, und dass do a Bewusstsein für österreichisches Deutsch erzeugt wird.

F5 ergänzte (1433): „Net nur in der Lehrerausbildung, sondern auch in den Lehrplänen. “ Und F4 (1434): „Die ganzen Schulbücher gehörn umgändert.“

Auch die SchülerInnen wurden gefragt, ob „österreichisches Deutsch“ für sie ein interessantes Thema sei. 73,1\% der SchülerInnen antworteten mit „Ja“. Auch das spricht dafür, das österreichische Deutsch im Unterricht und Lehrmaterialien zu thematisieren. Signifikante Unterschiede wurden hier nach den Muttersprachen ( $p=0,008$ ) festgestellt. 75,1\% der SchülerInnen, die Deutsch als Muttersprache haben, kreuzten an, das Thema „österreichisches Deutsch“interessant zu finden. Bei den SchülerInnen, die zweisprachig aufgewachsen sind, waren es nur 69,2\%, bei jenen, deren Muttersprache nicht Deutsch ist, waren es 64,2\%.

\subsection{Zusammenfassung der wichtigsten Ergebnisse der empirischen Erhebung an den Schulen ${ }^{22}$}

Welche Rolle das österreichische Standarddeutsch in der Unterrichtspraxis überhaupt spielt, sollte eine großangelegte Lehrer-Schüler-Befragung zeigen, nachdem die Dokumentenanalyse ergeben hatte, dass das Themenfeld „österreichisches Deutsch und standardsprachliche Variation“ in sämtlichen von uns untersuchten unterrichtsrelevanten Grundlagendokumenten unterbelichtet ist.

Die Aspekte, um die es bei der Befragung an den Schulen primär ging, waren die lehrer- und schülerseitigen Einstellungen gegenüber den Varietäten des Deutschen bzw. deren Konzeptualisierungen, die Variantenpräferenz bei Austriazismen und Deutschlandismen sowie das Normverständnis der Lehrkräfte. In diesem Zusammenhang war unsere Fragebogenerhebung die erste Untersuchung, die auf Basis von österreichweiten Befragungsdaten die Rolle des österreichischen Deutsch in seiner Funktion als Bildungssprache praxisnah beleuchtet hat.

Die Ergebnisse zeigen, dass die LehrerInnen im Spannungsfeld von Dialekt, Umgangssprache und Standarddeutsch mit Anforderungen konfrontiert sind,

22 Einen Überblick über Projektergebnisse bzw. die Analyse einzelne Aspekte finden sich auch in de Cillia 2016a, b, 2018, de Cillia/Fink/Ransmayr 2017, Fink 2016, Fink/Ransmayr/de Cillia 2017, Ransmayr 2015, 2017, Ransmayr/Fink 2016. 
auf die sie während des Studiums nicht ausreichend vorbereitet werden. $86 \%$ der befragten PädagogInnen sagten, dass sprachliche Variation in ihrer Ausbildung kaum ein Thema war. Nachdem auch die Lehrpläne und Lehrbücher (siehe Dokumentenanalyse Kap. 4) aufgrund fehlender Leitlinien keine Orientierungshilfe bieten, berichten die befragten LehrerInnen davon, dass sie sich bei Entscheidungen über die normative Sprachrichtigkeit im Zusammenhang mit sprachlicher Variation schwertun. Auch der von den Lehrkräften bei der Textkorrektur üblicherweise verwendete Kodex (ÖWB, Duden) kann nur bedingt weiterhelfen, sodass die DeutschlehrerInnen in Normfragen vielfach auf sich allein gestellt sind und nach eigenem Ermessen entscheiden.

Wie individuell unterschiedlich ausgeprägt dieser Ermessensspielraum in der Praxis ist, konnte anhand der Korrektur eines Erlebnisaufsatzes festgestellt werden. Dieser Schüleraufsatz, den die Lehrkräfte zu korrigieren hatten, war frei von jeglichen Rechtschreib- und Grammatikfehlern, enthielt aber eine Reihe von (im Kodex als standardsprachlich ausgewiesenen) Austriazismen und Deutschlandismen. Wie unterschiedlich das Empfinden in punkto normativer Sprachrichtigkeit hier von LehrerIn zu LehrerIn ausfällt, wurde zum einen durch die stark variierende Anzahl an Korrekturen sichtbar, zum anderen durch die Art der Korrekturen (Welle oder durchgestrichen):

Bei einer Textlänge von knapp 200 Wörtern wurden von einzelnen LehrerInnen bis zu 14 Normverletzungen markiert, also als "Fehler" angestrichen - in einem prinzipiell „fehlerfreien“ Text. Nimmt man die „Fehler" und die unterwellten Ausdrücke zusammen, so haben einige Korrektur-SpitzenreiterInnen sogar bis zu 25 Mal den Rotstift angesetzt. Andere LehrerInnen machten hingegen keine einzige Korrektur. Bei bestimmten Merkmalen des österreichischen Deutsch, z. B. beim Perfekt als Erzählzeit, beim Artikel mit Eigennamen und Verwandtschaftsbezeichnungen sowie bei Grenzfällen des Standards (z. B. fladern, Wimmerl, am Eck) wurde besonders häufig unterwellt und in geringerem Ausmaß korrigiert. Umgekehrt gab es auch einige Deutschlandismen, die häufig angestrichen wurden (z. B. an Weihnachten). Wenn der Rotstift zum Einsatz kam, dann wurden Austriazismen im Vergleich zu Deutschlandismen relativ häufiger als fehlerhaft empfunden: $8 \%$ der Austriazismen und rund $5 \%$ der Deutschlandismen wurden durchschnittlich als „Fehler“ korrigiert. Als stilistisch unpassend wurden jedoch auffallend mehr Deutschlandismen (33\%) unterwellt als Austriazismen (17\%).

Insgesamt haben die Ergebnisse gezeigt, dass die in der Literatur behauptete starke Exonorm-Orientiertheit bei der Textkorrektur in unserer Untersuchung bedingt bestätigt werden konnte. Trotzdem war die Normunsicherheit der LehrerInnen in ihrer Funktion als „Sprachnormautoritäten“ augenfällig - eine Tatsache, die als problematisch anzusehen ist. Die Gruppendiskussion der LehrerInnen und auch die Interviews zeigten, dass sich die teilnehmenden LehrerInnen der Widersprüchlichkeit ihrer Rolle als normsetzende Instanz und der Relativität von (Sprach-)Normen allerdings bewusst waren. Und dass in der Praxis der 
Klassenzimmer häufig ein durchaus reflektierter und differenzierter Umgang mit der Normproblematik im Sinn eines situativen, kommunikativen Normverständnisses die Regel sein dürfte.

Unsere Untersuchung sollte auch überprüfen, ob oder inwiefern der in der Literatur behauptete sprachliche „Minderwertigkeitskomplex“ (s. o.) der ÖsterreicherInnen im Hinblick auf das deutschländische Deutsch nachgewiesen werden kann. Auf die Frage, ob das österreichische Deutsch ebenso korrekt sei wie das deutsche Deutsch, antwortete zunächst die überwiegende Mehrheit der LehrerInnen (86\%) und ein Großteil der SchülerInnen (68\%) mit „Ja“. Eine differenzierte Nachfrage zum Stellenwert der beiden Varietäten im direkten Vergleich brachte jedoch eine gewisse Ambivalenz zum Vorschein: Nur mehr 45\% der LehrerInnen und etwa ein Drittel der SchülerInnen schätzten die beiden Varietäten als absolut gleich korrekt ein. Dieses Ergebnis unterstreicht, dass LehrerInnen wie SchülerInnen unsicher sind und beim Einstufen der Richtigkeit des österreichischen Deutsch Zweifel haben.

Unsere Untersuchung zeigt dennoch eines ganz klar: Trotz der festgestellten Normverunsicherung ist unter LehrerInnen und SchülerInnen ein plurizentrischer Zugang intuitiv feststellbar. Unsere Befragten unterstützten mit klarer Mehrheit (90\% der LehrerInnen und 80\% der SchülerInnen) die Aussage, dass Deutsch eine „Sprache mit Unterschieden in der Standardsprache zwischen den einzelnen Ländern“ ist. Unter den Lehrkräften mit Universitätsausbildung lag der entsprechende Wert sogar bei $95 \%$. Breite Zustimmung gab es von den LehrerInnen und SchülerInnen (80 \% bzw. 59\%) auch auf die Frage, ob es ein österreichisches Standarddeutsch gibt. Diese Befragungsergebnisse unterstreichen, dass die Mehrheit der LehrerInnen und SchülerInnen die deutsche Sprache intuitiv plurizentrisch konzeptualisieren, obwohl nur einem kleinen Teil der Befragten das Konzept der Plurizentrik als solches bekannt ist (LehrerInnen: 14,7 \%, SchülerInnen: 8,1\%). Interessant war, dass im Gegensatz zu den Lehrkräften in manchen österreichischen Regionen keineswegs alle SchülerInnen gleichermaßen von der Existenz eines österreichischen Standarddeutsch überzeugt waren: Unter Tiroler und Vorarlberger SchülerInnen liegen die Zustimmungsraten zu einem österreichischen Standarddeutsch signifikant unter den Werten der SchülerInnen aller anderen Bundesländer.

Was häufig ins Treffen geführt wird, wenn die Existenz eines österreichischen Deutsch diskutiert bzw. in Abrede gestellt wird, sind die regionalen Sprachunterschiede innerhalb Österreichs. Unsere Ergebnisse zeigen jedoch, dass die von uns befragten LehrerInnen und SchülerInnen mehrheitlich trotz regionaler Sprachunterschiede von einem österreichischen Standarddeutsch ausgehen. Die Behauptung, dass für ein eigenes österreichisches Standarddeutsch zu große regionalsprachliche Unterschiede innerhalb Österreichs vorhanden wären, wurde von der klaren Mehrheit der befragten LehrerInnen (82 \%) abgelehnt - von den Lehrkräften mit Universitätsausbildung oder mit plurizentrischem Vorwissen 
sogar fast einstimmig. Anders war es bei den SchülerInnen, die von einem regionenübergreifenden österreichischen Standarddeutsch weniger stark überzeugt waren $(57 \%)$ als die LehrerInnen, wobei unter den SchülerInnen erneut regional bedingte Meinungsunterschiede auffällig waren: Mehr als die Hälfte der befragten Vorarlberger (57\%) und Tiroler (55\%) SchülerInnen stimmten der oben genannten Behauptung nämlich zu.

Als explizit nach der Meinung zu einem „überregionalen österreichischen Standarddeutsch, das sich vom Deutsch Deutschlands in manchen Bereichen klar unterscheidet" gefragt wurde, fand weiterhin das Konzept eines „überregionalen österreichischen Standarddeutsch" unter der Mehrheit der PädagogInnen Zustimmung $(77 \%)$. Und erneut haben wir unter den Universitäts-AbsolventInnen die höchsten Zustimmungsraten ( $85 \%$ ) festgestellt.

Insgesamt wurde ersichtlich, dass innerösterreichische Sprachunterschiede für die Mehrheit der ProbandInnen kein Hindernisgrund sind, von einem überdachenden österreichischen Standarddeutsch auszugehen - was wiederum dafür spricht, dass in erster Linie eine plurizentrische Konzeptualisierung unter Österreichs Lehrkräften und (weniger stark) auch unter den SchülerInnen anzutreffen ist, die in zweiter Linie durch eine Form der regionalen Plurizentrik oder Pluriarealität ergänzt wird. Generell erschienen die SchülerInnen als sprachliche LaiInnen diesbezüglich unsicherer.

Die Durchsicht der Ergebnisse hat auch gezeigt, dass in punkto Konzeptualisierung der deutschen Sprache unter LehrerInnen andere Faktoren maßgeblich sind als bei den SchülerInnen. Als roter Faden hat sich durch die Konzeptualisierungsergebnisse gezogen, dass bei den Lehrkräften vor allem die Schulform respektive die Ausbildungsinstitution den konzeptionellen Blick auf das Deutsche signifikant beeinflusst - an den Universitäten ausgebildete Lehrpersonen tendierten stärker zu plurizentrischen Einstellungen.

Bei den SchülerInnen wurde je nach regionaler Herkunft die deutsche Sprache unterschiedlich konzeptualisiert. Signifikante regionale Unterschiede, vor allem zwischen östlichen und westlichen Bundesländern, zeigten sich bei den SchülerInnen darüber hinaus vom TV-Konsum in der Kindheit über die ÖWB-Verwendung bis hin zu den Einstellungen gegenüber den Standardvarietäten des Deutschen immer wieder, wobei Vorarlberger SchülerInnen häufig anders antworteten als Tiroler SchülerInnen.

Wie komplex die Konzeptualisierung der deutschen Sprache in Österreich ist, sieht man daran, dass unter den ProbandInnen keine Einigkeit darüber herrscht, was sie unter „österreichischem Deutsch“ verstehen. Dies ist angesichts der Tatsache, dass diese Frage weder in der LehrerInnenausbildung noch in den Schulbüchern Klärung erfährt, nicht weiter verwunderlich. Erkundigt man sich bei den LehrerInnen und SchülerInnen danach, mit welcher Varietät bzw. mit welchen Varietäten (Dialekte/Umgangssprache/standardnahe Mediensprache) sie österreichisches Deutsch primär assoziieren, so zeigen sich 
bemerkenswerte Auffassungsunterschiede zwischen den LehrerInnen- und SchülerInnen-Antworten: 70 \% der befragten Lehrkräfte verbinden mit dem österreichischem Deutsch in erster Linie die „Umgangssprache“, mit klarem Abstand folgen die standardnahe Mediensprache (47,6\%) und Dialekte (43,9\%). Auch die SchülerInnen assoziieren mit österreichischem Deutsch mehrheitlich die Umgangssprache (72,5\%). Anders als bei den LehrerInnen ist aber der Dialekt mit der Umgangssprache fast gleichauf (70,2\%), während die standardnahe Mediensprache, die eigentlich gemeinhin als typische Ausprägung des österreichischen Standarddeutsch gilt, bei den SchülerInnen eine klar untergeordnete Rolle spielt (20,5\%). Österreichisches Deutsch und die standardnahe Mediensprache bringen vor allem AHS-/Sek.-II-LehrerInnen (6o \%) bzw. Lehrkräfte, die an der Universität ausgebildet wurden $(61 \%)$, miteinander in Verbindung. Haben die PädagogInnen ihre LehrerInnenausbildung an einer PH/PädAk absolviert, tun sie das hingegen nur zu rund $33 \%$. Auffallend war auch, dass besonders VS-LehrerInnen (61\%) vorrangig „Dialekt“ mit österreichischem Deutsch assoziieren - eine Tendenz, die wir auch in der jüngsten Altersgruppe der LehrerInnen feststellen konnten: Rund 64\% der 22-32-jährigen PädagogInnen denken bei „österreichischem Deutsch“ an Dialekt.

Wie benennen die LehrerInnen und SchülerInnen eigentlich die Mehrheitssprache in Österreich? Etwa die Hälfte der LehrerInnen und SchülerInnen führten spontan „Deutsch“ an. Von knapp 20 \% der LehrerInnen wurde „österreichisches Deutsch“ genannt - darunter rund doppelt so häufig von UniversitätsabsolventInnen wie von AbgängerInnen einer PH/PädAk - und $11 \%$ der SchülerInnen. Was andere Angaben betrifft, so wurde „Dialekt“ bzw. „Mundart“ von ca. $9 \%$ der LehrerInnen und $5 \%$ der SchülerInnen angeführt. Unter der LehrerInnenschaft fällt dabei auf, dass vor allem jüngere Lehrkräfte bei dieser Frage zur Bezeichnung „Dialekt“ tendieren - von älteren LehrerInnen wurde „Dialekt“ praktisch kaum genannt. Bei den SchülerInnen findet man besonders häufig in Tirol und Vorarlberg die Bezeichnung „Dialekt“ für die Mehrheitssprache Österreichs, während SchülerInnen aus Ostösterreich mehrheitlich „Deutsch“ genannt haben.

Unsere Ergebnisse haben gezeigt, dass die Meinungen darüber, was österreichisches Deutsch ist, je nach Schultyp bzw. Ausbildungsinstitution und Alter stark variieren. Dies war auch anhand der Bezeichnungen erkennbar, mit denen die ProbandInnen die Mehrheitssprache in Österreich benannt haben. Insgesamt wurde jedoch klar, dass unsere ProbandInnen unter österreichischem Deutsch nicht - wie auf fachlicher Ebene üblich - primär die Standardsprache verstehen, sondern dass das gesamte sprachliche Varietätenspektrum Österreichs für die LehrerInnen und SchülerInnen österreichisches Deutsch ist - eine Erkenntnis, die in der Sprachwissenschaft Berücksichtigung finden sollte.

Ein weiteres Ergebnis unserer Untersuchung ist, dass sich in Österreich offensichtlich ein Sprachwandel vollzieht, der sich auf LehrerInnen und SchülerInnen auswirkt und sich etwa darin äußert, dass zunehmend mehr Deutschlandismen 
im österreichischen Deutsch Eingang finden. Markant sind die Unterschiede zwischen Jung und Alt bei der Verwendung von Austriazismen und Deutschlandismen. Ältere Lehrpersonen verwendeten eigenen Angaben zufolge in unserer (kleinen) Auswahl an Beispielen mehr Austriazismen als jüngere KollegInnen und SchülerInnen, die wiederum häufiger zur Verwendung von Deutschlandismen tendierten. Vermutlich finden sprachliche Substitutionsphänomene statt, indem manche nach dem Kodex als Austriazismen eingestufte Ausdrücke (z. B. das E-Mail, das Cola) tendenziell durch Deutschlandismen (die E-Mail, die Cola) ersetzt werden. Sowohl die ambivalente Einstellung zur Korrektheit des österreichischen Deutsch als auch die festgestellten Sprachwandeltendenzen können als Beleg dienen für die in der Literatur behauptete geringere Loyalität gegenüber der eigenen Varietät, sodass diese Annahme zum Teil bestätigt werden kann. Und auch der Widerspruch zwischen der wichtigen Bedeutung und der emotionalen Besetztheit des österreichischen Deutsch für Identitätskonstruktionen (vgl. de Cillia 1998, 2006, 2015, de Cillia/Wodak 2006) und dem ambivalenten und widersprüchlichen Umgang mit der eigenen Varietät hat sich in unseren Daten bestätigt. 


\section{Schlussbetrachtung und Ausblick}

\subsection{Zusammenfassung der Ergebnisse}

Das vorliegende Buch thematisiert die Rolle des österreichischen Deutsch als Unterrichts- und Bildungssprache im Kontext des Deutsch-als-MutterspracheUnterrichts an österreichischen Schulen sowie die Frage, welche Bedeutung dabei der Plurizentrik zukommt. Denn obwohl die deutsche Sprache bereits seit mehr als drei Jahrzehnten als plurizentrische Sprache konzeptualisiert wird und dieses Konzept im DaF-/DaZ-Unterricht mittlerweile durchaus präsent ist, gab es bisher noch keine Daten dazu, ob dies für den muttersprachlichen Deutschunterricht gleichermaßen der Fall ist.

Aus diesem Grund stand in einer ersten Projektsäule des hier dargestellten Projekts die Frage im Mittelpunkt, ob und wie in den österreichischen Lehrplänen, in den Unterrichtsmaterialien und in der DeutschlehrerInnenausbildung sprachliche Variation und dabei insbesondere standardsprachliche Variation im Deutschen behandelt wird. Da das plurizentrische Konzept im DaF-/DaZ-Bereich als etabliert gelten kann, war von Interesse, ob dies auch für den schulischen DaM-Kontext in Österreich gilt.

Weil Lehrkräfte die Spracheinstellungen ihrer SchülerInnen maßgeblich prägen, sollte in einer zweiten Projektsäule über quantitative und qualitative Befragungen eruiert werden, welche Spracheinstellungen unter österreichischen LehrerInnen und SchülerInnen vorherrschen, und wie die deutsche Sprache von SprachnormexpertInnen (den LehrerInnen) und linguistischen Laien (den SchülerInnen) konzeptualisiert wird. Ob sich die Spracheinstellungen nach außersprachlichen Faktoren wie etwa Region, Alter/Generationen oder Schultyp unterscheiden, sollte eine statistische Auswertung zeigen.

Überprüft werden sollten überdies die Annahmen aus der Literatur, dass österreichische LehrerInnen bei der Korrekturarbeit exonorm-orientiert seien und der eigenen Varietät wenig loyal gegenüberstehen würden.

In der Fachliteratur ist vielfach darauf hingewiesen worden, dass das österreichische Deutsch für die österreichische Bevölkerung identitätsstiftend ist. Sucht man jedoch im heimischen Bildungswesen nach dem Thema „österreichisches Deutsch", dann sucht man zumeist vergeblich: In den Lehrplänen und in den meisten Schulbüchern kommt die staatsspezifische standardsprachliche Variation im Deutschen praktisch nicht vor.

Unsere Dokumentenanalyse hat gezeigt, dass weder das österreichische Standarddeutsch als eigenständige Varietät des Deutschen noch allgemeine Hinweise auf standardsprachliche Variation im Deutschen in unterrichtsrelevanten Grundlagentexten, den Lehrplänen, verankert sind. Das hat zur Folge, dass sich auch 
Schulbücher nicht mit dieser Thematik beschäftigen. Nach wie vor scheint das heimische Bildungssystem von der Vorstellung geprägt zu sein, dass ein nicht näher definiertes „Hochdeutsch“ - quasi ein „Einheitsdeutsch“ - die Richtschnur für die DeutschlehrerInnen ist, obwohl in der Sprachwissenschaft niemand mehr ernsthaft daran zweifelt, dass es mehrere gleichwertige Varietäten der deutschen Sprache gibt, und demzufolge ein überregionales österreichisches Standarddeutsch existiert, das im Sinn einer plurizentrischen Auffassung dem Standarddeutsch der Bundesrepublik gleichwertig ist. Im DaF/DaZ-Unterricht ist dieses Konzept mittlerweile in die Lehrmittel-Gestaltung, die $\mathrm{DaF} / \mathrm{DaZ}$-LehrerInnenausbildung und die internationale Testentwicklung eingeflossen. Umso mehr verwundert es, dass der DaM-Bereich unseren Projektergebnissen zufolge davon bisher fast gänzlich unberührt geblieben ist. Dass sich die Plurizentrik auch in den ausschlaggebenden Grundlagentexten, wie die Lehr- und Studienpläne, nicht abbildet, ist im normativ orientierten Kontext der Schule als problematisch zu sehen.

Die Bildungsautoritäten verkennen, dass es weitreichende Konsequenzen hat, wenn die Varietäten-Thematik in den Lehrplänen und in der LehrerInnenausbildung fehlt. Eine Zahl aus unserer Fragebogenerhebung verdeutlicht, wie groß das Manko ist: 86 Prozent der befragten Lehrkräfte sagen, dass in ihrer Ausbildung das Thema sprachliche Variation wenig bis gar nicht vorgekommen ist (siehe Kap. 5.1). Die LehrerInnenschaft steht daher im Klassenzimmer-Alltag meist ohne fundiertes Wissen da, wie man mit sprachlicher Variation im Unterricht umgehen soll. Weil sich die LehrerInnen in der Thematik unsicher sind, wird das österreichische Deutsch auch dementsprechend selten im Unterricht aufgegriffen. Am ehesten wird der Themenbereich noch von AHS-/Sek.-II-LehrerInnen (rund $30 \%$ ) behandelt. Aber für die überwiegende Mehrzahl der LehrerInnen - vor allem der anderen Schulformen - ist österreichisches Deutsch selten bis nie ein Thema in der Deutschstunde. Auch was die Verwendung von Wörterbüchern betrifft, zeigt sich unter SchülerInnen und jüngeren LehrerInnen wenig Varietätenbewusstsein. Nach unseren Daten wird nur von den älteren Lehrkräften im Österreichischen Wörterbuch nachgeschlagen und von allen anderen Befragten eher auf den Duden und Internetquellen zugegriffen als auf das Österreichische Wörterbuch, das - einst als Teil eines österreichischen Kodex gegründet und von einem staatlichen Verlag herausgegeben - mittlerweile von einer deutschen Verlagsgruppe herausgegeben wird. Es ist auch im Internet nicht ohne Weiteres zugänglich - angesichts der Nachschlagegewohnheiten vor allem der SchülerInnen ein eindeutiges Manko. Dass das Variantenwörterbuch, das eigentlich in jeder Schulbibliothek stehen sollte, überhaupt nicht bekannt ist und von keiner einzigen Lehrperson erwähnt wurde, ergänzt dieses Bild.

Dazu kommt, dass die hierzulande gängigen Deutsch-Lehrwerke keine Unterstützung sind, wie unsere Untersuchung ergeben hat. Das österreichische Standarddeutsch wird in den von uns im Projektzeitraum untersuchten Lehrwerkserien entweder gar nicht erwähnt, oder es wird fälschlicherweise in den 
Dunstkreis eines sprachlichen Substandards gerückt. So fehlt den Lehrkräften der fachwissenschaftliche Rückhalt, dass das österreichische Standarddeutsch gleichermaßen „hochsprachlich“ ist und dass Zweifel an seiner Korrektheit unangebracht sind. Stattdessen werden die LehrerInnen beim tagtäglichen Umgang in der Klasse mit der Variantenvielfalt im Deutschen und den damit verbundenen Österreich-Spezifika im Stich gelassen. Schon allein bei der Frage, was österreichisches Deutsch ist, gehen die Ansichten der ProbandInnen auseinander: Mit gesprochenem österreichischem Deutsch werden alle drei österreichisch geprägten Sprachvarietäten (Dialekte, Umgangssprache, mediennahe Standardsprache) assoziiert, wobei SchülerInnen mit österreichischem Deutsch vor allem Dialekt und Umgangssprache verbinden - und erst in dritter Linie Standard. Auch bei den LehrerInnen tendieren die jüngeren im Unterschied zur Gruppe der älteren LehrerInnen zu dialektalen Varietäten.

Umso mehr überrascht es, dass LehrerInnen ganz klar (und in etwas schwächerer Form auch SchülerInnen) von einer österreichischen Standardvarietät ausgehen. Auf die Frage, ob es ein eigenes österreichisches Standarddeutsch gibt, antwortete die überwältigende Mehrheit der befragten LehrerInnen (8o \%) mit Ja. Und 90 \% der Lehrkräfte sagen, dass Deutsch eine Sprache mit Unterschieden in der Standardsprache zwischen den einzelnen Ländern sei. In unseren Daten zeigt sich im Übrigen bei den (weiblichen) Schülerinnen tendenziell eine relativ stärkere Wahrnehmung der Unterschiede zwischen österreichischem und deutschem Deutsch und eine relativ größere Zustimmung zu einem eigenen österreichischen Deutsch als bei den Schülern.

Zieht man in Betracht, dass nur etwa $15 \%$ der befragten LehrerInnen je vom Begriff „Plurizentrik“ gehört haben, gibt es also in der LehrerInnenschaft ein intuitives Verständnis für das Plurizentrik-Konzept. Innerösterreichische Sprachunterschiede ändern daran nichts, sie werden im Sinn einer regionalen Plurizentrik wahrgenommen. Damit bestätigt sich im Prinzip der Befund von Schmidlin (2011, 282), wonach sich die Landesgrenze sowohl als kognitive als auch als sich daraus ergebende sprachpragmatische Grenze auswirke.

Für einen souveränen didaktischen Umgang mit Phänomenen sprachlicher Variation reicht ein vages sprachliches Bauchgefühl freilich nicht aus. Deutlich wurde die lehrerseitige Unsicherheit in Bezug auf Sprachverwendung und den Umgang mit Sprachnormen in den Schulen in den Interviews und Gruppendiskussionen, die wir im Rahmen unserer Studie durchgeführt haben. Die Methodentriangulierung hat sich dabei als hilfreich herausgestellt. Die durch Gruppendiskussionen und Interviews erhobenen qualitativen Daten ergänzten die Ergebnisse der quantitativen Befragung immer wieder um feine Nuancen, indem sie beispielsweise Hinweise auf Unsicherheiten in der Normfrage und ambivalente Einstellungen gegenüber der eigenen Varietät lieferten, die aus den quantitativen Daten so nicht ersichtlich waren. Auch die teilnehmende Beobachtung zeigte, dass die Selbsteinschätzung der LehrerInnen bezüglich ihrer Varietätenverwendung nicht 
immer dem beobachteten Sprachverhalten entspricht. In den meisten Bereichen jedoch bestätigten die qualitativen Daten die Ergebnisse der quantitativen Erhebung und der Dokumentenanalyse. Viele LehrerInnen haben in den Interviews und Gruppendiskussionen berichtet, dass sie sich schwertun mit der Entscheidung, welche Austriazismen als standardsprachlich gelten, welche als umgangssprachlich einzuordnen sind - und welche als dialektal zu bewerten sind. Der Spagat zwischen dem eigenen Sprachempfinden und der diffusen, schwer greifbaren Norm verunsichert offenbar viele DeutschlehrerInnen. Der Rückgriff auf Nachschlagwerke bietet den LehrerInnen allerdings keine allzu große Hilfe. Denn die Kodifizierung des österreichischen Deutsch hat sich im Zuge unseres Projekts als lückenhaft erwiesen, was Befunde aus der Literatur bestätigt, wonach der österreichische Standard relativ wenig kodifiziert ist (z. B. Ender/Kaiser 2009, 268).

Dazu kommt, dass in der LehrerInnenschaft latente sprachliche Minderwertigkeitsgefühle gegenüber dem deutschen Standarddeutsch vorhanden sind, die unterschwellig zu widersprüchlichen Einstellungen führen: Einerseits ist es so, dass die meisten LehrerInnen in unserem Sample das österreichische Deutsch positiv bewerten und ihm große Bedeutung für die österreichische Identität beimessen. Auf die Frage, ob es ein österreichisches Standarddeutsch gebe, antworten $80 \%$ der LehrerInnen und knapp 60 \% der SchülerInnen mit ja. Ähnlich positiv sind die Reaktionen, wenn direkt nach der Korrektheit des österreichischen Deutsch gefragt wird: $86 \%$ der LehrerInnen und $68 \%$ der SchülerInnen halten das Standarddeutsch, das in Österreich verwendet wird, für gleichermaßen korrekt wie das Standarddeutsch, das in Deutschland verwendet wird. Wird jedoch differenzierter nachgefragt, dann bleibt dem österreichischen Deutsch die volle Anerkennung versagt. Nur $44 \%$ der LehrerInnen und $32 \%$ der SchülerInnen lehnen die Behauptung ab, dass das deutsche Deutsch korrekter wäre als österreichisches Deutsch. Demnach wird im Zweifelsfall das deutsche Standarddeutsch von vielen PädagogInnen doch als „richtiger“ empfunden.

Diese Ambivalenz äußert sich unserer Untersuchung zufolge auch beim Korrekturverhalten der LehrerInnen. Ein konstruierter Aufsatz, den die LehrerInnen zu korrigieren hatten und der eine Reihe an ausgewiesenen Austriazismen und Deutschlandismen enthielt, sollte Erkenntnisse über das Korrekturverhalten und die Normakzeptanz der DeutschlehrerInnen liefern. Grundsätzlich hat sich dabei gezeigt, dass österreichische LehrerInnen nicht so stark exonorm-orientiert korrigieren, wie dies aus den bisherigen Pilotstudien zu vermuten gewesen wäre, aber dass doch ein Teil der LehrerInnen dazu tendiert, Austriazismen als Fehler zu bewerten.

Unsere Untersuchung hat auch ergeben, dass LehrerInnen verschiedener Schulformen mit standardsprachlichen Varianten unterschiedlich umgehen: HS-/ NMS-LehrerInnen etwa strichen mit Abstand doppelt so häufig wie alle anderen LehrerInnen Austriazismen als "falsch“ an. Große Unterschiede kamen auch zwischen älteren und jüngeren LehrerInnen zum Vorschein: Je älter LehrerInnen 
sind, umso eher versehen sie Deutschlandismen mit Wellenlinien, während junge LehrerInnen Deutschlandismen weit weniger stören. Gleichzeitig werten JunglehrerInnen aber Austriazismen öfter als Fehler als ihre älteren KollegInnen. Hier spielt der schon erwähnte Umstand hinein, dass jüngere LehrerInnen unseren Daten zufolge unter dem österreichischen Deutsch eher Dialekt als Standard verstehen und ausgewiesene Austriazismen im Zweifelsfall für nicht normkonform halten. SchülerInnen assoziieren - ähnlich wie die jüngeren Lehrkräfte - am stärksten den Dialekt mit österreichischem Deutsch. Aus Sicht der älteren LehrerInnen steht hingegen in erster Linie die Umgangssprache für österreichisches Deutsch. Nach Ausbildungsinstitutionen betrachtet deuten die Ergebnisse darauf hin, dass die Universitäten stärker als die Pädagogischen Hochschulen am Standard orientiert sind: Mehr als $60 \%$ der LehrerInnen, die ihre Ausbildung an der Universität gemacht haben, verbinden die standardnahe Mediensprache mit österreichischem Deutsch, während nur knapp ein Drittel der VS- und HS/NMS-LehrerInnen die Standardsprache mit österreichischem Deutsch in Zusammenhang bringt.

Hinsichtlich des Alters verhält es sich ähnlich, wenn wir die Angaben zur Verwendung von Austriazismen und Deutschlandismen in den Blick nehmen. Zusätzlich haben sich auch innerösterreichische regionale Unterschiede bei der Sprachverwendung unter LehrerInnen und SchülerInnen gezeigt. Zum Abtesten von 26 Wortpaaren, die jeweils aus Doubletten in etwa gleichbedeutender Austriazismen und Deutschlandismen bestanden, wurde bewusst eine breite Auswahl an Beispielen getroffen, um feststellen zu können, wo tendenziell Sprachwandel stattfindet. Zwar können aufgrund des relativ kleinen Samples der 26 Doubletten keine allgemeingültigen Aussagen getroffen werden - dafür bräuchte es ein wesentlich umfassenderes Sample an Varianten. Es können aus den vorliegenden Daten aber sehr wohl Hinweise auf derzeit stattfindende Sprachentwicklungen in Österreich abgeleitet werden. Deutlich wurde, dass etliche Austriazismen durchaus stabil sind und keinerlei Sprachwandel unterliegen dürften, wie etwa Jänner, Schweinsbraten oder ich bin gestanden. Bei einigen anderen Beispielen konnten wir eine starke Tendenz hin zu den deutschländischen Varianten feststellen (die SMS statt das SMS, Pickel statt Wimmerl, die E-Mail statt das E-Mail).

Interessant ist dabei die generationsspezifische Sprachverwendung, die sich in unseren Daten andeutet. ${ }^{1}$ Die SchülerInnen stehen insgesamt Deutschlandismen offener gegenüber als ihre LehrerInnen: Sie verwenden beispielsweise nach eigenen Angaben häufiger „der Junge“ statt „der Bub“ oder „eine Cola“ statt „ein Cola“. Hier zeigte sich ein signifikanter Zusammenhang zwischen dem TV-Konsum von bundesdeutschen TV-Kanälen und der vermehrten Verwendung von Deutschlandismen. Aber auch innerhalb der LehrerInnenschaft gibt es in unseren Daten zwischen Jung und Alt deutliche Unterschiede bei der Sprachverwendung. Grundsätzlich überwiegt zwar die Loyalität gegenüber österreichischen Varianten.

1 Zur Altersvariable vgl. auch Pfrehm 2011a, de Cillia 2016a. 
Je jünger LehrerInnen jedoch sind, desto weniger geben sie im Vergleich zu älteren KollegInnen an, Austriazismen zu verwenden. Jüngere LehrerInnen akzeptieren Deutschlandismen eher bzw. integrieren diese in den eigenen Sprachgebrauch. Die Variantenloyalität unter den LehrerInnen ist somit altersabhängig. Dies kann als Indiz für einen generationsspezifischen Sprachwandel gedeutet werden, der derzeit stattfindet. Auch die jeweilige Region spielt bei den Angaben zur Variantenverwendung eine Rolle. Generell gilt für LehrerInnen und vor allem für SchülerInnen unabhängig vom Alter: Je weiter man in Österreich nach Westen geht, umso häufiger werden den Angaben der ProbandInnen zufolge auch Deutschlandismen verwendet. Und es zeigt sich in unseren Daten, dass in den Einstellungen innerösterreichische Unterschiede im Sinn einer regionalen Plurizentrik oder eines pluriarealen Ansatzes vorhanden sind: So verhalten sich bei den Einstellungen die fünf von uns angenommen Regionen Ost (Wien, Burgenland, Niederösterreich), Südost (Steiermark, Kärnten), Mitte (Oberösterreich, Salzburg), Tirol und Vorarlberg manchmal unterschiedlich. Dabei wiesen vor allem die Regionen Ost und West bzw. Vorarlberg signifikante Unterschiede auf, und es war wichtig, die in der Lexikographie meist als nur eine angenommene Region „Westösterreich“ in zwei Regionen, nämlich in die Regionen Tirol und Vorarlberg, zu unterteilen. Schließlich zeigte sich bei einer Befragung zu einer Reihe von Varianten und deren relativen Verwendung, dass der Prozentsatz von deren Verwendung auch zwischen LehrerInnen und SchülerInnen differiert. Was diese Relativität von Varianten betrifft, d.h. in welchem Ausmaß alternative Varianten verwendet werden, so müsste diese relative Variantenverwendung unseres Erachtens im plurizentrischen Zugang empirisch stärker berücksichtigt und differenzierter beschrieben werden. Ein Teil der präsumptiven Austriazismen schließlich dürfte zunehmend von Deutschlandismen abgelöst werden - ein Hinweis auf den schon angesprochenen, vom Medienverhalten beeinflussten altersspezifischen Sprachwandel.

Die parallele Verwendung von standardsprachlichen Austriazismen und Deutschlandismen ist aber nur eine Facette im Sprachenalltag in Österreichs Klassenzimmern. Sprachlich komplex ist die Situation dadurch, dass Dialekt und Umgangssprache sehr präsent sind und große Teile der Unterrichtskommunikation im Zeichen des Dialekt-Standard-Kontinuums stehen und ein ständiger Wechsel zwischen den Varietäten zu beobachten ist. Innere Mehrsprachigkeit ist im Unterricht Realität. LehrerInnen dürften - wie die teilnehmenden Unterrichtsbeobachtungen gezeigt haben - öfter zwischen Standard, Umgangssprache und Dialekt switchen, als ihnen bewusst ist. Müssen LehrerInnen schimpfen, tun sie das nicht im „Schönbrunner Deutsch“, sondern in einer umgangssprachlich oder dialektal gefärbten Diktion. Unsere Interviews und Gruppendiskussionen haben auch zu Tage gebracht, dass LehrerInnen nicht-standardsprachliche Äußerungen fast mit schlechtem Gewissen tätigen. Mehrfach haben die befragten PädagogInnen uns ihr „Bemühen“ geschildert, dass sie „schon versuchen“, Standardsprache zu sprechen und nach einer Nonstandard-Äußerung wieder zu dieser zurückzukehren. 


\subsection{Schlussfolgerungen und Empfehlungen}

Wir sind im vorliegenden Projekt von einem plurizentrischen Ansatz der sprachlichen Variation des Deutschen ausgegangen, der einerseits annimmt, dass staatliche Grenzen für sprachliche Variation und Spracheinstellungen eine wichtige Rolle spielen, andererseits aber auch Staatsgrenzen übergreifende und innerstaatliche Variation berücksichtigt. Was die Analyse von für den Unterricht an den Schulen zentralen Dokumenten und Texten betrifft (Lehrpläne, Studienpläne, DeutschLehrbücher), so zeigte sich, dass - entgegen der Wichtigkeit des österreichischen Deutsch im politischen und medialen Diskurs (z. B. EU-Beitritt, „Marmeladekrieg“ vgl. de Cillia 2006) - dem Konzept einer staatsbezogenen sprachlichen Variation in diesen Dokumenten keinerlei Bedeutung zukommt. Und obwohl dieser Zugang LehrerInnen und SchülerInnen kaum als wissenschaftliches Konzept bekannt ist, ist in den Spracheinstellungen, die mit unterschiedlichen Methoden der Befragung erhoben wurden, ein Bewusstsein von einem eigenen österreichischen Deutsch und eine intuitive plurizentrische Einstellung vorhanden. Dabei wird nicht in erster Linie und v.a. nicht ausschließlich die Standardvarietät mit österreichischem Deutsch assoziiert, sondern es sind eher dialektale und umgangssprachliche Varietäten. Letztlich umfasst für die ProbandInnen österreichisches Deutsch das gesamte österreichische Dialekt-Standard-Kontinuum.

Deutlich wurde auch, dass LehrerInnen als normsetzende Instanzen in der Zwickmühle stecken, weil sie mit Normfragen umgehen müssen, bei denen sie selber unsicher sind, die in ihrer Ausbildung nicht oder kaum thematisiert wurden und die bisher auch wissenschaftlich nicht zufriedenstellend beantwortet worden sind. Dies ist umso problematischer, als man von LehrerInnen nicht erwarten kann, dass sie das österreichische Deutsch im Unterricht einbeziehen, wenn sie kaum Hilfsmittel zur Hand haben oder diese nicht kennen. All das führt dazu, dass die LehrerInnen selbst nicht so recht wissen, was österreichisches Deutsch ist und was standardsprachliche plurizentrische Variation ausmacht.

Dazu kommt, dass die Grenzen zwischen den Varietäten, insbesondere zwischen der Standardsprache und der Umgangssprache, bekanntlich notorisch unscharf sind. Eine Folge davon ist, dass LehrerInnen in Zweifelsfällen - und umso mehr, wenn der Kodex mangelhaft ist - exonorm-orientiert korrigieren. Unsere Daten haben zwar die in Pilotstudien behauptete Exonorm-Orientiertheit nicht so stark bestätigt, aber die lehrerseitige Normverunsicherung war augenfällig. Gerade diese Entscheidungssicherheit angesichts der sprachlichen Vielfalt im Deutschen ist eine Kernkompetenz für DeutschlehrerInnen, denn - so die Deutschdidaktiker Steinig/Huneke - „ein Deutschlehrer muss fähig sein, nachvollziehbare Begründungen für die Bewertung mündlicher und schriftlicher Äußerungen seiner Schüler geben zu können“ (Steinig/Huneke 2015, 18).

Aufgrund der ungenügenden Berücksichtigung dieses Bereichs in der Ausbildung bleiben sprachliche Vorbehalte und Unsicherheiten erhalten. Das von 
manchen LehrerInnen empfundene Prestigedefizit des österreichischen Deutsch wird wohl in vielen Fällen unbewusst an die Lernenden weitergegeben, sodass SchülerInnen aufgrund variierender Zielsprache-Standards keine klare Linie im Unterricht erfahren - weder von den DeutschlehrerInnen noch aus dem Deutschbuch. Hier besteht Handlungsbedarf. Sowohl bei den Begrifflichkeiten als auch bei der Einordnung von Sprachvarietäten sollten in der LehrerInnenausbildung und auch in den Studienplänen von Anfang an die nötigen Leitlinien eingezogen werden. Dies wird mittlerweile bereits von verschiedenen LinguistInnen und DeutschdidaktikerInnen im deutschsprachigen Raum gefordert. So auch von Christa Dürscheid: „Es kann Gründe dafür geben, dass der plurizentrische Ansatz im Deutschunterricht nicht thematisiert wird. Es darf aber keine Gründe dafür geben, dass der plurizentrische Ansatz in der LehrerInnenausbildung nicht seinen festen Platz hat." (Dürscheid 2009, 68, zit. nach Kellermeier-Rehbein 2014, 219). In dieselbe Kerbe schlagen Schmidlin/Wyss/Davies (2017, 18), wenn sie betonen, „dass diese Problematik [der Varietäten, die Verf.] es verdient, in der DeutschlehrerInnenausbildung und im Deutschunterricht auf allen Stufen regelmäßiger thematisiert zu werden". Dass es eine Reihe an Legitimationsargumenten für das Einbeziehen von Variation im Sprachunterricht gibt, betont auch die Deutschdidaktikerin Eva Neuland. Ihr zufolge bietet sowohl der Themenschwerpunkt der Sprachnormen als auch „der Themenschwerpunkt der Sprachvariationen Anknüpfungspunkte nicht nur für den Lernbereich ,Reflexion über Sprache', sondern für alle grundlegenden Lernbereiche des Sprachunterrichts" (Neuland 2013, 211).

Unsere Befunde zeigen, dass - so wie im außerschulischen Alltag in Österreich - auch in der Schule und im Klassenzimmeralltag ein Dialekt-StandardKontinuum mit vielen Schattierungen existiert. Ein nicht näher definierter Standard wird dabei als bildungssprachliche Zielnorm angenommen. Unseres Erachtens sollte das gesamte Varietätenspektrum des Deutschen in Österreich auch in den Schulen verwendet werden (dürfen), auch von Lehrpersonen, ohne dabei ein schlechtes Gewissen haben zu müssen, und ein situations-, themen-, adressatenspezifischer Umgang mit Sprachnormen (vgl. Kap. 2.6) praktiziert werden. So ein reflektierter Umgang mit Varietäten und situativen Normen im Unterricht wäre auch wichtig, damit die SchülerInnen Sicherheit in der Verwendung der Standardsprache erlangen, was für ihr späteres Berufsleben im In- und Ausland wichtig wäre. Erschwerend kommt hinzu, dass vielfach noch keine Konzepte eines situationsadäquaten lehrerseitigen Sprachgebrauchs in die Deutsch-Fachdidaktik Einzug gehalten haben. Die pädagogisch sinnvolle Handhabung des Dialekt-Standard-Kontinuums bräuchte aber gerade in Österreich einen festen Platz in der Fachdidaktik-Ausbildung für DeutschlehrerInnen.

Im Rahmen unserer Untersuchung haben wir darüber hinaus festgestellt, dass eine begriffliche Unschärfe in LehrerInnenkreisen für Verwirrung sorgt: Auf ExpertInnenebene ist mit dem Terminus „österreichisches Deutsch“ in der Regel nur die Standardebene gemeint. LehrerInnen und SchülerInnen verstehen 
jedoch unter „österreichischem Deutsch“ nicht nur österreichisches Standarddeutsch, sondern vielmehr das gesamte Deutsch in Österreich - sprich das ganze Sprachspektrum von den in Österreich gesprochenen Dialekten über die Umgangssprache bis hin zur Standardvarietät.

Diese Definitionsproblematik führt zu unnötigen Auffassungsunterschieden darüber, was österreichisches Deutsch ist, und zu Missverständnissen zwischen SprachwissenschaftlerInnen und Nicht-SprachwissenschaftlerInnen, die unseres Erachtens dem österreichischen Deutsch als Unterrichts- und Bildungssprache abträglich sind. Durch das unterschiedliche Verständnis von Bezeichnungen verkompliziert sich das Bild davon, was österreichisches Deutsch ist, und bleibt gleichzeitig verschwommen. Uns scheint es daher sinnvoll, diese Begrifflichkeiten zu entflechten und im pädagogischen Diskurs klar zu definieren. Eine Möglichkeit wäre, vom „österreichischen Standarddeutsch“ zu sprechen, wenn die Standardebene gemeint ist, während „österreichisches Deutsch“ als Oberbegriff für alle österreichisch geprägten Sprachformen dienen könnte ${ }^{2}$. In Analogie dazu erscheint es naheliegend, auch von deutschem Standarddeutsch und vom Schweizer Standarddeutsch zu sprechen, wenn die Standardebene angesprochen wird.

Die Daten der vorliegenden Studie sind im Wesentlichen (mit Ausnahme der Dokumentenanalyse und der teilnehmenden Beobachtung) Selbstauskünfte der ProbandInnen und natürlich nicht unbedingt mit dem tatsächlichen Sprachverhalten gleichzusetzen. Was unter „Dialekt", „Umgangssprache“, „Standardsprache“ verstanden wird, variiert vermutlich von Person zu Person. Hier wären dringend empirische Erhebungen zur Sprachverwendung und zum tatsächlichen Sprachverhalten im Klassenzimmer und im Raum Schule mit systematischer teilnehmender Beobachtung notwendig (vgl. Steiner 2008 für die Schweiz; Einsatz von Videographie und Transkription der Unterrichtseinheiten, Methoden wie linguistic landscape etc.), um Aussagen treffen zu können, die über die Selbsteinschätzung der SprecherInnen hinausgehen. Und vertiefende empirische Untersuchungen zu arealen Unterschieden innerhalb Österreichs, v. a. Ost-West-Unterschiede, wären nach unseren Befunden ebenso wichtig.

Abschließend sei noch einmal festgestellt, dass in den handlungsleitenden Texten für den Deutschunterricht weder die standardsprachliche Variation im Deutschen noch der Dialekt und die Umgangssprache fundiert thematisiert werden. Da die Vermittlung eines differenzierten Sprachbewusstseins nicht allein über die Lehrkräfte laufen kann - weil angehende DeutschlehrerInnen wegen der fehlenden Stringenz im Lehrveranstaltungsangebot mit dieser Thematik während des Studiums nicht notwendigerweise in Berührung kommen -, ist es unerlässlich, dass Varietäten- und Konzeptualisierungsfragen in den unterrichtsrelevanten Basisdokumenten (Lehrpläne, Studienpläne), in der LehrerInnenausbildung und in den approbierten Unterrichtsmaterialien fachgerecht und explizit verankert werden.

2 Das was beispielsweise bei Ebner (2009, 442) „Deutsch in Österreich“ genannt wird. 
Dazu bedarf es einer Überarbeitung der Schulbücher, indem sachlich problematische Darstellungen und fachlich unscharfe Begriffsverwendungen modifiziert werden. Es sollten auch jene Kapitel bearbeitet werden, die der Sprachreflexion gewidmet sind, aber bislang die nötigen Ausführungen zu sprachlicher Variation vermissen lassen. Die vielen Beispiele für sprachliche Variation, die in den Schulbuchtexten bereits vorkommen, könnte man ohne allzu großen Aufwand didaktisch nutzen und - so wie es in der Fremdsprachendidaktik in manchen Englisch-Lehrwerken oder auch in DaF-Lehrwerken längst üblich ist - mit staatsspezifischen Varianten oder weiterführenden Texthinweisen versehen, die den Lernenden mehr Orientierung und eine klare Verortung im deutschen Sprachraum bieten. Das Sichtbarmachen plurizentrischer Variation birgt einen pädagogischen Mehrwert für die Lernenden und wäre für Lehrkräfte mit einfachen Mitteln umsetzbar, meint auch Dürscheid, die die Lektüre von Zeitungsartikeln aus Deutschland, Österreich und der Schweiz vorschlägt, in denen Unterschiede zwischen den Standardvarietäten thematisiert werden (Dürscheid 2009, 66f). Denn SchülerInnen können, betont etwa auch Kellermeier-Rehbein (2014, 210 f), „anhand der Plurizentrik Kenntnisse über die standardsprachliche Variation des Deutschen gewinnen und über sprachliche Normen sowie über eigenen und fremden Sprachgebrauch nachdenken". Und es sei sinnvoll, mit den SchülerInnen zunächst die Existenz mehrerer Nationalvarietäten zu erarbeiten und sie dafür zu sensibilisieren, was mit der Entwicklung von sprachlicher Toleranz und Respekt gegenüber dem fremdnationalen Sprachgebrauch einhergehen sollte.

Damit im Zusammenhang ist die Aus- und Weiterbildung für LehrerInnen in die Pflicht zu nehmen. Angehende Lehrkräfte brauchen nicht nur Fachwissen zum Umgang mit Dialekt, Umgangssprache und Standard im Deutschunterricht. LehrerInnen brauchen auch ein souveränes Auftreten in der Standardsprache, wenn sprachliche Variation zur Debatte steht. Dies kann durch einschlägige Pflichtlehrveranstaltungen zum Thema "Sprachliche Variation und Deutschunterricht" gewährleistet werden. Und da der Großteil der jetzt unterrichtenden Lehrpersonen in ihrer Ausbildung damit nicht in Berührung gekommen ist, ist natürlich auch die Weiterbildung gefordert. Veranstaltungen wie die vom österreichischen Bildungsministerium zum Thema „Welches Deutsch an Schulen. Österreichisches Deutsch und Plurizentrik“ (März 2014) oder „Österreichisches Deutsch im Bildungskontext“ (Oktober 2016) sollten verstärkt angeboten werden, um diese Lücke in der Ausbildung zu schließen. ${ }^{3}$

Ein Ziel des schulischen Deutschunterrichts sollte unseres Erachtens der reflektierte Umgang mit sprachlichen Unterschieden sein sowie die Schärfung der Wahrnehmung auf SchülerInnenseite, was den kompetenten Umgang mit

3 An der Ausarbeitung und Durchführung war das Projektteam beteiligt. Auch die vom Bildungsministerium herausgegebene Broschüre „(Österreichisches) Deutsch als Unterrichts- und Bildungssprache" (BMBF 2014a) ist in diesem Kontext entwickelt worden. 
sprachlichen Varietäten ausmacht. Dafür braucht es Lehrkräfte und Unterrichtsmaterialien, die der Thematik gerecht werden können. Heimische Lehrwerke und Lehrpläne sollten darauf abzielen, dass die Gleichwertigkeit der Varietäten der deutschen Sprache, wie sie etwa das plurizentrische Konzept beschreibt, umfassend verankert und nicht halbherzig angedeutet wird. Das würde den LehrerInnen und vor allem den SchülerInnen dabei helfen, ihre innere Mehrsprachigkeit im Rahmen des österreichischen Deutsch mit dem nötigen Selbstbewusstsein und der situativ gebotenen sprachlichen Sicherheit zu leben. 


\section{Anhang}

Im Rahmen dieses Projekts analysierte Lehrpläne für das Fach Deutsch an österreichischen Schulen

- Volksschule (Stand: Juni 2003): (Zugriff 3. 6. 2016): https://www.bmbf.gv.at/ schulen/unterricht/lp/hs22_886.pdf?4dzgm2

- Hauptschule, AHS Sek. I (Stand: Juli 2010) und Neue Mittelschule (Stand: Mai 2012) (Zugriff 3. 6. 2016): https://www.bmbf.gv.at/schulen/unterricht/lp/ ahs7_781.pdf?4dzgm2

- AHS Sek. II, AHS Sek. II Wahlpflichtfach Deutsch und AHS Sek. II DaZ (Stand: 2008) (Zugriff 3. 6. 2016): https://www.bmbf.gv.at/schulen/unterricht/ lp/lp_neu_ahs_23_11878.pdf?4dzgm2

- https://www.bmbf.gv.at/schulen/unterricht/lp/lp_neu_ahs_23_11878.pdf?4dz gm2

- https://www.bmbf.gv.at/schulen/unterricht/lp/deutschalszweitsprache_13838. pdf? 4 dzgm2

- BAKIP (Stand: August 2009)

- Bildungsplan-Anteil Sprache (Stand: Juni 2009) (Zugriff 3. 6. 2016): http:// www.sprich-mit-mir.at/app/webroot/files/file/bildungsplan.pdf

Im Rahmen dieses Projekts analysierte Studienpläne österreichischer Pädagogischer Hochschulen für das Fach Deutsch als Muttersprache

PH Wien
KPH Wien/Krems
PH Oberösterreich
PH Steiermark
PH Kärnten
PH Salzburg
PH Tirol
PH Wien
KPH Wien/Krems
PH Oberösterreich
PH Steiermark
PH Kärnten
PH Salzburg
PH Tirol

Studienplan für Volksschulen

Studienplan für Volksschulen

Studienplan für Volksschulen

Studienplan für Volksschulen

Studienplan für Volksschulen

Studienplan für Volksschulen

Studienplan für Volksschulen

Studienplan für Hauptschulen

Studienplan für Hauptschulen

Studienplan für Hauptschulen

Studienplan für Hauptschulen

Studienplan für Hauptschulen

Studienplan für Hauptschulen

Studienplan für Hauptschulen

$2008 / 09$
2008
2009
2011
2012
2009
2010
2009
2008
2009
2012
2012
2009
2010


Im Rahmen dieses Projekts analysierte Studienpläne österreichischer Universitäten für den Gegenstand Deutsch

- Wien (Studienplan 2002)

- Graz (Studienplan 2011)

- Innsbruck (Studienplan 2001)

- Salzburg (Studienplan 2011)

- Klagenfurt (Studienplan 2011)

Im Rahmen dieses Projekts analysierte Lehrwerke

Volksschule:

- Funkelsteine 4. Schulstufe von Gerlinde Fürnstahl, Verlag E. DORNER: Sprachbuch (11. Aufl. 2012), Arbeitsblätter (11. Aufl. 2011), Serviceteil (4. Aufl. 2012)

- Sprachlichter 4. Schulstufe von Christa Blumenschein, Veritas Verlag: Teil 1, Teil 2, Serviceteil (2. Aufl. 2012)

- Lilos 4. Schulstufe von Herbert Puchta und Renate Welsh, Helbing Verlag: Sprachbuch (4. Aufl. 2011), Übungsheft (3. Aufl. 2007), Lesewelt (2. Aufl. 2006), Serviceteil

Sekundarstufe I:

- Treffpunkt Deutsch 8. Schulstufe von Ursula Figl, Gudrun Natter und Stefan Schäfer, öbv: Sprachbuch, Arbeitsheft, Leseheft, Serviceteil (1. Aufl. 2007)

- Deutschstunde 8. Schulstufe von Wolfgang Pramper, Helmut Hammerschmid, Stefan Hochwind, Elisabeth Nömair, Veritas Verlag: Basisteil Standard, Basisteil Plus, Serviceteil (3. Aufl. 2012)

- ganz klar: Deutsch 8. Schulstufe von Wolfgang Gruber und Gertraud Hilger, Jugend und Volk Verlag: Sprachbuch (2007), Sprachbuch leicht, Fit und kompetent Beiheft (2007), Fit und kompetent Beiheft leicht, Übungsbuch A (2007) und B, Serviceteil

Sekundarstufe II:

- Aktion Sprache 11./12. Schulstufe von Eva und Gerald Rainer, Veritas Verlag: Sprachbuch 3-4 (5. Aufl. 2012), Serviceteil (3. Aufl. 2011)

- Das Sprachbuch 11./12. Schulstufe von Christian und Ulrike Schacherreiter, Veritas Verlag: Sprachbuch 3 bis zur Matura (2. Aufl. 2012), Serviceteil (1. Aufl. 2011)

- klar_Deutsch 11./12. Schulstufe von Gertraud Hilger und Maria Kiener, Jugend und Volk Verlag: Sprachbuch 7/8 (2011), Serviceteil (2011) 


\section{Schulstandorte der Fragebogenerhebungen}

\begin{tabular}{|c|c|c|}
\hline & SchülerInnen & LehrerInnen \\
\hline Burgenland & $\begin{array}{l}\text { BG/BRG/BORG Oberpullen- } \\
\text { dorf Franz Liszt } \\
\text { BG/BRG/BORG Oberschützen } \\
\text { Gymnasium der Diözese } \\
\text { Eisenstadt Wolfgarten }\end{array}$ & $\begin{array}{l}\text { BG/BRG/BORG Oberpullendorf Franz Liszt } \\
\text { BG/BRG/BORG Oberschützen } \\
\text { Gymnasium der Diözese Eisenstadt } \\
\text { Wolfgarten } \\
\text { NMS Groß-Warasdorf } \\
\text { VS Breitenbrunn am Neusiedlersee } \\
\text { VS Bahnstraße Eisenstadt } \\
\text { VS St. Georgen Eisenstadt }\end{array}$ \\
\hline Kärnten & $\begin{array}{l}\text { BG/BRG Mössingerstraße } \\
\text { Klagenfurt } \\
\text { BG/BRG für Slowenen } \\
\text { Klagenfurt } \\
\text { BG/BRG Feldkirchen }\end{array}$ & $\begin{array}{l}\text { BG/BRG Mössingerstraße Klagenfurt } \\
\text { BG/BRG für Slowenen Klagenfurt } \\
\text { BG/BRG Feldkirchen } \\
\text { VS Dr. Karl Renner Schule Klagenfurt } \\
\text { VS Richard-Wagnerstraße Klagenfurt } \\
\text { VS Schachterlweg Klagenfurt }\end{array}$ \\
\hline $\begin{array}{l}\text { Niederöster- } \\
\text { reich }\end{array}$ & $\begin{array}{l}\text { BG/BRG Piaristen Krems } \\
\text { BG/BRG Stockerau } \\
\text { BG Waidhofen an der Thaya } \\
\text { BORG an der Theresianischen } \\
\text { Militärakademie Wiener } \\
\text { Neustadt }\end{array}$ & $\begin{array}{l}\text { BG/BRG Piaristen Krems } \\
\text { BG/BRG Stockerau } \\
\text { BG Waidhofen an der Thaya } \\
\text { BORG an der Theresianischen Militär- } \\
\text { akademie Wiener Neustadt } \\
\text { VS Langenzersdorf } \\
\text { NMS Langenzersdorf } \\
\text { VS Groß-Enzersdorf } \\
\text { BG Stiftsgymnasium Sachsenbrunn }\end{array}$ \\
\hline $\begin{array}{l}\text { Oberöster- } \\
\text { reich }\end{array}$ & $\begin{array}{l}\text { BG/BRG Freistadt } \\
\text { BG/BRG Körnerstraße Linz } \\
\text { BG/BRG Rohrbach }\end{array}$ & $\begin{array}{l}\text { BG/BRG Freistadt } \\
\text { BG/BRG Körnerstraße Linz } \\
\text { BG/BRG Rohrbach } \\
\text { NMS Rainbach } \\
\text { NMS Rohrbach } \\
\text { VS Magdalenaschule Linz } \\
\text { VS Rainbach } \\
\text { VS Rohrbach }\end{array}$ \\
\hline Salzburg & $\begin{array}{l}\text { BG Nonntal Salzburg } \\
\text { BG Tamsweg }\end{array}$ & $\begin{array}{l}\text { BG Nonntal Salzburg } \\
\text { BG Tamsweg } \\
\text { VS Mauterndorf } \\
\text { VS Herrnau Salzburg } \\
\text { VS Leopoldskron-Moos Salzburg }\end{array}$ \\
\hline Steiermark & $\begin{array}{l}\text { Akademisches Gymnasium } \\
\text { Graz } \\
\text { Abteigymnasium Seckau } \\
\text { BG/BRG Weiz }\end{array}$ & $\begin{array}{l}\text { Akademisches Gymnasium Graz } \\
\text { Abteigymnasium Seckau } \\
\text { BG/BRG Weiz } \\
\text { Praxisvolksschule der PH Steiermark }\end{array}$ \\
\hline
\end{tabular}


Tirol
Abendgymnasium Innsbruck BG/BORG St. Johann/Tirol BORG Innsbruck

Tourismusschulen Villa Blanka Innsbruck
Abendgymnasium Innsbruck

BG/BORG St. Johann/Tirol BORG Innsbruck

Tourismusschulen Villa Blanka Innsbruck VS Fischer Straße Innsbruck VS Mariahilf Innsbruck VS Pradl-Ost Innsbruck

\begin{tabular}{lll} 
Vorarlberg & BORG Egg & BORG Egg \\
& BRG/BORG Dornbirn-Schoren & BRG/BORG Dornbirn-Schoren \\
& & VS Rieden Bregenz \\
& & VS Riedenburg Sacré Coeur Bregenz \\
\multirow{3}{*}{ Wien } & BAKIP Wien 21 (jetzt BAFEP & BAKIP Wien 21 (jetzt BAFEP Wien 21) \\
& Wien 21) & Bundesgymnasium Wien 19/Döblinger \\
Bundesgymnasium Wien 19/ & Gymnasium \\
Döblinger Gymnasium & BORG Hegelgasse14 \\
BORG Hegelgasse14 & BG/BRG Wien 3 Boerhaavegasse \\
BG/BRG Wien 3 & NMS Dietrichgasse \\
Boerhaavegasse & VS Friesgasse \\
& VS Speisinger Straße
\end{tabular}

Transkriptionskonventionen (HIAT)

\section{Pausen:}

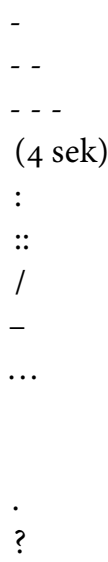

(Beispiel)

(xxx)

(lacht)

<zum Beispiel (lachend)>

VERSALIEN

Verschriftung gemäß der Aussprache der SprecherInnen:
(1 Sek.)

(2 Sek.)

(3 Sek.)

(4 Sek.)

Längung

sehr große Längung

Abbruch einer Konstruktion elliptisches Ende eines Turns

Äußerung wird abgebrochen, da anderes Ereignis eintritt oder ein/e andere/r SprecherIn mit einer neuen Äußerung beginnt fallende Satzintonation steigende Intonation mit interrogativer Bedeutung vermuteter Wortlaut unverständliches Wort Kommentar (lacht, hustet, räuspert sich etc.) Spitzklammern markieren den Bereich geänderter Äußerungsqualität Betonung

jetzt $\rightarrow$ jetz, also $\rightarrow$ oiso, gehört $\rightarrow$ ghört etc. 
$[\ldots]$

Kursivsetzungen
Auslassung von Teilen der Äußerung

dienen als Hervorhebung und verweisen auf im Textteil erwähnte inhaltliche Argumente

\section{Siglen Interviews}

Interviewer: INT, F: weibliche Sprecherin, M: männlicher Sprecher.

Wien: W, Burgenland: B, Niederösterreich: N, Oberösterreich: O, Steiermark: St, Kärnten: K, Salzburg: Sa, Tirol: T, Vorarlberg: V

Die SprecherInnen wurden nach zeitlicher Abfolge der Interviews im jeweiligen Bundesland durchnummeriert, Beispiel: FSt3: weibl. Sprecherin aus der Steiermark, 3. Interviewpartnerin in diesem Bundesland.

\section{Siglen Gruppendiskussionen}

MO: Moderator, F: weibliche Sprecherin, M: männlicher Sprecher.

Die SprecherInnen wurden nach zeitlicher Abfolge der Sprechbeiträge durchnummeriert, Beispiel: [ $\left.\mathrm{F}_{2}\right]$ : weibliche Sprecherin, meldete sich als zweite zu Wort. Die Zahlen verweisen auf die entsprechende Stelle im Transkript. Beispiel: F2, 892 = zweite Sprecherin, Fläche 892 im Transkript.

Dort, wo es für die Interpretation hlifreich schien, wurden die Flächen in den Transkripten angegeben.

Quelle: Rehbein et al. (2004): HIAT-Handbuch 


\section{Literatur}

Aichner, Herlinde/Schörkhuber, Wolfgang (2016): Kompetenz: Deutsch 7/8. Sprachbuch für allgemein bildende höhere Schulen. Wien: Hölder-PichlerTempsky Verlag. SBNr 175003.

Ammon, Ulrich (1991): Die Plurizentrizität der deutschen Sprache. In: Ekmann, Bjorn/Hauser, Hubert/Porsch, Peter/Wucherpfennig, Wolf (Hrsg.): Deutsch Eine Sprache? Wie viele Kulturen? Vorträge des Symposiums abgehalten am 12. und 13. Nov. 1990 an der Universität Kopenhagen (=Kopenhagener Kolloquien zur deutschen Literatur; 15). Kopenhagen/München: Wilhelm Fink, 14-34.

Ammon, Ulrich (1995): Die deutsche Sprache in Deutschland, Österreich und der Schweiz. Das Problem der nationalen Varietäten. Berlin u. a.: Walter de Gruyter. Ammon, Ulrich/Kellermeier, Birte (1997): Dialekt als Sprachbarriere passé? 25 Jahre danach: Versuch eines Diskussions-Erweckungsküsschens. In: Deutsche Sprache 1/1997, 21-38.

Ammon, Ulrich (1998): Plurinationalität oder Pluriarealität? Begriffliche und terminologische Präzisierungsvorschläge zur Plurizentrizität des Deutschen mit einem Ausblick auf ein Wörterbuchprojekt. In: Ernst, Peter/Patocka, Franz (Hrsg.): Deutsche Sprache in Raum und Zeit. Festschrift für Peter Wiesinger zum 6o. Geburtstag. Wien: Edition Praesens, 313-322.

Ammon, Ulrich (2000a): Sprache - Nation und die Plurinationalitat des Deutschen. In: Gardt, Andreas (Hrsg.): Nation und Sprache. Die Diskussion ihres Verhältnisses in Geschichte und Gegenwart. Berlin, New York: Walter de Gruyter, 509-524.

Ammon, Ulrich (20oob): Sprachnorm. In: Glück, Helmut (Hrsg.): Metzler Lexikon Sprache, 2. erw. Aufl. Stuttgart/Weimar: Metzler, 664-665.

Ammon, Ulrich/Bickel, Hans/Ebner, Jakob/Esterhammer, Ruth/Gasser, Markus/ Hofer, Lorenz/Kellermeier-Rehbein, Birte/Löffler, Heinrich/Mangott, Doris/ Moser, Hans/Schläpfer, Robert/Schlossmacher, Michael/Schmidlin, Regula/ Vallaster, Günter (2004): Variantenwörterbuch des Deutschen. Berlin/New York: Walter de Gruyter.

Ammon, Ulrich (2005): Standard und Variation. Norm, Autorität, Legitimation. In: Eichinger/Kallmeyer, 28-40.

Ammon, Ulrich/Dittmar, Norbert/Mattheier, Klaus J./Trudgill, Peter (2006) (Hrsg.): Sociolinguistics/Soziolinguistik. 2nd completely revised and extended edition/2., vollständig neu bearbeitete und erweiterte Auflage. Berlin, New York: Walter de Gruyter.

Ammon, Ulrich (2006): Die deutschsprachigen Länder/The German-Speaking Countries. In: Ammon/Dittmar/Mattheier/Trudgill, 3. Teilband, 1765-1772. 
Ammon, Ulrich (2008): Deutsch (German). In: Ammon, Ulrich/Haarmann, Harald (Hrsg.): Wieser Enzyklopädie. Sprachen des europäischen Westens Band I. Klagenfurt/Celovec: Wieser, 155-172.

Ammon, Ulrich/Bickel, Hans/Lenz, Alexandra Nicole (2016): Variantenwörterbuch des Deutschen. 2., völlig neu bearbeitete und erweiterte Auflage. Die Standardsprache in Österreich, der Schweiz, Deutschland, Liechtenstein, Luxemburg, Ostbelgien und Südtirol sowie Rumänien, Namibia und Mennonitensiedlungen. Berlin, Boston: Walter de Gruyter.

Atteslander, Peter (2000): Methoden der empirischen Sozialforschung. 9. neubearb. Aufl. Berlin: Walter de Gruyter.

Auer, Peter (2013): Enregistering pluricentric German. In: Augusto Soares da Silva (Hrsg.): Pluricentricity. Language Variation and Sociocognitive Dimensions. Berlin/Boston: de Gruyter - Mouton, 19-48.

Austin, John L. (1972): Zur Theorie der Sprechakte. Deutsche Bearbeitung von E. V. Savigny. Stuttgart: Suhrkamp.

Back, Otto/Fussy, Herbert/Steiner, Ulrike (Bearb.) (2012): ÖWB Österreichisches Wörterbuch. 42., neu bearb. Aufl., Wien: öbv \& hpt.

Barbour, Stephen/Stevenson, Patrick (1998): Variation im Deutschen: soziolinguistische Perspektiven. Berlin, New York: Walter de Gruyter.

Berend, Nina (2005): Regionale Gebrauchsstandards - Gibt es sie und wie kann man sie beschreiben? In: Eichinger/Kallmeyer, 143-170.

Botz, Gerhard/Sprengnagel, Gerald (1994) (Hrsg.): Kontroversen um Österreichs Zeitgeschichte. Verdrängte Vergangenheit, Österreich-Identität, Waldheim und die Historiker. Wien: campus.

Brenzinger, Mathias (1997): Language contact and language displacement. In: Coulmas, Florian (ed.): The Handbook of Sociolinguistics, Oxford: 273-284.

Bruckmüller, Ernst (1994): Österreichbewußtsein im Wandel. Identität und Selbstverständnis in den 9oer-Jahren. Wien: Signum.

Bruckmüller, Ernst (1996): Nation Österreich. Kulturelles Bewußtsein und gesellschaftspolitische Prozesse. Wien, Köln und Graz: Böhlau.

BMBF (2014a) (Hrsg.): (Österreichisches) Deutsch als Unterrichts- und Bildungssprache. Wien. https://bildung.bmbwf.gv.at/schulen/unterricht/oed.pdf?4endq2 (18. 12. 2018).

BMBF (2014b): Der muttersprachliche Unterricht in Österreich. Statistische Auswertung für das Jahr 2012/2013. Informationsblätter des Referats für Interkulturelles Lernen 5. Wien: BMBF.

BMBF (2016): SchülerInnen mit einer anderen Erstsprache als Deutsch. Statistische Übersicht Schuljahre 2008/2009 bis 2014/2015. Informationsblätter des Referats für Interkulturelles Lernen 2. Wien: BMBF.

Bußmann, Hadumod (1990): Sprachnormen. In: Lexikon der Sprachwissenschaft. Stuttgart: Kröner, 710.

Clyne, Michael G. (1992) (Hrsg.): Pluricentric Languages. Berlin: Walter de Gruyter. 
Clyne, Michael G. (1995): The German language in a changing Europe. Cambridge: Cambridge University Press.

Clyne, Michael G. (2005): Pluricentric Language/Plurizentrische Sprache. In: Ammon/Dittmar/Mattheier/Trudgill, 1. Teilband, 296-30o.

Davies, Winifred V. (2019): Sprachnormen und Sprachnormenvermittlung im muttersprachlichen Deutschunterricht: Theorie und Praxis und einige Überlegungen zur Relevanz für den DAF-Unterricht. In: Ransmayr, Jutta/Vasylchenko, Elena (2019) (Hrsg.): Sprachliche Variation im Deutschen. Unterrichtsdidaktische, deskriptive und internationale Perspektiven. Jahrbuch für internationale Germanistik. Reihe A. Berlin: Peter Lang.

Davies, Winifred V. (2017): Gymnasiallehrkräfte in Nordrhein-Westfalen als SprachnormvermittlerInnen und Sprachnormautoritäten. In: Davies et al., 123-146.

Davies, Winifred V./Häcki Buhofer, Annelies/Schmidlin, Regula/Wagner, Melanie/ Wyss, Eva Lia (2017) (Hrsg.): Standardsprache zwischen Norm und Praxis. Theoretische Betrachtungen, empirische Studien und sprachdidaktische Ausblicke. Tübingen: Narr Francke Attempto.

de Cillia, Rudolf/Reisigl, Martin/Wodak, Ruth (1999): The discursive construction of national identities. In: Discourse and Society 10/2, 149-173.

de Cillia, Rudolf (1995): Erdäpfelsalat bleibt Erdäpfelsalat. Österreichisches

Deutsch und EU-Beitritt. In: Muhr, Rudolf/Schrodt, Richard/Wiesinger, Peter (Hrsg.): Österreichisches Deutsch. Linguistische, sozialpsychologische und sprachpolitische Aspekte einer nationalen Variante des Deutschen. Wien: hpt, 121-131.

de Cillia, Rudolf (1997): „Alles bleibt, wie es ißt“. Österreichs EU-Beitritt und die Frage des österreichischen Deutsch. In: Jahrbuch Deutsch als Fremdsprache 23/1997, 239-258.

de Cillia, Rudolf (1998): „Burenwurscht bleibt Burenwurscht“. Sprachenpolitik und gesellschaftliche Mehrsprachigkeit in Österreich. Klagenfurt/Celovec: Drava.

de Cillia, Rudolf (2006): „Sieg im Marmeladekrieg“ - Das Protokoll Nr. 10 zehn Jahre danach. In: Fill, Alwin/Marko, Georg/Newby, David/Penz, Hermine (Hrsg.): Linguists (don't) only talk about it. Essays in Honour of Bernhard Kettemann. Tübingen: Stauffenburg, 123-139.

de Cillia, Rudolf (2008): „Ich bin verliebt in mein Österreich“. Die diskursive Konstruktion österreichischer Identität in zwei Gruppendiskussionen. In: de Cillia, Rudolf/Wodak, Ruth (2008) (Hrsg.): Gedenken im ,Gedankenjahr`. Zur diskursiven Konstruktion österreichischer Identitäten im Jubiläumsjahr 2005. Innsbruck, Wien, Bozen: StudienVerlag, 135-172.

de Cillia, Rudolf/Wodak, Ruth (2006): Ist Österreich ein „deutsches“ Land? Sprachenpolitik und Identität in der Zweiten Republik. Innsbruck u. a.: Studien Verlag. 
de Cillia, Rudolf/Wodak, Ruth (2009): Gedenken im ,Gedankenjahr‘. Zur diskursiven Konstruktion österreichischer Identitäten im Jubiläumsjahr 2005. Wien, Innsbruck: Studien Verlag.

de Cillia, Rudolf (2010): Mehrsprachigkeit statt Zweisprachigkeit - Argumente und Konzepte für eine Neuorientierung der Sprachenpolitik an den Schulen. In: de Cillia, Rudolf/Gruber, Helmut/Krzyzanowski, Michal/Menz, Florian (Hrsg.): Discourse - Politics - Identity. Diskurs - Politik - Identität. Festschrift für Ruth Wodak. Tübingen: Stauffenburg, 245-255.

de Cillia, Rudolf (2012): Sprache/n und Identität/en in Österreich. In: Der Sprachdienst 5/12, 166-179.

de Cillia, Rudolf (2013): Integrative Sprachenbildung an österreichischen Bildungsinstitutionen und SprachpädagogInnenbildung. In: Vetter, Eva (Hrsg.): Professionalisierung für sprachliche Vielfalt. Hohengehren: Schneider, 5-20. de Cillia, Rudolf/Fink, Elisabeth/Ransmayr, Jutta (2013): Österreichisches Deutsch als Unterrichts- und Bildungssprache an österreichischen Schulen. In: ÖDaF-Mitteilungen 2/2013. Horizonte. Sonderheft zur IDT 2013, 34-47. de Cillia, Rudolf (2013): Spracherwerb in der Migration. Informationsblätter des Referats für Migration und Schule Nr. 3/2013. 15. aktualisierte Auflage.

de Cillia, Rudolf (2015): Deutsche Sprache und österreichische Identität/en. In: Lenz, Alexandra/Ahlers, Timo/Glauninger, Manfed M. (Hrsg.): Dimensionen des Deutschen in Österreich. Variation und Varietäten im sozialen Kontext. Reihe „Schriften zur deutschen Sprache in Österreich“ 42. Frankfurt/M.: Peter Lang, 149-164.

de Cillia, Rudolf (2016a): Altersspezifischer/generationsspezifischer Sprachgebrauch in der österreichischen Varietät des Deutschen. In: Zhu, Jianhua/Zhao, Jin/Szurawitzki, Michael (Hrsg.): Beiträge des XIII. IVG-Kongresses Shanghai 2015. Frankfurt/M. u. a.: Peter Lang, 201-205.

de Cillia, Rudolf (2016b): Verwendung von Austriazismen und Deutschlandismen bei österreichischen Lehrer_innen und Schüler_innen. In: Schweiger, Hannes/Ahamer, Vera/Tonsern, Clemens/Welke, Tina/Zuzok, Nadja (Hrsg.): In die Welt hinaus. Festschrift für Renate Faistauer zum 65. Geburtstag. Wien: Praesens, 331-342.

de Cillia, Rudolf/Fink, Elisabeth/Ransmayr, Jutta (2017): Varietäten des Deutschen an österreichischen Schulen. Ergebnisse des Forschungsprojekts „Österreichisches Deutsch als Unterrichts- und Bildungssprache“. In: Davies et al. 2017, 207-234.

de Cillia, Rudolf (2018): Sprache(n) im Klassenzimmer. Varietätengebrauch und Spracheinstellungen in der Schule aus Sicht der Lehrer/innen und der Schüler/ innen. In: Dannerer, Monika/Mauser, Peter (Hrsg.): Formen der Mehrsprachigkeit. Sprachen und Varietäten in sekundären und tertiären Bildungskontexten. Tübingen: Stauffenburg, 67-85. 
Dittmar, Norbert (1997): Grundlagen der Soziolinguistik: ein Arbeitsbuch mit Aufgaben. Berlin, New York: Walter de Gruyter.

Dittmar, Norbert/Schmidt-Regener, Irena (2001): Soziale Varianten und Normen In: Helbig et. al, 520-534.

Dollinger, Stefan (in Druck): The Pluricentricity Debate. On Austrian German and other Germanic Standard Varieties. Routledge (FOCUS: short monographs). https://www.academia.edu/37714477/ (8.2.2019).

Dollinger, Stefan (2016): On parallels, differences and distortions in the pluricentricity of English and German. Vortrag bei der Tagung „Deutsch in Österreich“ am 7. Juli 2016. Scripted talk, written to be spoken. Zur Verfügung gestellt vom Autor.

Dollinger, Stefan (2017): TAKE UP \#9 as a semantic isogloss on the Canada-US border. In: World Englishes 2017, 80-103.

Dörnyei, Zoltán (2007): Oxford Applied Linguistics: Research Methods in Applied Linguistics. Cornelsen.

Dressler, Wolfgang U./de Cillia, Rudolf (2006): Spracherhaltung, Sprachverfall, Sprachtod. Language Maintenance, Language Decline and Language Death. In: Ammon/Dittmar/Mattheier/Trudgill, 3. Teilband, 2258-2271.

Duden (2008). Das große österreichische Schulwörterbuch. Mannheim, Leipzig, Wien, Zürich: Dudenverlag.

Durrell, Martin (2003): Register, Variation und Fremdsprachenvermittlung. Zum Problem des Deutschunterrichts in Großbritannien. In: Stickel, Gerhard (2003) (Hrsg.): Deutsch von außen. Jahrbuch des IDS 2002. Berlin, New York: Walter de Gruyter, 239-258.

Dürscheid, Christa (2009): Variatio delectat? Die Plurizentrizität des Deutschen als Unterrichtsgegenstand. In: Clalüna, Monika/Etterich, Barbara (Hrsg.): Deutsch unterrichten zwischen DaF, DaZ und DaM. Sondernummer Rundbrief AkDaF, 59-69.

Dürscheid, Christa/Elspaß, Stephan/Ziegler, Arne (2015): Variantengrammatik des Standarddeutschen. Konzeption, methodische Fragen, Fallanalysen. In: Lenz, Alexandra N./Glauninger, Manfred M. (Hrsg.): Standarddeutsch im 21. Jahrhundert - Theoretische und empirische Ansätze mit einem Fokus auf Österreich. Wien: Vienna University Press, 207-235.

Ebner, Jakob (1969): Wie sagt man in Österreich? Berlin: Bibliographisches Institut. Ebner, Jakob (1980): Wie sagt man in Österreich? Mannheim: Dudenverlag. Ebner, Jakob (2008): Österreichisches Deutsch. Eine Einführung von Jakob Ebner. Mannheim u. a.: Dudenverlag.

Ebner, Jakob (2009), Wie sagt man in Österreich? Wörterbuch der österreichischen Besonderheiten. 4., völlig überarb. Aufl. Mannheim: Dudenverlag.

Ebner, Jakob (2012): Wie erkennt man österreichisches Deutsch? In: Der Sprachdienst 5/12, 154-166. 
Ebner Jakob (2014): Österreichisches Deutsch. Ein Klärungsversuch. In: Bundesministerium für Bildung (Hrsg.): Österreichisches Deutsch als Unterrichtsund Bildungssprache.

Eichinger, Ludwig M./Kallmeyer, Werner (2005) (Hrsg.): Standardvariation. Wie viel Variation verträgt die deutsche Sprache? Jahrbuch des IDS 2004. Berlin/ New York: Walter de Gruyter.

Eichinger, Ludwig (2005): Deutsch in Österreich. In: German as a foreign language, $1,2-4$.

Elspaß, Stephan/Niehaus, Konstantin (2014): The standardization of a modern pluriareal language. Concepts and corpus designs for German and beyond. In: Orð og tunga 16, 47-67.

Ender, Andrea/Kaiser, Irmtraud (2009): Zum Stellenwert von Dialekt und Standard im österreichischen und Schweizer Alltag. In: ZGL 37/2, 266-295.

Ferguson, Charles A. (1982/1959): Diglossie. In: Anwendungsbereiche der Soziolinguistik. Darmstadt 1982, S. 253-276 (Übersetzung von: Diglossia. In: Word. Journal of the Linguistic Circle of New York. 15, 1959, 325-340).

Fielding, Nigel/Schreier, Margrit (2001): Introduction: On the Compatibility between Qualitative and Quantitative Research Methods. In: Forum: Qualitative Social Research, Vol.2 (1), 1-21.

Fink, Ilona Elisabeth (2016): Language loyalty to the Austrian variety of the German language. In: Muhr, Rudolf/Fonyuy, Kelen Ernesta/Ibrahim, Zeinab/ Miller, Corey (Hrsg.): Pluricentric Languages and Non-Dominant Varieties Worldwide: Volume 1: Pluricentric Languages across Continents - Features and Usage. Frankfurt/M., Wien u. a.: Peter Lang, 249-262.

Fink, Ilona Elisabeth/Ransmayr, Jutta/de Cillia, Rudolf (2017): „Also grammatisch würd ich fast sagen, dass die Österreicher inkorrekt sind, aber sonst eigentlich gar nicht. "Wahrnehmung von und Einstellungen gegenüber Varietäten des Deutschen bei österreichischen LehrerInnen und SchülerInnen. In: ÖDaF-Mitteilungen Juli 2017, Band 33, 79-96.

Fishman, Joshua A. (1964): Language maintenance and language shift as a field of inquiry. In: Linguistics 9, 32-70.

Fishman, Joshua A. (1975): Zur Soziologie der Sprache. München.

Friedrichs, Jürgen (1997): Methoden empirischer Sozialforschung. Opladen: Westdeutscher Verlag.

Gatta, Adriana (2017): Untersuchung des Korrekturverhaltens von Lehrpersonen auf der Sekundarstufe II in Bezug auf nationale Varianten der Schweizer Standardsprache. In: Davies, Winifred/Häcki Buhofer, A./Schmidlin, R./Wyss, Eva Lia (2017) (Hrsg.): Standardsprache zwischen Norm und Praxis. Theoretische Betrachtungen, empirische Studien und sprachdidaktische Ausblicke. Basler Studien zur deutschen Sprache und Literatur 99. Tübingen: Narr, 359-392.

Glauninger, Manfred M. (2007): Deutsch im 21. Jahrhundert: „pluri“-, „supra“oder „postnational“? Vortrag am II. Kongress des Mitteleuropäischen 
Germanistenverbandes (MGV), Olmütz, 13.-16. September 2007. http://homepage.univie.ac.at/manfred.Glauninger/Vorlesung/Glauninger\%20Olm\%FCtz. pdf (18.12. 2018).

Gogolin, Ingrid/Lange, Imke (2011): Bildungssprache und durchgängige Sprachbildung. In: Fürstenau, Sara/Gomolla, Mechthild (Hrsg.): Migration und schulischer Wandel: Mehrsprachigkeit. Wiesbaden: VS-Verlag, 107-127.

Griesmayer, Norbert (2004): Zur Sprachauffassung im neuen Lehrplan Deutsch für Österreichs Schulen der Zehn- bis Achtzehnjährigen. In: Trans. InternetZeitschrift für Kulturwissenschaften 15. Nr., Juni 2004. http://www.inst.at/ trans/15Nr/o6_1/griesmayer15.htm (9.1.2019).

Haas, Walter (2006): Die Schweiz/Switzerland. In: Ammon/Dittmar/Mattheier/ Trudgill, 3. Teilband, 1772-1787

Habermas, Jürgen (1971): Vorbereitende Bemerkungen zu einer Theorie der kommunikativen Kompetenz. In Habermas, Jürgen/Luhmann, Niklas (Hrsg.): Theorie der Gesellschaft oder Sozialtechnologie. Frankfurt/M.: Suhrkamp, 101-141.

Habermas, Jürgen (1977): Umgangssprache, Wissenschaftssprache, Bildungssprache. In: Jahrbuch der Max-Planck-Gesellschaft zur Förderung der Wissenschaften, 36-51.

Hägi, Sarah (2006): Nationale Varietäten im Unterricht Deutsch als Fremdsprache, Frankfurt /M.: Peter Lang.

Hartig, Matthias (1987): Sprachlegitimation und Sprachloyalität in ihrer Bedeutung für den Dialektwandel. In: Oksaar, Els (1987) (Hrsg.): Soziokulturelle Perspektiven von Mehrsprachigkeit und Spracherwerb. Tübingen: Narr, 30o-315.

Heer, Friedrich (1981): Der Kampf um die österreichische Identität. Wien, Köln, Graz: Böhlau.

Heinrich, Ilona Elisabeth (2010): Österreichisches Deutsch in Lehrbüchern der Sekundarstufe 1 für Deutsch als Muttersprache, Dipl. Univ. Wien.

Helbig, Gerhard/Götze, Lutz/Henrici, Gert/Krumm, Hans-Jürgen (2001) (Hrsg.): Deutsch als Fremdsprache. Ein internationales Handbuch. Berlin, New York: Walter de Gruyter.

Herrgen, Joachim (2015): Entnationalisierung des Standards. Eine perzeptionslinguistische Untersuchung zur deutschen Standardsprache in Deutschland, Österreich und der Schweiz. In: Lenz, Alexandra N./Glauninger, Manfred M. (Hrsg.): Standarddeutsch im 21. Jahrhundert (=Wiener Arbeiten zur Linguistik 1). Göttingen: V\&R unipress, 139-164.

Hirschfeld, Ursula (2009): Rezension Rudolf Muhr (2007): Österreichisches Aussprachewörterbuch. Österreichische Aussprachedatenbank. Frankfurt/M. u. a.: Peter Lang. In: Theorie und Praxis 12/2008, 205-212.

Höbelt, Lothar (1994): Österreich $=$ deutsch $\neq$ bundesrepublikanisch. In: Botz/ Sprengnagel (1994), 338-345. 
Hochholzer, Rupert (2004): Konfliktfeld Dialekt. Das Verhältnis von Deutschlehrerinnen und Deutschlehrern zu Sprache und ihren regionalen Varietäten. Regensburg: edition vulpes.

Hymes, Dell (1972): Towards communicative competence. Philadelphia. ide - Informationen zur Deutschdidaktik 3/97: Fremde Muttersprachen Deutsch. Kellermeier-Rehbein, Birte (2014): Plurizentrik. Eine Einführung in die nationalen Varietäten des Deutschen. Berlin: Erich Schmidt.

Kleineidam, Hartmut (1986): Fremdsprachengrammatik: Analysen und Positionen. Beiträge mit dem Schwerpunkt Französisch. Tübingen: Gunter Narr. Kloss, Heinz (1978): Die Entwicklung neuer germanischer Kultursprachen seit 180o. Düsseldorf: Schwann.

Konecky, Angelika (2017): Pfiati sagt leise Servus. Diachrone Untersuchung ausgewählter Austriazismen in österreichischen Medien, Dipl. Univ. Wien.

Kreissler, Felix (1984): Der Österreicher und seine Nation. Ein Lernprozeß mit Hindernissen. Wien, Köln, Graz.

Lamnek, Siegfried (1989): Qualitative Sozialforschung. Band 1: Methodologie. Band 2: Methoden und Techniken. München: Beltz.

Landesinstitut für Statistik (2012). Volkszählung 2011. Berechnung des Bestandes der drei Sprachgruppen in der Autonomen Provinz Bozen-Südtirol/Censimento della popolazione 2011. Determinazione della consistenza dei tre gruppi linguistici della Provincia Autonoma di Bolzano-Alto Adige. Bozen. Astat info 38, 06/2012.

Legenstein, Christian (2008): Das Österreichische Deutsch im Deutschunterricht. Eine empirische Untersuchung, Dipl. Univ. Graz.

Lenz, Alexandra N./Glauninger, Manfred M. (2015): Standarddeutsch im 21. Jahrhundert. Theoretische und empirische Ansätze mit einem Fokus auf Österreich. Göttingen V\&R.

Lewis, M. Paul/Simons, Gary F./Fennig Charles D. (2014, 2015, 2016): Ethnologue: Ethnologue: Languages of the world. Seventeenth, eighteenth, nineteenth editions. Dallas: SIL International: https://www.ethnologue.com/archive (18. 12. 2018).

Löffler, Heinrich (2005): Wieviel Variation verträgt die deutsche Standardsprache? Begriffsklärung: Standard und Gegenbegriffe. In: Eichinger, Ludwig M./ Kallmeyer, Werner (Hrsg.): Standardvariation. Wie viel Variation verträgt die deutsche Sprache? Berlin, New York: Walter de Gruyter, 143-171.

Löffler, Heinrich (2016): Germanistische Soziolinguistik. 5. neu bearb. Aufl. Berlin: Erich Schmidt.

Lüdi, Georges/Werlen, Iwar (2005): Eidgenössische Volkszählung 200o. Sprachenlandschaft in der Schweiz. Neuchâtel: Bundesamt für Statistik.

Markhardt, Heidemarie (2005): Das österreichische Deutsch im Rahmen der EU. Frankfurt/M. u. a.: Peter Lang. 
Mayring, Philipp (2001). Kombination und Integration qualitativer und quantitativer Analyse. Forum Qualitative Sozialforschung/Forum Qualitative Social Research (Online-Journal), 2(1).

Mohn, Dennis (2017): Konvergenz und Divergenz im standardnahen Sprachgebrauch in Bayern und Österreich. Eine empirische Untersuchung zur Standardaussprache von Rundfunksprechern von BR und ORF. Vortrag gehalten am 2. 8. 2017 bei der IDT 2017 in der Sektion „Sprachliche Variation im Deutschen" (D9).

Mohn, Dennis (in Druck): Die Standardaussprache von Nachrichtensprechern des Bayerischen Rundfunks (BR) und Österreichischen Rundfunks (ORF). In: Ransmayr, Jutta/Vasylchenko, Elena (Hrsg.): Sprachliche Variation im Deutschen. Unterrichtsdidaktische, deskriptive und internationale Perspektiven. Jahrbuch für internationale Germanistik. Reihe A. Berlin: Peter Lang.

Moosmüller, Sylvia (1991): Hochsprache und Dialekt in Österreich. Soziophonologische Untersuchungen zu ihrer Abgrenzung in Wien, Graz, Salzburg und Innsbruck. Wien, Köln, Weimar: Böhlau.

Moosmüller, Sylvia (2015): Methodisches zur Bestimmung der Standardaussprache in Österreich. In: Lenz/Glauninger, 165-184.

Muhr, Rudolf (1982): Österreichisch. Anmerkungen zur linguistischen Schizophrenie einer Nation. In: Klagenfurter Beiträge zur Sprachwissenschaft 8, 306-319.

Muhr, Rudolf (1989): Deutsch und Österreich(isch): Gespaltene Sprache - Gespaltenes Bewußtsein - Gespaltene Identität. In: ide 2, 74-98.

Muhr, Rudolf (1995): Zur Sprachsituation in Österreich und zum Begriff „Standardsprache“ in plurizentrischen Sprachen. Sprache und Identität in Österreich. In: Muhr, Rudolf/Schrodt, Richard/Wiesinger, Peter (Hrsg.): Österreichisches Deutsch. Linguistische, sozialpsychologische und sprachpolitische Aspekte einer nationalen Variante des Deutschen. Wien: hpt, 75-110.

Muhr, Rudolf (1997): Welche Sprache wird an österreichischen Schulen unterrichtet? Sprachnormen, Spracheinstellungen und Sprachwirklichkeit im Deutschunterricht. In: ide 1997/3, 32-46.

Muhr, Rudolf (200o): Österreichisches Sprachdiplom Deutsch. Lernzielkataloge. Wien: öbv \& hpt.

Muhr, Rudolf (2005a) (Hrsg.): Standardvariationen und Sprachideologien in verschiedenen Sprachkulturen der Welt. Standard Variations and Language Ideologies in Different Language Cultures around the World. Frankfurt/M.: Peter Lang.

Muhr, Rudolf (2005b): Language Attitudes and language conceptions in nondominating varieties of pluricentric languages. In: Muhr, Rudolf (Hrsg.): Standardvariationen und Sprachideologien in verschiedenen Sprachkulturen der Welt. Standard Variations and Language Ideologies in Different Language Cultures around the World. Frankfurt/M.: Peter Lang, 11-20. 
Muhr, Rudolf (2007): Österreichisches Aussprachewörterbuch und Österreichische Aussprachedatenbank. Frankfurt/M., Wien: Peter Lang.

Muhr, Rudolf (2013): Zur sprachenpolitischen Situation des Österreichischen Deutsch 200o-2012. In: de Cillia, Rudolf/Vetter, Eva (Hrsg.): Sprachenpolitik in Österreich. Bestandsaufnahme 2011. Frankfurt/M. u. a.: Peter Lang, 257-305. Neuland, Eva (2006) (Hrsg.): Variation im heutigen Deutsch: Perspektiven für den Sprachunterricht. Zur Einführung. Frankfurt/M. u. a.: Peter Lang, 9-27.

Neuland, Eva/ Peschel, Corinna (2013): Einführung in die Sprachdidaktik. Stuttgart: Metzler.

Niehaus, Konstantin (2017): Die Begrenztheit plurizentrischer Grenzen: Grammatische Variation in der pluriarealen Sprache Deutsch. In: Davies, Winifred V./ Häcki Buhofer, Annelies/Schmidlin, Regula/Wagner, Melanie/Wyss, Eva Lia (Hrsg.): Standardsprache zwischen Norm und Praxis.Theoretische Betrachtungen, empirische Studien und sprachdidaktische Ausblicke. Tübingen: Narr Francke Attempto, 61-88.

Peter, Klaus (2015): Sprachliche Normvorstellungen in Österreich, Deutschland und der Schweiz. In Lenz, Alexandra N./Ahlers, Timo/Glauninger, Manfred M. (Hrsg.): Dimensionen des Deutschen in Österreich. Variation und Varietäten im sozialen Kontext. Frankfurt/M. u. a.: Peter Lang, 123-147 (= Schriften zur Deutschen Sprache in Österreich Band 42).

Peyer, Ann/Portmann, Paul R./Brütsch, Edgar/Gallmann, Peter/Lindauer, Thomas/Linke, Angelika/Nussbaumer, Markus/Looser, Roman/Sieber, Peter (1996): Norm, Moral und Didaktik. Die Linguistik und ihre Schmuddelkinder. In: Peyer, Ann/Portmann, Paul R. (Hrsg.): Norm, Moral und Didaktik - Die Linguistik und ihre Schmuddelkinder. Eine Aufforderung zur Diskussion. Tübingen: Max Niemeyer, 9-46.

Pfrehm, James W. (2007): An Emipirical Study of the Pluricentricity of German: Comparing German and Austrian Nationals' Perception of the Use, Pleasantness, and Standardness of Austrian Standard and German Standard Lexical Items. Dissertation: University of Wisconsin-Madison.

Pfrehm, James W. (2011a): The role of age in Austrians' perceptions of the frequency of use and likeability of lexical Teutonisms and Austriacisms. In: Folia Linguistica. Band 44, Heft 2, 439-470.

Pfrehm, James W. (2011b): The Pluricentricity of German: Perceptions of the Standardness of Austrian and German Lexical Items. In: Journal of Germanic Linguistics 23.1 (2011), 37-64.

Pohl, Heinz-Dieter (1997): Gedanken zum Österreichischen Deutsch (als Teil der pluriarealen deutschen Sprache). In: Muhr, Rudolf/Schrodt, Richard (1997) (Hrsg.): Österreichisches Deutsch und andere nationale Varietäten plurizentrischer Sprachen in Europa. Wien: öbv \& hpt, 67-88. 
Polenz, Peter von (1987): Nationale Varianten der deutschen Hochsprache. Podiumsdiskussion auf der VIII. Internationalen Deutschlehrertagung in Bern am 5. August 1986. In: ZGL 15.1987, 101-103.

Polenz, Peter von (1988): ,Binnendeutsch' oder plurizentrische Sprachkultur. Ein Plädoyer für Normalisierung in der Frage der, nationalen' Varietäten. In: ZGL 16/1988, 198-218.

Pollak, Wolfgang (1992): Was halten die Österreicher von ihrem Deutsch? Eine sprachpolitische und soziosemiotische Analyse der sprachlichen Identität der Österreicher, Wien: ÖGS/ISSS.

Protze, Helmut (2001): Das Deutsche in Deutschland und seine regionalen Varianten. In: Helbig et. al: 505-520.

Putz, Martin (2002): Österreichisches Deutsch als Fremdsprache? Kritische Überlegungen, in: GFL-Journal 3/2002,48-76 (http://www.gfl-journal.de/3-2002/ putz.html [18.12.2018]).

Ransmayr, Jutta (2005): Das österreichische Deutsch und sein Status an Auslandsuniversitäten. Eine empirische Untersuchung an Germanistikinstituten in Frankreich, Großbritannien, Tschechien und Ungarn. Wien: Diss. Phil.

Ransmayr, Jutta (2006): Der Status des Österreichischen Deutsch an Auslandsuniversitäten. Eine empirische Untersuchung. Frankfurt/M. u. a.: Peter Lang. Ransmayr, Jutta/Fink, Ilona Elisabeth (2014): Das österreichische Deutsch als Unterrichts- und Bildungssprache. Vorläufige Ergebnisse des FWF-Projekts. In: Ransmayr, Jutta/Moser-Pacher, Andrea/Fink, Ilona Elisabeth (2014) (Hrsg.): „Österreichisches Deutsch und Plurizentrik“. In: ide 3/2014, 40-53.

Ransmayr, Jutta (2015): Wie normal ist österreichisches Deutsch? Über das Normverständnis im Inland und im Ausland. In: Krausneker, Verena/Volgger, MarieLuise (Hrsg.): Sprache im Kontext 43: Meine Sprachen, meine Forschung und ich. Festschrift für Rudolf de Cillia. Wien: Peter Lang, 181-193.

Ransmayr, Jutta/Fink, Ilona Elisabeth (2016): Umgang mit Varietäten im Unterricht von Deutsch als Muttersprache/Bildungssprache. In: Rückl, Michaela (2016) (Hrsg.): Sprachen und Kulturen: vermitteln und vernetzen. Beiträge zu Mehrsprachigkeit und Inter-/Transkulturalität im Unterricht, in Lehrwerken und in der Lehrerbildung. Münster: Waxmann (Salzburger Beiträge zur Lehrerbildung: Der Dialog der Fachdidaktiken mit Fach- und Bildungswissenschaften 2), 166-179.

Ransmayr, Jutta/Mörth, Karlheinz/Durco, Matej (2017): AMC (Austrian Media Corpus) - Korpusbasierte Forschungen zum österreichischen Deutsch. In: Resch, Claudia/Dressler, Ulrich (Hrsg.): Digitale Methoden der Korpusarbeit in Österreich. Linguistik und Kommunikationsforschung. Wien: Verlag der Österreichischen Akademie der Wissenschaften, 27-38.

Ransmayr, Jutta/Fink, Ilona Elisabeth/de Cillia, Rudolf (2017): Österreichisches Deutsch als Unterrichts- und Bildungssprache an österreichischen Schulen. In: Hans Drumbl/Geraldo de Cavalho/Jörg Klinner (Hrsg.): Sprachenpolitik 
und Sprachenvielfalt. Konferenzbeiträge/Atti/Proceedings IDT 2013, Band 8, Sektionen G1, G2, G3, G4, G5. Bozen, 151-167.

Ransmayr, Jutta (2017): Insiders' and outsiders' views on German from Austria's perspective: Austrian Standard German and German Standard German - the odd couple. In: Nuolijärvi, Pirkko/Stickel, Gerhard (Hrsg.): Stereotypes and linguistic prejudices in Europe. Contributions to the EFNIL Conference 2016 in Warsaw, 187-206.

Ransmayr, Jutta/Vasylchenko, Elena (Hrsg.) (in Druck): Sprachliche Variation im Deutschen. Unterrichtsdidaktische, deskriptive und internationale Perspektiven. Reihe A. Wien: Peter Lang.

Rastner, Eva-Maria (1997): Sprachvarietäten im Unterricht. Eine Umfrage unter Österreichs LehrerInnen zu Standardsprache - Umgangssprache - Dialekt. In: ide 1997/3, 80-93.

Rehbein, Jochen/Schmidt, Thomas/Meyer, Bernd/Watzke, Franziska/Herkenrath, Annette (2004): Handbuch für das computergestützte Transkribieren nach HIAT. Arbeiten zur Mehrsprachigkeit 56/2004. Folge B. Hamburg: Universität Hamburg.

Reich, Hans H./Krumm, Hans-Jürgen (2013): sprachbildung und mehrsprachigkeit. Ein Curriculum zur Wahrnehmung und Bewältigung sprachlicher Vielfalt im Unterricht. Münster u. a.: Waxmann.

Redl, Klaus (2014): Plurizentrik im Deutschunterricht? Eine empirische Untersuchung bei Wiener UnterrichtspraktikantInnen, MA-Arbeit Univ. Wien.

Reiffenstein, Ingo (1973): Österreichisches Deutsch. In: Haslinger, Adolf (Hrsg.): Deutsch heute. München: Hueber, 19-26.

Reiffenstein, Ingo (2001): Das Problem der nationalen Varietäten. Rezensionsaufsatz zu Ulrich Ammon. Die deutsche Sprache in Deutschland, Österreich und der Schweiz. Das Problem der nationalen Varietäten. In: Zeitschrift für deutsche Philologie 120/1, 78-89.

Rheindorf, Markus/Wodak, Ruth (2014): Der Wandel des österreichischen Deutsch: Eine textsorten-bezogene Pilotstudie (1970-2010). In: Deutsche Sprache 42/2014, 139-167.

Scharloth, Joachim (2005): Zwischen Fremdsprache und nationaler Varietät. Untersuchungen zum Plurizentrizitätsbewusstsein der Deutschschweizer. In: Muhr (2005a), 21-44.

Scheuringer, Hermann (1985): The state border as a dialect border - on the necessity of dialect geography in dialectology. In: Warkentyne, Henry J. (Hrsg.): Papers from the Fifth International Conference on Methods in Dialectology. Victoria: University of Victoria, 443-455.

Scheuringer, Hermann (1988): Powidltatschkerl oder Die kakanische Sicht aufs Österreichische. In: Jahrbuch für Internationale Germanistik XX/Heft 1, 63-70. 
Scheuringer, Hermann (1990): Sprachentwicklung in Bayern und Österreich: Eine Analyse des Substandardverhaltens der Städte Braunau am Inn (Österreich) und Simbach (Bayern) und ihres Umlandes. Hamburg: Buske.

Scheuringer, Hermann (1992): Deutsches Volk und deutsche Sprache. In: Österreich in Geschichte und Literatur 1992/3-4a; 162-173.

Scheuringer, Hermann (1996): Das Deutsche als pluriareale Sprache: Ein Beitrag gegen staatlich begrenzte Horizonte in der Diskussion um die deutsche Sprache in Österreich. In: Die Unterrichtspraxis/Teaching German 2/29, 147-153.

Scheuringer, Hermann (2001): Die deutsche Sprache in Österreich. In: KnipfKomlósi, Elisabeth/Berend, Nina (Hrsg.): Regionale Standards. Budapest. Dialog Campus Kiadó, 95-120.

Scheuringer, Hermann (2016): Bayerisches Standarddeutsch. Des Faktischen normative Schwäche. In: Annual Review of the Faculty of Philosophy, Novi Sad, Volume XLI-1 (2016), 369-384.

Schlobinski, Peter (1996): Empirische Sprachwissenschaft. Opladen: Westdeutscher Verlag.

Schmid, Georg (1990): ... SAGEN DIE DEUTSCHEN. Annäherung an eine Geschichte des Sprachimperialismus. In: Rathkolb, Oliver/Schmid, Georg/ Heiß, Gernot (Hrsg.): Österreich und Deutschlands Größe. Ein schlampiges Verhältnis. Salzburg: Otto Müller, 23-34.

Schmidlin, Regula (2011): Die Vielfalt des Deutschen: Standard und Variation. Gebrauch, Einschätzung und Kodifizierung einer plurizentrischen Sprache. Berlin, Boston: Walter de Gruyter.

Schmidlin, Regula (2013): Gebrauch und Einschätzung des Deutschen als plurizentrische Sprache. In: Schneider-Wiejowski, Karina/Kellermeier-Rehbein, Birte/Haselhuber, Jakob (Hrsg.): Vielfalt, Variation und Stellung der deutschen Sprache. Berlin, Boston: Walter de Gruyter, 23-41.

Schmidlin, Regula/Wyss, Eva Lia/Davies, Winifred V. (2017): Plurizentrik revisited - aktuelle Perspektiven auf die Variation der deutschen Standardsprache. In: Davies, Winifred V./Häcki Buhofer, Annelies/Schmidlin, Regula/Wagner, Melanie/Wyss, Eva Lia (Hrsg.): Standardsprache zwischen Norm und Praxis. Theoretische Betrachtungen, empirische Studien und sprachdidaktische Ausblicke. Tübingen: Narr Francke Attempto, 7-20.

Schmidlin, Regula (2017): Normwidrigkeit oder Variationsspielraum? Die Varianten des Standarddeutschen als sprachliche Zweifelsfälle. In: Davies, Winifred V./ Häcki Buhofer, Annelies/Schmidlin, Regula/Wagner, Melanie/Wyss, Eva Lia (Hrsg.): Standardsprache zwischen Norm und Praxis. Theoretische Betrachtungen, empirische Studien und sprachdidaktische Ausblicke. Tübingen: Narr Francke Attempto, 41-6o.

Schneider-Wiejowski, Karina/Kellermeier-Rehbein, Birte/Haselhuber, Jakob (2013) (Hrsg.): Vielfalt, Variation und Stellung der deutschen Sprache. Berlin, Boston: Walter de Gruyter. 
Searle, John J. (1971): Sprechakte - ein sprachphilosophischer Essay. Frankfurt/M.: Suhrkamp.

Seifter, Thorsten/Seifter, Ingolf (2015): Warum die Frage, ob sich „pfiati vertschüsst“, keine linguistische ist. Zur Fundamentalkritik am „Österreichischen Deutsch“. In: Lüger, Heinz-Helmut (Hrsg.): Beiträge zur Fremdsprachenvermittlung. Landau: Verlag Empirische Pädagogik, 65-90.

Spiekermann, Helmut (2005): Regionale Standardisierung, nationale Destandardisierung. In: Eichinger/Kallmeyer, 100-125.

Spiekermann, Helmut (2008): Sprache in Baden-Württemberg. Merkmale des regionalen Standards, Tübingen: Niemeyer.

Spiekermann, Helmut (2010): Variation in der deutschen Sprache. In: Krumm, Hans-Jürgen et al. (Hrsg.): Deutsch als Fremd- und Zweitsprache. 1. Halbband. Berlin, Boston: Walter de Gruyter, 343-359.

Stangel, Johann/Schäfer, Stefan (2013): Sprachräume 3, Deutsch für die AHS-Oberstufe. Wien: öbv. SBNr 165246.

Statistik Austria (2002): Volkszählung 2001. Hauptergebnisse I - Österreich, Wien. Statistik Austria (2012): Adult Education Survey 2011/12.

Statistik Austria (2013): migration \& integration. zahlen. daten. indikatoren.

Statistik Austria (2015): Bildung in Zahlen 2013/14. Schlüsselindikatoren und Analysen. Wien.

Statistisches Bundesamt (2017): Bevölkerung auf einen Blick. https://www.destatis. de/DE/ZahlenFakten/GesellschaftStaat/Bevoelkerung/Bevoelkerung.html (18.12.2018).

Steinegger, Guido (1998): Sprachgebrauch und Sprachbeurteilung in Österreich und Südtirol: Ergebnisse einer Umfrage. Frankfurt/M. Wien u. a.: Peter Lang.

Steiner, Astrid (2008): Unterrichtskommunikation. Eine linguistische Untersuchung der Gesprächsorganisation und des Dialektgebrauchs in Gymnasien der Deutschschweiz. Tübingen: Gunter Narr.

Steinig, Wolfgang/Huneke, Hans-Werner (2015): Sprachdidaktik Deutsch. Eine Einführung. Berlin: Erich Schmidt Verlag.

Störig, Hans Joachim (1987): Abenteuer Sprache: ein Streifzug durch die Sprachen der Erde. London: Insight Guides.

Tatzreiter, Herbert (1988): Besonderheiten der Morphologie in der deutschen Sprache in Österreich. In: Wiesinger, Peter (Hrsg.): Das österreichische Deutsch. Wien, Köln, Graz: Böhlau, 71-98.

Vetter, Eva (2008): Sprachenbewusstheit von FranzösischlehrerInnen. Habilitationsschrift Univ. Wien.

Wandruszka, Mario (1979): Die Mehrsprachigkeit des Menschen. München: Piper. Wiesinger, Peter (1983): Sprachschichten und Sprachgebrauch in Österreich. In: Zeitschrift für Germanistik 4, 184-195.

Wiesinger, Peter (1985): Die Entwicklung des Verhältnisses von Mundart und Standardsprache in Österreich. In: Besch, Werner/Reichmann, Oskar/Sonderegger, 
Stefan (Hrsg.): Sprachgeschichte. Ein Handbuch zur Geschichte der deutschen Sprache und ihrer Erforschung. 2. Halbband. Berlin, New York: Walter de Gruyter, 1939-1949.

Wiesinger, Peter (1988a) (Hrsg.): Das österreichische Deutsch. Wien, Köln, Graz: Böhlau (= Schriften zur deutschen Sprache in Österreich 12).

Wiesinger, Peter (1988b): „Die deutsche Sprache in Österreich. Eine Einführung.“ In: Wiesinger, Peter (Hrsg.): Das österreichische Deutsch. Wien, Köln, Graz: Böhlau, 9-30.

Wiesinger, Peter (2006/2014): Das österreichische Deutsch in Gegenwart und Geschichte. 1. Aufl. 2006, 2. Aufl. 2008. 3. aktualisierte und neuerlich erweiterte Auflage 2014. Wien, Berlin u. a.: LitVerlag.

Wiesinger, Peter (2006): Das österreichische Deutsch in der Diskussion. In: Wiesinger (2006): 203-218.

Wiesinger, Peter (2009): Die Standardaussprache in Österreich. In: Krech, EvaMaria et al. (Hrsg.): Deutsches Aussprachewörterbuch. Berlin, New York: Walter de Gruyter, 229-258.

Wiesinger, Peter (2010): Deutsch in Österreich: Standard, regionale und dialektale Variation. In: Krumm, Hans-Jürgen et al. (Hrsg.): Deutsch als Fremd- und Zweitsprache. 1. Halbband. Berlin, Boston: Walter de Gruyter, 360-372.

Wissik, Tanja (2014): Terminologische Variation in der Rechts- und Verwaltungssprache Deutschland - Österreich - Schweiz. Berlin: Frank Timme.

Wodak, Ruth (1994): Wir sind nicht Duden-Land. In: Wiener Journal, Juni 1994, $26-27$.

Wodak, Ruth/de Cillia, Rudolf/Reisigl, Martin/Liebhart, Karin/Hofstätter, Klaus/ Kargl, Maria (1998): Zur diskursiven Konstruktion nationaler Identität. Frankfurt/M.: Suhrkamp.

Wodak, Ruth/de Cillia, Rudolf/Reisigl, Martin/Liebhart, Karin (2009): The discursive construction of national identity. 2nd ed. Edinburgh: Edinburgh University Press.

Wodak, Ruth/Krzyzanowski, Michal (2008): Qualitative Discourse Analysis in the Social Sciences. Palgrave Macmillan.

Wolf, Norbert R. (1994): Österreichisches zum Österreichischen Deutsch. In: ZDL 61, 66-76.

Wolf, Norbert R. (2012): Die deutsche Sprache im Zeitalter der Globalisierung. In: Neuphilologische Mitteilungen 113; Nr. 4.2012, 497-509.

Wyss, Eva Lia (2017): Sprachnormurteile im Dilemma. Deutschlehrerinnen und Deutschlehrer an Deutschschweizer Gymnasien beurteilen Sprachkompetenzen, Sprachgebrauch und Zweifelsfälle. In: Davies, Winifred V./Häcki Buhofer, Annelies/Schmidlin, Regula/Wagner, Melanie/Wyss, Eva Lia (Hrsg.): Standardsprache zwischen Norm und Praxis.Theoretische Betrachtungen, empirische Studien und sprachdidaktische Ausblicke. Tübingen: Narr Francke Attempto, 147-188. 


\section{Verzeichnis der Tabellen und Abbildungen}

Tab. 1: Empirische Erhebung

Tab. 2: Analysierte Datensätze

Tab. 3: Korrekturvorschläge/Anmerkungen zu Deutschlandismen

Tab. 4: Korrekturvorschläge/Anmerkungen zu Austriazismen

Tab. 5: In den 30 Sätzen enthaltene Varianten zur Auswahl

Abb. 1: Erhebungsstandorte der Fragebogenerhebungen unter LehrerInnen und SchülerInnen

Abb. 2: Alter der befragten LehrerInnen (in \%)

Abb. 3: Schulformen der LehrerInnen (in \%)

Abb. 4: Dienstjahre der LehrerInnen (in \%)

Abb. 5: Dienstort nach Bundesländern (in \%)

Abb. 6: Dienstort nach Regionen (in \%)

Abb. 7: Unterrichtsfächer (in \%)

Abb. 8: Höchster Ausbildungsabschluss der LehrerInnen (in \%)

Abb. 9: Österreichisches Deutsch: Thema im Unterricht (Antworten LehrerInnen) (in \%)

Abb. 10: Verwendete Nachschlagwerke (Antworten LehrerInnen) (in \%)

Abb. 11: War sprachliche Variation (Standardvarietäten/Umgangssprache/Dialekt)

ein Thema in Ihrer Ausbildung? (in \%)

Abb. 12: Wurde sprachliche Variation in der Ausbildung ausreichend thematisiert? (in \%)

Abb. 13: Altersverteilung SchülerInnen (in \%)

Abb. 14: Schulstandorte der befragten SchülerInnen (in \%)

Abb. 15: Muttersprachen SchülerInnen (in \%)

Abb. 16: Muttersprachen SchülerInnen mit Kombinationsnennungen (in \%)

Abb. 17: Muttersprachen Eltern (in \%)

Abb. 18: Muttersprache SchülerInnen - Muttersprache Eltern (in \%)

Abb. 19: TV-Kanäle aktuell: SchülerInnen (in \%)

Abb. 20: Nur österreichische TV-Kanäle nach Region („TV aktuell“ SchülerInnen) (in \%)

Abb. 21: Nur deutsche TV-Kanäle nach Region („TV aktuell“ SchülerInnen) (in \%) Abb. 22: Bevorzugte TV-Kanäle Kindheit (SchülerInnen) (in \%)

Abb. 23: Nur österreichische TV-Kanäle („TV Kindheit“) nach Region (SchülerInnen) (in \%)

Abb. 24: Nur deutsche TV-Kanäle Kindheit nach Region (SchülerInnen) (in \%)

Abb. 25: Nachschlagwerke SchülerInnen (in \%)

Abb. 26: Plurizentrisches Konzept bekannt (SchülerInnen) (in \%) 
Abb. 27: Wie wird die Mehrheitssprache in Österreich benannt (LehrerInnen/ SchülerInnen)

Abb. 28: SchülerInnen-Antworten nach Regionen: Sprache, die in Österreich gesprochen wird: Deutsch (in \%)

Abb. 29: SchülerInnen-Antworten nach Bundesländern: Sprache, die in Österreich gesprochen wird: Dialekt/Mundart (in \%)

Abb. 30: Varietät, die mit österreichischem Deutsch verbunden wird (in \%)

Abb. 31: Sprachliche Unterschiede zwischen Österreich, Deutschland und der Schweiz: LehrerInnen-SchülerInnen-Vergleich (in \%)

Abb. 32: Das in Österreich gesprochene Deutsch unterscheidet sich von dem in Deutschland gesprochenen Deutsch in einer mündlichen, formellen Situation ... (in \%)

Abb. 33: Das in Österreich gesprochene Deutsch unterscheidet sich von dem in der Schweiz gesprochenen Deutsch in einer mündlichen, formellen Situation ... (in \%)

Abb. 34: Das im Osten Österreichs gesprochene Deutsch unterscheidet sich von dem im Westen Österreichs gesprochenen Deutsch in einer mündlichen, formellen Situation ... (in \%)

Abb. 35: Wahrgenommene Unterschiede zwischen Ost- und Westösterreich (sehr stark/stark)

Abb. 36: Deutsch ist ... (in \%)

Abb. 37: Glauben Sie, dass es ein österreichisches Standarddeutsch (Hochdeutsch) gibt? (in \%)

Abb. 38: Ja, es gibt ein österreichisches Standarddeutsch (nach Bundesländern; SchülerInnen) (in \%)

Abb. 39: Innerhalb Österreichs gibt es zu große regionale sprachliche Unterschiede (z. B. zwischen Ost- und Westösterreich), als dass es ein eigenes österreichisches Standarddeutsch geben kann. (in \%)

Abb. 40: Es gibt ein überregionales österreichisches Standarddeutsch, das sich vom Deutsch Deutschlands in manchen Bereichen klar unterscheidet. (in \%) Abb. 41: Österreich und Süddeutschland haben sprachlich mehr gemeinsam als Nord- und Süddeutschland. (in \%)

Abb. 42: SchülerInnen: Österreich und Süddeutschland haben sprachlich mehr gemeinsam als Nord- und Süddeutschland. (in \%)

Abb. 43: Österreichisches Deutsch gleich korrekt wie deutsches Deutsch? - LehrerInnen/SchülerInnen (in \%)

Abb. 44: LehrerInnen: Deutsches Deutsch korrekter? (in \%)

Abb. 45: Deutsches Deutsch korrekter? LehrerInnen/SchülerInnen (in \%)

Abb. 46: Gibt es Ihrer Meinung nach ein besonders gutes Deutsch? (in \%)

Abb. 47: Wo (in welchem Land/in welcher Region) wird besonders gutes Deutsch gesprochen? (in \%) 
Abb. 48: Was wäre eher der Fall, wenn Sie mit einem/er deutschen Gesprächspartner/in sprechen? (in \%)

Abb. 49: LehrerInnen: Wie wirkt in Österreich, in Deutschland und in der Schweiz gesprochenes Standarddeutsch auf Sie?

Abb. 50: LehrerInnen/SchülerInnen: Wie wirkt in Österreich gesprochenes Standarddeutsch auf Sie?

Abb. 51: Österreichisches Deutsch: Das ist das, wo ich mich wohlfühle, es gehört zu meiner Identität. Inwiefern trifft diese Aussage auch auf Sie zu? (in \%)

Abb. 52: Wenn auf Speisekarten österreichischer Restaurants Wörter wie Rinderbraten, Quarktasche oder Schorle stehen - stört Sie das? (in \%)

Abb. 53: Wenn Sie beim Einkaufen auf Produkten Wörter wie Brötchen oder Blumenkohl lesen - stört Sie das? (in \%)

Abb. 54: Wenn ein/e deutsche/r Nachrichtensprecher/in die österreichischen Nachrichten verlesen würde - würde Sie das stören? (in \%)

Abb. 55: Meinen Sie, dass über die deutschen Fernsehsender der Sprachgebrauch in Österreich beeinflusst wird? (in \%)

Abb. 56: Wenn Sie Tschüss hören - stört Sie das? (in \%)

Abb. 57: Austriazismen und Deutschlandismen: korrigiert und unterwellt im Durchschnitt (in \%)

Abb. 58: Deutschlandismen: am häufigsten korrigiert oder unterwellt (in \%) Abb. 59: Austriazismen: am häufigsten korrigiert oder unterwellt (in \%) Abb. 6o: Unterwellt: Unterschiede zwischen den Altersgruppen (in \%) Abb. 61: Unterwellt: Unterschiede zwischen den Schulformen (in \%) Abb. 62: Unterwellt: Unterschiede zwischen den Regionen (in \%) Abb. 63: Korrigiert: Unterschiede zwischen den Regionen (in \%) Abb. 64: Korrigiert: Unterschiede nach Altersgruppen (in \%) Abb. 65: Korrigiert: Unterschiede zwischen den Schulformen (in \%) Abb. 66: LehrerInnen und SchülerInnen wählen mehrheitlich Austriazismus oder gemeindeutschen Ausdruck

Abb. 67: LehrerInnen wählen mehrheitlich Austriazismus oder gemeindeutschen Ausdruck, SchülerInnen Deutschlandismus oder gemeindeutschen Ausdruck Abb. 68: LehrerInnen und SchülerInnen wählen mehrheitlich Deutschlandismus oder gemeindeutschen Ausdruck

Abb. 69: Antwortverhalten LehrerInnen/SchülerInnen

Abb. 70: Am häufigsten bevorzugte Deutschlandismen: LehrerInnen/SchülerInnen (in \%)

Abb. 71: Am häufigsten bevorzugte Austriazismen: LehrerInnen/SchülerInnen (in \%)

Abb. 72: Mündlicher Abschiedsgruß: LehrerInnen/SchülerInnen (in \%)

Abb. 73: Mündlicher Abschiedsgruß „Servus" und „Tschüss": „Generationen“ (in \%) 
Abb. 74: Austriazismen/Deutschlandismen: Unterschiede zwischen den Altersgruppen (in \%)

Abb. 75: Am häufigsten gewählte Deutschlandismen: Unterschiede nach „Generationen" (in \%)

Abb. 76: Am häufigsten gewählte Austriazismen: „Generationsunterschiede“ (in \%)

Abb. 77: LehrerInnen: gewählte Austriazismen und Deutschlandismen - Unterschiede zwischen den Regionen (in \%)

Abb. 78: SchülerInnen: gewählte Austriazismen und Deutschlandismen - Unterschiede zwischen den Regionen (in \%)

Abb. 79: SchülerInnen: TV-Konsum und Präferenz von Deutschlandismen und Austriazismen (in \%)

Abb. 8o: Herkunftssprachen und Varietäten der SchülerInnen (in \%)

Abb. 81: Sprachverwendung LehrerInnen (Selbsteinschätzung) (in \%)

Abb. 82: Dialektverwendung der SchülerInnen in verschiedenen schulischen Situationen (in \%) (nach Einschätzung der LehrerInnen)

Abb. 83: LehrerInnen-Reaktion auf schülerseitigen Dialektgebrauch: „Korrektur/ Hinweis“ oder „lasse gelten“" (in \%)

Abb. 84: LehrerInnen: Wie sprechen Sie mit wem? (in \%)

Abb. 85: SchülerInnen: Wie sprichst du mit wem? (in \%)

Abb. 86: SchülerInnen, die nie/immer Dialekt und Standard sprechen (in \%)

Abb. 87: SchülerInnen: in keiner Situation verwendete Varietäten (nach Region) (in \%)

Abb. 88: SchülerInnen: in allen Situationen (Unterricht, Familie, Freundeskreis) verwendete Varietäten (nach Region) (in \%) 


\section{Sachregister}

A

AHS Allgemeinbildende Höhere Schule $56,63,68-72,83,90,95,99,100,102$, 104, 107, 108, 115, 118, 119, 122, 125, 126, 140, 141, 171, 172, 176, 200, 201, 212, 220, 223, 233, 251

Akzeptanz (von Varianten) 40, 41, 57, 72, $159,160,175,180,187,225$

Alemannisch 17, 21, 31, 35, 44, 49

Alter (Variable), altersspezifisch 14, 22, $36,54,64,66,68,93-96,100,104,105$, $115,120-123,145,170,172,173,190,191$, 194-197, 201, 220, 222, 226, 227, 253, 255,256

ambivalent 146, 162, 180, 221, 224

Amtssprache, nationale, regionale 17-20, 26,41

A-Nation 28, 29

Artefakt (als Sprachkonzept) 162

Asymmetrie (zwischen den Varietäten), asymmetrisch 28, 29, 39, 148, 164

Ausbildung, Ausbildungsinstitution, Ausbildungsort, Ausbildungsabschluss 5 , $11,12,39,59,60,62-64,66,68,73-75$, $89,93,94,97-99,101-104,115,121$, $122,126,131,132,136,140,145,164,212$, 216-220, 222, 223, 226, 228-231, 253

außerordentliche SchülerInnen 64, 94, 108

Ausgleichsvarietät 24

Aussprache, Standardaussprache, Aussprachevarianten 44, 50, 71, 131, 133, 151, 163

Austrian Media Corpus AMC 50, 194, 248

Austriazismus 6, 13, 28, 29, 42, 43, 45, 47, $49,59,77-80,81,82-87,91,92,127,155$, $159,164,165,167-174,179,180,182-197$, 200, 216, 217, 221, 225-227, 241, 253, 255,256

B

BAFEP Bildungsanstalt für Elementarpädagogik; BAKIP Bildungsanstalt für Kinderkartenpädagogik 62, 73, 95, 236 Bairisch 17, 44
Basisdialekt 47

Bayern, Bayrisch 42-44, 49, 143, 206, 246, 250

Befragung 5, 10, 12-14, 30, 32, 34, 47-49, 58, 61, 65-67, 90, 94-96, 114-116, 121, $134,182,186,194,199,214,216,218$, 222, 224, 227, 228

Benrather Linie 17

Beobachtung, teilnehmende 6, 12, 13, 65-68, 90, 116, 118-120, 182, 184, 208, 211, 212, 224, 230

Beobachterparadoxon 118

Beobachtungsraster 120

BHS Berufsbildende Höhere Schule 68, 90, 95, 104, 107, 115, 118, 119, 126, 171-173, 212

Bildungsinstitution 16, 26, 241

Bildungskontext 231

Bildungsministerium BMBF BMBWF 88 , 93, 106, 231, 233, 239

Bildungsstandard 79

Bildungssprache 9, 11, 16, 22, 25, 53, 56, $59,75,87,88,93,102,175,216,222,230$, 231, 239, 241, 243, 244, 248

Bildungssystem, Bildungswesen 16, 222, 223

Binnendeutsch 17, 248

Binnenkodex, Binnenkodifizierung 29, 150

BKS 105,106

BRD (Bundesrepublik Deutschland)

Deutschland 17, 18, 20, 21, 27-29, 31, $34-39,41,43,45,46,49,78,81,82,86$, $89,92,121,132-134,144-146,149,151$, $154,160,162,165,166,174,178,195$, 197, 212, 214, 225, 231, 238, 239, 244, 247-249, 252, 254, 255

Bundesland 10, 13, 52, 56, 64, 68, 78, 90, 93, 94, 96, 97, 109, 111, 113, 115, 117, 118, $123,124,126-128,134,135,137,139,142$, 171, 180, 192, 193, 200, 204, 209-211, 214, 218, 219, 237, 253, 254

Burgenland 56, 94, 97, 104, 117, 128, 131, 171, 179, 180, 193, 196, 199, 200, 202, 204, 211, 213, 215, 227, 235, 237 
C

Chi-Quadrat-Test 66

Code-Switching 195

Curriculum, Lehrplan 5, 10, 11, 16, 22, $48,55,57-59,61,63,64,66,68-75,77$, $85-89,93,181,214-217,222,223,228$, 230, 232, 233, 244, 249

\section{D}

DDR (Deutsche Demokratische Republik) 26, 35

deskriptiv (im Gegensatz zu präskriptiv)/ Statistik 23, 37, 39, 54, 66, 94, 240, 246, 249, 260

De-Standardisierung 24

Deutsch als Erstsprache 15

DaM Deutsch als Muttersprache 12, 58-60, 64, 76, 89, 223, 242, 257

DaZ Deutsch als Zweitsprache 12, 58, 59, $61,69,72,93,101,102,108,222,223$, 233, 242, 257

Deutsch in Österreich DiÖ 51

Deutsche Sprache 5, 16-18, 20, 22, 26, 27, $38,58,107,138,139,218,219,222,238$, 241, 243, 245, 249, 250, 252

Deutsches Deutsch, Deutsches Standarddeutsch 13, 84, 92, 127, 145-147, 150, $163,168,195,254$

DeutschdidaktikerInnen 229

deutschländisch, deutschländisches Deutsch 129, 146, 151, 1532, 153, 163, $165,169,188,198,218,226$

Deutschlandismus 6, 28, 47, 59, 77, $78,80-87,91,92,109,155,157,159$, $163-165,167,168,170-173,179,180$, $182-194,196,198,200,216,217,220$, $221,225-227,241,253,255,256$

DeutschlehrerInnen, Deutschlehrkräfte, Deutschlehrende 5, 56, 59, 62, 73, 75, $76,88,89,94,100,101,148,164,169$, $171,175,176,179,180,195,212,217,222$, 223, 225, 228-230, 245, 252

Deutschlehrplan 62, 64, 69, 71-73

Deutsch-Lehrwerke, -Lehrbücher, -Lehrwerkserie 5, 10, 12, 68, 76, 223, 228

deutschsprachig 5,14-22, 26-28, 31, 33, $35-38,41,42,45,59,81,85,86,89-91$, $107,108,132,136,229,238$
Deutschunterricht $6,9,11,14,49,56,58$, $63,64,88,92-94,99,108,164,175,180$, 214, 222, 229-231, 240, 242, 245, 246, 249

Dialekt, siehe auch Mundart, dialektal 6 , $14-18,20,22,24,30,31,33,35,36,42$, $44-49,51-53,55,56,60,62,63,69,71$, $73-75,77-88,92-94,99,101,103,106$, $116,119,120,122-132,136,143,144,150$, $151,153,160,163,169,175,180-182,196$, 198-216, 219-221, 224-231, 238, 239, 243-246, 249, 251-254, 256

Dialektabbau, Dialektschwund 24, 18 Dialektologie, dialektologisch 31, 43, 46 Dialektgleichsetzung 9, 151, 163 dialektnahe 24

Dialekt-Standard-Kontinuum 18-21, 44, $48,51,129,150,206-208,212,227-229$

Dialektgebrauch 18, 19, 51, 205, 206, 256 diastratisch 22

diatopisch 22, 24

didaktische Grundsätze 70-73

Dienstjahr 64, 96, 100

Diglossie, diglossal 14, 16, 19, 24, 44, 47, $48,127,211,243$

Diskurs 65, 75, 228, 230, 241

diskursanalytisch $32,60,67$

diskursiv 16, 45, 65, 161, 196, 240, 241

Diversität 29

D-Nation 28, 29

Dokumentenanalyse $5,50,65,68,88,116$, $128,132,216,217,222,225,230$

Doublette 82, 92, 182-186, 188, 194, 199, 226

Duden 33, 43, 44, 50, 55, 59, 77, 100, 101, $103,113,114,165,178,179,217,223,242$, 253

E

eigennational 164, 179, 193

Eigenvariante 31

Eloquenz 151-153, 163

endonormativ 27

Erstsprache 11, 15, 16, 56, 64, 71, 73, 94, 106, 115, 119, 121, 145, 239

exonormativ, exonorm-orientiert 13,27 ,

$59,64,90,91,113,164,217,222,225,228$ 
ExpertInnenbefragung,

ExpertInneneinschätzung,

ExpertInnen-Interview 5, 14, 16, 58, 60,61

extralinguistische, außersprachliche

Variable, Faktor 13, 22, 40, 64, 99, 201, 222

$\mathrm{F}$

Fachdidaktik 9, 58, 229, 248

Fächerkombination 64, 97, 99, 121

Fachsprache 22, 81, 82, 87

Fehler, fehlerhaft, fehlerfrei 30, 70, 85, 92, $139,165,167,174,217,225,226$

formell, informell 16, 19, 21, 23, 24, 35, 48, $51,56,120,127,133-135,207,254$

Fragebogen, Fragebogenerhebung 6, 10, $12,13,30,43,45,50,51,65-68,89-92$, 94, 99, 103, 104, 115, 116, 118, 120, 121, $128,129,138,143,145,148,149,160$, $162,163,165,173,174,181,182,199,204$, 206-208, 212, 216, 223, 235, 253

fremdnational 59, 164, 231

Fremdsprache 5, 11, 12, 20, 29, 30, 44, 58, 99, 108, 114, 118, 138, 231, 240, 242, 244, $245,248,249,251$

Fugenmorphem 166, 183

\section{G}

Gebrauchsstandard 35, 37, 42, 47, 239

Geburtsort 93, 104

Gemeindeutsch 28, 164, 165, 169, $182-186,188,189,255$

Gemeinsprache 22, 41

Gender, genderspezifisch 22, 64

Generation, generationsspezifisch 14, 34, 51, 108, 117, 131, 158, 190-193, 195, 197, 222, 226, 227, 241, 255, 256

Germanismus 28

Germanizismus 28

Geschlecht 22, 66, 67, 93, 94, 104, 114, 123, $124,134,140-142,145,212$

gesprächsanalytisch 67

Grammatik, grammatikalisch 22, 23, 29, $36,37,41,42,50,55,77,87,93,101,132$, $133,148,151,163,166,174,179,217,242$, 245

Grenzfall des Standards 24, 30, 36, 82, $164,168,179,217$ großdeutsch 40

Grundschule siehe Volksschule

Gruppendiskussion 6, 10, 12, 65-68, 91, 116-118, 127, 128, 147, 148, 150-152, 160-164, 175, 180, 181, 194-197, 203, $205,206,215,217,224,225,227,237,240$

Gymnasium, gymnasial, siehe auch AHS $63,64,72,117,235,236,240$

$\mathrm{H}$

Halbzentrum 17, 26, 27

Hauptschule 63, 64, 69, 74, 95, 115, 233

Hauptschul-Curriculum 74

Helvetismus 28, 29, 59, 77-79, 81, 82, $84-87,164,179$

Herkunftssprache 11, 57, 70, 71, 74, 93, 99, 196, 199, 200, 256

Hesitation 129, 130, 152

HIAT Halbinterpretative Arbeitstranskription 116, 236, 237, 249

Hochdeutsch, hochdeutsch 11, 12, 17-19, $25,30,39,45,46,51-53,56,91,92,108$, 127-129, 136-138, 145-147, 202-204, 223, 254

Hochsprache, hochsprachlich 16, 22-24, $34,51-53,56,60,79,128,129,180,202$, 224, 246, 248

I

Identität, Sprache $6,60,62,72,92,139$, 155-161, 163, 164, 198, 221, 222, 225, 241, 246, 248, 255

Identität, nationale $13,19,21,33,34,38$, $87,91,116,196,239-241,244,252$

Ideologie 32, 33, 35, 40, 246

inferenzstatistisch 49

informell siehe formell

innere Mehrsprachigkeit 5, 15, 60, 72, 74, 130, 227, 232

integrativer Sprachunterricht 88, 93, 102, 241

interkulturell, interkulturelles Lernen 118, 239

Internet, Internetnutzung $39,75,78,93$, $113,114,215,223$

Interview, Interviewerhebung, Leitfadeninterview, Einzelinterview 6, 9, 10, 12, 13, 57-60, 62, 65-68, 90, 102, 115-116, $118,127,128,131,143,144,152,153,155$, 
$156,163,174,175,178-181,194,195,198$, 199, 202, 206, 208, 211-213, 215, 217, $224,225,227,237$

Isoglosse 17,31

\section{J}

Jugendsprache 22, 80-82, 87, 93, 99

\section{K}

Kabel-TV 87, 109, 111, 195

Kärnten 39, 56, 57, 74, 75, 94, 96, 97, 104, $115,117,119,123,127,193,194,204,210$, 211, 227, 233, 235, 237

Kinderliteratur, Kinderbuch 170, 109, 112 Kodex, Sprachkodex 12, 13, 23, 29, 50, 53, $54,60,72,77,88,100,178,179,182,187$, 217, 222, 223, 228

Kodifizierung, kodifiziert 13, 23-25, 29, $35,41,46,47,50,55,60,77,88,130,175$, $178,225,250$

kognitiv 16, 45, 46, 224

Kommunikation, schriftlich, mündlich 6, 21, 52, 70, 116, 131, 164, 180, 199, 204, 207, 208, 227, 250

Kommunikationsradius, Kommunikationsreichweite, kommunikative Reichweite, Kommunikationssituation, Kommunikationsverhalten 23, 24, 57, 120, 132, 180, 182, 201,

kommunikative Kompetenz 23, 55, 244, kommunikative Sprachnorm, Angemessenheit 54-56, 218

kommunikative Validierung 116

Konsensualnation 38

Konsonant 84

Kontrollfrage 141, 145, 146, 162

Konzeptualisierung 5, 6, 11-15, 25-46, 48, $55,58,63,69,72-75,84,87,89-91,94$, $116,121-144,217,219,230$

Korpus 36, 37, 50, 65

Korpusplanung 49

Korpuslinguistik, korpuslinguistisch 35, 94, 186, 248

Korrektheit 6, 53, 92, 145-152, 162, 175, 221, 224, 225

Korrekturarbeit 93, 100, 101, 222

Korrekturverhalten 6, 12, 13, 59, 61, 91, $115,116,164-180,187,225,243$
Korrekturvorschläge 168, 169, 253

KPH Katholische Pädagogische Hochschule 74, 75, 233

Kruskal-Wallis-Test 66, 94

Kulturbürokratie 33, 41

Kurdisch 105, 106, 108, 117

L

LaiInnen 14, 36, 49, 133, 141, 165, 219, 222

länderspezifisch 63

Lehramtsstudierende 75

Lehramtsstudienplan, Lehramtscurriculum 63,75

Lehramtsausbildung, LehrerInnenausbildung 11, 63, 64, 66, $68,73,74,97,101,102,131,132,219,220$, 222, 223, 229, 230

Lehrplan, Curriculum 5, 10, 11, 13, 16, 22, $48,55-59,61-64,66,68,69-73,74,75$, $77,85-89,93,181,214-217,222,223$, 228, 230, 232, 233, 244, 249

Lehrbuch, Lehrbuchserie, LehrerInnenhandbuch, Lehrwerk 5, $10-13,32,48,58,59,61-63,65,66,68$, $69,76-86,87-89,93,101,132,187,215$, $217,218,223,231,232,234,244,248$

Lehrmaterialien $16,22,63,215,216$

Lehrveranstaltung $64,66,68,69,75,89$, 93, 101, 103, 230, 231

Lehrziel 69,72

Leitfadeninterview siehe Interview

Lektor, Lektorin 23, 53

Letzeburgisch 17,19

Lexikographie 46, 139, 227

Lexikon 42, 49, 238, 239

Liechtenstein 17, 19, 26, 27, 33, 36, 49, 239

linguistic cringe 29, 39

Loyalität (in Bezug auf eigene Varietät), Sprachloyalität, Variantenloyalität 13 , $29-31,45,59,60,64,90,145,149,163$, $187,193,194,221,226,227,244$

Luxemburg 17, 19, 26, 27, 36, 105, 239

M

Mann-Whitney-U-Test 66

Markierung 24

Matura 76, 78, 80, 82, 84, 86, 181, 215, 234

Mediensprache $87,125,127,144,219,220$, 226 
Medienkonsum 109, 187

Medienverhalten 93, 195, 227

Mehrheitssprache 6, 15, 33, 121, 122, 124, 144, 220, 254

Mehrsprachigkeit

- innere, innersprachliche siehe innere Mehrsprachigkeit

- äußere 15, 72

- lebensweltliche 15

Minderheitensprache, Sprachminderheit $15,18,20,108$

Minderwertigkeitskomplex, -gefühl 9 , $30,60,62,145,148,162,213,218,225$

Mitteldeutsch 17, 18, 35, 43, 44

Mittelwertsvergleich 67

Mobilität 51

Modell, Modellierung, modelliert 14-16, $22,24-27,32,37,38,46-48,51,89$

Modellsprecher 23, 55,

Modelltext 23, 55

monozentrisch $12,17,25,38,61,64,71$, $76,80,82,87,88,121,137$

Morphologie, morphologisch 17, 49, 54, 77, 165, 251

Mundart, siehe auch Dialekt 19, 24, 70, $73,78,79,81,82,85,87,115,122-124$, $127,169,220,252,254$

Muttersprache $9,11,12,17,31,33,56,58$, 59, 64, 70, 88, 91, 94, 104-108, 111, 115, $117-123,127,134,136,138-142,216,222$, $233,244,245,248,253$

\section{$\mathrm{N}$}

Nachschlagwerk 12, 21, 23, 27, 55, 61, 63, $69,72,88,93,101,113,114,178,225,253$

Nationalbewusstsein $34,38,148$

Nationalsprache 19, 23, 85

Nationalstaat 26, 38, 47

Nationalvarietät, nationale Varietät, Variante 27, 28, 31, 35, 36, 46, 62, 75, $86,152,164,231,243,244,247,248$

Niederdeutsch 17,18

Niederösterreich 94, 96, 97, 117, 119, 152, 153, 171, 193, 195, 198, 200, 204, 208, 211, 227, 235, 237

NMS Neue Mittelschule 69-71, 90, 95, 98-100, 102, 108, 122, 126, 140, 141, 171-173, 180, 200, 201, 212, 225, 226, 235, 236
Non-Standard (auch Nonstandard), Nonstandard-Varietät 28, 36, 82, 53, $54,74,81,86,88,117,128,212,227$

Norddeutschland 24

Normauffassung, Normerwartung, Normverständnis, Normvorstellung $45,55-57,59,63,116,169,180,216,218$, 247,248

Normautorität, Sprach- 14, 23, 29, 55, 145 , 217,240

normgerecht $28,70,79,86,180$

Normkonzept $11,53,77,88,89,176$

normsetzende Instanz 23, 55, 57, 131, 175,

$177-179,193,217,228$

Normtoleranz 29, 57, 59

Normverletzung 45, 167, 179, 217

$\mathrm{O}$

Oberdeutsch 17, 18, 49, 81, 86

Oberösterreich $56,74,75,94,97,117,123$, $127,174,193,204,208,210,211,227,233$, 235, 237

Oberstufe, gymnasiale, siehe auch Sekundarstufe II 63, 69, 72, 90, 95, 104, 214, 251

ORF Österreichischer Rundfunk 39, 44, 50, 109, 111, 122, 246

Orthographie 77,87

Ostbelgien 17, 20, 27, 36, 239

Ostösterreich, ostösterreichisch - siehe Region Ostösterreich

Österreichisch (Sprache) 12, 82, 106, 122-124, 246

Österreichisches Standarddeutsch 92, 129, 137-143, 145, 208, 218, 223-225, 254

Österreichische Standardsprache 72, 73, 80,213

Österreichisches Wörterbuch (ÖWB) 21, $33,43,49,50,59-61,100,101,103,113$, $114,139,161,165,182,215-217,219$

Ostmitteldeutsch 17

Ost-West-Unterschiede 3, 143, 230

$\mathrm{P}$

Pädagogische Hochschule PH, 64, 74, 89, 94, 97, 116, 118, 122, 123, 126, 136, 140, 212, 220, 233, 235 
Pädak Pädagogische Akademie 94, 97, $136,140,220$

Perfektbildung 166, 183

Perfekt als Erzählzeit 83, 85, 170, 174, 176, 217

Peripherie 17

Perspektive 6, 42, 65, 132

pluriareal $5,12,14,15,17,22,25,31-38$, $40-42,46,64,81,84,87-89,227,243$, 247,250

Pluriarealität $5,11,16,32-40,42,46,84$, $86,139,219,238$

plurinational $14,17,27,35-38,42,87$,

Plurizentrik 5, 9, 10, 16, 25-32, 37, 39, 45, $46,59,63,69,70,73,75,81,82,85,88$, $89,94,99,100,103,121,122,139,140$, $218,219,222-224,227,231,245,248$, 249

Plurizentrizität $28,32,35,42,84,87,238$, 242

plurizentrisch $5,6,9,12,14,15,17,22$, $25-28,31-35,37,38,40-44,46,47$, $58-62,64,70,75,76,79-82,85-89$, 93, 94, 99, 101, 104, 114, 121, 132, 135, $136-145,165,214,218,219,222,223$, 227-229, 231, 232, 240, 246-248, 250, 253

Polaritätsprofil 6, 92, 153, 163

polyzentrisch 25

Prädikation 127

Präferenz 6, 178, 197, 182-199, 205, 256,

Pragmatik 49, 54, 75, 132, 133

präskriptiv (im Gegensatz zu deskriptiv)

23, 41, 54, 56, 178

Präteritum als Erzählzeit 81, 83, 85, 86, 174-177

Prestige (Sprach-) 10, 16, 29, 35, 45, 56

Protokoll Nr. 10 20, 21, 87, 240

\section{Q}

qualitativ $43,65,67,115,116,121,143$, $222-225,243,245,246,252$

quantitativ $43,65-67,121,143,222,224$, $225,243,246$

Quellenstudium 67

\section{$\mathrm{R}$}

Radiokonsum 64, 94

Rechtschreiben 70, 72, 79
Referenzwerk 55, 60, 77, 165, 179

Region Mitte (Österreich) 36, 49, 94, 97, $110,114,123,135,137,138,140,143,171$, 172, 193, 194, 227

Region Ostösterreich, ostösterreichisch 28, 49, 52, 109, 111, 137, 139, 144, 171, 210, 220

Region Südostösterreich 36, 49, 94, 97 , $110,114,123,137,138,140,172,1903$, 194, 227

Region Westösterreich, westösterreichisch 12, 21, 33, 36, 49, 92, 135, 136, 139, 140, 143, 144, 182, 183, 193, 199, 200, 227, 254

Regionalsprache, regionalsprachlich 18 , 24, 218

regionale Plurizentrik, Plurizentrizität 32, 139, 219, 224, 227

regionaler Standard, Gebrauchsstandard $35,42,75,239,250,251$

regionale Umgangssprache 70

regionale Varietät, Variation, Varianz 24, $28,35,46,48,78-80,84,165,245,248$, 252

Register, sprachlich $16,22,48,72,78,150$, 181, 206, 208, 242

Relativierung $152,174,180$

Romanes 106

\section{$\mathrm{S}$}

Salzburg 49, 53, 56, 74, 75, 94, 97, 104, 116, $117,123,127,131,152,174,178,181,193$, 204, 208, 210, 211, 213, 215, 227, 233, 234 235, 237, 246

Satz(ab)bruch 129, 130, 152, 202

Schibboleth 44

schichtspezifisch 18, 22, 52 .

Schizophrenie, sprachliche 30, 246

Schreibnorm 71, 72

Schreibrichtigkeit $71,72,85$

Schriftsprache 22

Schülertext 93, 131, 164, 179

Schulform 93-95, 100, 120, 122, 126, 140, $141,145,170-173,201,212,219,223,225$, 226, 253, 255

Schulstandort 104, 105, 235, 253, 255

Schulstatistik 104

Schultyp $61,64,68,89,90,94,95,122$, 212, 200, 220, 222 
Schweizerdeutsch 19, 30

Schweizer Standarddeutsch, Schweizerhochdeutsch $6,12,16,19,30,84,153$, 230,

Sekundarstufe I, II 12, 63, 65, 68, 69, $71-73,76,80,82,83,90,97,176,234$, 243, 244,

Selbsteinschätzung 151, 194, 201, 208, 224, 230, 256

Selbstwahrnehmung 65

Semantik 49

situative Norm 55, 57, 80, 176, 218, 229

Slowakisch 21, 105, 106, 109

Slowenisch 21, 105, 106, 108, 109, 118, 119, 211

sozial (Schicht, Herkunft, Status) 19, 51-53,

sozial erwünscht 145, 202, 206,

soziale Norm 18, 53-55, 132, 242

Sozialisation $45,161,162$

Soziolekt 22, 82, 83, 213

spezifische Variante (Austriazismus,

Deutschlandismus) 20, 28, 37, 42, 45, 49, 77-84, 164, 165, 182, 183

Sprachauffassung 57,63,69, 244

Sprachbewusstsein $30,45,52,62,69,78$, 198, 230

Sprachbiographie 116, 118

Sprachebene 49,81

Spracheinstellung 6, 9, 12, 13, 30, 36, 43-45, 58-6o, 64, 90, 94-98, 115, $145-164,222,228,241,246$,

Sprachempfinden 9, 147, 225

SprachexpertInnen 23, 55, 165, 187

sprachextern - extralinguistisch 22, 94, 104, 122

Sprachgemeinschaft $16,31,54,55$

Sprachidentität 139,161

Sprachkenntnisse 118

Sprachkodex 23, 29

Sprachkonzept 63

Sprachloyalität siehe Loyalität 13, 30, 31, $45,59,60,64,90,145,244$

Sprachnation 33,40

Sprachnorm, Sprachnormautorität, SprachnormexpertInnen 5, 14, 15, 23, 29, 53-56, 64, 65, 71, 131, 143, 145, 217, 222, 224, 229, 238-240, 246

Sprach/en/politik 49, 240, 241, 247, 248
Sprach/en/unterricht 5, 9, 15, 23, 53, 56,

$57,93,102,229,247$,

Sprachreflexion $62,69,72,87,231$

Sprachrepertoire 106, 109, 121

Sprachrichtigkeit, sprachrichtig 13 ,

$71-73,79,86,88,93,100,166,167,217$

Sprachvariation, sprachliche Variation 9,

$11,12,15,16,22,42,46,47,51,61-64$,

$69,74,76,78,79,81,84,93,99,102$,

$103,132,214,217,222,223,228,229,231$,

240, 246, 249, 253,

Sprachverwendung 6, 13, 44, 48, 57, 118, $12 \mathrm{O}, 182,186,194,201,203,206,208$,

211, 224, 226, 230, 256

Sprachwandel 14, 190, 193, 220, 226, 227

Sprachwissen 63,164

Sprechstandard 18, 35, 48

SPSS Statistical Package for Social

Sciences 66,67

Staatsnation, staatsnational $33,38,39$

staatsspezifische Variation $27,38,59,62$,

$63,69,72,73,75,78,82,85,222,231$

Standardaussprache 41,246

Standarddeutsch 5, 6, 12, 13, 16, 18, 19, $25,29,30,33,41,47,50,60,62,64,83$,

$84,92,94,99,120,121,125,127,129$,

$137-143,145,146,150,153,154,162,163$,

171, 176, 182, 199-201, 207-211, 216,

218-220, 222-225, 230, 242, 244, 245,

250, 254, 255

Standardisierung 25-27, 251

Standardorientiertheit 123, 126, 211

Standardsprache 10-12, 16-19, 21-26, $30,32,35,36,39,41,43-48,51-53$, $55-57,59,63,70-75,78-88,91,93,101$, $119,120,125,128-131,136,137,139,141$, $142,151-153,163,180-182,200-203$, 206-209, 211-213, 215, 218, 220, 224, 226-231, 239, 240, 243-247, 249, 250, 252

Standardvariante 198

Standardvarietät $18,19,22,23,27,30,35$, $36,47,48,53,59,60,78,82,83,85,88$, $141,143,150,198,224,228,230$

Standardvariation $11,63,69,116,243,245$, 246

Status, sprachlicher $16,17,20,27,28,42$, $45,53,55,58,84,248$ 
Steiermark 56, 74, 75, 88, 94, 97, 104, 117, 123, 127, 128, 152, 174, 179, 181, 193, 199, 203, 204, 210, 211, 214, 227, 233, 235, 237

Stereotyp 29

Stichprobe 6, 67, 94, 96, 104

Stigmatisierung 38

Studienfach 93, 94, 212

Studienplan 5, 10, 11, 48, 58, 60, 62-64, $66-69,73-75,79,86,88,89,223$, $228-230,233,234$

Substandard 22, 24, 30, 42, 59, 198

Süddeutschland 24, 33, 49, 81, 92, 142, 143,254

Südostösterreich 49,1172 - siehe auch Region Südostösterreich

Südtirol 17-19, 26, 27, 33, 36, 49, 51, 239, 245,251

systemlinguistische Norm 54, 56

Syntax 42, 49, 54

\section{T}

teilnehmende (Unterrichts-) Beobachtung $6,67,68,118,119,224$

Testverfahren, statistisch $66,67,94$

Teutonismus 28, 29, 43, 87, 247

Textsorte, textsortenabhängig, -spezifisch, -abhängig, -bezogen $33,57,168$, $169,174,176,249$

Tirol 9, 36, 49, 56, 74, 75, 94, 97, 104, $105,109-114,117,123,124,127,130,131$, $135-138,140,142-144,152,172,174,178$, $181,193,194,200,202,204,208-211$, $213-215,218-220,227,233,236,237$

Transkription, Transkript 118, 230, 237

Triangulation, Daten-, Methoden- 64, 67

Triglossie 16

Tschechisch 21, 105, 106, 108, 109, 119

T-Test für unabhängige Stichproben 67 , 94

TV, TV/Fernseh-Gewohnheiten, -Kanal 64, 87, 91, 94, 109-113, 125-128, 195, $196,219,226,253,256$

Türkisch 21, 105, 106, 109, 117-119

Turnwechsel 129
U

überregional 18, 19, 23, 24, 30, 47, 52, 53, $92,131,141,142,219,223,254$

Umgangssprache, umgangssprachlich 6 , $12,14,16,17,20-22,24,25,35,43,47$, $48,51,52,55-57,62,63,69-71,73-75$, $80-84,86-88,92,93,99,101,103,116$, 119, 120, 125-131, 136, 144, 151, 164, 169, 175, 181, 182, 186, 198, 199, 201-204, 206-209, 211, 212, 216, 219, 220, 224-228, 230, 231, 244, 249, 253

Ungarisch 21, 106, 108, 109

Universität, Universitätsausbildung, -absolventInnen, -lehrende, -studium $11,45,58,59,62-65,68,73-75,94,97$, $115,123,126,136,140,196,212,218-220$, 226, 234,

Unterbrechung 129

unspezifische Variante (Austriazismus, Deutschlandismus) 28, 49, 77, 778, $79-82,84,86,165,182$

Unterrichtserfahrung 93, 116

Unterrichtsfach $68,93,97,98,118,203$, 204, 253

Unterrichtssprache $5,20,56,58,60,102$ Unterrichtskommunikation 199, 227, 251 Unterrichtssituation 93, 182, 201, 205, 206 Untersuchungsdesign 5, 62, 64

V

Variable $13,22,27,28,40,44,51,64,66$, $67,94,99,104,111,126,132,172,201$, 204

Variante, spezifisch, unspezifisch 9, 22, $26-28,30,31,35-37,42,45,46,48,49$, $51,52,59,77-81,83,84,86,92,129$, 164-166, 169, 171, 174, 179, 182, 183, 185-188, 191, 193-195, 198, 213, 214, 225-227, 231, 242, 243, 246, 248, 250, 253

Variantengrammatik $36,37,414,42,50$, 242

Variantenloyalität 31, 45, 187, 193, 194, 227

Variantenwörterbuch VWB 17, 32, 36, 42, $43,49,77,88,165,168,182,199,223$, 238, 239

Varianzanalyse 67 
Variation, sprachliche 3, 5, 6, 9-12, $14-16,18,20,22,24,25,32,34-38$, $40-42,46,47,49,50,55,58-64,69$, $72-79,81,82,84,85,87-91,93,94$, 99, 101-103, 115, 116, 118, 121, 128, 129, $131,132,141,144,145,194,198,121,214$, $216,217,222-224,228-231,238-243$, 245-247, 249-253

Varietätenbewusstsein 11, 206, 223

Varietätengebrauch 6, 199, 200, 209, 241

Varietätenloyalität 31

Varietätenspektrum, Variationsspektrum $25,48,220,229$

Varietätenverständnis 65

Verkehrsdialekt 47

Viertelzentrum 26, 27

Vollzentrum 17, 27

Volksschule 12, 56, 63, 65, 69, 70, 73, 74, $77,79,95,108,115,162,171-173,176$, 215, 233-235

Vorarlberg 21, 33, 36, 44, 45, 49, 56, 94, 97, 104, 110, 113, 114, 117, 123, 127, 135, 137, 138, 140, 142-144, 171, 180, 193, 195, 200, 204, 209-211, 214, 220, 227, 236, 237
W

Wahlpflichtfach Deutsch 69, 72, 233

Weiterbildung 11, 231

Westösterreich, westösterreichisch - siehe auch Region Westösterreich 12, 21, 33, $36,49,92,135,136,139,140,143,144$, $182,183,193,199,200,227,254$

Wien 10, 39, 52, 53, 56, 58, 74, 75, 80, 94, 97, 104, 115-117, 119, 144, 153, 171, 174, 193-195, 200, 202, 204, 207, 211, 215, $227,233,234,236,237$

Wohnregion 104

Wortabbruch 131

Wortatlas der deutschen Umgangssprachen 84

Wortschatz $18,19,49,77,79,80,84,87$, 131-133

\section{Z}

Zentrum, sprachliches 22, 26, 27, 29, 165

Zweifelsfall 59, 77, 93, 175, 179, 225, 226, 228, 250, 252

Zweisprachigkeit, zweisprachig 16, 18, 71, $108,117,119,211,216,241$

Zweitsprache 11, 12, 21, 56, 71, 88, 93, 101, 233, 251, 252 